Portland State University

PDXScholar

Dissertations and Theses

Dissertations and Theses

Winter 3-16-2017

\title{
An AFM-SIMS Nano Tomography Acquisition System
}

Richard William Swinford

Portland State University

Follow this and additional works at: https://pdxscholar.library.pdx.edu/open_access_etds

Part of the Physics Commons

Let us know how access to this document benefits you.

Recommended Citation

Swinford, Richard William, "An AFM-SIMS Nano Tomography Acquisition System" (2017). Dissertations and Theses. Paper 3485.

https://doi.org/10.15760/etd.5369

This Dissertation is brought to you for free and open access. It has been accepted for inclusion in Dissertations and Theses by an authorized administrator of PDXScholar. Please contact us if we can make this document more accessible: pdxscholar@pdx.edu. 
An AFM-SIMS Nano Tomography Acquisition System

by

Richard William Swinford

A dissertation submitted in partial fulfillment of the requirements for the degree of

Doctor of Philosophy

in

Applied Physics

Dissertation Committee:

Erik J. Sánchez, Chair

John Freeouf

Derek Nowak

Rajendra Solanki

Dean Atkinson

Portland State University

2017 
(C) 2017 Richard William Swinford 


\begin{abstract}
An instrument, adding the capability to measure 3D volumetric chemical composition, has been constructed by me as a member of the Sánchez Nano Laboratory. The laboratory's in situ atomic force microscope (AFM) and secondary ion mass spectrometry systems (SIMS) are functional and integrated as one instrument. The SIMS utilizes a Ga focused ion beam (FIB) combined with a quadrupole mass analyzer. The AFM is comprised of a 6-axis stage, three coarse axes and three fine. The coarse stage is used for placing the AFM tip anywhere inside a $\left(13 \times 13 \times 5 \mathrm{~mm}^{3}\right)(\mathrm{xyz})$ volume. Thus the tip can be moved in and out of the FIB processing region with ease. The planned range for the Z-axis piezo was $60 \mu \mathrm{m}$, but was reduced after it was damaged from arc events. The repaired Z-axis piezo is now operated at a smaller nominal range of $18 \mu \mathrm{m}(16.7 \mu \mathrm{m}$ after pre-loading), still quite respectable for an AFM. The noise floor of the AFM is approximately $0.4 \mathrm{~nm} \mathrm{R} \mathrm{R}_{\mathrm{q}}$. The voxel size for the combined instrument is targeted at 50 $\mathrm{nm}$ or larger. Thus $0.4 \mathrm{~nm}$ of xyz uncertainty is acceptable. The instrument has been used for analyzing samples using FIB beam currents of $250 \mathrm{pA}$ and $5.75 \mathrm{nA}$. Coarse tip approaches can take a long time so an abbreviated technique is employed. Because of the relatively long thro of the $\mathrm{Z}$ piezo, the tip can be disengaged by deactivating the servo PID. Once disengaged, it can be moved laterally out of the way of the FIB-SIMS using the coarse stage. This instrument has been used to acquire volumetric data on AlTiC using AFM tip diameters of $18.9 \mathrm{~nm}$ and $30.6 \mathrm{~nm}$. Acquisition times are very long, requiring multiple days to acquire a 50-image stack. New features to be added include auto stigmation, auto beam shift, more software automation, etc. Longer term upgrades
\end{abstract}


to include a new lower voltage Z-piezo with strain-gauge feedback and a new design to extend the life for the coarse XY nano-positioners. This AFM-SIMS instrument, as constructed, has proven to be a great proof of concept vehicle. In the future it will be used to analyze micro fossils and it will also be used as a part of an intensive teaching curriculum. 
This work is dedicated to my family, Leslie and Carl. 


\section{ACKNOWLEDGMENTS}

I have spent many years working on the individual components of the instrument described in this document. I have constructed most of the components and software myself although leveraging existing designs where possible This has been a very fulfilling experience in that constructing many of the pieces of this machine pushed me outside my comfort zone and forced me to learn things I may never have otherwise.

During the writing of this dissertation, it was more difficult deciding what to leave out than what to include. Over the course of working in the Sánchez Nano Development Laboratory at PSU, I worked on many other experiments and instruments which are not discussed in this dissertation. I started on this PhD after having a decent 9-year career start as a software engineer. While software engineering can be very rewarding both intellectually and monetarily, I missed working on actual hardware and on Physics.

At my job I have had access to some of the world experts in the field of charged particle optics and Software/Systems Engineering so that I could make repairs and modifications on the machine that is the topic of this work. I am thankful that my advisor agreed to take me on as a non-traditional graduate student, working a full-time job while attending PSU part-time. He was a non-traditional student himself, having worked as an engineer at a distant place of work.

I would like to thank these organizations; Sunstone, National Instruments, PSU, and the NSF. The author is indebted to David Tuggle, Dustin Laur, Tom Brandt, Patrick McBride, Mark Utlaut, Justin Zimmerman, Brad Larson, Chris Edwards, Reinier Warshauer, Judy Green, Dan Cook, Steven Linerooth, Chris Sanak, Greg Schwind, 
Mostafa Maazouz, Bob Lieb, Jim McGinn, Philip Witham, Fredrick DeArmond, A.J. Lawrence, and Jeff Doughty, Chris Halseth, Alex Challey and my advisor, Erik Sanchez for helping to make this work possible.

Bob Lee from R5D3 and Mike Carter from Surplus Gizmos were instrumental in helping me find some of the refurbished equipment which was used to construct this AFM-SIMS acquisition system.

Finally, I would like to thank FEI Company, now Thermo Fisher Scientific, for providing me with continuous employment, for enabling my pursuit of this dream, access to tools during off-hours, work schedule flexibility, and for reimbursing a large portion of my tuition while I pursued this $\mathrm{PhD}$. 


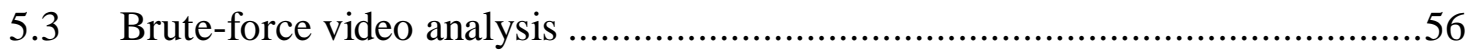

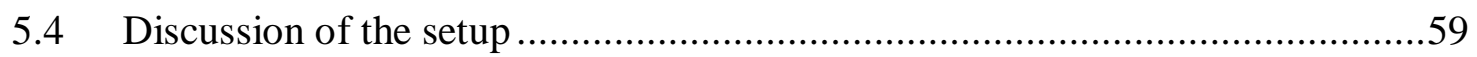

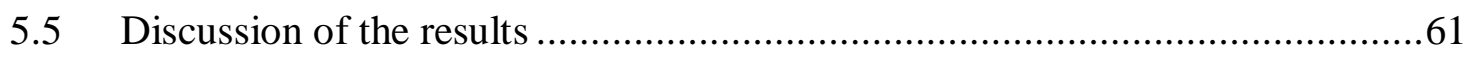

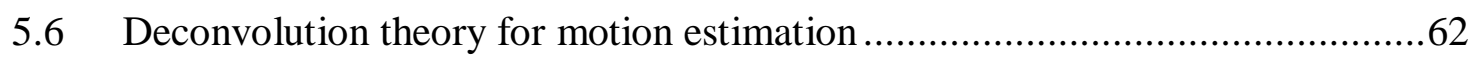

5.7 Uniform linear motion removal from an image in the time domain ..................66

5.8 Practical usage of the frequency domain deconvolution formulas ....................70

5.9 Examples of images with motion blur and its removal ...................................72

5.10 Estimating complex motion blur by deconvolution ........................................75

5.11 Demonstration of the tuning parameter on deconvolution performance …….....78

5.12 Deconvolution technique for estimation of motion from single images ............80

5.13 Discussion of deconvolution as applied to motion estimation...........................83

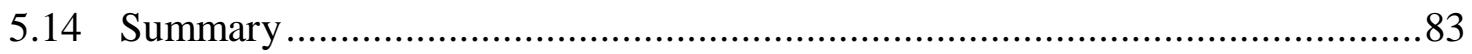

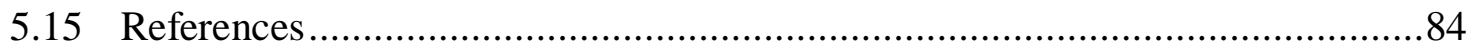

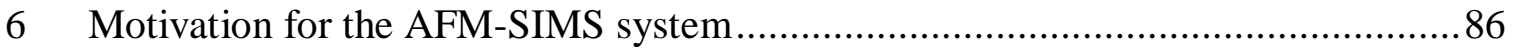

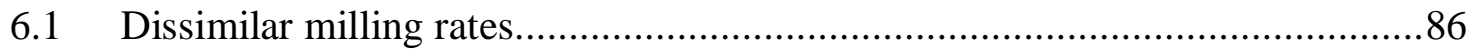

6.2 More details about a common sample - AlTiC ……….................................93

6.3 SRIM predictions for components of AITiC ……........................................97

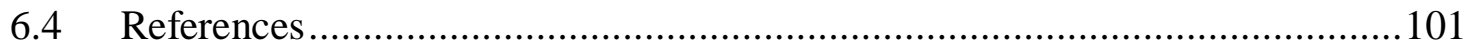

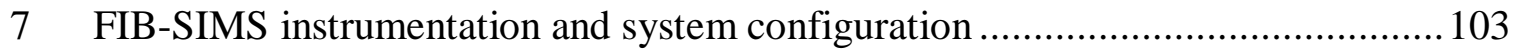

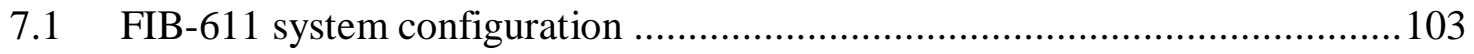

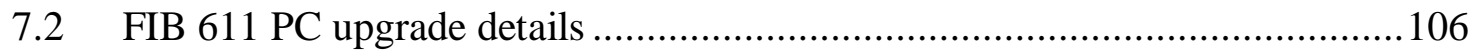

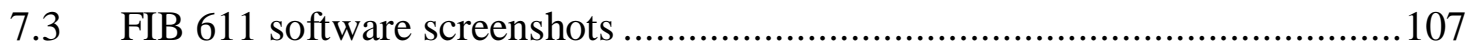

7.4 FIB modifications for AFM integration ……............................................... 108

7.5 FIB system enhancements: Magnum FIB column ........................................112

7.6 FIB system enhancements: Auto focus software ……................................117

7.7 Future enhancements to AutoLibControlApp.exe ……..................................123

7.8 FIB system enhancements: Stage control software ........................................123

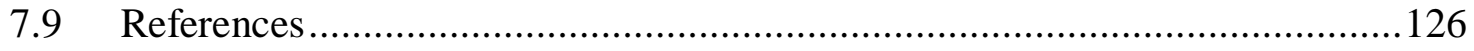

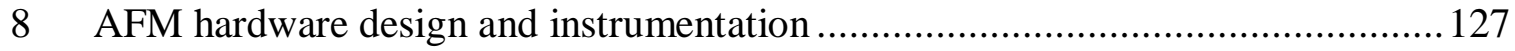

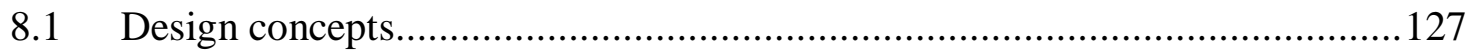

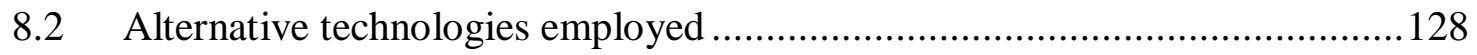

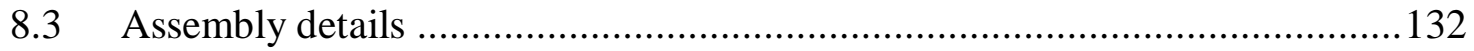

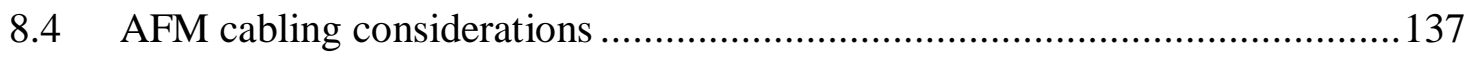




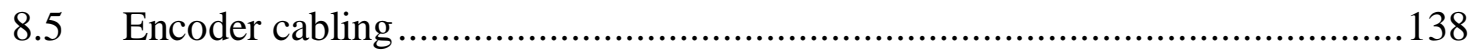

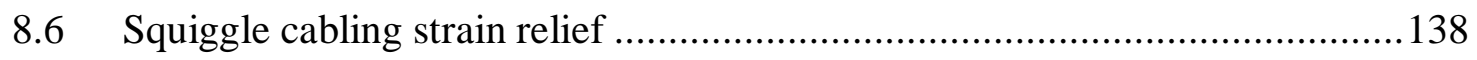

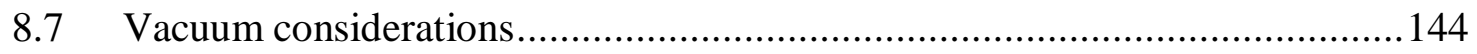

8.8 National Instruments Reconfigurable Input/Output (RIO) ..............................144

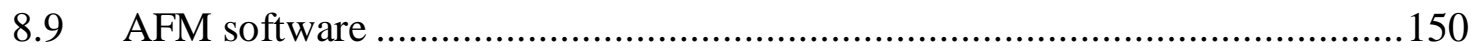

8.10 Modifications to the FPGA code and host software......................................151

8.11 One quirk with National Instruments Labview FPGA development ................151

8.12 NewScale Technology motors and control software.......................................151

8.13 Ad-hoc changes to intended AFM design..................................................153

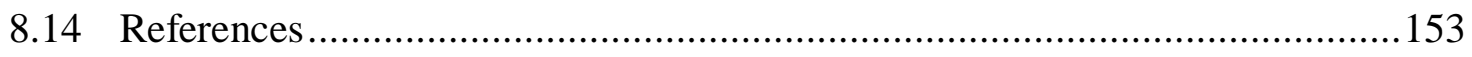

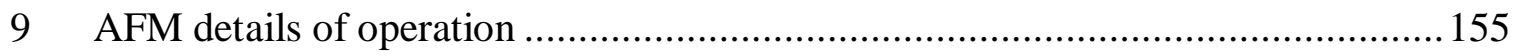

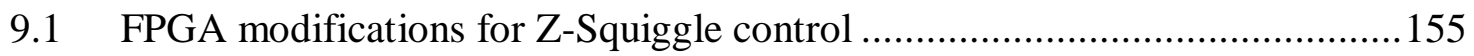

9.2 Polarity modifications to the FPGA code .................................................... 158

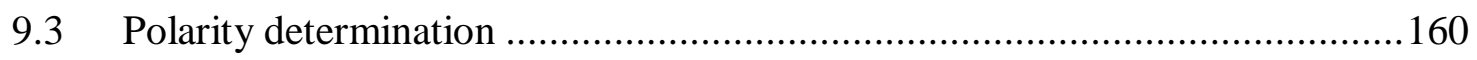

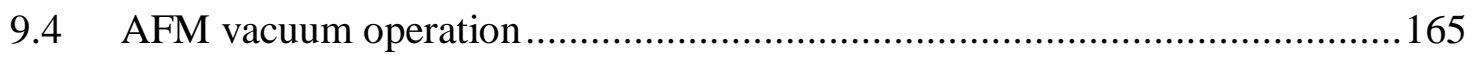

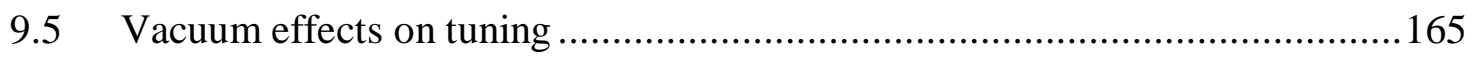

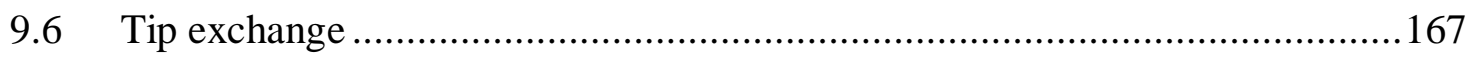

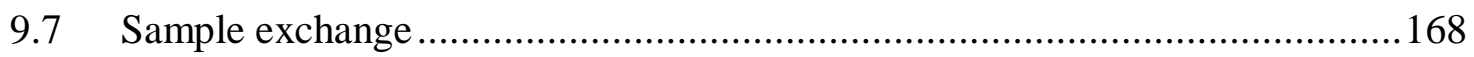

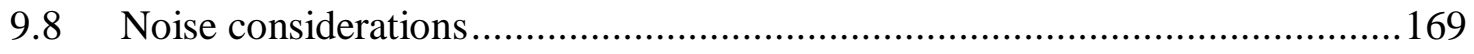

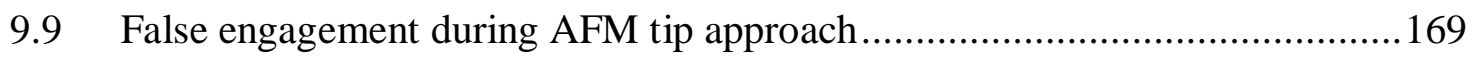

9.10 Possibility for reducing errors in an AFM scan using phase data ....................170

9.11 Removal of other AFM image artifacts ....................................................... 178

9.12 Noise floor assessment with XY-scanning turned off .....................................179

9.13 Noise assessment of AFM images on AlTiC .................................................. 182

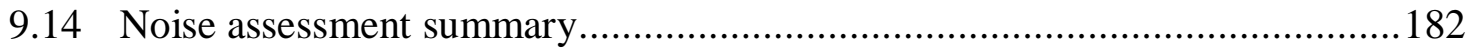

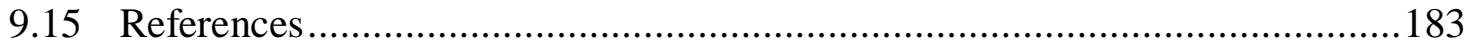

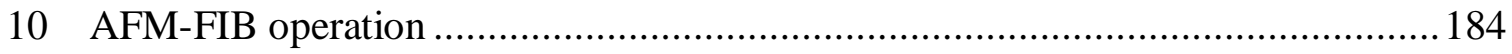

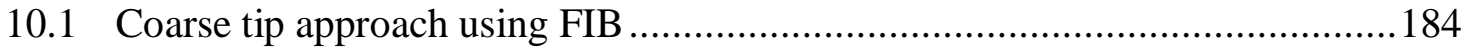

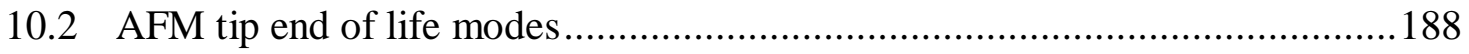

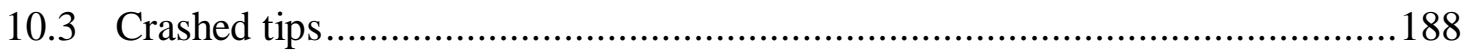

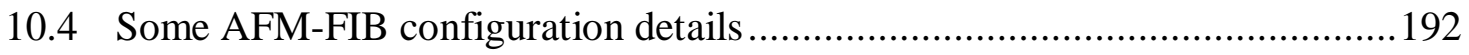

10.5 Experiment \#1: Low beam current milling using 250 pA beam ..................... 192

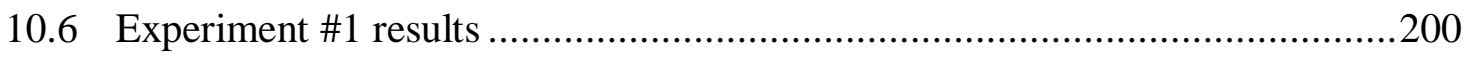


10.7 Experiment \#2: High beam current milling using the $5.75 \mathrm{nA}$ beam plus image integration 201

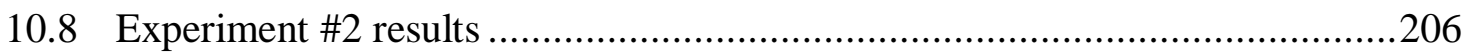

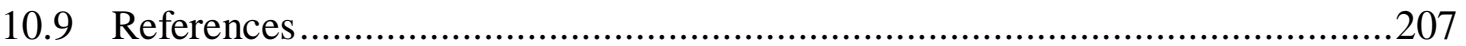

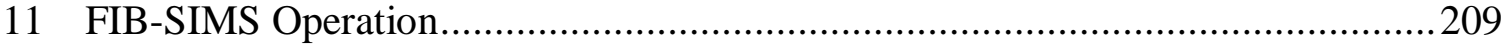

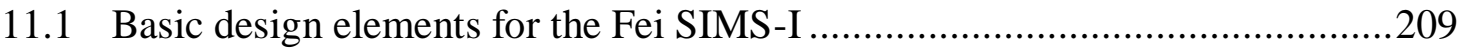

11.2 Benninghoven voltage ratios for SIMS energy filter ....................................211

11.3 Optimization of the SIMS optics voltages ...................................................213

11.4 Practical considerations for optimal operation...............................................214

11.5 Possible artifacts related to SIMS-I geometry or by mixing of species ...........216

11.6 Experiment \#1: Low resolution, high milling rate SIMS …...........................218

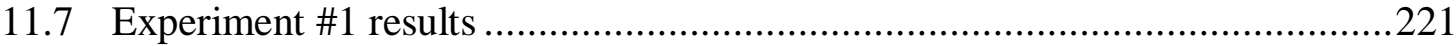

11.8 Experiment \#2: High resolution, low milling rate SIMS ..............................222

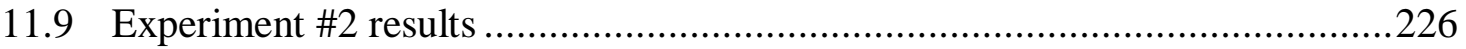

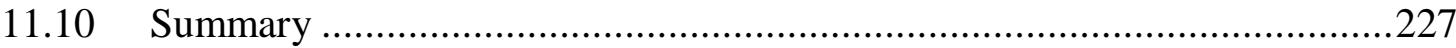

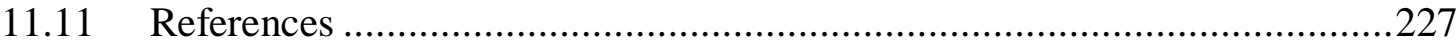

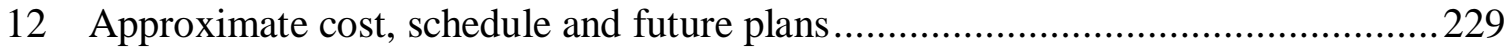

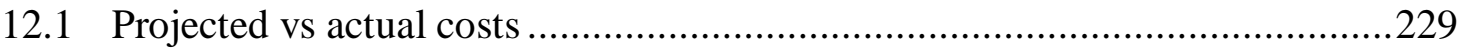

12.2 Decision not to open source the AFM-SIMS design ....................................230

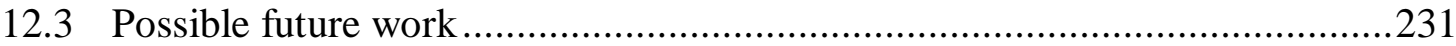

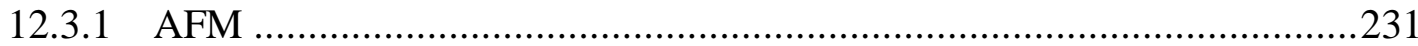

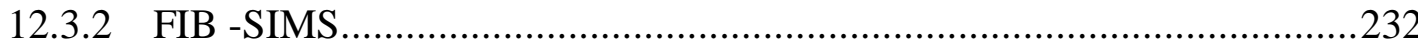

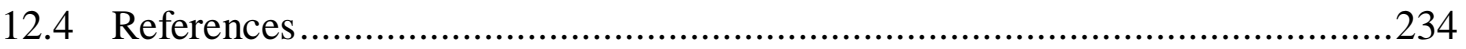

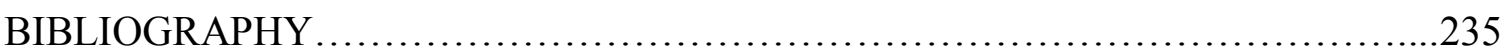

\section{APPENDICES}

Appendix A. Miscellaneous software...............................................245

Appendix B. Selected g-code snippets for CNC operation............................256 


\section{LIST OF TABLES}

Table 2.1. Typical operating regimes for a Ga-based two lens FIB operating at $25 \mathrm{keV} .11$

Table 2.2. Approximate behavior for electron and ion yields with increasing sample atomic number, $Z_{2}$. Electron yield increases in SEM and decreases in FIB with increasing $\mathrm{Z}_{2}$.

Table 6.1. As the range in relative milling rates is increased in a multi-component material, reconstruction is less accurate, particularly with mill depth. Notice how the reconstructed image is a compressed version of the original. Distortion of the amorphous features is somewhat difficult to see when the milling rates are similar. The distortion of the spherical features illustrates the effect even when the mill rates are comparable.

91

Table 6.2. Summarized milling rate predictions for some of the materials contained in AlTiC, .99

Table 7.1. Tabulated values used on the 2LI column for about one year prior to upgrading the column to the Magnum. Omitted values for Lens 2 were not recorded in the lab notebook. EVA stands for electrically variable aperture.

Table 7.2. Some settings used on the Magnum. Missing entries were not recorded.....114

Table 7.3. This listing of parameters and functions describes much of the useful capabilities needed for a useful auto focus used during FIB - SIMS operation.

Table 7.4. Typical run times for an auto focus comprising: 1 starting image, 3 throwaway images, 11 focus sweep images and 1 final image. There is also a $200 \mathrm{~ms}$ delay between each lens 2 setting and image acquisition.

Table 7.5. Descriptions for the stage control UI elements. .125

Table 9.1. Recorded values which can be used to estimate the Phase Sensitivity factors shown in Table 9.2. The Phase Sensitivity is the factor which can be multiplied to the phase error which is then added to the topographic image to reduce noise.

Table 9.2. Computed quantities, in real-world units, for the final determination of the Phase Sensitivity factor for minimizing topographic errors. Data shown include starting and ending values, demonstrating the stability of the system for a given set of PID parameters. 174

Table 9.3. Absolute $\mathrm{Z}$ noise floor determination with XY scanning turned off. The minimum $\mathrm{Rq}$ values here are computed by adding a multiple of the phase error image. 181

Table 11.1. SIMS pixel dwells used for experiment \#1. 219 
Table 11.2. SIMS pixel dwells used for experiment \#2.

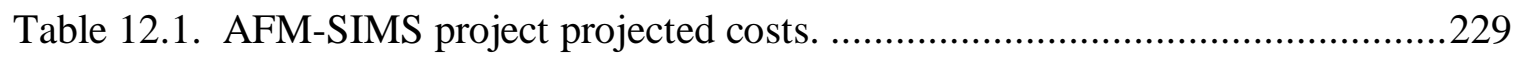

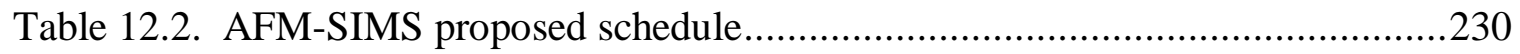




\section{LIST OF FIGURES}

Figure 2.1. Illustration showing the milling effect of the ion beam on a two-component sample. Most ejected atoms are neutrals. Ejected ions can be positive or negative, depending on the substrate atom composition and primary ion species. The drawing attempts to illustrate the non-uniformity of the beam of incoming ions. The sample lattice can be damaged by the beam and also will contain some of the atoms from the beam as interstitials.

Figure 2.2. FIB column diagrams showing the collimated mode (left) and crossover mode (right) of an early FEI FIB column having a fixed BDA. Each mode is schematically represented. In collimated mode, both lens 1 and lens 2 are relatively weak and the column magnification is less than 1, resulting in a demagnification of the virtual source. In crossover mode, lens 1 is relatively strong, focusing more ions through the BDA than in collimated mode. A crossover is necessarily created and it can be adjusted up or down to control the beam current and to a lesser degree, column magnification can be greater than 1 .

Figure 2.3. SEM image of a Ga sphere which has accumulated at a nucleation site on bare Si during repeated FIB imaging. .

Figure 2.4. SEM image at 52 degrees of the crater formed during repeated FIB imaging. This is the result of acquiring 50 images using $30 \mathrm{kV}, 80 \mathrm{pA}$ at $30 \mu$ s pixel dwell. Approximately $3 \mathrm{~nm}$ per FIB image was removed.

Figure 2.5. SEM image at 45 degrees of the crater formed during repeated FIB imaging. This is the result of acquiring 50 images using $30 \mathrm{kV}, 790 \mathrm{pA}$ at $30 \mu$ s pixel dwell. Note that the crater bottom is uneven. The $\mathrm{Al}_{2} \mathrm{O}_{3}$ material on the right has milled faster and has caused the $\mathrm{Al}_{2} \mathrm{O}_{3}-\mathrm{TiC}$ interface to mill even faster. Approximately $45 \mathrm{~nm}$ per FIB image was removed. Also note that the side-walls of the crater is likely covered with re-deposited material since none of the underlying sample structure is visible. 15

Figure 2.6. SEM images of the $80 \mathrm{pA}$-milled crater in Figure 2.4, showing the FIB drift from right-to-left during milling. It is more likely that the stage drifted from left to right during the mill. (left) Top-left corner of the crater. (right) Top-right corner of the crater clearly showing the ramp created by the drift.

Figure 2.7. Image stack constructed into a 3D volumetric rendering using ImageJ. .......17

Figure 2.8. FIB electron (left) and ion (right) images of pencil lead which is commonly used for FIB column alignments. Image time is 10 seconds at $75 \mathrm{pA}$. The images are slightly shifted w.r.t. each other, since the image appears to shift to the left and down when the detector is in ion mode. The shift is larger 
than the field of view so beam shift is used to move the ion image to the right. They do not match up perfectly, demonstrating that it can be difficult for a human to match random features. A simple computer algorithm, cross-correlation, can easily find the matching features between these two images.

Figure 2.9. Low magnification image illustrating the typical amount of shift between electron and ion images due to the polarity of the CEM collector voltage. The shift shown here is approximately $18 \mu \mathrm{m}$ in $\mathrm{X}$ and $8 \mu \mathrm{m}$ in $\mathrm{Y}$. 19

Figure 2.10. FIB images of $\mathrm{Au}$ on $\mathrm{C}$ on a chipped corner. Note the superior signal to noise on the electron image (left). The ion image (right) exhibits a stronger shadowing effect, particularly in the chipped region near the micron marker. Both images appear to be illuminated from the left because the CEM is located to the right. 20

Figure 2.11. FIB images of a dust particle using detected electrons and ions. The particle in the electron image (left) has become so strongly charged that its image and even the surrounding Si grid appears distorted. The Si grid is out of focus in the bottom third of the image. The particle in the ion image (right) appears to be unaffected by the beam and it might even be difficult to convince yourself that these are images of the same feature. .21

Figure 2.12. The SRIM program can be used to estimate sputter yields for FIB systems. For the example shown, SRIM predicts that $3.69 \mathrm{Al}$ atoms will be sputtered for each incoming $\mathrm{Ga}+$ ion at $25 \mathrm{kV}$ and normal incidence. .24

Figure 2.13. Stopping power computed using SRIM for $25 \mathrm{kV}$ primary beam $\mathrm{Z} 1=\mathrm{Ga}+$ for elements 3-84 at normal incidence. Stopping power and melting point are roughly correlated. .25

Figure 2.14. Stopping power computed using SRIM for $25 \mathrm{kV}$ primary beam $\mathrm{Z} 1=\mathrm{Ga}+$ for elements 3-84 a normal incidence. Stopping power and target density show some relatively strong correlations over certain intervals. .25

Figure 2.15. There are relatively strong correlations between nuclear stopping power and target density over certain ranges in sample atomic number $(\mathrm{Ga} 25 \mathrm{keV}$ primary beam). .26

Figure 2.16. Correlations are more tenuous for electronic stopping power using the same atomic number ranges as for the nuclear in Figure 2.15 (Ga $25 \mathrm{keV}$ primary beam). .26

Figure 3.1. FIB images of a passivated Si chip. The square regions are 100x100 $\mu \mathrm{m} 2 \mathrm{Au}$ pads. Note the lack of material contrast on the ion image. 31

Figure 3.2. Rough illustration of the FEI FIB SIMS-I energy analyzer and ion transfer optics. There are eight individual controls which need to be optimized for the highest possible ion count rate. 32

Figure 3.3. Sample of a three-species SIMS composite image. The contributing images 
have been registered, Gaussian blurred and histogram stretched in order to create the final colored image.

Figure 3.4. SIMS spectrum on a dirty Al sample. Notice the numerous contaminants, compounds, and multiply ionized species. 35

Figure 3.5. SIMS spectrum on a dirty Ti sample. Note the many compounds and contaminants.

Figure 4.1, Fine positioning elements of the AFM. a) The Z-axis piezo tube rests on an $\mathrm{XY}$ nano positioner for the XY raster. b) As the sample is scanned in XY, constant tip-sample distance is maintained by the $\mathrm{Z}$-axis piezo tube. c) The Z-position which is required to maintain constant tip-sample distance is recorded synchronously with the XY position during the raster. The result is a 3D image of the sample surface. .38

Figure 4.2. Two primary AFM tip configurations. (left) Tip oscillates vertically, perpendicular to the sample. This mode is preferred in vacuum environments where it might tend to stick to the sample. It also saves space between the SIMS-I and sample. (center \& right) The tip is operated having its can rotated 90 degrees wrt the vertical configuration and oscillates laterally, parallel to the sample. The TENOM for which the AFM in this work is based, uses this configuration. 39

Figure 4.3. Schematic illustration of the AFM tip being moved along a sample which has uneven topography. The sample is moved up or down to maintain a constant tip-sample distance. The shape of the tip determines how finely the surface features can be resolved. 40

Figure 4.4. Parabolic relationship of $\mathrm{f}_{\mathrm{o}}$ vs temperature for a quartz tuning fork. The data for this curve was extracted from the tuning fork data sheet using a software program named 'Data Thief".

Figure 4.5. Illustration of the resonance phenomenon for a quartz tuning fork in its sealed can using a function generator. The yellow curves are the excitation and the blue are the tuning fork response which is always sinusoidal. (top) Sinusoidal excitation. (Middle) Triangle wave excitation. (Bottom) Square wave excitation.

Figure 5.1. SEM images of a typical electro-chemically etched Au tip and its mounting in the shear-mode configuration on its tuning fork. Note the bumps created by the Au coating .52

Figure 5.2. Electrical setup for manual tuning fork excitation and measurement. Later, the oscilloscope was replaced by a true rms digital multimeter. .53

Figure 5.3. (left) Photograph of the electrostatic transmission electron microscope used in this work. (right) Drawing of the ETEM optics. This system was designed by Gert Rempfer in the 1970's for applications in biology. It has some features which were innovative for the time, including 3D 
visualization using shutter glasses. (images reproduced from original

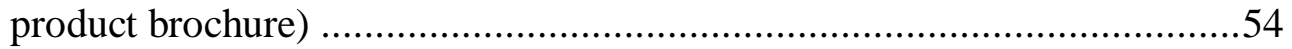

Figure 5.4. Photograph (left) of the ETEM sample holder which has been modified to hold tuning fork based AFM sensors, including the MESFET and scan head preamp. Photograph (right) showing a tuning fork being inserted into the ETEM.

Figure 5.5. Setup for recording video of vibrating AFM tips inside the ETEM. Initially, the AFM controller was used to drive the tuning fork. Later the UD-B Direct Digital Synthesis (DDS) function generator was used...................55

Figure 5.6. Video processing steps. The output from the video analysis is the raw measurements for each image in the movie. There are generally 1000 or more images in a video, so the data go through some smoothing and averaging in a spreadsheet. Finally, a plot of the data is generated............57

Figure 5.7. Direct measurement of an AFM tip mounted on a tuning fork. The tuning fork was driven using $200 \mathrm{mV}$ P-P. Normal operation is typically $2-7 \mathrm{mV}$ r.m.s. Image horizontal field of view is approximately $7.5 \mu \mathrm{m}$. This is sample \#19. 1740 images were extracted from the acquired video for analysis. The large difference between $\mathrm{Q}$ measured electrically and from video are still not understood entirely. This experiment demonstrates the feasibility of using ETEM only qualitatively so far. .58

Figure 5.8. The result of tracking the peak amplitude at resonance vs applied voltage (sample \#16). The line fit suggests $0.6 \mathrm{~nm}$ per $\mathrm{mV}$ of excitation and an intercept of $2.3 \mathrm{~nm}$. We might expect a y-intercept in the pm range.........60

Figure 5.9. (left) Image motion blurred using a 64 pixel uniform motion vector. (center) Image of the 64 pixel uniform motion vector. (right) Motion blur removed using eq. (5.14).

Figure 5.10. Comparison of errors in the initial estimate of motion blur using eq. (5.14) on an image blurred using a 16 pixel uniform motion blur vector along $\mathrm{x}$. (left) Restored image using 15 pixels. (center) Restored image using 16 pixels. (right) Restored image using 17 pixels.

Figure 5.11. Comparison of errors in the initial estimate of motion blur using eq. (5.14) on an image blurred using a 4 pixel uniform motion blur vector along $\mathrm{x}$. (left) Restored image using 3 pixels. (center) Restored image using 4 pixels. (right) Restored image using 5 pixels.

Figure 5.12. Application of eq. (5.14) to a noisy motion-blurred image. (left) Image with 32 pixels of motion blur and 50\% additive Gaussian noise. (center) 32 pixel uniform motion vector. (right) Result of removing 32 pixels of uniform motion. The original image is not discernable. ..................................70

Figure 5.13. Screenshot of the software developed to evaluate convolution and deconvolution. In this screenshot, the upper-left image has been convolved 
with the upper-right image representing a uniform motion vector as a white line. The motion blurred result is in the lower-left.................................73

Figure 5.14. The motion in an image can be estimated if the ideal image is known. (left) Noiseless, motion blurred image. (center) Ideal non-blurred template image. (right) Image which is the result of applying eq 4.13 using an epsilon of 0.001 .

Figure 5.15. Demonstration of estimating the motion in an image using a non-ideal template image. The parts of the template image which cannot be found in the blurred image show up as structured noise in the motion image (right). The estimated motion vector is shifted from the center of the deconvolved image. Its center of mass corresponds to the relative shift between the word "Image" in each of the two images. Thus the deconvolution process can also be used to perform pattern-matching operations. (epsilon 0.001)....74

Figure 5.16. Example of adding 50\% Gaussian noise to the motion-blurred image. The motion vector is buried in the random noise of the deconvolved image and is irretrievable. (epsilon 0.001) .75

Figure 5.17. Example of adjustment of the epsilon parameter to a value of 100 , representing the noise to signal ratio, to reduce the effect of the $50 \%$ Gaussian noise in the motion-blurred image. The motion vector can be readily retrieved from the deconvolved image. 75

Figure 5.18. (left) An image convolved with a hand-drawn spiral and corrupted with $50 \%$ additive Gaussian noise. (middle) Ideal template image. (right) Deconvolved image revealing the hand-drawn spiral. (epsilon 50) ........76

Figure 5.19. (left) Probability of finding a SHO in a particular position along a peak to peak oscillation of 72 pixels. The curve is scaled in gray levels for creation of motion vectors as images, $\mathrm{h}(\mathrm{x}, \mathrm{y})$ (right). .77

Figure 5.20. (left) Image motion blurred using a 72 pixel SHO motion vector image in Figure 5.19. (center) Uniform 72 pixel motion vector. (right) SHO motion blurred image with uniform motion removed. (epsilon 10) ........78

Figure 5.21. Demonstration of an image blurred using a motion vector of 16 pixels, which has been deconvolved using (left) 15 pixels, (center) 16 pixels, and (right) 17 pixels. (epsilon $~ 0.001)$........................................................79

Figure 5.22. Demonstration of an image blurred using a motion vector of 16 pixels, which has been deconvolved using (left) 15 pixels, (center) 16 pixels, and (right) 17 pixels. (epsilon 1.0)

Figure 5.23. Illustration of what a 72 pixel, uniform linear motion of the AFM tip would look like. .81

Figure 5.24. AFM tip as it would look it if were in 72 pixel, pure simple harmonic motion. Notice that the tip image is very clear at the ends of motion and that there is a nearly uniform "ghost" in between. .81 
Figure 5.25. (left) Static ETEM image of an AFM tip mounted on a crystal tuning fork. This is to be treated as the template image, $f(x, y)$. (center) Same AFM tip, except that it has been set in oscillatory motion near its resonance frequency. It is to be treated as $\mathrm{g}(\mathrm{x}, \mathrm{y})$. The images were extracted from videos described in section 5.3. Additionally, the image gray levels were inverted so that the "signal pixels" are of the tip itself. (right) Motion vector image, $h(x, y)$ using eq. (5.14). 82

Figure 5.26. (left) Intensity profile along $\mathrm{x}$ in $\mathrm{h}(\mathrm{x}, \mathrm{y}=128)$ in Figure 5.25. (right) 5-point slope of $\mathrm{h}(\mathrm{x}, \mathrm{y}=128)$ profile. Peaks of the slope image suggest that the motion peak to peak is 72 pixels. 82

Figure 6.1. (left) 2D representation of FIB imaging, illustrating the removal of a thin layer of the sample with each successive FIB image. The white material mills away 3 times faster than the black material. With each successive FIB image, the white material recedes farther and farther from the mean etch plane. In an amorphous sample, the result can be very complex. (right) Simulated depth data, taken for every 10th pass with the Ion Beam. The surface roughness appears to increase with each pass. .86

Figure 6.2. Plot of SRIM computed sputter rates vs incident angle for $25 \mathrm{kV} \mathrm{Ga}$ ions on Si. Each point was computed by flying only 256 ions, for time considerations. Some error is evident, but the trend shows high sputter rates for steep angles of incidence. This tells us that high aspect structures can sputter up to ten times faster than flat ones. 88

Figure 6.3. 2D representation of FIB sputtering on a 6-component sample in which each component is has a relative sputter rate of $1 \mathrm{X}, 2 \mathrm{X}, 3 \mathrm{X}, 4 \mathrm{X} 5 \mathrm{X}$ and $6 \mathrm{X}$, as indicated by its gray level. The plot represents the milled surface for every 10 th pass (and the last one) of the ion beam.

Figure 6.4. Plot of SRIM computed sputter rates at normal incidence for $25 \mathrm{kV} \mathrm{Ga+} \mathrm{ions}$ on elements 1-92. Each point was computed by flying only 256 ions, for time considerations. Notice the trend of increasing sputter yield with increasing Z2. This amounts to about 0.05 (atoms per incident ion) per AMU of target mass. 92

Figure 6.5. SEM image of a fractured piece of AlTiC. The angular grains inside the material are not readily evident from the polished surface. 95

Figure 6.6. FIB image of a crater made by successive FIB images on the surface of a hard drive head air bearing surface. This image illustrates some distinguishing characteristics of the air bearing material and the effect of imaging using the FIB-SIMS. Image contrast has been enhanced slightly.96

Figure 6.7. FIB SE image of AlTiC taken just as the beam has milled through the DLC layer. The bright borders surrounding the gray islands are likely due to edge-enhanced secondary electron yield caused by the step created during the grain recession processing. These bright borders quickly fade away 
with successive FIB imaging.

Figure 6.8. SRIM predictions for $25 \mathrm{keV} \mathrm{Ga}+$ ion sputtering of $\mathrm{Al}_{2} \mathrm{O}_{3}$. Nearly two Oxygen atoms per Aluminum atom is sputtered away, leaving an Aluminum rich surface. 100

Figure 6.9. SRIM predictions for $25 \mathrm{keV} \mathrm{Ga+} \mathrm{ion} \mathrm{sputtering} \mathrm{of} \mathrm{TiC.} \mathrm{Nearly} \mathrm{two}$ Titanium atoms per Carbon atom are sputtered, leaving a Carbon rich surface. 100

Figure 7.1. FIB xP 2.25 control software controlling the FIB 611. The magnifications have been customized to match the ones on the 611's deflection controller. Pixel dwell presets are shown in the small windows in the center of the screen. 108

Figure 7.2. Original stage layout showing the original location of the slip-ring brush mount. The block at the center of the image holds the rotational limit switch. A replacement was made for this block which includes a new slipring brush mount.

Figure 7.3. Relocation of the Teflon slip ring brush holder involved combining a smaller version of it with the homing block. The original block was not damaged so that it can be reused if the AFM is moved to a different system later......111

Figure 7.4. New FIB stage layout showing the relocated slip-ring brush block and the AFM at the corner of the stage. The original sample stage was elevated to the level of the AFM. Samples can be analyzed on either the AFM or original sample stages. The encoder plate to the left has not yet been removed. 112

Figure 7.5. Biasing schematic for the Magnum FIB column. Lens 1, Lens 2 and the Beam Voltage are all ground referenced, while the Extractor and Suppressor are referenced to the Beam. Heating current to the LMIS filament requires an isolation transformer which is capable of standing off the beam Voltage $(25-30 \mathrm{kV})$. .116

Figure 7.6. Screenshot of the AutoLibControlApp.exe. Among its most useful functions is auto focus. Multiple auto focus runs are shown in the graph display. This program has some useful functionality to not only perform the auto focus function, but also to setup and recover the system. 121

Figure 7.7. Stage controller software user interface showing (left) powered off and (right) powered on modes. 124

Figure 8.1. Artist's rendering of the AFM unit. The tuning fork holder with preamp is on a plate which is moved vertically with a mini piezo motor on the back for coarse approach of the tip onto the surface. The tip coarse XY positioner moves the tip over a location of interest somewhere on the sample (unit has a range of $+/-3.5 \mathrm{~mm}$ ). The XY sample scanner moves the sample for high resolution topographic imaging, and the $\mathrm{Z}$ piezo sample scanner moves the 
sample up or down through a feedback from the tuning fork signal 128

Figure 8.2. Illustration of an AFM scan across a surface which was imaged/milled using a FIB. Some of the materials are milled faster than the others and make for a rough surface. Knowledge of the differing milling rates for each of these materials is crucial for $3 \mathrm{D}$ reconstruction of the sample. 130

Figure 8.3. AFM coarse XYZ design, as drawn using TurboCAD 17. Dimensions of the completed AFM are approximately $60 \mathrm{~mm}$ x $60 \mathrm{~mm}$ x $80 \mathrm{~mm}$. Not shown is the nano-postioning XY scan bed. 131

Figure 8.4. Coarse Z-axis designs. (left) original flexure design having $<=5 \mathrm{~mm}$ range and large cosine error. (right) New design which was actually implemented. The plate which contains the tuning fork is supported by two linear bearings and a small squiggle motor $(7 \mathrm{~mm}$ diameter) in a tripod arrangement. It has a range $>5 \mathrm{~mm}$ and virtually no cosine error. .132

Figure 8.5. AFM showing coarse $\mathrm{Z}$ axis removed (inset). The coarse $\mathrm{Z}$ stage uses the linear bearings and Squiggle motor in a tripod arrangement. 134

Figure 8.6. Assembly of the Coarse XY stage using a pre-load jig. One bearing rail is aligned with the middle block's edge and the other is pressed against it using screws to apply a uniform pressure before tightening the mounting screws. The image shown is the prototype mechanism after the adjustable pieces have been removed. The black spacer is still inserted. 135

Figure 8.7. Final AFM design showing the coarse $\mathrm{Z}$ stage in its lowest position. This design can accommodate a large range in sample thickness and tip length, limited only by the length of the bearing posts, Squiggle motor travel and encoder range. 136

Figure 8.8. Fully assembled AFM hardware illustrating its size w.r.t. a dollar bill. The coarse- $Z$ axis is shown in its highest position. The material used was Aluminum for its ease of machining and for its non-magnetic qualities. The cutouts for the XY return springs were not implemented for improved stiffness. .137

Figure 8.9. Image showing the strain relief points and their associated zip-ties. Each strain relief point is positioned to minimize the force of the cable on the associated stage axis. All strain reliefs, except the squiggle are shown before tightening. 139

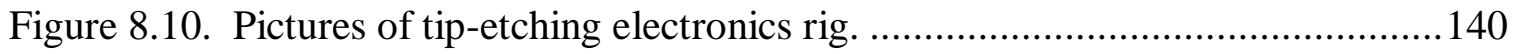

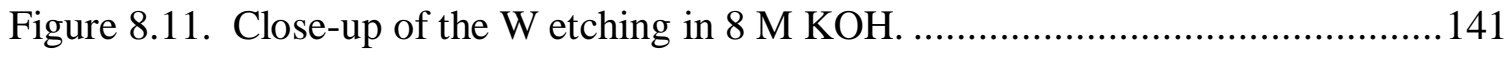

Figure 8.12. Typical setup for gluing etched tips to tuning forks. .............................. 142

Figure 8.13. Side-view of the AFM test setup. Notice that the tuning fork can is clamped in using a set-screw. This arrangement was difficult to tune so it was hastily remade. Now the tuning fork is supported by its electrical leads 
only. This has a de-tuning effect giving lower Q's, allowing for faster

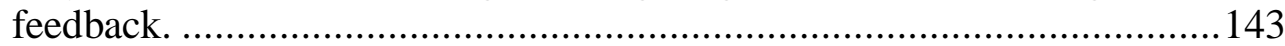

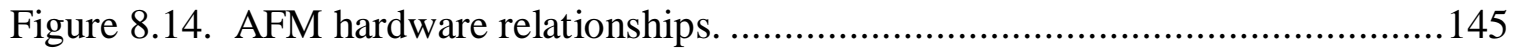

Figure 8.15. AFM modules and their connections shown as one or two-way................146

Figure 8.16. Main UI for accessing the AFM functionality. Many of the ANSOM functions have been removed for simplicity. .........................................146

Figure 8.17. Motor Control UI. Items added to this control are Motor ON Time (us), Motor Step Counts, Position (counts), Position $(\mu \mathrm{m})$, Zero Encoder Button and Approach Read Delay (us).............................................................. 147

Figure 8.18. Quadrature decoding directly on the FPGA........................................... 148

Figure 8.19. Piezo Control UI in normal mode. Items added to this control are Avg Delay (us), \# Avgs, and the Advanced toggle switch. 148

Figure 8.20. FIB image showing the $550 \mathrm{~nm}$ lateral shift of the sample during an AFM ramp sequence in $Z$. This lateral shift can be corrected inside the FPGA code by activating the $\mathrm{XY}$ vs $\mathrm{Z}$ functionality and adjusting the sliders for nulling image shift while FIB imaging.

Figure 8.21. Z-Piezo Control UI showing the XY vs Z fly-out which can be used to calibrate and then activate the feature. 149

Figure 8.22. FPGA code for determination of the $\mathrm{X}$ vs $\mathrm{Z}$ and $\mathrm{Y}$ vs $\mathrm{Z}$ factors which can optionally be applied to the scan bed to minimize the effect seen in Figure 8.20 . 150

Figure 9.1. Original FPGA stepper motor code which is designed to control the stepper motor coils individually. This code exhibits a flaw in which the "Step" input is read before the "Direction". The result is that direction reversals are not possible until one or more steps are executed in the prior direction.

Figure 9.2. Modified FPGA stepper motor control which retains the original, individual coil control and adds a step-direction output for the Z-Squiggle controller. Many stepper motor drivers also have step-direction inputs, increasing the possible number of configurations. This code also fixed a race condition where reversing the direction of stepping resulted in one or more extra steps in the prior direction, potentially resulting in crashed tips. 157

Figure 9.3. Electrical signals which form the AFM tip approach sequence. The surface is sought using alternating Z-piezo ramps and coarse-Z motor bursts. 157

Figure 9.4. Original ANSOM FPGA high-level PID control showing only one output to the Z-Piezo. 158

Figure 9.5. Modified FPGA high-level PID control showing an additional inverted output to an extra DAC output on the Controller Board (J9-1). This bit of code also reflects other changes made elsewhere. The gap in the middle 
was intentionally made for inserting low-pass and notch filters during debugging sessions. 158

Figure 9.6. Labview code showing the PID control and how it is linked to the variables and control variable which is usually the phase error signal. 159

Figure 9.7. Using the coarse $\mathrm{Z}$ encoder to measure the piezo sensitivity by touching it to the sample stub allowing the spring to cause constant contact during the measurement. The response is approximately $56.854 \mathrm{~nm} /$ Volt. 160

Figure 9.8. Typical frequency sweep for the tuning fork used in Figure 9.9 to acquire a scan. The phase detection circuit is adjusted for a negative phase response to frequency and for maximum dynamic range. In this case the phase range is about 35 degrees, but only 20 degrees is usable for feedback, due to the high curvature of the phase response. 161

Figure 9.9. An early AFM scan in air on a MetroCal wafer piece. The left monitor shows the two video feeds used for coarse tip placement. The smaller one is the microscope image at $\sim 500$ um Horizontal FOV and the other is from a borescope camera mounted at oblique. The right monitor shows the scan in progress. The left (pink\&yellow) and right (gray) AFM images are the socalled trace and retrace images, respectively. The AFM is operating correctly when these images are very similar. 162

Figure 9.10. (left and right, respectively) Trace and retrace images $(50 \times 50 \mu \mathrm{m} 2)$ of one of the first unambiguous scans on a MetroCal wafer in air (shown in Figure 9.9). The fast scan axis is shown along Y. Some processing was performed to align the scan lines and to level the vertical scale. Some distortion is evident along the fast scan axis. This is a result of operating the XY scan bed in open-loop mode.

Figure 9.11. Electronic phase adjustment for positive slope in the phase response (red curve). This is the sweep is for tip \#B-002 in air. Notice how it is adjusted for saturation at $+/-90$ degrees and that zero phase is close to the center of the range. 164

Figure 9.12. AFM operation in air after installation into FIB system. The FIB system is mechanically stable enough that the AFM can be used in air with the FIB chamber door hanging open. The computer monitor on the lower-right shows the AFM scan in progress. .164

Figure 9.13. Demonstration of vacuum effect on tuning of the cantilever and tip\#B-002. The settings are the same as in Figure 9.11. Note that the amplitude (white curve) is saturated and requires that the Drive Voltage $(\mathrm{mV})$ be reduced. 166

Figure 9.14. Tuning for tip\#B-002 in vacuum. The Drive Voltage $(\mathrm{mV})$ has been reduced from $5 \mathrm{mV}$ to $4 \mathrm{mV}$. Also notice that the peak frequency has shifted up by $9.8 \mathrm{~Hz}$. The peak frequency will continue to move up slowly as the adhesive outgases inside the vacuum. For AFM operation, the Center Frequency $(\mathrm{Hz})$ is adjusted so that the nominal phase is about +5 degrees. 
Figure 9.15. Tip\#B-002 tuned and ready for AFM operation. The nominal phase here reads 5.9024 degrees in this static photo, but will be continuously changing, as there is a lot of noise in the phase read back when the tip is not engaged.

Figure 9.16. Illustration of multiplying the (left) phase image by the factor $6.773 \mathrm{~nm} / \mathrm{deg}$, then adding it to the (middle) topography image to obtain the (right) calculated image. The starting $\mathrm{R}_{\mathrm{q}}$ for the topography image is $12.02 \mathrm{~nm}$. The calculated image's $R_{\mathrm{q}}$ is $10.71 \mathrm{~nm}$. The factor was read off the table for image \#7, thus illustrating that these factors can be pre-computed if some extra data are recorded for each AFM scan. 175

Figure 9.17. Illustration of reducing noise in an AFM topgraphy trace image by adding a multiple of the phase feedback data. (top-left) Original topography ROI image. (top-right) Phase ROI image. (bottom-left) Image having the smallest $\mathrm{Rq}$ which is the result of adding 4.76 times the phase ROI to the topography ROI. (bottom-right) Plot of rms roughness vs phase ROI image multiplier. A parabola has been fit to determine its vertex which corresponds to the lowest $\mathrm{Rq}$ and also the \# nm per degree of phase, 4.76. The noise is greatly reduced, although spikes from the sharp edges remain. 176

Figure 9.18. Same calculation as in Figure 9.17 for the retrace image using a scale factor of $4.686 \mathrm{~nm} / \mathrm{deg}$. The noise for the retrace image is smaller than for the trace image. This may be due in part to the fact that the trace image is effectively moving up due to a positive slope and the retrace image is effectively moving down due to the negative slope in that direction. In a later image comparison, the trace image has some curvature which tends to increase $\mathrm{Rq}$. 177

Figure 9.19. The results of identifying the spikes and sharp edges, then interpolating under them using the solution to Laplacian to remove the spikes and soften the edges. (left) Trace image whose $\mathrm{Rq}$ has been reduced from $4.93 \mathrm{~nm}$ to $4.36 \mathrm{~nm}$. (right) Retrace image whose $\mathrm{Rq}$ has been reduced from $4.38 \mathrm{~nm}$ to $3.68 \mathrm{~nm}$. The dark regions are recessed by about $8.5 \mathrm{~nm}$ for both images. 178

Figure 9.20. The result of (left) simply averaging the trace and retrace images, giving an Rq of $4.38 \mathrm{~nm}$, compared with (right) keeping the minimum pixel value between the two. Its $\mathrm{Rq}$ is $4.35 \mathrm{~nm}$. The minimum image looks surprisingly good to the eye, as nearly all of the spikes have been removed. 179

Figure 9.21. Determination of the system noise floor using a scan acquired while the tip is engaged but without $\mathrm{XY}$ scanning. The initial $\mathrm{R}_{\mathrm{q}}$ is measured as $3.9 \mathrm{~nm}$. It can be reduced to $1.18 \mathrm{~nm}$ using a multiplier of 9.36 to the phase data and adding to the height data. In this case, the proportional gain was 0.1 and the 
integral gain was 0.075. The derivative gain was set to zero. (Tip\#B-004)

181

Figure 9.22. The result of masking the (left) $\mathrm{Al}_{2} \mathrm{O}_{3}$ surface and (right) TiC surface to compute their roughness. The $\mathrm{Al}_{2} \mathrm{O}_{3}$ and $\mathrm{TiC}$ roughness values are computed as $1.23 \mathrm{~nm}$ and $1.40 \mathrm{~nm} \mathrm{R}$, respectively. On the $\mathrm{Al}_{2} \mathrm{O}_{3}$ image, the roughness of a $64 \times 64$ pixel rectangle having its origin at $(105,50)$ pixels has a roughness of $0.98 \mathrm{~nm} R_{\mathrm{q}}$. Individual TiC grains average to $0.98 \mathrm{~nm}$ $\mathrm{R}_{\mathrm{q}}$. 182

Figure 10.1. Close-up of the mounting of tip\#B-004, showing the slight angle from perpendicular. This tilt establishes a relatively safe distance between the quartz tuning fork and the ion beam path. With this particular tip, only the edge of the shank could be imaged using the FIB. The tip is shown approximately $4 \mathrm{~mm}$ from the sample surface. 186

Figure 10.2. Tip\#B-004 showing that the shank to tip distance is about $150 \mu \mathrm{m}$. The FIB is imaged on the edge of the shank and an estimate to the surface is made by recording the FIB lens 2 Voltage on both, the shank and sample surface, applying $\sim 7 \mu \mathrm{m} / \mathrm{V}$. This is a very conservative estimate and the coarse $\mathrm{Z}$-axis can be driven without the concern of crashing. 187

Figure 10.3. FIB images of the AFM tip shank (left) far from the sample surface making it look blurry and (right) engaged. The total distance in $\mathrm{Z}$ is $3190 \mu \mathrm{m}$ and the difference in lens 2 is about $396 \mathrm{~V}$. 187

Figure 10.4. FIB image of a tip\#A-010 just after it was slammed into the sample while tuning the Z-Squiggle motor. The motor had gotten stuck at $-425.8 \mu \mathrm{m}$. During Squiggle motor tuning it moved another $-1600 \mu \mathrm{m}$, driving the tip into the sample and breaking the adhesive.

Figure 10.5. (left) This particular tip\#A-010 had touched the sample during installation and so was curled up at the very end. It was initially used for some testing of the XY Squiggle motors while scanning. (right) Later, it was repaired once by cutting off the curled portion in order to continue debugging the ZSquiggle motor tuning. AFM scans after repair were better than the crashed tip but were still of low quality. This tip had a long enough taper that it was possible to view it using a FIB image. The original tip which was blunt, having a diameter of $179.7 \mathrm{~nm}$. 191

Figure 10.6. (left) AFM tip\#A-008 image of the tip's taper being pressed against a slider body to reveal the tip past its shank. It has picked up some contamination. (right) The same tip after milling with the FIB to remove the contamination. Later, it was revealed that the contamination simply moved to another location on this tip and still caused imaging artifacts. 191

Figure 10.7. (left) Low-mag image of AFM tip\#A-005 showing a crook in the end. (right) The tip has a bulbous shape, but is still relatively sharp at about 65.1 $\mathrm{nm}$ diameter on the very end. This tip was fortunately mounted such that 
the crook caused it to be nearly perpendicular to the sample surface 194

Figure 10.8. Starting AFM scan, using tip\#A-005, which is $25.01 \times 25.01 \mu \mathrm{m}^{2}$ before FIB imaging/milling. The vertical axis is the fast axis. At least two pinholes are apparent even before using the FIB. The darker islands are $\mathrm{TiC}$ and the surrounding material is $\mathrm{Al}_{2} \mathrm{O}_{3}$. These darker islands are recessed from the lighter regions by about $8.5 \mathrm{~nm}$. 194

Figure 10.9. Sequence of nine $25 \times 25 \mu \mathrm{m}^{2}$ AFM scans, each following a FIB image. FIB images are scanned left-to-right, top-to-bottom and are not perfectly rotated to 90 degrees w.r.t. the AFM. FIB scanning artifacts are clearly visible as nearly horizontal streaks inside the crater. FIB HFW is $16 \mu \mathrm{m}$. 195

Figure 10.10. Image of the AFM tip lifted from the FIB crater edge. During the milling process, the AFM tip is parked on the left side of the crater, as can be seen by its shadow left by the FIB beam tails. The AFM scan slow axis is from left to right. This allows the AFM to scan to unambiguously detect FIB scan artifacts. Image contrast has been enhanced. 196

Figure 10.11. Auto focus image stacks averaged together.

Figure 10.12. Runscript image stacks averaged together. 198

Figure 10.13. (left to right, top to bottom) AFM images inside the crater. 199

Figure 10.14. SEM image of a distinctive TiC island inside a FIB-milled crater. This feature is upside-down w.r.t. the corresponding FIB image. The bright circular features are Ga spheres remaining from the FIB imaging. These Ga spheres survived venting and exposure to air for months before this SEM image was taken. 200

Figure 10.15. Comparative images of the distinctive feature in Figure 10.14. Each has been adjusted to have similar aspect ratios. (left) SEM image, (center) AFM image and (right) FIB image. 200

Figure 10.16. (left) Low magnification image of AFM tip\#B-002, showing a blob of insulating contamination on the tapered region. (right) High magnification image showing small bits of contamination on the taper, but a rather sharp tip, about $30.6 \mathrm{~nm}$ diameter. Installed inside the FIB, this blob blocked FIB imaging of the tip and charged excessively. 202

Figure 10.17. (left to right, top to bottom) FIB image acquisition of 51, $5.75 \mathrm{nA}$ images. Pixel dwell is $300 \mathrm{~ns}$ and HFW is $20 \mathrm{um}$. Images were acquired using increasing frame integration counts in order to determine the count necessary for $50 \mathrm{~nm}$ material removal. 203

Figure 10.18. (left to right, top to bottom) AFM acquisition of 51 images, each occurring after its corresponding FIB image, shown in Figure 10.17. AFM images are $40 \mathrm{x} 40 \mu \mathrm{m}^{2}$ in size. Contrast has been normalized so that features can be seen on the sample surface and inside the FIB crater. .204 
Figure 10.19. Analysis of the AFM images shown in Figure 10.18 for crater depth and roughness as a function of total integrated FIB image count. FIB images acquired for auto focus are not shown, because the goal for this experiment is to determine the number of integrations, being a power of 2 , which leads to an average removal of $50 \mathrm{~nm}$ to include the auto focus overhead. The result is 64 . 205

Figure 10.20. (left) AFM image \#10, taken as the last image before stopping for the day. (center) AFM image \#10a, taken as the first image of the next day before the FIB milling began. (right) Mutual crop difference image, highlighting the relative affine distortion between the two. These images have had their contrast levels normalized so that features on the surface and inside the crater can be viewed. 205

Figure 11.1. Rough illustration of the FEI FIB SIMS-I energy analyzer and ion transfer optics and AFM tuning fork/tip assembly. There are eight individual SIMS controls which need to be optimized for the highest possible ion count rate. Once the three elements comprising the energy analyzer, EXTL1, EXTL2 and EXTL3, are determined, the others are easily found. 209

Figure 11.2. Low magnification image of two hard drive sliders which are made from AlTiC. The air bearing surfaces are the triangular regions to the left and the upper \& lower smooth regions. The AFM tuning fork can be seen in the bottom right. The FIB's deflection controller Vernier setting has been turned all the way CCW for the largest possible field of view, so the scale shown is incorrect. The faint outline of the SIMS circular aperture is also visible. 211

Figure 11.3. SIMS optics tuning window showing typical operating Voltages on the AlTiC sample using $25 \mathrm{keV}$ and $250 \mathrm{pA}$. 214

Figure 11.4. Low magnification images showing pairs of imaging rectangles associated with the deflection caused when the SIMS optics are turned on. Deflections shown resulted from using the SIMS optics settings shown in Figure 11.3. Deflection $(\mathrm{X}, \mathrm{Y}) \sim(-1.8 \mu \mathrm{m},+21.4 \mu \mathrm{m})$. 215

Figure 11.5. Example of possible re-deposition inside the crater during SIMS. The central area of the crater was imaged after 2037 seconds of FIB exposure with the SIMS optics turned off to reveal the higher contrast features under the re-depositied material. 216

Figure 11.6. Massive sample re-deposition and scalloping inside the crater floor which occurred during three consecutive SIMS maps using $250 \mathrm{pA}$ at $4 \mathrm{~ms}$ pixel dwell and $10 \mu \mathrm{m}$ HFW. 218

Figure 11.7. SIMS spectrum of AlTiC using the $5.75 \mathrm{nA}$ beam. Peaks used for SIMS maps are the $\mathrm{Mg}+(24 \mathrm{amu}), \mathrm{Al}+(27 \mathrm{amu})$ and $\mathrm{Ti}+(48 \mathrm{amu})$. Traces of Carbon, Oxygen and doubly ionized Aluminum can be seen.

Figure 11.8. (left, center, right) Aluminum, Titanium and Magnesium SIMS maps shown 
in the order taken. These are the first set of SIMS maps to be merged into the first image of the 10-image sequence shown in Figure 11.12 ...........220

Figure 11.9. (left to right, top to bottom) Aluminum SIMS maps at $50 \mu \mathrm{m}$ HFW. The left $2 / 3$ of the $1^{\text {st }}$ image is darkened by a thin layer of DLC. Raw images are shown. .220

Figure 11.10. (left to right, top to bottom) Titanium SIMS maps at $50 \mu \mathrm{m}$ HFW. Raw images are shown. 220

Figure 11.11. (left to right, top to bottom) Magnesium SIMS maps at $50 \mu \mathrm{m}$ HFW. Raw images are shown. 221

Figure 11.12. (left to right, top to bottom) SIMS maps using a large beam current of 5.75 $\mathrm{nA}$ at $50 \mu \mathrm{m}$ HFW. The first image shows the effect of the auto dwell determination as little dots on the DLC layer of the AlTiC surface.

Figure 11.13. SIMS spectrum of AlTiC using the $250 \mathrm{pA}$ beam. Peaks used for SIMS maps are the $\mathrm{Mg}+(24 \mathrm{amu}), \mathrm{Al}+(27 \mathrm{amu}), \mathrm{Ti}+(48 \mathrm{amu})$ and $\mathrm{Ga}$ (69 amu).

Figure 11.14. (left to right, top to bottom) Al SIMS maps. Images have been processed to boost contrast. 223

Figure 11.15. (left to right, top to bottom) Magnesium SIMS maps. Images have been processed to boost contrast. .224

Figure 11.16. Ti SIMS maps. Images have been processed to boost contrast. .224

Figure 11.17. (left to right, top to bottom) SIMS maps showing Mg (red), $\mathrm{Al}$ (green) and Ti (blue). .225

Figure 11.18. (left to right, top to bottom) Ga SIMS maps. Images have been processed to boost contrast. 225

Figure 11.19. (left) Last FIB SE image acquired inside the crater with the SIMS optics turned on. Its contrast is adversely affected by energizing the SIMS optics. (right) FIB SE image after turning off the SIMS optics and re-centering using the stage. Both images have been processed to boost contrast. .....226

Figure 11.20. (left) Ga SIMS map for run \#000, which is the last one in the \#000 set. (right) Al SIMS map, which is the $1^{\text {st }}$ one in run \#001, thus being taken directly after the Ga SIMS map on the left. These images are very similar, showing nearly identical TiC regions as dark islands. The main differences are the light regions in the Al SIMS map, which are locations for Mg. ....226 


\section{LIST OF ACRONYMS AND UNITS OF MEASURE}

\section{ACRONYMS}

2D

2LI

3D

$\dot{\mathrm{A}}, \mathrm{A}$

$\mathrm{a}_{0}$

AFM

AlTiC

ANSOM

API

BAA

BDA

$\mathrm{BNC}$

$\mathrm{C}$

$\mathrm{C}++$

$\mathrm{C \#}$

CEM

CMOS

$\mathrm{CNC}$

CAM

d50

DI

DC

DDS

DLC

dll

DPA

DRO

$\mathrm{e}^{-}$

ESR

ETEM

EVA

EXE

$\mathrm{f}_{0}$

FIB

FOV

GIS

HFW
Two-dimensional

Two-Lens Ion Column

Three-dimensional

Angstrom, equal to $10^{-10}$ meters

Bohr radius, $0.529 \dot{A}$

Atomic Force Microscopy

Shorthand for $\mathrm{X} \% \mathrm{Al}_{2} \mathrm{O}_{3}-\mathrm{Y} \%$ TiC, nominally $75 \%$

$\mathrm{Al}_{2} \mathrm{O}_{3}-25 \% \mathrm{TiC}$

Aperture-less Near-Field Optical Microscopy

Application Programming Interface

Beam Acceptance Aperture

Beam Defining Aperture

Bayonet Neill-Concelman connector

$\mathrm{C}$ Programming Language

$\mathrm{C}++$ Programming Language

C-Sharp Programming Language

Channel Electron Multiplier

Complementary Metal Oxide Semiconductor

Computer Numerical Controlled

Computer Aided Machining

Diameter of the circle which encloses $50 \%$ of the

ion/electron beam at the sample surface

Deionized, as in deionized water

Direct Current

Direct Digital Synthesis

Diamond-Like Carbon

Dynamic Link Library

Differential Pumping Aperture

Digital Read-Out

Electron

Equivalent Series Resistance

Electrostatic Transmission Electron Microscope

Electronically Variable Aperture

Executable file

Resonant frequency of an oscillator (tuning fork)

Focused Ion Beam

Field of View

Gas Injection System

Horizontal Field of View 


HST
HVPS
IPA
KOH
LEMO
LMIS
MVA
NI
PE
PI
PRAL
pwm
Q
RIO
RMS
Ra
RF
Rq
SE
SEM
SHO
SIM
SIMS
SRIM
TENOM
TEM
TOF SIMS
TRIM
UI
UI1280
XP
XP
W
w.r.t
Z

Hubble Space Telescope

High Voltage Power Supply

Isopropyl Alcohol

Potassium Hydroxide solution

A brand/type of electrical connnector

Liquid Metal Ion Source

Mechanically Variable Aperture

National Instruments

Program Executable file such as a dll or exe

Physik Instrumente (company)

Projected Range Algorithm

Pulse-width modulation

Quality factor in a resonant circuit

Reconfigurable Input/Output

Root mean square

Average Roughness

Radio Frequency

RMS Roughness

Secondary Electron

Scanning Electron Microscope

Simple Harmonic Oscillator

Scanning Ion Microscope

Secondary Ion Mass Spectrometry

Stopping Range of Ions in Matter

Tip Enhanced Near-Field Scanning Probe

Microscope

Transmission Electron Microscope

Time of Flight SIMS

Transport of Ions in Matter

User interface (software)

User interface for the FEI XP 2.25 software

Refers to the FEI XP 2.25 server software

Refers to Windows XP

Tungsten

with respect to

Represents the number of protons in an atomic species

Typically represents the \# protons in the focused ion beam species

Typically represents the \# protons in the sample species 


\section{UNITS OF MEASURE}

$\begin{array}{ll}\mathrm{C} & \text { Degrees Celsius } \\ \mu ” & \text { micro-inch, equal to } 25.4 \mathrm{~nm} \\ \mathrm{kcounts} & \text { kilo counts per second } \\ \mathrm{m} & \text { Meters } \\ \mathrm{cm} & \text { Centimeters, } 10^{-2} \mathrm{~m} . \\ \mathrm{mm} & \text { Millimeters, } 10^{-3} \mathrm{~m} . \\ \mu \mathrm{m} & \text { Micrometers, } 10^{-6} \mathrm{~m} . \\ \mathrm{nm} & \text { Nanometers, } 10^{-9} \mathrm{~m} . \\ \mathrm{pm} & \text { Pico meters, } 10^{-12} \mathrm{~m} . \\ \mathrm{eV} & \text { Electron Volt } \\ \mathrm{Hz} & \text { Frequency in cycles per second } \\ \mathrm{J} & \text { Energy in Joules } \\ \mathrm{N} & \text { Force in Newtons } \\ \mathrm{sr} & \text { Steradian measure of solid angle } \\ & \\ \omega & \text { Angular frequency in radians per second } \\ \Omega & \text { Ohms } \\ \mathrm{s} & \text { seconds } \\ \mathrm{V} & \text { Volts } \\ \mathrm{W} & \text { Power in Watts }\end{array}$

xxix 


\section{PREFACE}

The topic of this work is the development of a combined instrument composed of a focused ion beam based Secondary Ion Mass Spectrometer (SIMS) and an Atomic Force Microscope (AFM). This composite instrument referred to as AFM-SIMS is capable of acquiring the data necessary for creating 3D chemical maps of multi-component

materials down to $50 \times 50 \times 50 \mathrm{~nm}^{3}$ voxels or less. The AFM is based on the design created for the ANSOM project.

The intent of this project was to create a prototype instrument capable of creating 3D chemical reconstructions of micro fossils. The result was a successful analytical instrument prototype. It is not by any means, perfect and so will require future upgrades to make it into a sustainable machine which can be easily replicated. The instrument will be described in three sections.

There are some planned new features, some of which have been started and will not be finished before this dissertation is completed. Other features have not been started and will be integrated when it is convenient. The demonstration of this instrument's capability marks the beginning of the research to come.

This is a project which could have kept an entire team of researchers busy with its various topics. I, however, was the sole researcher and so there are many topics which could still use a good deal of further investigation. What is presented is a best-effort to touch on each topic to the level necessary needed to create a functional instrument. I see this dissertation as a reference to my future self and to anyone else interested in constructing an instrument like the one I am about to describe. 
Chapters 1-4 are introductory and can be skipped by those familiar with FIB, SIMS and AFM. The first part of chapter 5 contains the first set of data analyzed and can be interesting to those curious about the AFM tip oscillations. The second part of chapter 5 introduces a unique application of image deconvolution to measure AFM tip oscillations from video. Chapter 6 describes some of the issues with FIB milling topdown and some predictions for a particular sample, AlTiC, are made. Chapter 7 is an introduction to the FIB-SIMS system hardware. Some discussion of upgrades and modifications are also in this chapter. Chapter 8 discusses the AFM which was integrated into the FIB-SIMS system. Chapter 9 briefly details AFM operation in air and then goes on to discuss the necessary changes from the ANSOM implementation. A detailed noise measurement analysis is presented for usage in existing experiments and for future consideration of upgrades. Chapter 10 presents the usage of the AFM and FIB without the SIMS. A simple experiment is presented where the AFM is used to characterize the crater formed by the FIB. Chapter 11 discusses the practical usage of the FIB and SIMS and summarizes the issues addressed in combining AFM-FIB-SIMS. Chapter 12 briefly discusses the project cost and schedule and then ends with future work which is planned for this AFM-SIMS instrument. 


\section{Introduction}

The earliest life on Earth likely started out as microorganisms, some of which has been discovered in the fossil record. It stands to reason that it is possible that life on other planets within our solar system might have followed a similar path. Their oldest life could then have been fossilized long ago and somehow ejected from the host planet to roam the solar system as meteors. The orbits of these meteors may have had a large eccentricity due to their method of creation and so could impact the Earth from time to time. This torturous journey may sound farfetched, but this has occurred many times.

Such a meteorite indeed fell to Earth in the Antarctic region 13000 years ago, having originated on Mars 4 billion years ago, which was then ejected 17 million years ago. It was discovered in December of 1984. Much excitement over some of the features on this meteorite named ALH84001 originated with these little features which resembled microscopic bacteria. Some structural analysis using SEM and EDS hinted that these structures might indeed be the fossilized remains of early microscopic life on Mars some 4 billion years ago during the time that there was water.

ALH84001 is named for the location where it was discovered, the Allan Hills of Antarctica. Many others, 98 of them, have been discovered and positively identified as

originating from Mars. Locations where these meteorites were found include Antarctica, France, Africa, Egypt and India.

There can be some difficulty in definitively concluding that the features on these meteorites are signs of ancient Martian life. It is helpful to consider similar terrestrial 
fossils. To be considered evidence of early life, a mineral fossil candidate should exhibit eight distinct signs. These are:

- The geology of the sample should be consistent with the particular living creatures when they were alive.

- The sample's place in the stratigraphic layer should be consistent with the type of life form present.

- The structures contained in the sample should contain some evidence of cellular morphology.

- The sample should contain colonies of structures containing evidence of cellular morphology, perhaps exhibiting additional group morphology.

- The sample should contain minerals created by living creatures, as evidenced by different mineral stoichiometry and structure than non-organic minerals.

- Isotopic analysis of the sample should reveal isotopic patterns which can be traced to biologic origin.

- The sample should contain some organic biomarkers.

- The features found on the sample should be from the sample. Any contaminants must have a good explanation.

Terrestrial fossil candidates have been evaluated using the criteria above using a variety of specialized instrumentation. Two examples include

- Scanning Electron Microscopy (SEM), utilizing backscattered electron detection and Energy Dispersive Spectroscopy (EDS) for structural characterization, relative elemental analysis and absolute elemental analysis, respectively.

- Time of Flight Secondary Ion Mass Spectrometry (TOF SIMS) for bulk isotopic and 2D chemical analysis.

AH84001 has been evaluated using many of the criteria listed above using the same specialized instrumentation as the terrestrial samples. To date, AH84001 has not been definitively proven to contain ancient life on Mars. It has also not been proven not to.

What if there was a way to deconstruct an Earthly micro fossil so that its internal 
structure and chemistry were known? This same technique could be used to do the same analysis on candidate non-terrestrial fossils and verify or refute whether there is or has been life elsewhere in our solar system. One of the pieces to this analytical technique is the topic of this dissertation. The addition of an Atomic Force Microscope to an existing SIMS system would allow for 3D isotopic and chemical analysis of the samples mentioned above. Such instruments have been constructed for well-funded laboratories.

\subsection{References}

Altermann, Wladyslaw, and Józef Kazmierczak. "Archean Microfossils: A Reappraisal of Early Life on Earth.” Research in Microbiology 154, no. 9 (November 2003): 611-17. doi:10.1016/j.resmic.2003.08.006.

Brasier, Martin D, Owen R Green, John F Lindsay, Nicola McLoughlin, Andrew Steele, and Cris Stoakes. "Critical Testing of Earth's Oldest Putative Fossil Assemblage from the 3.5 Ga Apex Chert, Chinaman Creek, Western Australia." PRECAM Precambrian Research 140, no. 1 (2005): 55-102.

Cady, Sherry L., Jack D. Farmer, John P. Grotzinger, J. William Schopf, and Andrew Steele. "Morphological Biosignatures and the Search for Life on Mars." Astrobiology 3, no. 2 (2003): 351-368.

Curnutt, J, E Gomez, and K.E Schubert. "Patterned Growth in Extreme Environments." ASTRONOMICAL SOCIETY OF THE PACIFIC CONFERENCE SERIES 420 (2009): 233-38.

E. K. Gibson Jr., F. Westall, D. S. McKay, K. Thomas-Keprta, S. Wentworth, and C. S. Romanek, "EVIDENCE FOR ANCIENT MARTIAN LIFE", Mail Code SN2, NASA Johnson Space Center, Houston TX 77058, USA.

Itay Halevy1,2, Woodward W. Fischer, and John M. Eiler, "Carbonates in the Martian meteorite Allan Hills 84001 formed at $18+/-4{ }^{\circ} \mathrm{C}$ in a near-surface aqueous environment", PNAS | October 11, 2011 | vol. 108 | no. 41 | 16899

Osterhout, Jeffrey T. "Diversity of Microfossils and Preservation of Thermally Altered Stromatolites from Anomalous Precambrian Paleoenvironments." University of Cincinnati, 2016. http://rave.ohiolink.edu/etdc/view?acc_num=ucin1470753351. 
J. William Schopf, Anatoliy B. Kudryavtsevb, Andrew D. Czaja, Abhishek B. Tripathi, "Evidence of Archean life: Stromatolites and microfossils", Precambrian Research 158 (2007) 141-155

Schopf, J. William, and Anatoliy B. Kudryavtsev. "Biogenicity of Earth's Earliest Fossils: A Resolution of the Controversy." Gondwana Research 22, no. 3-4 (November 2012): 761-71. doi:10.1016/j.gr.2012.07.003.

Steele, Andrew, David T. Goddard, Dave Stapleton, Vanessa Peters, Virginia Bassinger, George Sharples, David d Wynn-Williams, and others. "Investigations into an Unknown Organism on the Martian Meteorite Allan Hills 84001." Meteoritics \& Planetary Science 35, no. 2 (2000): 237-241.

Thomas STEPHAN, Elmar K. JESSBERGER, Christian H. HEISS, and Detlef ROST, "TOF-SIMS analysis of polycyclic aromatic hydrocarbons in Allan Hills 84001", Meteoritics \& Planetary Science 38, Nr 1, 109-116 (2003)

K.L. Thomas-Keprta, S.J. Clemett, D.S. McKay b, E.K. Gibson, S.J. Wentworth, "Origins of magnetite nanocrystals in Martian meteorite ALH84001", Geochimica et Cosmochimica Acta 73 (2009) 6631-6677

Toporski, J. K. W., A. Steele, F. Westall, R. Avci, D. M. Martill, and D. S. McKay. "Morphologic and Spectral Investigation of Exceptionally Well-Preserved Bacterial Biofilms from the Oligocene Enspel Formation, Germany." Geochimica et Cosmochimica Acta 66, no. 10 (2002): 1773-1791.

White, Lauren M., Everett K. Gibson, Kathie L. Thomas-Keprta, Simon J. Clemett, and David S. McKay. "Putative Indigenous Carbon-Bearing Alteration Features in Martian Meteorite Yamato 000593.” Astrobiology 14, no. 2 (February 2014): 170-81. doi:10.1089/ast.2011.0733. 


\section{Introduction to Focused Ion Beams (FIB)}

A Focused Ion Beam (FIB) system is one in which a very narrow beam of high energy ions is used for imaging and processing samples in similar fashion to Scanning Electron Microscopy (SEM). The beam diameter can be smaller than $5 \mathrm{~nm}$ and can be larger than $1 \mu \mathrm{m}$. Image fields of view typically range from about $1 \mu \mathrm{m}$ to $1 \mathrm{~mm}$. Ions are typically generated from a liquid metal ion source (LMIS). While many metals are commercially available for LMIS's, the most common one in use is Gallium. Gallium has a number of desirable qualities which make it the best choice of materials for LMIS's in commercial systems. A few of these desirable qualities are:

- Room temperature operation. Gallium melts at $29.8 \mathrm{deg} \mathrm{C}$ and can remain in liquid form at room temperature, below its melting point.

- Low viscosity/surface energy. This allows Ga to wet the LMIS support structure which is usually made from Tungsten. Also it readily flows from the reservoir to to replenish the field-evaporated ions leaving the tip.

- Low vapor pressure. This contributes to long life sources, which operate with little wasted material.

A typical FIB column operates between 500 and 50,000 electron Volts (eV). Ions are emitted from the source in a diverging cone, through the Beam Admittance Aperture (BAA), which determines the maximum available current. From the BAA, the ions are focused by lens 1 , which acts as the condenser lens. The ions then travel through the Beam Defining Aperture (BDA). From the BDA, the ions are focused to a small spot by lens 2 , which acts as the objective lens. 


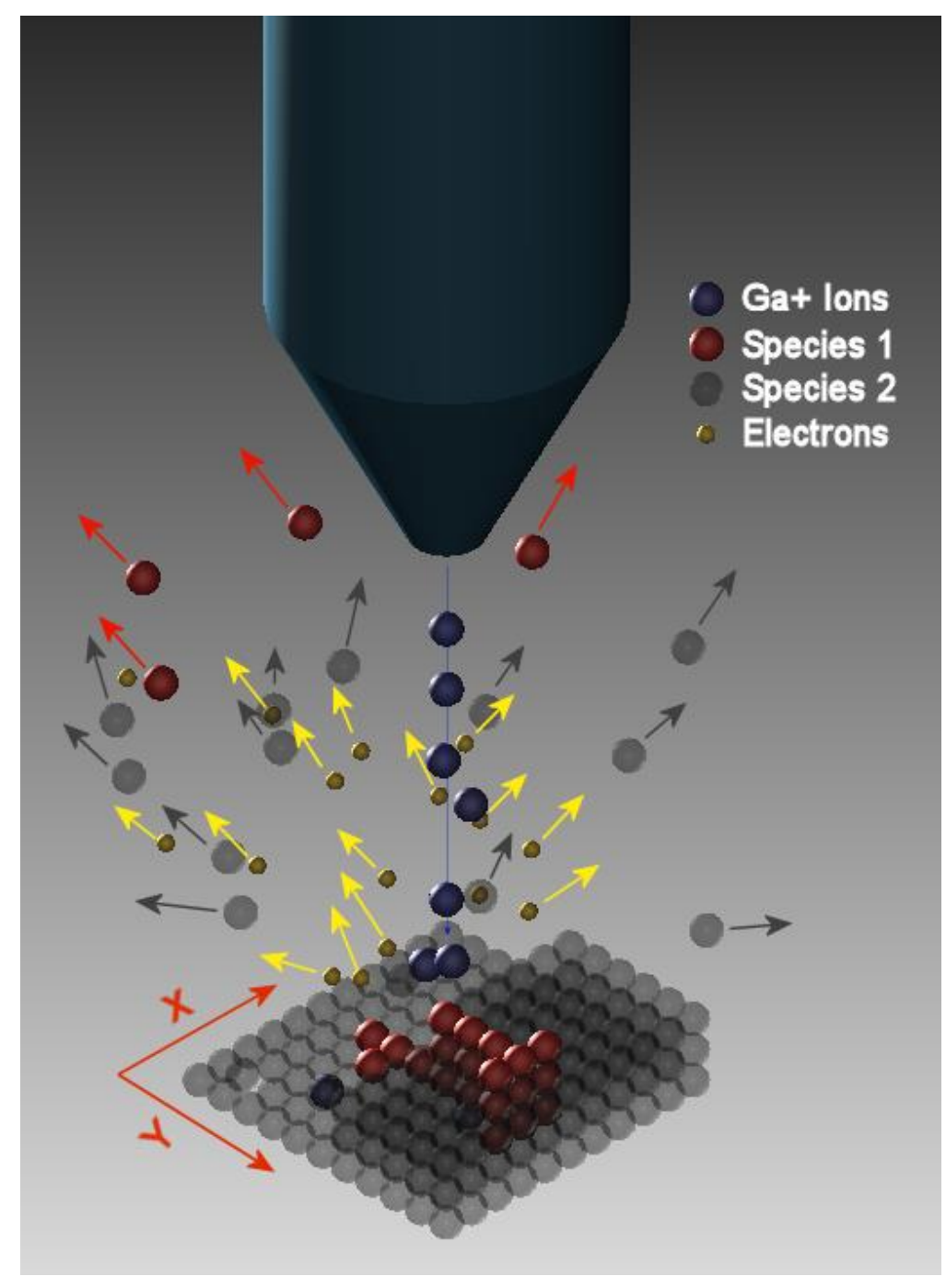

Figure 2.1. Illustration showing the milling effect of the ion beam on a two-component sample. Most ejected atoms are neutrals. Ejected ions can be positive or negative, depending on the substrate atom composition and primary ion species. The drawing attempts to illustrate the non-uniformity of the beam of incoming ions. The sample lattice can be damaged by the beam and also will contain some of the atoms from the beam as interstitials.

Two distinct modes of operation are collimated and crossover. The collimated mode is used to obtain low beam currents and is optimal for imaging at high resolution. Collimated mode is obtained by focusing lens 1 near infinity. Crossover mode is used for generating high beam currents by making lens 1 strong so that the beam is focused below the BDA, possibly forming a crossover above lens 2 . However, because of this 
crossover, the ions experience strong electrical repulsion, referred to as space charge effects. This limits the smallest possible diameter of the beam and so is mostly used for generating high currents for fast milling operations and its imaging quality is relatively

poor.

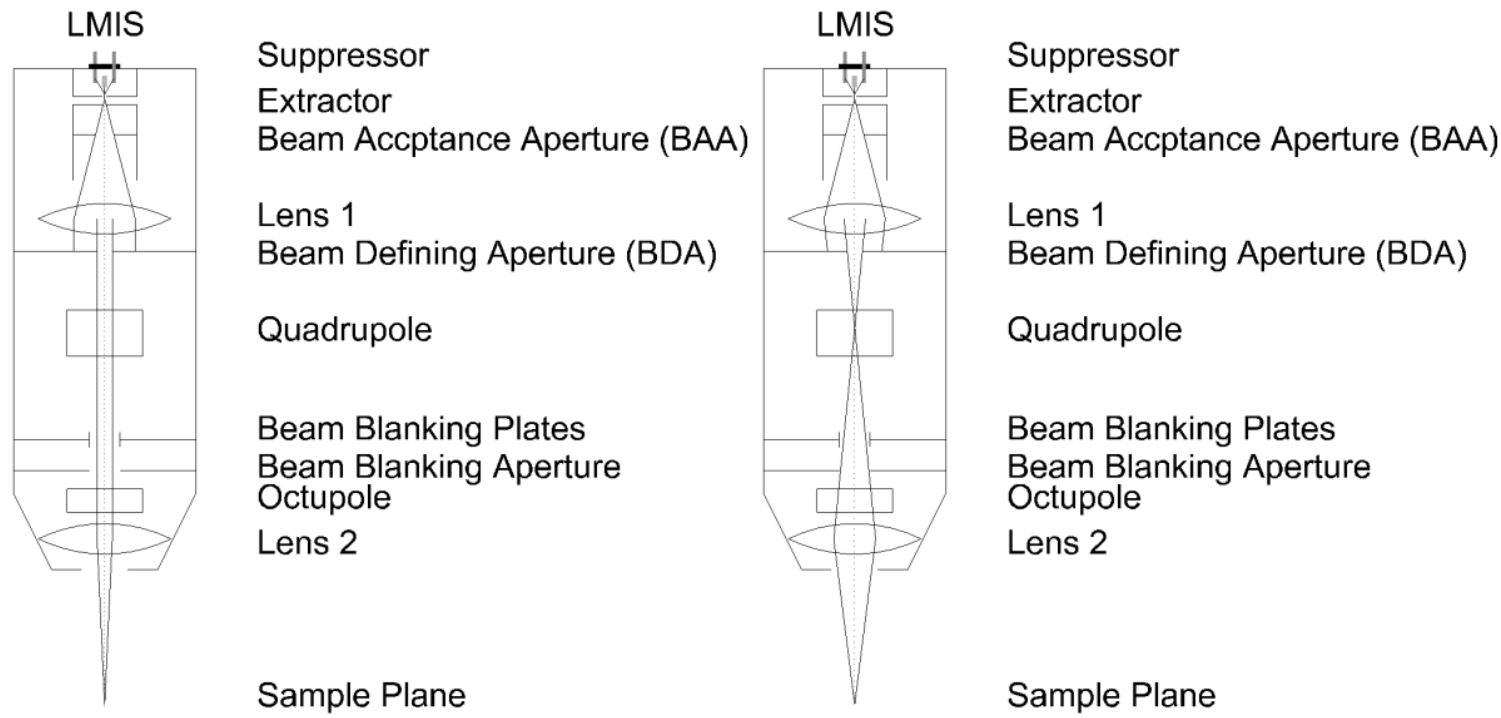

Figure 2.2. FIB column diagrams showing the collimated mode (left) and crossover mode (right) of an early FEI FIB column having a fixed BDA. Each mode is schematically represented. In collimated mode, both lens 1 and lens 2 are relatively weak and the column magnification is less than 1 , resulting in a demagnification of the virtual source. In crossover mode, lens 1 is relatively strong, focusing more ions through the BDA than in collimated mode. A crossover is necessarily created and it can be adjusted up or down to control the beam current and to a lesser degree, column magnification can be greater than 1 .

The lens elements for FIB's are usually electrostatic, three-element Einsel lenses. The focusing power of these electrostatic lenses is the same for electrons and ions, as the mass term does not appear in the lens equations. The electrical biasing of these lenses will be discussed later. Traditional light microscope systems operate so that they magnify the light rays leaving the sample. It may not be evident from Figure 2.2, but FIB and SEM columns are typically operated in such a way as to de-magnify the source of ions/electrons. This serves two basic 
functions: 1) source movements/vibrations have a small effect on image quality and 2) the image of the source is a contributor to the final spot size at the sample plane. For LMIS's the point of ion emission has been estimated to be on the order of $5 \mathrm{~nm}$, but due to the close proximity of ions leaving this point, and because of their relatively slow speed at this emission point, the effective source size is larger. This larger source size is used for calculation of the final spot size and is referred to as the virtual source size. For Gallium LMIS's it is about $50 \mathrm{~nm}$.

\subsection{Barth \& Kruit power-root-sum formula for spot size determination}

Ultimately, the user is interested in the smallest beam diameter at the sample for a given beam current. In an ideal world, the beam diameter would simply be a simple geometrical de-magnified image of the source. This means that the probe size at the sample plane would be related by a simple geometric relationship between the source semi angle, $\alpha_{\text {source, }}$ and the probe or object semi angle, $\alpha_{\text {probe }}$. Analysis by Barth $\&$ Kruit takes into account the size of the beam at the sample, $d_{\text {probe }}$, where amount of current is $50 \%$ of the total. This is usually referred to as $\mathrm{d}_{50}$.

$$
d_{\text {probe }}^{2}=\left[\left(d_{\text {spherical }}^{4}+d_{\text {diffraction }}^{4}\right)^{1.3 / 4}+d_{\text {source }}^{1.3}\right]^{2 / 1.3}+d_{\text {chromatic }}^{2}
$$

Where,

The contribution to spherical aberration is 


$$
d_{\text {spherical }}=\left(\frac{1}{2}\right)^{5 / 2} C_{s} \propto_{\text {probe }}^{3}
$$

The contribution to diffraction of the beam defining aperture (BDA), taking into account relativistic effects is

$$
\begin{aligned}
d_{\text {diffraction }}= & 0.54 \frac{\lambda}{\alpha_{\text {probe }}}=\frac{0.54}{\alpha_{\text {probe }}}\left(\frac{h}{p}\right) \\
& =\frac{0.54}{\alpha_{\text {probe }}} \frac{h}{m v} \sqrt{1-\frac{v^{2}}{c^{2}}}
\end{aligned}
$$

Where $\mathrm{h}$ is Plank's constant, $\mathrm{p}$ is the ion momentum, $\mathrm{v}$ is the ion speed and $\mathrm{c}$ is the speed of light. The contribution to the source demagnification is

$$
d_{\text {source }}=\left(\frac{2}{\pi}\right)\left(\frac{1}{\beta}\right)^{1 / 2} \frac{1}{\alpha_{\text {probe }}}
$$

Where $\beta$ is the source brightness and is related to the divergence angle of the source, $\alpha_{\text {source, }}$ and the emission current density, $\mathrm{J}$.

$$
\beta=\frac{J}{\pi \alpha_{\text {source }}^{2}}
$$

For a given geometrical configuration and applied voltage arrangement, $\beta$ is treated 
as a constant.

And the contribution to chromatic aberration is

$$
d_{\text {chromatic }}=0.34 C_{c} \frac{\Delta V}{V} \propto_{\text {probe }}
$$

$\mathrm{C}_{\mathrm{c}}$ is chromatic aberration and $\Delta \mathrm{V}$ is the energy spread in the beam energy, $\mathrm{V}$, of the $\mathrm{Ga}$ ions. Focused ion beams typically operate at or below $50 \mathrm{keV}$ which results in a very small de Broglie wavelength so it is usually ignored. The probe size calculation is thus simplified to

$$
d_{\text {probe }}^{2}=\left[d_{\text {spherical }}^{1.3}+d_{\text {source }}^{1.3}\right]^{2 / 1.3}+d_{\text {chromatic }}^{2}
$$

Virtual source size for a Gallium LMIS is $50 \mathrm{~nm}$ and the $\Delta \mathrm{V}$ of the source resulting in chromatic aberrations is $5 \mathrm{eV}$ for a $2 \mathrm{uA}$ extraction current.

Optimization of the probe size is then a minimization of the source demagnification which tends to decrease with increasing $\alpha_{\mathrm{p}}$, while chromatic and spherical contributions increase probe size with increasing $\alpha_{\mathrm{p}}$.

For small beam currents, $1 \mathrm{pA}$ to $1 \mathrm{nA}$, the column is operated so as to de-magnify the source since the lens chromatic and spherical aberrations are relatively small. The range of column magnifications ranges from 0.1 to 0.5 for these current ranges which result in probe sizes ranging between $5 \mathrm{~nm}$ and $50 \mathrm{~nm}$. Spot sizes for currents between 1 
$\mathrm{nA}$ and $5 \mathrm{nA}$ are a mix of source demagnification, spherical and chromatic aberrations. Probe sizes range from $50 \mathrm{~nm}$ to $100 \mathrm{~nm}$. For currents above $5 \mathrm{nA}$, chromatic and spherical aberration increasingly dominate the probe size so the column can be operated such that the source is slightly magnified (1.0-1.25). Probe sizes range from about 100 $\mathrm{nm}$ to $500 \mathrm{~nm}$. In general, image angles are very small, being defined by the BDA and lens 2 working distance, and ranges from about 1-10 mrad.

Table 2.1. Typical operating regimes for a Ga-based two lens FIB operating at $25 \mathrm{keV}$.

\begin{tabular}{|c|c|c|c|}
\hline $\begin{array}{c}\text { Beam Current } \\
\text { Range }\end{array}$ & $\begin{array}{c}\text { Typical d50 Spot Size } \\
\text { Range }\end{array}$ & $\begin{array}{c}\text { Typical Column } \\
\text { Magnification Range } \\
\text { (unit-less) }\end{array}$ & $\begin{array}{c}\text { Dominant } \\
\text { Contributor to Spot } \\
\text { Size }\end{array}$ \\
\hline $\mathbf{1} \mathbf{~ p A}$ to $\mathbf{1} \mathbf{~ n A}$ & 5 to $50 \mathrm{~nm}$ & 0.1 to 0.5 & $\begin{array}{c}\text { Source } \\
\text { Demagnification and } \\
\text { Chromatic }\end{array}$ \\
\hline $\mathbf{1}$ to $\mathbf{5} \mathbf{~ n A}$ & 50 to $100 \mathrm{~nm}$ & 0.5 to 1.0 & $\begin{array}{c}\text { Mix of Spherical } \\
\text { and } \\
\text { Chromatic }\end{array}$ \\
\hline $\mathbf{5}$ to $\mathbf{2 0} \mathbf{~ n A}$ & 100 to $500 \mathrm{~nm}$ & 1.0 to 1.5 & Spherical \\
\hline
\end{tabular}

The quadrupole element in Figure 2.2 is configured as a pair of flat plates and is used to correct for imperfections in the manufacturing of the column. In particular, concentricity misalignment and out of parallel alignment between lens 1 and lens 2 can be corrected using this mid-column quadrupole. The octupole is used for shaping the beam and for rastering the beam in $\mathrm{X}$ and $\mathrm{Y}$. The beam is rastered over the sample in a rectangular fashion using electrical ramps shaped like triangles. The rastered beam can be used for either imaging or for milling to remove material. More sophisticated rasters can be created by modifying the triangular electrical ramps to form complex patterns to compensate for things like ion time-of-flight, field distortions and beam-pixel overlap. 
The primary utility of a FIB system is its milling capability, also referred to as sputtering. The FIB system can be programmed to make various cut patterns in samples. Typical uses are TEM sample preparation, where thin samples, as thin as $50 \mathrm{~nm}$ or less, are cut from semiconductor and data storage wafers. These thin samples are called lamella. Skilled FIB users can routinely create lamella thinner than $10 \mathrm{~nm}$.

Figure 2.1 illustrates that the FIB milling process gives ions and electrons from the interaction of the beam with the sample. Many electrons are emitted and can be collected during milling to form an image of the surface. If the sample is repeatedly imaged with the FIB, a rectangular crater is formed. The sputtered ions can be collected and analyzed to determine the chemical makeup of the sample.

The primary ions can become incorporated into the sample, either in solution such as is the case with Aluminum, or as interstitials in the case of a crystalline sample such as Silicon. Sometimes conditions are right for the primary atoms to become neutralized and pool on the sample surface. In the case of Gallium, these pools tend to accumulate into little spheres being 10 's of $\mathrm{nm}$ in diameter. They can be seen using a second imaging beam such as a SEM, accumulating over nucleation sites or in places where the FIB doesn't readily mill them away. If conditions are even better, a nucleation site can cause the Gallium sphere to grow as large as a few microns in diameter. One of these large Gallium spheres can be seen in Figure 2.3. The outside of it has apparently begun to freeze, while repeated top-down FIB imaging has caused it to continue to grow and crack its shell. Some stress wrinkles about its "equator" are also evident, making it somewhat resemble a beach ball. Repeated SEM imaging eventually causes these stress wrinkles to go away completely. 


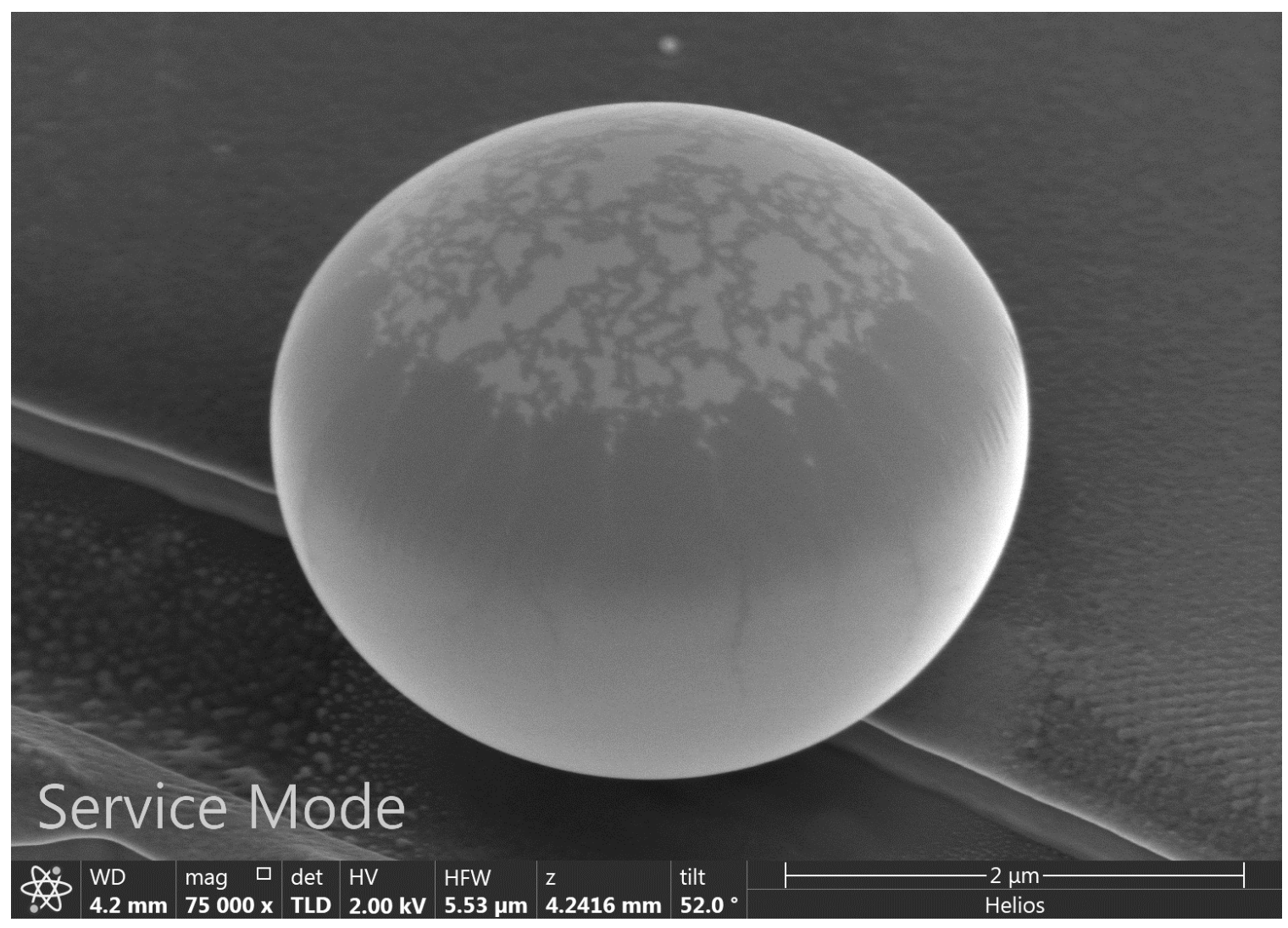

Figure 2.3. SEM image of a Ga sphere which has accumulated at a nucleation site on bare Si during repeated FIB imaging.

\subsection{Examples of FIB milling}

A beam current of $41 \mathrm{pA}$ corresponds to roughly $3.56 \times 10^{8}$ ions per second hitting the target. Thus each pixel in an image having $1024 \times 1024$ pixels acquired in one second was made with about $256 \mathrm{Ga}+$ ions. It can be difficult to predict the milling rates and ionization yields for a given sample. However, the SRIM program can be used to establish orders of magnitude. For example, for $25 \mathrm{kV}$ Ga ions on Al, the SRIM estimate for the penetration range is about $20.1 \mathrm{~nm}$. The spot size for the $41 \mathrm{pA}$ beam on the FEI FIB 611 is less than $50 \mathrm{~nm}$. Al has a density of $2.7 \mathrm{~g} / \mathrm{cm}^{3}$. From the SRIM screen shot in 
Figure 2.12, we can estimate the number of ejected $\mathrm{Al}$ atoms/ions for 256 incoming $\mathrm{Ga}+$ ions as $3.69 * 256=944 \mathrm{Al}$ atoms. Thus we can estimate that a one second FIB image, containing $1024 \times 1024$ pixels and having a field of view of $10 \mu \mathrm{m}$ to remove only 0.175 monolayers of Al. It will be necessary to image for 6 seconds to remove one monolayer and 60 seconds to remove ten (approximately $22 \mathrm{~nm}$ ).

As the sample is imaged over the same area multiple times, a rectangular crater is formed. Composite materials imaged with FIB will show regions of bright and dark, depending on the imaging mode used. SRIM can be used to estimate sputter yields for different materials and thereby determine the so-called differential rates for composite materials. Sputter rates can also be different for various grain crystal orientation in polycrystalline materials.

Differential milling rates can lead to an uneven crater floor. This can be seen in Figure 2.4 and Figure 2.5. The material is $\mathrm{Al}_{2} \mathrm{O}_{3}-\mathrm{TiC}$, a ceramic used in making hard drive heads. The ratio of $\mathrm{Al}_{2} \mathrm{O}_{3}$ to $\mathrm{TiC}$ is about 3 , by volume. The material surface is polished to a roughness on the order of $1 \mathrm{~nm}$ rms roughness $(\mathrm{Rq})$. SRIM sputter estimates indicate that $\mathrm{Al}_{2} \mathrm{O}_{3}$ should mill faster than the TiC. This is consistent with the image of the crater at 45 degrees. Although it is difficult to say with certainty, the material comprising the smaller volume, TiC (dark regions inside the crater), appears to be sticking out of the surrounding $\mathrm{Al}_{2} \mathrm{O}_{3}$. In Figure 2.5, to the right of the $\mathrm{Al}_{2} \mathrm{O}_{3}-\mathrm{TiC}$ is nearly pure $\mathrm{Al}_{2} \mathrm{O}_{3}$. The $\mathrm{Al}_{2} \mathrm{O}_{3}$ clearly mills faster than the ceramic material to the left, and causes the AlTiC to mill faster near the interface. This can be attributed to the enhanced sputter rates at steeper angles. 


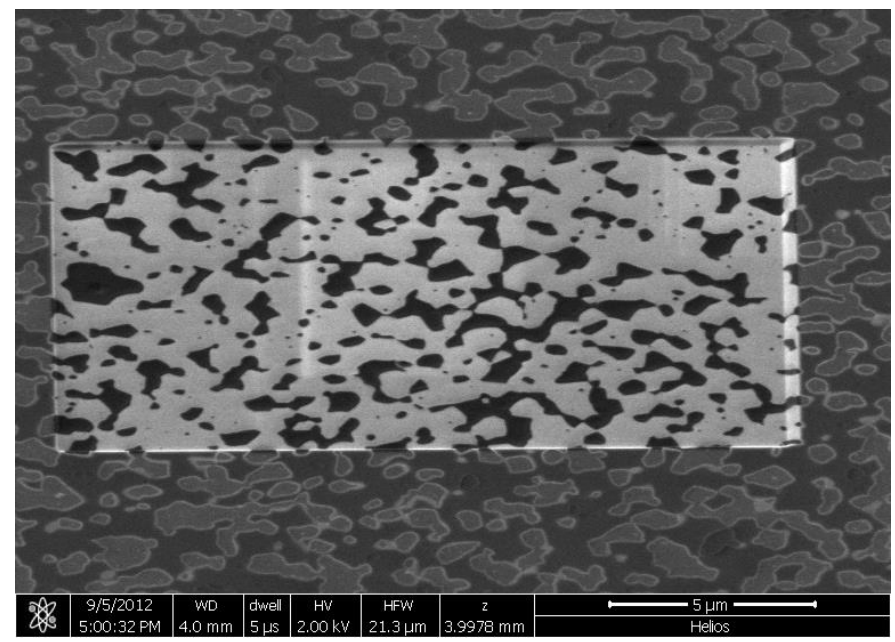

Figure 2.4. SEM image at 52 degrees of the crater formed during repeated FIB imaging. This is the result of acquiring 50 images using $30 \mathrm{kV}, 80 \mathrm{pA}$ at $30 \mu$ s pixel dwell. Approximately $3 \mathrm{~nm}$ per FIB image was removed.

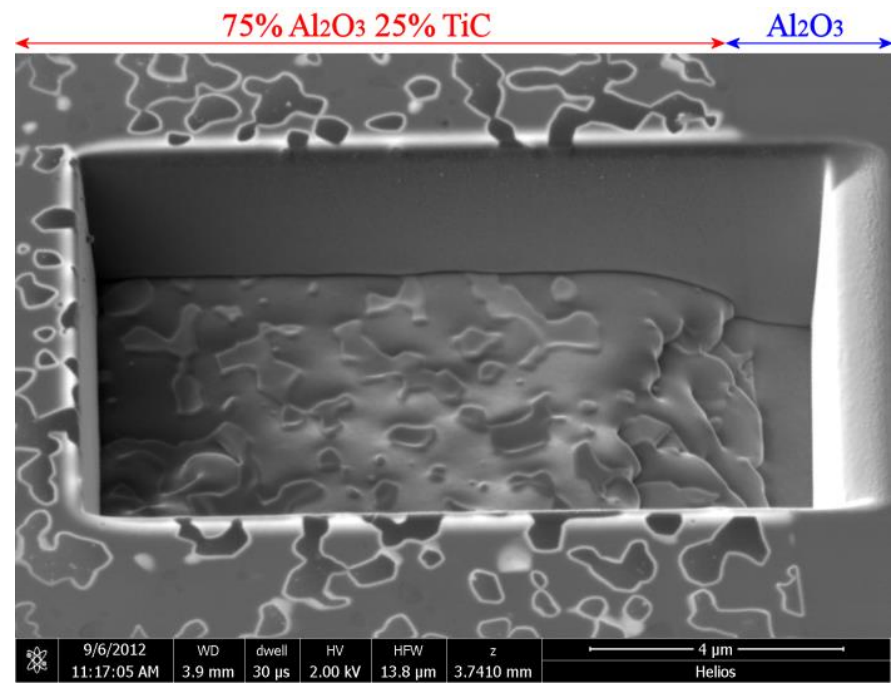

Figure 2.5. SEM image at 45 degrees of the crater formed during repeated FIB imaging. This is the result of acquiring 50 images using $30 \mathrm{kV}, 790 \mathrm{pA}$ at $30 \mu$ sixel dwell. Note that the crater bottom is uneven. The $\mathrm{Al}_{2} \mathrm{O}_{3}$ material on the right has milled faster and has caused the $\mathrm{Al}_{2} \mathrm{O}_{3}$ - $\mathrm{TiC}$ interface to mill even faster. Approximately $45 \mathrm{~nm}$ per FIB image was removed. Also note that the side-walls of the crater is likely covered with re-deposited material since none of the underlying sample structure is visible. 
Other artifacts evident in Figure 2.4 and Figure 2.5 include a slight sample drift from left to right, leading to the walls at the right being sloped more than the left ones. Sources for this drift typically come from the stage, thermal gradients and from charging. Stage drift typically starts out fast and asymptotically approaches a steady state. If the stage has been recently disassembled, loose bearing races can contribute. It is important that they are preloaded and lubricated adequately. Thermal gradients are usually due to difference in temperature between the elements making up the chamber, stage and FIB column. For this reason, systems in industrial and laboratory settings are usually left running continuously. Sample exchanges will still introduce small thermal gradients but these should come to equilibrium within an hour for even critical applications. Charging is sometimes unavoidable for insulating samples. Thin metallic coatings on the sample will offer some charge control but will likely fail once the FIB has milled into the sample. Conducting samples will charge up and drift if there is a poor electrical connection between the sample and stage. Ultimately, some drift can affect certain applications and cannot be removed. In this case, schemes in which the milling is periodically corrected with a fiducial are very common. 


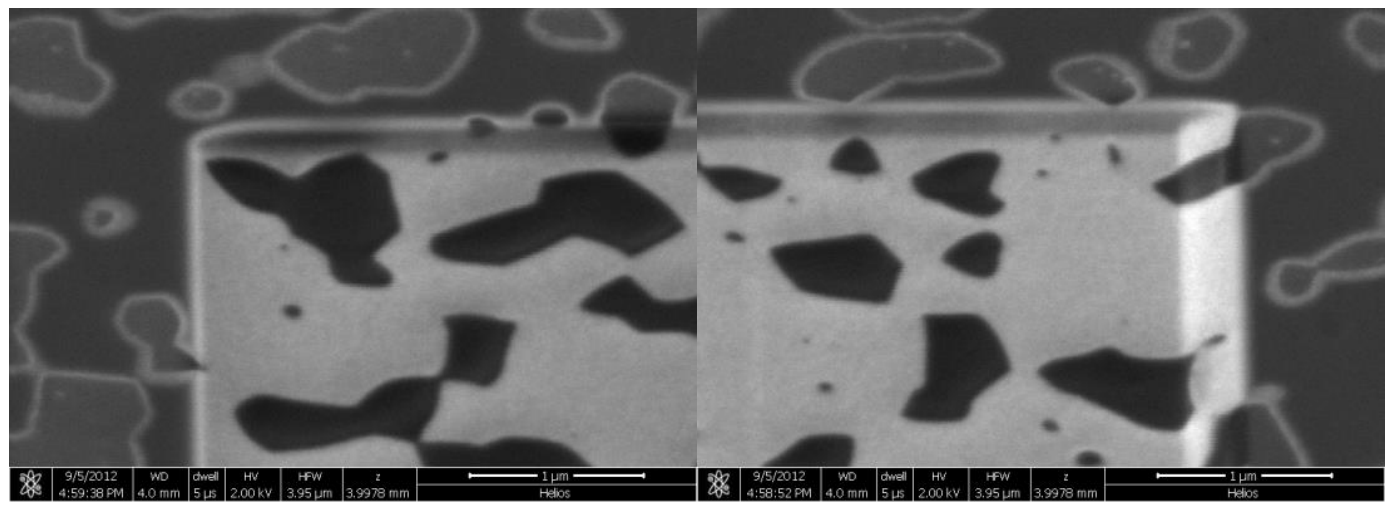

Figure 2.6. SEM images of the $80 \mathrm{pA}$-milled crater in Figure 2.4, showing the FIB drift from rightto-left during milling. It is more likely that the stage drifted from left to right during the mill. (left) Top-left corner of the crater. (right) Top-right corner of the crater clearly showing the ramp created by the drift.
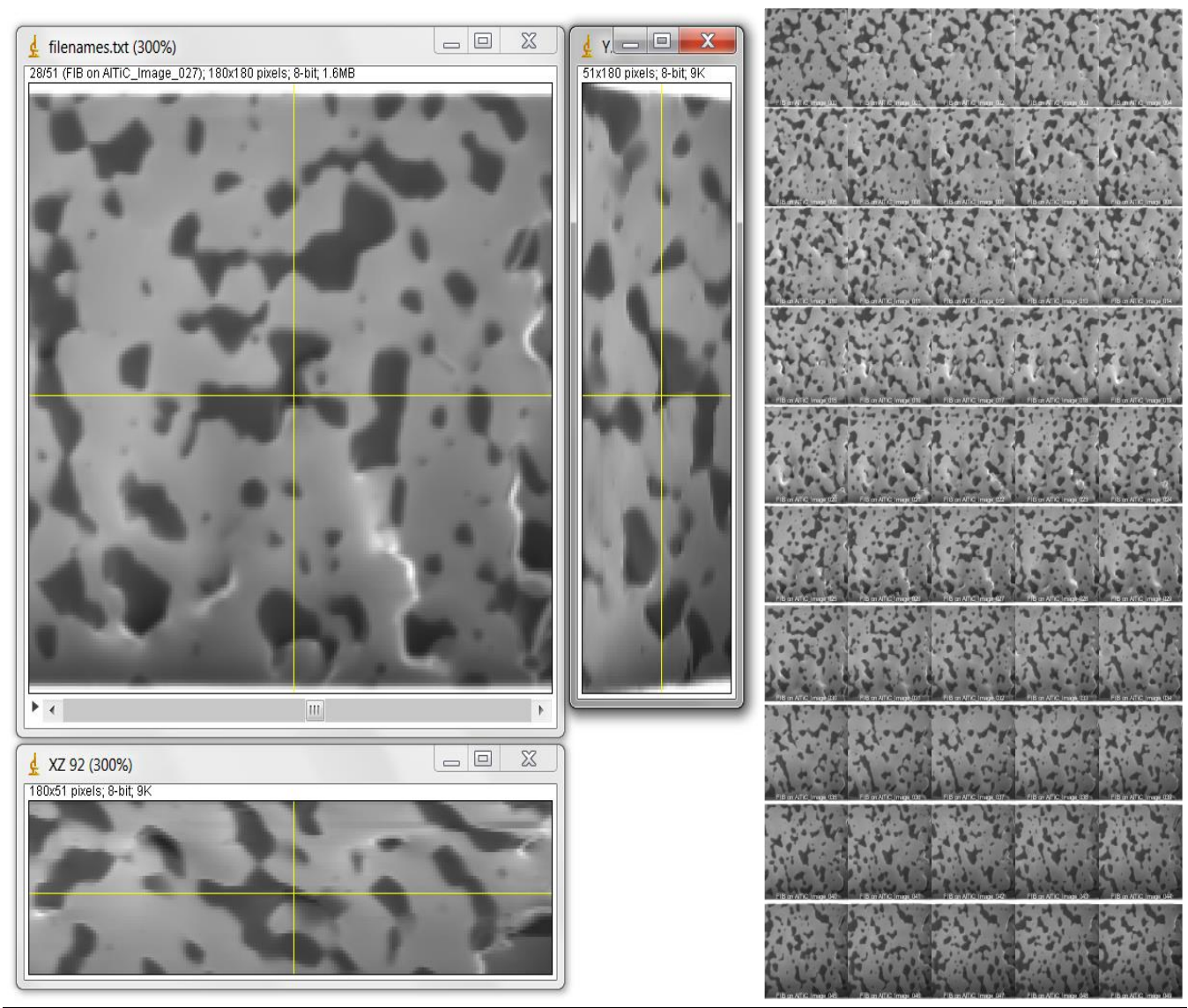

Figure 2.7. Image stack constructed into a 3D volumetric rendering using ImageJ. 


\subsection{Electron and ion FIB imaging modes}

Figure 2.1 illustrates that ions and electrons are ejected during the FIB raster. Two or more electrons per sputtered atom are generated. The actual number is dependent on the sample being imaged. Thus for the one-second image in the example above, 2000 or more electrons are generated for each pixel. Because electrons have low mass, they can be collected very efficiently. On the other hand, only about one in one hundred (or less) sputtered atoms are ionized. I one-second image will have a lot of dark pixels and the electronically amplified image will appear grainy.

Images are formed by reading the output of a Channel Electron Multiplier (CEM). A positive bias causes it to pull in the electrons emitted during milling. A negative bias attracts positive ions instead. At the end of the CEM, only electrons come out as a current which is then amplified and interpreted as a video signal. Some image pairs, electron and ion, of the same region on some samples follow in Figure 2.8, Figure 2.10 and Figure 2.11. They were all collected using $25 \mathrm{keV}$ primary beam, $75 \mathrm{pA}$ beam current, using a 10 us pixel dwell. 


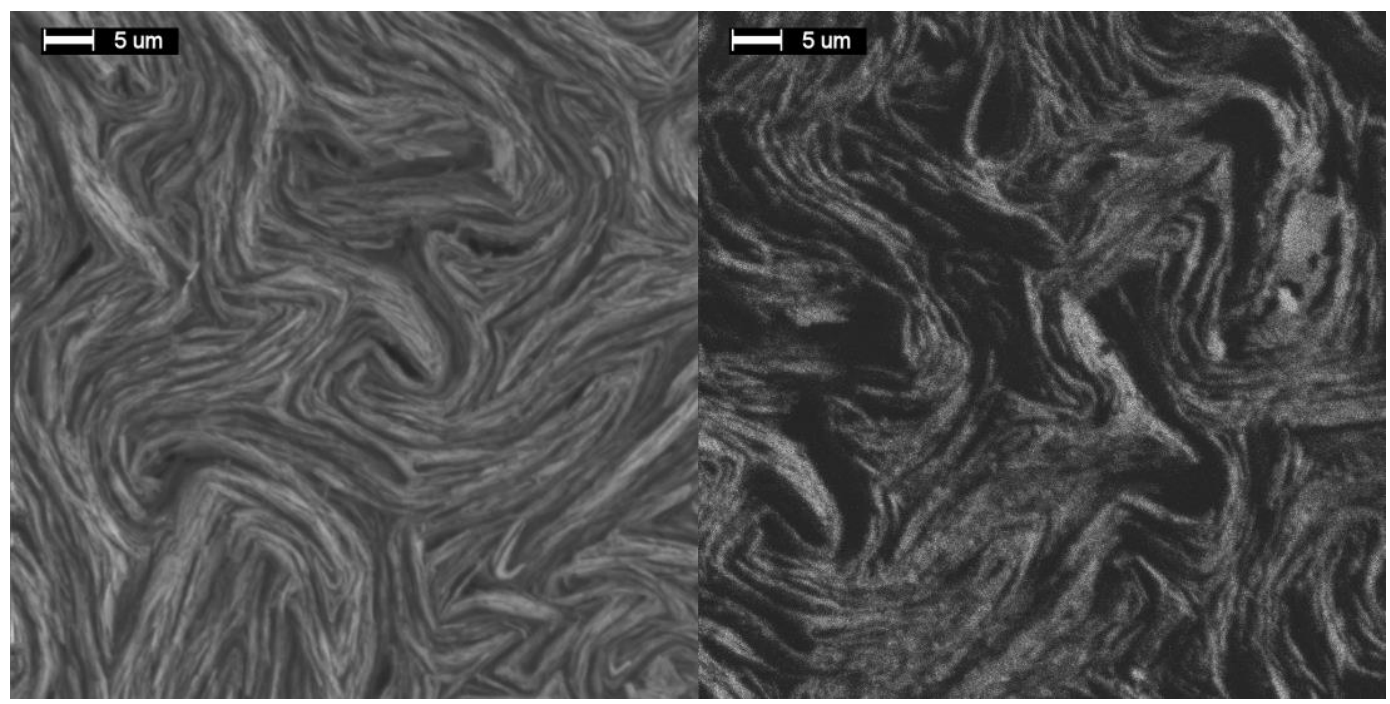

Figure 2.8. FIB electron (left) and ion (right) images of pencil lead which is commonly used for FIB column alignments. Image time is 10 seconds at 75 pA. The images are slightly shifted w.r.t. each other, since the image appears to shift to the left and down when the detector is in ion mode. The shift is larger than the field of view so beam shift is used to move the ion image to the right. They do not match up perfectly, demonstrating that it can be difficult for a human to match random features. A simple computer algorithm, cross-correlation, can easily find the matching features between these two images.

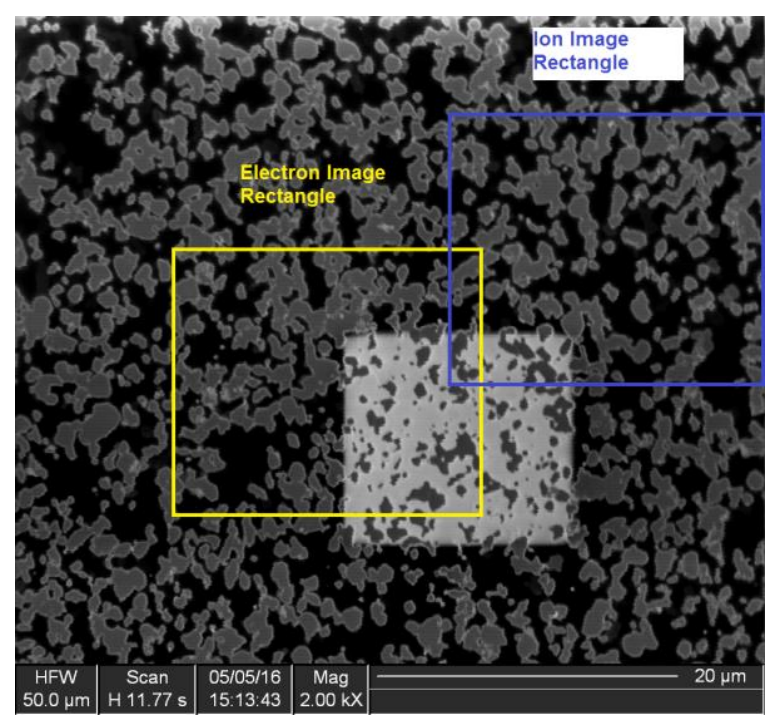

Figure 2.9. Low magnification image illustrating the typical amount of shift between electron and ion images due to the polarity of the CEM collector voltage. The shift shown here is approximately $18 \mu \mathrm{m}$ in $\mathrm{X}$ and $8 \mu \mathrm{m}$ in $\mathrm{Y}$. 


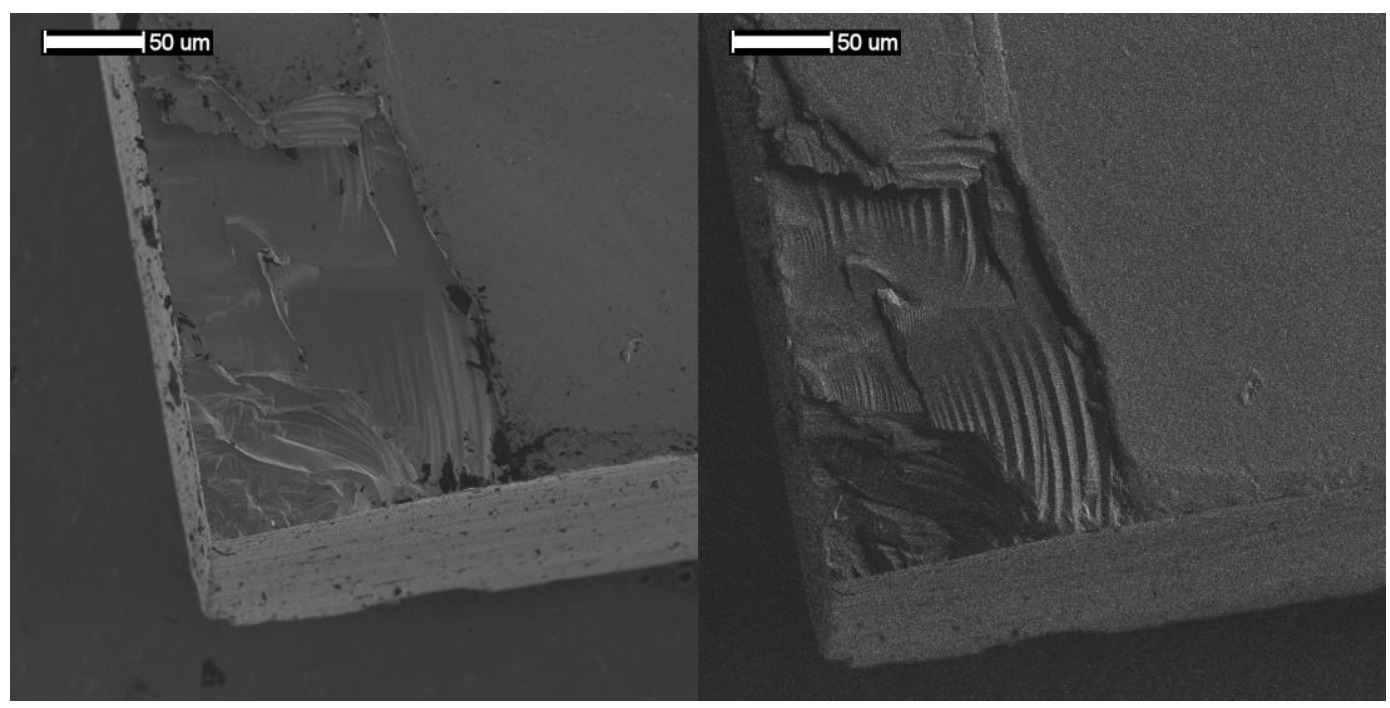

Figure 2.10. FIB images of Au on $\mathrm{C}$ on a chipped corner. Note the superior signal to noise on the electron image (left). The ion image (right) exhibits a stronger shadowing effect, particularly in the chipped region near the micron marker. Both images appear to be illuminated from the left because the CEM is located to the right.

\subsection{Charging effects on FIB imaging}

For many samples, electron imaging gives superior image quality. These secondary electron images are characterized by their high contrast and low noise. However, there are materials which cannot be reliably imaged in electron mode. One of the simplest examples is a dust particle resting on a conductive substrate such as Silicon. Electrons leaving the particle cause it to become positively charged. It may appear dark as a result. As the particle is imaged over and over, the charge build up may discharge in short bursts, causing bright regions. Due to the distortions created by its trapped charge, the particle may appear to oscillate or change shape during this process. Non uniform fields from the trapped charge may cause distortions in other parts of the image area. In extreme cases, the charge build up will cause the particle to spontaneously fly from the substrate and land millimeters away on another region of the sample. Figure 2.11 
demonstrates some of the phenomenon mentioned above.

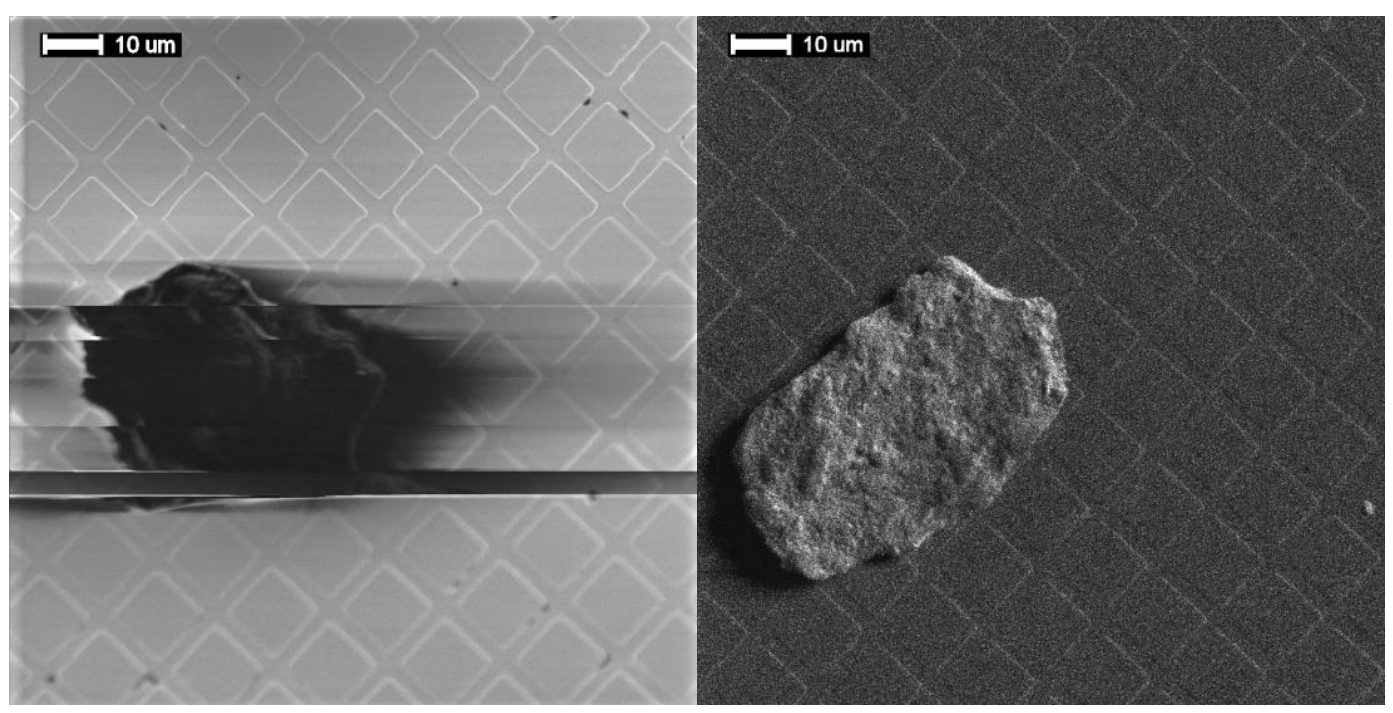

Figure 2.11. FIB images of a dust particle using detected electrons and ions. The particle in the electron image (left) has become so strongly charged that its image and even the surrounding Si grid appears distorted. The Si grid is out of focus in the bottom third of the image. The particle in the ion image (right) appears to be unaffected by the beam and it might even be difficult to convince yourself that these are images of the same feature.

\subsection{Theory of ion beam-sample interactions}

Chemical bonds are on the order of a few eV for most elements and compounds and are characterized by their lattice binding energy. Removing an atom from its spot inside a solid typically requires breaking more than one bond, which then raises the threshold for removal to tens of $\mathrm{eV}$. This is characterized by the displacement energy and is more or less representative of an atom's average neighbor count and separation distance. A similar argument for the number of neighbors being more than one, but less than inside the bulk predicts that the energy required to remove an atom from the surface is typically more than the lattice binding energy but is less than the displacement energy. 
This is usually characterized by the surface binding energy. Thus $25 \mathrm{kV}$ Ga ions have enough energy to break chemical bonds of all known chemical elements and compounds. Ions striking the sample surface will penetrate, interacting with each sample atom as if it is alone in free space. This is termed the binary collision model. As the ion loses energy due to collisions, it follows a tortuous path through the material and finally stops somewhere inside. The cascade caused by this ion leads to sample damage, knock-on dislocations, sputtering, etc. The interaction is also responsible for the generation of electrons, ions and photons which is important for the detection and measurement of the physical processes occurring during this interaction. The original primary Ga atoms, many of which are now in the sample, may later be sputtered from the sample and can be detected using SIMS. The peaks resulting from Ga sputtering is frequently used to calibrate the mass setting in the SIMS instrument.

It is fortunate that ions $\mathrm{Ga}$ are relatively massive and so relativistic effects are very small. Thus classical theory is used to describe ion interactions with samples. Because of the large number of possible ion/sample combinations, it is advisable to consider the creation of a universal formula to describe their interactions. Ziegler et al have created such a technique and it is in a software package named SRIM, which stands for the Stopping Range of Ions in Matter.

When $25 \mathrm{keV}$ Ga ions enter a sample, they interact little with the sample's electrons, effectively interacting with nuclei more often. While inside the sample, these ions are diverted from their incident path only slightly until their kinetic energy drops below some threshold which it then begins dropping quite fast.

Stopping power is the term used to describe the energy loss cascade in the ion- 
sample interaction. Stopping power is correlated to the sample density and is thus related to the periodic nature of the elements.

Sputtering yield, Y, is dependent on the source and sample species and can be roughly correlated with the sample melting point. Energy transfer is another factor. The better matched the source and target species result in higher sputter yields. Sputter yield can then be related to the periodic nature of the elements.For Scanning Electron Microscopes (SEM's) the Secondary electron yield, SE, is roughly correlated to the sample proton count, $\mathrm{Z}_{2}$. This is the opposite for Scanning Ion Microscopes (SIM's).

Table 2.2. Approximate behavior for electron and ion yields with increasing sample atomic number, $Z_{2}$. Electron yield increases in SEM and decreases in FIB with increasing $Z_{2}$.

\begin{tabular}{|c|c|c|c|}
\hline Primary Species & $\mathbf{Z}_{2}$ & $\gamma_{\mathrm{e}}$ & $\gamma_{\text {ion }}$ \\
\hline e- & $\uparrow$ & $\uparrow$ & N.A. \\
\hline $\mathbf{G a}^{+}$ & $\uparrow$ & $\downarrow$ & $\uparrow$ \\
\hline
\end{tabular}




\subsection{Stopping Range of Ions in Matter (SRIM)}

Software accompanying the SRIM book can provide useful insight into ion-solid interactions. In general, it provides good estimates for things like milling rates of single and multi-component samples. It provides estimates of secondary ion creation. Although it does not provide estimates for secondary electron creation, it does reveal some insight into the behavior shown in Table 2.2. Ion penetration with increasing $Z_{2}$ tends to decrease, leading to increased secondary electron yield.

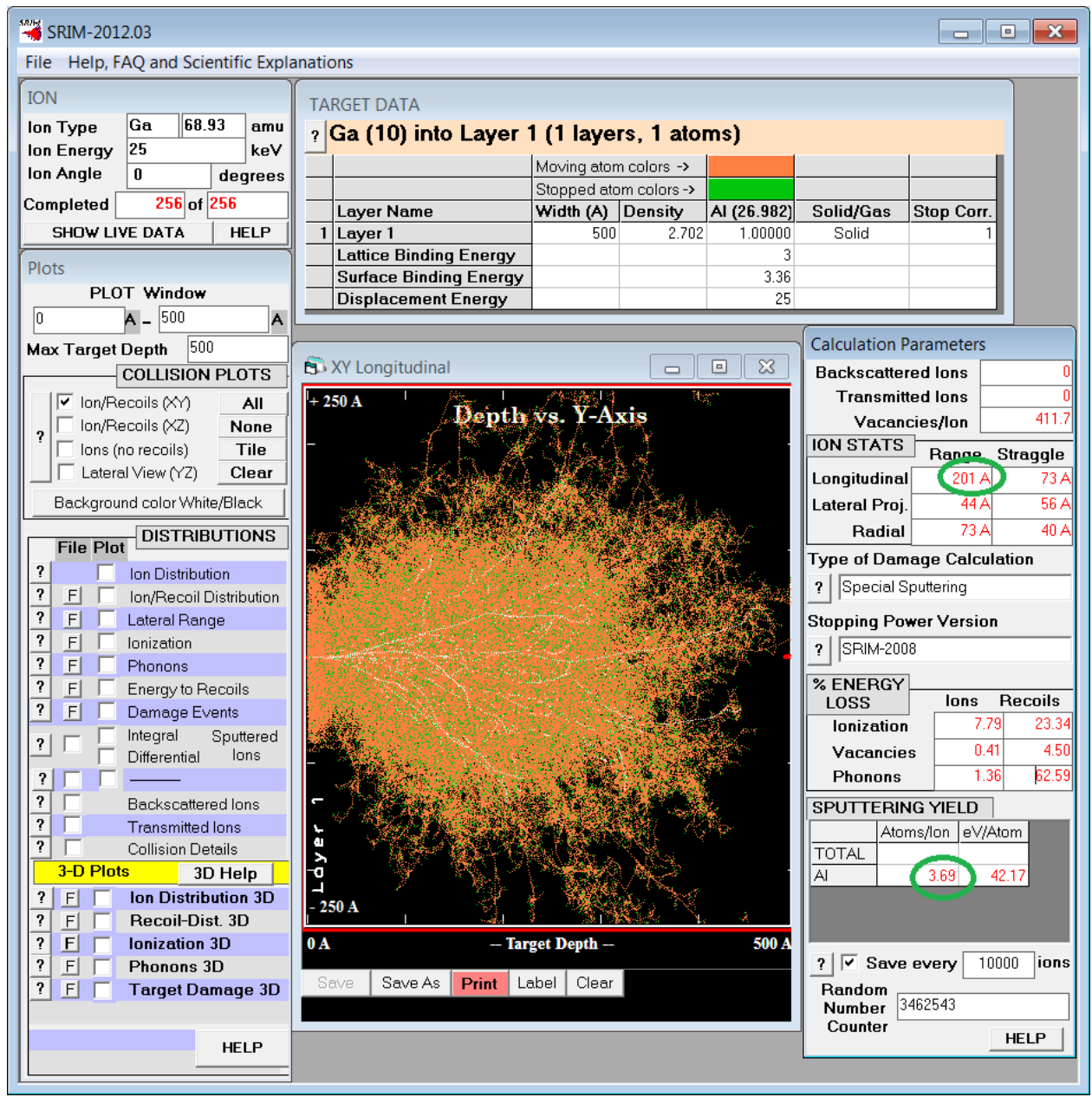

Figure 2.12. The SRIM program can be used to estimate sputter yields for FIB systems. For the example shown, SRIM predicts that 3.69 Al atoms will be sputtered for each incoming $\mathrm{Ga}+$ ion at 25 $\mathrm{kV}$ and normal incidence. 


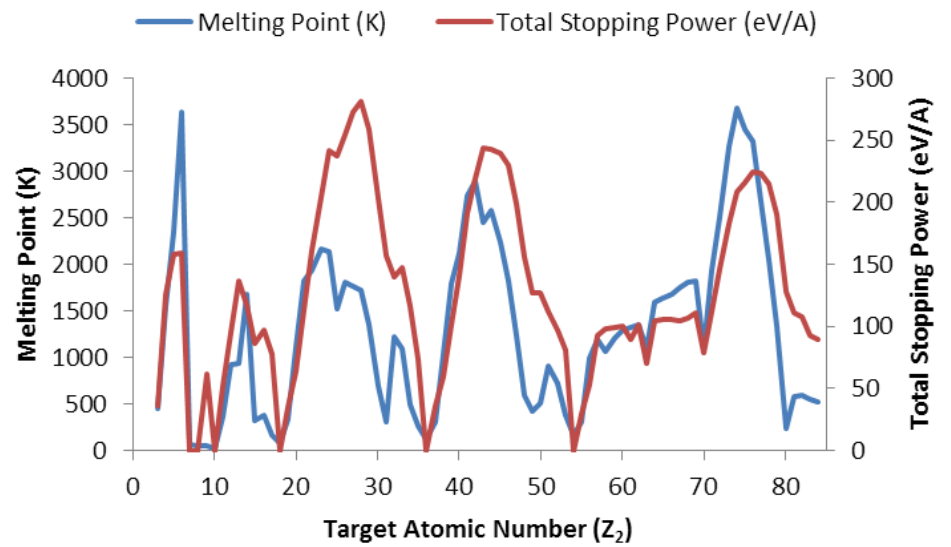

Figure 2.13. Stopping power computed using SRIM for $25 \mathrm{kV}$ primary beam $\mathrm{Z1}=\mathrm{Ga}+$ for elements 3-84 at normal incidence. Stopping power and melting point are roughly correlated.

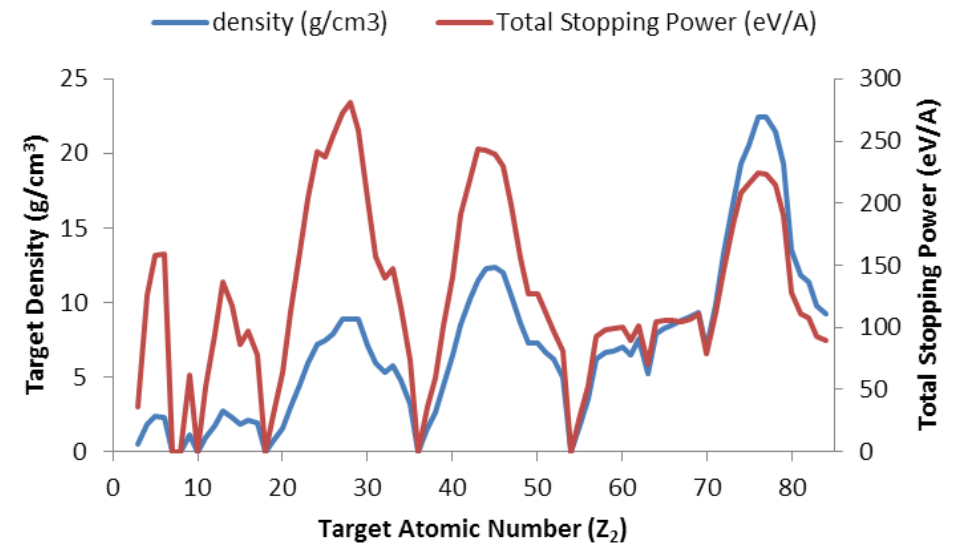

Figure 2.14. Stopping power computed using SRIM for $25 \mathrm{kV}$ primary beam $\mathrm{Z1}=\mathrm{Ga}+$ for elements 3-84 a normal incidence. Stopping power and target density show some relatively strong correlations over certain intervals. 


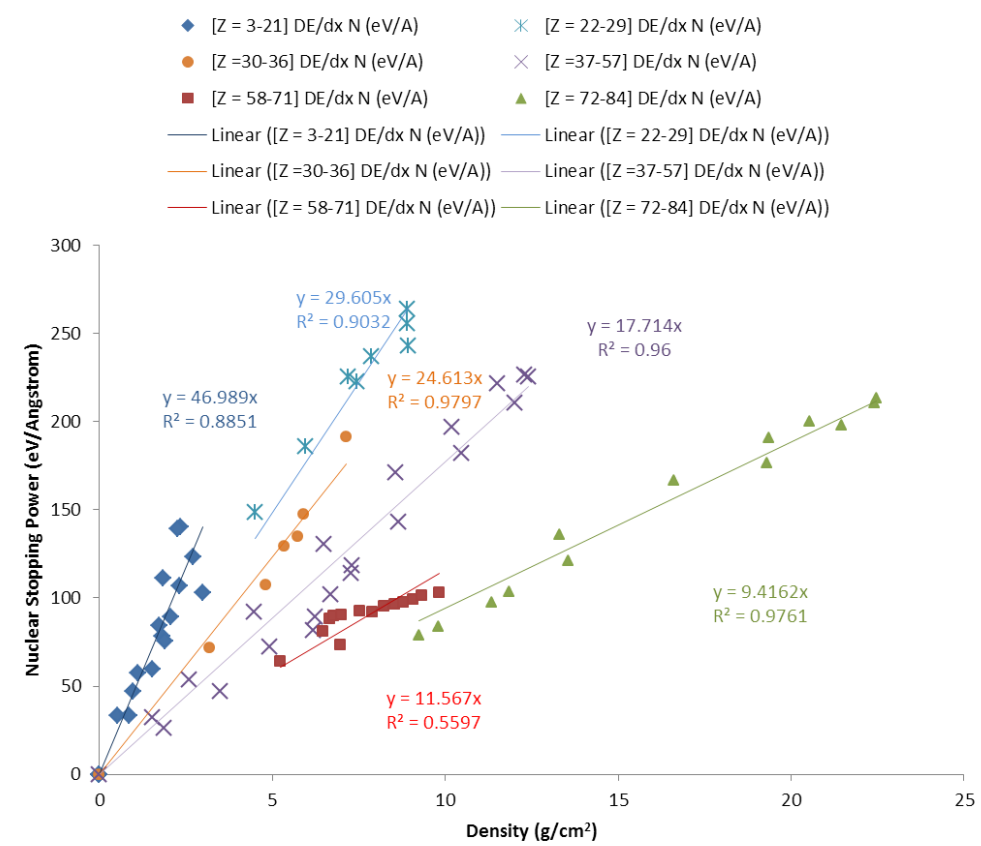

Figure 2.15. There are relatively strong correlations between nuclear stopping power and target density over certain ranges in sample atomic number (Ga $25 \mathrm{keV}$ primary beam).

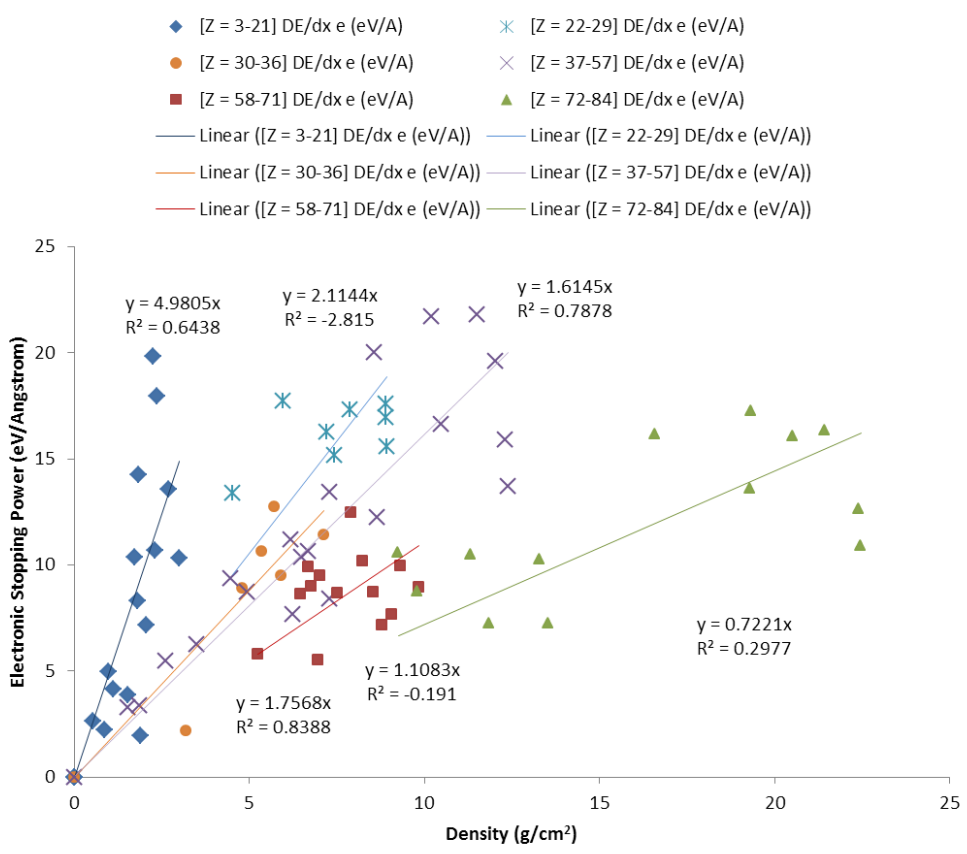

Figure 2.16. Correlations are more tenuous for electronic stopping power using the same atomic number ranges as for the nuclear in Figure 2.15 (Ga $25 \mathrm{keV}$ primary beam). 


\subsection{References}

Ando, Masaaki, and Julius J. Muray. "Spatial Resolution Limit for Focused Ion-Beam Lithography from Secondary-Electron Energy Measurements." Journal of Vacuum Science \& Technology B: Microelectronics Processing and Phenomena 6, no. 3 (1988): 986-988.

Baroody, E. M. "A Theory of Secondary Electron Emission from Metals.” Physical Review 78, no. 6 (1950): 780.

Barth, J. E, and P Kruit. "Addition of Different Contributions to the Charged Particle Probe Size.” OPTIK -STUTTGART-101, no. 3 (1996): 101-9.

Bigler, Jeff. "Periodic-Table.xls," n.d. http://www.mrbigler.com/documents/PeriodicTable.xls.

Castaldo, V., C.W. Hagen, and P. Kruit. "Simulation of Ion Imaging: Sputtering, Contrast, Noise." Ultramicroscopy 111, no. 8 (July 2011): 982-94.

doi:10.1016/j.ultramic.2011.03.019.

Giannuzzi, L.A, and M Utlaut. "Non-Monotonic Material Contrast in Scanning Ion and Scanning Electron Images.” Ultramicroscopy Ultramicroscopy 111, no. 11 (2011): 156473.

Giannuzzi, Lucille A, and F. A Stevie. Introduction to Focused Ion Beams:

Instrumentation, Theory, Techniques, and Practice. New York: Springer, 2005.

Hagen, C. W., E. Fokkema, and P. Kruit. "Brightness Measurements of a Gallium Liquid Metal Ion Source." Journal of Vacuum Science \& Technology B: Microelectronics and Nanometer Structures 26, no. 6 (November 2008): 2091-96. doi:10.1116/1.2987958.

Harriott, L. R. "Focused ion beam secondary ion mass spectrometry: Ion images and endpoint detection." J. Vac. Sci. Technol. B Journal of Vacuum Science \& Technology B: Microelectronics and Nanometer Structures 7, no. 2 (1989): 181.

Ishitani, T, T Yamanaka, K Inai, and K Ohya. "Secondary Electron Emission in Scanning Ga Ion, He Ion and Electron Microscopes." Vacuum Vacuum 84, no. 8 (2010): 1018-24.

Kanaya, Koichi, and Susumu Ono. "Secondary Electron Emission from Solid Surfaces by Bombardment with Charged Particles." Jpn. J. Appl. Phys. Japanese Journal of Applied Physics 13, no. 6 (1974): 944-49. 
Khursheed, Anjam. Scanning Electron Microscope Optics And Spectrometers. World Scientific, 2011.

Ohya, K, K Inai, H Kuwada, T Hayashi, and M Saito. "Dynamic Simulation of Secondary Electron Emission and Charging up of an Insulating Material." Surface \& Coatings Technology. 202, no. 22 (2008): 5310.

Ohya, K, and T Ishitani. "Simulation Study of Secondary Electron Images in Scanning Ion Microscopy." Nuclear Instruments and Methods in Physics Research Section B: Beam Interactions with Materials and Atoms Nuclear Instruments and Methods in Physics Research Section B: Beam Interactions with Materials and Atoms 202 (2003): 305-11.

Ohya, K., T. Yamanaka, K. Inai, and T. Ishitani. "Comparison of Secondary Electron Emission in Helium Ion Microscope with Gallium Ion and Electron Microscopes." Nuclear Instruments and Methods in Physics Research Section B: Beam Interactions with Materials and Atoms 267, no. 4 (February 2009): 584-89.

doi:10.1016/j.nimb.2008.11.003.

Ohya, K. "Monte Carlo Simulation of Heavy Ion Induced Kinetic Electron Emission from an Al Surface." Nuclear Instruments \& Methods in Physics Research. Section B, Beam Interactions with Materials and Atoms. 195, no. 3 (2002): 281.

Ohya, K. "Comparative Study of Target Atomic Number Dependence of Ion Induced and Electron Induced Secondary Electron Emission." Nuclear Instruments \& Methods in Physics Research. Section B, Beam Interactions with Materials and Atoms. 206, no. 1 (2003): 52.

Ohya, Kaoru, and Tohru Ishitani. "Monte Carlo Study of Secondary Electron Emission from $\mathrm{SiO} 2$ Induced by Focused Gallium Ion Beams." Applied Surface Science 237, no. 1-4 (October 2004): 602-6. doi:10.1016/j.apsusc.2004.06.133.

Orloff, Jon, L W Swanson, and M Utlaut. "Fundamental Limits to Imaging Resolution for Focused Ion Beams." Journal of Vacuum Science \& Technology. B, Microelectronics Processing and Phenomena 14, no. 6 (1996): 3759.

Orloff, Jon. Handbook of Charged Particle Optics. Boca Raton, Fla.: CRC Press, 1997.

Sakai, Y., T. Yamada, T. Suzuki, and T. Ichinokawa. "Contrast Mechanisms of Secondary Electron Images in Scanning Electron and Ion Microscopy." Applied Surface Science 144 (1999): 96-100. 
Sakai, Y, T Yamada, T Suzuki, T Sato, H Itoh, and T Ichinokawa. "Contrast Mechanisms in Scanning Ion Microscope Imaging for Metals." APPLIED PHYSICS LETTERS 73, no. 5 (1998): 611-13.

Sigmund, Peter, and Andreas Schinner. "Binary Theory of Electronic Stopping." Nuclear Instruments and Methods in Physics Research Section B: Beam Interactions with Materials and Atoms 195, no. 1 (2002): 64-90.

Sigmund, Peter. "Stopping Power in Perspective." Nuclear Instruments and Methods in Physics Research Section B: Beam Interactions with Materials and Atoms 135, no. 1 (1998): 1-15.

Smith, Roger. Atomic and Ion Collisions in Solids and at Surfaces: Theory, Simulation and Applications. Cambridge, U.K.; New York, NY, USA: Cambridge University Press, 1997.

Sternglass, E. J. "Theory of Secondary Electron Emission by High-Speed Ions.” Phys. Rev. Physical Review 108, no. 1 (1957): 1-12.

Tsipinyuk, Ba, and Vi Veksler. "Energy Spectra of Secondary Ions and Secondary Ion Emission (SIE) Mechanisms." Vacuum 29, no. 4-5 (January 1979): 155-67. doi:10.1016/S0042-207X(79)80747-2.

Volkert, Cynthia A., and Andrew M. Minor. "Focused Ion Beam Microscopy and Micromachining." MRS Bulletin 32, no. 05 (2007): 389-399.

Yi, Whikun, Taewon Jeong, Segi Yu, Jeonghee Lee, Sunghwan Jin, Jungna Heo, and J M Kim. "Study of the Secondary-Electron Emission from Thermally Grown SiO2 Films on Si." Thin Solid Films. 397, no. 1 (2001): 170.

Ziegler, J. F, J. P Biersack, and Matthias D Ziegler. SRIM, the Stopping and Range of Ions in Matter. Chester, Maryland: SRIM Co., 2008. 


\section{Introduction to Secondary Ion Mass Spectrometry (SIMS)}

Recall that the number of electrons and ions released during the imaging process is material dependent. The effect on the image contrast can be dramatic for electron images. Ion images tend to display the same dull gray distribution on differing materials and it can be difficult to discern regions of differing material composition. Typically only the material boundaries themselves are evident, as ion images are sensitive to topography. Topographic contrast can be useful for certain applications. This makes ion images not so useful for material analysis in this case. Still, we have shown using SRIM, that the number of atoms sputtered during the imaging process is material dependent.

In order to improve ion image material contrast, the ions sputtered at each pixel can be sent through a mass filter. Ions are filtered according to their mass to charge ratio and make it to a Channel Electron Multiplier (CEM). Thus, an image can be acquired in which the bright regions represent the amount of the single species the SIMS detector is set for. The surrounding pixels will be black. Two dimensional chemical maps can be made by imaging over the same area multiple times, each with the SIMS detector set for a unique species.

As was stated earlier, less than one in one hundred atoms removed during FIB milling is ionized. This, combined with the less than unity collection efficiency, leads to long acquisition times. Another consequence is that the minimum practical volume element, or voxel, is also bounded by the ionization fraction times the collection efficiency. These factors are currently unknown for our instrument, but we expect voxels on the order of 25-50 $\mathrm{nm}$ on a side. 


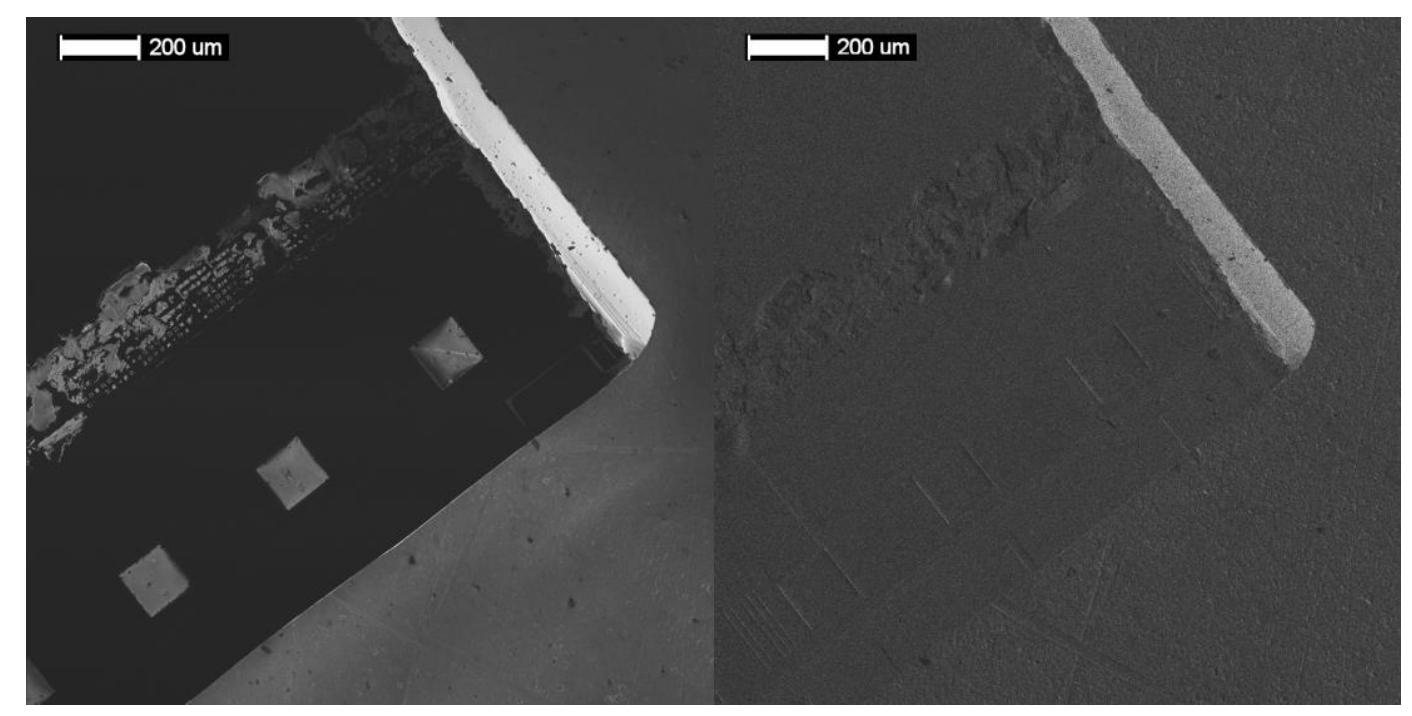

Figure 3.1. FIB images of a passivated Si chip. The square regions are 100x100 $\mu \mathrm{m} 2$ Au pads. Note the lack of material contrast on the ion image.

Since SIMS mass spectra represent mass to charge ratio, information about multiply-ionized species and singly ionized clusters can be determined. For example, Aluminum has a mass of 27 and a large peak can be seen in Figure 3.4. If the primary peak at $\mathrm{m} / \mathrm{q}=27$ is large enough, we can expect to see another smaller peak at 13.5 and one at 54 . Indeed, for a primary peak of $38295 \mathrm{kcounts}$ at $\mathrm{m} / \mathrm{q}=27$, we see 4 counts at $\mathrm{m} / \mathrm{q}=13.5$ and 17 counts at $\mathrm{m} / \mathrm{q}=54$. Thus there is a small but nonzero probability for generating doubly ionized $\mathrm{Al}$ and about 4 times more singly ionized $\mathrm{Al}$ pairs from the Ion Milling process.

\subsection{Mass spectrometry instrumentation}

For larger $\mathrm{m} / \mathrm{q}$ ratios, it can be important to consider the reduction in ion transmission through the quadrupole mass analyzer. 


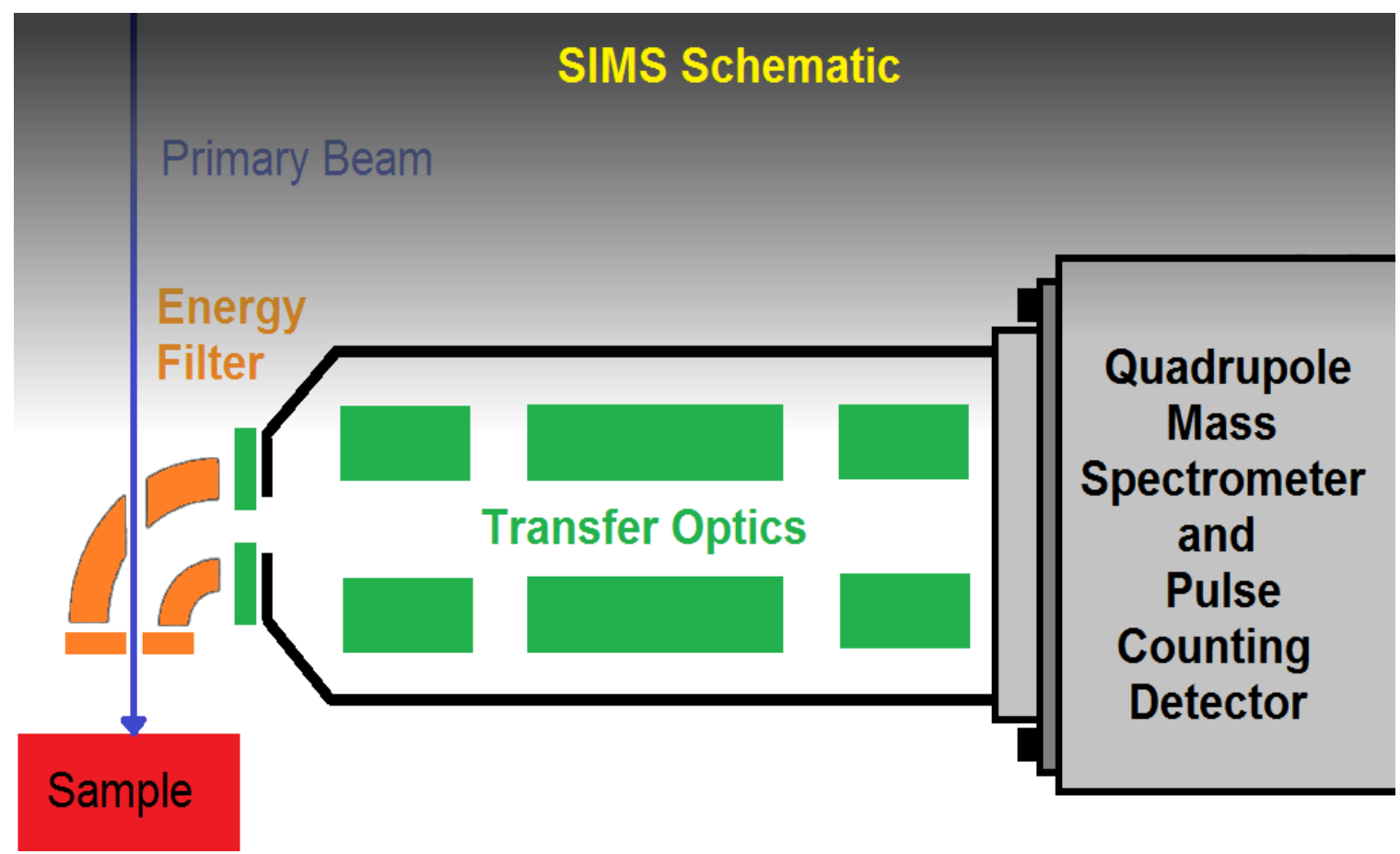

Figure 3.2. Rough illustration of the FEI FIB SIMS-I energy analyzer and ion transfer optics. There are eight individual controls which need to be optimized for the highest possible ion count rate.

Our configuration consists of a spherical energy analyzer in front of a quadrupole mass analyzer feeding a CEM which is optimized for pulse counting. The total range for the quadrupole mass analyzer is $200 \mathrm{amu}$. The images are combined after some processing. For example, the sample may have drifted slowly during the long process of imaging. The images must be somehow registered so that when overlaid, the final image is a good representation. SIMS ion counts can be somewhat sparse and the instrument sensitivities will not be the same for all species. The effect is rather grainy raw images. Some processing may be necessary to make each species stand out. For example, an image may be Gaussian-blurred and have its histogram stretched so as to amplify the apparent signal. Finally, each species is assigned a color and the images are combined 
using a software package such as Paint Shop Pro, GIMP, ImageJ, etc.

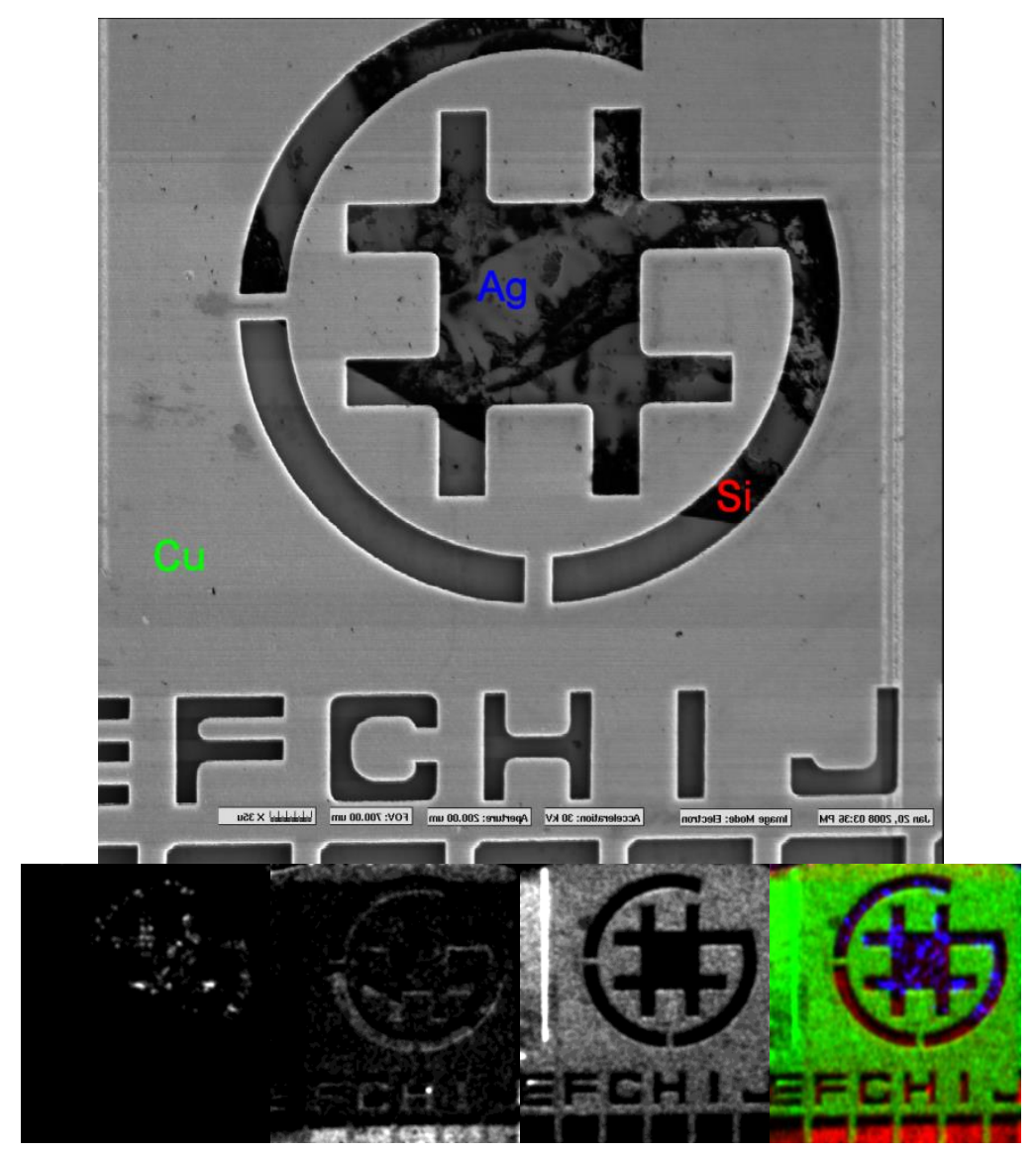

Figure 3.3. Sample of a three-species SIMS composite image. The contributing images have been registered, Gaussian blurred and histogram stretched in order to create the final colored image. (bottom row images, left to right) $\mathrm{Au}, \mathrm{Si}, \mathrm{Cu}$ and composite.

If there are only three species to combine, each species can be assigned a RedGreen-Blue (RGB) channel for the final image. More species may be added by assigning each a color which is one of three complimentary colors in another color scheme. The author will likely need to experiment with these schemes.

All three images can be created at once using sufficiently long pixel dwells. In this scheme, the SIMS detector's mass setting is switched to each species of interest 
during the FIB beam dwell at each pixel. Species giving stronger signals can be given smaller time slices in order to acquire longer on the less sensitive species. The maximum scan rate of the $\mathrm{C} 50$ Quadrupole SIMS detector is $1000 \mathrm{AMU} / \mathrm{s}$. The actual slew rate may be higher, but $1000 \mathrm{AMU} / \mathrm{s}$ represents a worse case specification we can use to predict the feasibility of switching masses at each pixel. Because the range of our SIMS is 0-200 AMU, the absolute worst case mass switching time is $200 \mathrm{~ms}$. Elements close to each other in mass can be switched in a few ms or so. During the mass switch, the detector output must be ignored and is thus wasted. The impact of losing the SIMS signal during the species switch may be prohibitive for samples having large differences in atomic mass.

\subsection{Characteristics of the FEI SIMS-I 2D system}

Benefits of this configuration include:

1) The energy analyzer effectively obscures the line-of-site path from the sample surface to the detector. This has two benefits:

a. Only charged particles will make it through the energy analyzer so neutrals will not contribute to the measured ion counts.

b. No light can make it to the detector to cause false counts.

2) Ion energy distribution studies can be made.

Drawbacks of this configuration are:

1) The primary drawback for this quadrupole mass spectrometer configuration is that only one mass can be set at a time for SIMS maps.

2) The energy analyzer passes a band of ion energies, reducing the already small signal, thus reducing sensitivity to trace elements in the sample.

3) The conductance through the energy analyzer and transfer optics is small enough that the local vacuum may be much poorer than the chamber's. This can lead to accelerated deterioration of the electron multiplier.

4) Ion transmission through the quadrupole mass analyzer is mass dependent, decreasing for increasing mass.

5) Ion detection is mass dependent, roughly exponentially decreasing for increasing mass. 


\subsection{SIMS spectra on some typical samples}

Pure samples are available for calibrating the SIMS. The sample in our lab is very old and has been contaminated by persons handling without gloves. Cleaning with DI water and IPA, although clean to the eye, does not remove the microscopic contaminants. Figure 3.4 and Figure 3.5 show typical SIMS spectra from two elemental samples contained on this stub.

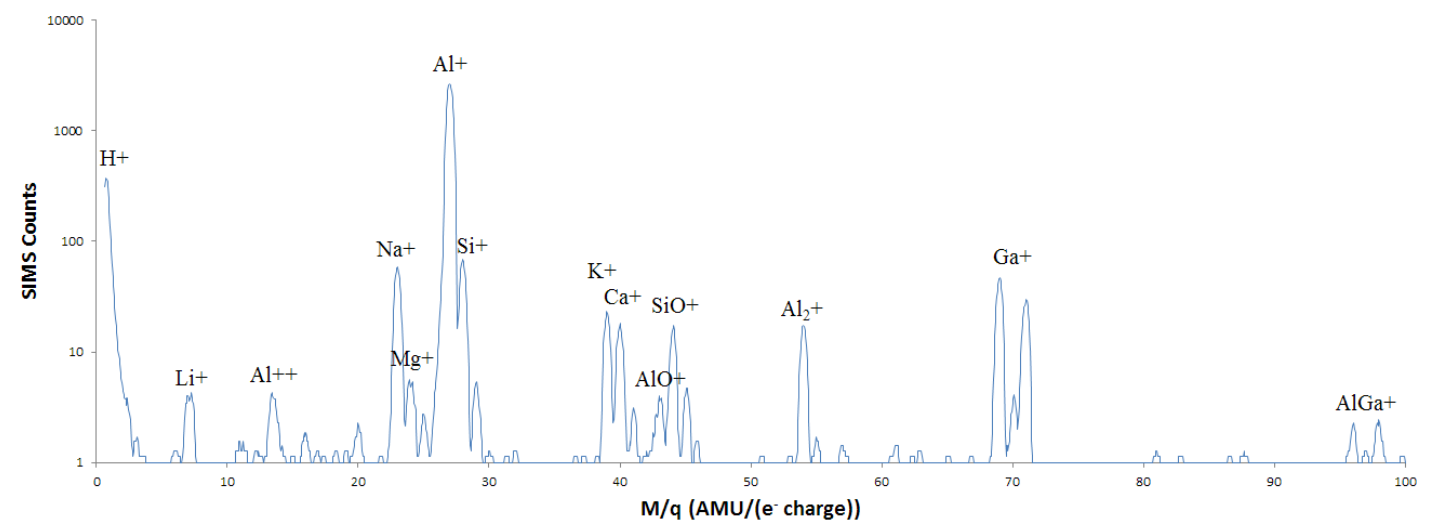

Figure 3.4. SIMS spectrum on a dirty Al sample. Notice the numerous contaminants, compounds, and multiply ionized species.

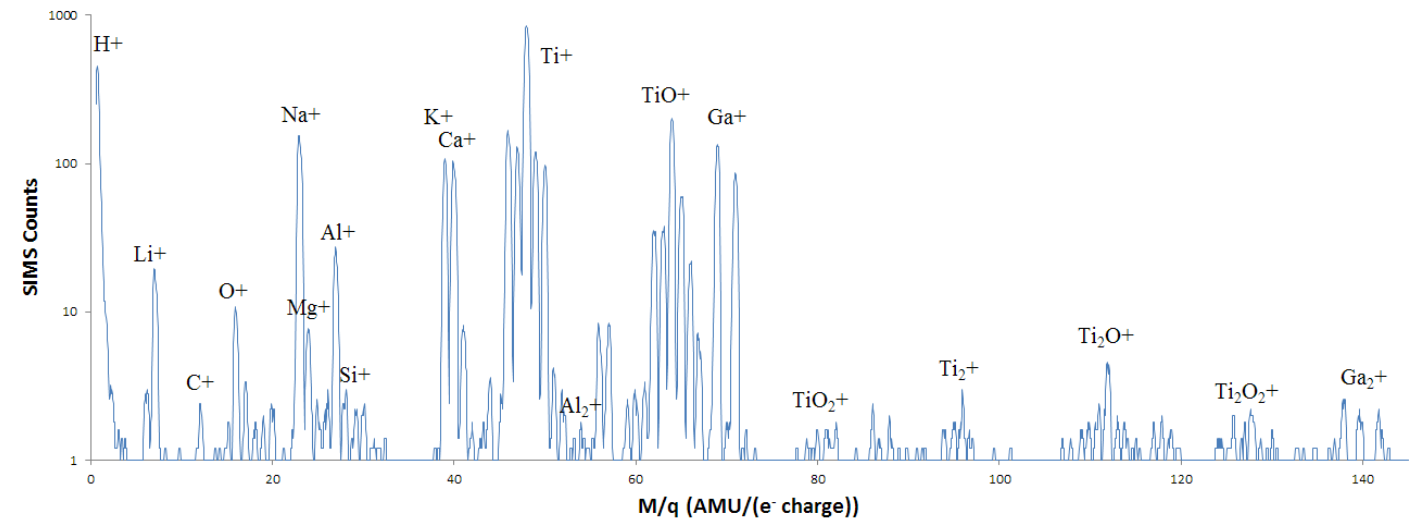

Figure 3.5. SIMS spectrum on a dirty Ti sample. Note the many compounds and contaminants. 


\subsection{References}

Bardi, U, S.P Chenakin, A Lavacchi, C Pagura, and A Tolstogouzov. "Sputter Depth Profiling by Secondary Ion Mass Spectrometry Coupled with Sample Current Measurements.” APSUSC Applied Surface Science 252, no. 20 (2006): 7373-82.

Benninghoven, A, F. G Rüdenauer, and H. W Werner. Secondary Ion Mass Spectrometry: Basic Concepts, Instrumental Aspects, Applications, and Trends. New York: J. Wiley, 1987.

Bigler, Jeff. "Periodic-Table.xls," n.d. http://www.mrbigler.com/documents/PeriodicTable.xls.

Extrel Corporation, "Model C50 Mass Spectrometer User Reference Manual”, P.O. Box 11512 Pittsburgh, Pennsylvania 15238

Galileo Electro-Optics Corporation, "Channeltron Electron Multiplier Handbook for Mass Spectrometry Applications”, Galileo Park, P.O. Box 550 Sturbridge, MA 01566

Harriott, L. R. "Focused ion beam secondary ion mass spectrometry: Ion images and endpoint detection.” J. Vac. Sci. Technol. B Journal of Vacuum Science \& Technology B: Microelectronics and Nanometer Structures 7, no. 2 (1989): 181.

Lawrence Livermore National Laboratory, United States, Department of Energy, United States, Department of Energy, and Office of Scientific and Technical Information. Phase Separation of Lipid Membranes Analyzed with High-Resolution Secondary-Ion Mass Spectrometry. Washington, D.C: United States. Dept. of Energy, 2009. http://www.osti.gov/servlets/purl/973328-jjrQQb/.

Russo, Michael F, Mostafa Maazouz, Lucille A Giannuzzi, Clive Chandler, M Utlaut, and Barbara J Garrison. "Trench Formation and Lateral Damage Induced by Gallium Milling of Silicon.” Applied Surface Science. 255, no. 4 (2009): 828.

Werner, H. W., and P. R. Boudewijn. "A Comparison of SIMS with Other Techniques Based on Ion-Beam Solid Interactions." Vacuum 34, no. 1-2 (1984): 83-101. 


\section{Introduction to Atomic Force Microscopy (AFM)}

An atomic force microscope is a scanning probe instrument in which the probe is a very sharp metal, semiconducting or non-conducting tip. This sharp tip is used to probe the topography of a sample surface, resulting in 2D images. Metal tips are made sharp by electro-chemical etching. Non-conducting glass tips can be made by the pulling process in which a heated rod/tube is pulled apart leaving two very sharp tips/pipettes. FIB can be used to make custom tips, such as those used in the TENOM. In operation, the tip is brought very close to the sample and a tuned feedback loop keeps the tip at a constant distance from the sample surface. The sample is then scanned in a raster pattern while the tip is maintained at a constant distance. In this way the tip follows the contours of the sample and is recorded synchronously with the $\mathrm{X}$ and $\mathrm{Y}$ position of the sample. A 3D image of the surface is formed as a result. 


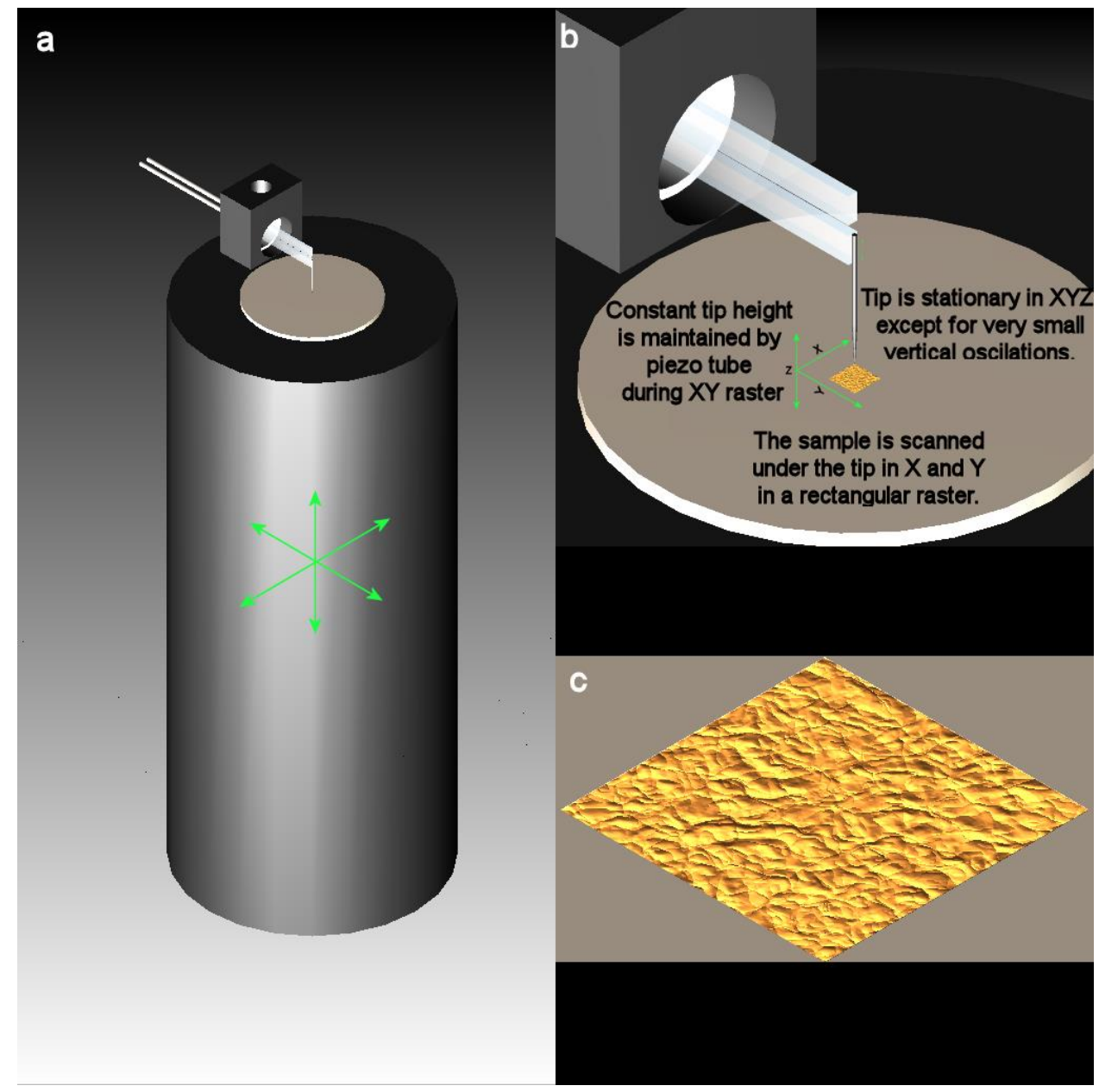

Figure 4.1, Fine positioning elements of the AFM. a) The Z-axis piezo tube rests on an XY nano positioner for the $\mathrm{XY}$ raster. b) As the sample is scanned in $\mathrm{XY}$, constant tip-sample distance is maintained by the $Z$-axis piezo tube. c) The $Z$-position which is required to maintain constant tipsample distance is recorded synchronously with the $\mathrm{XY}$ position during the raster. The result is a 3D image of the sample surface.

The feedback loop works by monitoring the difference in phase between the drive signal and the return signal in a common quartz tuning fork, operating near resonance. As the tip interacts with the sample surface, its effective resonance frequency changes slightly. This can be measured as a difference in phase between the drive signal and the return signal. The tuning fork used in our instrument has a resonance at $32,768 \mathrm{~Hz}$ and is very inexpensive compared to a typical commercial AFM cantilever. 
The AFM electronics design used for this work is a modified version of the opensource TENOM, developed earlier in our lab. Because the TENOM was designed to operate in air or fluids, it utilizes the shear mode of operation, where the tuning fork is mounted vertically and the tip oscillates in the X or Y direction. The oscillations are small enough that they are not measureable in a near-field optical microscope. The AFM configuration for our in system will utilize the up-down oscillation mode of the tuning fork for vacuum operation.

AFM lateral resolution is limited in part by the tip sharpness. Tip radii on the order 10 's of nm are common. During operation, the tip sometimes comes in contact with the sample surface, which can cause sample damage and tip wear. To combat tip wear, a hard material such as Tungsten is commonly used. A compromise in resolution and wear can be made by using a blunt tip, having a radius greater than $50 \mathrm{~nm}$.

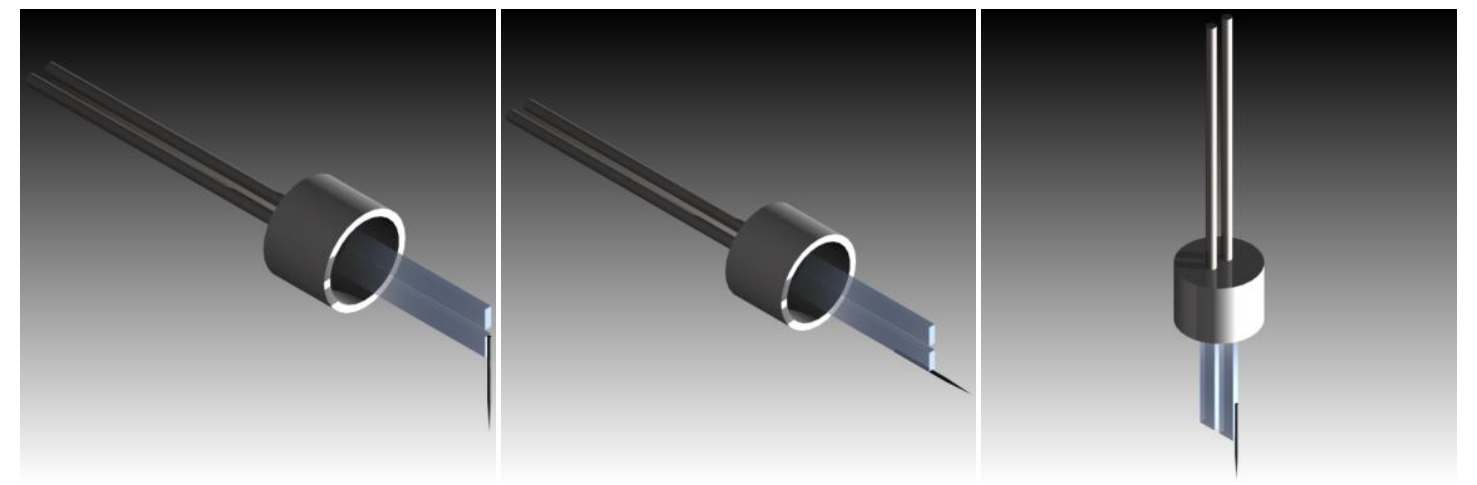

Figure 4.2. Two primary AFM tip configurations. (left) Tip oscillates vertically, perpendicular to the sample. This mode is preferred in vacuum environments where it might tend to stick to the sample. It also saves space between the SIMS-I and sample. (center \& right) The tip is operated having its can rotated 90 degrees wrt the vertical configuration and oscillates laterally, parallel to the sample. The TENOM for which the AFM in this work is based, uses this configuration.

To minimize sample-tip contact events, slow scans are used. Typical scan dwell is on the order of $1 \mathrm{~ms} /$ pixel. A 512x512 pixel image will take 262 seconds, or 4.36 
minutes. Scans as long as 30 minutes to a few hours are common for certain applications.

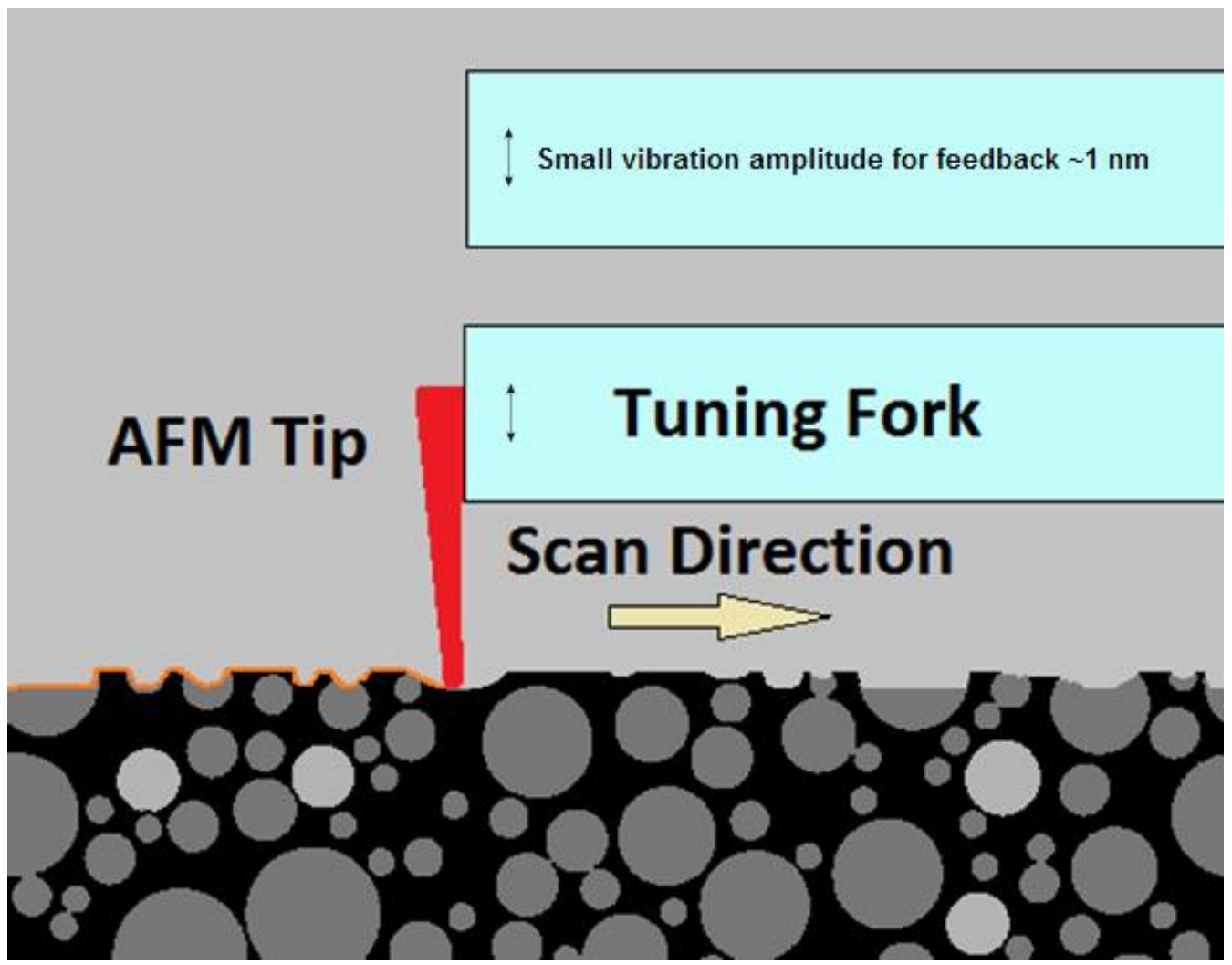

Figure 4.3. Schematic illustration of the AFM tip being moved along a sample which has uneven topography. The sample is moved up or down to maintain a constant tip-sample distance. The shape of the tip determines how finely the surface features can be resolved.

\subsection{Tip-sample distance control using tuning fork oscillations}

The implementation of the AFM used in this work relies on tip-surface feedback of the simple quartz tuning fork. These devices are manufactured in large quantities for the electronics industry and can be purchase very cheaply. The ones used in this work have a nominal resonance frequency of $32768 \mathrm{~Hz}$. Novotny demonstrates that the tuning fork can be treated using the Simple Harmonic Oscillator (SHO) model. 


$$
m \ddot{x}(d, t)+m \gamma \dot{x}(d, t)+m \omega_{0}^{2}(d) x(d, t)=F e^{-i \omega t}
$$

Where $m$ is the effective mass being oscillated, $\gamma$ is the damping coefficient in $\mathrm{rad} / \mathrm{s}$ and $\omega_{0}$ is the natural resonant frequency of the tuning fork. The mass, $\mathrm{m}$, requires special treatment. The tuning fork is essentially a uniform beam, fixed at one end, supporting a mass on its free end. The dots above the x's denote time derivatives. F is the peak amplitude of excitation and $\omega$ is the drive frequency. The left side of eq. 3.1 describes the tuning fork's intrinsic motion properties, while the right side describes an external sinusoidal driving force.

The dependence on the tip-sample distance, $\mathrm{d}$, can be used to derive interaction forces and interaction induced damping. This is an interesting topic, but is not needed for this discussion. Solution of this equation far from the sample surface, $x(d \rightarrow \infty, t)$ goes to $\mathrm{x}(\mathrm{t})$ ), giving a sinusoidal relation which is enclosed by an envelope defined by the SHO parameters $\mathrm{F}, \mathrm{m}, \omega_{0}$ and $\gamma$.

$$
x(t)=\frac{F / m}{\omega_{0}^{2}-\omega^{2}-i \gamma \omega} e^{-i(\omega t+\varphi)}=A e^{-i(\omega t+\varphi)}
$$

Thus the motion of the tuning fork has the same frequency as the drive frequency but the amplitude of vibration is greatly affected by $\omega_{0}$ and $\gamma$. The induced vibrational motion lags the drive force by a phase, $\varphi$. The oscillatory dependence can be removed to give the amplitude as a function of $\omega$. 


$$
A(\omega)=\frac{F / m}{\sqrt{\left(\omega_{0}^{2}-\omega^{2}\right)^{2}+\gamma^{2} \omega^{2}}}
$$

The phase can be found using

$$
\begin{gathered}
\tan \varphi=\left(\frac{\gamma \omega}{\omega_{0}^{2}-\omega^{2}}\right) \\
x(t) \approx \frac{F / m}{\omega_{0}^{2}-\omega^{2}-\frac{i \omega_{0} \omega}{\sqrt{3} Q}}
\end{gathered}
$$

The $\sqrt{ } 3$ factor arises from the tuning fork being a uniform beam, fixed at one end. The other end is free to oscillate and has the AFM tip attached. The quality factor, Q, is easily determined from a plot of the amplitude vs drive frequency, usually referred to as a frequency sweep.

$$
Q=\frac{f_{0}}{\Delta f}
$$

Where $\Delta \mathrm{f}$ is the full-width at half max of a frequency sweep. The time constant of the interaction is approximately 


$$
\tau \approx \frac{2 \sqrt{3} Q}{\omega_{0}}=\frac{\sqrt{3}}{\pi \Delta f}
$$

Where $\omega_{0}=2 \pi \mathrm{f}_{0}$. For a particular configuration, it is sometimes useful to have an estimate of $\gamma$.

$$
\gamma \approx \frac{2 \pi f_{0}}{Q \sqrt{3}}=\frac{2 \pi \Delta f}{\sqrt{3}}
$$

Fowles goes a little further in demonstrating that although the steady-state motion has frequency $\omega_{0}$, the amplitude resonant frequency, $\omega_{\mathrm{r}}$, is related to $\gamma$ making it lower.

$$
\omega_{r}=\sqrt{\omega_{0}^{2}-\frac{\gamma^{2}}{2}}
$$

thus,

$$
f_{r}=\sqrt{f_{0}^{2}-\frac{\gamma^{2}}{8 \pi^{2}}}
$$

This is the peak of the envelope which is actually measured in a typical frequency sweep. So for large damping, the amplitude resonant frequency can be considerably different from $\mathrm{f}_{0}$. An example is a tuning fork/AFM tip having a relatively low $\mathrm{Q}$ factor 
of 1375 . This Tuning fork's envelope peak is at $28609.1 \mathrm{~Hz}$ and its FWHM is about 20.8 Hz. The damping factor is about $75.45 \mathrm{~s}^{-1}$, resulting in a shift of $72.1 \mathrm{~Hz}$. The actual resonant frequency for this arrangement is thus $28681.2 \mathrm{~Hz}$. It is worth noting that these operating frequencies are considerably lower than $32768 \mathrm{~Hz}$ due to the relatively large mass of the AFM tip and some other intentional de-tuning.

The interaction time constant for amplitude changes is about $26.5 \mathrm{~ms}$. A scan of 256x256 pixels using amplitude feedback would require nearly 58 hours to acquire!

In principle, the phase has an instantaneous response to changes in F. In practice, it requires at least two cycles to measure. The measurement speed is further reduced by the phase detection electronics which convert the phase difference between excitation and driven waveforms into pwm pulses which are then passed through a low-pass filter having a time constant of $1 / 1.7 \mathrm{kHz}$. This gives a minimum response time on the order of $0.59 \mathrm{~ms}$ for the feedback system.

This is acceptable for acquiring AFM scans of 1024x1024 pixels at $0.25 \mathrm{~Hz}$, translating to $0.512 \mathrm{~second} /$ pixel, requiring about 70 minutes. Thus a larger number of pixels can be scanned in less time using phase rather than amplitude as feedback.

\subsection{Temperature dependence of oscillator frequency}

It might be tempting to use eq 3.10 to determine $\gamma$ from air \& vacuum measurements of $\omega_{\mathrm{r}}$. Recall that $\omega_{0}$ is a relatively strong function of temperature so careful measurements require good knowledge of T. Also, the cyano-acrolate adhesive slowly outgases and tends to lose mass, changing $\omega_{0}$ inside the vacuum. Further, the electronics are greatly affected by the vacuum and would need to be accounted for.

The data sheet is available for the $32768 \mathrm{~Hz}$ tuning forks used in this project. The 
temperature dependence of $\mathrm{f}_{0}=\omega_{0} /(2 \pi)$ is:

$$
\frac{\Delta f}{f_{0}}=-0.03 T^{2}+1.5 T-18.75
$$

Where $\mathrm{T}$ is the fork's temperature in degrees Celsius. $\Delta \mathrm{f}$ here is the change in frequency due to a temperature different from $25 \mathrm{C}$. The resulting temperature dependence for the operating frequency is parabolic. Thus for an operation range of $+/-$ $45 \mathrm{C}, \Delta \mathrm{f}$ is $+/-2 \mathrm{~Hz}$.

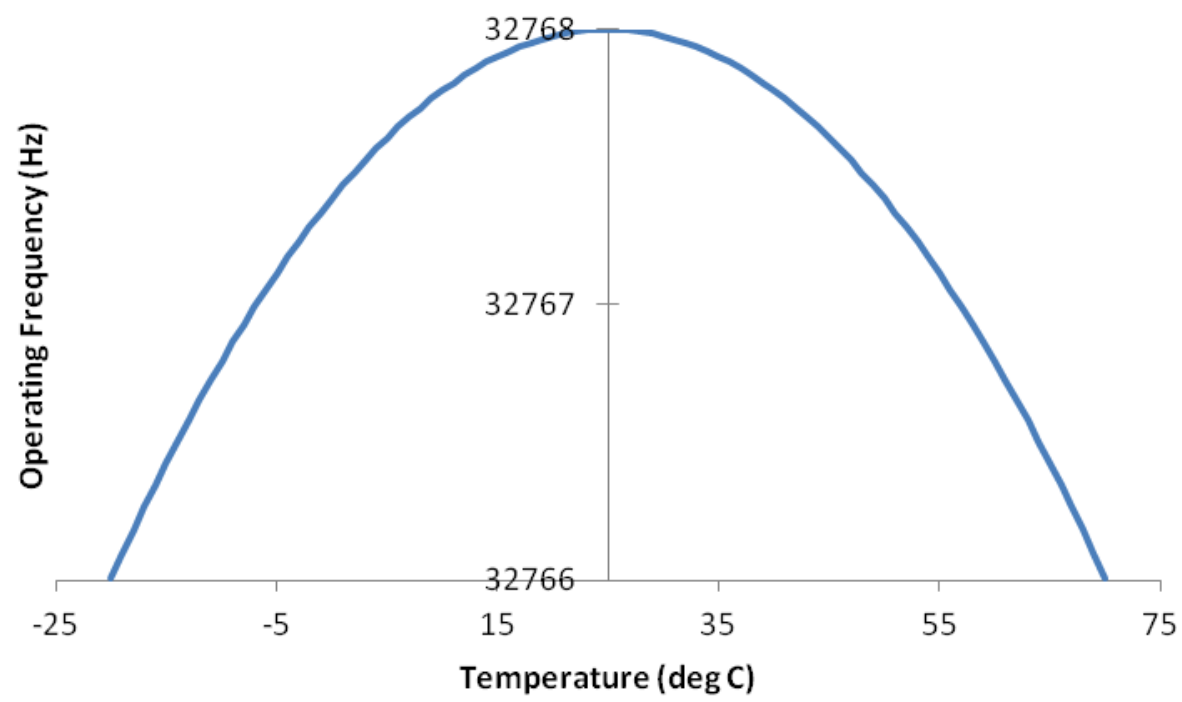

Figure 4.4. Parabolic relationship of $f_{0}$ vs temperature for a quartz tuning fork. The data for this curve was extracted from the tuning fork data sheet using a software program named "Data Thief".

Operating temperature range is $-20 \mathrm{C}$ to $+60 \mathrm{C}$. Using (tuning fork)/(AFM tip) configurations having Q's of between 1000 and 4000 requires $f_{o}$ stability on the order of $0.1 \mathrm{~Hz}$. Operation in a vacuum will lead to an appreciable sensitivity to temperature, 
requiring a long warm-up time for the feedback system.

\subsection{Non-sinusoidal driving forces}

Eq. 3.2 illustrates that the frequency of oscillation is the same as the driving frequency. This is also true for non-sinusoidal driving forces. For example, a tuning fork can be driven as in Figure 5.2, where one lead is connected to the driving force and the other to a monitor such as a high-impedance oscilloscope.

Q can be alternatively interpreted as the number of cycles it takes for the amplitude to drop to 1/e times the peak amplitude once the driving force is removed. Elements having high Q's then require a large number of cycles for their amplitudes to die down. Another property of high Q elements is that their response to changing force applied, beit amplitude or frequency, is slow.

So if you imagine the application of a shaped periodic signal at $f_{0}$ to a high $Q$ crystal, it will experience a push at each time $\tau$, corresponding to $1 / \mathrm{f}_{0}$. Each push then results in a damped oscillation of $\mathrm{Q}$ sinusoidal cycles, where $\mathrm{x}>\mathrm{x}_{0} / \mathrm{e}$. It receives the next push only one cycle later and so its amplitude actually increases a little if the energy contained in the push is greater than the same amplitude sine wave. This occurs with each cycle until the little pushes balance the decay and steady state is reached.

The result of this little discussion is that a tuning fork having a high $\mathrm{Q}$ can be driven by almost any type of waveform and it will simply execute sinusoidal motion. Thus it can be driven by a white noise signal and still, clean sinusoidal motion of the tuning fork is the result. 


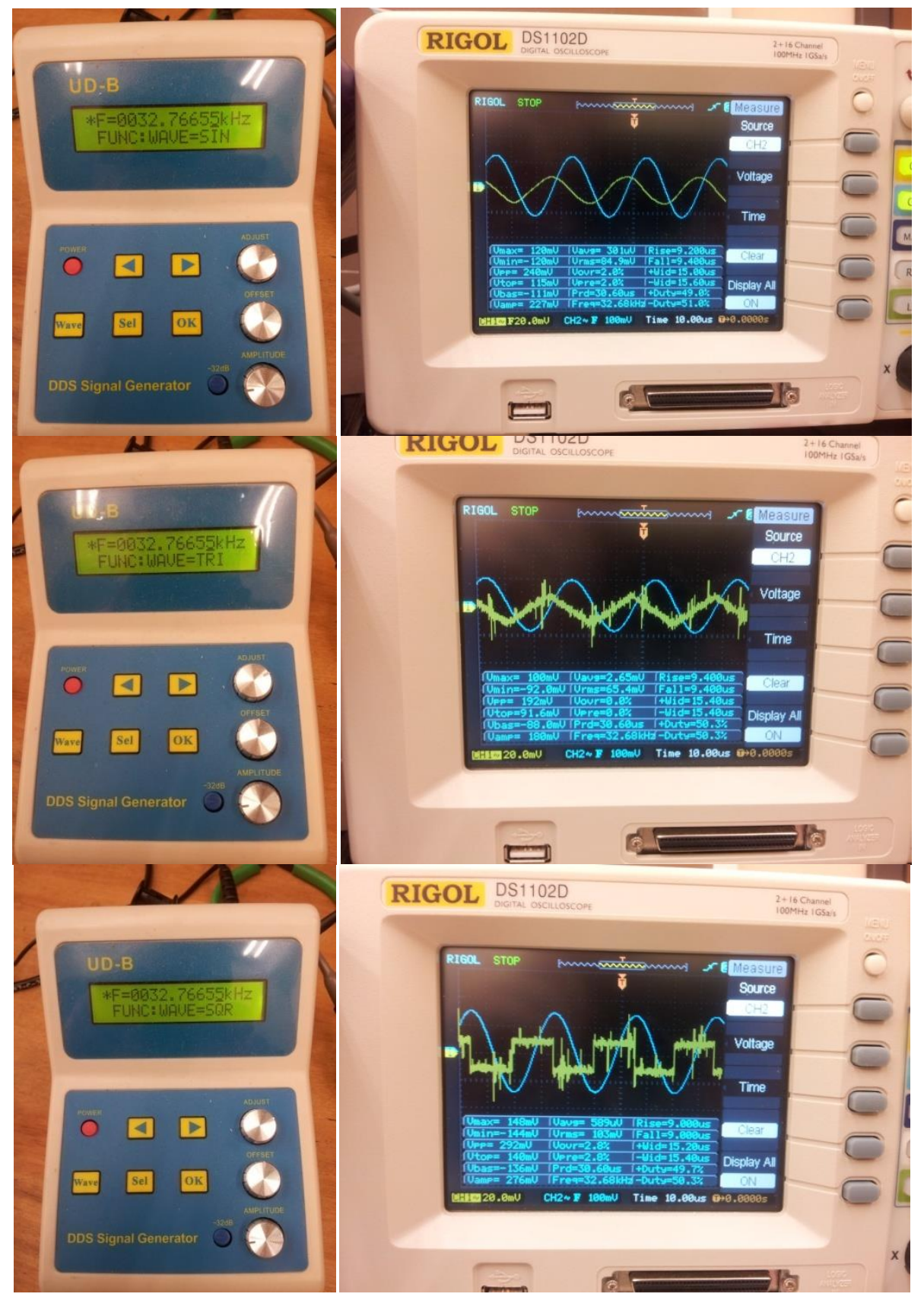

Figure 4.5. Illustration of the resonance phenomenon for a quartz tuning fork in its sealed can using a function generator. The yellow curves are the excitation and the blue are the tuning fork response which is always sinusoidal. (top) Sinusoidal excitation. (Middle) Triangle wave excitation. (Bottom) Square wave excitation. 
In this discussion higher order and lateral vibrational modes of the (tuning fork)/(AFM tip) system were ignored. The AFM tips are rather long, being 3-5 $\mathrm{mm}$ in length. The tuning fork is installed at an angle so that the tip may be seen by the FIB. The tuning fork itself is suspended by its leads, sort of detuning it giving smaller Q's. Future consideration of these effects would lead a more complete understanding of the AFM constructed.

\subsection{References}

Bielefeldt, H, and F J Giessibl. "A Simplified but Intuitive Analytical Model for Intermittent-Contact-Mode Force Microscopy Based on Hertzian Mechanics." Surface Science. 440, no. 3 (1999): L863.

Binnig, Gerd, Calvin F. Quate, and Ch Gerber. "Atomic Force Microscope.” Physical Review Letters 56, no. 9 (1986): 930.

Doughty, Jeffrey Jon. "Symmetric Near-Field Probe Design and Comparison to Asymmetric Probes," 2010. http://pdxscholar.library.pdx.edu/open_access_etds/390/.

Edwards, Hal, Larry Taylor, Walter Duncan, and Allan J Melmed. "Fast, HighResolution Atomic Force Microscopy Using a Quartz Tuning Fork as Actuator and Sensor." Journal of Applied Physics. 82, no. 3 (1997): 980.

Fowles, Grant R. Analytical Mechanics. Philadelphia: Saunders, 1986.

Fox Electronics, "Thru-Hole Tuning Fork Data Sheet”, 2012

Giessibl, F. J, and M Tortonese. "Self-Oscillating Mode for Frequency Modulation Noncontact Atomic Force Microscopy.” APPLIED PHYSICS LETTERS 70, no. 19 (1997): 2529-31.

Giessibl, F. J. “A Direct Method to Calculate Tip-Sample Forces from Frequency Shifts in Frequency-Modulation Atomic Force Microscopy." APPLIED PHYSICS LETTERS 78 (2001): 123-25.

Giessibl, F. J. “AFMs Path to Atomic Resolution." MATERIALS TODAY-OXFORD THEN KIDLINGTON- 8, no. 5 (2005): 32-41. 
Giessibl, Franz J. "Forces and Frequency Shifts in Atomic-Resolution Dynamic-Force Microscopy." Physical Review. B, Condensed Matter. 56, no. 24 (1997): 16010.

Giessibl, Franz J. “Atomic Resolution on Si (111)-(7x7) by Noncontact Atomic Force Microscopy with a Force Sensor Based on a Quartz Tuning Fork." Applied Physics Letters 76, no. 11 (2000): 1470-1472.

Giessibl FJ, Herz M, and Mannhart J. "Friction Traced to the Single Atom." Proceedings of the National Academy of Sciences of the United States of America 99, no. 19 (2002): 12006-10.

Giessibl F.J, Pielmeier F, Eguchi T, An T, and Hasegawa Y. "Comparison of Force Sensors for Atomic Force Microscopy Based on Quartz Tuning Forks and LengthExtensional Resonators.” Phys. Rev. B Condens. Matter Mater. Phys. Physical Review B - Condensed Matter and Materials Physics 84, no. 12 (2011).

Grober, Robert D., Jason Acimovic, Jim Schuck, Dan Hessman, Peter J. Kindlemann, Joao Hespanha, A. Stephen Morse, Khaled Karrai, Ingo Tiemann, and Stephan Manus. "Fundamental Limits to Force Detection Using Quartz Tuning Forks." Review of Scientific Instruments 71, no. 7 (July 2000): 2776-80. doi:10.1063/1.1150691.

Herz, M, F. J Giessibl, and J Mannhart. "Probing the Shape of Atoms in Real Space." Phys. Rev. B Physical Review B 68, no. 4 (2003).

T. Ihn, T. Vancura, A. Baumgartner, P. Studerus, K. Ensslin, " Operating a phase-locked loop for controlling a high-Q tuning fork sensor for scanning force microscopy", arXiv: cond-mat/0112415v1 [cond-mat.mes-hall] 21 Dec 2001

Jannis Lübbe, Matthias Temmen, Holger Schnieder, and Michael Reichling. "Measurement and Modelling of Non-Contact Atomic Force Microscope Cantilever Properties from Ultra-High Vacuum to Normal Pressure Conditions.” Measurement Science and Technology 22, no. 5 (2011).

Khaled Karrai, Robert D. Grober, "Piezoelectric tip-sample distance control for near field optical microscopes”, 1942 Appl. Phys. Lett. 66 (14), 3 April 1995

Khaled Karrai, Robert D. Grober, "Piezo-electric tuning fork tip-sample distance control for near field optical microscopes”, Ultramicroscopy 61 (1995) 197-205

Jinquan Liu, Andrea Callegari, Marting Stark, Majed Chergui, "A simple and accurate method for calibrating the oscillation amplitude of tuning-fork based AFM sensors", UltraMicroscopy 109 (2008) 81-84 
K Saitoh, K Hayashi, Y Shibayama, and K Shirahama. "A Low Temperature Scanning Probe Microscope Using a Quartz Tuning Fork.” Journal of Physics: Conference Series 150, no. 1 (2009).

Liu, Jinquan, Andrea Callegari, Martin Stark, and Majed Chergui. "A Simple and Accurate Method for Calibrating the Oscillation Amplitude of Tuning-Fork Based AFM Sensors." Ultramicroscopy. 109, no. 1 (2009): 81.

Novotny, Lukas, and Bert Hecht. Principles of Nano-Optics. Cambridge: Cambridge University Press, 2006.

Nowak, Derek Brant. The Design of a Novel Tip Enhanced near-Field Scanning Probe Microscope for Ultra-High Resolution Optical Imaging, 2010. http://adsabs.harvard.edu/abs/2010PhDT.........38N.

Serway, Raymond A. Physics for Scientists and Engineers. Philadelphia [etc.: Saunders College Publishing, 1992.

Tambade, Popat S. "Harmonic Oscillator Wave Functions and Probability Density Plots Using Spreadsheets." Latin-American Journal of Physics Education 5, no. 1 (2011): 7.

Ternes, Markus, Christopher P. Lutz, Cyrus F. Hirjibehedin, Franz J. Giessibl, and Andreas J. Heinrich. "The Force Needed to Move an Atom on a Surface." Science 319, no. 5866 (2008): 1066-1069.

Torbrugge S, Lubbe J, Troger L, Cranney M, Reichling M, Eguchi T, and Hasegawa Y. "Improvement of a Dynamic Scanning Force Microscope for Highest Resolution Imaging in Ultrahigh Vacuum." Rev. Sci. Instrum. Review of Scientific Instruments 79, no. 8 (2008).

West, Paul, and Natilia Starostina. "A Guide to AFM Image Artifacts." Pacific Nanotechnology, Inc. 3350 Scott Blvd \#29 Santa Clara, CA 95054-3105, n.d.

Whitehouse, D. J. "Nanometrology." Contemporary Physics 49, no. 5 (September 2008): 351-74. doi:10.1080/00107510802611251.

Young, Warren C, Richard G Budynas, and Ali M Sadegh. Roark's Formulas for Stress and Strain. New York: McGraw-Hill, 2012. 


\section{Direct measurement of tuning fork motion using Electrostatic TEM (ETEM)}

This chapter is a slight diversion from the topic of AFM-SIMS. There is some interest in observing AFM tip motion directly. We constructed an experiment to observe this motion directly and estimated some parameters of the tuning fork from videos of the tip motion. Others have conducted measurements of tuning fork oscillations using interferometery and have confirmed that the tuning amplitude of oscillation is on the order of $1 \mathrm{pm}$ per $\mathrm{mV}$ of drive. An alternative technique would be to measure the oscillations directly by an electron microscope.

\subsection{Direct measurement of tuning fork motion using ETEM.}

AFM lateral resolution is also limited by the noise on the XY scanner. As the

tip/sample is scanned, XY positioning noise will cause an averaging effect over a roughly circular region centered on the tip in motion. The result is a somewhat blurry AFM scan. In shear mode, the oscillation amplitude of the tip may contribute to image blur in the direction of oscillation. There have been attempts to predict the vibration amplitude of the tuning fork and measurements have been made utilizing interferometric methods.

Our group has made some attempts to directly measure the amplitude of vibration, utilizing Electrostatic Transmission Electron Microscopy (ETEM). Gold tips were electro chemically etched and attached to tuning forks. A thin coating of Gold was sputtered onto the end of the tuning fork to minimize charging. The tips were inserted into the ETEM and operated in air so that their baseline resonance curve was measured electronically. Once pumped down in the ETEM, another electronic resonance curve was measured. The tuning fork was then operated while imaging with the ETEM. Movies were recorded of the tuning fork through resonance, for a range of drive amplitudes. 
Field of view calibration was performed by correlation of the tip image in the ETEM with SEM images taken using an FEI Nova Nanolab system.

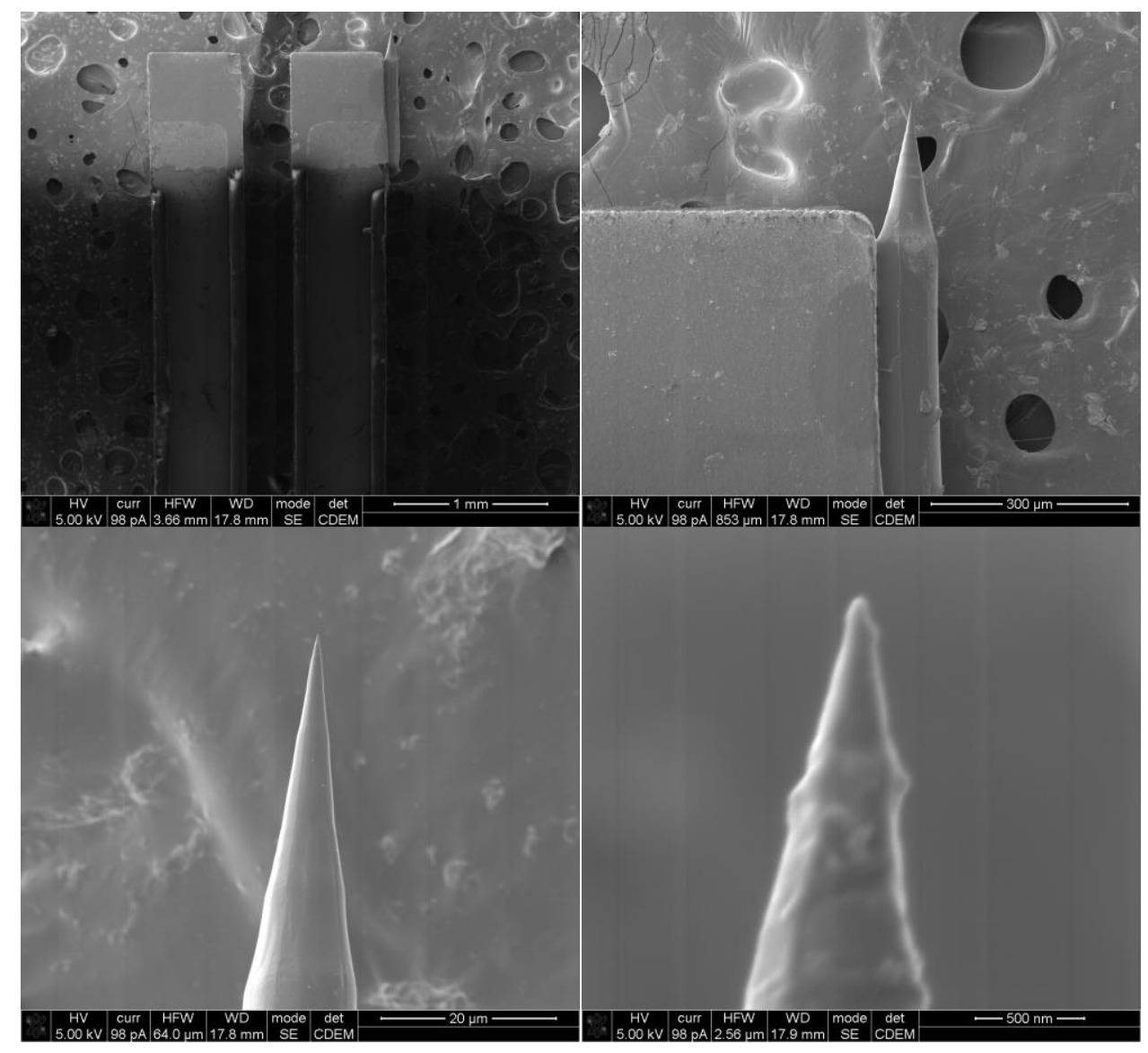

Figure 5.1. SEM images of a typical electro-chemically etched Au tip and its mounting in the shearmode configuration on its tuning fork. Note the bumps created by the Au coating.

At the beginning of the experiment, the TENOM AFM controller electronics and computer were used for data acquisition. Unfortunately, about 1 in 20 of the frequent arcs from the ETEM coupled into the AFM computer and caused various issues. Finally, the AFM controller electronics were damaged and could no longer be used without repair. In order to reduce further risk of damage to the AFM controller electronics, the experiment was conducted using manual measurements. Refer to the setup in Figure 5.2. 


\subsection{Experimental setup}

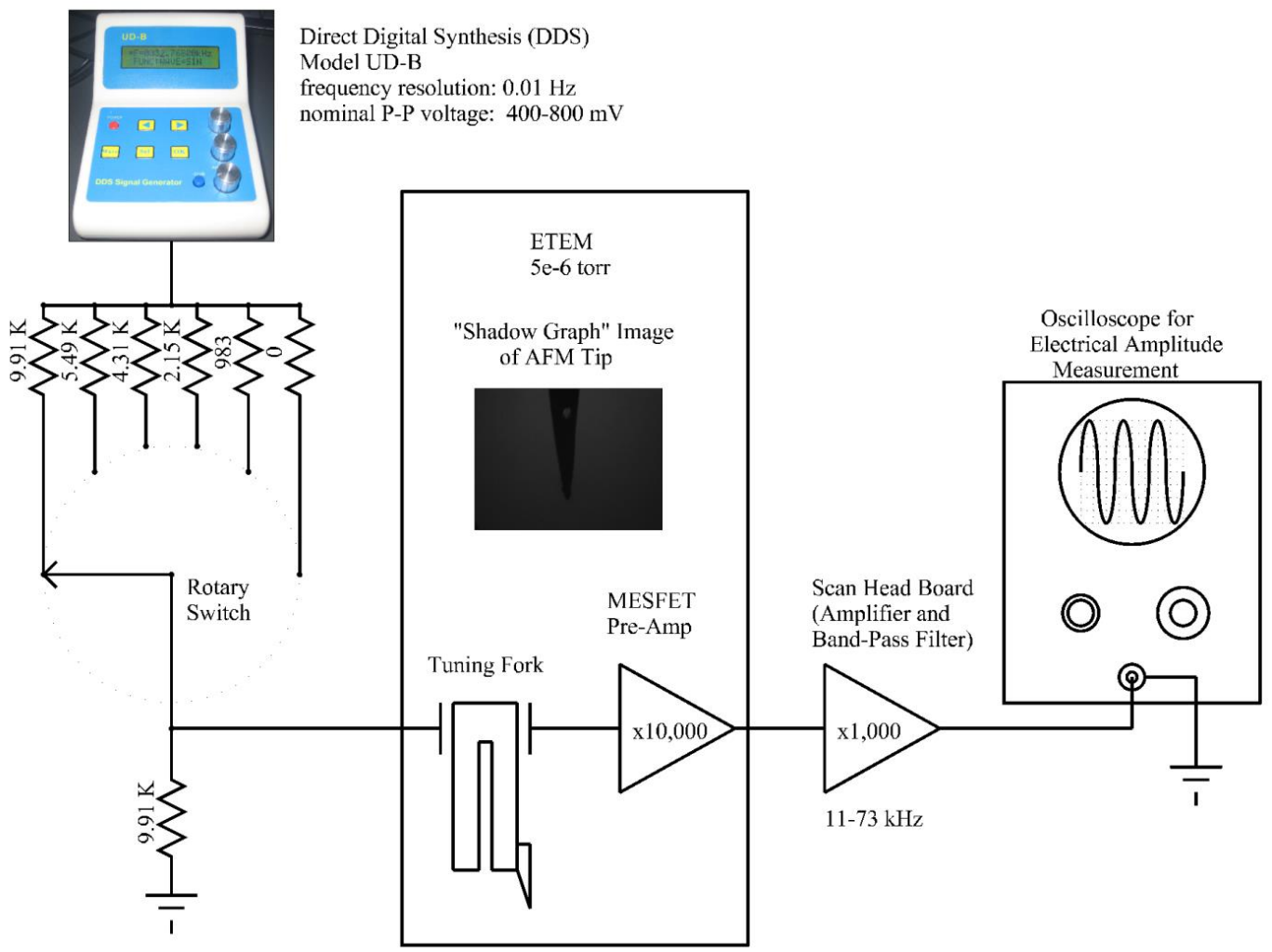

Figure 5.2. Electrical setup for manual tuning fork excitation and measurement. Later, the oscilloscope was replaced by a true rms digital multimeter.

Two basic experiments were performed, 1) Ramp the drive frequency at constant amplitude through resonance and record video of the event and 2) At resonance, ramp the drive voltage and record video of the event. Video recordings of the events utilized a SenTech STC-400 machine vision camera, aimed at the ETEM preview screen. Camera video was brought into the computer using a LinXcel VC-211V USB 2.0 Video Grabber (DVD Maker) Adapter. The software used to record the video was AmCap.exe. With this combination of hardware and software, movies were recorded at 30 frames/second, each frame having $640 \times 480$ pixels. Fields of view used ranged between $5 \mu \mathrm{m}$ and 10 
$\mu \mathrm{m}$. Video processing was carried out in multiple steps in order to maximize contrast, reduce the noise and remove imaging artifacts. These steps are detailed in

Figure 5.6. The deflection measurement utilized the fact that the image on the ETEM screen is essentially a time integrated shadow and the intensity of this shadow can be measured to determine the vibration amplitude.
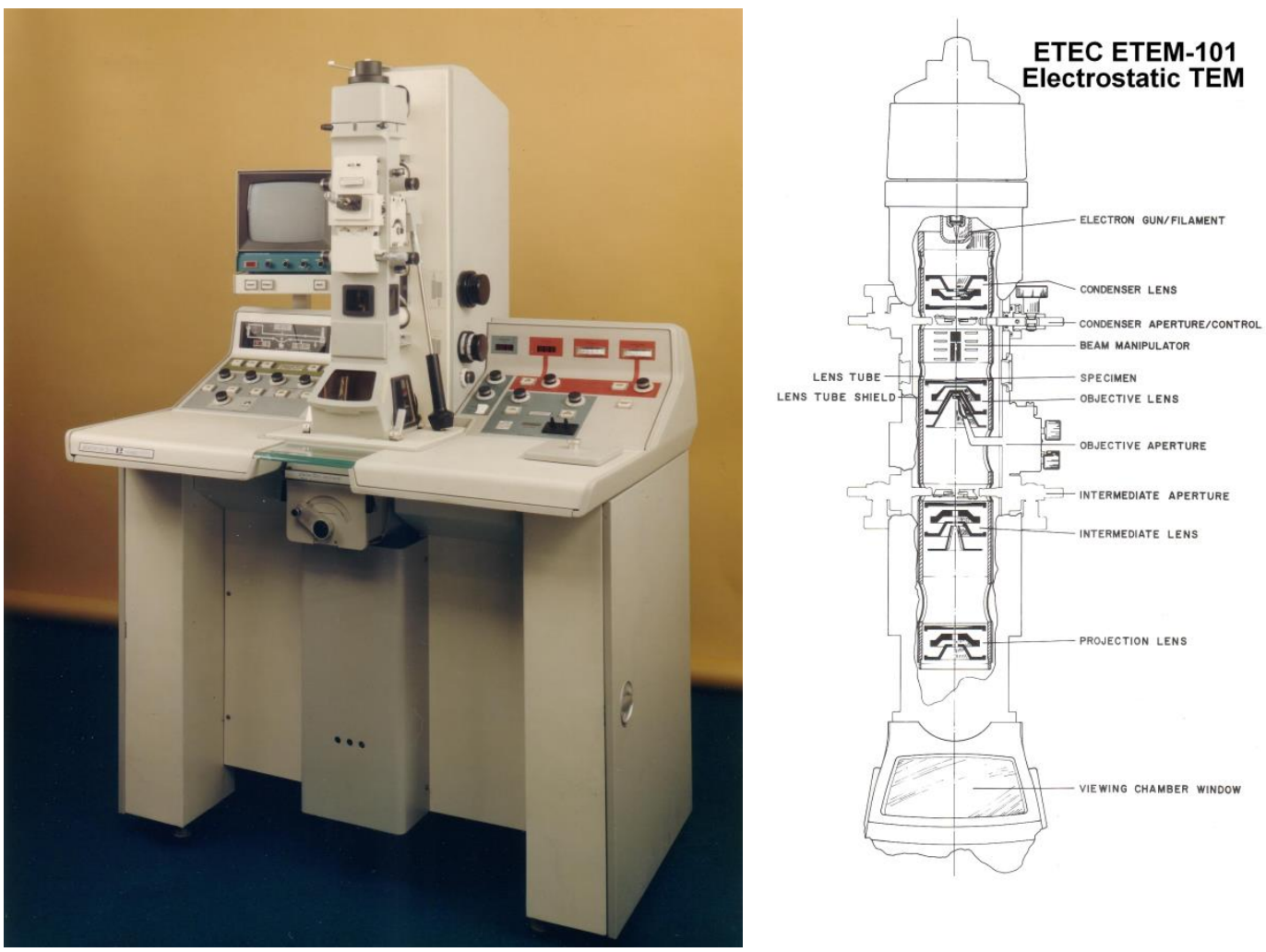

Figure 5.3. (left) Photograph of the electrostatic transmission electron microscope used in this work. (right) Drawing of the ETEM optics. This system was designed by Gert Rempfer in the 1970's for applications in biology. It has some features which were innovative for the time, including 3D visualization using shutter glasses. (images reproduced from original product brochure) 

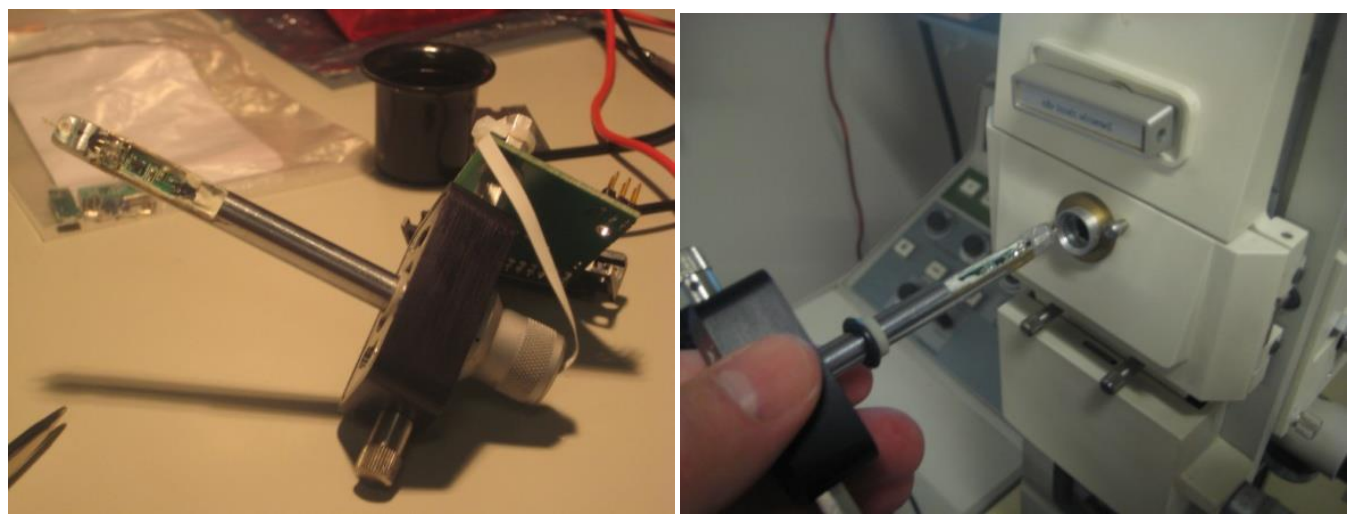

Figure 5.4. Photograph (left) of the ETEM sample holder which has been modified to hold tuning fork based AFM sensors, including the MESFET and scan head preamp. Photograph (right) showing a tuning fork being inserted into the ETEM.

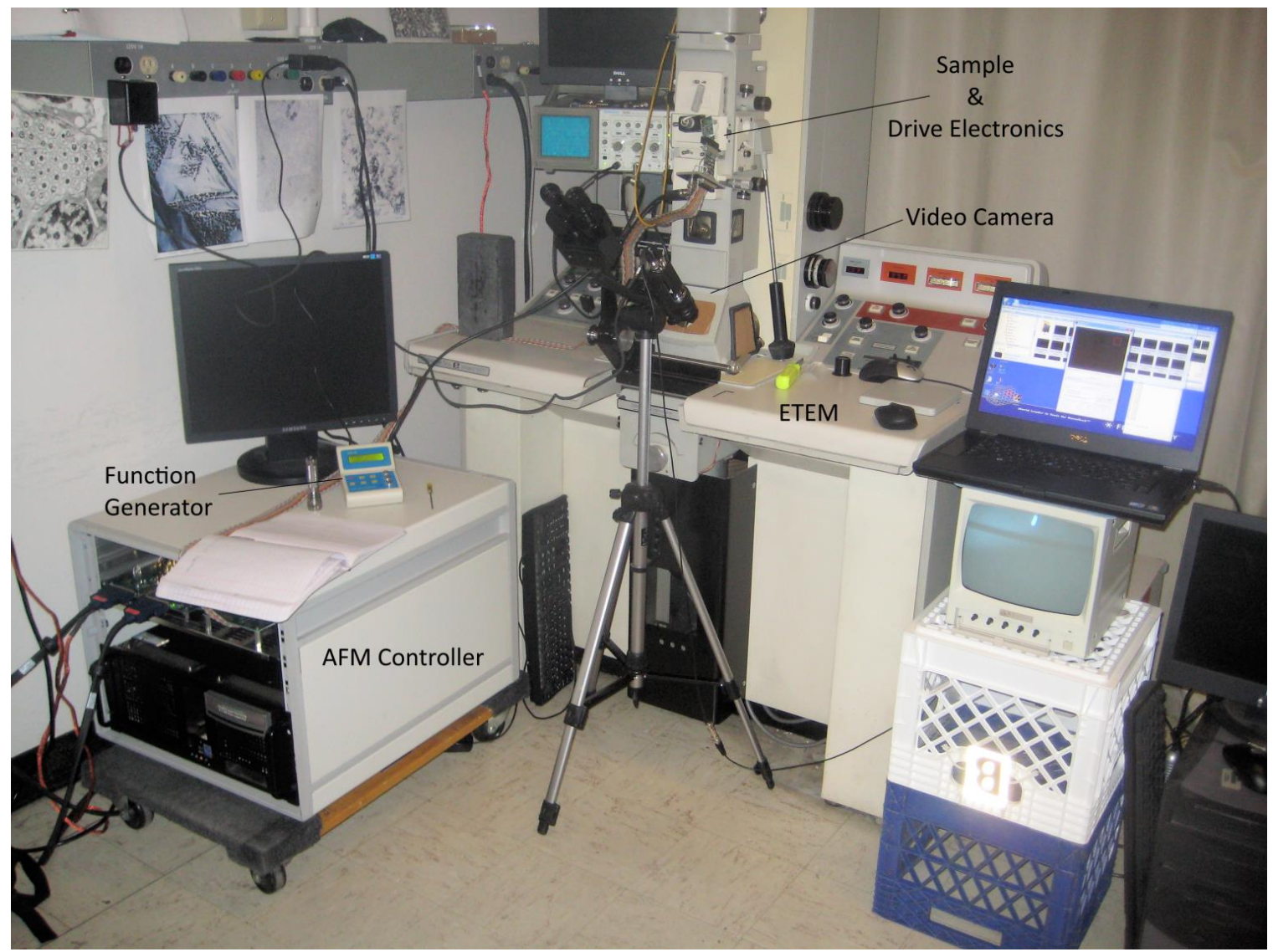

Figure 5.5. Setup for recording video of vibrating AFM tips inside the ETEM. Initially, the AFM controller was used to drive the tuning fork. Later the UD-B Direct Digital Synthesis (DDS) function generator was used. 


\subsection{Brute-force video analysis}

Once the video has been collected, the individual frames can be extracted, filtered and have an estimated background removed. Then they are analyzed for vibration of the tip. The method chosen is slope. The outside edges of the tip are computed and

monitored for every image. Then these edges are used to determine the effective width of the tip and are later correlated with the frequency or amplitude as appropriate. 
Acquire video of oscillating afm tip performing an amplitude or frequency sweep. Save in avi format.

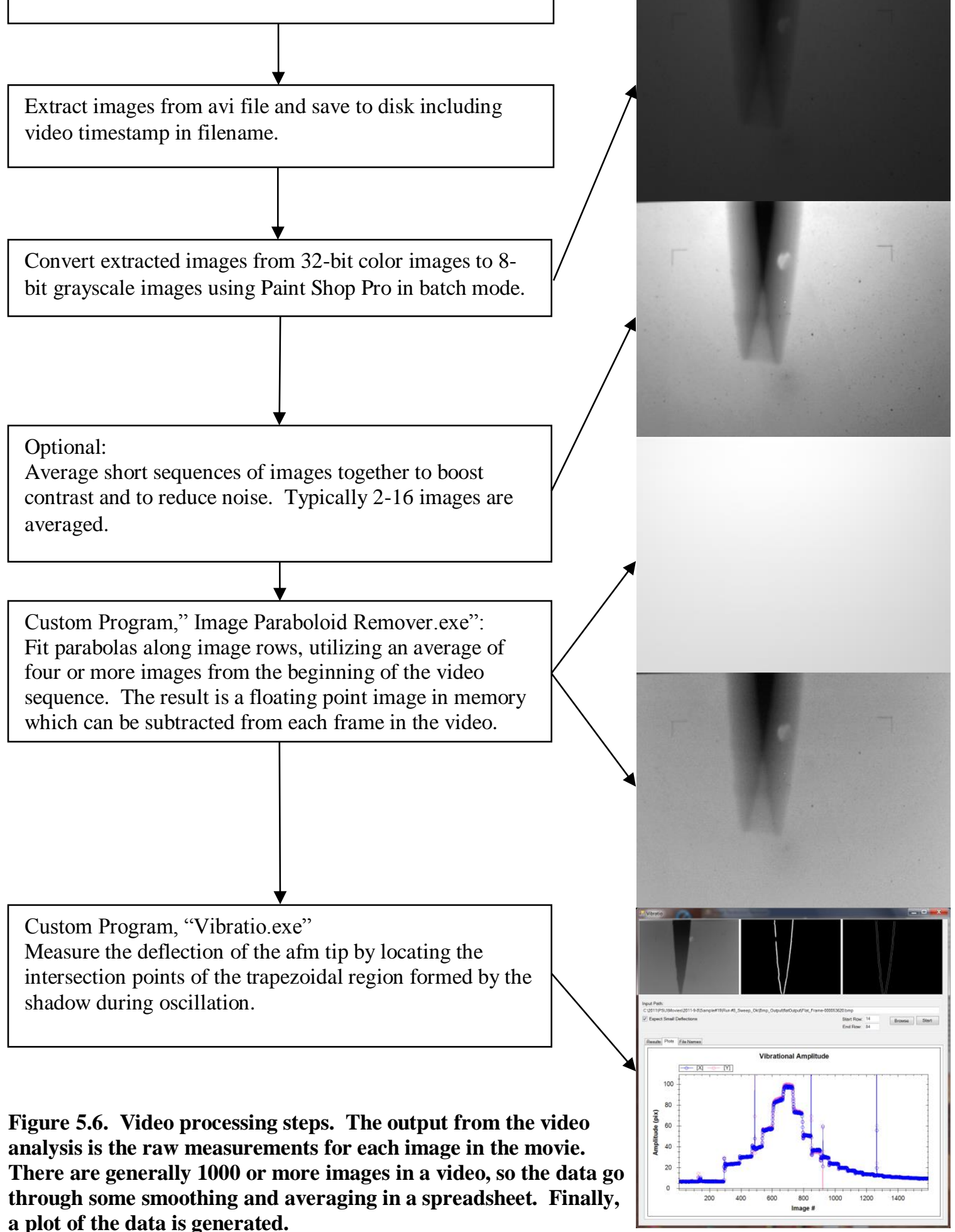



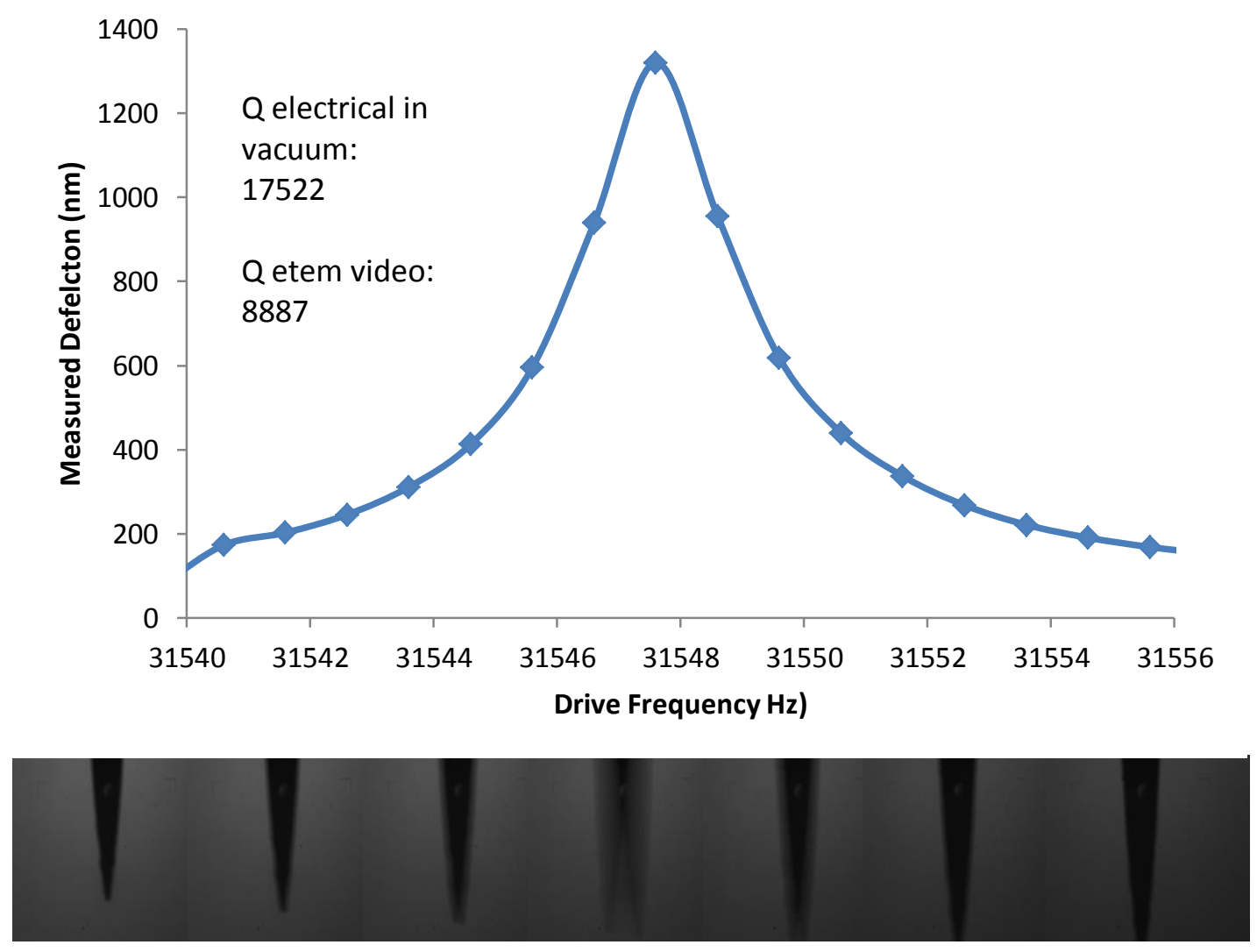

Figure 5.7. Direct measurement of an AFM tip mounted on a tuning fork. The tuning fork was driven using $200 \mathrm{mV}$ P-P. Normal operation is typically 2-7 $\mathrm{mV}$ r.m.s. Image horizontal field of view is approximately $7.5 \mu \mathrm{m}$. This is sample \#19. 1740 images were extracted from the acquired video for analysis. The large difference between $Q$ measured electrically and from video are still not understood entirely. This experiment demonstrates the feasibility of using ETEM only qualitatively so far.

Frequency sweeps at constant drive voltage should resemble Figure 5.7. The Qfactor is simply the peak frequency divided by the full-width at half max (FWHM). Voltage sweeps at resonance resemble Figure 5.8. If the tuning fork is operated in its linear regime, we expect a linear plot of measured tip deflection vs. drive voltage. The yintercept should be very close to zero. 


\subsection{Discussion of the setup}

This experiment was conducted using excitation voltages which are about 100x those used during normal AFM operation so that the motion was clearly visible using an FOV which could simultaneously accommodate the size of the tip and the vibration magnitude. The tuning fork data sheet is available for determining the maximum drive Voltage [Fox]. This tuning fork can be driven at $1 \mu \mathrm{W}$, its capacitance is $12.5 \mathrm{pF}$ and has a $35 \mathrm{k} \Omega$ equivalent series resistance (ESR). The average power in a mostly RLC circuit is

$$
P_{\text {avg }}=\frac{V_{r m s}^{2} R}{R^{2}+\left(X_{L}-X_{C}\right)^{2}}=\frac{\left(\frac{V_{\text {peak }}}{\sqrt{2}}\right)^{2} R}{R^{2}+\left(X_{L}-X_{C}\right)^{2}}
$$

Assuming the inductor reactance is negligible, the peak Voltage is

$$
V_{\text {peak }}=\sqrt{\frac{2 P_{\text {avg }}\left(R^{2}+\left(\frac{1}{2 \pi f C}\right)^{2}\right)}{R}}
$$

Where $\mathrm{R}$ is the Equivalent Series Resistance (ESR). The result is Vpeak $\sim 2.95$ Volts for operation at $1 \mu \mathrm{W}$. Operation at Voltages smaller than this are assumed to be in the linear range of operation. 


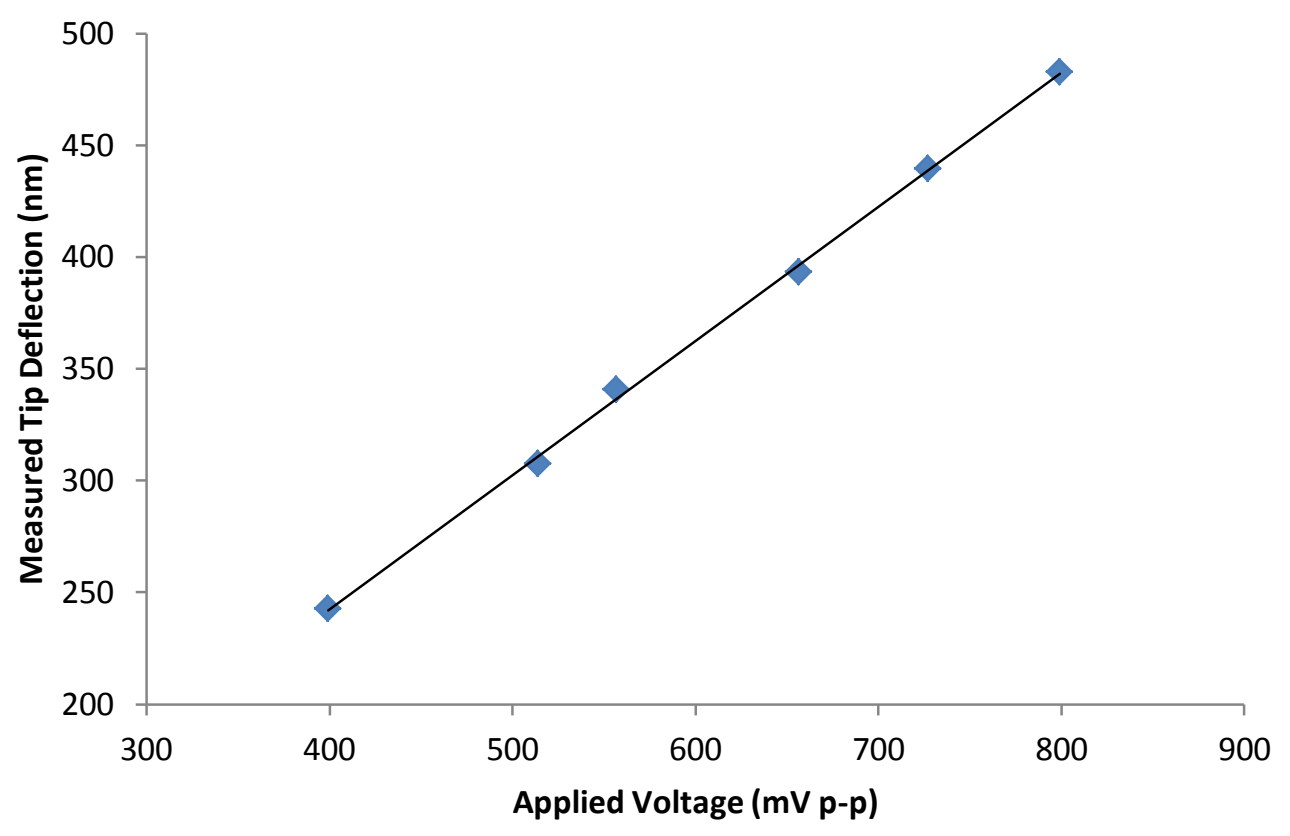

Figure 5.8. The result of tracking the peak amplitude at resonance vs applied voltage (sample \#16). The line fit suggests $0.6 \mathrm{~nm}$ per $\mathrm{mV}$ of excitation and an intercept of $2.3 \mathrm{~nm}$. We might expect a yintercept in the pm range.

The $2.3 \mathrm{~nm}$ y-intercept in Figure 5.8 represents an error of up to $1 \%$. Typical drive voltages for AFM operation are as low as $2 \mathrm{mV}$, meaning that the peak tuning fork amplitude during AFM operation could be as high as $1.2 \mathrm{~nm}$. Drive voltages up to $8 \mathrm{mV}$ would then lead to amplitudes close to $10 \mathrm{~nm}$. In operation, the frequency is set near the resonance peak, about partway down so that the phase response is strong and nearly linear.

The discussion above suggests that AFM operating amplitudes aver $5 \mathrm{~nm}$ are likely. This would mean that shear force mode may not be good for an AFM needing high resolution. Recall that these measurements were taken in vacuum, where the $\mathrm{Q}$ factor is nearly double what it is in air. The drive voltage is low enough that the vibration is expected to be in the linear response range of the tuning fork. 


\subsection{Discussion of the results}

As stated earlier, complications from arcing in the ETEM forced manual operation of the scans through resonance. Sample heating was excessive for certain condenser settings. The resistor voltage divider was removed from the drive circuit so that the drive amplitude was increased by a factor of 100. Manual sweeps through resonance were done by switching out resistors in a voltage divider and the return signal was measured on an oscilloscope.

Some useful things were learned from this work. In no particular order, they are as follows:

- 30 frames/s appears to be adequate for image analysis.

- We will need to take more precautions to minimize sample heating.

- A dedicated experiment, including automated acquisition might be better than using the AFM controller, or manual operation of the DDS and oscilloscope.

- The DDS is very stable and this work demonstrated that a frequency resolution of 0.1 $\mathrm{Hz}$ or less is required for measurements in vacuum.

- The resonance frequency, $\mathrm{f}_{0}$, generally increased during a set of measurements. This may have been due to evaporation of the tip adhesive. The measured peak amplitude at resonance may have been adversely impacted by this drift, causing the measured slope to be lower than actual.

- The electronically measured Q-factor nearly doubled in vacuum.

- Similar experiments could be performed using SEM or FIB, although the video processing may need to be modified.

- The author was a novice on the ETEM and should have spent more time understanding how to optimize the imaging and sample dose.

- The "shadow graph" technique makes the image analysis relatively easy although the very poor image quality required extensive processing.

- Actual amplitudes of vibration during AFM operation may be larger than is commonly believed.

Some unknowns include:

- Effect of the phosphor time constant (it's yellowish-green, possibly P31).

- Slopes were seen on the on individual steps in some of the frequency sweeps. Faster, automated measurements may eliminate these. 
- The temperature of the tuning fork during imaging is unknown. The tuning fork cantilever has a temperature coefficient of $-0.04 \mathrm{ppm} / \mathrm{K}$. This is a parabolic function, peaked at $298 \mathrm{~K}$, meaning that an increase in temperature will result in a reduced resonant frequency.

- Sample electron dose was not measured, but is likely very high.

- We expect the sweeps at resonance to have intercepts very close to zero. Many of these plots, not presented here, do not show this trend.

This work was put on hold in order to repair the AFM controller electronics used in the early steps of the experiment and, to begin construction of the AFM hardware. The author is planning to conduct similar experiments using smaller excitation amplitudes. This experiment may also be easier to perform on an SEM or FIB. Once completed, the results of this work could offer an alternative to interferometric measurements.

\subsection{Deconvolution theory for motion estimation}

Some may recall the launch of the Hubble Space Telescope (HST) in 1990.

Scientists had been predicting the benefits of launching telescopes into space since the 1920 's, so there was great anticipation in the high quality images to be taken by the HST. Unfortunately, its main mirror, although having been ground very precisely, had been made too flat. It was $2.2 \mu \mathrm{m}$ flatter than intended, causing massive spherical aberrations. During the three years it took to repair the HST's optics, NASA was still able to get useful images of bright objects. The effect of the flattened mirror made good images of bright objects, but they had a large background so that contrast was reduced. The big problem was with imaging faint diffuse objects, as the signal to be acquired distributes the signal over the entire mirror, which blurred the images. Scientists were able to sharpen some of these images of faint diffuse objects into useful sharp images using the deconvolution techniques known at the time [Jansson]. 
The mathematics of image processing can be as interesting as the Physics that the images themselves contain. The deconvolution operation can be utilized for more things than just making sharper images. This will be shown later. It is well known that images can be deconvolved from their point spread function representing an instrument's aberrations [Gonzales, Woods]. Using the notation from [Gonzales, Woods], an image $g(x, y)$ can be modeled as an idealistic image $f(x, y)$, convolved with an instrumental point-spread-function $h(x, y)$, with noise $\eta(x, y)$ added. In the time or space domain, this is represented as

$$
g(x, y)=h(x, y) \otimes f(x, y)+\eta(x, y)
$$

The $\otimes$ symbol is to be interpreted as the convolution operation.

Convolution is itself an integral operation, requiring integration over infinity.

$$
g(x, y)=\int_{-\infty}^{+\infty} \int_{-\infty}^{+\infty} h\left(x-x^{\prime}, y-y^{\prime}\right) f(x, y) d x^{\prime} d y^{\prime}+\eta(x, y)
$$

The task of deconvolution involves the determination of $f(x, y)$, given $g(x, y)$ and estimates for $h(x, y)$ and $\eta(x, y)$. Inverting this integral can be computationally intensive, so to obtain $f(x, y)$, it is generally better to use the frequency domain. Each function has its representation in the frequency domain. 


$$
\begin{aligned}
f(x, y) & \Leftrightarrow F(u, v) \\
g(x, y) & \Leftrightarrow G(u, v) \\
h(x, y) & \Leftrightarrow H(u, v) \\
\eta(x, y) & \Leftrightarrow N(u, v)
\end{aligned}
$$

The representation in the frequency domain can utilize any orthogonal basis. A particular choice of basis is determined by a balance of symmetry, convergence and ease of computation. The convolution operation becomes multiplication in the frequency domain.

$$
G(u, v)=H(u, v) F(u, v)+N(u, v)
$$

The degraded image $G(u, v)$, is the output of a particular imaging device such as a microscope or camera. The ideal image $\mathrm{F}(\mathrm{u}, \mathrm{v})$ is what is usually sought after. The point spread function $\mathrm{H}(\mathrm{u}, \mathrm{v})$ is also sought after but usually only to establish the instrument calibration. The noise $\eta(\mathrm{u}, \mathrm{v})$ is usually treated as a nuisance, but generally also estimated only to establish instrument noise characteristics. Simple inversion gives

$$
F(u, v)=\frac{G(u, v)-N(u, v)}{H(u, v)}=\frac{G(u, v)}{H(u, v)}-\frac{N(u, v)}{H(u, v)}
$$

Thus if the point spread function and noise distribution are known or can be estimated, the ideal image $\mathrm{F}(\mathrm{u}, \mathrm{v})$ can be reconstructed in a point by point manner over the 
range $(\mathrm{u}, \mathrm{v})$. Noise is commonly treated as having a Poisson distribution which can be mapped to a Gaussian distribution for large enough variances. Certain situations allow for the noise to be treated as a uniform distribution.

$$
[\eta(x, y)=\text { uniform }] \Leftrightarrow[N(u, v)=\delta(u, v)]
$$

Thus in the frequency domain, equation (5.3) can be written as

$$
G(u, v)=H(u, v) F(u, v)+\text { Const }
$$

Instrumental point spread functions, $\mathrm{H}(\mathrm{u}, \mathrm{v})$, are typically treated as single-peaked functions such as Gaussians, Laplacians, etc. Each pixel can have a unique point spread function although it is usually taken to be the same function at all pixels for many types of images.

The topic of this section, however, is to measure simple linear motion from two images. $H(u, v)$ can be any sort of function which causes imperfections in an image, including linear motion.

$$
H(u, v)=\frac{G(u, v)-\text { Const }}{F(u, v)}
$$

The optimal inverse filter in the least-squares sense is the Weiner inverse. The estimated deconvolved image is 


$$
\hat{F}(u, v)=\frac{G(u, v)}{H(u, v)}\left\{\frac{|H(u, v)|^{2}}{|H(u, v)|^{2}+\frac{S_{\eta}(u, v)}{S_{f}(u, v)}}\right\}
$$

The ratio $\frac{S_{f}(u, v)}{S_{\eta}(u, v)}$ is the signal to noise ratio of the ideal image and $S_{\mathrm{f}} \& \mathrm{~S}_{\eta}$ are power spectra of $\mathrm{F}$ and $\mathrm{N}$, respectively. When the signal to noise ratio has a weak spectral dependence, the Weiner inverse can be written as

$$
\hat{F}(u, v)=\frac{G(u, v)}{H(u, v)}\left\{\frac{|H(u, v)|^{2}}{|H(u, v)|^{2}+K}\right\}
$$

Where $\mathrm{K}$ is the inverse of the signal to noise ratio and is the same for all frequency components.

A similar expression for the point spread function estimate looks like this

$$
\widehat{H}(u, v)=\frac{G(u, v)}{F(u, v)}\left\{\frac{|F(u, v)|^{2}}{|F(u, v)|^{2}+K}\right\}
$$

\subsection{Uniform linear motion removal from an image in the time domain}

Anyone who has accidentally taken a picture with a little jerk to the camera knows the result. The resulting photograph is motion blurred. Although a photograph 
taken like this is not perfect uniform motion, it can to first order be treated as such.

To begin with, it is worth noting that linear motion can be removed from an image in the time domain. [Gonzales, Woods] demonstrated that if the motion blur were characterized by uniform linear motion, the deconvolution problem can be cast as a time domain solution.

Using the same notation for ideal and degraded images, $f(x, y)$ and $g(x, y)$, respectively and the slope image, $\mathrm{g}^{\prime}(\mathrm{x}, \mathrm{y})$, uniform motion along $\mathrm{x}$ can be removed using the following formula.

$$
f(x, y) \approx A-\frac{1}{K} \sum_{k=0}^{K-1} \sum_{j=0}^{k} g^{\prime}[x-m a+(k-j) a, y]+\sum_{j=0}^{m} g^{\prime}(x-j a, y)
$$

Where A approaches the mean of the output image $f(x, y)$. In practice, $A$ is instead often estimated to be the mean of the input image $g(x, y)$. Assuming a total motion blur of a pixels, $\mathrm{K}$ is the number of times that a fits into the starting image. Thus for large motion blurs, $\mathrm{K}$ is small and vise-versa.

Using eq. (5.14) requires intimate knowledge of the motion distance and the results of the motion removal depend heavily on the fraction of the image taken up by the motion. Better performance may be obtained by up-sampling the image before motion removal. This formula can be modified to accommodate non-uniform linear motion, curved motion, etc. A software program implementing these features would likely require the evaluation of $f(x, y)$ 's quality in an iterative fashion for best performance. A program implementing eq. (5.14) was written to measure its performance in 
less than ideal situations. Figure 5.9 shows the result of applying eq. (5.14) to remove 64 pixels of uniform motion blur. The result is rather good for being solely calculated in the time domain. Figure 5.10 and Figure 5.11 show the results of removing motion blur only one pixel different from what was put in and they are not good reconstructions. Thus the amount of blur must be known very well to retrieve the original un-blurred image using eq. (5.14). One can imagine a scheme where the blur is unknown and a range of motion vectors is evaluated so that the best result is chosen using some criterion of image quality.

Figure 5.12 illustrates the effect of noise corruption on the performance of eq. (5.14). Having no provision for noise rejection, it is no surprise that its performance is poor. It is so poor in fact, that the original image is not recovered at all. 

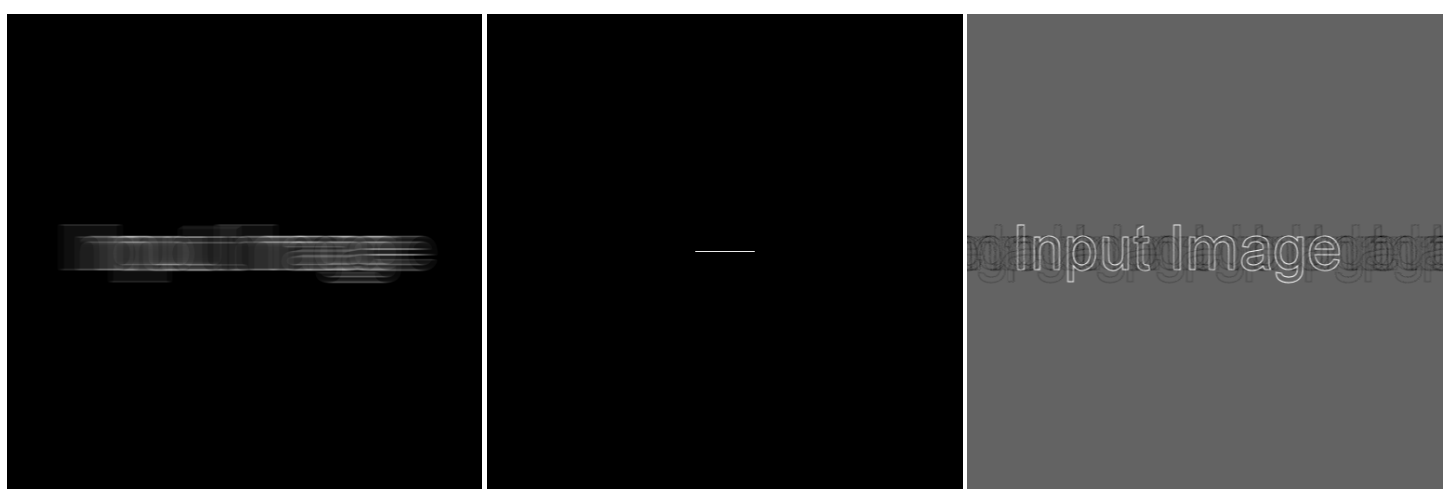

Figure 5.9. (left) Image motion blurred using a 64 pixel uniform motion vector. (center) Image of the 64 pixel uniform motion vector. (right) Motion blur removed using eq. (5.14).
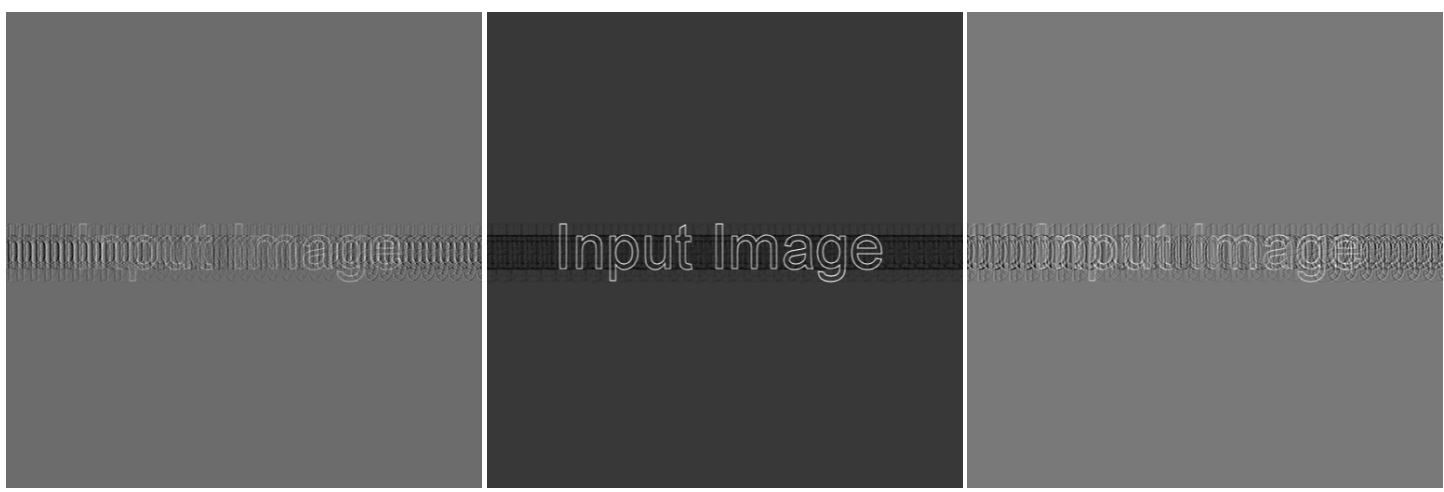

Figure 5.10. Comparison of errors in the initial estimate of motion blur using eq. (5.14) on an image blurred using a 16 pixel uniform motion blur vector along $x$. (left) Restored image using 15 pixels. (center) Restored image using 16 pixels. (right) Restored image using 17 pixels.
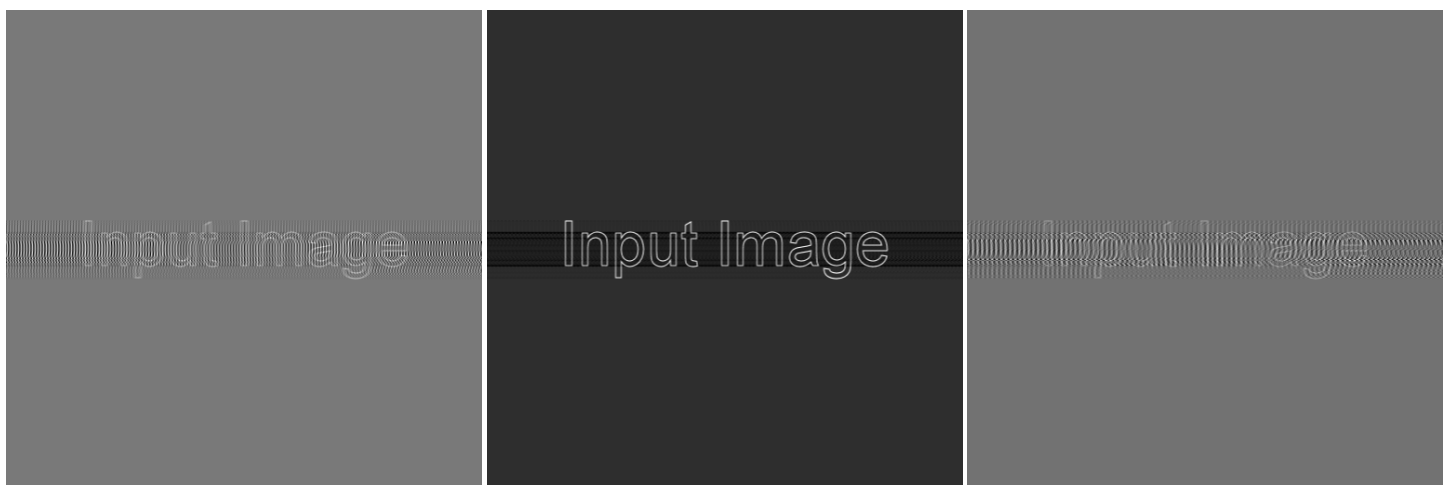

Figure 5.11. Comparison of errors in the initial estimate of motion blur using eq. (5.14) on an image blurred using a 4 pixel uniform motion blur vector along $x$. (left) Restored image using 3 pixels. (center) Restored image using 4 pixels. (right) Restored image using 5 pixels. 


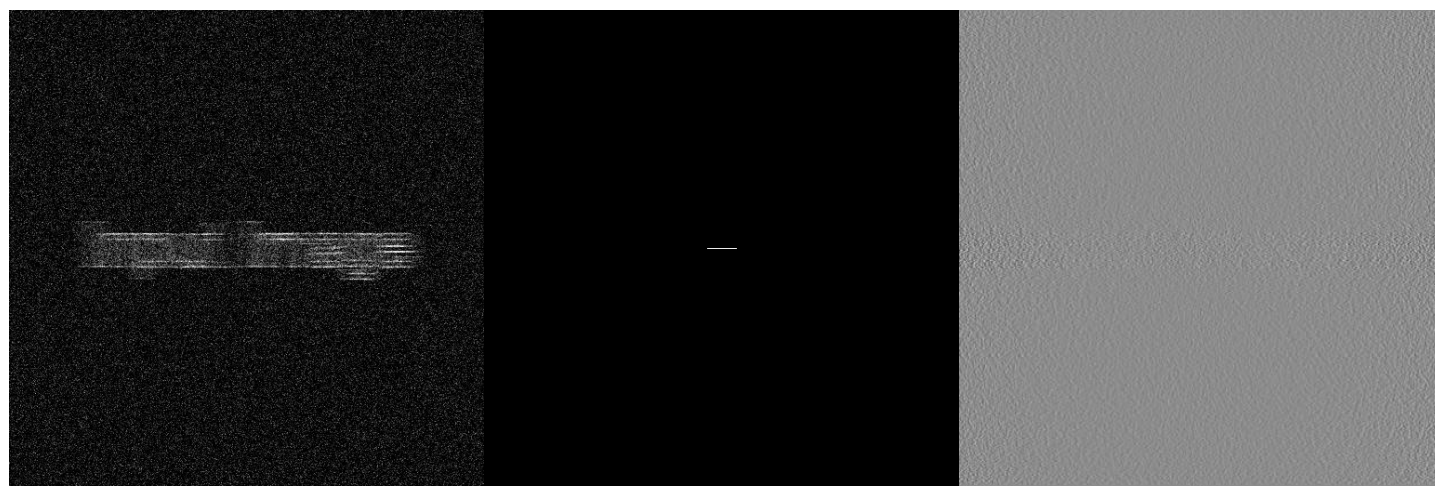

Figure 5.12. Application of eq. (5.14) to a noisy motion-blurred image. (left) Image with 32 pixels of motion blur and 50\% additive Gaussian noise. (center) 32 pixel uniform motion vector. (right) Result of removing 32 pixels of uniform motion. The original image is not discernable.

Eq. (5.14)'s time to execute depends on the magnitude of the motion being removed. Large shifts are computed faster than small ones. Small errors in the commanded shift vector to be removed result in large reconstruction errors. Eq. (5.14) is interesting because it performs the motion deconvolution in the time domain. However, its usefulness is doubtful in light of the maturity of the frequency domain algorithms.

\subsection{Practical usage of the frequency domain deconvolution formulas}

Equations 4.8 and 4.11 can be used to estimate the point spread function $\mathrm{H}(\mathrm{u}, \mathrm{v})$, given $\mathrm{G}(\mathrm{u}, \mathrm{v})$ and $\mathrm{F}(\mathrm{u}, \mathrm{v})$. Division by zero can be avoided by inspecting the denominator and taking appropriate action. For example if at a particular pixel in the frequency domain if $\mathrm{G}=\mathrm{a}+\mathrm{bi}$ and $\mathrm{F}=\mathrm{c}+\mathrm{di}$, the ratio is computed as a complex number as follows.

$$
\frac{G}{F}=\frac{a+b i}{c+d i}\left[\frac{c-d i}{c-d i}\right]=\frac{a c-b d+(b c-a d) i}{c^{2}+d^{2}}
$$

So the real and complex portions of the ratio both contain $c^{2}+\mathrm{d}^{2} \geq 0$ in their 
denominators. It is a simple matter to add a positive number to this denominator to avoid the zero case. Ignoring the constant in the numerator, eq. 4.8 turns into

$$
H(u, v) \approx \frac{G(u, v) F^{*}(u, v)}{F(u, v) F^{*}(u, v)+\text { epsilon }}
$$

Equation 4.14 is an example of simple inversion and it can be used in a variety of image processing applications with much success. Equation 4.11 and 4.14 tend to have similar performance, allowing for the adjustable parameters $\mathrm{K}$ and epsilon, respectively. Equation 4.14 has the divide by zero protection so it is favored for this project. The epsilon can be a proxy for the signal to noise and so has been made a variable parameter in the development of the software.

Additive noise can totally wreck the deconvolution process in complex imaging systems. It turns out that there is no unique solution to most deconvolution problems involving additive noise. Such problems are termed "ill-posed". To combat these illposed problems, many practitioners have developed iterative techniques, relying on regularization for fast convergence. These iterative techniques are not discussed here because simple inversion works satisfactorily. A short list of other useful techniques include 
- Van Cittert

- Jansen technique

- Richardson-Lucy

- Bracewell reverse diffusion

- etc.

These approaches are generally used for improving image resolution. Our approach is a little different in that we have a copy of the sharp image and instead want the blurring function. In our case this blurring function is caused by motion of the sample which is then integrated by the imaging system to form the final, motion-blurred image.

\subsection{Examples of images with motion blur and its removal}

A software program, named Image Motion Remover has been developed for the purpose of evaluating the process of image convolution and deconvolution for the express application where an ideal image is known. A screenshot of it performing the simulation of motion during image acquisition is shown in Figure 5.13. 


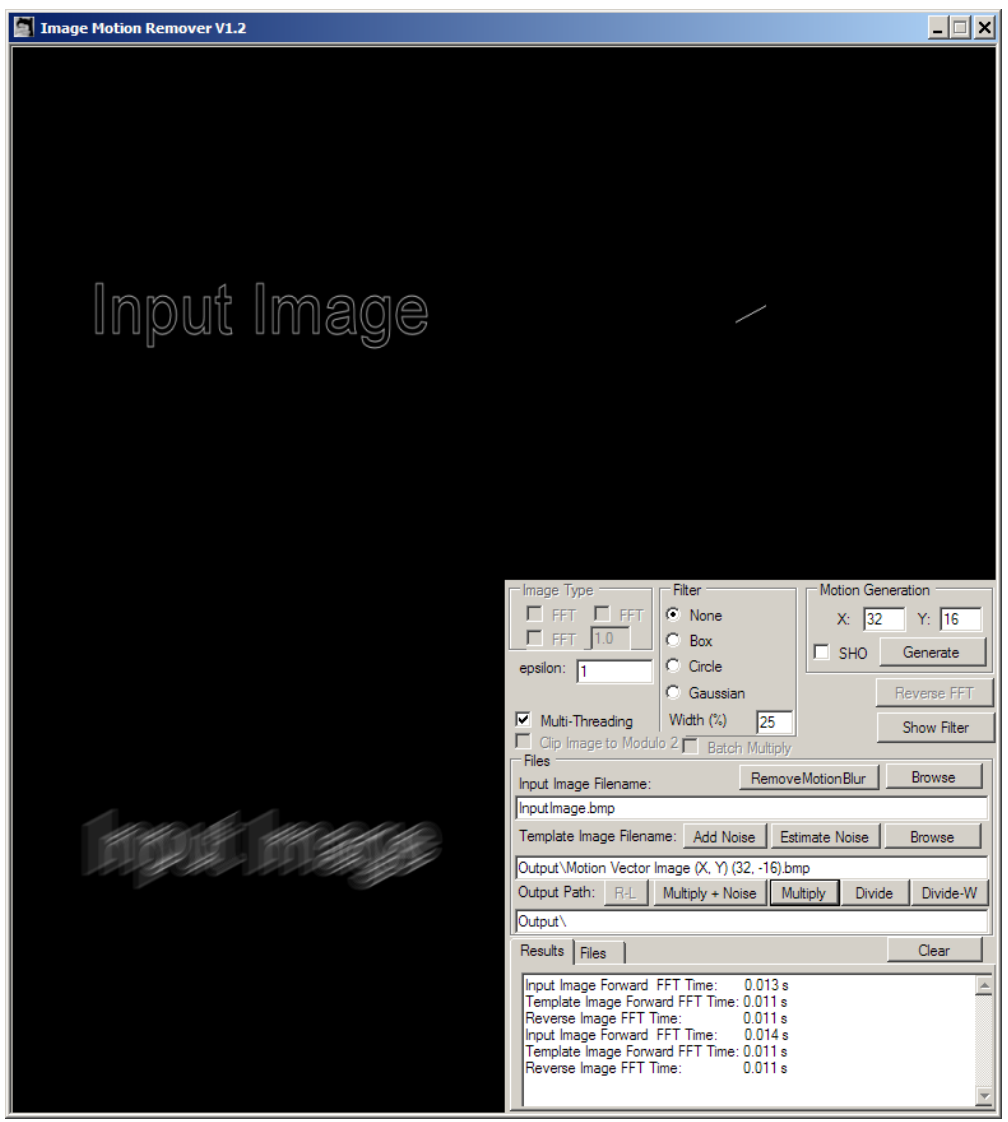

Figure 5.13. Screenshot of the software developed to evaluate convolution and deconvolution. In this screenshot, the upper-left image has been convolved with the upper-right image representing a uniform motion vector as a white line. The motion blurred result is in the lower-left.

The motion-blurred image can be easily inverted if the template image containing the motion vector is known. Similarly, the motion vector is easily retrievable, given the motion blurred image and its non-motion blurred template. Figure 5.14, Figure 5.15 and Figure 5.16 demonstrate some of the considerations in using image deconvolution to retrieve the motion vector from a motion blurred image and its template using eq. (5.16). 

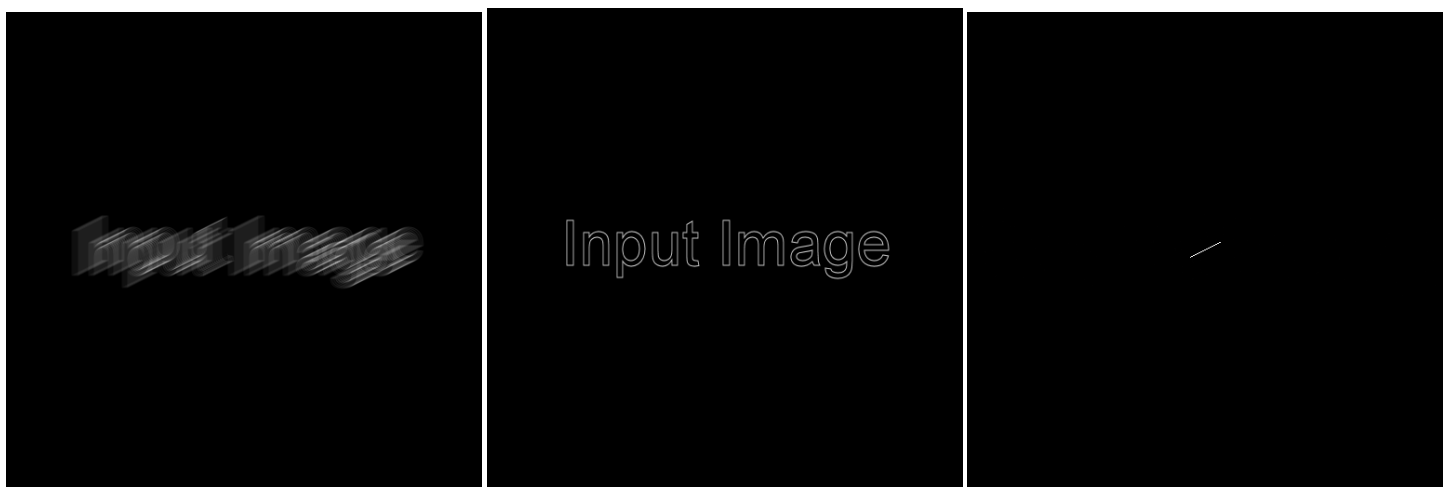

Figure 5.14. The motion in an image can be estimated if the ideal image is known. (left) Noiseless, motion blurred image. (center) Ideal non-blurred template image. (right) Image which is the result of applying eq 4.13 using an epsilon of 0.001 .

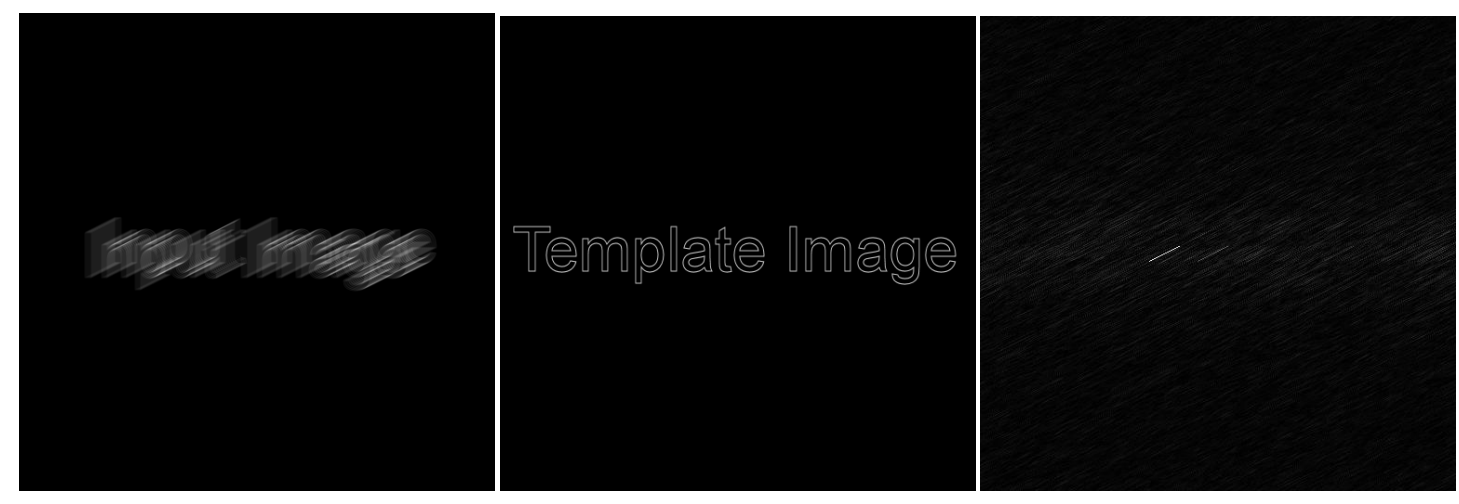

Figure 5.15. Demonstration of estimating the motion in an image using a non-ideal template image. The parts of the template image which cannot be found in the blurred image show up as structured noise in the motion image (right). The estimated motion vector is shifted from the center of the deconvolved image. Its center of mass corresponds to the relative shift between the word "Image" in each of the two images. Thus the deconvolution process can also be used to perform patternmatching operations. (epsilon $\sim 0.001$ ) 

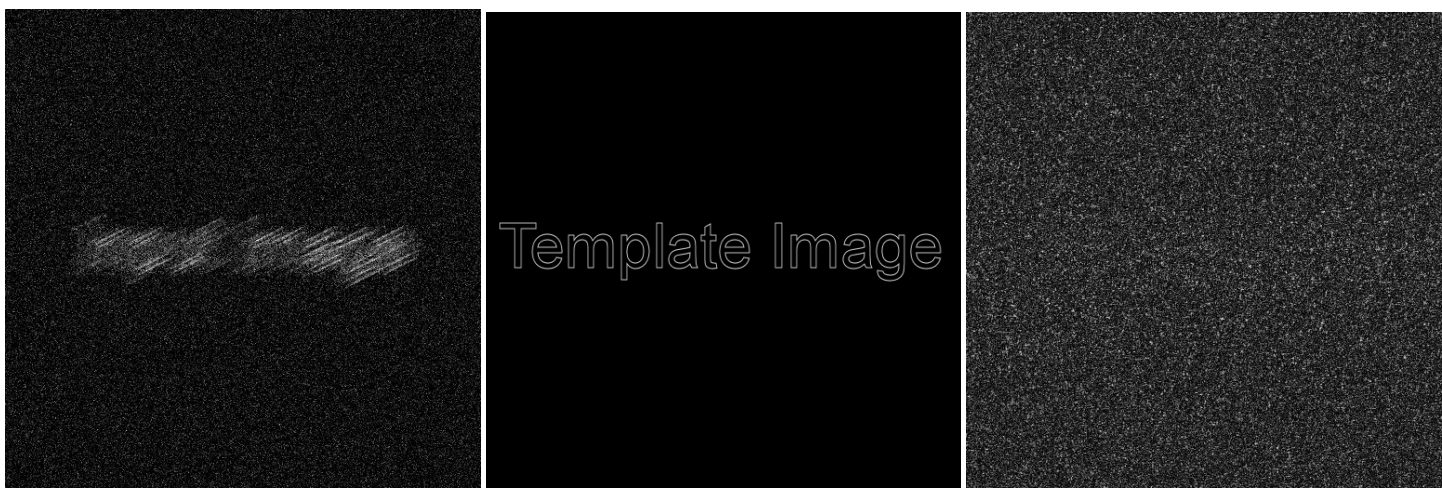

Figure 5.16. Example of adding $50 \%$ Gaussian noise to the motion-blurred image. The motion vector is buried in the random noise of the deconvolved image and is irretrievable. (epsilon $\sim 0.001$ )
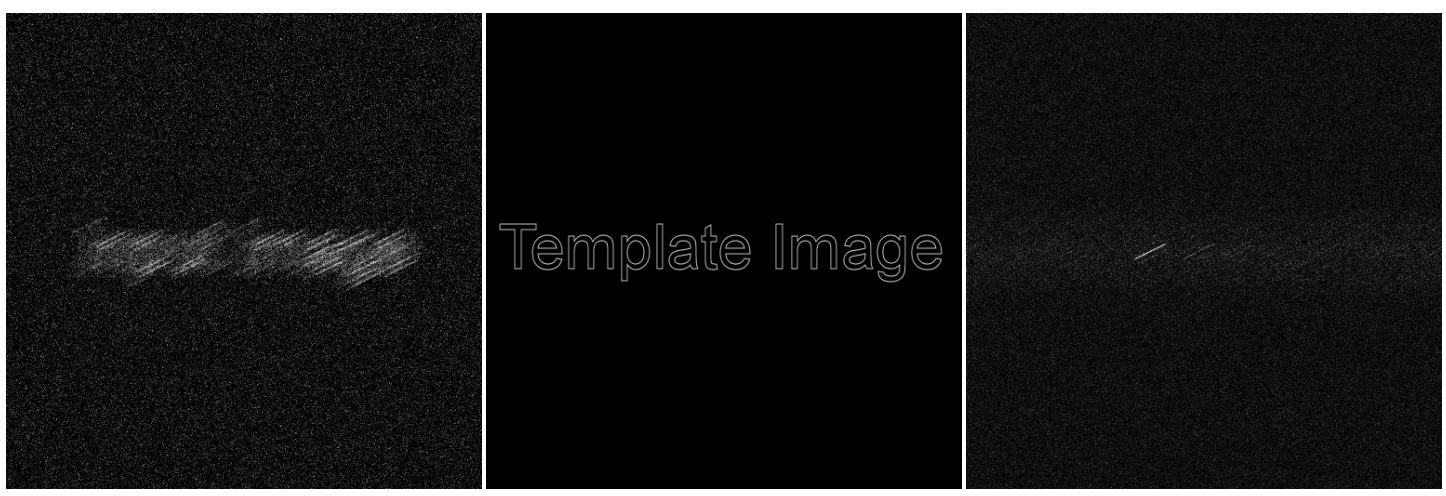

Figure 5.17. Example of adjustment of the epsilon parameter to a value of 100, representing the noise to signal ratio, to reduce the effect of the $50 \%$ Gaussian noise in the motion-blurred image. The motion vector can be readily retrieved from the deconvolved image.

\subsection{Estimating complex motion blur by deconvolution}

The deconvolution technique for motion estimation can be a very powerful tool for certain applications. The motion demonstrated in the previous section was uniform linear motion but we are by no means limited to just that. For example, imagine that an image undergoes spiral motion during an integrated acquisition. Noise further obfuscates the image to the point where it is not clear just what type of motion occurred. In certain situations an ideal image can be estimated and the motion can be determined using image inversion. 

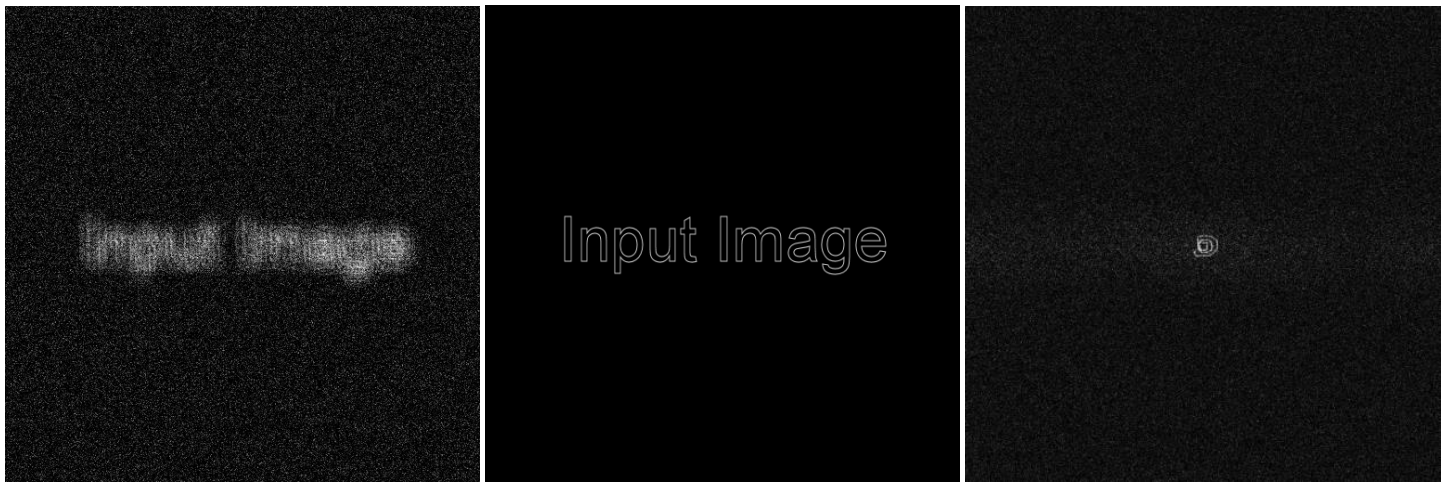

Figure 5.18. (left) An image convolved with a hand-drawn spiral and corrupted with $50 \%$ additive Gaussian noise. (middle) Ideal template image. (right) Deconvolved image revealing the handdrawn spiral. (epsilon 50)

The image representing the motion vector can also represent non-uniform motion. For example, an image feature undergoing sinusoidal motion in $\mathrm{x}$ would be represented by a line whose gray levels correspond to the amount of time spent at each pixel during acquisition.

A great deal of fun can be had making up image pairs for deconvolution and the sometimes counterintuitive results. For example, acquisition of a feature undergoing simple harmonic motion which executes about 1000 cycles per image will be a motion blurred version of the starting image. It can be shown that the probability density function of finding the feature whose peak amplitude is a, is related to position $\mathrm{x}$, by

$$
\operatorname{Probability}_{S H O}(x)=\frac{1}{\pi \sqrt{a^{2}-x^{2}}}
$$

To determine the probability at a given pixel, eq. (5.17) must be integrated between the centers of each adjacent pixel pair. For example, if the desired range is 72 
pixels, the integrated function is determined at each point. Since a range of \pm 36 pixels is computed using 73 points, including zero, the interval probabilities are the differences between these 73 points, yielding 72 points for the motion vector.

The integral of eq. (5.17) over an interval $\left[\mathrm{x}_{1}, \mathrm{x}_{2}\right]$ is

$$
\operatorname{Probability}_{S H O}\left(x_{1}, x_{2}\right)=\int_{x_{1}}^{x_{2}} \frac{d x}{\pi \sqrt{a^{2}-x^{2}}}=\left.\frac{1}{\pi} \sin ^{-1}\left\{\frac{x}{a}\right\}\right|_{x_{1}} ^{x_{2}}
$$

This function resembles the curve in Figure 5.19. Since the oscillations reach the end points only once per cycle and twice for all others, the probability values for these inside points are multiplied by two. Figure 5.19 illustrates that the vibrating feature is nearly five times more likely to be found at the ends of its oscillatory motion than in the center. This makes sense because the end points are where it is moving the slowest.
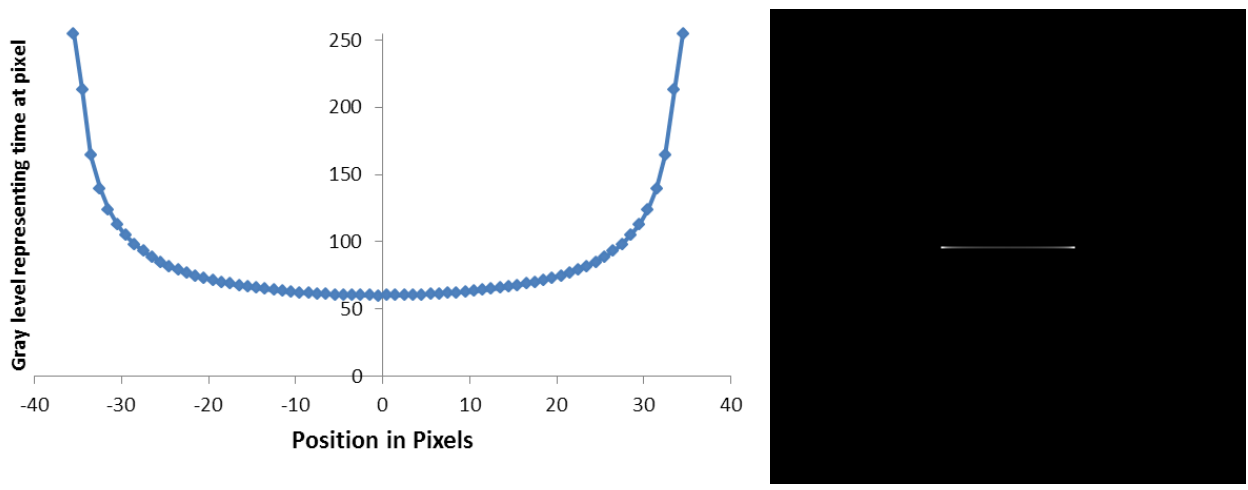

Figure 5.19. (left) Probability of finding a SHO in a particular position along a peak to peak oscillation of 72 pixels. The curve is scaled in gray levels for creation of motion vectors as images, $\mathbf{h}(\mathbf{x , y})$ (right). 
The SHO motion vector is quite a bit different than the uniform linear motion vector. For example, an image motion blurred using the SHO motion vector can be unblurred using the linear vector to see which artifacts remain.

Figure 5.20 demonstrates that it is possible to retrieve the un-blurred input image using an epsilon of 10 on a noise-free SHO motion-blurred image. Ghost images appear in the recovered image, representing the uncorrected motion elements. Thus it is possible to recover useful un-blurred images even when the model for the motion is not perfect.
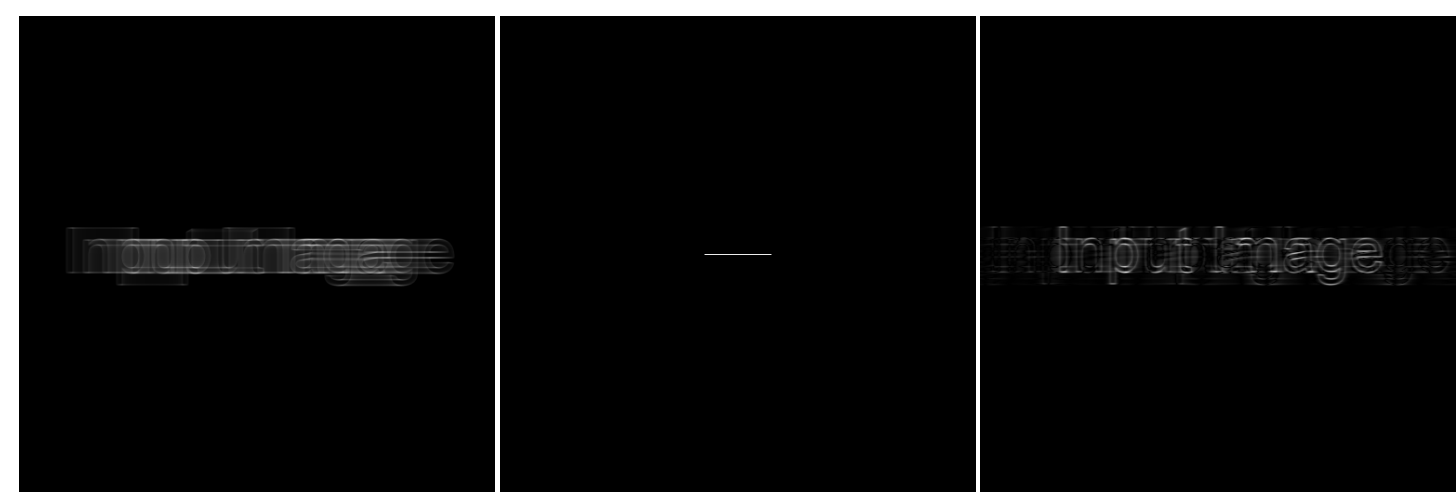

Figure 5.20. (left) Image motion blurred using a 72 pixel SHO motion vector image in Figure 5.19. (center) Uniform 72 pixel motion vector. (right) SHO motion blurred image with uniform motion removed. (epsilon 10)

\subsection{Demonstration of the tuning parameter on deconvolution performance}

Figure 5.9 illustrates that the image motion blur must be well known to use the time domain eq. (5.14) in order to recover the image. There are no adjustable parameters which can be used to improve its performance. In the frequency domain, equations (5.11), (5.12) and (5.16) have more adjustable parameters which can be used to cause faster convergence to the most pleasing estimate. For example, Figure 5.21 illustrates the need for accurate estimates of the uniform motion vector, being able to accurately 
deconvolve only the exact amount of motion as was originally put in. The same thing happens using the frequency domain technique using small values of epsilon, representing large signal to noise ratios.

Figure 5.21 demonstrates that the frequency domain technique could be considered worse than the corresponding time domain solutions in Figure 5.10 and Figure 5.11. Simply increasing the epsilon factor to a value of 1.0 causes the image inversion to recover the original image.

Figure 5.22 almost completely recovers the original image with all three motion vectors of 15, 16 and 17 pixels using an epsilon of 1.0. This is a remarkable result, given how badly the deconvolutions worked with the small epsilon. Notice that the deconvolution using the 16 pixel motion vector is also degraded slightly, so that accuracy is traded for robustness. This is the nature of ill-posed problems so one must be careful to control as many factors as is possible for a given application.

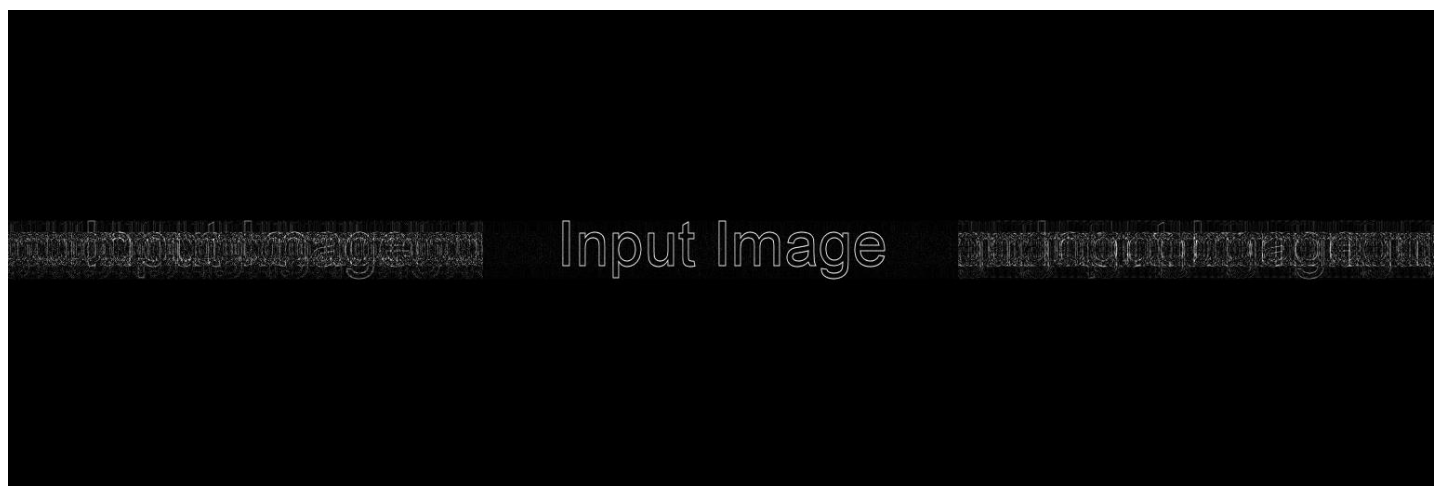

Figure 5.21. Demonstration of an image blurred using a motion vector of 16 pixels, which has been deconvolved using (left) 15 pixels, (center) 16 pixels, and (right) 17 pixels. (epsilon 0.001) 


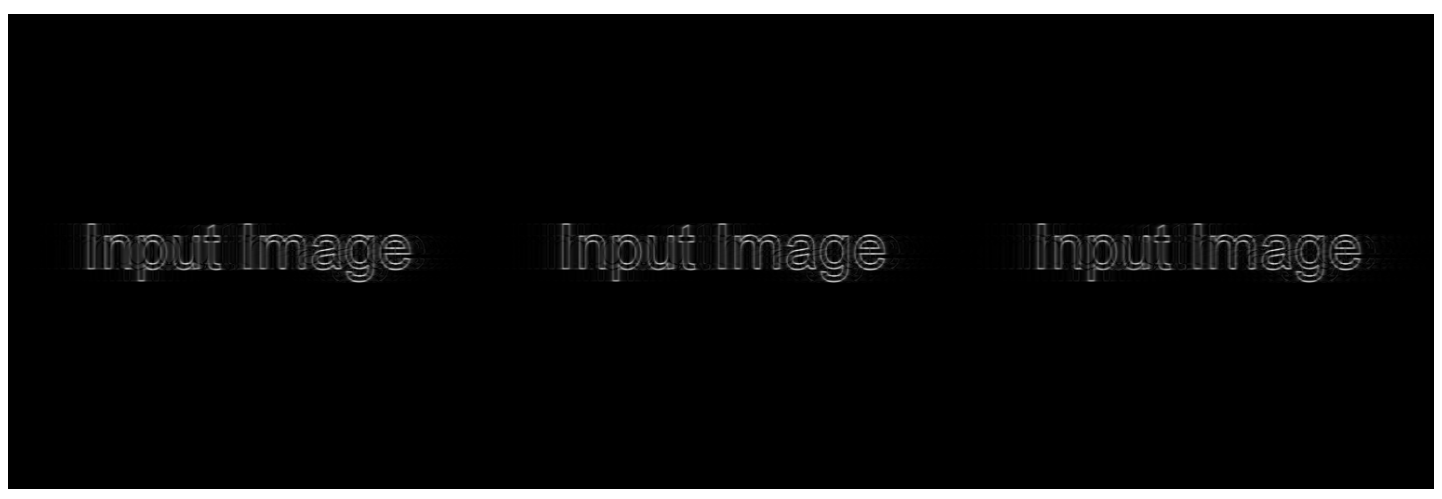

Figure 5.22. Demonstration of an image blurred using a motion vector of 16 pixels, which has been deconvolved using (left) 15 pixels, (center) 16 pixels, and (right) 17 pixels. (epsilon 1.0)

An apodization filter can also be applied during deconvolution. This feature has also been included in the ImageMotionRemover app but will not be discussed here in detail. Suffice it to say that it is one more parameter which requires tuning for a particular application.

\subsection{Deconvolution technique for estimation of motion from single images}

Section 5.10 illustrated how motion which is more complex than uniform linear motion can be represented in the motion vector image $h(x, y)$. If the images captured in the ETEM videos represent simple harmonic motion, then it should be possible to demonstrate. It was mentioned earlier that the role of the phosphor screen's persistence was unknown. Anecdotally, some of the movies acquired started with the AFM tip offcenter and the ETEM sample stage needed to be adjusted to the center of the screen.

During quick manual position adjustments, there were no persistent "ghost" images of the tip after it was moved. Thus the persistence effect may be small. A small but measurable persistence effect in the phosphor might still contribute to a uniform linear motion component in the blurring of the motion image. Its image might resemble Figure 5.23. 
The simple harmonic motion blurred image is shown in Figure 5.24. A comparison of the images in Figure 5.23 and Figure 5.24 with the center image in Figure 5.25 shows that the simple harmonic motion dominates, as it looks the most similar.

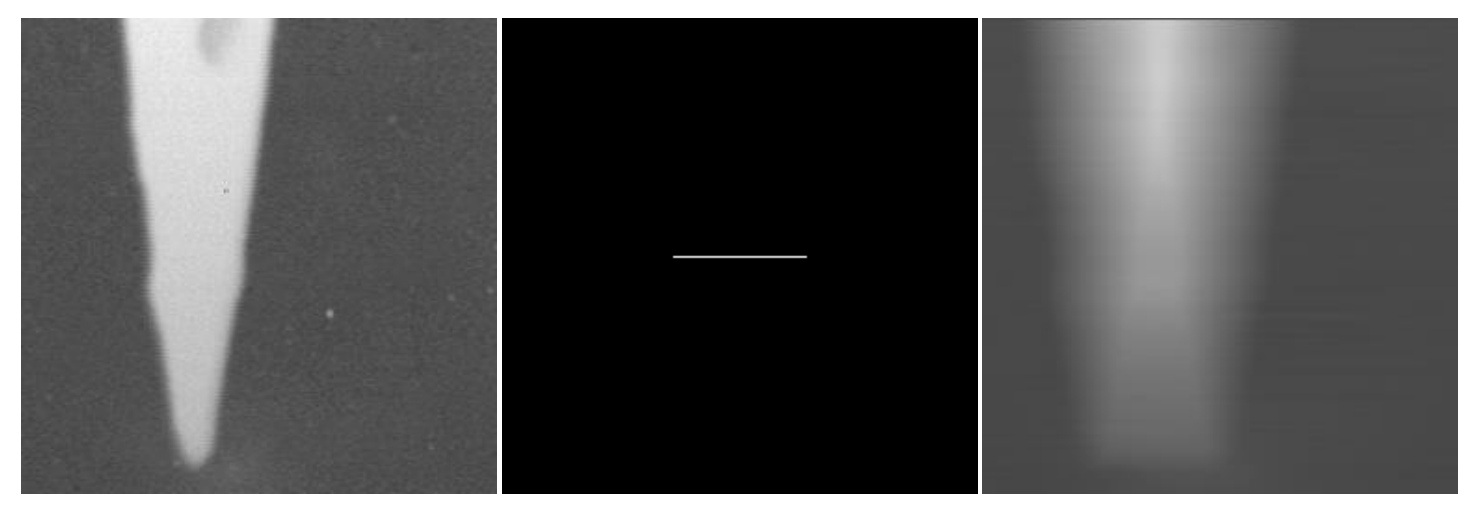

Figure 5.23. Illustration of what a 72 pixel, uniform linear motion of the AFM tip would look like.

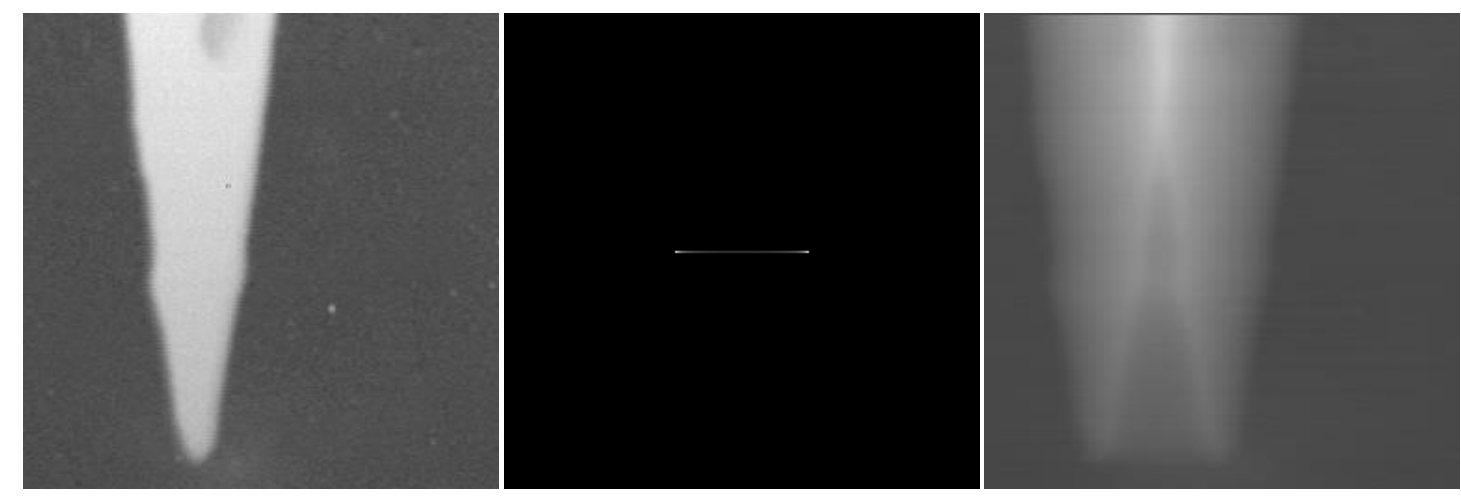

Figure 5.24. AFM tip as it would look it if were in 72 pixel, pure simple harmonic motion. Notice that the tip image is very clear at the ends of motion and that there is a nearly uniform "ghost" in between. 

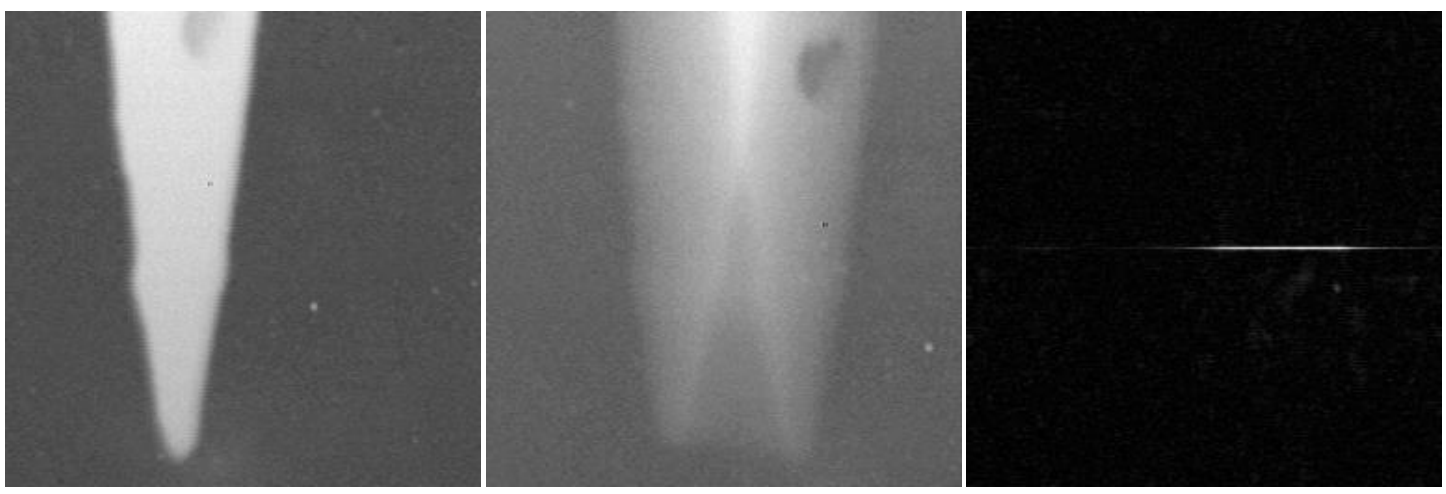

Figure 5.25. (left) Static ETEM image of an AFM tip mounted on a crystal tuning fork. This is to be treated as the template image, $f(x, y)$. (center) Same AFM tip, except that it has been set in oscillatory motion near its resonance frequency. It is to be treated as $g(x, y)$. The images were extracted from videos described in section 5.3. Additionally, the image gray levels were inverted so that the "signal pixels" are of the tip itself. (right) Motion vector image, $h(x, y)$ using eq. (5.14).
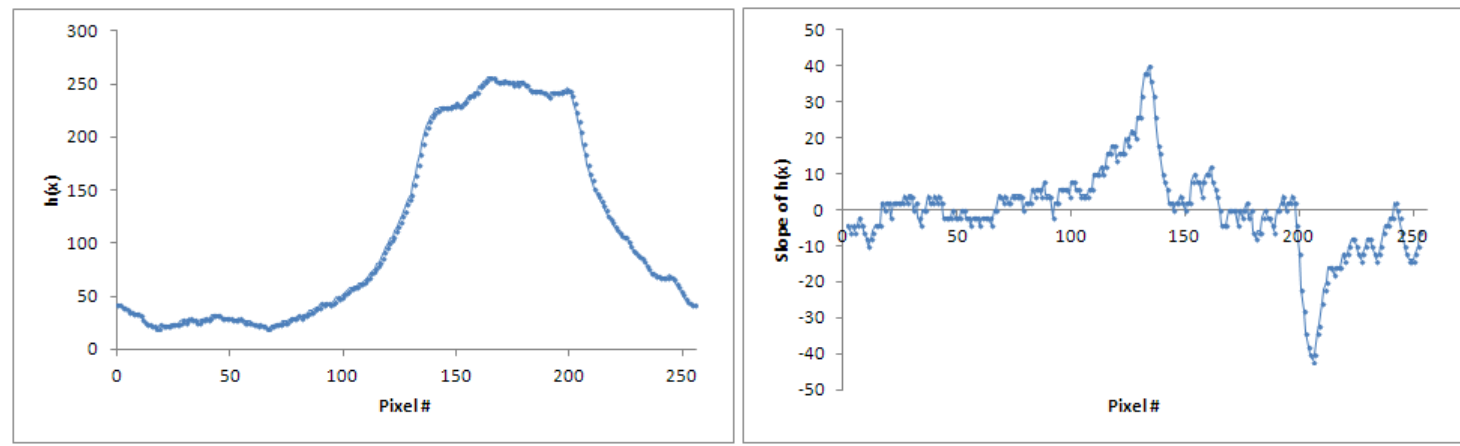

Figure 5.26. (left) Intensity profile along $x$ in $h(x, y=128)$ in Figure 5.25. (right) 5-point slope of $h(x, y=128)$ profile. Peaks of the slope image suggest that the motion peak to peak is 72 pixels.

Figure 5.25 is a deconvolution of the motionless AFM tip from the tip in oscillatory motion. Its motion vector is taken as the profile of the pixel data and is shown in Figure 5.26. The motion vector does not definitively look like either uniform motion or simple harmonic motion, as illustrated in Figure 5.23 and Figure 5.24. Instead, its profile looks like that in Figure 5.26. Its slope indicates that the peak to peak amplitude is approximately 72 pixels. Applying SHO motion blur of 72 pixels in Figure 5.24 creates a motion blurred image which greatly resembles the actual one in Figure 5.25.

The video images were heavily processed to obtain the static images prior to this 
analysis and it is possible that unwanted artifacts were introduced. In particular, the parabola removal operation was applied to the entire image, even where the shadow of the tip blocked the phosphor screen. This is the most likely culprit. It is also possible that the assumption of uniform image and noise spectral densities is inaccurate.

It is remarkable that the synthesized image using a measurement of peak slope, translated into simple harmonic motion, gives an image which resembles the actual vibrating image better than the uniform linear motion. This method can likely be used asis to perform these oscillation measurements on the video collected so far.

\subsection{Discussion of deconvolution as applied to motion estimation}

There are many books and tutorials on the topic of image deconvolution. There are also many software programs which demonstrate various algorithms. I have found most of them difficult to use and are difficult to tune for a particular application. For video analysis of a single movie, the deconvolution must run unattended for 1800 images or more. Algorithms requiring more than 2-3 tuning parameters quickly become unmanageable. This is the explanation for the simple approach presented. The application is very specific and can in principle be optimized for use with only one or two parameters.

\subsection{Summary}

It is clear that the motion of an AFM tuning fork can be observed using a TEM. Recorded video can be analyzed to determine the amplitude using brute-force slope analysis or by the more sophisticated techniques of deconvolution. Many of the movies collected contain more than 2000 images. For the deconvolution technique, batch-mode will need to be added to the ImageMotionRemover app along with the associated results 
and analyses. Not mentioned is the role of the drift of the AFM tip in the videos. For the deconvolution scheme to be successful, the vertical position of the tip in every single image should be in the same position w.r.t the first template image. To do this, a smaller image is clipped out of the video, centered on the center of mass of the AFM tip. An observant person will notice that since the images are of a phosphor screen, little imperfections are seen as little spots which have the same relative positions. Alignment fiducials can also be seen in the full-size images. These little imperfections contribute significant power to the frequency spectrum and so represent structured noise in the deconvolution process. Very careful inspection of the rightmost image in Figure 5.25 reveals a small dot in the deconvolved image. This dot's intensity represents the amount of power contained in these little imperfections. The vector from the image center to this dot represents the amount of image drift which occurred from the first frame.

\subsection{References}

Andrews, Harry C, and Bobby Ray Hunt. Digital Image Restoration. Englewood Cliffs, N.J.: Prentice-Hall, 1977.

Bracewell, Ronald N. Fourier Analysis and Imaging. New York: Springer, 2006.

Castleman, Kenneth R. Digital Image Processing. Englewood Cliffs, N.J.: Prentice-Hall, 1979.

Doughty, Jeffrey Jon. "Symmetric Near-Field Probe Design and Comparison to Asymmetric Probes,” 2010. http://pdxscholar.library.pdx.edu/open_access_etds/390/.

Elektros. "ETEM User Manual.pdf.” Elektros, circa 1975.

Fraser, Stephen. Pro Visual C++/CLI and the .NET 2.0 Platform. Berkeley, CA; New York: Apress ; Springer-Verlag [distributor], 2006. 
Gonzalez, Rafael C, and Richard E Woods. Digital image processing. Reading, Mass.: Addison-Wesley, 1993.

Hansen, Per Christian. Discrete Inverse Problems: Insight and Algorithms. Philadelphia: Society for Industrial and Applied Mathematics, 2010.

Jansson, Peter A. Deconvolution of Images and Spectra. San Diego: Academic Press, 1997.

Liu, Jinquan, Andrea Callegari, Martin Stark, and Majed Chergui. "A Simple and Accurate Method for Calibrating the Oscillation Amplitude of Tuning-Fork Based AFM Sensors." Ultramicroscopy. 109, no. 1 (2009): 81.

Novotny, Lukas, and Bert Hecht. Principles of Nano-Optics. Cambridge: Cambridge University Press, 2006.

Nowak, Derek Brant. The Design of a Novel Tip Enhanced near-Field Scanning Probe Microscope for Ultra-High Resolution Optical Imaging, 2010.

http://adsabs.harvard.edu/abs/2010PhDT........38N.

Serway, Raymond A. Physics for Scientists and Engineers. Philadelphia [etc.: Saunders College Publishing, 1992.

Tambade, Popat S. "Harmonic Oscillator Wave Functions and Probability Density Plots Using Spreadsheets.” Latin-American Journal of Physics Education 5, no. 1 (2011): 7. 


\section{Motivation for the AFM-SIMS system}

AFM data can be useful in characterizing FIB milling. This is typically performed exsitu, where the FIB and AFM are two distinct instruments. There can be substantial time lags and sample contamination issues using this ex-situ scheme. The addition of SIMS necessitates sample cleanliness, since freshly exposed material will invariably oxidize when the SIMS chamber is vented.

\subsection{Dissimilar milling rates}

As described in chapter 2, different materials will exhibit differing sputter rates during ion imaging /milling. Consider a two-component material, where the darker substrate mills three times slower than the bits of white material. The result is represented schematically in Figure 6.1.
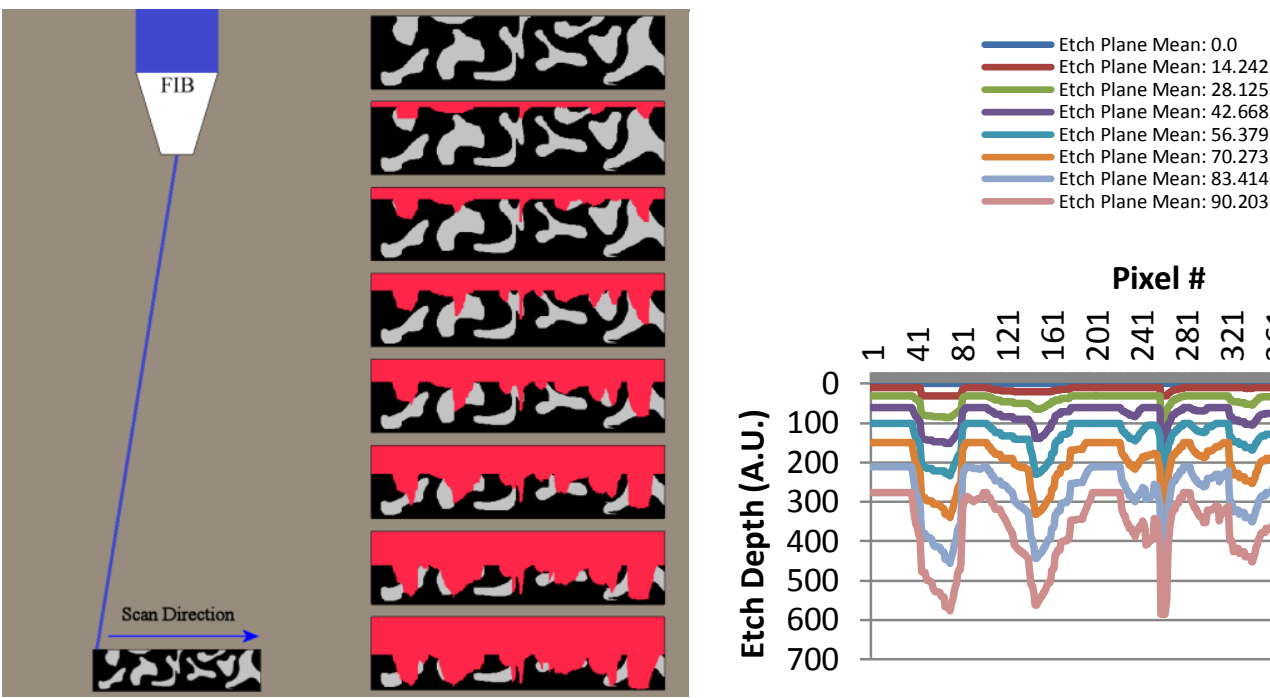

Pixel \#

Figure 6.1. (left) 2D representation of FIB imaging, illustrating the removal of a thin layer of the sample with each successive FIB image. The white material mills away 3 times faster than the black material. With each successive FIB image, the white material recedes farther and farther from the mean etch plane. In an amorphous sample, the result can be very complex. (right) Simulated depth data, taken for every 10th pass with the Ion Beam. The surface roughness appears to increase with each pass. 
The situation in Figure 6.1 has been somewhat overstated. For example, the tall pointy structures are known to have higher mill rates than the bulk of the same material. A series of SRIM runs on Silicon, varying angle of incidence shows a peak at about 82 degrees (Figure 6.2). This demonstrates that high aspect ratio structures can mill up to about ten times faster than flat ones. Thus the surface roughness may not grow without bounds and may reach some steady state. A study of this phenomenon could lead to some interesting results.

From Figure 6.2, it may be the case that sharp structures are not favored during the milling process. However, buried structures or voids may lead to both low and high frequency variations in the mean etch surface. We expect to encounter complex structures containing many components, each having its own sputter rate. Geological samples are prime candidates.

For a more complex example, consider a material composed of 6 distinct types of little grains, each denoted by a different gray level. Further, material \#1 has a unity sputter rate; material $\# 2$ has $2 \mathrm{X}$ and so on. From the side, it might look like Figure 6.3. It is difficult to predict the actual evolution of the surface, but Table 6.1 gives use a clue how we might use the predictions in reverse, as if we measured them during a sputtering process. 


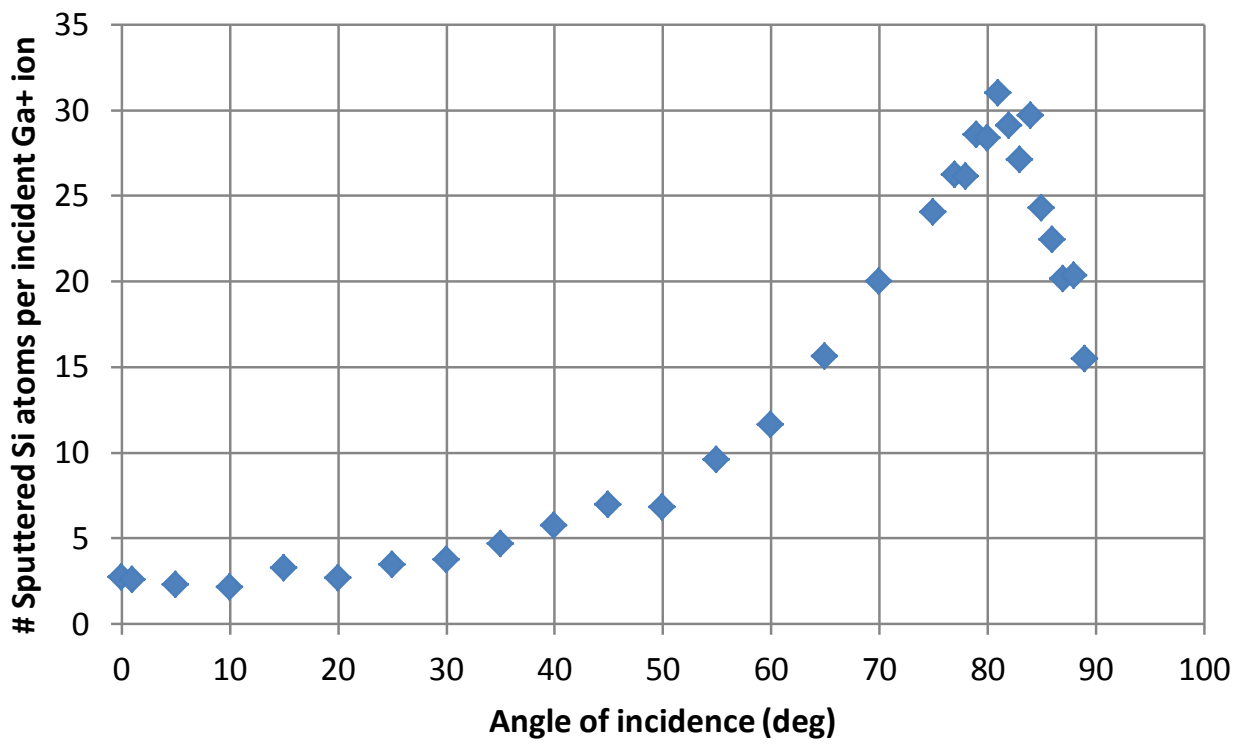

Figure 6.2. Plot of SRIM computed sputter rates vs incident angle for $25 \mathrm{kV}$ Ga+ ions on Si. Each point was computed by flying only 256 ions, for time considerations. Some error is evident, but the trend shows high sputter rates for steep angles of incidence. This tells us that high aspect structures can sputter up to ten times faster than flat ones.

To visualize this process, imagine the situation where we have a material as in Figure 6.1 and we attempted to reconstruct the 3D volume from images obtained during the milling process. In Table 6.1, images on the left represent the side-view of a 3D material and images on the right represent how a reconstructed volume might look if we blithely used the top-down intensity at each pixel. It is important to remember that when we remove layer by layer, we can only see the current layer as an intensity plot, from the top as in a FIB or SIMS image. If the material milling rates were identical, we could reconstruct the volume by simply recording the signal intensity at each pixel with each milling pass. Now imagine that the milling rates are not equal. The side view of the reconstructed volume might look like the images on the right.

In general, the faster milling materials will be the least accurately reconstructed. 
They will be thinner in $\mathrm{Z}$ and will exhibit more distortions from the layers above.

Combining this reconstruction scheme with AFM measurements at each FIB pass will allow for more accurate reconstructions. In this way we intend to develop a system for using AFM data, acquired alternately with FIB images, to perform 3D volume reconstructions of complex samples.
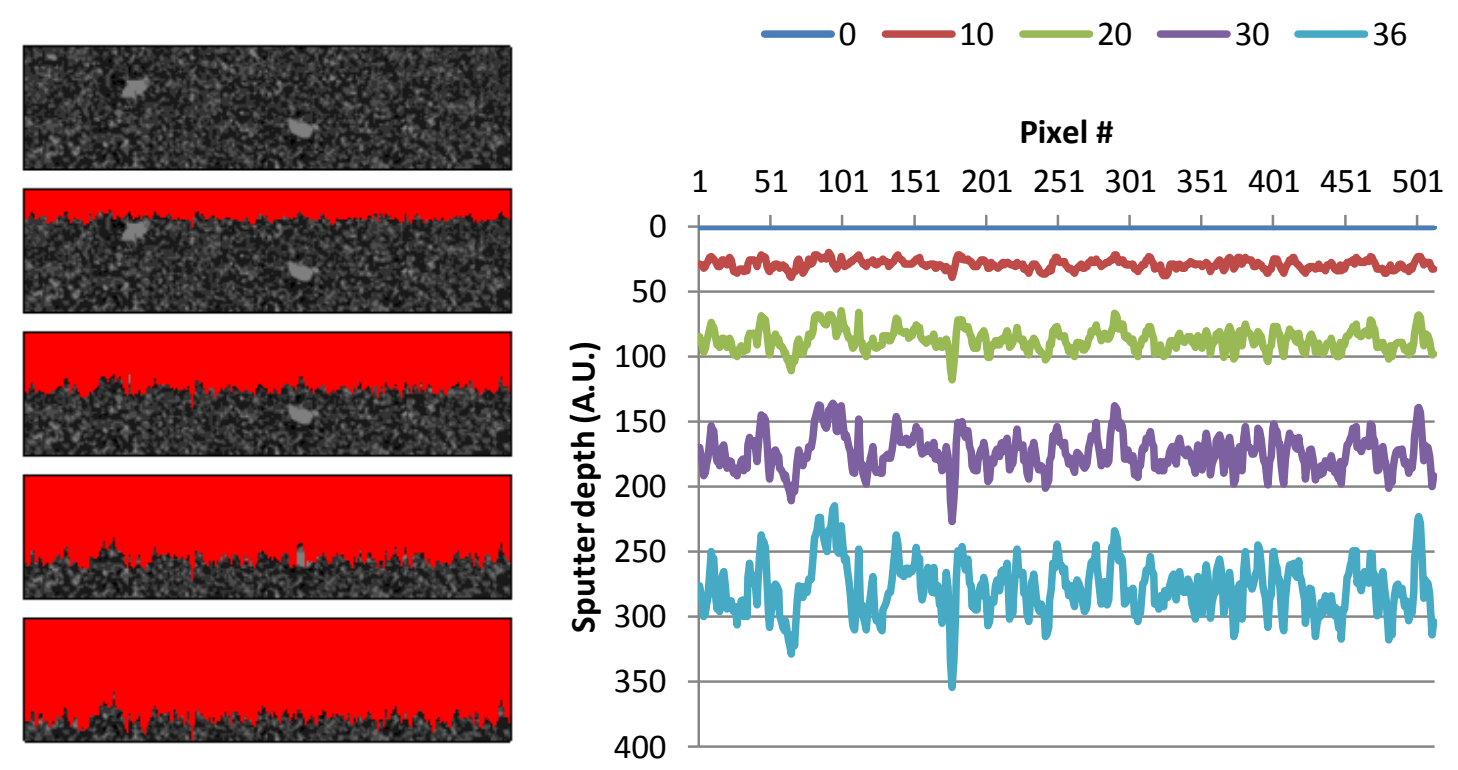

Figure 6.3. 2D representation of FIB sputtering on a 6-component sample in which each component is has a relative sputter rate of $1 X, 2 X, 3 X, 4 X 5 X$ and $6 X$, as indicated by its gray level. The plot represents the milled surface for every 10th pass (and the last one) of the ion beam.

The images in Table 6.2 were generated using a program created for the purpose, named DissimilarMillingCalculator.exe, which reads grayscale bitmaps and interprets each gray level as a different milling rate. The user can use presets for the milling rates, or can manually adjust them before starting the simulation. Input images are created using MS Paint, Gimp, Paint Shop Pro, etc. Although DissimilarMillingCalculator.exe can interpret as many as 256 gray levels, it is only practical to use up to six or so. 
Ultimately, the plan is to port this program to operate on 3D data and eventually, to incorporate AFM height data. It will also be important to incorporate topographical slope to accurately reconstruct material boundaries. The ability to reconstruct $3 \mathrm{D}$ data sets will also be possible using only the gray levels and each material's relative milling rate if known from prior runs incorporating AFM. In this way, the AFM is only needed for new samples and the rates disseminated for use on FIB systems without AFM or SIMS. 
Table 6.1. As the range in relative milling rates is increased in a multi-component material, reconstruction is less accurate, particularly with mill depth. Notice how the reconstructed image is a compressed version of the original. Distortion of the amorphous features is somewhat difficult to see when the milling rates are similar. The distortion of the spherical features illustrates the effect even when the mill rates are comparable.

\begin{tabular}{|c|c|c|}
\hline Description & Actual Side-View Image & $\begin{array}{c}\text { Reconstructed Side-View } \\
\text { Image }\end{array}$ \\
\hline $\begin{array}{l}\text { Two-component material in } \\
\text { which the grains are } \\
\text { relatively large. Relative mill } \\
\text { rates are } 1.0 \text { and } 1.1 \text {. }\end{array}$ & & \\
\hline $\begin{array}{l}\text { Two-component material in } \\
\text { which the grains are } \\
\text { relatively large. Relative } \\
\text { mill rates are } 1.0 \text { and } 1.5 .\end{array}$ & & \\
\hline $\begin{array}{l}\text { Two-component material in } \\
\text { which the grains are } \\
\text { relatively large. Relative } \\
\text { mill rates are } 1.0 \text { and } 2.0 .\end{array}$ & & \\
\hline $\begin{array}{l}\text { Two-component material in } \\
\text { which the grains are } \\
\text { relatively large. Relative } \\
\text { mill rates are } 1.0 \text { and } 3.0 .\end{array}$ & & \\
\hline $\begin{array}{l}\text { Six-component material in } \\
\text { which the relative mill rates } \\
\text { are } 1.0,1.1,1.2,1.3,1.4 \text { and } \\
1.5 \text {. }\end{array}$ & & \\
\hline $\begin{array}{l}\text { Six-component material in } \\
\text { which the relative mill rates } \\
\text { are } 1,1.5,2.0,2.5,3.0 \text { and } \\
3.5 \text {. }\end{array}$ & & \\
\hline $\begin{array}{l}\text { Six-component material in } \\
\text { which the relative mill rates } \\
\text { are } 1,2,3,4,5 \text { and } 6 .\end{array}$ & & \\
\hline $\begin{array}{l}\text { Three component material, } \\
\text { containing spherical } \\
\text { materials. The relative mill } \\
\text { rates are } 1.0,1.1 \text {, and } 1.2 \text {. }\end{array}$ & & \\
\hline $\begin{array}{l}\text { Three component material, } \\
\text { containing spherical } \\
\text { materials. The relative mill } \\
\text { rates are } 1,1.5 \text {, and } 2.0 .\end{array}$ & & \\
\hline $\begin{array}{l}\text { Three component material, } \\
\text { containing spherical } \\
\text { materials. The relative mill } \\
\text { rates are } 1,2 \text {, and } 3 \text {. }\end{array}$ & & \\
\hline
\end{tabular}




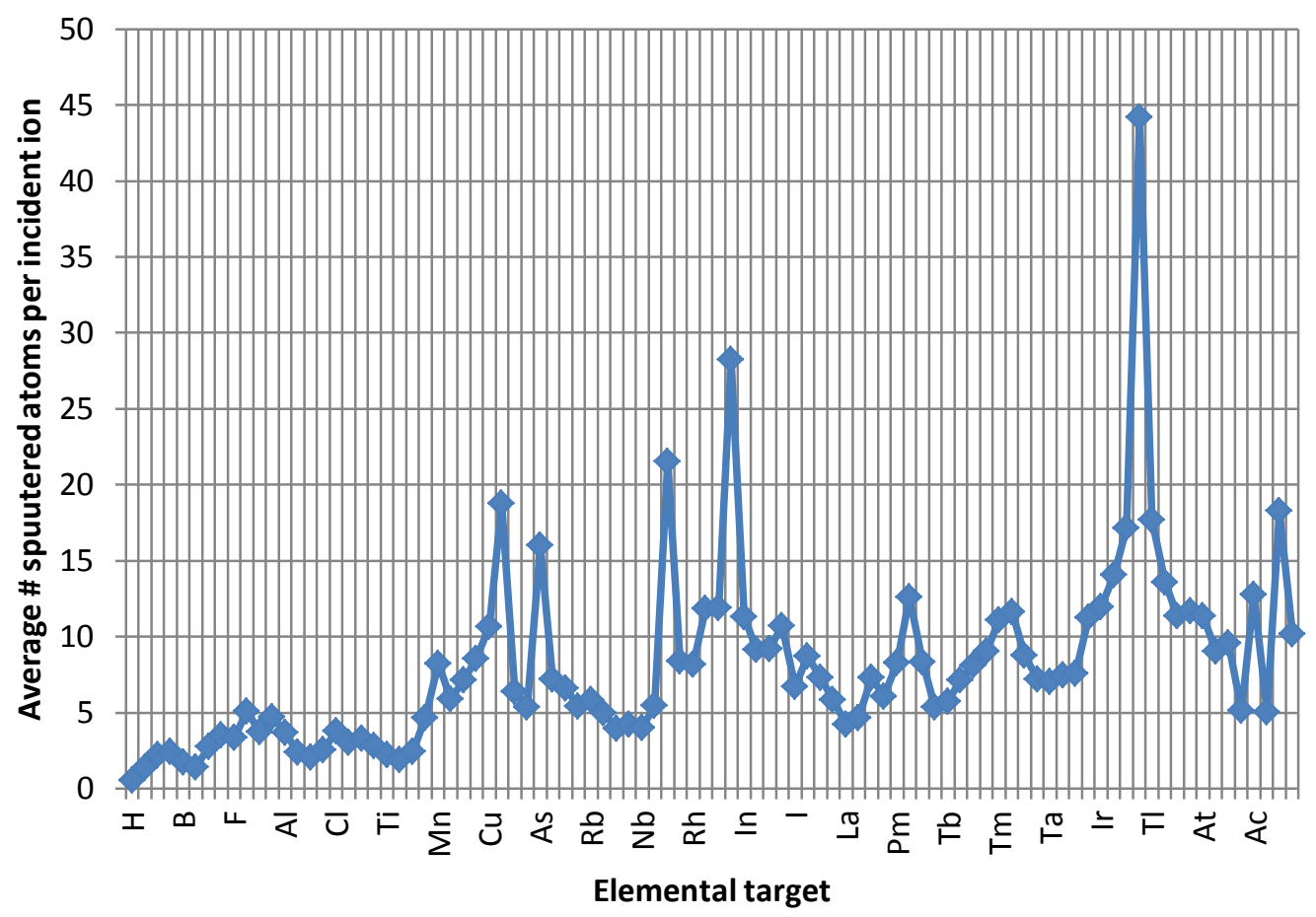

Figure 6.4. Plot of SRIM computed sputter rates at normal incidence for $25 \mathrm{kV} \mathrm{Ga+} \mathrm{ions} \mathrm{on}$ elements 1-92. Each point was computed by flying only 256 ions, for time considerations. Notice the trend of increasing sputter yield with increasing $\mathrm{Z2}$. This amounts to about 0.05 (atoms per incident ion) per AMU of target mass.

The milling rates in Table 6.1 may seem extreme at first, but a SRIM calculation of the sputter rates for elements 1-92 shows that the range for virtually any composite material can span an order of magnitude. This, coupled with the angular dependence can lead to more than two orders of magnitude in some samples.

Figure 6.4 illustrates one more feature of ion milling and that is that the milling rate exhibits a gradual increase as a function of target atomic mass. Recall that secondary electron yield has the opposite trend.

Finally, another factor in dissimilar sputter rates is matrix effects. Put simply, a given material may exhibit a different sputter rate than its bulk rate when it is in a multi- 
component material. Matrix effects are difficult to predict and can be affected by morphology and chemical makeup. This is one of our motivations for combining an AFM and a SIMS into one instrument.

It would be useful if the sample surface topography could be measured at some interval during milling of the crater. AFM height data could then be correlated with FIB image intensity and with SIMS elemental maps to form accurate 3D volumetric reconstructions.

\subsection{More details about a common sample - AITiC}

In order to verify the various pieces of the AFM-SIMS functionality, it is important to use samples which are both representative of the actual use-case and which present similar difficulties as real samples. Certain aspects of the real sample can be eliminated, such as starting surface roughness and conductivity. The target sample for this AFM-SIMS instrument is a micro-fossil. Micro-fossils are composed of very hard materials, minerals in a sort of a matrix having features on the order of microns to millimeters.

Perhaps one of the most challenging human-made samples is this ceramic having a nominal composition of $75 \% \mathrm{Al}_{2} \mathrm{O}_{3}-25 \%$ TiC. These ratios can be varied to a large degree and additional components added so oftentimes this material is simply referred to as AlTiC. AlTiC is a very hard material so it is mainly used in the construction of cutting tools. In the last thirty years, the data storage industry has adapted the advanced grinding and polishing techniques to the high-technology processes of thin film deposition and active device fabrication to create the hard-drive read/write head. This read/write head uses AlTiC for the construction of the hard drive head slider body. The slider body is a 
rectangular block whose surface has been polished to a mirror finish which can be less than $1 \mathrm{~nm} \mathrm{R} \mathrm{R}_{\mathrm{q}}$. This polished surface interfaces to the rotating disk, which is typically made of Aluminum coated with Nickel-Iron. Often a lubricant material coats the disk. The slider body interface to the disk is patterned with recessed features to create a dynamically stable air bearing surface which keeps the read/write head portion less than 1 $\mu$ ", or $25.4 \mathrm{~nm}$ above the disk during operation. The slider surface is often coated with a thin layer of Diamond-Like Carbon (DLC) for its hardness, lubricating and thermal properties.

Early consumer hard drives were prone to developing a problem with static friction between the slider and disk which was strong enough to keep the disk from spinning up during computer startup. These drives could sometimes be started by tapping on the hard drive's case. Many strategies were implemented to reduce this static friction. One of these strategies is the reduction in contact surface area between slider and disk. First is the shaping of the slider air bearing surface to have a cylindrical profile along its short width. Another is a process called grain recession, where one of the AlTiC components is preferentially etched using a plasma or other means. The result is that the slider air bearing surface has a bimodal topographical distribution and a reduced surface area of contact.

FIB images of AlTiC using SE's clearly delineate its composition with high contrast. Figure 6.6 shows a FIB image of a crater which was made by successive FIB imaging using the long pixel dwells associated with SIMS. Between each image, an 11image autofocus was performed. The rectangular walls of the crater exhibit a very high contrast due to the higher SE yield at physical steps. The right wall is dimmer than the 
left because the CEM detector is located to the right of the sample and many electrons leaving this wall are lost due to the effect detector shadowing. Most of the image is black and is due to the DLC coating. This coating is thin enough that the tails of the FIB have milled it away during the repeated auto focus operations and the very long pixel dwells during SIMS image acquisitions. One would expect that this halo of etched DLC would be perfectly centered on the rectangle but it is not. This is due to the fact that the autofocus operation utilizes a smaller dwell than the images used for making the rectangular crater and there is a system dependent image shift for different pixel dwells.

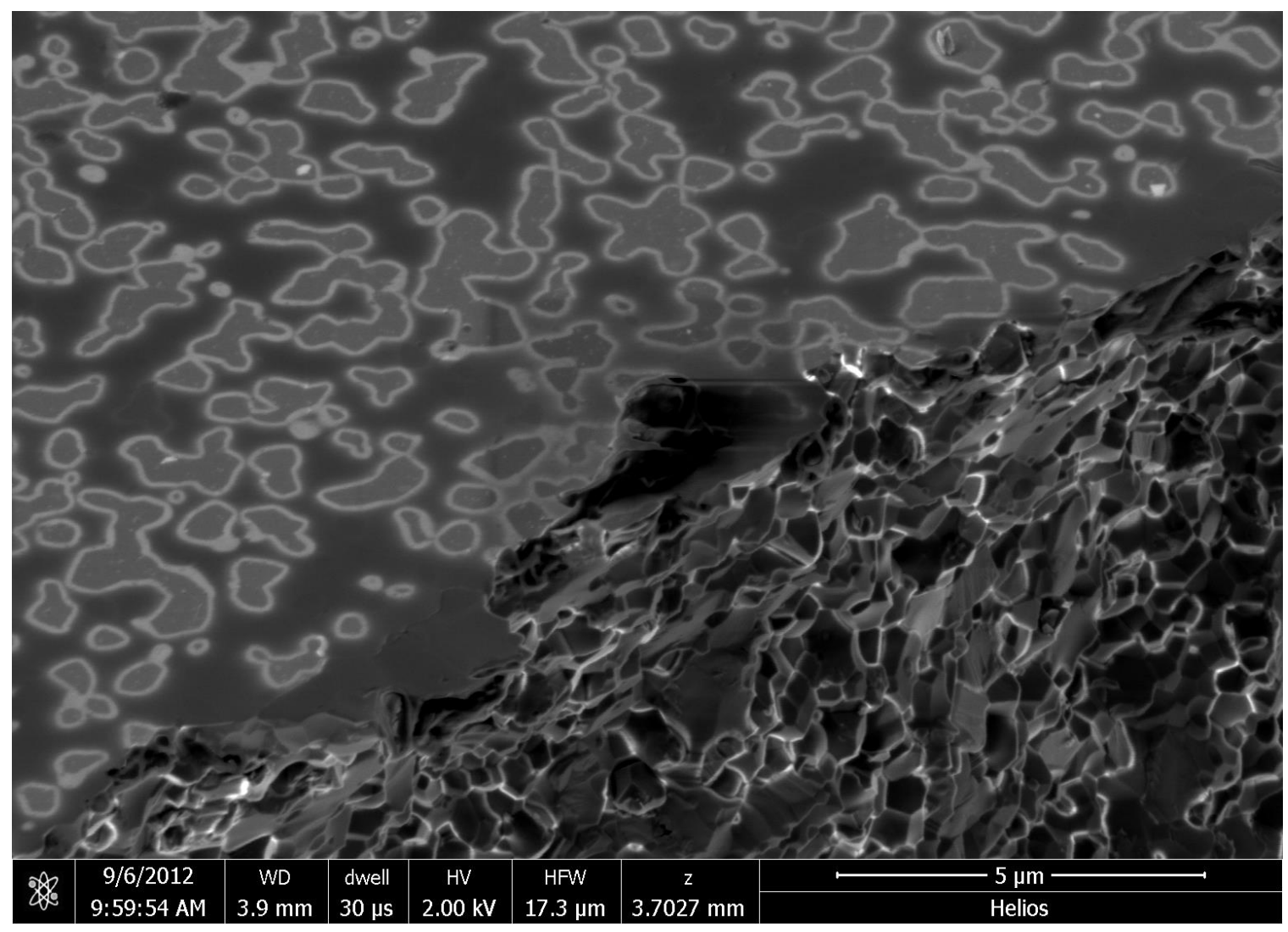

Figure 6.5. SEM image of a fractured piece of AITiC. The angular grains inside the material are not readily evident from the polished surface. 


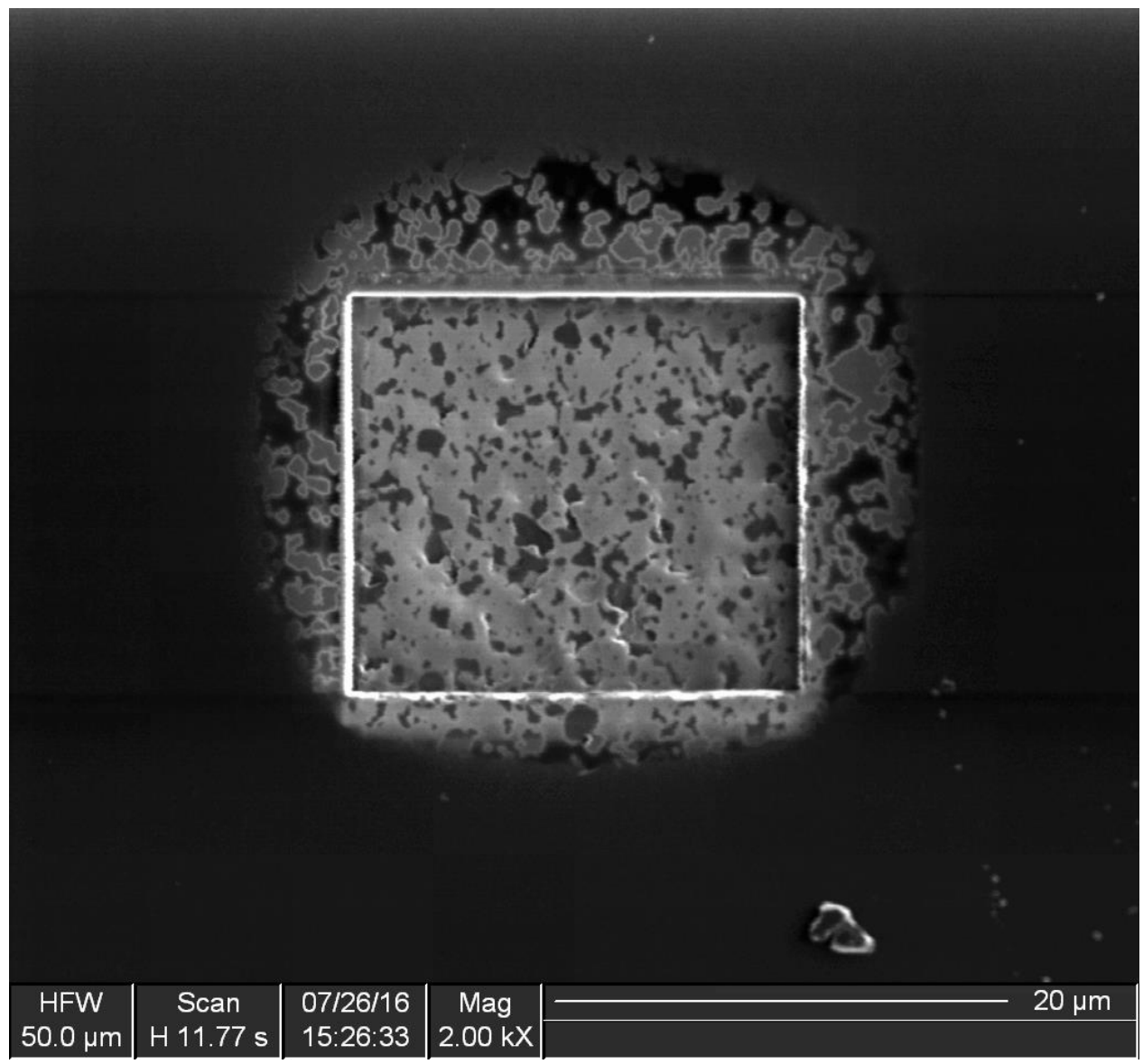

Figure 6.6. FIB image of a crater made by successive FIB images on the surface of a hard drive head air bearing surface. This image illustrates some distinguishing characteristics of the air bearing material and the effect of imaging using the FIB-SIMS. Image contrast has been enhanced slightly.

The imaging effects of the grain recession can be seen in Figure 6.6 and Figure 6.7 outside the border of the crater where the DLC has been removed. The islands contain these bright borders which signify an enhanced SE yield. The DLC coating is likely conformal and possibly even fills in these recessed regions to form a nearly level surface. As the DLC is removed, the borders become steps where the SE yield is high. Corresponding regions inside the crater show that there are virtually no bright borders so these regions are no longer appreciably recessed. 


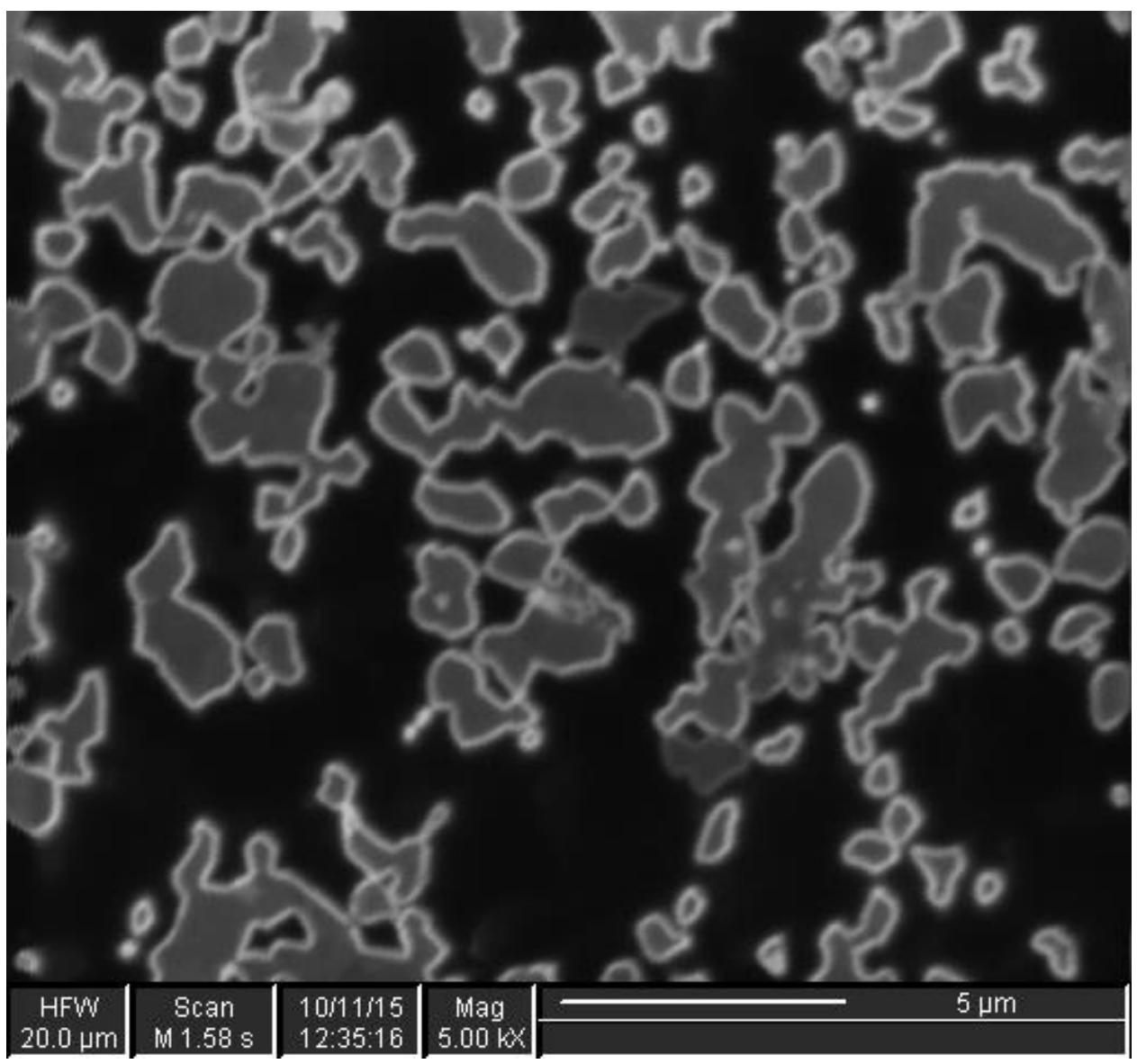

Figure 6.7. FIB SE image of AITiC taken just as the beam has milled through the DLC layer. The bright borders surrounding the gray islands are likely due to edge-enhanced secondary electron yield caused by the step created during the grain recession processing. These bright borders quickly fade away with successive FIB imaging.

\subsection{SRIM predictions for components of AITiC}

$\mathrm{Al}_{2} \mathrm{O}_{3}$ has a density of approximately $4 \mathrm{~g} / \mathrm{cm}^{3}$ which is greater than the density of its constituents, $\rho(\mathrm{Al}) \approx 2.7 \mathrm{~g} / \mathrm{cm}^{3} \rho\left(\mathrm{O}_{\text {liquid }}\right) \approx 1.4 \mathrm{~g} / \mathrm{cm}^{3}$. TiC has a density of $4.93 \mathrm{~g} / \mathrm{cm}^{3}$, while $\rho(\mathrm{Ti}) \approx 4.506 \mathrm{~g} / \mathrm{cm}^{3}, \rho\left(\mathrm{C}_{\text {amorphous }}\right) \approx 2.1 \mathrm{~g} / \mathrm{cm}^{3}, \rho\left(\mathrm{C}_{\text {graphite }}\right) \approx 2.267 \mathrm{~g} / \mathrm{cm}^{3}$ and $\rho\left(\mathrm{C}_{\text {diamond }}\right) \approx 3.515 \mathrm{~g} / \mathrm{cm}^{3}$. These parameters can be used with the SRIM calculations to estimate sputter rates in nm per incident ion. For example, 


$$
\begin{aligned}
\rho\left(\frac{\text { atoms }}{\mathrm{nm}^{3}}\right) \approx & \rho\left(\frac{\mathrm{g}}{\mathrm{cm}^{3}}\right) * \frac{6.022 * 10^{23} \text { atoms }}{\text { mole }} \\
& * \frac{1}{\text { atomic_weight }}\left(\frac{\text { mole }}{\mathrm{g}}\right) \frac{\mathrm{cm}^{3}}{\left(10^{7} \mathrm{~nm}\right)^{3}}
\end{aligned}
$$

And the monolayer thickness in nm can be estimated as

$$
\text { monolayer_thickness }(\mathrm{nm}) \approx \sqrt[3]{\frac{1}{\rho\left(\frac{\text { atoms }}{n m^{3}}\right)}}
$$

For example, an estimate for Titanium is

$$
\rho_{T i}=\frac{4.506 \mathrm{~g}}{\mathrm{~cm}^{3}} * \frac{6.022 * 10^{23} \text { atoms }}{\text { mole }} * \frac{\text { mole }}{47.867 \mathrm{~g}}=56.6886 \frac{\mathrm{Ti} \text { atoms }}{\mathrm{nm}^{3}}
$$

And

$$
\text { monolayer_thickness }_{T i} \approx 0.260 \mathrm{~nm} / \text { atom }
$$

SRIM estimates that $25 \mathrm{kV} \mathrm{Ga}^{+}$ion sputtering for Titanium is $\sim 2.189$ atoms/ion. The estimated sputter rate in $\mathrm{nm} /$ ion is 


$$
\text { sputter_rate } \approx \frac{\text { SRIM_sputtering_Yield }}{\text { \#atoms per molecule }} * \text { monolayer_thickness }
$$

Then

$$
\text { sputter_rate }_{T i} \approx 0.5698(\mathrm{~nm} / \text { ion })
$$

Table 6.2. Summarized milling rate predictions for some of the materials contained in AITiC,

\begin{tabular}{|c|c|c|c|c|c|c|}
\hline Material & $\begin{array}{c}\text { Melting } \\
\text { point } \\
\text { (K) }\end{array}$ & $\begin{array}{c}\text { \# atoms or } \\
\text { molecules } \\
\text { per } \mathbf{n m}^{\mathbf{3}}\end{array}$ & $\begin{array}{c}\text { SRIM sputtering } \\
\text { yield in atoms } \\
\text { per ion }\end{array}$ & $\begin{array}{c}\text { \# atoms } \\
\text { per } \\
\text { molecule }\end{array}$ & $\begin{array}{c}\text { Monolayer } \\
\text { thickness } \\
\text { in nm }\end{array}$ & $\begin{array}{c}\text { Sputtering } \\
\text { rate in nm } \\
\text { per ion }\end{array}$ \\
\hline $\mathbf{M g}$ & 923 & 43.062 & 4.404 & 1 & 0.285 & 1.256 \\
\hline $\mathbf{A l}$ & 933 & 60.22 & 3.609 & 1 & 0.255 & 0.921 \\
\hline $\mathbf{T i}$ & 1941 & 56.689 & 2.189 & 1 & 0.260 & 0.570 \\
\hline $\mathbf{A l}_{\mathbf{2}} \mathbf{O}_{\mathbf{3}}$ & 2345 & 23.625 & 4.926 & 5 & 0.349 & 0.343 \\
\hline $\mathbf{T i C}$ & 3430 & 49.572 & 2.345 & 2 & 0.272 & 0.319 \\
\hline $\mathbf{C}$ & 3915 & 113.661 & 1.352 & 1 & 0.206 & 0.279 \\
\hline
\end{tabular}

The milling rates in table Table 6.2 follow a trend which is expected in terms of melting points. We expect lower sputter rates with higher melting points. A careful look at Figure 6.8 and Figure 6.9 shows that the ratio of species liberated during sputtering is not stoichiometric with the sample composition. Thus $\mathrm{Al}_{2} \mathrm{O}_{3}$ becomes Aluminum rich with 3.31 Oxygen atoms per 1.62 Aluminum atoms sputtered. TiC becomes Carbon rich with 1.51 Titanium atoms per 0.837 atoms Carbon sputtered. The effective milling rate is likely affected, particularly with $\mathrm{TiC}$, as Carbon has a very low sputter rate. Also, the surface may be affected enough to change the secondary electron yield during normal SE imaging so the relative image contrast may change with depth during milling. Thus the prediction is that the $\mathrm{Al}_{2} \mathrm{O}_{3}$ will become bright and the TiC will become dark with repeated FIB imaging. 


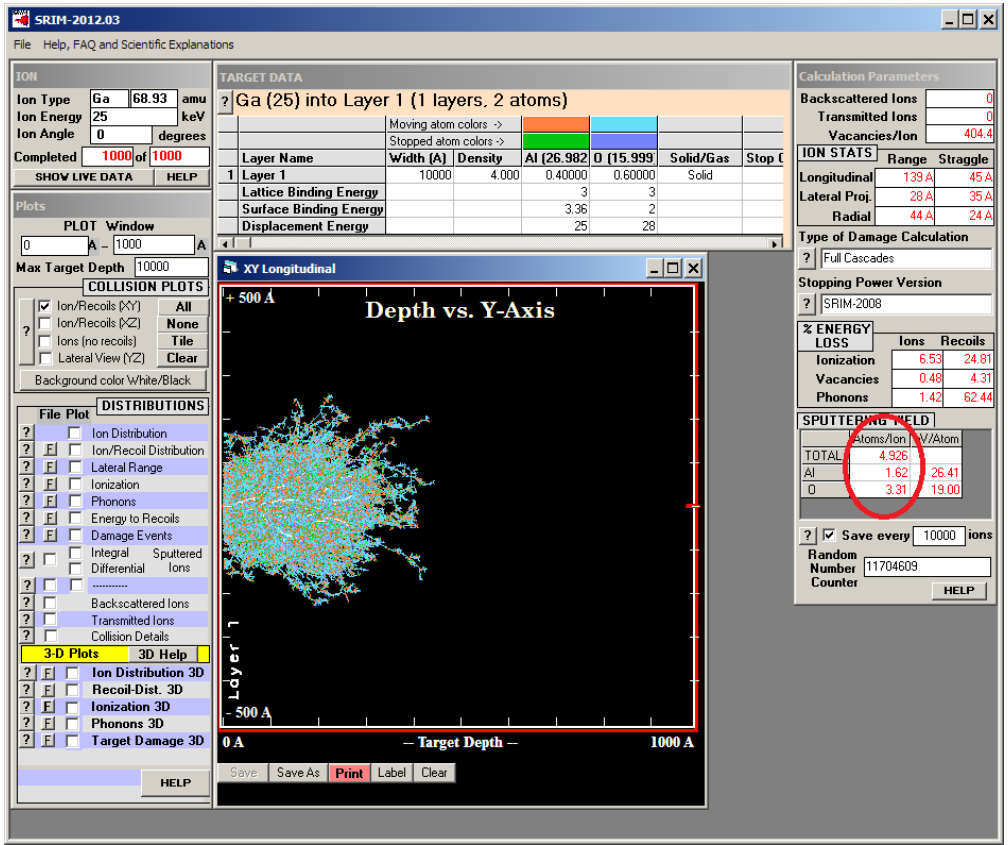

Figure 6.8. SRIM predictions for $25 \mathrm{keV} \mathrm{Ga}+$ ion sputtering of $\mathrm{Al}_{2} \mathrm{O}_{3}$. Nearly two Oxygen atoms per Aluminum atom is sputtered away, leaving an Aluminum rich surface.

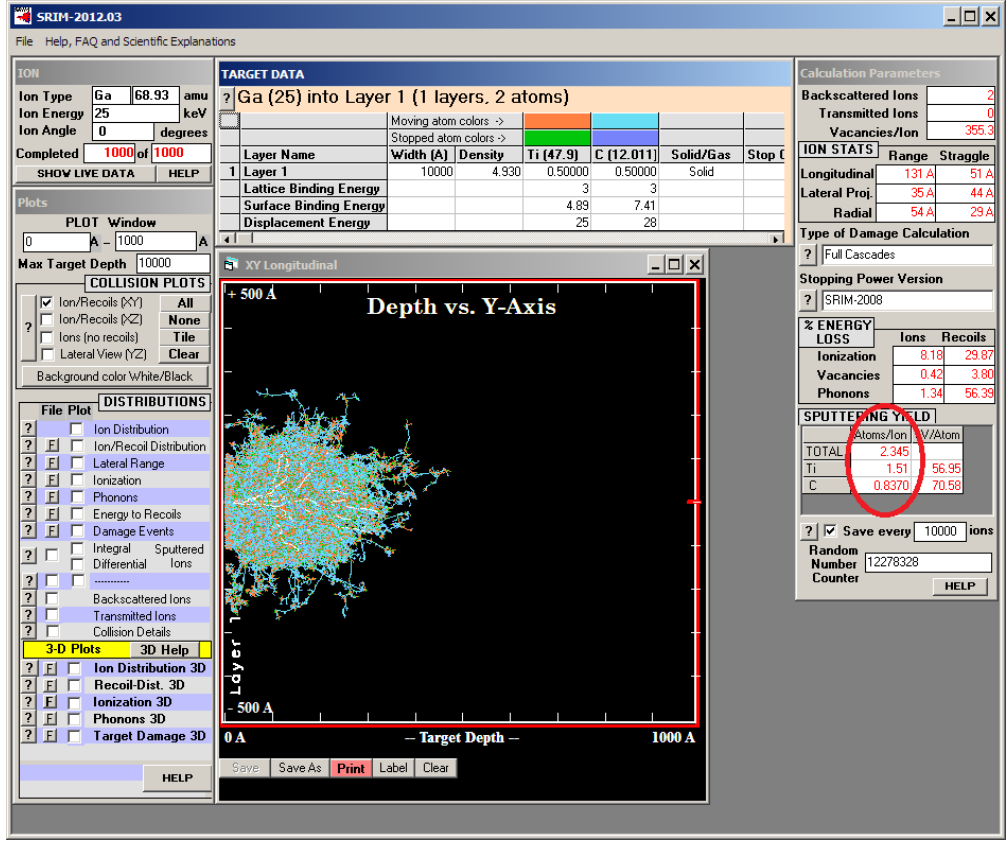

Figure 6.9. SRIM predictions for $25 \mathrm{keV} \mathrm{Ga}+$ ion sputtering of TiC. Nearly two Titanium atoms per Carbon atom are sputtered, leaving a Carbon rich surface. 


\subsection{References}

Azarian, M. H., C. L. Bauer, and M. S. Jhon. "Tribology of Thin-Film Media in Both Flying and Sliding Modes.” Wear 168, no. 1 (September 1, 1993): 59-76.

doi:10.1016/0043-1648(93)90199-V.

Bhushan, Bharat. "Chemical, Mechanical and Tribological Characterization of Ultra-Thin and Hard Amorphous Carbon Coatings as Thin as 3.5 Nm: Recent Developments."

Diamond and Related Materials 8, no. 11 (1999): 1985-2015.

Bhushan, Bharat. "Micro/Nanomechanics of Magnetic Storage Devices," 1999. http://dlia.ir/nano.tech/english/Books/09/131.pdf.

Bigler, Jeff. "Periodic-Table.xls," n.d. http://www.mrbigler.com/documents/PeriodicTable.xls.

Cai, K. F., D. S. McLachlan, N. Axen, and R. Manyatsa. "Preparation, Microstructures and Properties of Al 2 O 3-TiC Composites." Ceramics International 28, no. 2 (2002): 217-222.

Clark, B.K. "The effect of slider roughness on asperity testing of thin-film media." IEEE Trans. Magn. IEEE Transactions on Magnetics 29, no. 1 (1993): 235-40.

Cutler, Raymond A., Andrew C. Hurford, and Anil V. Virkar. "Pressureless-Sintered Al 2 O 3-TiC Composites.” Materials Science and Engineering: A 105 (1988): 183-192.

Li, Jing, Yan-sheng Yin, Rui-xia Shi, Lai-peng Ma, and Jian Li. "Microstructure and Mechanical Properties of Al2O3-TiC-4vol.\%Co Composites Prepared from Cobalt Coated Powders." Surface and Coatings Technology 200, no. 12-13 (March 2006): 3705-12. doi:10.1016/j.surfcoat.2005.02.165.

Liu, N., M. Shi, Y.D. Xu, X.Q. You, P.P. Ren, and J.P. Feng. "Effect of Starting Powders Size on the A12O3-TiC Composites." International Journal of Refractory Metals and Hard Materials 22, no. 6 (November 2004): 265-69. doi:10.1016/j.ijrmhm.2004.09.001.

Sourty, E., J. L. Sullivan, and M. D. Bijker. "The Tribology of Advanced Digital Recording (ADR) Systems.” Tribology International 33, no. 9 (2000): 629-637.

Yamamoto, Takayuki, Yoshiharu Kasamatsu, and Hiroyuki Hyodo. "Advanced StictionFree Slider and DLC Overcoat." Fujitsu Scientific and Technical Journal 37, no. 2 (2001): 201-11. 
Zhang, Yanfeng, Lianjun Wang, Wan Jiang, Guangzhao Bai, and Lidong Chen. "Effect of Fabrication Method on Microstructure and Properties of Al2O3-TiC Composites." Materials Transactions 46, no. 9 (2005): 2015-2019.

Ziegler, J. F, J. P Biersack, and Matthias D Ziegler. SRIM, the Stopping and Range of Ions in Matter. Chester, Maryland: SRIM Co., 2008. 


\section{FIB-SIMS instrumentation and system configuration}

Initially, the target FIB system was to be a Micrion model 2500 FIB system fitted with the so-called $5 \mathrm{~nm}$ "Saucer" column. The target SIMS system was to be the FEI SIMSIII and a surplus industrial pc containing the original microscope boards and FEI xP 2.25 software. At some point during the AFM development, an FEI FIB 611 became available for this project. This FIB 611 was already fitted with an FEI SIMS-I and was running FEI Ion Mill software version 5 using Windows 3.11. The SIMS control was performed by a dedicated computer and communication to the host pc running Ion Mill used an Ethernet connection. Unfortunately, its SIMS did not function with the available software so the similar scheme as used on the Micrion was employed, whereby the separate industrial computer was used to control it.

This same SIMS pc can also control the scanning and patterning functions. Thus the Windows $3.11 \mathrm{pc}$ was phased out, giving all of the FIB-SIMS control to the industrial pc running Windows XP and FEI xP2.25 software. Some additional controls were needed to make the system fully functional and are described below.

\subsection{FIB-611 system configuration}

The FIB system consists of a vacuum console, an electronics rack for FIB control and a SIMS rack for SIMS control. Samples are mounted onto a rotatable stub holder and can be moved in XYZ and Tilt. Thus the stage has five axes, XYZRT. The rotation axis is very small so it was decided that the AFM be mounted along-side it rather than on top of it. This reduces the range of sample motion in $\mathrm{Z}$ but simplifies the design. The AFM was placed on the far corner of the XY stage so that the original rotation axis and its sample holder could remain undisturbed. This arrangement also positioned the AFM 
in a relatively safe location from crashing into the FIB, SIMS and GIS's. The brush for the slip-ring did need to be redesigned and moved to the other side to accommodate the AFM.

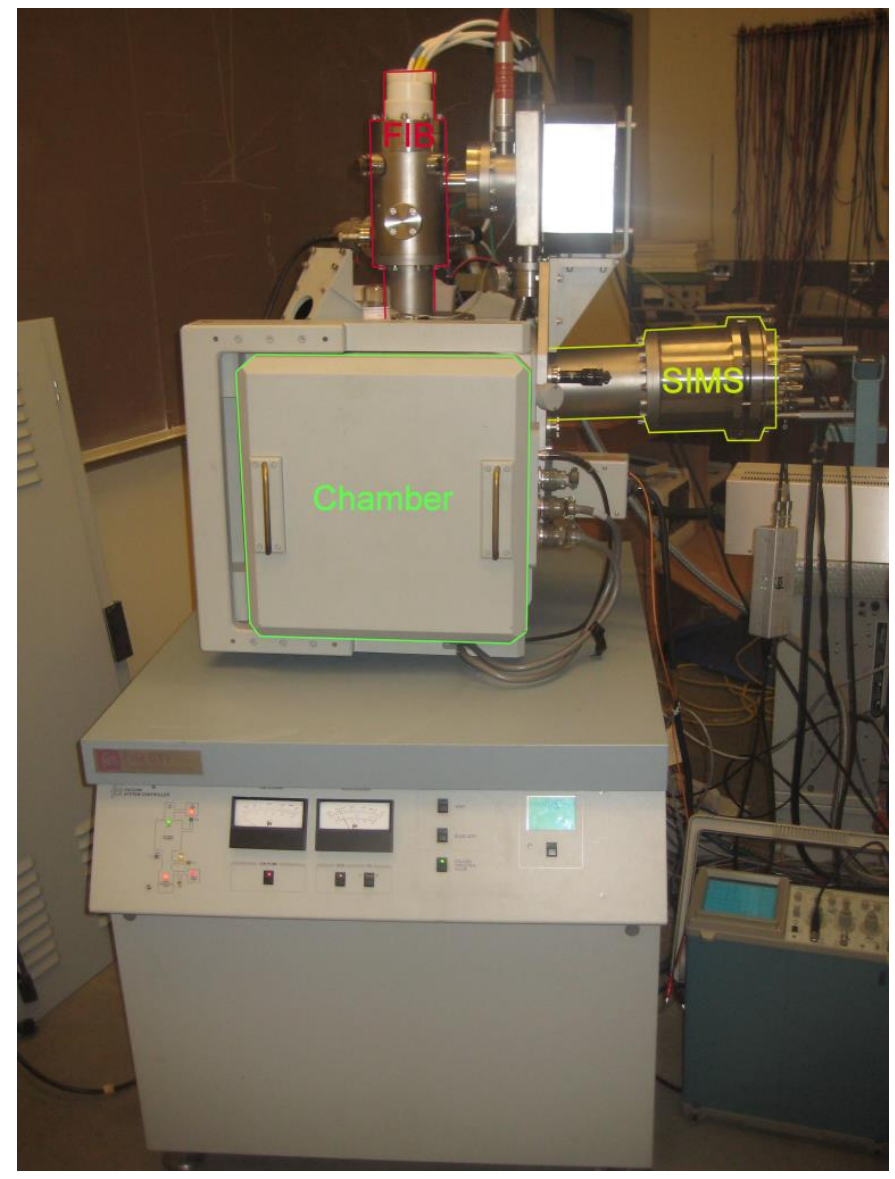

Figure 7.1. FIB 611 vacuum console showing air isolation table, chamber, 2LI FIB column and SIMS-I analyzer. 


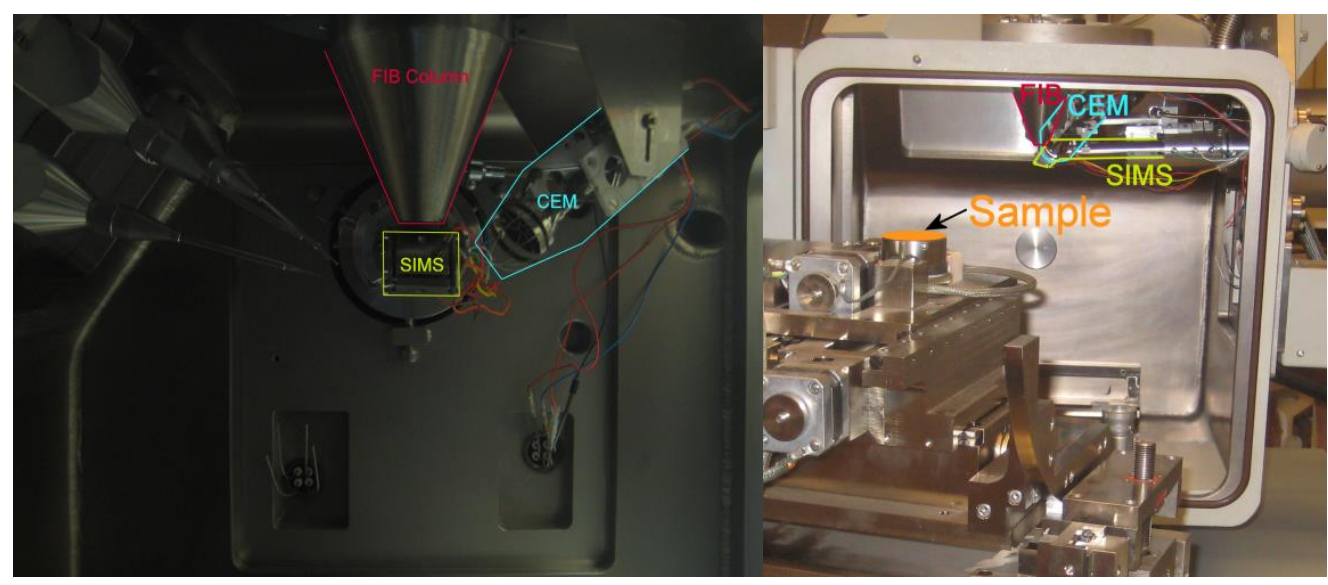

Figure 7.2. Photographs of the FEI FIB 611. a) Exterior, showing the FIB and SIMS columns. b) View from the front with the chamber door open. The 5-axis stage is mounted on the chamber door. c) View from the left, showing how the FIB, CEM and SIMS are mounted above the sample in a typical configuration.

The original Fei FIB 611 system was manufactured between 1990 and 1995. It has a 5-axis XYZTR stepper motor stage whose $\mathrm{XY}$ travel is 6 inches and $\mathrm{Z}$ can move more than 4 inches. It can tilt 60 degrees and rotate the sample more than 360 degrees. The stage is a robust crossed-roller lead-screw design and its controller is designed so that the motors are powered down when not in motion.

The vacuum console is a more or less self-contained subsystem which can perform the functions normally associated with a vacuum controller. It controls the interlocks for the valves, gauges and pumps. These interlocks are available to the system control rack and imaging PC. Thus if the system were operating at the time of a vacuum failure, all systems would be shut down and valves would be set to their safe positions. Hardware components included in the FIB 611

- Vacuum console

○ Turbo pump \& backing pump

- Gauges: Thermocouple, Cold Cathode, Ion Pump Current

- Gas Injectors

- Deflection amplifier/blanker control 
- Descriminator

- Vacuum chamber

- FIB column

- SIMS analyzer

- Channel electron multiplier (CEM) for imaging

- Rack items (left)

- Gas Injection System (GIS) controllers

- Live monitor display

$\circ \quad$ PC with Ion Mill 5.0 $\rightarrow$ XP 2.25 software

- Industrial PC cards (PCI)

- Coreco S3 video

- Coreco F64 Frame-Grabber

- $\mathrm{DSPG} \rightarrow \mathrm{DSPB}$

- Frame Grabber $\rightarrow$ F64 frame grabber

- Industrial PC cards (ISA)

- DAC card, 16 channels, 12 bits (SIMS optics)

- Pulse counting PC card

- IEE488 card

- RocketPort card, 16 RS-232 channels

- OMS PC38 motion controller card

○ Video controller

- SIMS optics controller

- Rack items (right)

- Picoammeter

- 2LI supply

- Deflection controller

○ Ion pump supply

- Stage controller

- Mass filter electronics rack

○ DC supply

O RF supply

○ Descriminator supply

\subsection{FIB 611 PC upgrade details}

The original microscope computer was an Intel 486, capable of dual cpu's although it ran only one. The Windows 3.11 operating system was used for its ethernet capability. The SIMS was a separate Intel 486 PC linked to the microscope PC via Ethernet. It had no UI and was treated as an autonomous controller. The last version of 
FEI software with SIMS support was xP2.25, released in August of 2000. It ran MS Windows NT 4.0. We were able to upgrade to Windows XP SP3. The microscope computers of this era were industrial rack-mounted PC's made by ICS containing 9 ISA slots and 9 PCI slots. It was obtained on the resale market, fully populated with the pc cards and software which were originally installed on the microscope it went to (sans SIMS cards). SIMS cards and stage controller came from the old pc. The microprocessor can be either a Slot or Single Board Computer (SBC) configuration. Ours is a Pentium-II running at $266 \mathrm{MHz}$ and has $256 \mathrm{MB}$ RAM. Many configurations are possible with all of these slots so only ours is presented.

\subsection{FIB 611 software screenshots}

As mentioned earlier, FIB and software control uses xP 2.25 to control the scanning and patterning. Much of the hardware intended for microscopes running xP 2.25 is not installed on the older FIB 611. However, the $\mathrm{xP}$ software can be configured to run by simulating the missing hardware components. 


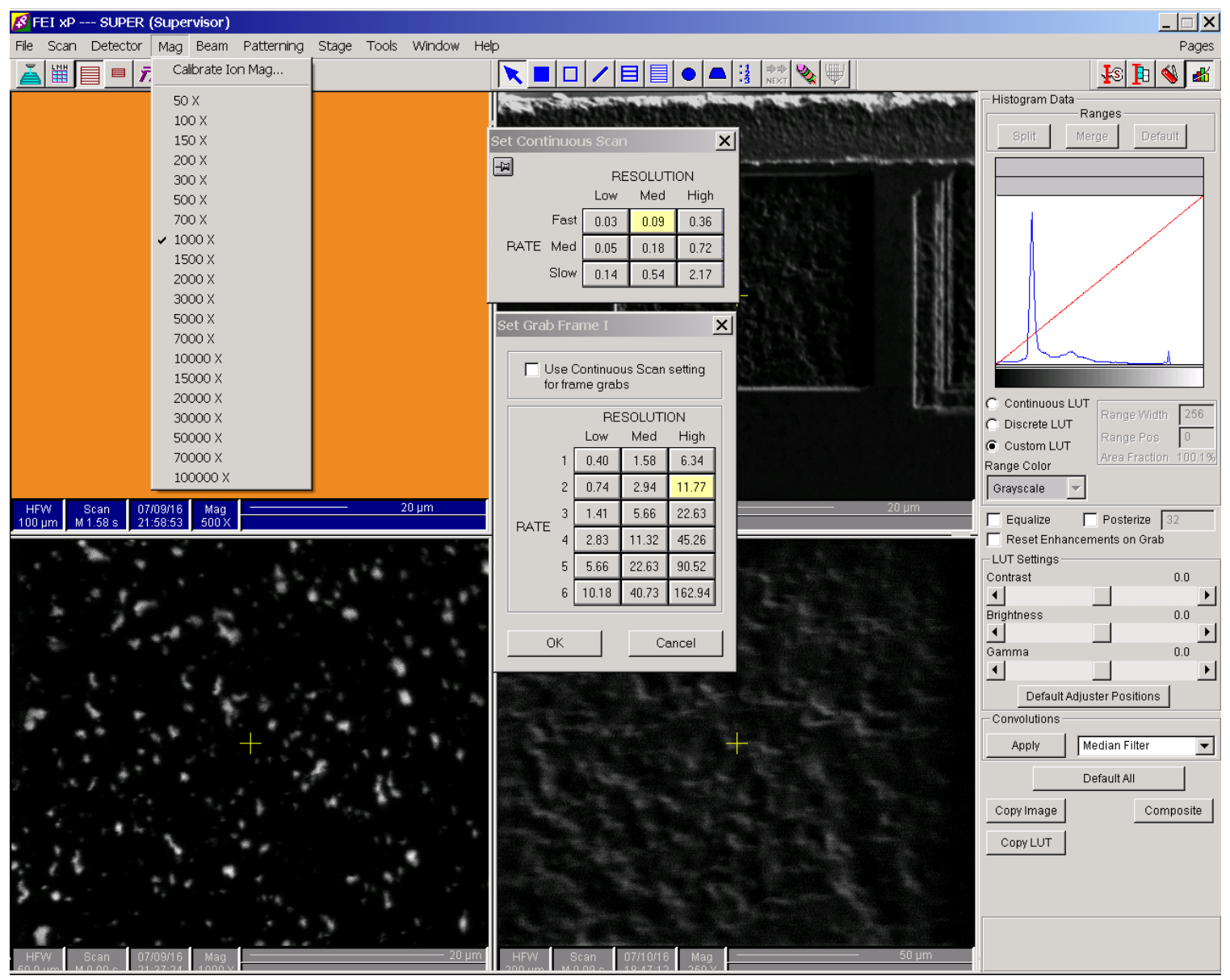

Figure 7.1. FIB xP 2.25 control software controlling the FIB 611. The magnifications have been customized to match the ones on the 611's deflection controller. Pixel dwell presets are shown in the small windows in the center of the screen.

\subsection{FIB modifications for AFM integration}

The 611 sample stage is large enough to accommodate the original sample holder and the AFM. First, the stub holder was elevated using standoffs to reach the approximate height of the AFM sample stub. The next step was to relocate the block containing the Carbon brush for the slip-ring portion of the sample grounding mechanism. It is a block of Teflon with mounting holes and a slot for the Carbon brush \& wire. So that new holes would not need to be drilled and threaded, an existing block 
which supports the theta home switch was modified to support the Teflon block as well. Additionally, there was a metal plate on the South of the XY stage which is intended to hold a glass encoder scale. This plate did not have the glass scale, nor was the encoder read-head installed. This plate was removed to accommodate the electrical connectors to the AFM.

Once the AFM was fully integrated, the base pressure has increased to approximately $3 \times 10^{-6}$ torr. Pump down time is also increased. In the past, operation of the FIB/SIMS was ok at $5 \times 10^{-6}$ torr. With the addition of the AFM, it is better to wait for the pressure to drop below $1 \times 10^{-5}$ torr to avoid the generation of coronas inside the $\mathrm{Z}$ piezo tube. 


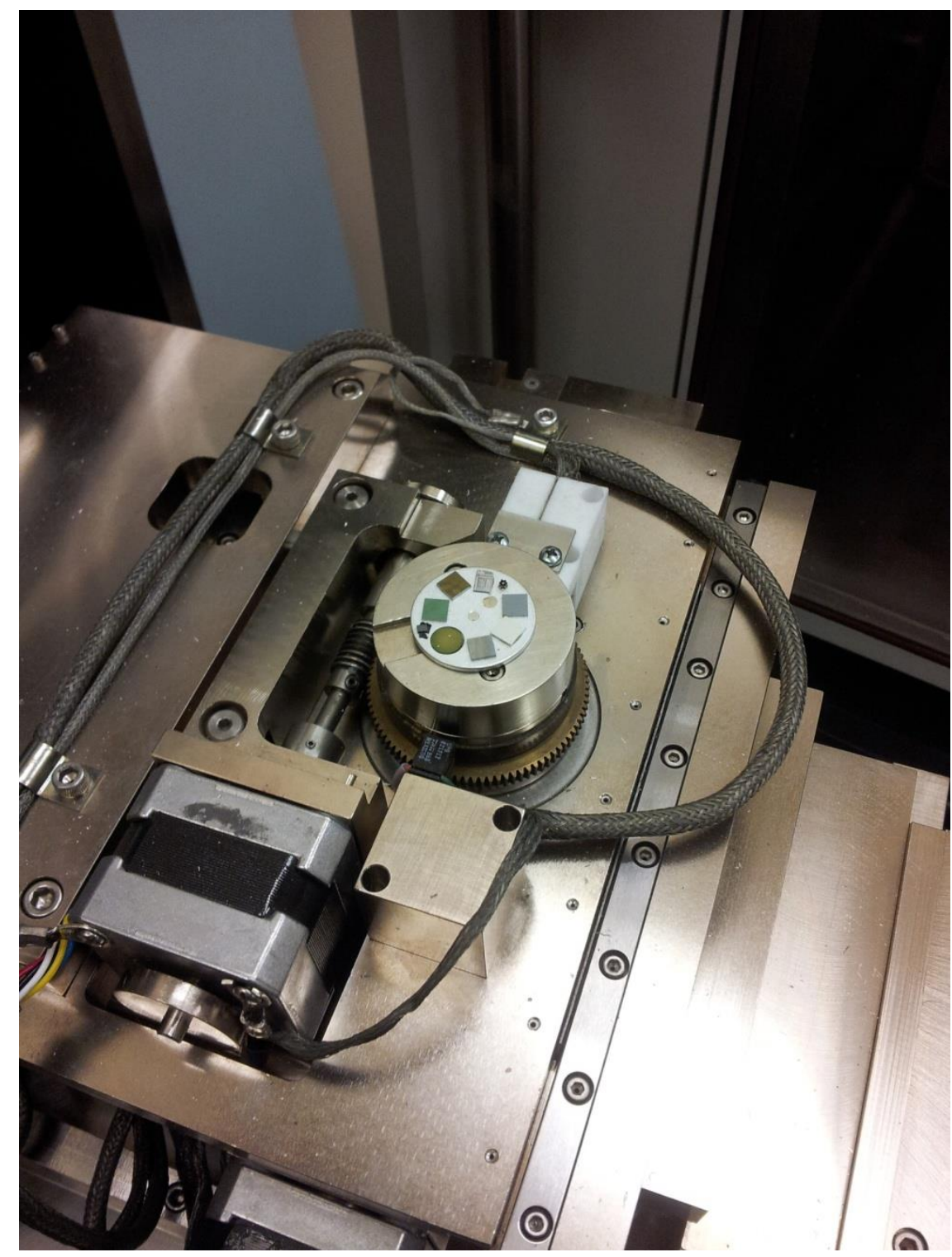

Figure 7.2. Original stage layout showing the original location of the slip-ring brush mount. The block at the center of the image holds the rotational limit switch. A replacement was made for this block which includes a new slip-ring brush mount. 


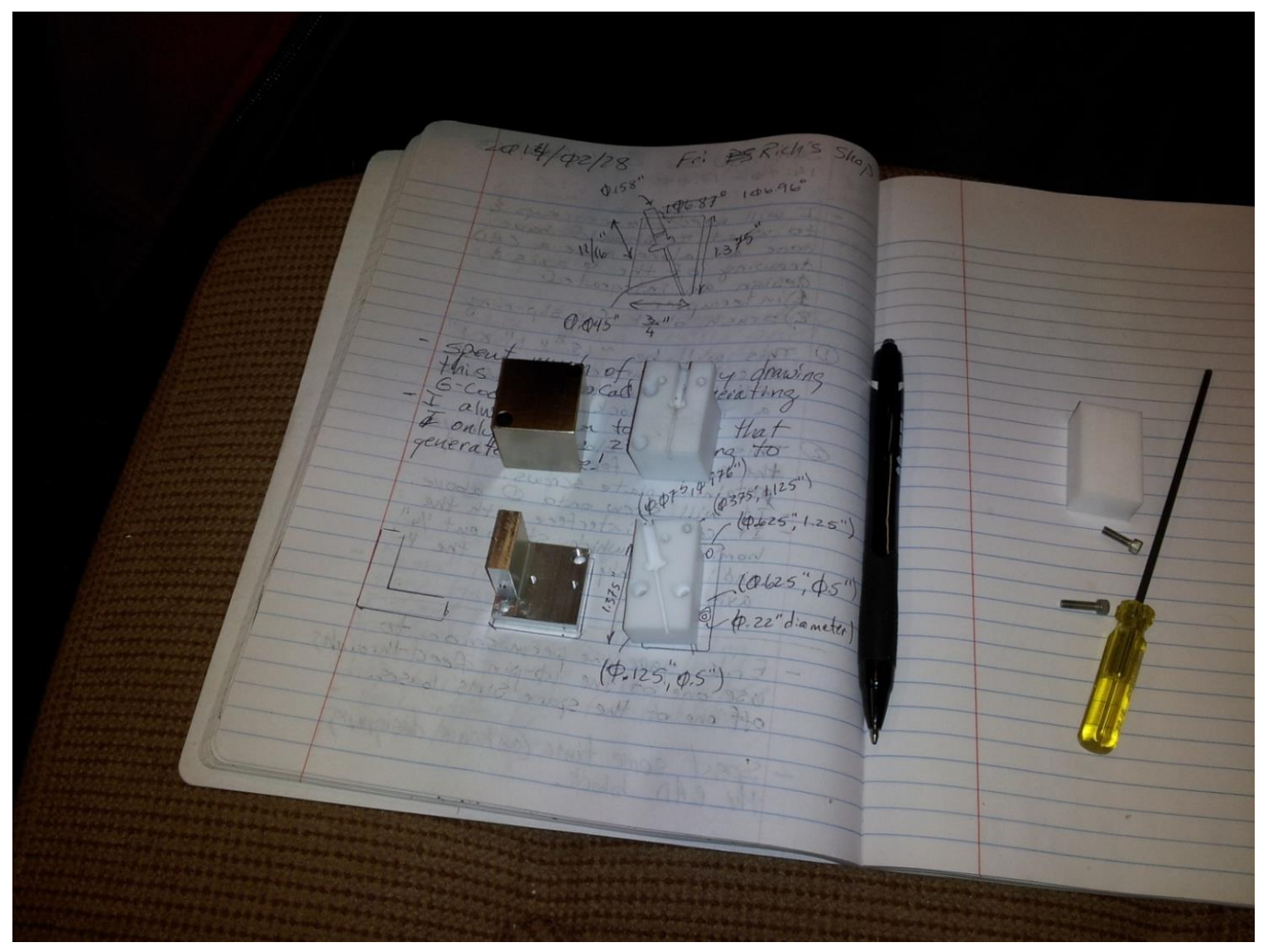

Figure 7.3. Relocation of the Teflon slip ring brush holder involved combining a smaller version of it with the homing block. The original block was not damaged so that it can be reused if the AFM is moved to a different system later. 


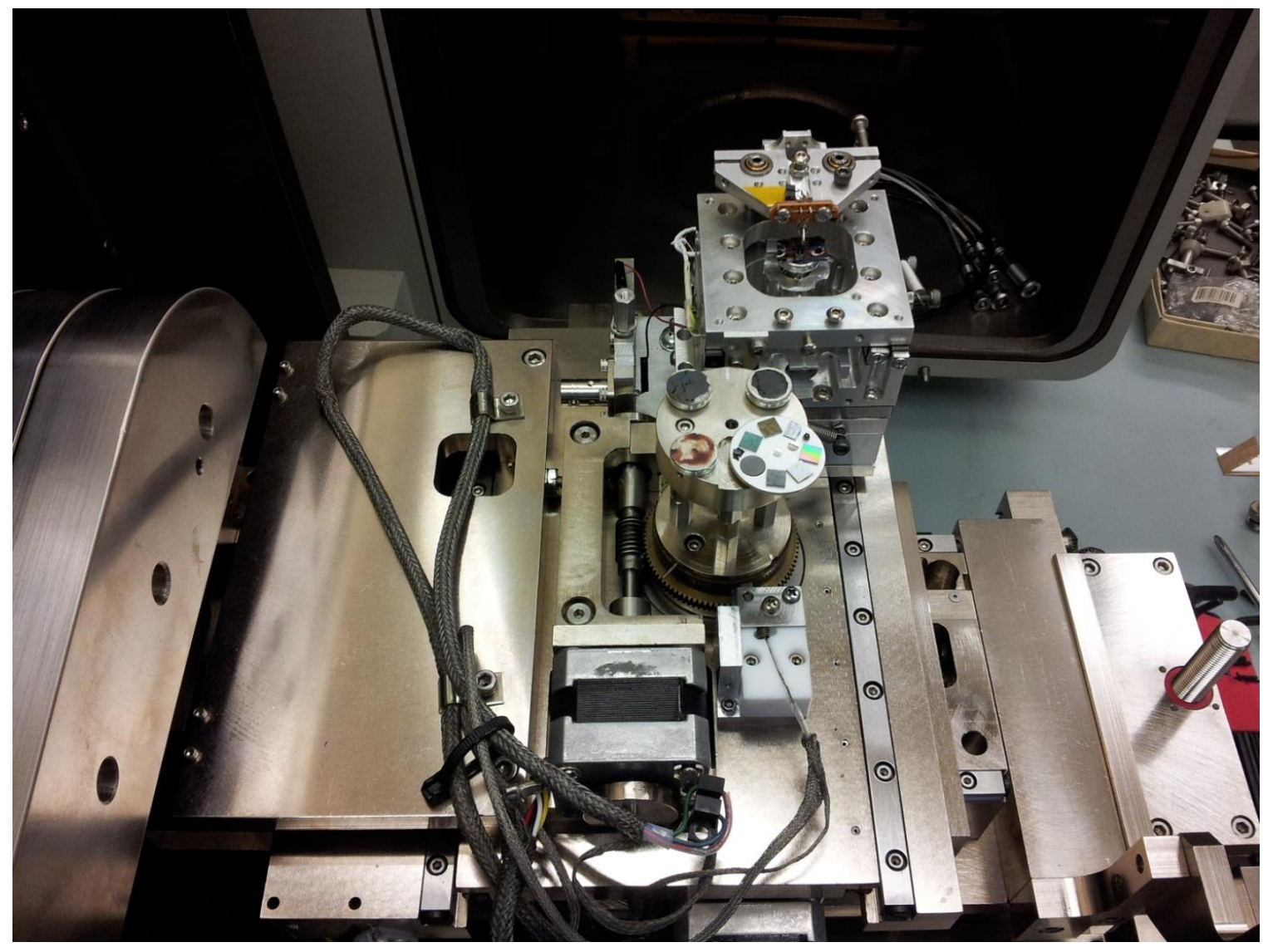

Figure 7.4. New FIB stage layout showing the relocated slip-ring brush block and the AFM at the corner of the stage. The original sample stage was elevated to the level of the AFM. Samples can be analyzed on either the AFM or original sample stages. The encoder plate to the left has not yet been removed.

As discussed above, upgrading to the newer industrial pc, its SIMS control and scanning system simplified some things but forced us to make additional changes/improvements. These changes include a new FIB column, auto focus functionality, and software to control the stage.

\subsection{FIB system enhancements: Magnum FIB column}

Although the originally planned for, Micrion system was capable of superior milling and imaging performance, substantial difficulties in keeping it operational made 
the choice to use the Fei FIB 611 more attractive. Sometime after getting the SIMS to work on the 611 however, its turbo pump crashed. Repairs took a considerable amount of time in part because we were presented with and took the opportunity to upgrade the FIB column to an FEI Magnum FIB model. The Magnum has these improved features over the 2LI:

1) Beam voltage operation to $30 \mathrm{kV}$

2) Beam Defining Aperture (BDA) array after Lens 1 for creating optimal beam profiles

3) Ground-referenced Lens 1 for larger differential potentials, giving higher performance than tip-referenced Lens 1 designs.

4) Beam currents up to $20 \mathrm{nA}$.

5) Extractor operation optimized for $12 \mathrm{kV}$

6) Bipolar suppressor operation for long operation between source heats

7) Bipolar Lens 2 operation for better performance at low beam energies

All of the Magnum column's capability was not implemented during integration into the

FIB 611. A brief list of these items is:

1) Beam voltage on our system is limited to $25 \mathrm{kV}$ max using the original 2LI HVPS. The Bertan supplies inside the 2LI HVPS are capable of $30 \mathrm{kV}$ and the external control electronics can drive them. However, even though the cabling can likely be operated at this higher Voltage, it was deemed safer to stay within the original operating ranges to minimize the chances of damage due to arcing. Possibly more importantly, sputtering rates typically level off at about $20 \mathrm{kV}$ for Ga.

2) The Suppressor Voltage is monopolar, ranging from 0 to -2000 Volts.

3) Extractor operation is typically set to $-9500 \mathrm{~V}$, due to the single polarity suppressor. This is the voltage that the source tends to turn on following a source heating procedure. When the source is nearing the need for a heat, it can be operated at $12 \mathrm{kV}$.

4) Lens 2 is monopolar, being a decelerating (positive) lens for all beam voltages. In general, beam energies below $10 \mathrm{kV}$ would have better performance using a negative (accelerating) lens2.

The calculations used in earlier chapters were based on the FEI 2LI column. Some typical operating Voltages which were recorded over the period of about a year are 
shown for comparison with those later used on the Magnum.

Table 7.1. Tabulated values used on the $2 \mathrm{LI}$ column for about one year prior to upgrading the column to the Magnum. Omitted values for Lens 2 were not recorded in the lab notebook. EVA stands for electrically variable aperture.

\begin{tabular}{|c|c|c|c|c|c|c|c|}
\hline $\begin{array}{c}\text { Aperture } \\
\text { Diameter } \\
(\boldsymbol{\mu m})\end{array}$ & $\mathbf{V}_{\text {ext }}(\mathbf{V})$ & $\mathbf{V}_{\text {supp }}(\mathbf{V})$ & $\mathbf{I}_{\text {ext }}(\mathbf{u A})$ & $\begin{array}{c}\text { Lens } \mathbf{1} \\
(\mathbf{V})\end{array}$ & $\begin{array}{c}\text { Lens } 2 \\
(\mathbf{V})\end{array}$ & $\begin{array}{c}\mathbf{V}_{\text {beam }} \\
(\mathbf{k V})\end{array}$ & $\begin{array}{c}\mathbf{I}_{\text {beam }} \\
(\mathbf{p A})\end{array}$ \\
\hline EVA & -8264 & -1303 & 2 & -1026 & & 24.98 & 5 \\
\hline EVA & -8264 & -1303 & 2 & -2447 & & 24.98 & 20 \\
\hline EVA & -8264 & -1303 & 2 & -2857 & 9674 & 24.98 & 40 \\
\hline EVA & -8767 & 0 & 2 & -2998 & 10947 & 24.98 & 50 \\
\hline EVA & -8356 & -996 & 2 & -3002 & 11524 & 24.98 & 60 \\
\hline EVA & -8264 & -1303 & 2 & -3172 & & 24.98 & 80 \\
\hline EVA & -8065 & -1000 & 2 & -3163 & 11367 & 24.98 & 100 \\
\hline EVA & -8092 & -1000 & 2 & -3463 & 11434 & 24.98 & 250 \\
\hline EVA & -8264 & -1303 & 2 & -3745 & & 24.98 & 800 \\
\hline EVA & -8259 & -1106 & 2 & -3813 & 11564 & 24.98 & 1100 \\
\hline
\end{tabular}

Table 7.2. Some settings used on the Magnum. Missing entries were not recorded.

\begin{tabular}{|c|c|c|c|c|c|c|c|}
\hline $\begin{array}{c}\text { Aperture } \\
\text { Diameter } \\
(\boldsymbol{\mu m})\end{array}$ & $\mathbf{V}_{\text {ext }}(\mathbf{V})$ & $\mathbf{V}_{\text {supp }}(\mathbf{V})$ & $\mathbf{I}_{\text {ext }}(\mathbf{u A})$ & $\begin{array}{c}\text { Lens 1 } \\
(\mathbf{V})\end{array}$ & $\begin{array}{c}\text { Lens } 2 \\
(\mathbf{V})\end{array}$ & $\begin{array}{c}\mathbf{V}_{\text {beam }} \\
(\mathbf{k V})\end{array}$ & $\mathbf{I}_{\text {beam }}(\mathbf{p A})$ \\
\hline $\mathbf{2 0}$ & -9505 & -910 & 2 & -10829 & 13079 & 24.98 & 9.9 \\
\hline $\mathbf{7 5 ?}$ & -9500 & -1803 & 2 & -13928 & 13204 & 24.99 & 250 \\
\hline & & & & & & & \\
\hline $\mathbf{7 5} \boldsymbol{3}$ & -9500 & -1480 & 2 & -20191 & 12845 & 24.97 & 300 \\
\hline $\mathbf{3 6 0}$ & & & & & & 24.99 & 5750 \\
\hline $\mathbf{4 3 0}$ & -9502 & -391 & 2.4 & -23783 & 9020 & 24.98 & 10312 \\
\hline & -10319 & & 2 & -20682 & 5127 & 10 & 2267 \\
\hline & -12006 & & 2 & -16657 & 2829 & 5 & 1694 \\
\hline & -12006 & & 2 & -11304 & 1488 & 2.75 & 1448 \\
\hline & -12006 & & 2 & -7965 & 1088 & 2 & 1146 \\
\hline
\end{tabular}

The Magnum column is constructed using 3-element Einsel lenses. Lens 1 is constructed so that its $1^{\text {st }}$ element is tied to the extractor. This asymmetrical design 
provides more consistent beam current density at the sample if the extractor is held at a constant Voltage. Extractor current is controlled by the suppressor, which is also negative w.r.t. the beam. Lens 1, lens 2 and the beam Voltages are all referenced to ground. The sample is shown as grounded, but it can be biased w.r.t. Earth ground in order to affect ion landing energies and secondary ion/ $\mathrm{e}^{-}$detection. Extractor and suppressor are both negative and are referenced to the beam Voltage. The LMIS can be heated via low Voltage isolation transformer. Its primary coil is connected to the line Voltage. The secondary coil consists of only a few turns of $30 \mathrm{kV}$ high Voltage wire and can generate up to 4 Amps of heating current. A new source is typically heated at $3.2 \mathrm{~A}$ for about 45 seconds. Source heating is generally needed when it either will not emit at all, or if $2 \mathrm{uA}$ of emission current cannot be obtained using normal extractor Voltage. 


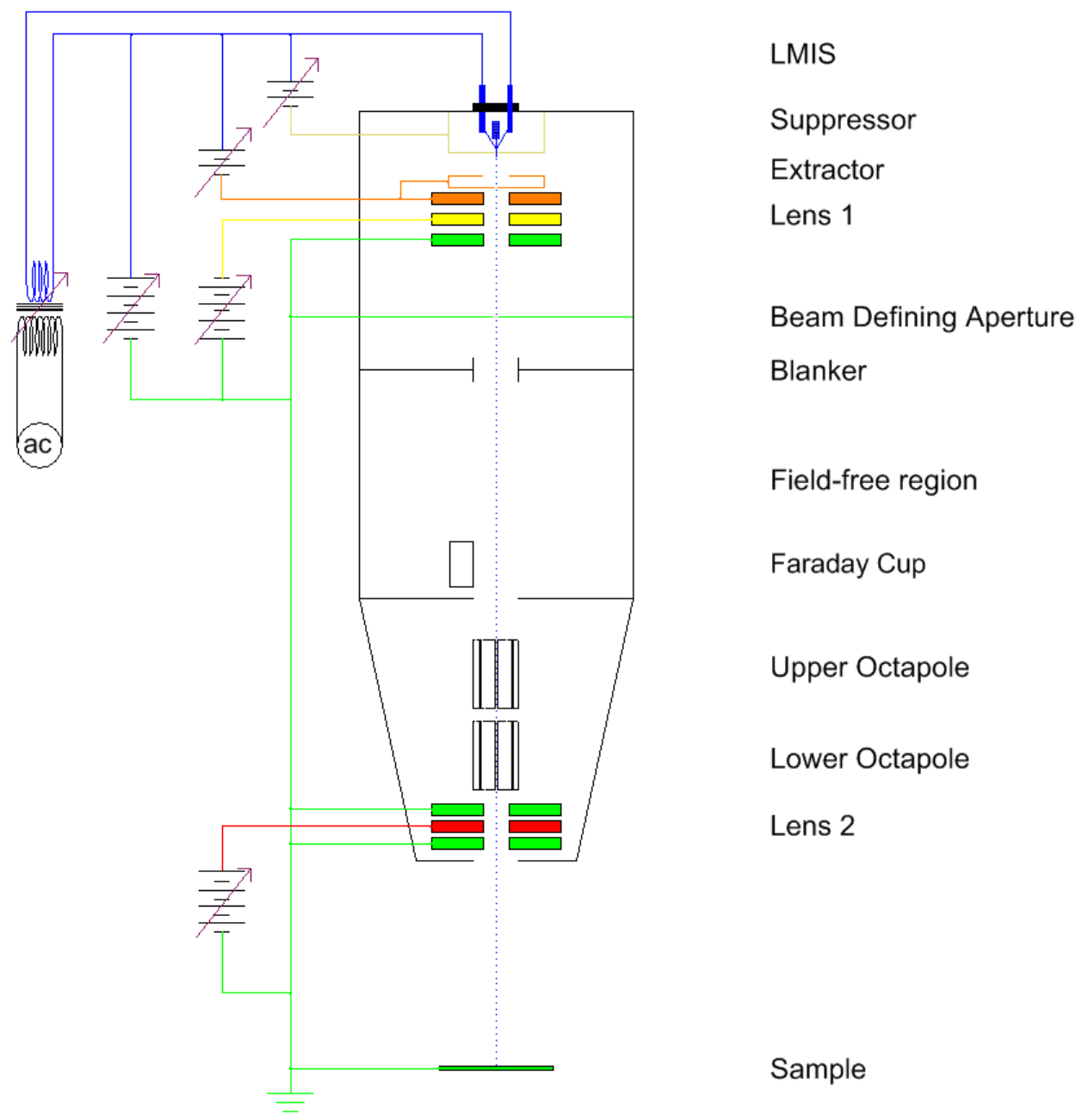

Figure 7.5. Biasing schematic for the Magnum FIB column. Lens 1, Lens 2 and the Beam Voltage are all ground referenced, while the Extractor and Suppressor are referenced to the Beam. Heating current to the LMIS filament requires an isolation transformer which is capable of standing off the beam Voltage $(25-30 \mathrm{kV})$.

Magnum column deflection and beam blanking utilize the existing FIB-611

hardware. The deflection cable on the original FIB-611 terminates on a 20-pin

Amphenol military style connector. Magnum deflection input is a DB-25 connector. The Magnum is constructed without mid-column steering and its dual octapoles are cross- 
wired, so only 10 signal wires are needed to deflect and stigmate the beam. The Faraday cup current is wired to its own BNC electrical feed-through.

\subsection{FIB system enhancements: Auto focus software}

The analog 2LI HVPS was not upgraded to accommodate the Magnum FIB column and in fact needed some repairs. During this repair process, two external control inputs were added. These are inputs for the beam and the Lens2 Voltages. With the beam input, arbitrary beam landing energies are possible for experiments requiring different ones from those provided on the $\operatorname{HVPS}(5,10,15,20$ and $25 \mathrm{kV})$. More importantly for normal operation however, is the Lens2 control. The analog HVPS tends to drift pretty fast for an hour or more during warm up and will continue to drift more slowly afterwards. The effect of this drift is blurry images, thus requiring the user to refocus periodically during operation. Although it is relatively easy to focus blurry images using Lens 2 manually, it is preferable to utilize an automated unbiased sharpness estimator. Moreover, since each image contributes to the total dose of the sample during milling, an auto focus will give a reasonably constant dose every time while a manual user will tend to take a variable number of images to focus with each pass. Thus it is easier to accurately compute the total FIB dose to the sample using auto focus.

The FEI xP2.25 software comes with a scripting interface. This scripting interface provides functions for automating some of the more mundane operations of the microscope. The format of the scripting language somewhat resembles the $\mathrm{C}$ programming language. Scripts may be typed into a text editor and executed through the program named RunScript.exe. Some rather sophisticated applications are possible using the scripting interface. However, it would be somewhat difficult to implement an auto 
focus routine using RunScript so an alternative technique was used.

RunScript makes its calls to a C-style Program Executable (PE) dll, named autolib.dll to provide the scripting capability. Calls to this dll can be made directly if the function prototypes can be determined. Although the interface to this dll is not documented, some of its function prototypes can be determined using a Microsoft tool named Depends.exe.

When run on autlib.dll, the output from Depends.exe contains the so-called "mangled" function names as entries like this:

\section{_ALInit@12}

_ALExit@0

_ALGetScanParameters@32

_ALSetScanParameters@36

$\leftarrow$ 4-bytes less than "Set" function below

_ALGetImageSharpness@4

\section{$\leftarrow 4$-bytes more than "Get" function above}

_ALSaveImage@8

_ALSaveUIImage@8

There are many more but these are the particular ones which are used to perform auto focus.

The scripting and its documentation did perform a valuable purpose for this development. The number which follows the "@” sign in each mangled function name represents the number of bytes used by the parameters in the function's call stack. For this type of dll, these bytes typically represent integers, or integer pointers which can point to integers, floats, doubles, and character arrays. The FEI AutoScript Technical Note contains detailed documentation on the structure of script calls. Some of the script function calls sort of resemble these functions and so the parameter list can be determined 
with some trial and error. For example, there is no script call for setscanparameters, but the call to grabframe takes seven parameters while another takes eight.

grabframe $[\mathrm{x} 1, \mathrm{y} 1, \mathrm{x} 2, \mathrm{y} 2, \mathrm{scanres}, \mathrm{dwe}$ ll,filter $]$

And the other looks like this

grabframe $\mathrm{n}[\mathrm{x} 1, \mathrm{y} 1, \mathrm{x} 2, \mathrm{y} 2, \mathrm{scanres}, \mathrm{dwell}$, filter $]$

where:

- $\mathrm{n}$ is the \# frames to integrate

- $(\mathrm{x} 1, \mathrm{y} 1)$ is the pixel location of the upper-left corner of the scan

- $(\mathrm{x} 2, \mathrm{y} 2)$ is the pixel location of the lower-right corner of the scan

- scanres is a enumeration having the following meaning

○ $0 \rightarrow 256 \times 221$ (low res)

- $1 \rightarrow 512 \times 442$ (medium res)

○ $2 \rightarrow 1024 \times 884$ (high res)

- filter is a either 0 or 1 , for optimal or existing filters, respectively

Another script call, named getscan, is better resembles_ALGetScanParameters in that its signature looks like:

getscan [xstart, ystart, xsize, ysize, res, scandwell, filterstate]

This can be interpreted as

getscan[x1, y1, w-(x2-x1), h-(y2-y1), res, dwell, filter]

where $\mathrm{w}$ and $\mathrm{h}$ are the complete image width and height determined from res.

After some trial and error (and some hard crashes), the function signature was found to be a little different from the calls to grabframe and getscan but it took only a few iterations to determine that it looks like this:

typedef__declspec(dllimport) long (_stdcall* farptrALSetScanParameters)(long w, long $\mathrm{h}$, long $\mathrm{x} 0$, long $\mathrm{y} 0$, long $\mathrm{x} 1$, long $\mathrm{y} 1$, double dwell_s, long q); 
Determinining that the dwell parameter was a double precision floating point value and not an integer or 4-byte float was revealed in the fact that_ALGetScanParameters takes 4-bytes less, meaning that it must be a pointer to an 8-byte double precision number. Its function signature looks like this:

typedef __declspec(dllimport) long (__stdcall* farptrALGetScanParameters)(long* w, long* $\mathrm{h}$, long* $\mathrm{x} 0$, long* y0, long* $\mathrm{x} 1$, long* y1, double* dwell_s, long* q);

where:

- $(\mathrm{w}, \mathrm{h})$ is the nominal image resolution in pixels

- $(\mathrm{x} 0, \mathrm{y} 0)$ is the upper-left corner of the scan

- $(\mathrm{x} 1, \mathrm{y} 1)$ is the lower-right corner of the scan

- dwell_s is the pixel dwell in seconds

- $\mathrm{q}$ is likely the filter parameter as in the grabframe script function, always set to zero

Because integers and long integers are the same size (4-bytes) on 32-bit machines, long integers were chosen. They can just as well be defined as int.

The function signatures for the other mangled functions were determined in a similar manner and a reasonably robust program for performing auto focus was created. Once the function signatures are known, the dll is loaded using LoadLibrary and each function gets a function pointer by calling GetProcAddress on the mangled function name. This kind of programming is sometimes considered hacking. It is not for the faint of heart and it takes some time to get good at it. A professional $\mathrm{C} / \mathrm{C}++$ programmer will need to do this sort of thing at some point in his/her career to either work around a problem or to simply obfuscate the production code. For example, there are many api calls in the Windows platform for which header files and lib files are not available, i.e. the crypto dlls. The technique described above can be tweaked for the particular problem at hand and it is worth mentioning that there are a few other ways to call dll functions when headers and libs are unavailable. 


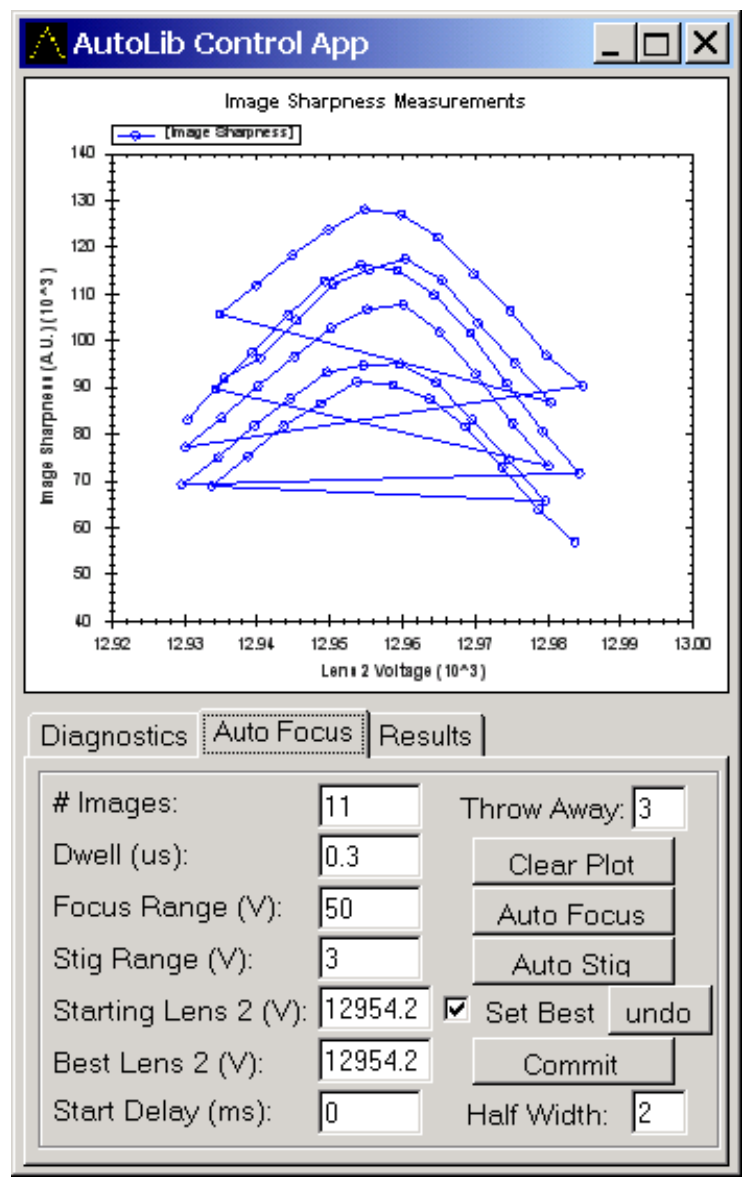

Figure 7.6. Screenshot of the AutoLibControlApp.exe. Among its most useful functions is auto focus. Multiple auto focus runs are shown in the graph display. This program has some useful functionality to not only perform the auto focus function, but also to setup and recover the system.

Many of the autolib.dll function prototypes were determined and an app was made to call them. The name of this app is AutoLibControlApp.exe. While it can perform more functions than just auto focus, only auto focus will be described here. The code listing for the auto focus function can be found in Appendix A.

When the app starts, the user has some flexibility in the choice of parameters in order to perform auto focus runs. These are listed in Table 7.3. A brief discussion of the usage of this app is needed to illustrate its full potential. 
Table 7.3. This listing of parameters and functions describes much of the useful capabilities needed for a useful auto focus used during FIB - SIMS operation.

\begin{tabular}{|c|c|c|}
\hline UI Element Name & Type & Notes \\
\hline \# Images & Parameter & $\begin{array}{l}\text { This will be the number of images acquired through the sweep of } \\
\text { Lens } 2 \text { through the focus range parameter. A typical \# images is } \\
11 .\end{array}$ \\
\hline Dwell (us) & Parameter & $\begin{array}{l}\text { This is the pixel dwell used for performing the auto focus. It can } \\
\text { be made negative to tell auto focus to use the dwell used last in } \\
\text { UI1280. }\end{array}$ \\
\hline Focus Range (V) & Parameter & $\begin{array}{l}\text { This is the focus range to search for best focus. It is important to } \\
\text { choose a range which is suitable for finding the best focus to } \\
\text { sufficient accuracy. Using } 11 \text { images, typical values for coarse, } \\
\text { medium and fine focus are } 200 \mathrm{~V}, 100 \mathrm{~V} \text { and } 50 \mathrm{~V} \text {, respectively. }\end{array}$ \\
\hline Stig Range (V) & Parameter & Not implemented yet, and may get its own tab. \\
\hline Starting Lens 2 (V) & Parameter & $\begin{array}{l}\text { This is the nominal lens } 2 \text { used for imaging. After auto focus } \\
\text { completes, this value will be set to the Best Lens } 2 \text { if the "Set Best" } \\
\text { check box is checked. }\end{array}$ \\
\hline Best Lens $2(V)$ & Parameter & $\begin{array}{l}\text { When auto focus completes it will update this field. If "Set Best" } \\
\text { is checked, the Starting Lens } 2 \text { will also be set and this will be the } \\
\text { system's working lens } 2 \text { value. If "Set Best" is not checked, Best } \\
\text { Lens } 2 \text { will still be updated and its value may be set to the system's } \\
\text { working lens } 2 \text { value by pressing the "Commit" button. } \\
\text { The user can also manually enter a lens } 2 \text { number and press } \\
\text { "Commit" to set the system's working lens } 2 \text { value. }\end{array}$ \\
\hline Start Delay (ms) & Parameter & Delay in ms after Lens 2 is set, before the image is acquired. \\
\hline Throw Away & Parameter & $\begin{array}{l}\text { A common problem with FIB imaging is that the first few images } \\
\text { in a series will tend to be brighter than the others to follow. The } \\
\text { sharpness metric calculation is usually a larger value for these } \\
\text { starting images and the auto focus determination will usually fail. } \\
\text { This parameter allows the user to ignore or throw away the first n } \\
\text { images. Typically set to } 3 \text {. }\end{array}$ \\
\hline Half Width & Parameter & $\begin{array}{l}\text { The auto focus determination utilizes a simple center of mass about } \\
\text { the sharpest image. It is often better to use one or two images } \\
\text { about the sharpest image for reliable results. }\end{array}$ \\
\hline Clear Plot & Button & $\begin{array}{l}\text { The sharpness curves accumulate each time Auto Focus is run. } \\
\text { This allows the user to compare the current run to previous ones. } \\
\text { The plot tends to get cluttered after many auto focus runs and so } \\
\text { needs to be cleared from time to time. }\end{array}$ \\
\hline Auto Focus & Button & $\begin{array}{l}\text { When pressed, performs an auto focus operation. If UI1280 is live } \\
\text { imaging before this button is pressed, it will stop live imaging } \\
\text { before the auto focus operation begins. }\end{array}$ \\
\hline Auto Stig & Button & Not implemented yet. \\
\hline Set Best & Parameter & $\begin{array}{l}\text { When checked, this checkbox causes the "Best Value" determined } \\
\text { by the auto focus routine to also set the "Starting Lens 2" which } \\
\text { sets the system's working lens } 2 \text { value. }\end{array}$ \\
\hline Undo & Button & $\begin{array}{l}\text { If the auto focus run was unsatisfactory for some reason, the } \\
\text { previous working focus value can be restored by pressing this } \\
\text { button. }\end{array}$ \\
\hline Commit & Button & $\begin{array}{l}\text { Pressing "Commit" sets the system's working lens } 2 \text { to the value } \\
\text { entered in the "Best Lens 2" field. }\end{array}$ \\
\hline
\end{tabular}


Table 7.4. Typical run times for an auto focus comprising: 1 starting image, 3 throwaway images, 11 focus sweep images and 1 final image. There is also a 200 ms delay between each lens 2 setting and image acquisition.

\begin{tabular}{|c|c|c|c|}
\hline Speed/Pixel Res & Low & Medium & High (UI Med res) \\
\hline Fast & $24.2 \mathrm{~s}$ & 31.3 & 58.9 \\
\hline Medium & 25.4 & 33.2 & 65.1 \\
\hline Slow & 26.2 & 40.2 & 88.4 \\
\hline
\end{tabular}

\subsection{Future enhancements to AutoLibControlApp.exe}

Auto focus has proven to be indispensable for AFM-FIB operation. AFM-SIMS operation will be more useful with the addition of the auto stigmator function. This is because there is a significant astigmation introduced into the FIB image when the SIMS energy analyzer is turned on. Also since it is typically electrically isolated, the AFM tip will become charged if it is imaged for a significant period of time. This charge will also cause a large astigmation of the FIB image which slowly fades away. Thus auto stigmation is one of the upgrades with high desirability.

\subsection{FIB system enhancements: Stage control software}

The FEI xP2.25 software was not intended to run on a FIB 611 and so cannot control its stage. Various options were evaluated until it was discovered that the PC38 and PC48 motion controllers from Oregon Micro Systems are still supported. In fact the drivers are available for download along with each's user manual. The instruction set for these controllers is virtually identical and the same source code can be compiled to control either. At first, a test app was created with the intent of turning it into a dll for integration into other apps. In practice however, the test app became the primary software used for stage control in our system. It is pretty robust and has not crashed once during operation so we continue to use it as is. 
Its functions are pretty simple. The homing sequence and find limits sequences move the stage in such a way that it has the least danger of crashing the AFM into the FIB column, CEM detector, SIMS and GIS needles. In normal operation the tilt and Z axis are not activated.

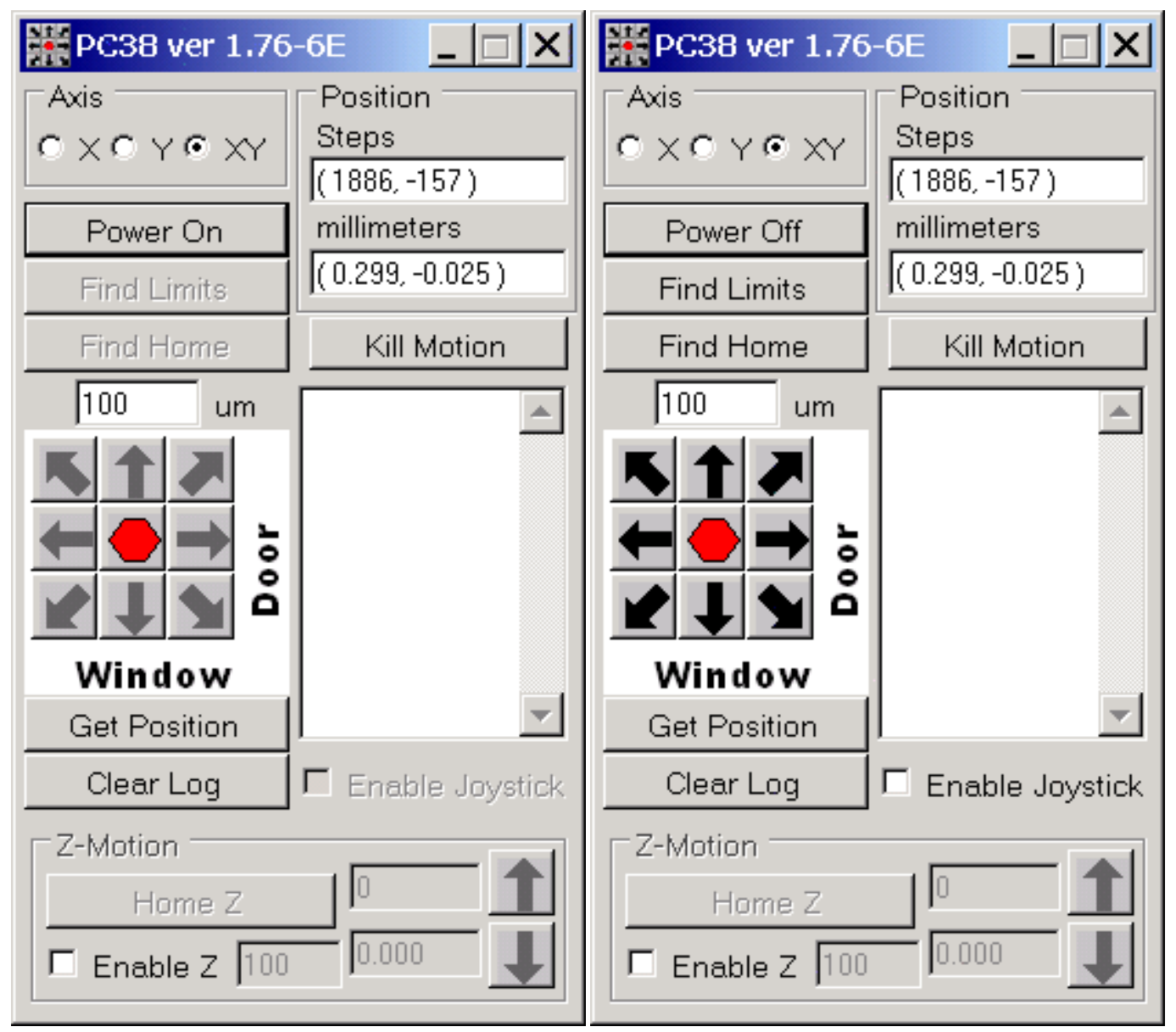

Figure 7.7. Stage controller software user interface showing (left) powered off and (right) powered on modes. 
Table 7.5. Descriptions for the stage control UI elements.

\begin{tabular}{|c|c|c|}
\hline Name & Type & Notes \\
\hline Window Title Bar & Indicator & $\begin{array}{l}\text { When the program starts up, it will attempt to connect to the PC38 } \\
\text { controller. If the connection succeeds, the name of the controller and } \\
\text { firmware version will be displayed in the title bar. Otherwise, it will } \\
\text { display "Not Connected". }\end{array}$ \\
\hline Axis Radio Buttons & Selector & $\begin{array}{l}\text { Selects either } \mathrm{X} \text { axis, } \mathrm{Y} \text { axis or both } \mathrm{X} \text { and } \mathrm{Y} \text { for the "Find Limits" } \\
\text { and "Find Home" buttons. }\end{array}$ \\
\hline Power On/Off & Button & $\begin{array}{l}\text { Use this button to enable/disable stage control. When powered on, } \\
\text { the pertinent buttons/controls will be enabled allowing the user to } \\
\text { move the stage. The stage can be powered down for critical } \\
\text { applications where the user might accidentally press one of the } \\
\text { buttons, causing damage to the sample or AFM. }\end{array}$ \\
\hline Find Limits & Button & $\begin{array}{l}\text { When pressed, causes the controller to seek the negative limit sensor } \\
\text { for X, Y or both, depending on which axis radio button is selected. }\end{array}$ \\
\hline Find Home & Button & $\begin{array}{l}\text { When pressed, causes the controller to seek the home sensors for X, } \\
\text { Y or both, depending on which axis radio button is selected. The } \\
\text { home sensors are located in the centers of their respective axes. Once } \\
\text { located, the position is zero'ed where the home sensor was } \\
\text { encountered and motion decelerates to a stop. Thus the stage will not } \\
\text { be exactly at the zero location after a home operation. }\end{array}$ \\
\hline $\begin{array}{l}\text { XY Position in Steps } \\
\text { and millimeters }\end{array}$ & Indicators & $\begin{array}{l}\text { The position is automatically read back from the controller every } 100 \\
\text { ms to update these indicators. }\end{array}$ \\
\hline Kill Motion & Button & $\begin{array}{l}\text { Sends a "Kill Motion" command to the controller which is } \\
\text { immediately executed and stops all motion. Use this only if the stage } \\
\text { is in motion and is in danger of crashing into something delicate. } \\
\text { Otherwise, use the hexagonal "Stop" button. }\end{array}$ \\
\hline XY Step Size & Parameter & $\begin{array}{l}\text { This is the amount the stage will move when one of the "XY Motion } \\
\text { Buttons" is pressed. Steps smaller than } 20 \mu \mathrm{m} \text { are unreliable. }\end{array}$ \\
\hline XY Motion Buttons & Buttons & $\begin{array}{l}\text { Press one of these buttons to move the stage in the direction indicated } \\
\text { by the "XY Step Size". }\end{array}$ \\
\hline Stop Motion & Button & When pressed, causes the stage to decelerate to a stop. \\
\hline Get Position & Button & $\begin{array}{l}\text { When pressed, will read back the stage position and update the } \\
\text { indicators. This button is redundant and may be eliminated. }\end{array}$ \\
\hline Clear Log & Button & Clears the Joystick Log. \\
\hline Joystick Log & Indicator & $\begin{array}{l}\text { The joystick is read every } 200 \mathrm{~ms} \text { and the stage is moved } \\
\text { accordingly. Observation of this log is useful for determining if the } \\
\text { zero point of the joystick is set correctly. }\end{array}$ \\
\hline Enable Joystick & Checkbox & $\begin{array}{l}\text { Normally the joystick is disabled. This is because the stage will tend } \\
\text { to execute very small stage moves in one direction which is exhibited } \\
\text { as image drift when using the FIB. }\end{array}$ \\
\hline Home Z & Button & $\begin{array}{l}\text { When pressed, causes the controller to seek the negative limit and } \\
\text { then decelerates to a stop. }\end{array}$ \\
\hline Enable Z & Checkbox & $\mathrm{Z}$ motion is normally disabled. \\
\hline Z Step Size & Parameter & $\mathrm{Z}$ motion step size in microns \\
\hline $\begin{array}{l}\mathrm{Z} \text { Position in Steps } \\
\text { and millimeters }\end{array}$ & Indicators & $\begin{array}{l}\text { The position is automatically read back from the controller every } 100 \\
\text { ms to update these indicators. }\end{array}$ \\
\hline Move Z Up/Down & Buttons & $\begin{array}{l}\text { Moves the stage up or down by the distance set in the "Z Step Size" } \\
\text { parameter. }\end{array}$ \\
\hline
\end{tabular}


Both programs discussed in this chapter for stage control and for auto focus were written using $\mathrm{C}++/ \mathrm{cli}$ in MS Visual Studio 2008. $\mathrm{C}++$ is a powerful language which can provide low-level access to PE-style dll's, while at the same time, provide easy to manage user interface elements.

\subsection{References}

FEI Company, "SD Gallium LMIS High Speed Blanking MVA Focusing Column with Electronics User's Guide”, PN 16887 rev A, 1993

Fraser, Stephen. Pro Visual C++/CLI and the .NET 2.0 Platform. Berkeley, CA; New York: Apress ; Springer-Verlag [distributor], 2006.

Oregon Micro Systems, Inc., 'User's Manual Intelligent Motor Controllers PC38 Family", 1989, 1990, 1996, 1997, 1999 Oregon Micro Systems, Inc., A Pro-Dex Company. 


\section{AFM hardware design and instrumentation}

AFM's come in a variety of configurations. Ours is one in which the tip is held in one position while the sample is scanned in XYZ. The AFM is essentially a 6-axis stage, comprising a coarse and fine stage as one. The coarse stage has a range in $(\mathrm{X}, \mathrm{Y}, \mathrm{Z})$ of (13.5 mm, $13.5 \mathrm{~mm}, 7.0 \mathrm{~mm})$, each having encoder resolution of $100 \mathrm{~nm}$. The fine stage has a target range in $(\mathrm{X}, \mathrm{Y}, \mathrm{Z})$ of $(100 \mu \mathrm{m}, 100 \mu \mathrm{m}, 60 \mu \mathrm{m})$, each having a resolution of 1 nm or better.

Some adherence to the original ANSOM hardware was observed. The AFM design for this work is simpler than the original NSOM, thus it is very possible to construct this AFM using the original ANSOM electronics without modifications to the circuit board layouts. In fact, there are enough extra I/O lines broken out in the original ANSOM design create many types of AFM configurations. The main differences between the ANSOM design and ours are:

1) The ANSOM design scans the sample in $X Y$, but its $Z$ piezo is attached to the tip.

2) The ANSOM design utilizes a bipolar $Z$ piezo and drive electronics.

3) The ANSOM design uses the sheer mode of operation of its tuning fork.

4) The ANSOM design has a smaller Z-range $\sim 4 \mu \mathrm{m}$, giving it more $Z$ resolution.

5) ANSOM coarse tip positioning hardware utilizes large stepper motors.

\subsection{Design concepts}

The design used for this work is based on the conceptual design by Erik Sánchez. This original design called for a 2D stage and controller made by AttoCube for the coarse XY tip positioner, costing approximately $\$ 30,000.00$. We were unable to purchase one of these stages due to budgetary constraints. This coarse XYZ tip positioning stage is 
mounted to an XY scan bed from Physik Instrumente (PI). Fine Z motion is performed using a piezo stack, also from PI. It has a nominal range of $60 \mu \mathrm{m}$ with 1000 Volts applied.

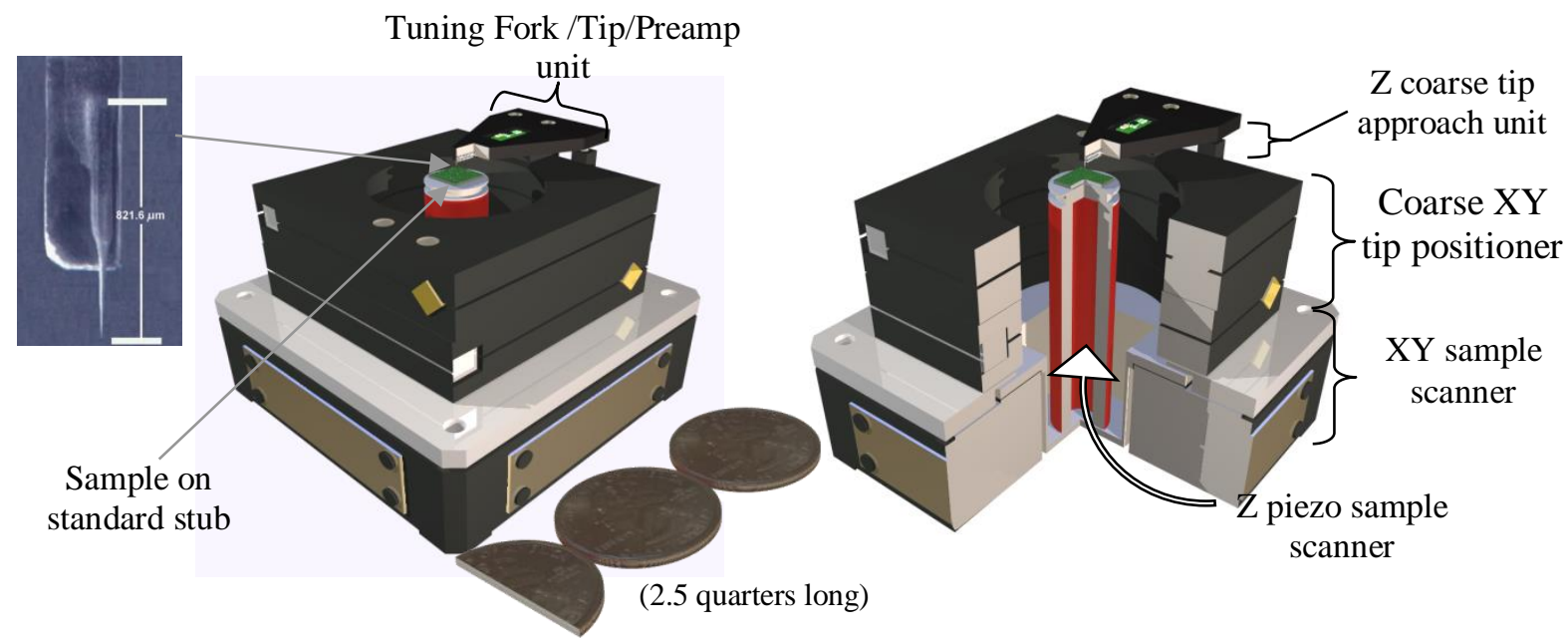

Figure 8.1. Artist's rendering of the AFM unit. The tuning fork holder with preamp is on a plate which is moved vertically with a mini piezo motor on the back for coarse approach of the tip onto the surface. The tip coarse XY positioner moves the tip over a location of interest somewhere on the sample (unit has a range of $+/-3.5 \mathrm{~mm}$ ). The XY sample scanner moves the sample for high resolution topographic imaging, and the $Z$ piezo sample scanner moves the sample up or down through a feedback from the tuning fork signal

The conceptual design shown in Figure 8.1 was lacking some details on the coarse Z portion of the stage, so the initial design incorporated a flexure for the Z-axis instead.

This can be seen in Figure 8.4.

\subsection{Alternative technologies employed}

Elimination of the AttoCube stage from the design required finding alternative motion components which are suitable for nano-positioning in a vacuum environment. 
While providing coarse XY motion for the tip positioner, the AttoCube stage had no Z axis. A solution for coarse $\mathrm{Z}$ motion was needed even if the AttoCube stage would have been used in the AFM design. Squiggle motors made by NewScale Technologies were chosen for the in-house designed coarse XYZ tip positioning stage. Squiggle Motors provide nano-positioning capability exhibiting these features:

1) Small size

2) Large force

3) Simple design

4) Non-magnetic capability

5) Piezo-ceramic

6) Easy to use controller interface

The in-house designed XY coarse tip positioning stage's range is larger than the AttoCube's, $3.5 \times 3.5 \mathrm{~mm}^{2}$, being $6.75 \times 6.75 \mathrm{~mm}^{2}$. This allows for navigation over the entire area of a standard $12 \mathrm{~mm}$ SEM stub.

The AttoCube stage would have contained its own position feedback and control. Instead, incremental encoders were chosen as the position feedback technology to be integrated into the in-house designed coarse XYZ tip positioning stage. MicroE Technologies has been making very small incremental encoders for over 15 years now and have become a very popular choice for those integrating them into very small designs. The encoders chosen were the MicroE Systems S200, readily available on the surplus market. These encoders are connected to interpolators which can be programmed to provide interpolations from 1-256 of the $20 \mu \mathrm{m}$ encoder line pitch. For example, when the interpolation value is set to 256 , the encoder resolution becomes $20 \mu \mathrm{m} / 256=$ $78.125 \mathrm{~nm}$. The coarse stage interpolators were set to a value of 200 , for a resolution of 
$100 \mathrm{~nm}$.

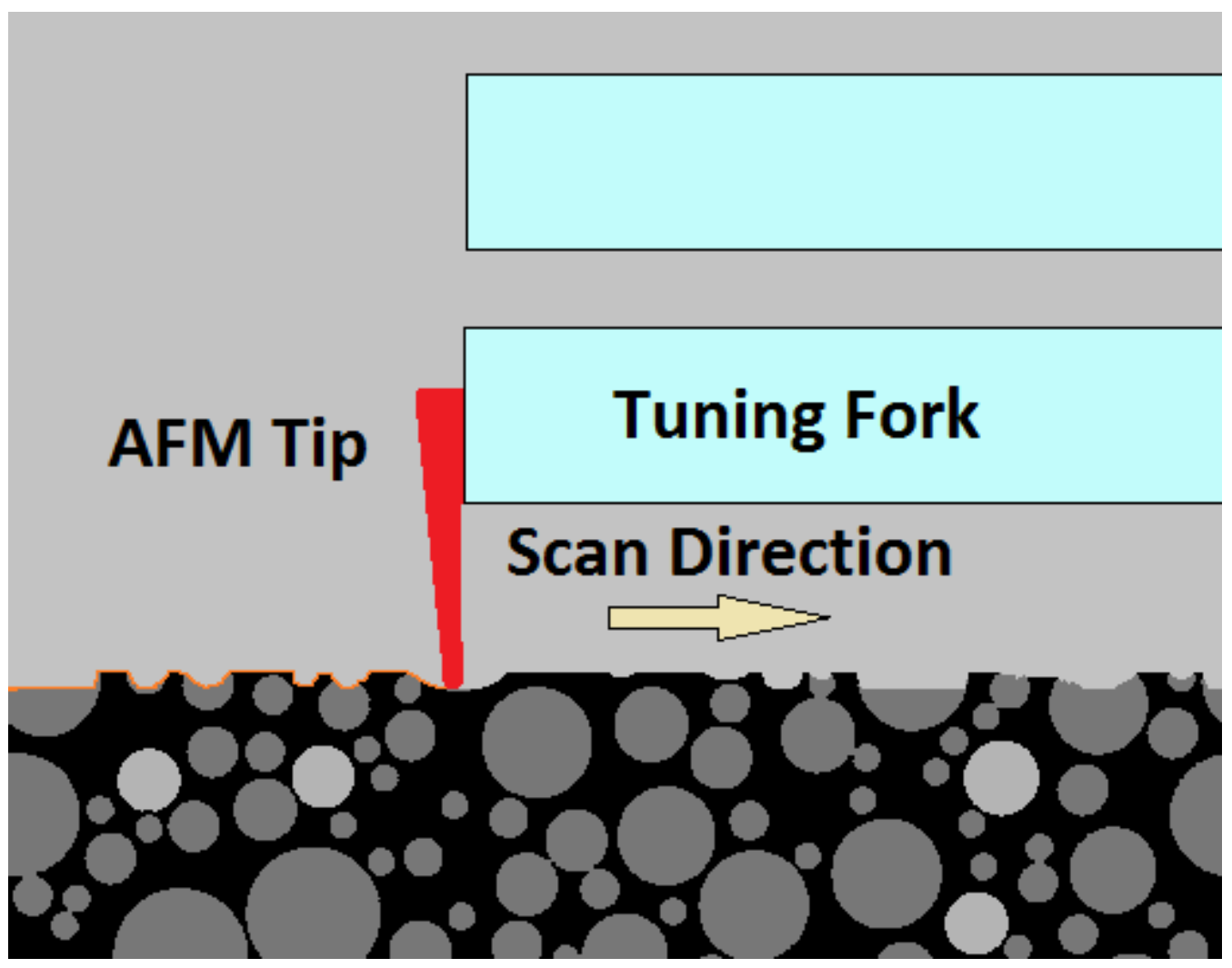

Figure 8.2. Illustration of an AFM scan across a surface which was imaged/milled using a FIB. Some of the materials are milled faster than the others and make for a rough surface. Knowledge of the differing milling rates for each of these materials is crucial for $3 \mathrm{D}$ reconstruction of the sample. 


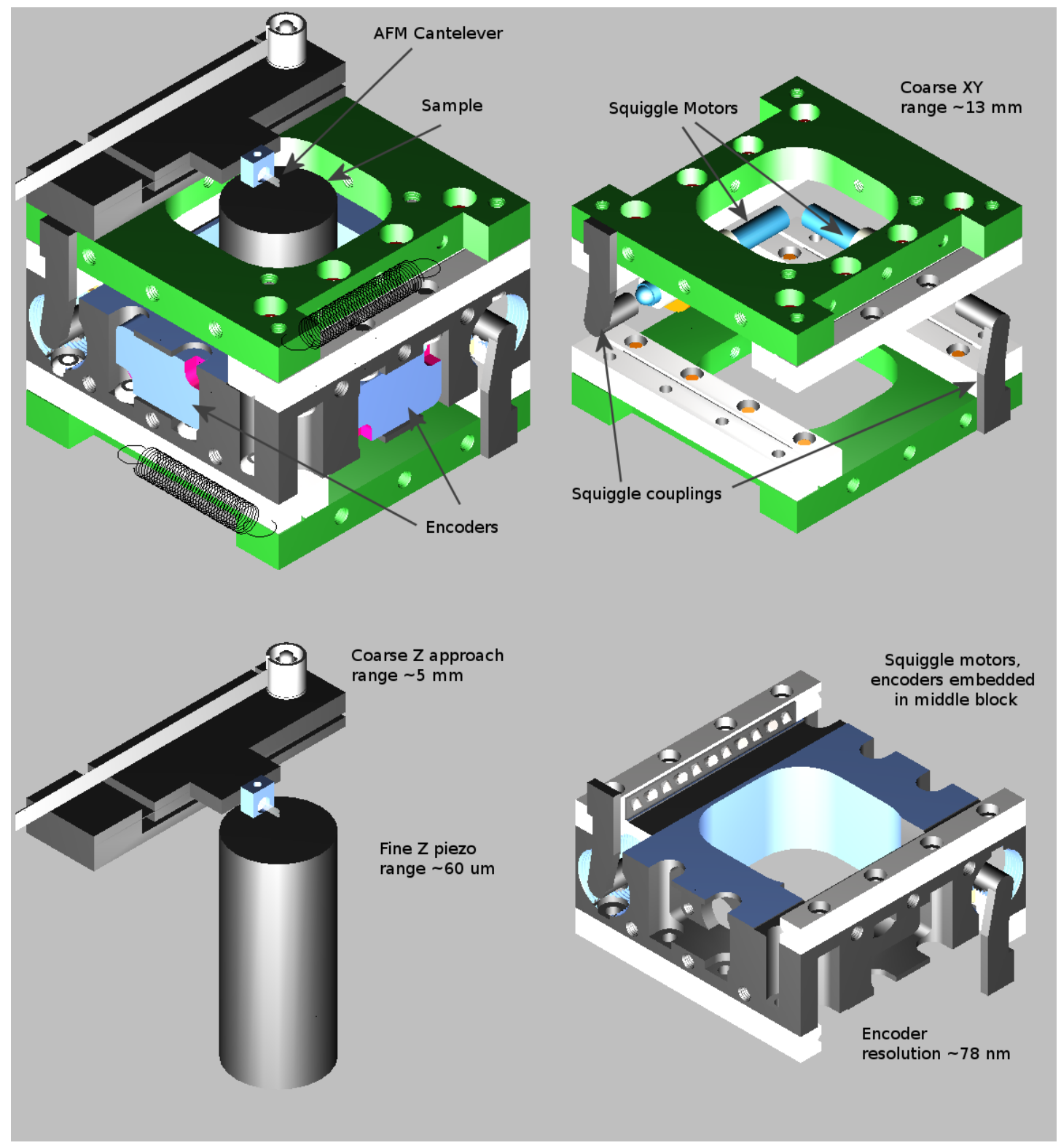

Figure 8.3. AFM coarse $X Y Z$ design, as drawn using TurboCAD 17. Dimensions of the completed AFM are approximately $60 \mathrm{~mm} \times 60 \mathrm{~mm} \times 80 \mathrm{~mm}$. Not shown is the nano-postioning XY scan bed.

Later, we found that we wanted more than $5 \mathrm{~mm}$ travel for the coarse $\mathrm{Z}$, so it was redesigned again before a prototype was constructed. The final design incorporates a linear bearing arrangement for the coarse $\mathrm{Z}$-axis. This arrangement allows for a large travel and has a very small lateral error. Figure 8.5 shows the how the two bearings form 
a tripod with the squiggle motor. Also, the original design included cutouts for the XY return springs. These cutouts caused the Top and Bottom Plates, made out of Aluminum, to be weak resulting in a less rigid mechanism.
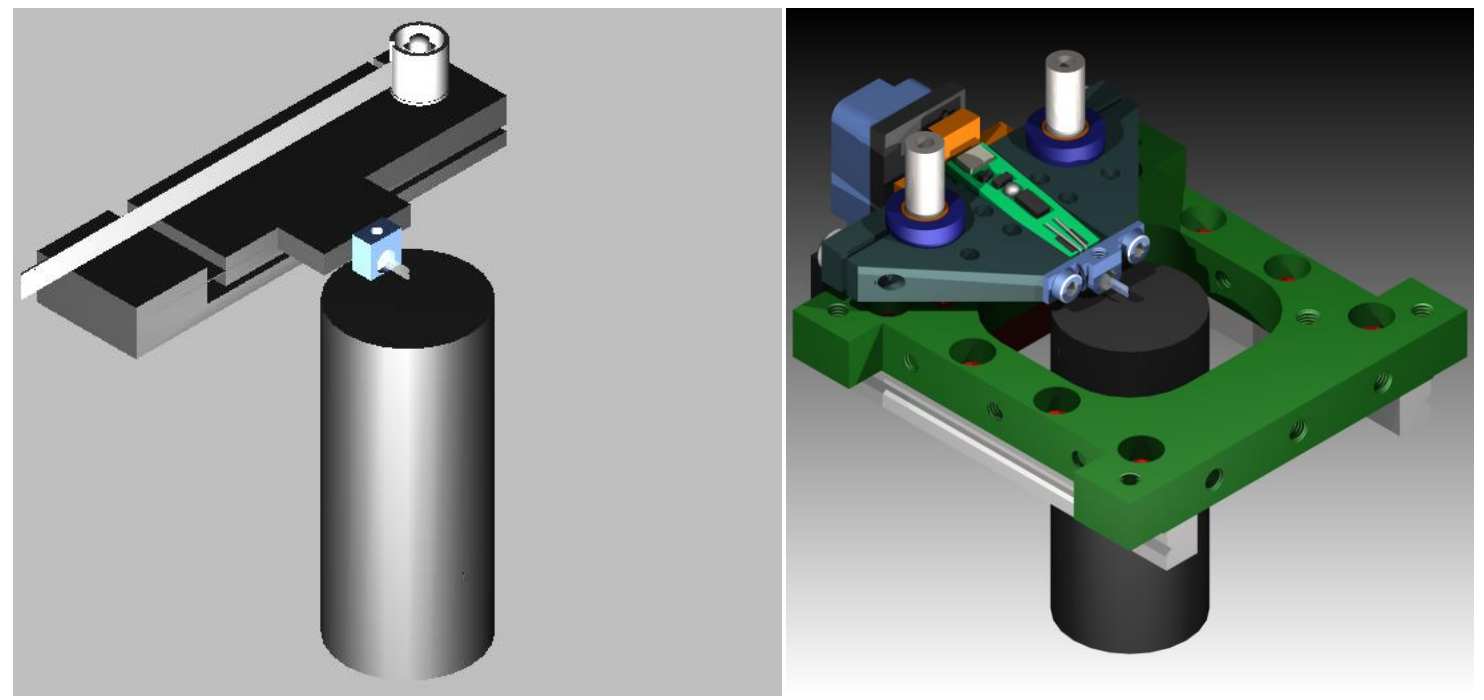

Figure 8.4. Coarse Z-axis designs. (left) original flexure design having $<=5 \mathrm{~mm}$ range and large cosine error. (right) New design which was actually implemented. The plate which contains the tuning fork is supported by two linear bearings and a small squiggle motor ( $7 \mathrm{~mm}$ diameter) in a tripod arrangement. It has a range $>5 \mathrm{~mm}$ and virtually no cosine error.

\subsection{Assembly details}

The coarse stage is essentially a nanopositioner and so must be assembled very carefully. To provide repeatable motion, the crossed roller bearings must be aligned and preloaded. A jig was constructed for this purpose. The idea behind this preload jig is to apply pressure while the mounting screws are loose such that the axis motion is smooth and on the threshold of cogging. Cogging is felt as periodic spots of resistance through the bearing motion. Once the bearing race mounting screws are tightened, the jig can be released and the springiness of the plates will cause them to bend very slightly and the 
cogging should go away. This can be an iterative process which requires some practice to master. 
Cogging in the coarse $\mathrm{Z}$ axis is unavoidable in the final assembly. This is due to the slight bowing of the top plate (X-axis). This causes the posts to be a little off from parallel. This cogging can be reduced to a minimum by installing the $\mathrm{Z}$ axis starting with the bearing cages only halfway on the posts. Cogging for this arrangement is further reduced by mounting the return spring so that it acts opposite the Squiggle, creating a torque about the plane formed by the posts. This arrangement also reduces Z-drift during the approach sequence. After final assembly this Z-axis can be commanded in steps as small as $10 \mathrm{~nm}$, greatly exceeding expectations. During the development of the approach algorithm, it was necessary to approach manually in $10 \mathrm{~nm}$ steps using this coarse zstage.

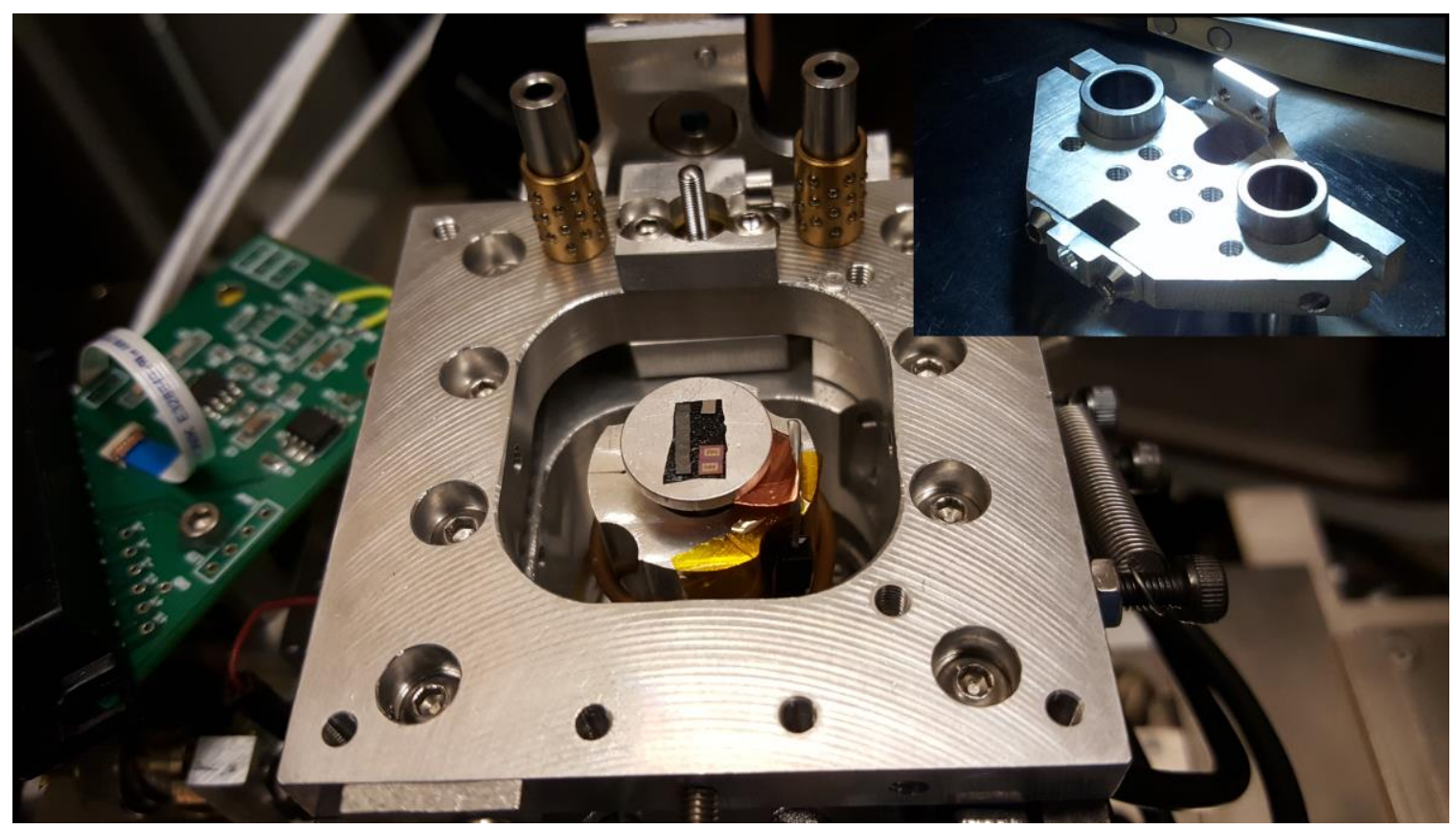

Figure 8.5. AFM showing coarse $Z$ axis removed (inset). The coarse $Z$ stage uses the linear bearings and Squiggle motor in a tripod arrangement. 


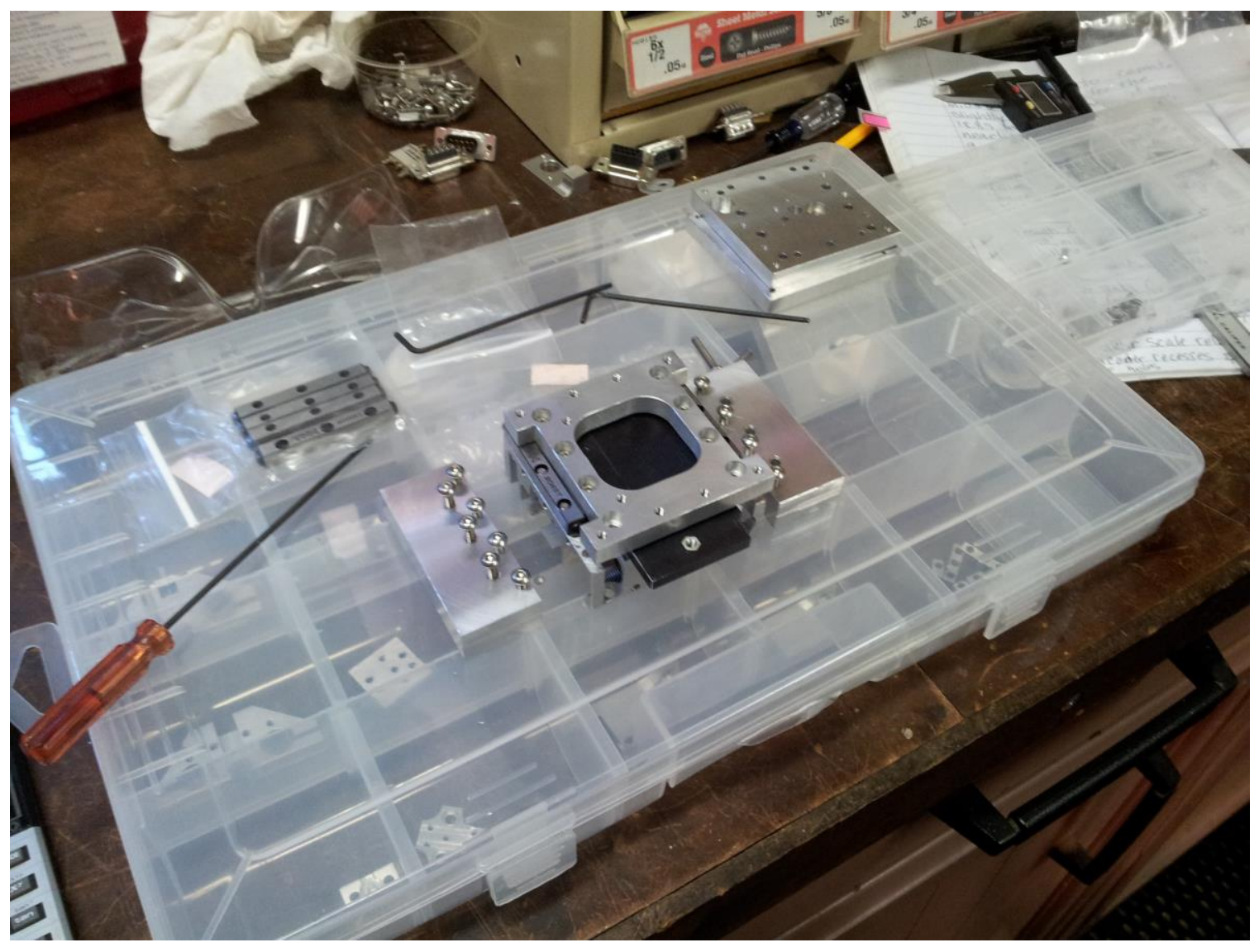

Figure 8.6. Assembly of the Coarse XY stage using a pre-load jig. One bearing rail is aligned with the middle block's edge and the other is pressed against it using screws to apply a uniform pressure before tightening the mounting screws. The image shown is the prototype mechanism after the adjustable pieces have been removed. The black spacer is still inserted. 


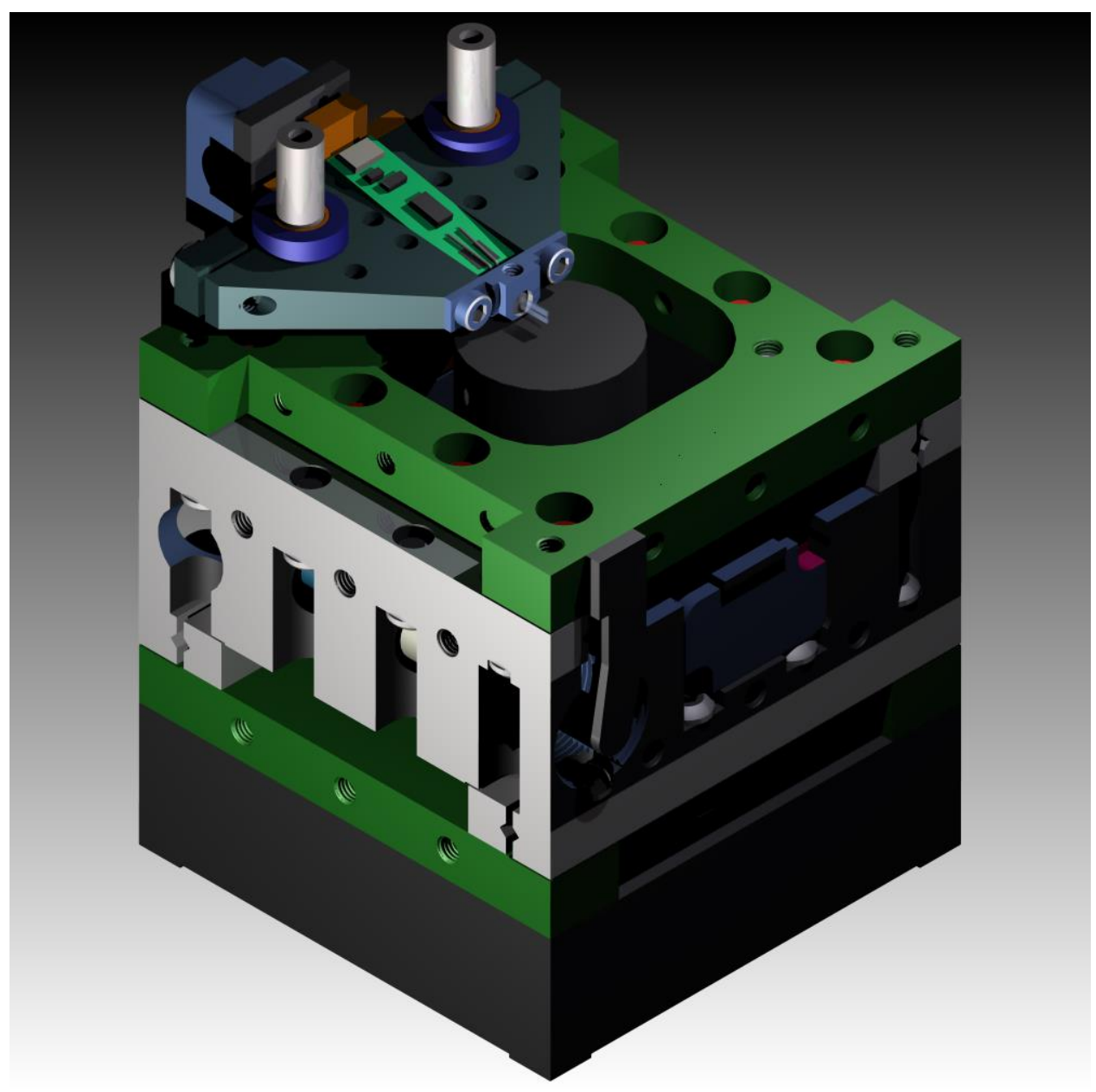

Figure 8.7. Final AFM design showing the coarse $Z$ stage in its lowest position. This design can accommodate a large range in sample thickness and tip length, limited only by the length of the bearing posts, Squiggle motor travel and encoder range. 


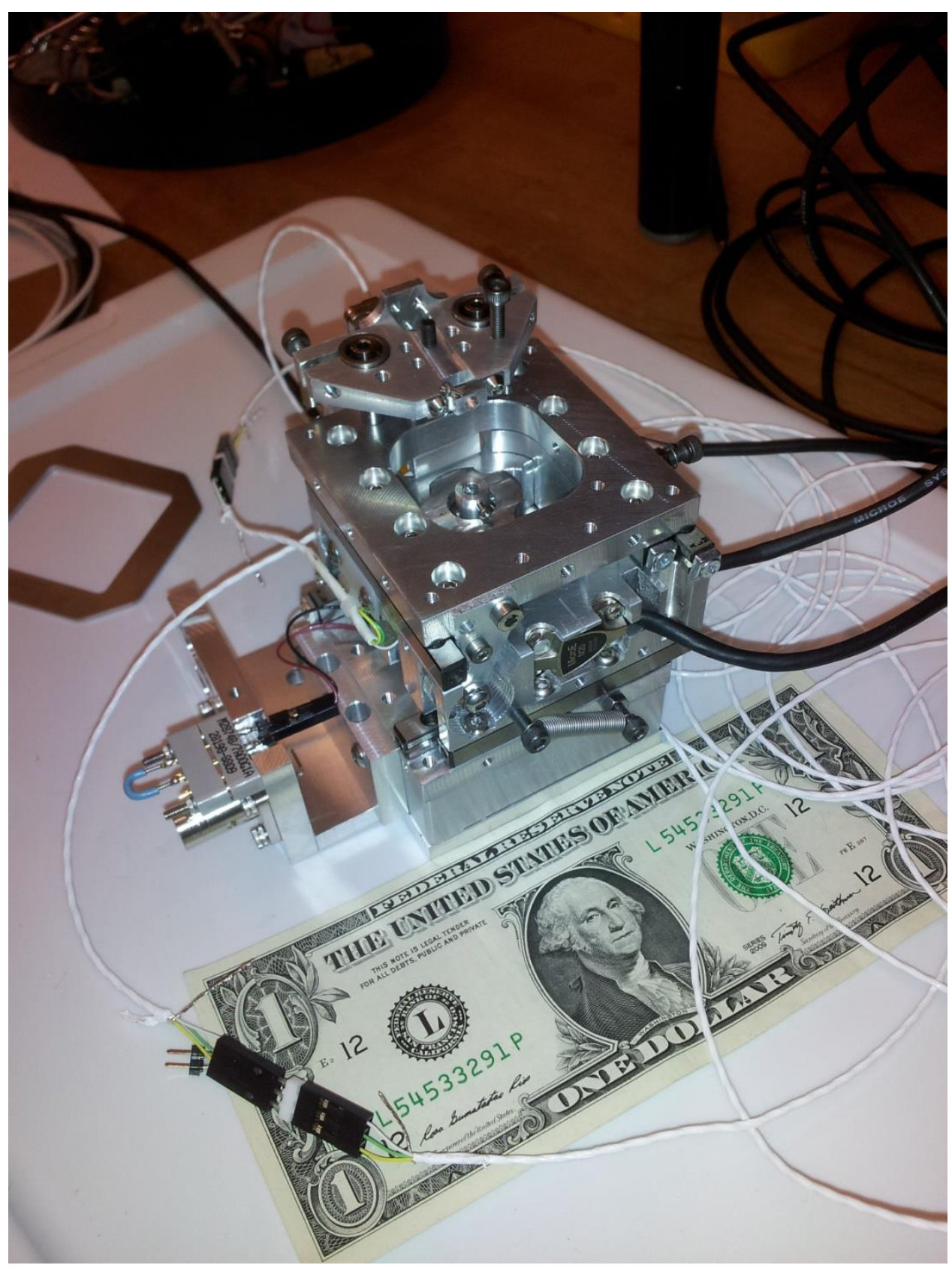

Figure 8.8. Fully assembled AFM hardware illustrating its size w.r.t. a dollar bill. The coarse-Z axis is shown in its highest position. The material used was Aluminum for its ease of machining and for its non-magnetic qualities. The cutouts for the XY return springs were not implemented for improved stiffness.

\subsection{AFM cabling considerations}

The AFM has been integrated from custom and off the shelf components. 
Original cabling for these off the shelf components required modifications either to reduce the number of connector pins or simply to feed into the vacuum chamber.

\subsection{Encoder cabling}

The thick cables connecting to the encoders must then be carefully routed so that their influence on the coarse stage motion is that of a damped spring.

\subsection{Squiggle cabling strain relief}

The Squiggle motors can apply a force of $1 \mathrm{~N}$ for $\mathrm{Z}$ and $5 \mathrm{~N}$ for $\mathrm{X}$ and $\mathrm{Y}$. This translates to approximately $100 \mathrm{~g}$ and $500 \mathrm{~g}$, respectively. The cables leading to the Squiggle motors themselves are very thin and have little effect on the overall spring constant of the return springs.

The cabling provided with the squiggle motors is very fragile so it was necessary to include strain relief. For the $\mathrm{X}$ and $\mathrm{Y}$ squiggle motors, two holes were punched into a simple zip-tie tag and it was screwed into the center block. The $\mathrm{Z}$ axis required more attention. The $7 \mathrm{~mm}$ hole drilled into the top plate was given a slight recess so that the ribbon cable could be folded on itself and the whole thing gently inserted. The motor then is supported on the bottom using a block which is screwed to the bottom of the top plate. This block has a slot milled into it for the ribbon cable to exit. The ribbon cable is then attached to the ribbon-to-header adaptor board which is screwed into the center block. 


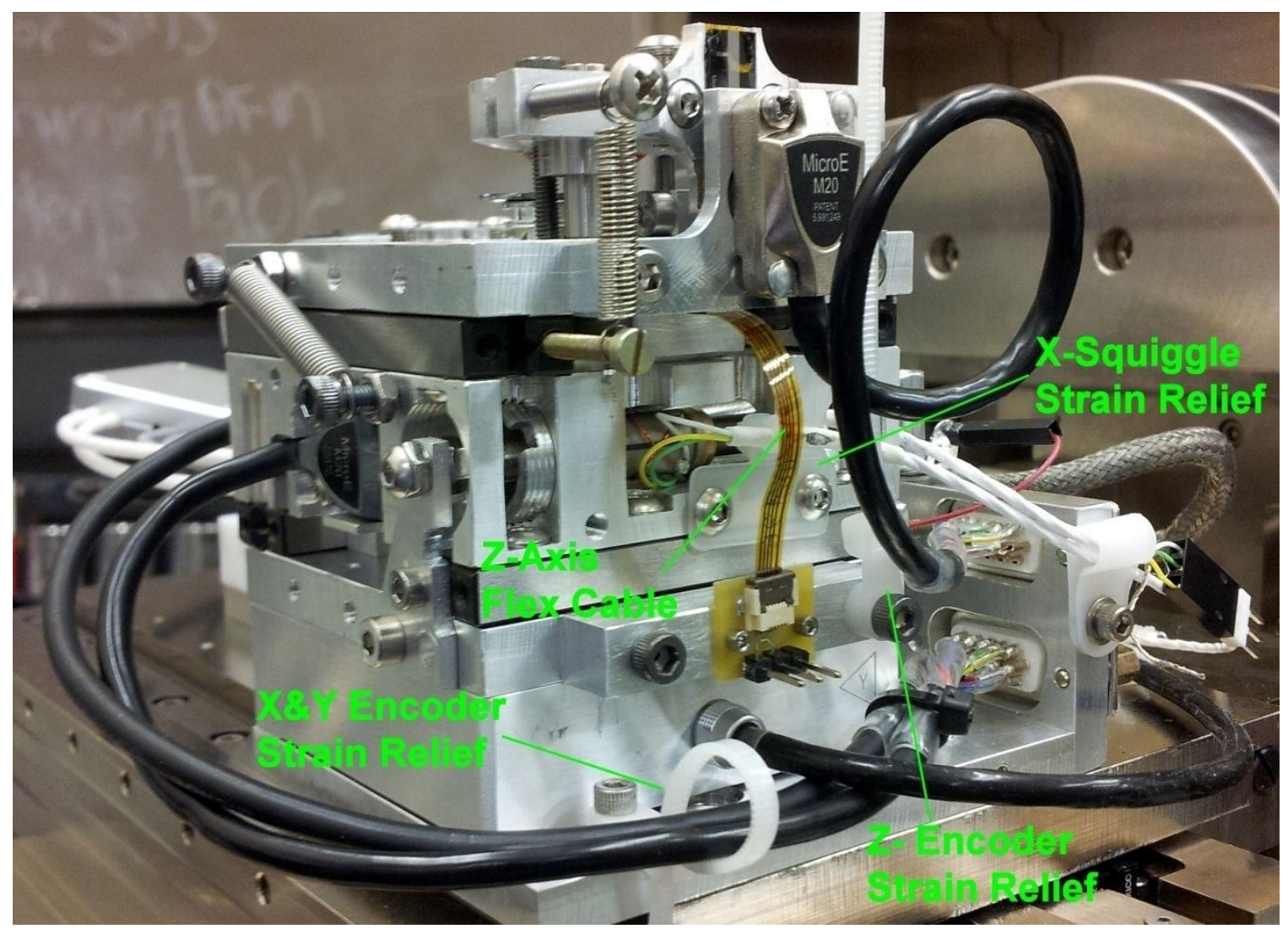

Figure 8.9. Image showing the strain relief points and their associated zip-ties. Each strain relief point is positioned to minimize the force of the cable on the associated stage axis. All strain reliefs, except the squiggle are shown before tightening.

The AFM tips are fabricated from $80 \mu \mathrm{m}$ diameter $\mathrm{W}$ wire using a DC electrochemical etch process. Tip diameters range from $15 \mathrm{~nm}$ to $1000 \mathrm{~nm}$ or more. Smaller diameters are generally better for lateral resolution and large ones are more durable. The best range of diameters for this application is $30-100 \mathrm{~nm}$. The FIB, as will be discussed later, will be operated using beam currents ranging between $100 \mathrm{pA}$ and $6 \mathrm{nA}$. Optimal beam current for AFM-SIMS operation is between $250 \mathrm{pA}$ and $1 \mathrm{nA}$. These beams have a small enough spot size and large enough current for strong signals and for fast milling. 


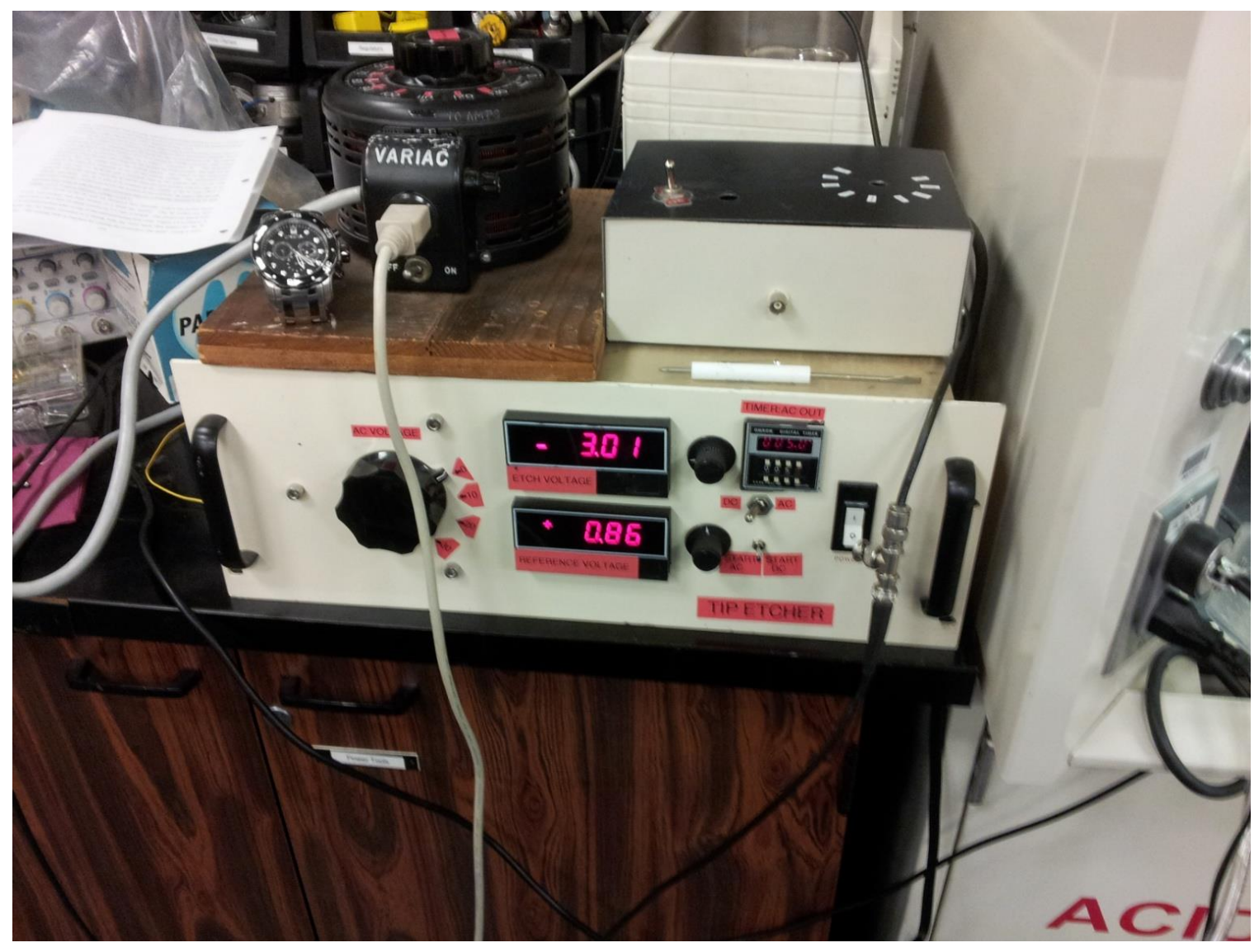

Figure 8.10. Pictures of tip-etching electronics rig. 


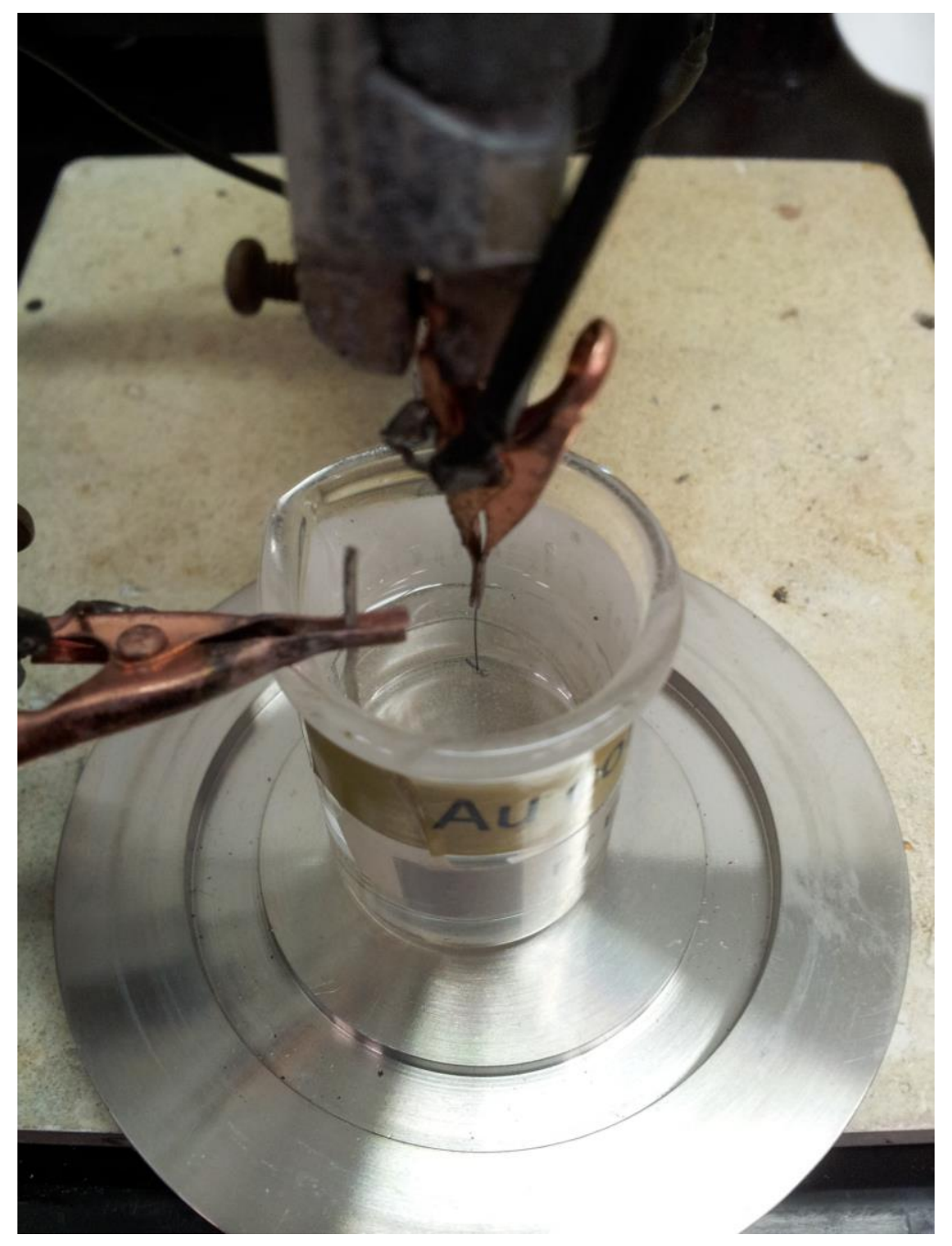

Figure 8.11. Close-up of the $\mathrm{W}$ etching in $8 \mathrm{M} \mathrm{KOH.}$ 


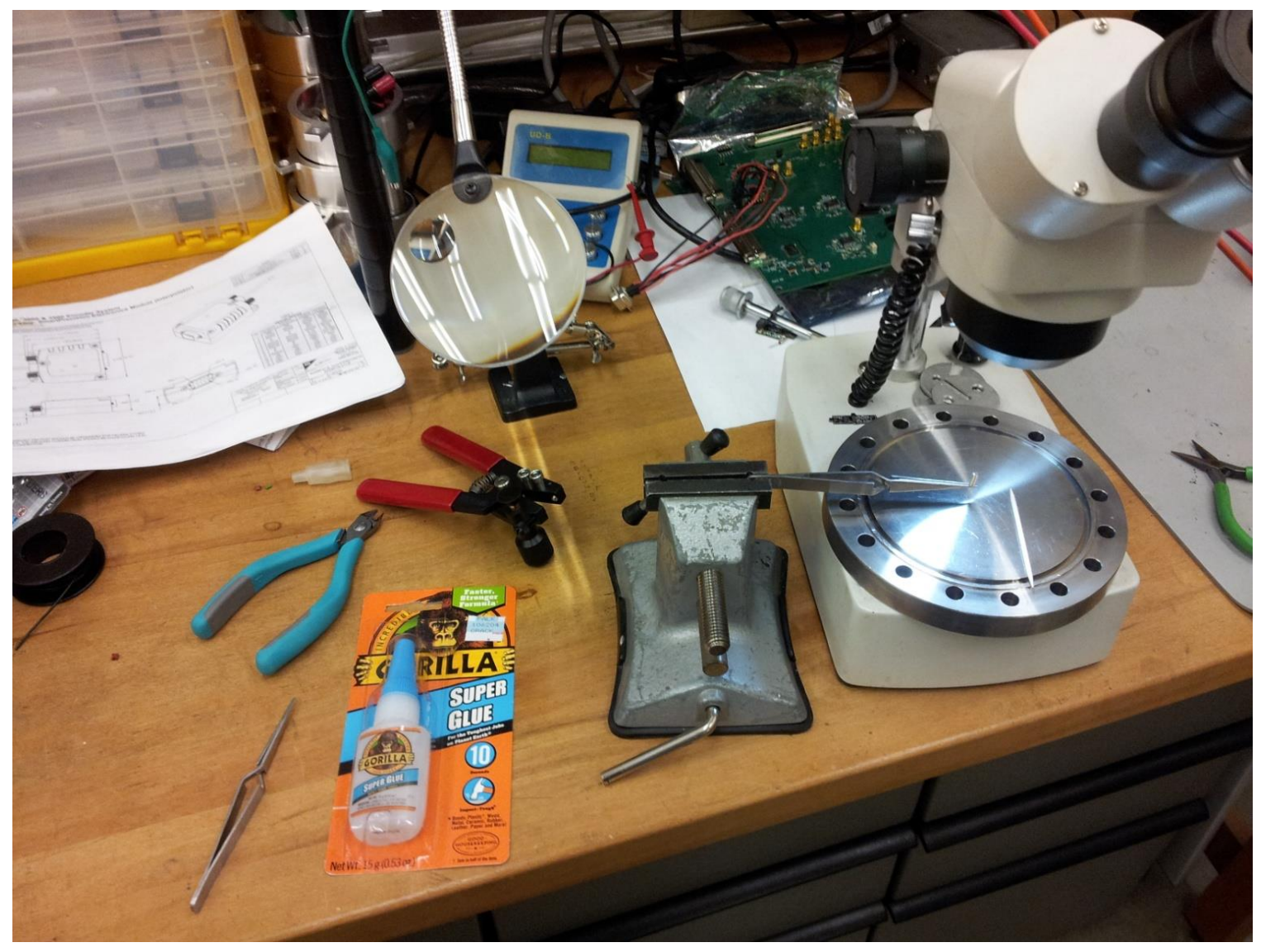

Figure 8.12. Typical setup for gluing etched tips to tuning forks.

Tips are typically glued to the tuning fork using Cyanocrolate or two-part epoxy. Most of the tips used in this work were attached using Gorilla Super- Glue. As the glue ages, it tends to form stringers more easily. These stringers tend to align themselves along the wire body of the $\mathrm{W}$ tip and will interfere with the feedback process. They are difficult to remove so it is better to avoid creating them altogether if possible. 


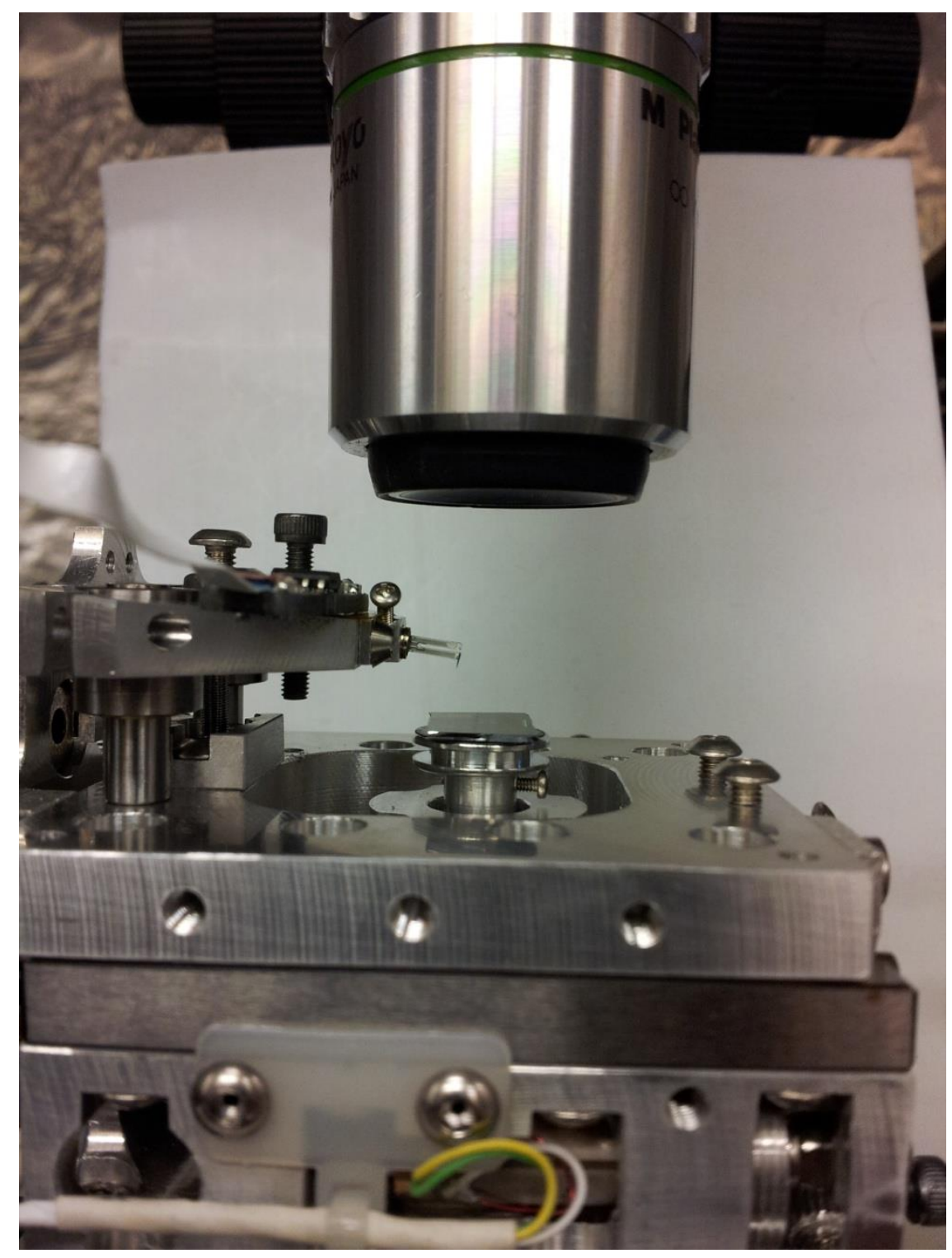

Figure 8.13. Side-view of the AFM test setup. Notice that the tuning fork can is clamped in using a set-screw. This arrangement was difficult to tune so it was hastily remade. Now the tuning fork is supported by its electrical leads only. This has a de-tuning effect giving lower Q's, allowing for faster feedback.

Ideally, the tip will be maintained at a constant distance above the sample surface as the sample is scanned in XY. In practice, the servo loop cannot always respond quickly enough to large steps and so the tip will make contact from time to time. These frequent contacts with the sample will degrade the XYZ data, damage the sample and 
will eventually wear out the tip itself. Of course, tip crashes can be catastrophic and the usual behavior is a curled tip.

\subsection{Vacuum considerations}

Operation in vacuum requires that the materials be compatible and nonmagnetic wherever possible. The AFM body of the coarse stage is made of Aluminum. Connecting screws are vented stainless steel wherever possible. The AFM tip is operated in the so-called "tapping mode" where it oscillates vertically perpendicular to the sample surface. The tip is prone to sticking to the surface.

Steps were taken to minimize the chances of igniting a corona in the regions where high potentials are required for AFM operation. These are the XY scan bed and the Zaxis piezo stack. The XY scan bed has its own power switch so that it can be powered off during vent/pump cycles while the rest of the low Voltage circuits remain powered. The Z-axis piezo stack is driven by a PI E-107, thus it can be powered off during vent cycles.

\subsection{National Instruments Reconfigurable Input/Output (RIO)}

The electronics design was leveraged from the ANSOM Project and is discussed completely therein. However, enough changes to the running code were made for this project that a discussion is necessary in this dissertation. The National Instruments NI PCI-7833R RIO was chosen for the central AFM control. The 7833R is a mixed-mode development platform in which complex digital logic elements can be programmed to communicate with the outside world through analog and digital interfaces. Most of the original functionality of the ANSOM code was either used directly or enhanced for this project. Functionality which can be turned into digital logic such as control loops, pixel 
raster, stepper/pulsed motor control and positional encoder quadrature decoding are examples of the things needed from this card in order to control an AFM.

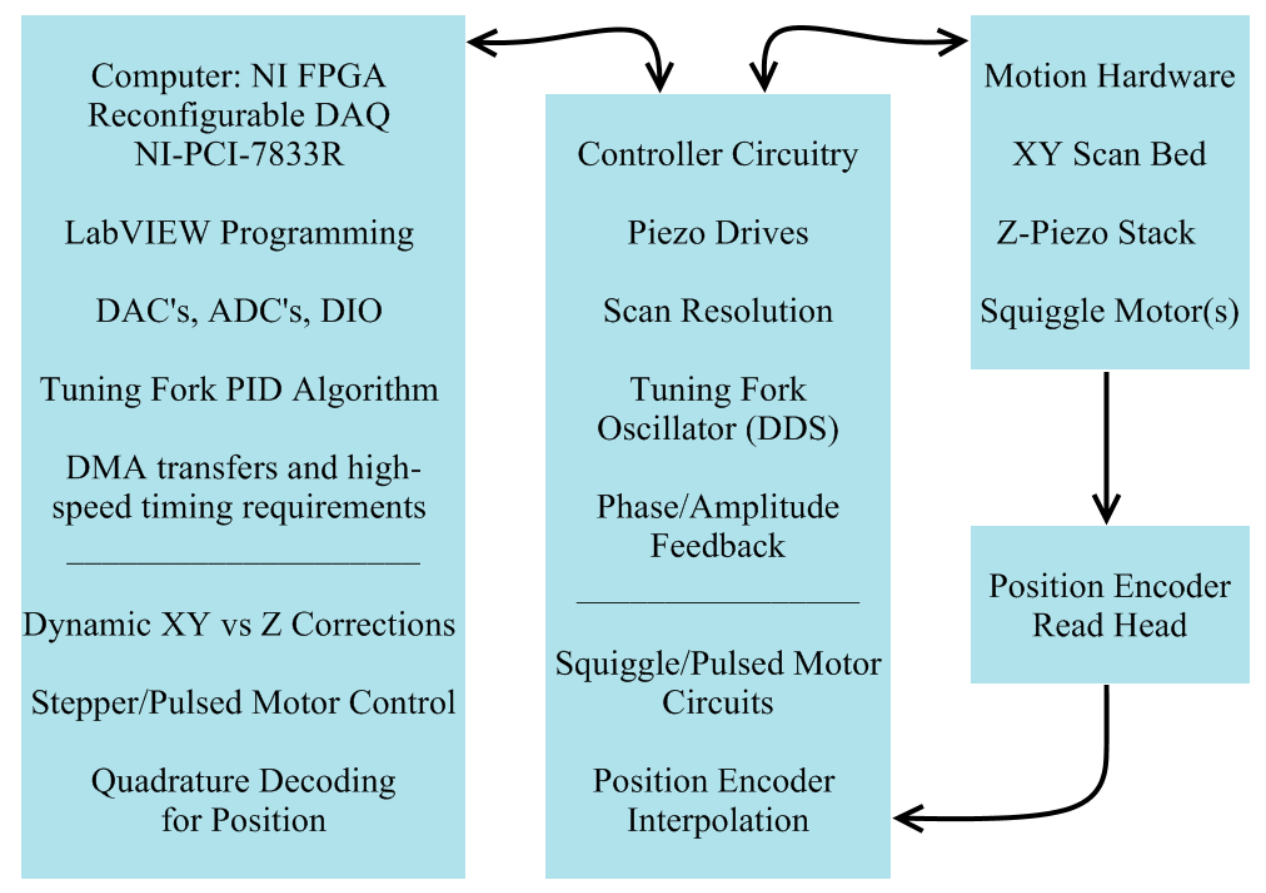

Figure 8.14. AFM hardware relationships. 


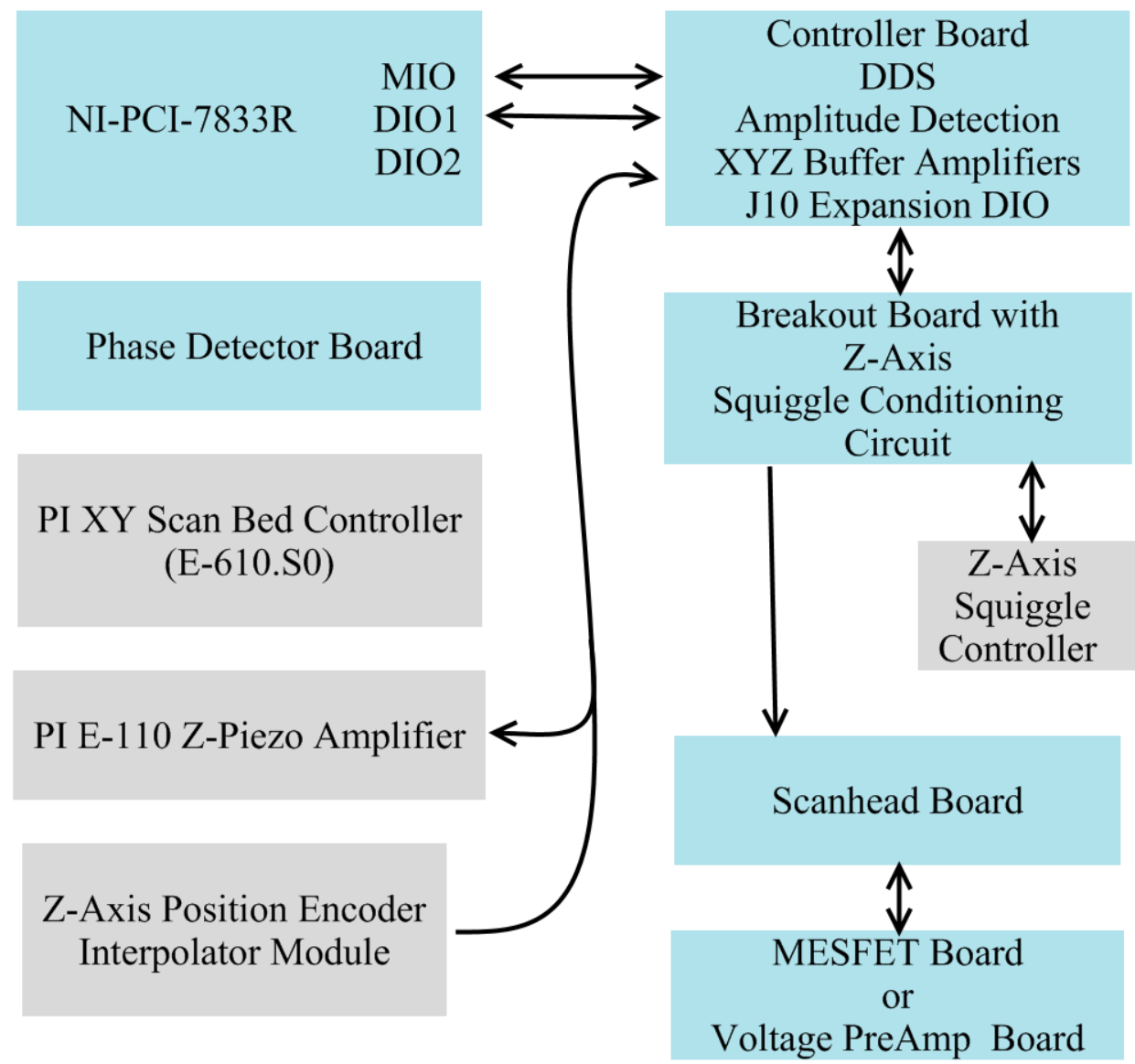

Figure 8.15. AFM modules and their connections shown as one or two-way.

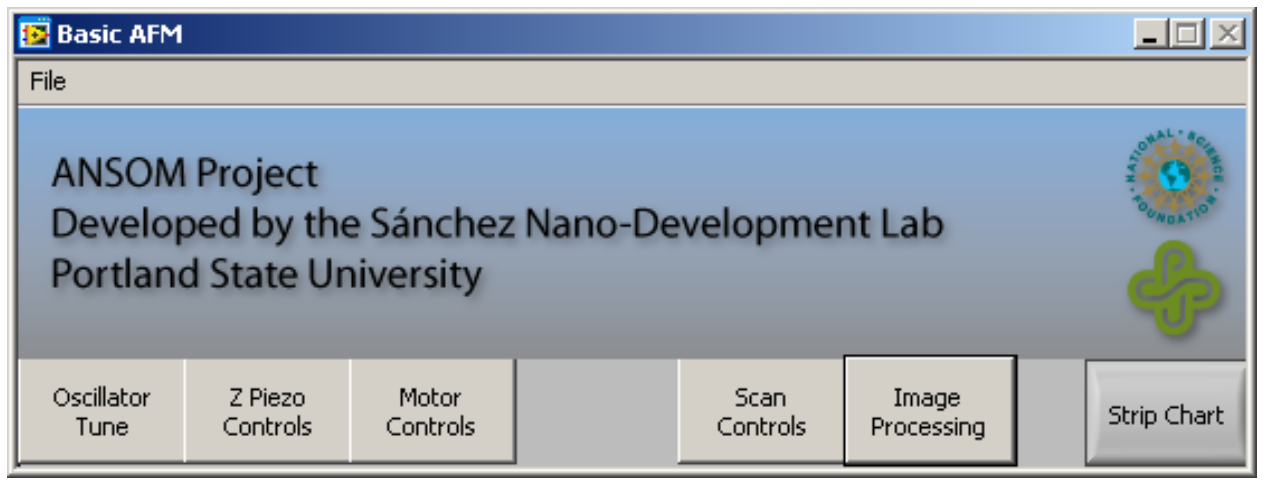

Figure 8.16. Main UI for accessing the AFM functionality. Many of the ANSOM functions have been removed for simplicity. 


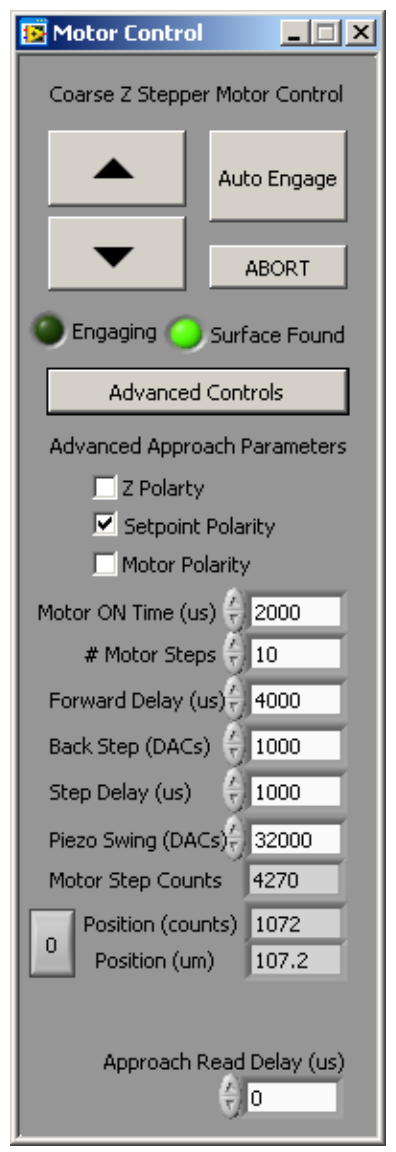

Figure 8.17. Motor Control UI. Items added to this control are Motor ON Time (us), Motor Step Counts, Position (counts), Position ( $\mu \mathrm{m})$, Zero Encoder Button and Approach Read Delay (us).

Interpolator output is digital quadrature. For the $\mathrm{Z}$ axis, an FPGA function reads and decodes the quadrature directly. The decoded position is available for the Stepper Motor Control vi to display in real time. 


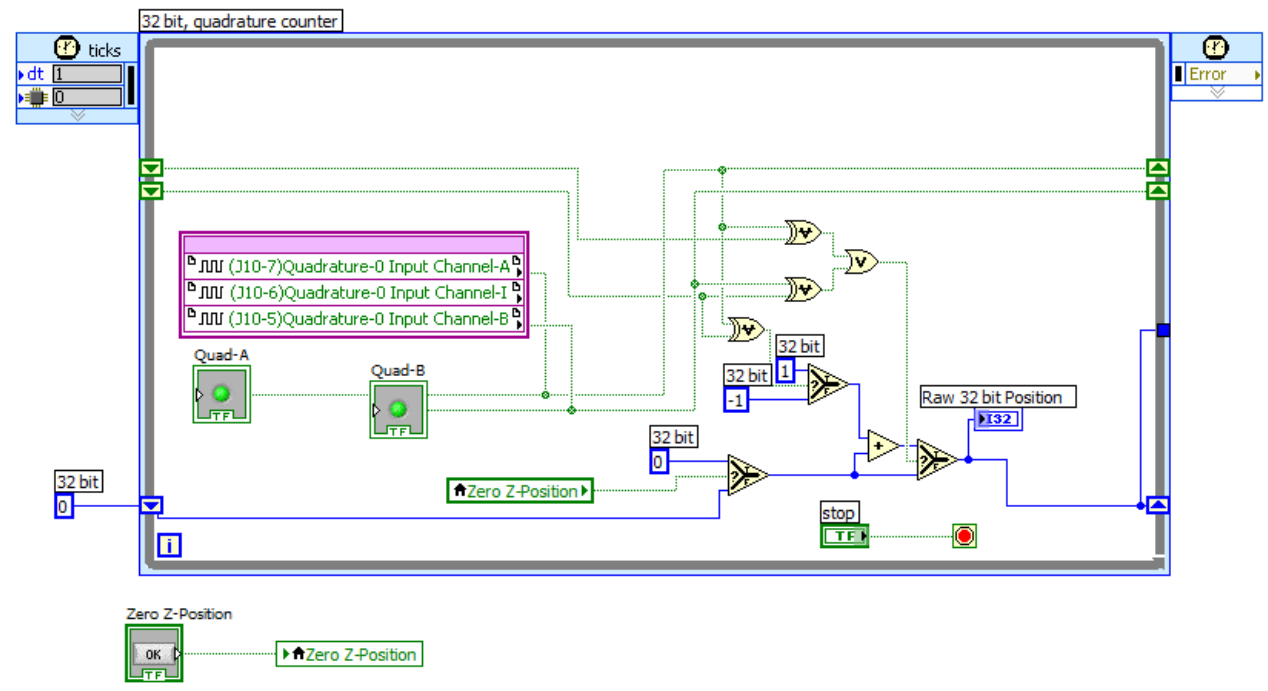

Figure 8.18. Quadrature decoding directly on the FPGA.

\begin{tabular}{|c|c|c|}
\hline 粪 Z-Piezo Control & & $-10 x$ \\
\hline z-Piezo Voltage & z-Piezo (nm) & Avg Delay (us) $\frac{\hat{r}}{5}$ \\
\hline 300 & $\begin{array}{l}1 \\
-300\end{array}$ & $\#$ Avgs $\frac{1}{5} 0$ \\
\hline Proportional $\frac{f}{\tau}, 0.00000$ & Feedback Signal Phase & $\square$ High Resolution Z \\
\hline Integral $\frac{\hat{r}}{0.00000}$ & Feedback Value 90 & $\begin{array}{l}\text { Manual Z Control } \\
\text { Negative Z-Polarity }\end{array}$ \\
\hline Differential $\frac{7}{5}, 0.00000$ & Feedback Setpoint $\frac{\theta}{v} 0$ & Advanced $\longrightarrow$ \\
\hline
\end{tabular}

Figure 8.19. Piezo Control UI in normal mode. Items added to this control are Avg Delay (us), \# Avgs, and the Advanced toggle switch. 


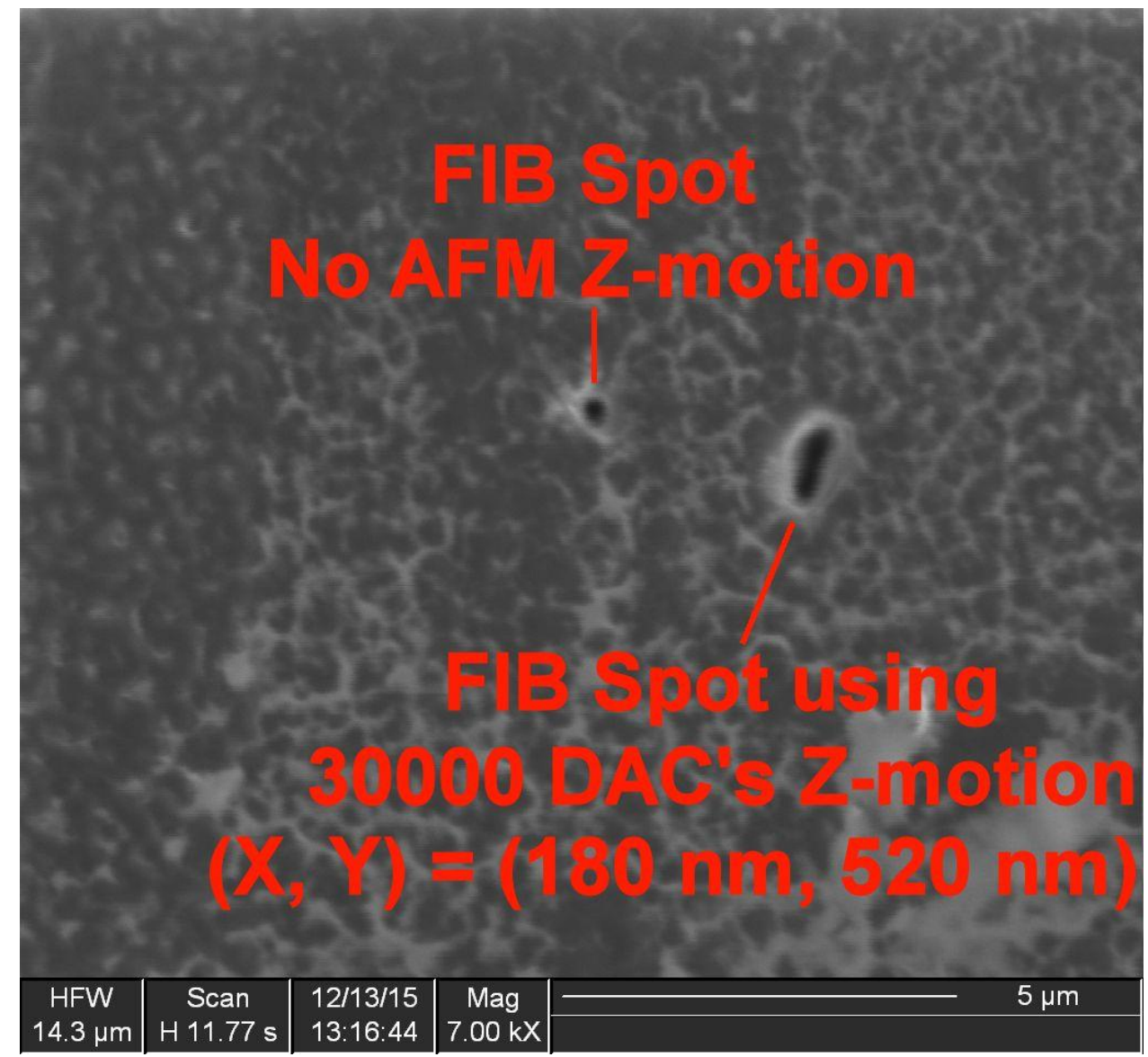

Figure 8.20. FIB image showing the $550 \mathrm{~nm}$ lateral shift of the sample during an AFM ramp sequence in $Z$. This lateral shift can be corrected inside the FPGA code by activating the XY vs $Z$ functionality and adjusting the sliders for nulling image shift while FIB imaging.

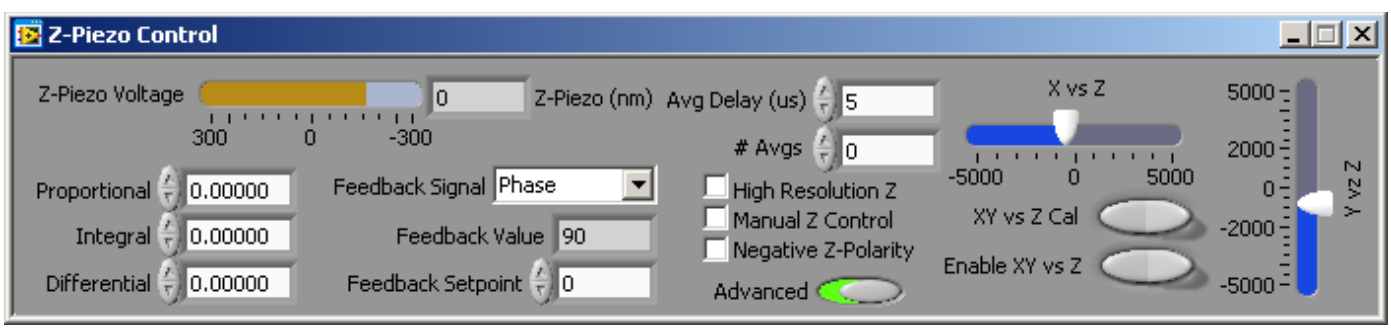

Figure 8.21. Z-Piezo Control UI showing the $X Y$ vs $Z$ fly-out which can be used to calibrate and then activate the feature. 


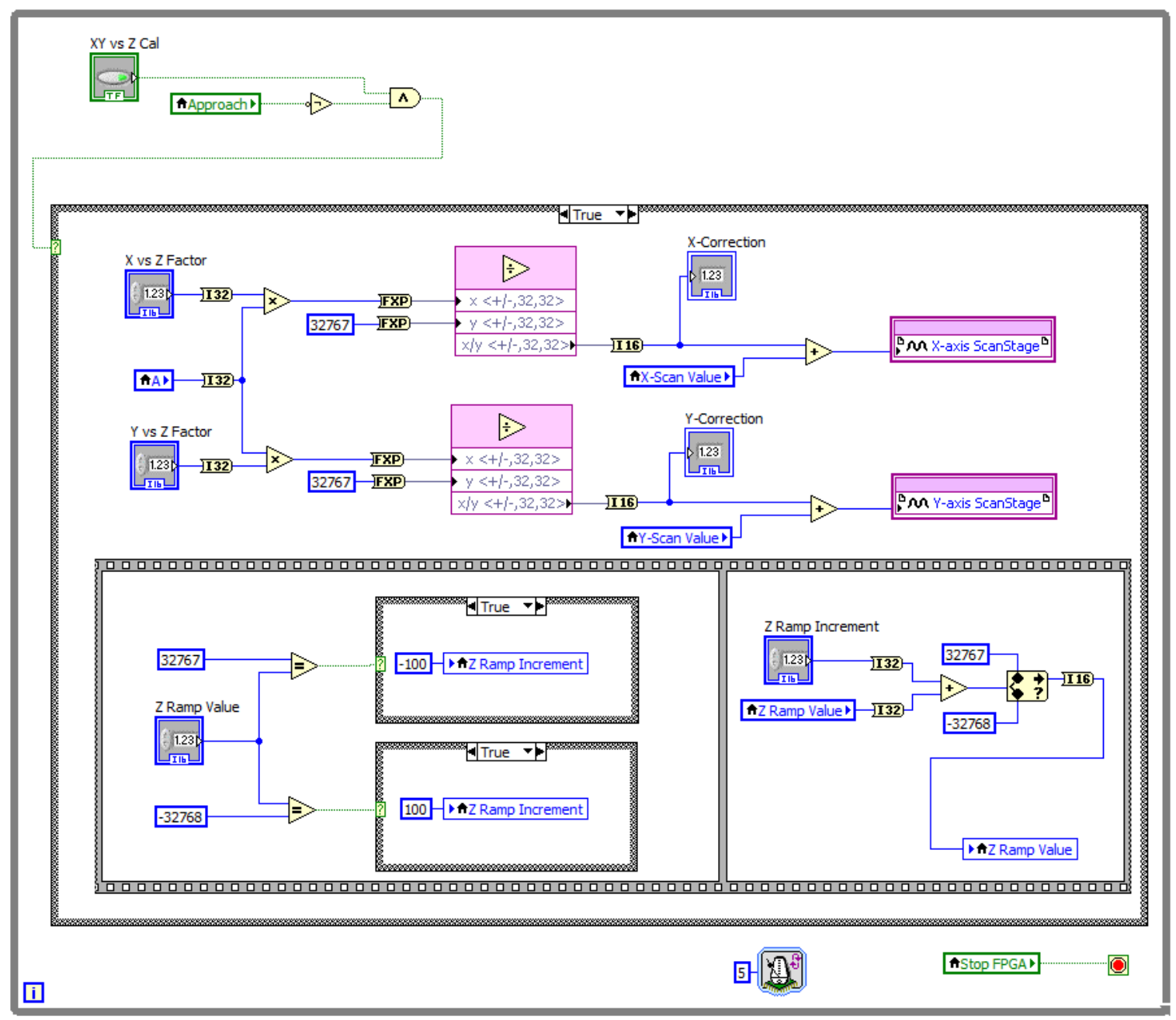

Figure 8.22. FPGA code for determination of the $X$ vs $Z$ and $Y$ vs $Z$ factors which can optionally be applied to the scan bed to minimize the effect seen in Figure 8.20.

\subsection{AFM software}

National Instruments Labview is used for both PC host and fpga controller. It provides a Rapid Application Development environment (RAD), giving the user the flexibility to create rich UI's while at the same time the power to create real-time controller modules. Labview is a proper high level programming language containing all of the constructs necessary to create programs which are compiled to optimized executable code. 


\subsection{Modifications to the FPGA code and host software}

Numerous modifications to the NSOM FPGA code and associated host programs were needed to improve the performance of this AFM. These include, quadrature decoder add-ins for the coarse $\mathrm{Z}, \mathrm{XY}$ vs $\mathrm{Z}$ dynamic correction, etc.

\subsection{One quirk with National Instruments Labview FPGA development}

On many occasions, I have modified the FPGA interface and have found no surefire way to force a build of the UI vi's. These projects do not display a "Rebuild" context menu item and so will not always perform builds when the fpga interface changes. This can lead to catastrophic behavior since the remapped fpga interface receives calls from an outdated UI. This outdated interface also does not seem to change in a predictable way, i.e. adding to the end of a large data structure. It is important to make sure that the afm hardware elements are in safe states before attempting to use recompiled software with the power turned on.

\subsection{NewScale Technology motors and control software}

The Coarse $\mathrm{Z}$ axis squiggle control software has one little bug which can be a nuisance if the user forgets about it. Once the Coarse $\mathrm{Z}$ controller is powered on, it is important to switch to the Axis Attributes tab and uncheck the "Auto Tuning" checkbox. With this version of software it generally will auto tune to a frequency which does not cause motion of the motor. It is better to use the "alt-p" page to determine the best tuning. This tuning will remain valid as long as there are no major changes to the coarse$\mathrm{Z}$ axis system. A major change can be one or more of these things: 
1) Crashing the motor into its negative stop

2) Preload spring changes

3) Dissasembly \& reassembly of the mechanism can change the cogging behavior of the linear bearings

The Squiggle motors provide very good performance in terms of resolution and stability. The electrical and software controls allow for great design flexibility. It is really unfortunate that the Squiggle motors we used for this design are no longer available. This is the main reason that the XY Squiggle control was not fully integrated into the FPGA code. Only the minimum integration required for automated approach was implemented for coarse Z. The company which made them, Newscale Technology, no longer makes these "large" format squiggle motors. Newscale has consolidated its product offerings to more reliable assemblies and high-end custom nanomanipulators. They now integrate the smaller Squiggle motors into linear stages which can be stacked to provide complex motion. On our AFM, the X-axis Squiggle motor has broken and its repair is a very delicate operation. A more sustainable solution will be needed for future work and for possible replication of this design. We are pursuing two possibilities. 1) Pico motors by Newport are cost effective, robust and are commercially successful enough that they are available for purchase new or used. They are also about $\$ 500.00$ apiece which is cheaper than the Squiggles we paid $\sim \$ 1000.00$ apiece. 2) Inexpensive tiny stepper motors are available for less than $\$ 10.00 /$ axis. 3) There is still the possibility there is one more chance to repair our X-axis Squiggle motor by making a threaded piece which is mounted without adhesive. This approach may extend the life of both motors as Newscale recently described this as a failure mechanism, i.e. relatively soft steel threaded shaft running inside a similarly soft steel threaded ring. 


\subsection{Ad-hoc changes to intended AFM design}

Some changes to the original design were made either by necessity or simply just to get something working. As was mentioned earlier, the FIB 611 system has a larger stage than the Micrion, originally intended as the target system, so some of the size constraints were relaxed in this implementation. The return springs for the coarse XY and the encoder cables were intended to fit entirely inside the $60 \times 60 \mathrm{~mm}^{2}$ envelope. To accommodate the XY return springs, the top and bottom plates were originally designed with cutouts so that they would fit inside the envelope. This would mean that they could not be made out of Aluminum for rigidity concerns. Additionally, the coarse Z-axis coupling was intended to fit inside the mechanism so that the MESFET/VPreAmp board could fit into the groove. It was unknown at the time whether the scan head board could operate inside the vacuum so the original plan was for it to be outside the chamber.

Finally, the original tuning fork holder was very rigid and had a strong coupling to the rest of the coarse XY stage making tuning very difficult. Now it simply plugs into a board with tiny sockets. This makes tip changes very easy and also has the effect of detuning the mechanical circuit so that tuning is much easier and scans are faster.

\subsection{References}

IMSI. TurboCAD Version 19 User's Guide. 25 Leveroni Court Novato, CA 94949: IMSI/Design, 2012. www.TurboCAD.com.

Morawski, Ireneusz, Józef Blicharski, and Bert Voigtländer. "Voltage Preamplifier for Extensional Quartz Sensors Used in Scanning Force Microscopy." Review of Scientific Instruments 82, no. 6 (June 2011): 063701. doi:10.1063/1.3594103.

Novotny, Lukas, and Bert Hecht. Principles of Nano-Optics. Cambridge: Cambridge University Press, 2006. 
Nowak, Derek Brant. The Design of a Novel Tip Enhanced near-Field Scanning Probe Microscope for Ultra-High Resolution Optical Imaging, 2010. http://adsabs.harvard.edu/abs/2010PhDT.........38N.

Sanguinetti, B, and B.T.H Varcoe. "Use of a Piezoelectric SQUIGGLE ${ }^{\circledR}$ Motor for Positioning at $6 \mathrm{~K}$ in a Cryostat." JCRY Cryogenics 46, no. 9 (2006): 694-96.

Shrotriya, Archana. "Writing Win32 Dynamic Link Libraries (DLLs) and Calling Them from LabVIEW." NI LabVIEW User's Manual, 1999. http://citeseerx.ist.psu.edu/viewdoc/download?doi=10.1.1.452.8789\&rep=rep1\&type=pdf

Silberman, Donn M. "Dynamic Alignment, Tolerances, and Metrology Fundamentals at the Nano and Micro Scales." In SPIE Optical Engineering+ Applications, 95820B95820B. International Society for Optics and Photonics, 2015.

http://proceedings.spiedigitallibrary.org/proceeding.aspx?articleid=2436526. 


\section{AFM details of operation}

Before attempting vacuum operation, the assembled AFM was tested in air inside an enclosure on an air table. Additionally, in order to verify the coarse XY stage, the AFM was attached to a microscope using coaxial illumination via red LED. The objective is a 20X Mitutoyo, having a numerical aperture of 0.42 and an approximate FOV of $500 \mu \mathrm{m}$. The imaging camera is a Lumenera LU105 USB-based machine-vision camera having $1280 \times 1024$ native pixels on a 1/2" CMOS sensor.

A few things needed addressing during the verification in air:

1) The FPGA needed modification to use the Z-Squiggle motor for the automated tip approach sequence.

2) Determining the polarity of the PID, Z-piezo and the phase adjustment which is easy to use and repeatable.

3) Finding stable PID values.

4) Z-piezo arcing occurred due to an electrical short of the stack's electrodes with the stub mount. The stack could no longer be operated at 1000 Volts. It could only be operated at 300 Volts, reducing the maximum theoretical range of motion from $60 \mu \mathrm{m}$ to $18 \mu \mathrm{m}$.

\subsection{FPGA modifications for Z-Squiggle control}

The ANSOM FPGA code provides three separate stepper motor interfaces. The one we are interested in is the Z-axis stepper control. This controller is used for part of the tip-sample approach sequence. Our AFM utilizes a very small Squiggle motor. Its outer diameter is $7 \mathrm{~mm}$ and it has a travel range exceeding $5 \mathrm{~mm}$. It can push a load up to 100 grams. However, it is not a stepper motor. NewScale software provides numerous ways to integrate their controller software into custom software designs. A short list includes: 
1) Base application program. This is the program which can control all of the functions of one or more Squiggle motors.

2) Active-X control which can be simply be dropped onto a Windows WinForm or LabView window which could be part of a larger program. Once the WinForm app is running, the Active- $\mathrm{X}$ control has the same functionality as the base application program.

3) Direct API calls to the controller via dlls

4) Analog control of the controller.

The method chosen was \#4, the analog control interface. The motor is pulsed with a

CMOS logic level.

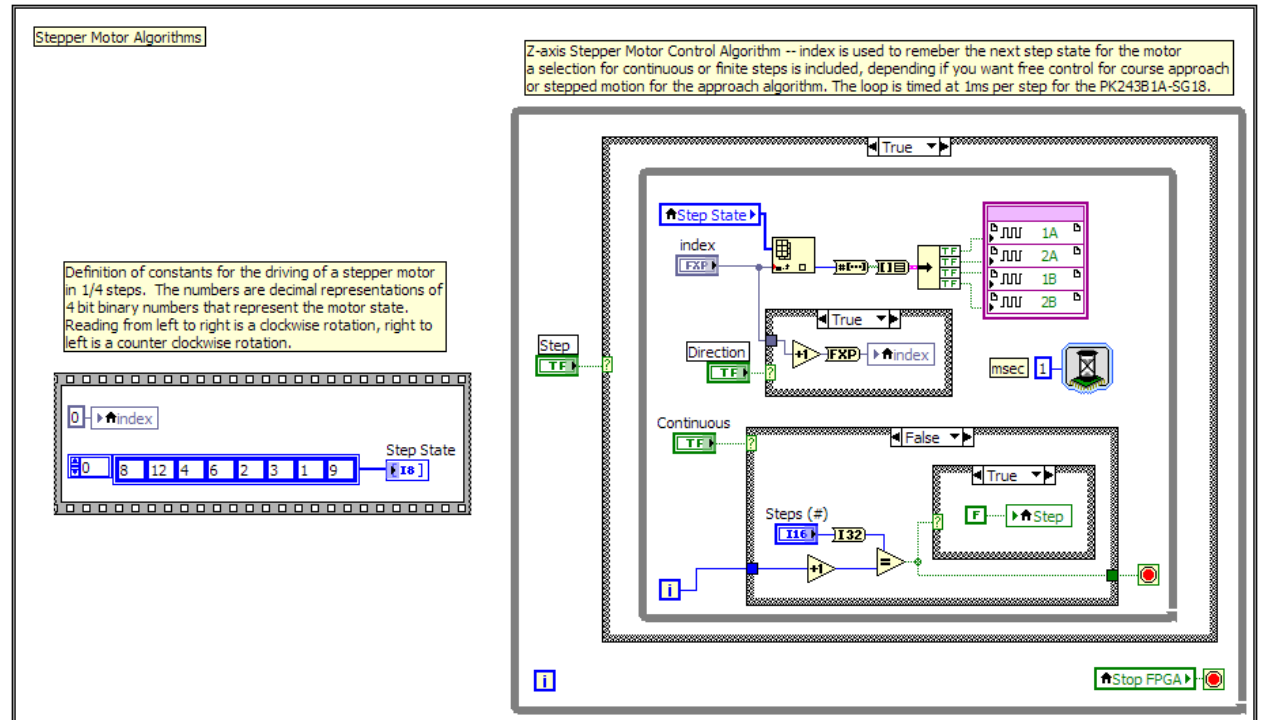

Figure 9.1. Original FPGA stepper motor code which is designed to control the stepper motor coils individually. This code exhibits a flaw in which the "Step" input is read before the "Direction". The result is that direction reversals are not possible until one or more steps are executed in the prior direction. 


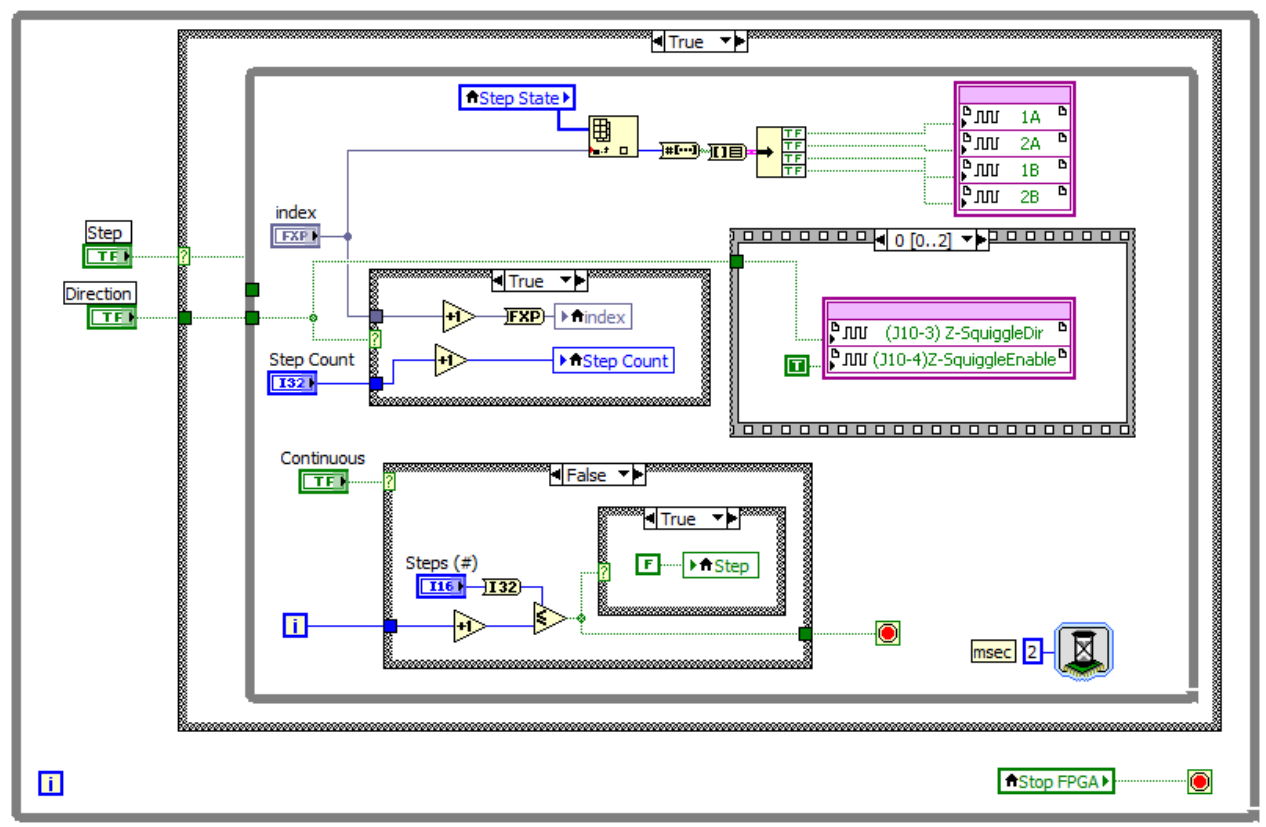

Figure 9.2. Modified FPGA stepper motor control which retains the original, individual coil control and adds a step-direction output for the Z-Squiggle controller. Many stepper motor drivers also have step-direction inputs, increasing the possible number of configurations. This code also fixed a race condition where reversing the direction of stepping resulted in one or more extra steps in the prior direction, potentially resulting in crashed tips.

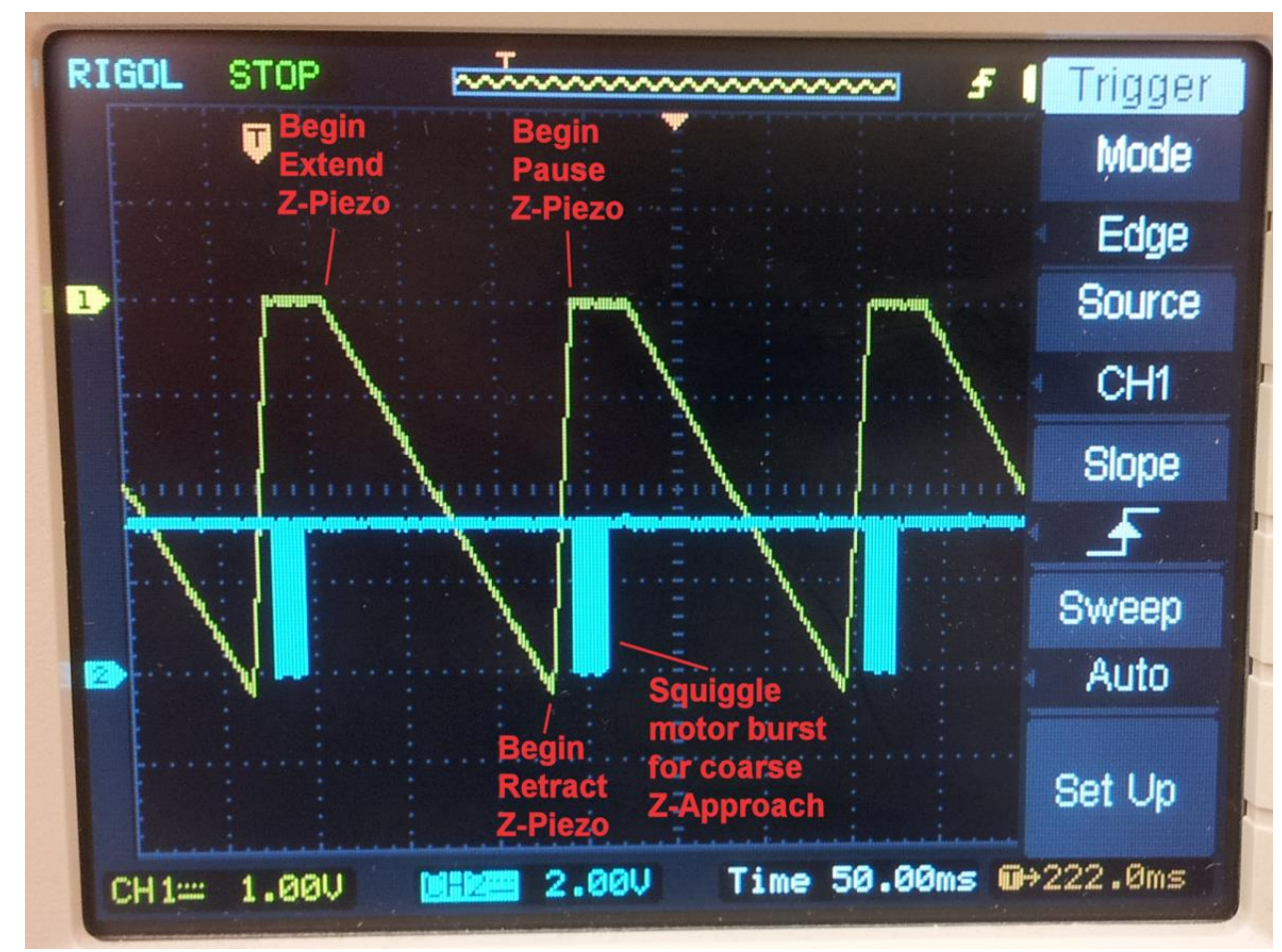

Figure 9.3. Electrical signals which form the AFM tip approach sequence. The surface is sought using alternating $\mathrm{Z}$-piezo ramps and coarse-Z motor bursts. 


\subsection{Polarity modifications to the FPGA code}

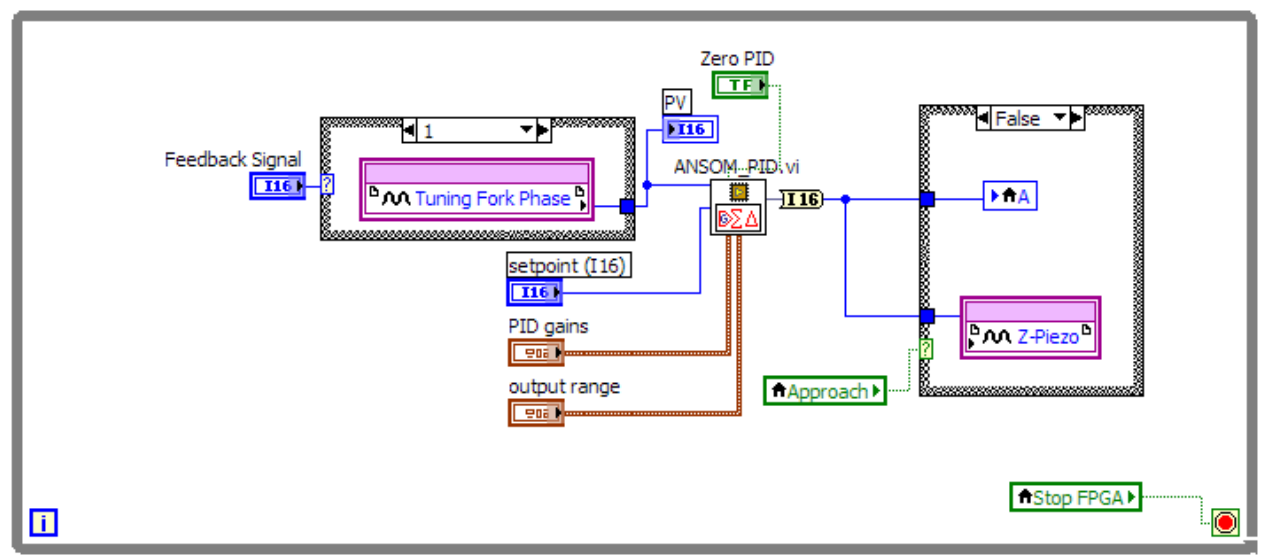

Figure 9.4. Original ANSOM FPGA high-level PID control showing only one output to the Z-Piezo.

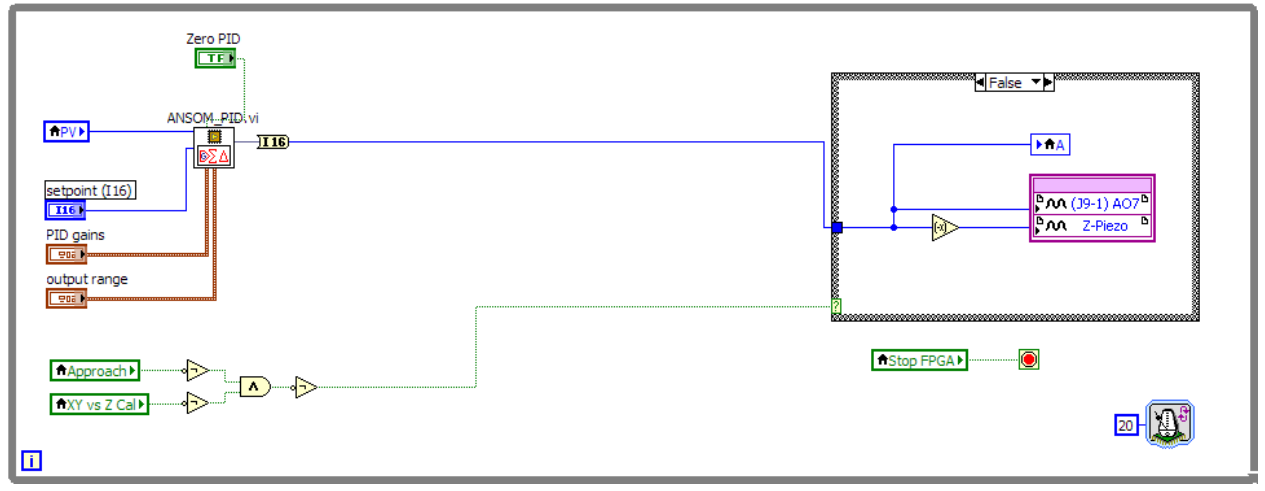

Figure 9.5. Modified FPGA high-level PID control showing an additional inverted output to an extra DAC output on the Controller Board (J9-1). This bit of code also reflects other changes made elsewhere. The gap in the middle was intentionally made for inserting low-pass and notch filters during debugging sessions. 


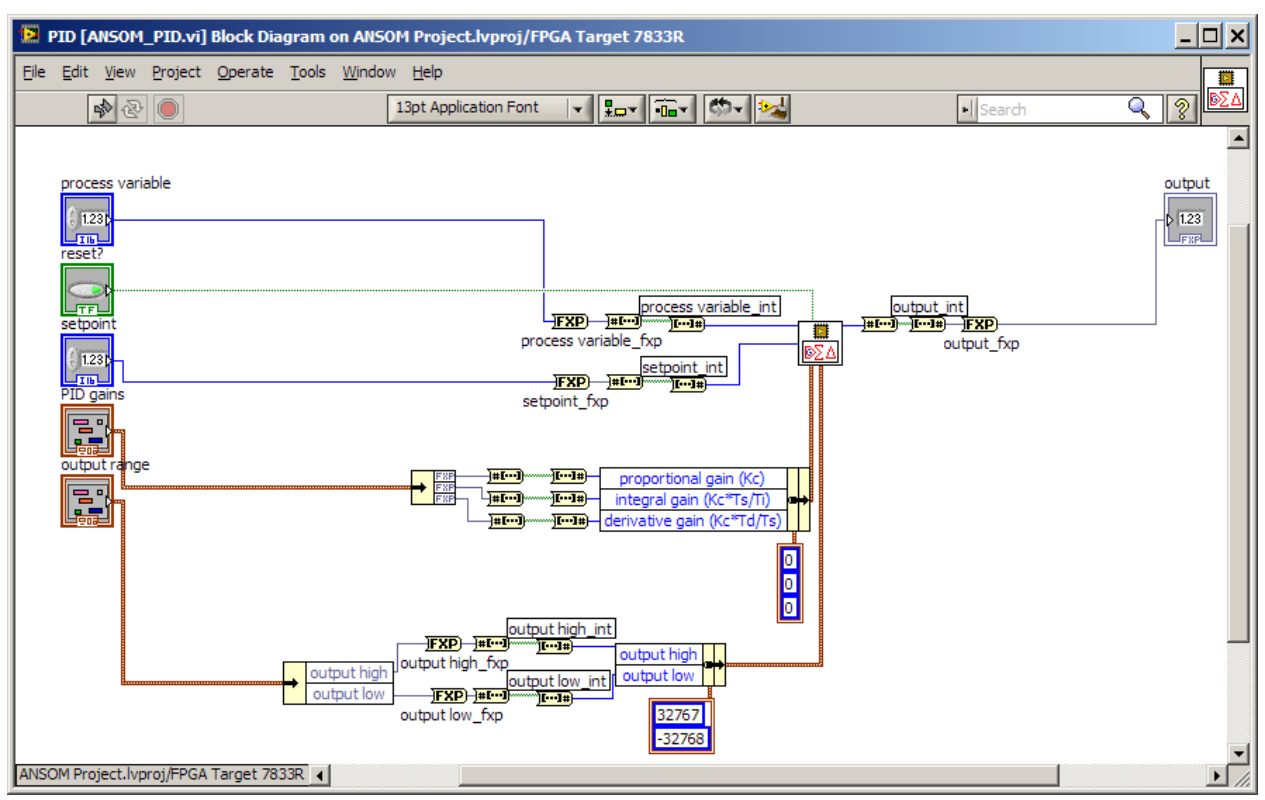

Figure 9.6. Labview code showing the PID control and how it is linked to the variables and control variable which is usually the phase error signal.

The motion of the Z-piezo is not only reduced by the smaller applied maximum voltage, it is also reduced as a function of the preload applied.

$$
f=\frac{k_{\text {Piezo }}}{k_{\text {Piezo }}+k_{\text {Preload }}}
$$

Once the preload was applied, the range of motion was reduced from $18 \mu \mathrm{m}$ to $16.7 \mu \mathrm{m}$, allowing for an estimate of the preload spring constant. The spring constant for the piezo stack is $29 \mathrm{~N} / \mu \mathrm{m}$. For our preloaded mechanism, $\mathrm{f}=(16.7 \mu \mathrm{m}) /(18 \mu \mathrm{m})=0.9278$.

$$
k_{\text {Preload }}=k_{\text {Piezo }}\left(\frac{1}{f}-1\right)
$$


Thus $\mathrm{k}_{\text {Preload }}$ is about $2.26 \mathrm{~N} / \mu \mathrm{m}$.
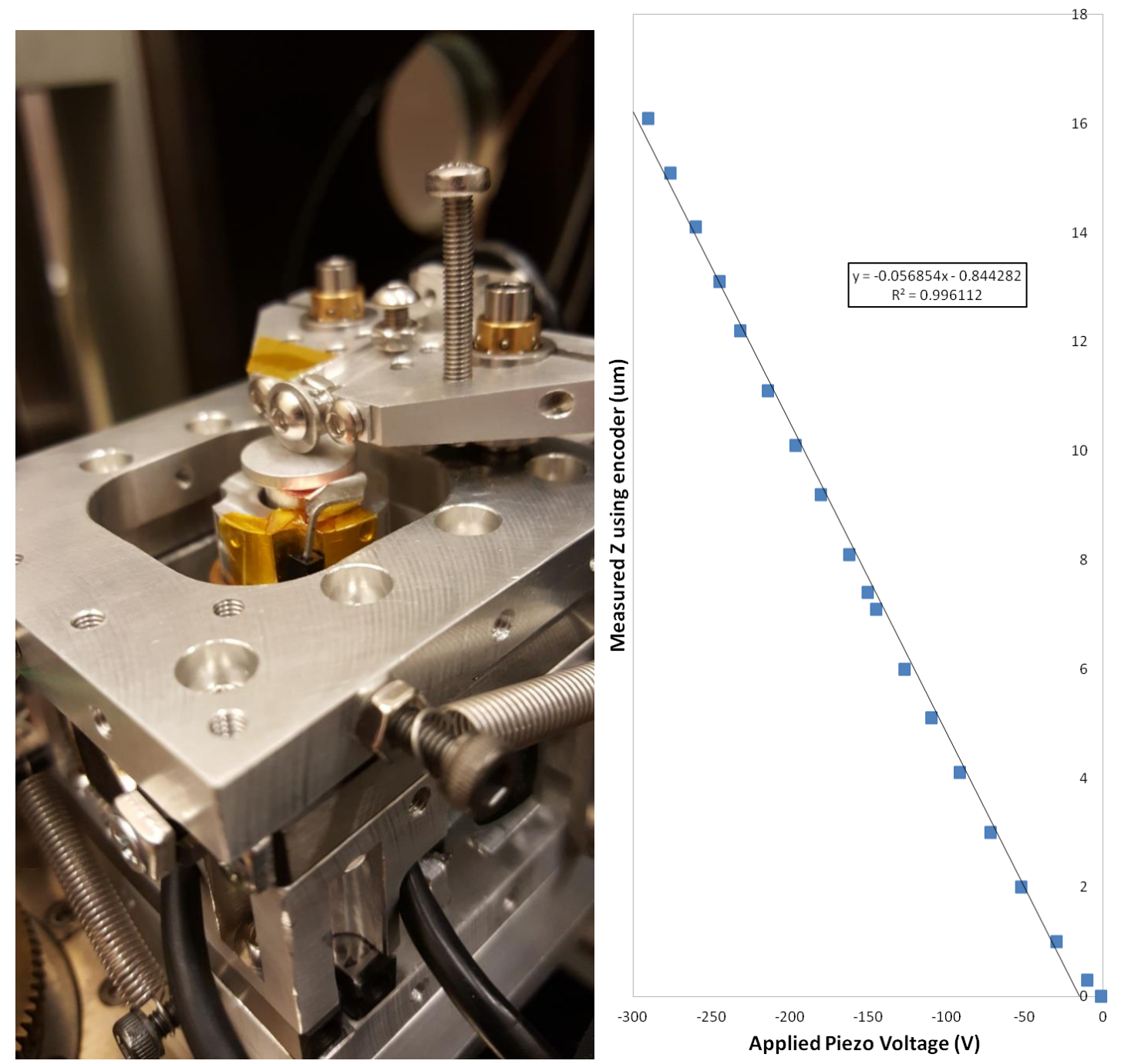

Figure 9.7. Using the coarse $Z$ encoder to measure the piezo sensitivity by touching it to the sample stub allowing the spring to cause constant contact during the measurement. The response is approximately $56.854 \mathrm{~nm} /$ Volt.

\subsection{Polarity determination}

The TENOM height feedback utilizes a phase profile which goes from positive to negative as frequency is swept from low to high. This was the starting point for this AFM during the initial scans in air on Si wafer pieces. Figure 9.8 shows a typical 
frequency sweep.

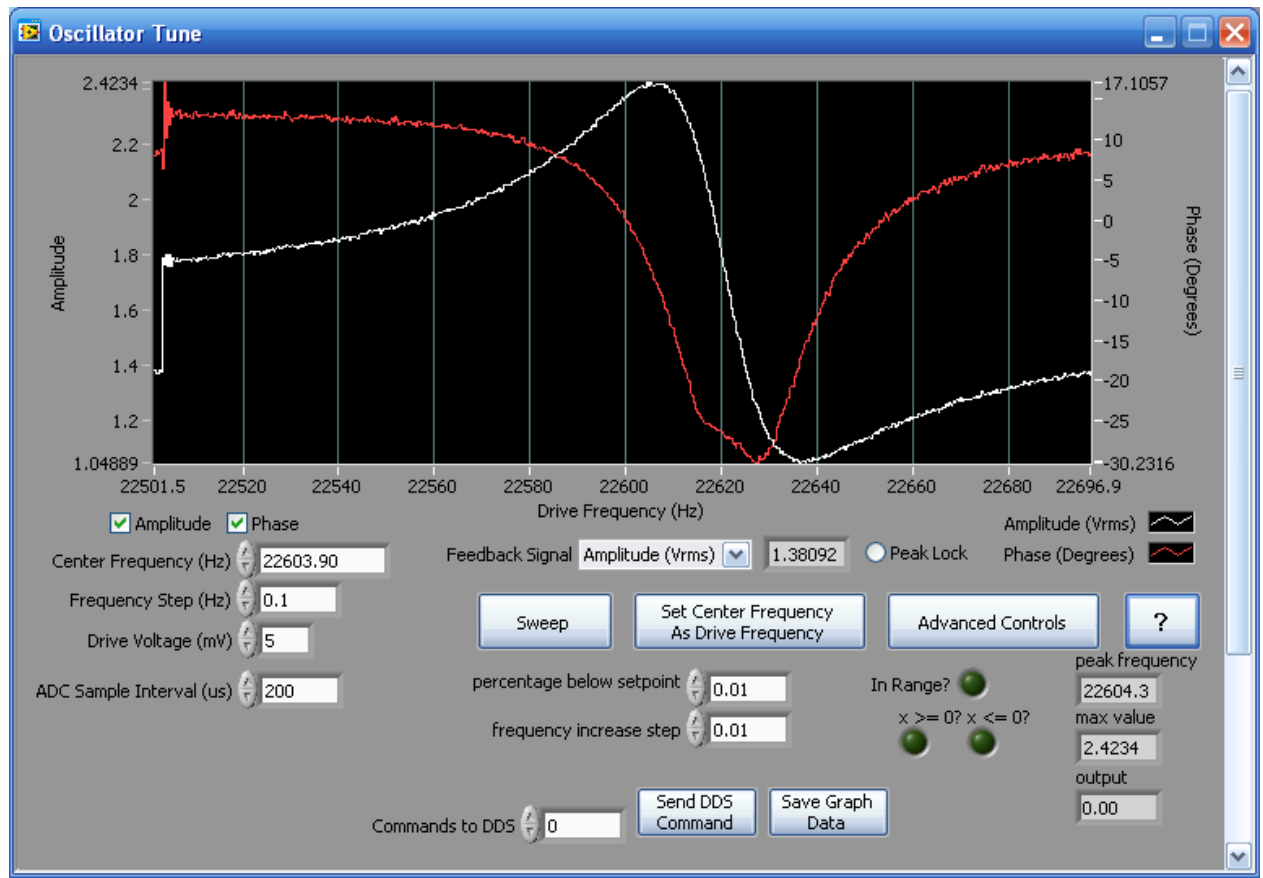

Figure 9.8. Typical frequency sweep for the tuning fork used in Figure 9.9 to acquire a scan. The phase detection circuit is adjusted for a negative phase response to frequency and for maximum dynamic range. In this case the phase range is about 35 degrees, but only 20 degrees is usable for feedback, due to the high curvature of the phase response.

It was necessary to operate using a set point of +5 degrees. Z-polarity was set to negative and the PID values used were $(-0.5,-0.001,0.0)$. The dynamic range for phase is very small, effectively 25 degrees, and its response to frequency has a large curvature over its operating range. Still a good image was taken using this arrangement (Figure 9.10). Using negative PID settings in addition to negative Z Polarity with a positive set point can be a little confusing. If any of these parameters are set wrong before an approach sequence is initiated, the tip may crash. For this reason, some effort was spent in creating a consistent polarity scheme for PID settings and $\mathrm{Z}$.

Figure 9.11 shows the electronic adjustment for a positive phase response with 
frequency. This, combined with positive PID parameters, negative set point and a negative $\mathrm{Z}$ polarity give a very consistent way to operate the AFM.

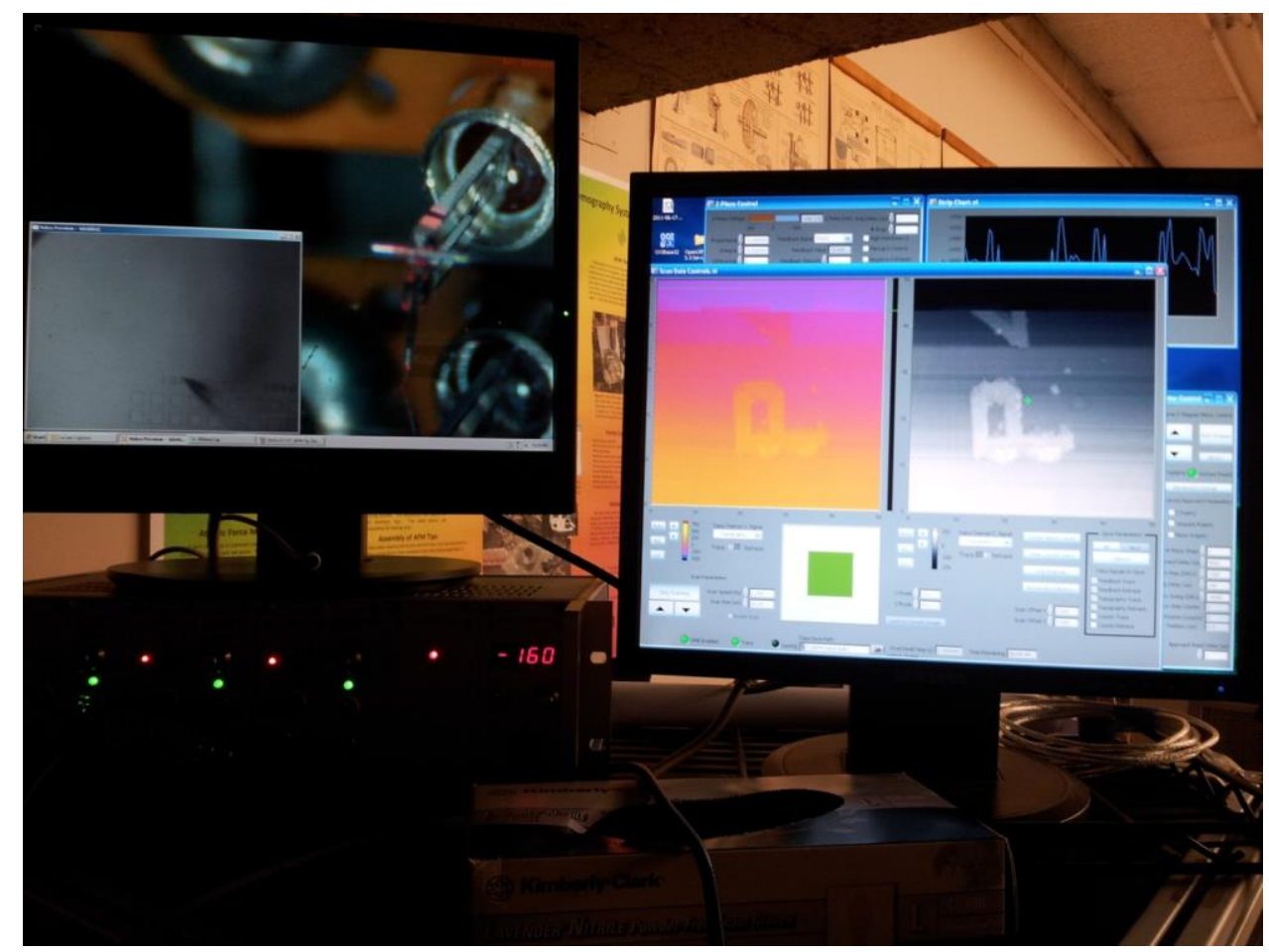

Figure 9.9. An early AFM scan in air on a MetroCal wafer piece. The left monitor shows the two video feeds used for coarse tip placement. The smaller one is the microscope image at $\sim 500 \mathrm{um}$ Horizontal FOV and the other is from a borescope camera mounted at oblique. The right monitor shows the scan in progress. The left (pink\&yellow) and right (gray) AFM images are the so-called trace and retrace images, respectively. The AFM is operating correctly when these images are very similar.

As stated earlier, the $\mathrm{Q}$ factor for crystal tuning forks can be very large and tends to double in vacuum. [Novotny] demonstrates that high Q's result in slow response of the feedback system. The tuning forks used for this AFM therefore are de-tuned in two ways. First a long tip tends to decrease both the resonant frequency, $\mathrm{f}_{0}$, and the $\mathrm{Q}$ of the mechanical system. Further detuning is accomplished by suspending the tuning fork by its own leads rather than rigidly mounting the can via set screw. The frequency sweeps from this arrangement, along with a suitable adjustment give a positive-sloped phase 
response. See Figure 9.11 for an example of the positive phase response.

In chapter 5 it was demonstrated that Q factors of 18000 are possible in vacuum. The detuning discussed above reduced the Q factors by a factor of 10 . For example, tip\#B-002 was used to acquire over 70 AFM images. Its Q in air was 1561 and its Q in vacuum was 1851 . With this reduced $\mathrm{Q}$, the drive voltage was increased by changing the resistor in the Voltage divider from $146.2 \mathrm{k} \Omega$ to $14.6 \mathrm{k} \Omega$.

There is enough adjustment range on the phase detector circuit to saturate the phase measurement at $+/-90$ degrees. This serves two purposes: 1) The phase curve is approximately linear at zero phase, near where the AFM will operate. 2) If the tip reaches these points during operation, the saturation zones reduce the chances of a reversed phase response, which would likely lead to a crashed tip.
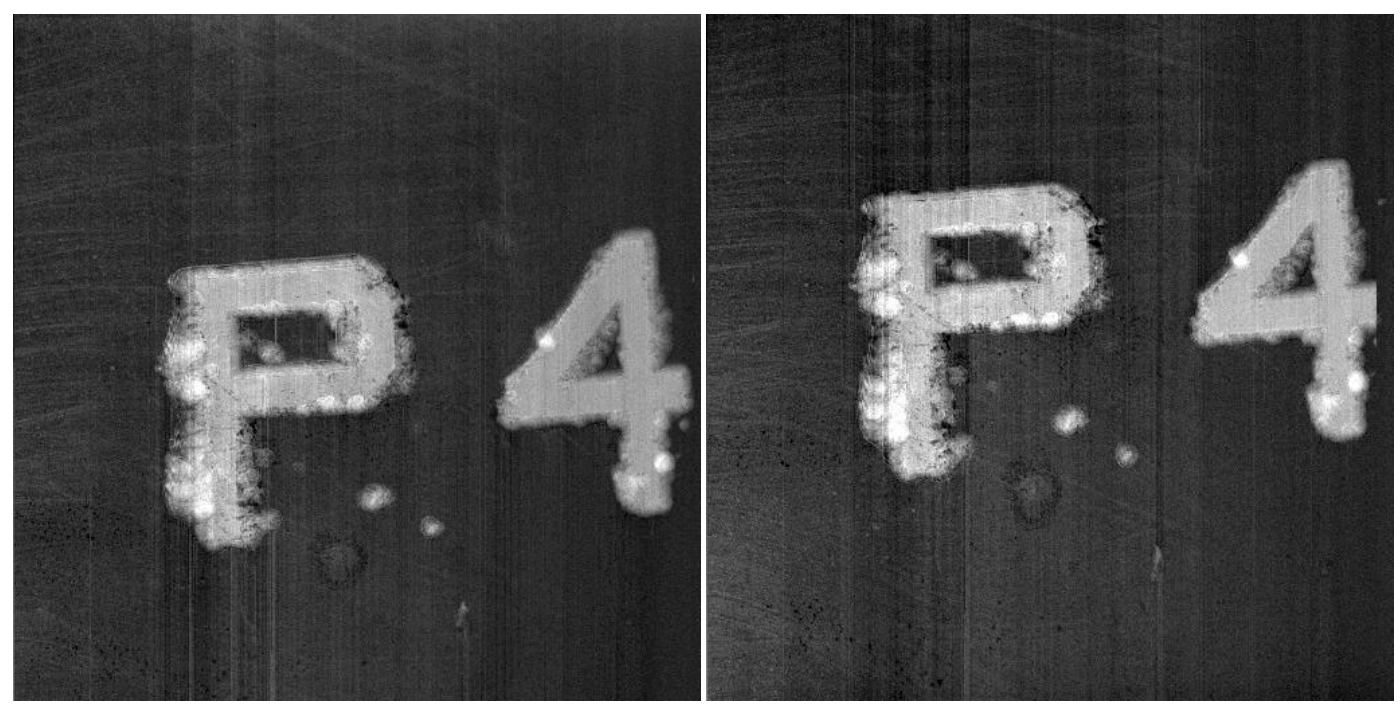

Figure 9.10. (left and right, respectively) Trace and retrace images $(50 \times 50 \mu \mathrm{m} 2)$ of one of the first unambiguous scans on a MetroCal wafer in air (shown in Figure 9.9). The fast scan axis is shown along Y. Some processing was performed to align the scan lines and to level the vertical scale. Some distortion is evident along the fast scan axis. This is a result of operating the XY scan bed in openloop mode. 


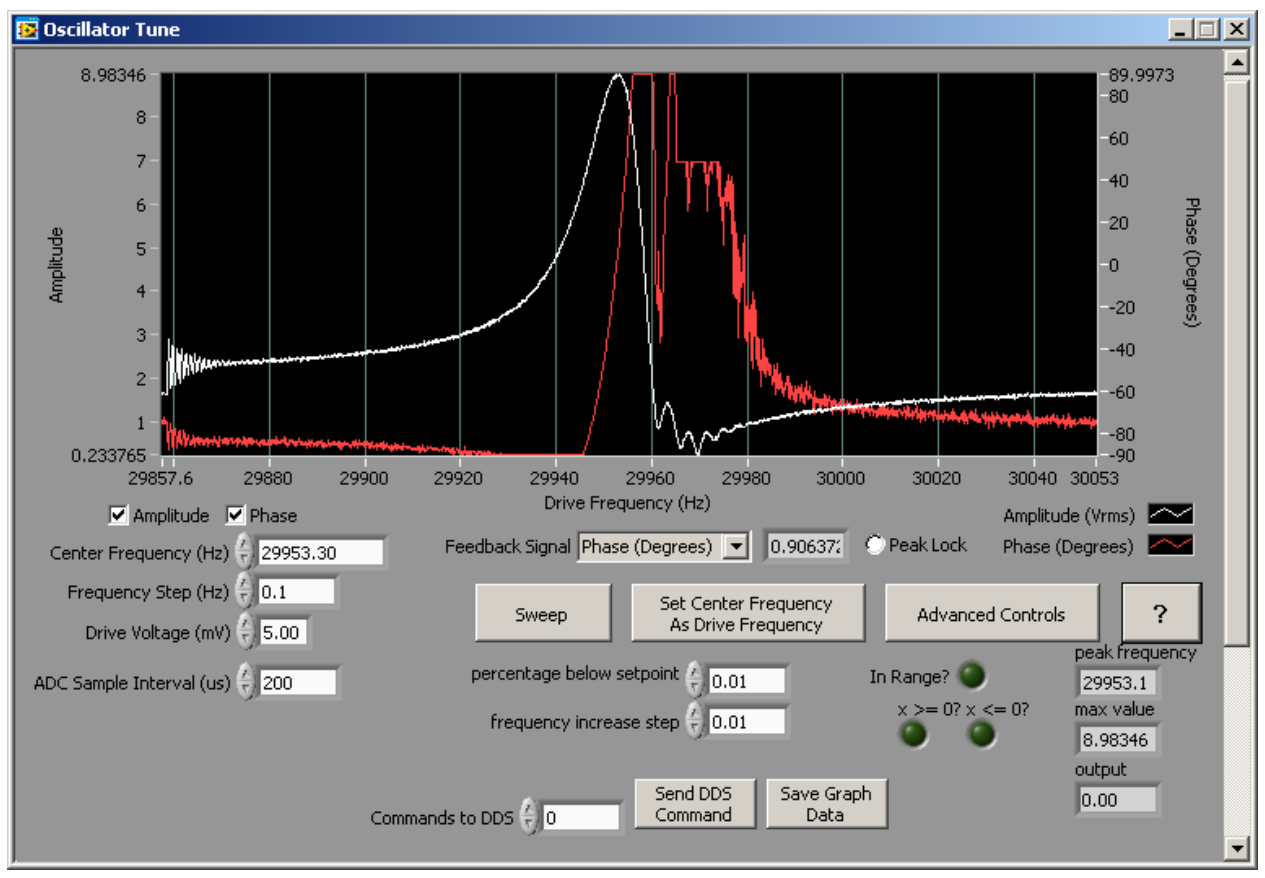

Figure 9.11. Electronic phase adjustment for positive slope in the phase response (red curve). This is the sweep is for tip \#B-002 in air. Notice how it is adjusted for saturation at $+/-90$ degrees and that zero phase is close to the center of the range.

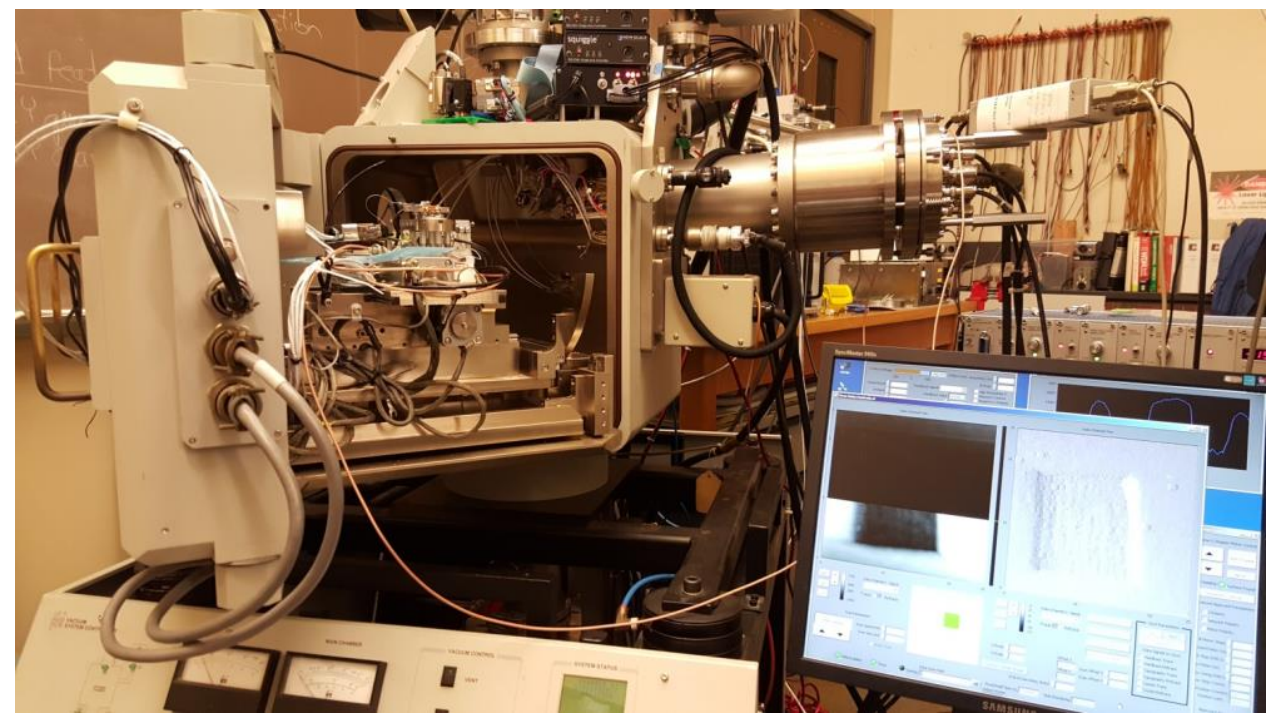

Figure 9.12. AFM operation in air after installation into FIB system. The FIB system is mechanically stable enough that the AFM can be used in air with the FIB chamber door hanging open. The computer monitor on the lower-right shows the AFM scan in progress. 


\subsection{AFM vacuum operation}

Shortly after acquiring what looked like a good scan in air, preparations for vacuum operation began. Some configuration choices were made.

1) The scan head board and the preamp are both mounted inside the vacuum, very close to the AFM. Ultimately, this proved to be a good choice. Both boards draw very little power, however there is a 2-hour warm up time.

2) Electrical feedthroughs used are:

a. The round 26-pin Amphenol connector on the FIB 611 door (see Figure 9.12)

b. A home-made feedthrough plate for the AFM scan bed and Z-piezo, integrating LEMO and BNC vacuum connectors.

c. A 10-pin conflat feedthrough for Squiggle Motor control.

3) Encoders are powered from the AFM Controller via switch

4)

5) A box containing:

a. Z-Squiggle motor control,

b. encoder power,

c. scan head \& preamp board power

d. Breakout board, including default logic circuit

6) From earlier experience with tuning forks in vacuum, only minor tuning changes were required.

\subsection{Vacuum effects on tuning}

Operation in vacuum tended to double the $\mathrm{Q}$ factor for the electronic frequency sweep for high Q tuning forks in section 5.5. Because of the intentional detuning of the AFM tuning fork, the Q does not appear to double but it still increases enough that it needs attention. Figure 9.11 shows tuning in air, having a peak amplitude of 8.98 Volts and Figure 9.13 shows the same in vacuum, but saturated at 9.99 Volts. Both sweeps were acquired using a drive Voltage setting of $5 \mathrm{mV}$. Setting the vacuum drive Voltage to $4 \mathrm{mV}$ reduced the peak amplitude to 8.81 Volts (Figure 9.14). 


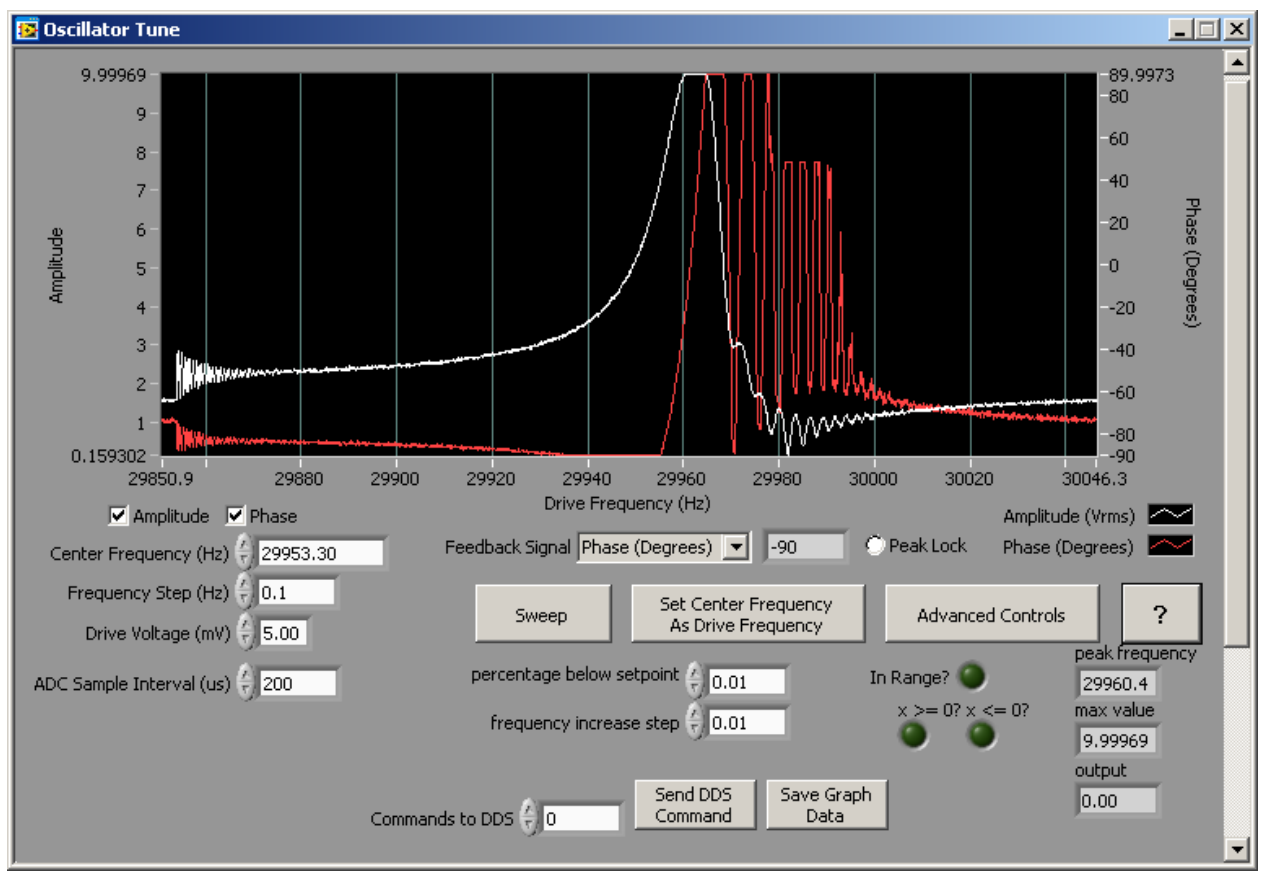

Figure 9.13. Demonstration of vacuum effect on tuning of the cantilever and tip\#B-002. The settings are the same as in Figure 9.11. Note that the amplitude (white curve) is saturated and requires that the Drive Voltage $(\mathrm{mV})$ be reduced.

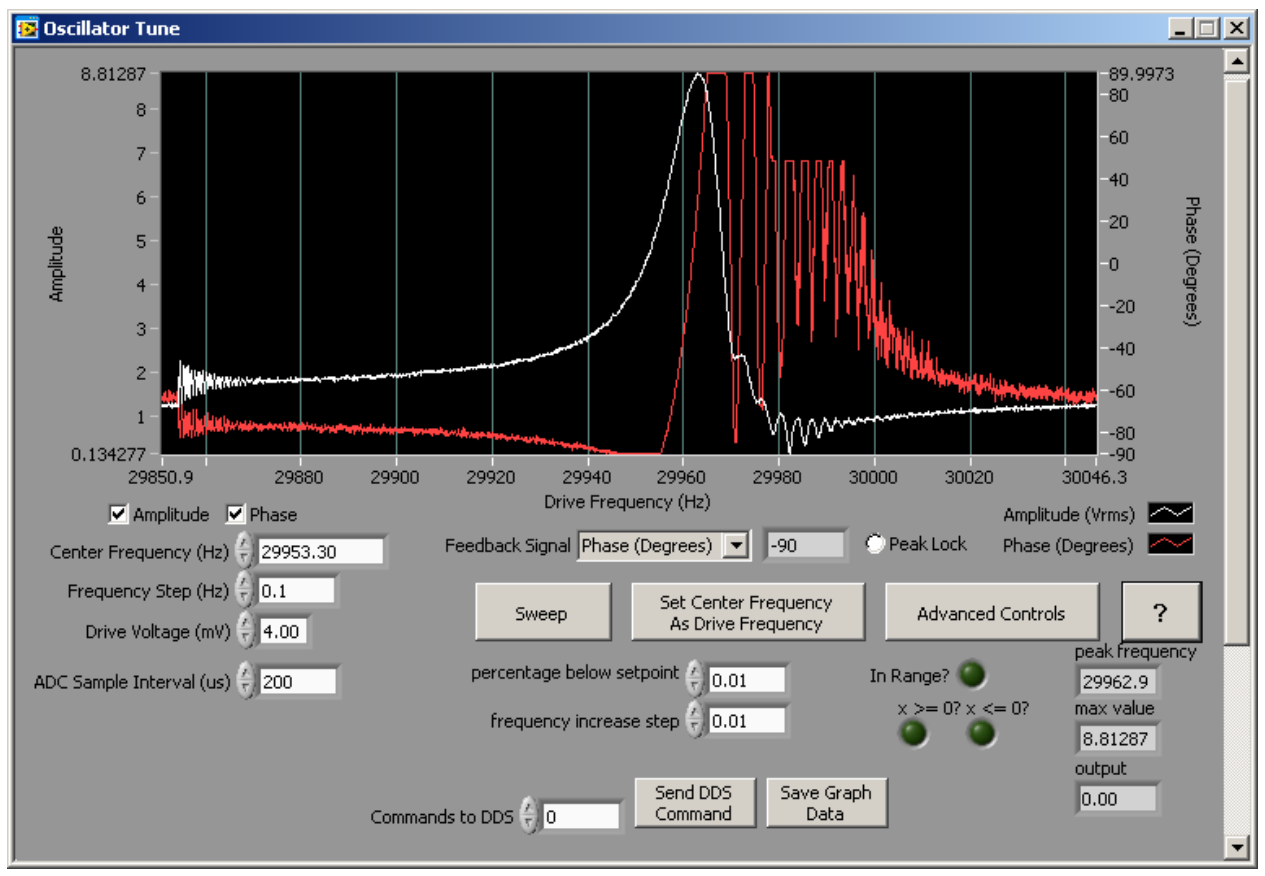

Figure 9.14. Tuning for tip\#B-002 in vacuum. The Drive Voltage $(\mathrm{mV})$ has been reduced from $5 \mathrm{mV}$ to $4 \mathrm{mV}$. Also notice that the peak frequency has shifted up by $9.8 \mathrm{~Hz}$. The peak frequency will continue to move up slowly as the adhesive outgases inside the vacuum. For AFM operation, the Center Frequency $(\mathrm{Hz})$ is adjusted so that the nominal phase is about +5 degrees. 


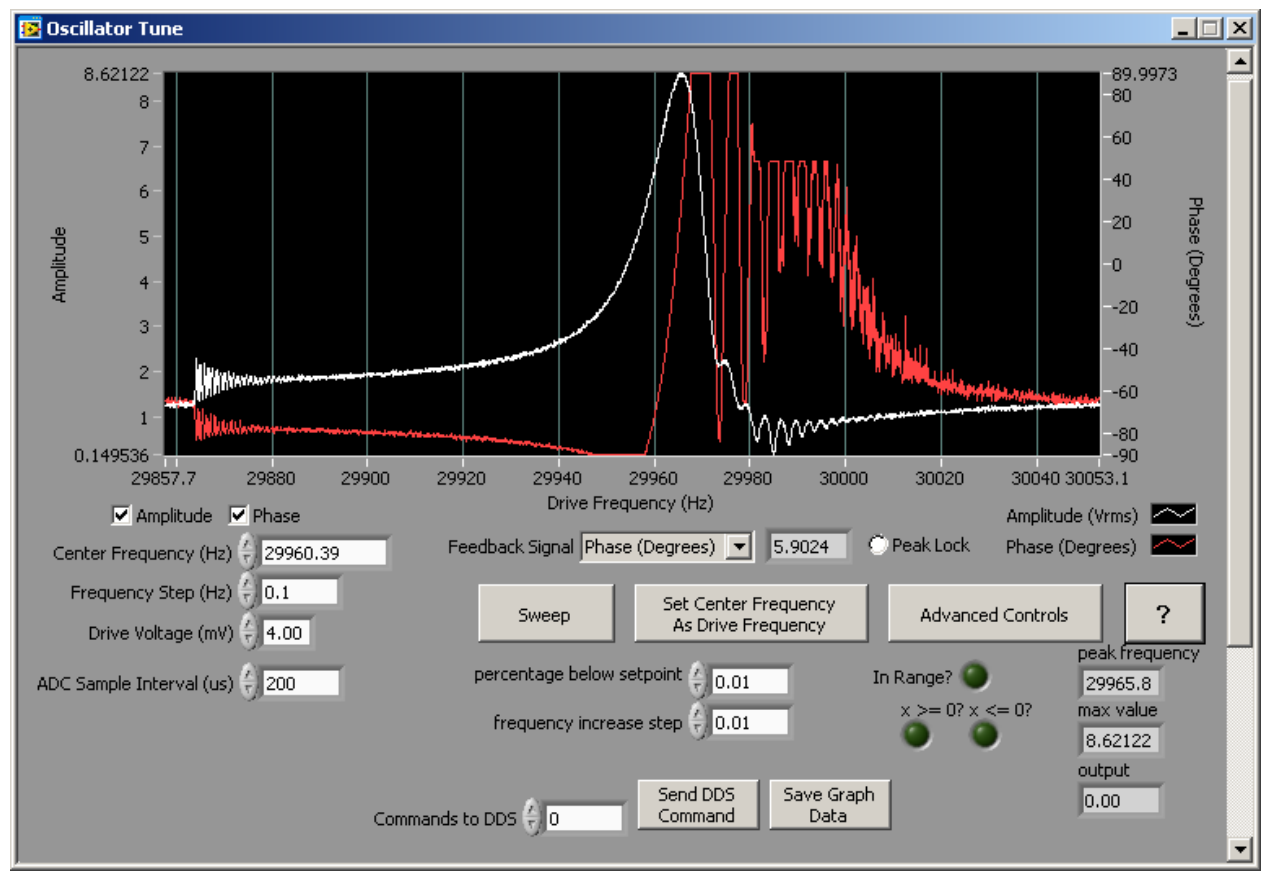

Figure 9.15. Tip\#B-002 tuned and ready for AFM operation. The nominal phase here reads 5.9024 degrees in this static photo, but will be continuously changing, as there is a lot of noise in the phase read back when the tip is not engaged.

\subsection{Tip exchange}

The AFM's coarse-Z axis can be moved under Squiggle motor control over a range exceeding $7 \mathrm{~mm}$. Its position can be known to $100 \mathrm{~nm}$ using its encoder. Finer motion steps less than $10 \mathrm{~nm}$ are possible and are knowable via tip engagement with the sample surface, or by counting the \# motor pulses needed to move one, $100 \mathrm{~nm}$ encoder step. Given this good resolution performance, the tradeoff is that the Squiggle motor itself is very delicate. The design is intended to minimize the chances for damage to the Z Squiggle in these ways: 
1) Constrained lateral motion

2) Hard-stop just above the Squiggle's bottom of motion

3) Long travel of mechanism, $\sim 10 \mathrm{~mm}$, past the range of the Squiggle

4) Simple mechanism for disengaging the Squiggle during tip/sample exchanges

The procedure for tip exchange is as follows:

1) Drive the Z-Squiggle up to $>100 \mu \mathrm{m}$ from sample surface

2) Insert the long $3 \mathrm{~mm} \times 0.5 \mathrm{~mm}$ screw into the $Z$-axis plate

3) Turn the screw until the tip is $>3 \mathrm{~mm}$ from the sample surface

4) Exchange the tip, being careful not to touch it on anything

5) Adjust the new tip's orientation using pliers by grabbing the tuning forks's can base

6) Lower the tip to within $\sim 1 \mathrm{~mm}$ from the sample surface using the long screw

7) Drive the Z-Squiggle up until it engages the plate and visibly disengages the long screw

8) Remove the long screw

Thus exchanging the AFM tuning fork/tip assembly is as simple as inserting a long screw into the Z-axis coarse stage plate and turning it until the tip is $3-5 \mathrm{~mm}$ from the surface. The tuning fork is removed using locking tweezers and the new one inserted. Once inserted, the orientation of the tip can be adjusted by grabbing the tuning fork can base with pliers and either rotate or tilt it. The tip is usually mounted at a slight angle from vertical to keep the tuning fork away from the FIB and for imaging the shank just at the start of the etched taper for coarse tip approach.

\subsection{Sample exchange}

Exchanging the sample is essentially the same as the tip exchange procedure and usually requires moving the coarse XY so that the stub clears the tuning fork/tip assembly. It is usually better to remove the tuning fork/tip assembly with locking tweezers, setting it aside while exchanging the sample stub. The stub is secured using a \#4-40 set screw in the stub holder assembly. 


\subsection{Noise considerations}

ANSOM development included a noise analysis of the electronics. The National Instruments data sheet reports the noise levels as follows: $\mathrm{ADC}$ noise $=+/-7.78 \mathrm{mV}$ and DA nosie $=+/-5.88 \mathrm{mV}$. Ultimately, this will translate to noise in the AFM images. Both ADC's and DAC's on the 7833R are fixed at $+/-10.0$ Volts. This translates to about $305.180 \mathrm{uV}$ per ADC/DAC value. Thus the noise constitutes approximately 25.5 and 19.3 bits of noise for ADC's and DAC's respectively.

Phase feedback is read into the FPGA where +/- 90 degrees of phase translates to +/- 10.0 Volts, giving 0.111 V/degree. This is also about $364 \mathrm{ADC}$ values per degree giving a resolution of 0.00275 degrees. This suggests an input electrical noise floor of 0.053 degrees.

$\mathrm{Z}$ resolution is determined by the sensitivity of the piezo stack. Using the max deflection of $16.7 \mu \mathrm{m}$ for an applied DAC of 65535 gives an approximate resolution of $0.255 \mathrm{~nm}$ per DAC bit. This suggests an output electrical noise floor of $+/-4.9 \mathrm{~nm}$.

In practice, the input phase noise appears much larger than one degree but scans having much less than $4.9 \mathrm{~nm}$ are common. It is clear that the Z-noise is a strong function of the proportional gain. We might the conclude that much of the expected 4.9 nm of noise is low enough in frequency for the servo loop to correct and that some of it has higher frequencies than the servo loop can respond to.

\subsection{False engagement during AFM tip approach}

Once a tip has been installed, it can be 3 or more $\mathrm{mm}$ from the sample surface. The tip can be lowered to within $\sim 1 \mathrm{~mm}$ by eye. If the approach sequence discussed in 8.x is used from this far, it can take an hour or more to reach the 
surface. This would not be so bad if the system could be left over lunch performing the tip approach automatically, except that false engagements occur frequently, requiring the user to restart the approach sequence many times.

False engagement occurs as a result of the large phase noise and from the little abrupt stops and starts of the coarse Z Squiggle motor coupling into the phase response. Also, as was mentioned earlier, the tuning fork is mechanically coupled to the rest of the AFM hardware. Reduction of this coupling by hanging the tuning fork on its own leads dramatically reduces this coupling, however the nominal phase position is still affected by the coarse $\mathrm{Z}$ position and so must be monitored and corrected during approach. This coarse Z-position influence on the nominal phase appears to be related to the cogging of the bearings, causing alternating points of weak and strong mechanical coupling during the downward motion. Anecdotally, this effect can cause the nominal phase to vary by $+/-5$ degrees of phase during motion and can lead to a phase offset greater than 10 degrees over a 1 mm approach.

\subsection{Possibility for reducing errors in an AFM scan using phase data}

Tip-sample interaction typically begins at about $20 \mathrm{~nm}$ and gets stronger as the tip gets closer. As the tip is scanned across the sample and the servo loop attempts to maintain a constant phase defined by the set point. It does this by moving the sample up or down at each pixel until the set point is reached. This constant phase is assumed to correspond to a constant tip-sample distance, and for a short range can be linearly correlated to the tip-sample distance. Invariably, the servo cannot maintain a perfectly constant phase, leading to errors in the topographic image. Abrupt topographical changes 
in the surface are the largest contributor to these errors, as the physical hardware and servo loop are bandwidth limited. These errors are recorded in the feedback image, corresponding to the measured phase difference from the set point at each pixel. Also, there are sources of noise, common-mode electrical noise, and noise related to the phase measurement, are seen in the feedback error images. As long as the servo is still engaged when these errors are recorded, the phase error is correlated to the actual distance from the set point at each pixel.

The servo will typically disengage briefly when the tip makes contact with the sample surface, leading to streaks in the topographic data and where the phase data are not linearly correlated to the tip-sample distance. These cases will not be correctable using the strategy presented.

If a few more tidbits of information are recorded for each AFM scan, the errors in the height data may be reduced considerably. The idea is that the phase error is proportional to the tip distance error and the phase response is approximately linear near the set-point when engaged. Thus all that is needed is to multiply the phase error data by a factor and then add it to the height data. A fast way is to record a few things before the scan begins. They are as follows: 
1) Nominal $Z$ piezo Voltage: The $Z$-piezo is a unipolar device and must be operated in the middle of its range by applying an offset, typically $-150 \mathrm{~V}$.

2) Phase error, while disengaged and Proportional Gain is nonzero: The tuning fork frequency is typically set to about +5 degrees when the Proportional Gain is zero and the tip is not in contact. Once the Proportional Gain is set to a nonzero value, the measured phase error determines the slope of the $\mathrm{Z}$ response to phase error.

3) Phase error when engaged: This should correspond to the setpoint.

4) Phase setpoint, which can be retrieved from the saved data files as a DAC value.

5) Z piezo Voltage when engaged: The sample is typically set so that it requires between -5 and -20 Volts to engage. In this way, the sample is between 284 and $1137 \mathrm{~nm}$ from the tip when disengaged.

These pieces of information can be used to determine a $1^{\text {st }}$-order $Z$ to phase ratio which can then be multiplied by the phase image and added to the height data to reduce the height data error. Higher order corrections are possible but may reach the point of diminishing returns. Figure 9.16 shows the result of using the scheme described above to reduce the noise using calculated $\mathrm{nm} /$ degree for a given tuning fork-tip configuration. 


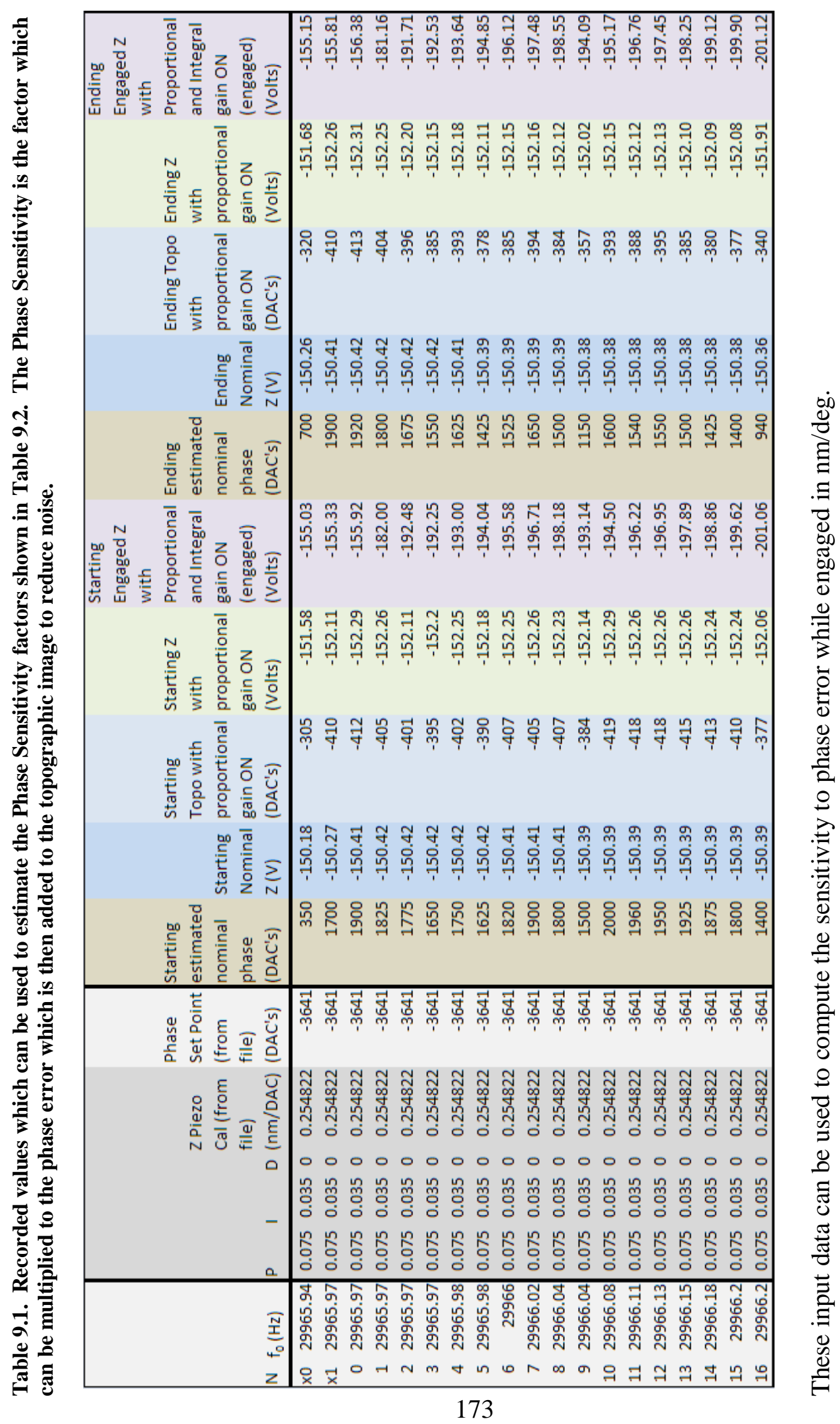




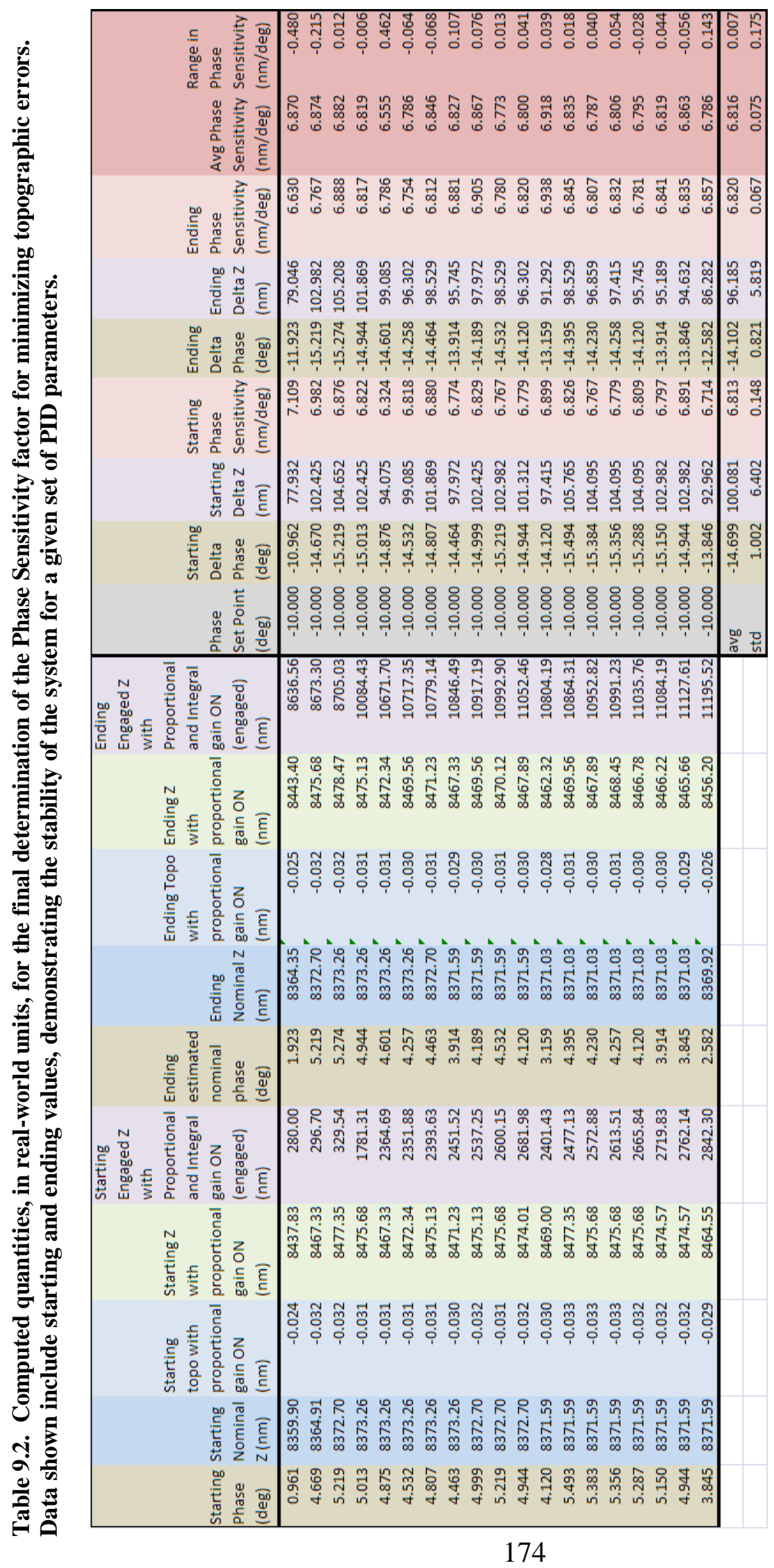



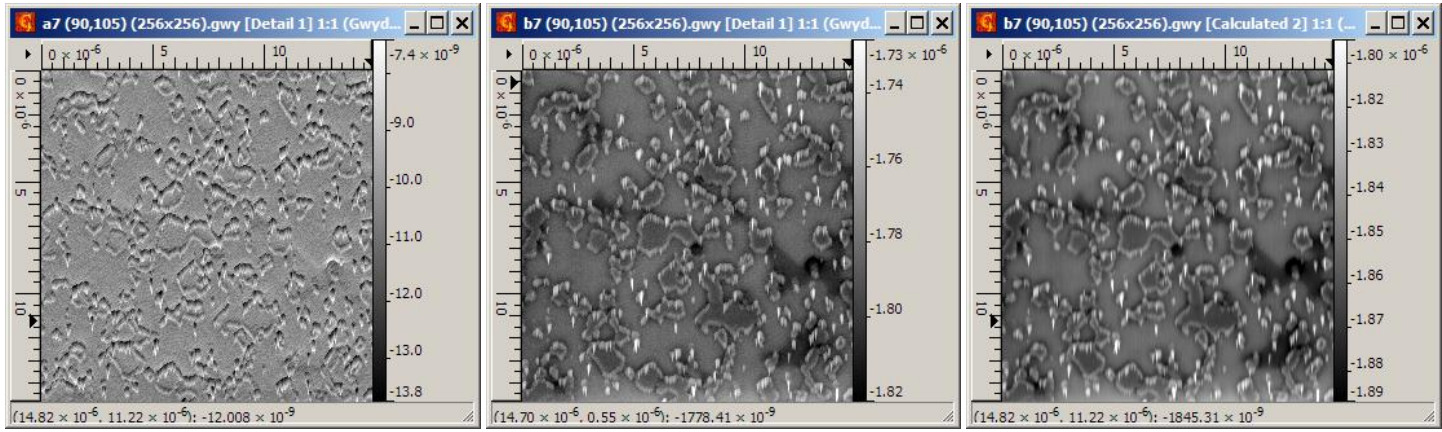

Figure 9.16. Illustration of multiplying the (left) phase image by the factor $6.773 \mathrm{~nm} / \mathrm{deg}$, then adding it to the (middle) topography image to obtain the (right) calculated image. The starting $R_{q}$ for the topography image is $12.02 \mathrm{~nm}$. The calculated image's $R_{q}$ is $10.71 \mathrm{~nm}$. The factor was read off the table for image \#7, thus illustrating that these factors can be pre-computed if some extra data are recorded for each AFM scan.

Typically, the five pieces of information above are not collected. Even if they are, some of these readings must be carefully observed at the correct time and errors are possible. Thus an alternative method is presented using the phase error and topography images. If the order of magnitude for the \# nm per degree of phase can be estimated, then the actual scale factor can be found quickly using a program like Gwyddian. We will refer to this number as "scale" in the following discussion.

The procedure is as follows:

1) Find a representative Region of Interest (ROI) from an AFM scan and clip it out.

2) Level it.

3) Clip out the exact same ROI from the corresponding phase error plot.

4) Set the scale to 0.

5) Compute the rms roughness in the topographic ROI, $\mathrm{R}_{\mathrm{q}}$, plot against the scale

6) Increment the scale by some multiple of the original estimated.

7) Multiply the phase image ROI by scale.

8) Add this to the original topography ROI.

9) Go to step \# 5 until the $R_{q}$ passes through a minimum.

10) Fit a parabola and compute its vertex. The $x$ coordinate of this vertex is the multiplier which will give the smallest $R_{q}$ in the topographic image. 


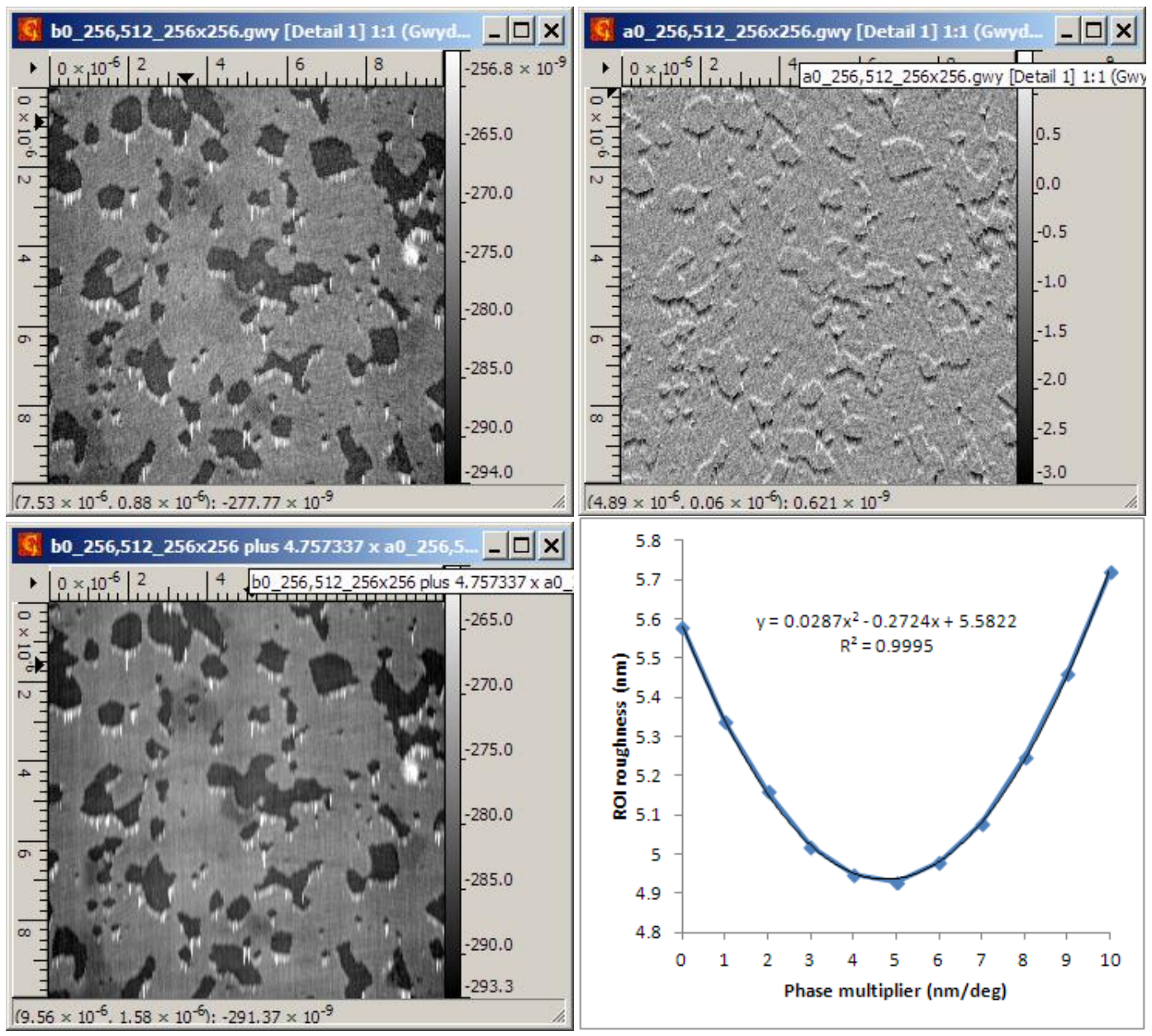

Figure 9.17. Illustration of reducing noise in an AFM topgraphy trace image by adding a multiple of the phase feedback data. (top-left) Original topography ROI image. (top-right) Phase ROI image. (bottom-left) Image having the smallest $\mathrm{Rq}$ which is the result of adding 4.76 times the phase ROI to the topography ROI. (bottom-right) Plot of rms roughness vs phase ROI image multiplier. A parabola has been fit to determine its vertex which corresponds to the lowest Rq and also the \# $\mathbf{n m}$ per degree of phase, 4.76. The noise is greatly reduced, although spikes from the sharp edges remain.

Since the AFM topography noise is a strong function of proportional gain, this phase multiplication factor is also related to proportional gain. Thus for a given tuning fork/tip assembly, these factors can be effectively computed once and used on an entire stack of AFM topographic images. For example, Figure 9.17 demonstrates that the phase multiplication factor for tip\#B-004 is $4.76 \mathrm{~nm} / \mathrm{deg}$ when the proportional gain is set to 
0.05. On another scan using the same tip, having a proportional gain of 0.1 , the phase multiplier is determined to be 9.36 so then has a ratio of 1.966 , almost exactly the same as the ratio of proportional gains between the two AFM images.

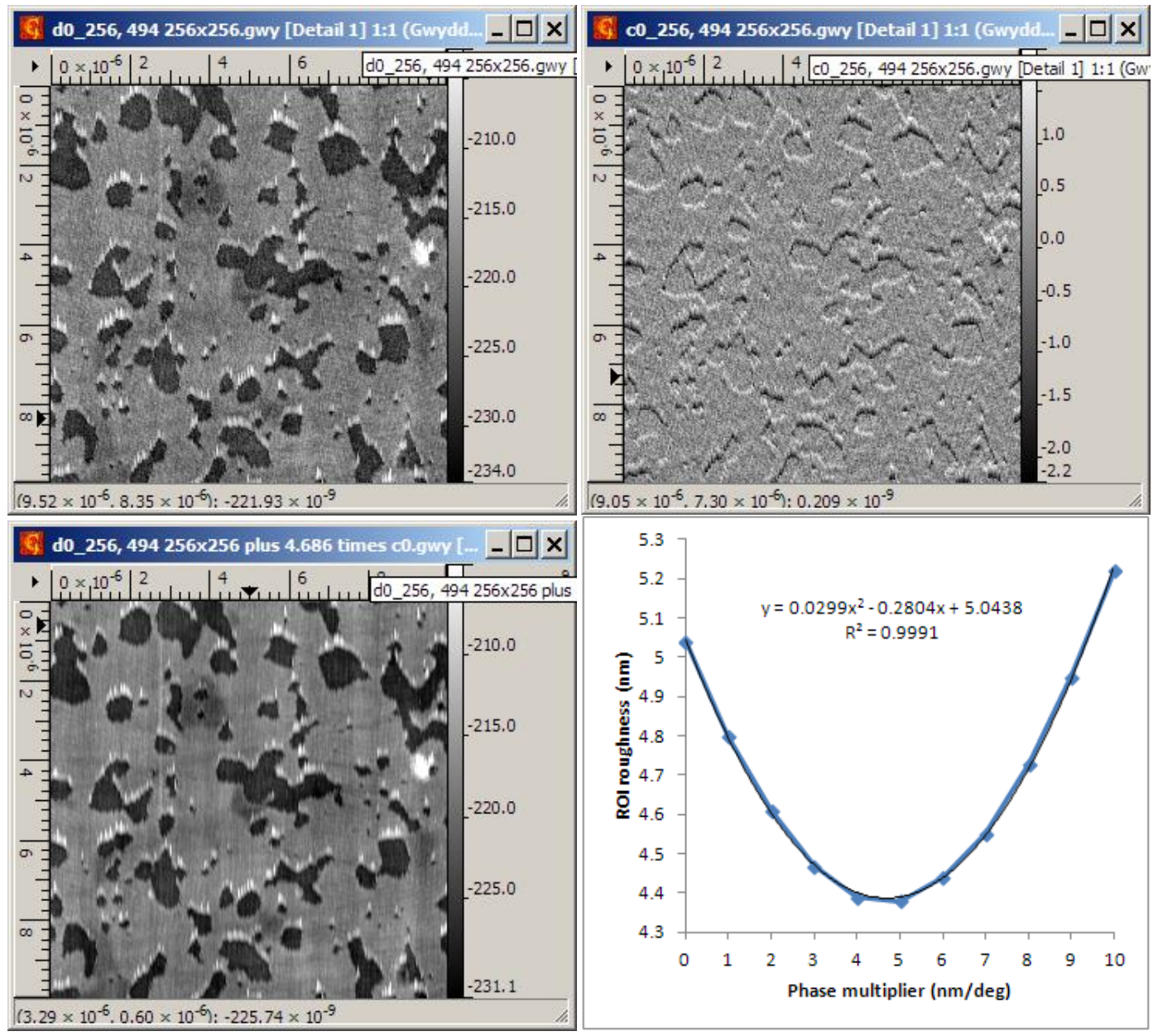

Figure 9.18. Same calculation as in Figure 9.17 for the retrace image using a scale factor of 4.686 $\mathrm{nm} / \mathrm{deg}$. The noise for the retrace image is smaller than for the trace image. This may be due in part to the fact that the trace image is effectively moving up due to a positive slope and the retrace image is effectively moving down due to the negative slope in that direction. In a later image comparison, the trace image has some curvature which tends to increase Rq.

The net result is that more than $0.5 \mathrm{~nm} \mathrm{R}_{\mathrm{q}}$ can be removed using this technique. However, this technique has not been employed in all of the data presented in this work. 
Sometimes it is of more important to remove the artifacts which cannot be removed using knowledge of the acquired data. The most problematic artifacts are the spikes at the sharp steps evident in many of the AFM scans on AlTiC.

\subsection{Removal of other AFM image artifacts}

These spikes occur when the tip effectively disengages from the sample surface as the servo over-reacts to the very sharp edges. Because of this effective tip-sample-servo disengagement there is no information in either the topographic or phase data which can be used to remove these spikes. Thus the application of a heuristic technique to the trace and retrace images must be used instead. The results of such a method are shown in Figure 9.19.
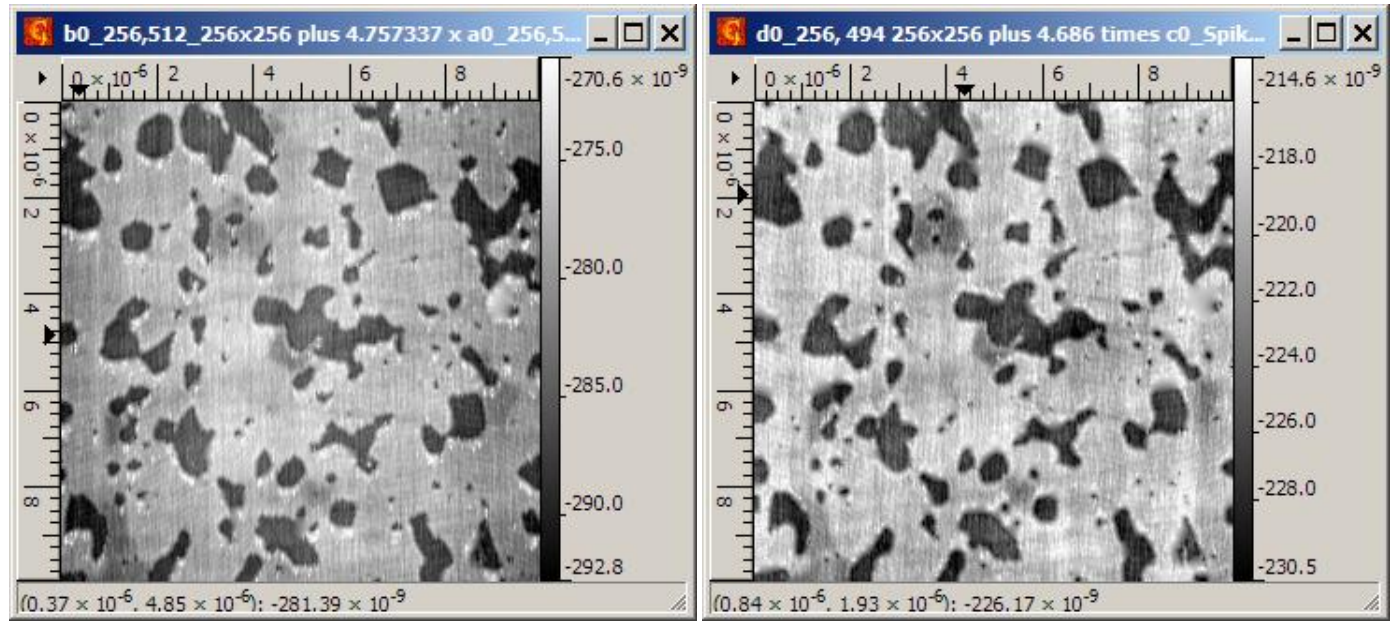

Figure 9.19. The results of identifying the spikes and sharp edges, then interpolating under them using the solution to Laplacian to remove the spikes and soften the edges. (left) Trace image whose $\mathrm{Rq}$ has been reduced from $4.93 \mathrm{~nm}$ to $4.36 \mathrm{~nm}$. (right) Retrace image whose $\mathrm{Rq}$ has been reduced from $4.38 \mathrm{~nm}$ to $3.68 \mathrm{~nm}$. The dark regions are recessed by about $8.5 \mathrm{~nm}$ for both images.

Given sufficient similarity between trace and retrace images, they can be combined so that the artifacts caused by abrupt positive and negative steps can be 
eliminated. Thus the combined image becomes the best of each contributor. The result of performing outImage $=\min (b 0, d 0)$ is shown in Figure 9.20 on the right. To be successful at this, the images must be aligned with each other and leveled using the same fitting plane. Alignment of the trace and retrace can be automatically determined using the Gwyddion multi-data "Mutual Crop" function. This function will crop two images equally, only where their features overlap. Levelling by three-point plane-fit works suitably using the "Set plane zero" option.
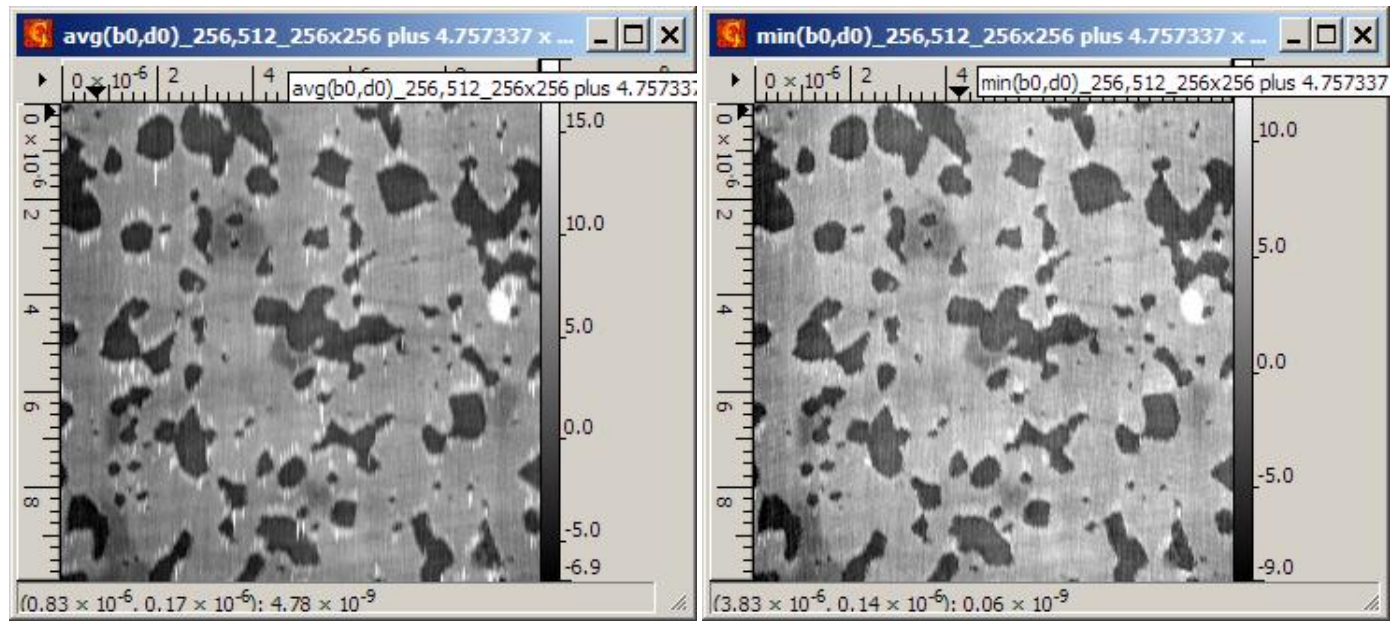

Figure 9.20. The result of (left) simply averaging the trace and retrace images, giving an Rq of 4.38 $\mathrm{nm}$, compared with (right) keeping the minimum pixel value between the two. Its Rq is $4.35 \mathrm{~nm}$. The minimum image looks surprisingly good to the eye, as nearly all of the spikes have been removed.

\subsection{Noise floor assessment with XY-scanning turned off}

It is useful to measure the noise floor of the Z-servo without scanning. In this scenario, all of the AFM sub modules are powered ON except the scan bed. The tip is engaged to the surface using the normal procedure and the resulting images are analyzed. The procedure is as follows: 
1) Find a spot to engage the tip using the coarse $X Y$ positioning and make sure that the XY scan bed power is turned off.

2) Engage the tip using the proportional \& integral gain and acquire a $1024 \times 1024$ pixel AFM image. Save the trace, retrace phase and topography data to disk. There will be four images total. Naming convention goes like this: a for trace phase image, $b$ for trace topography, $c$ for retrace phase image and $d$ for retrace topo image.

3) For each dataset, clip out the middle $512 \times 512$ pixel image.

4) For the trace and retrace images, align the rows using "Matching" to remove the low-frequency trend. Optionally level the topography trace. It is usually not required for the phase image. This step can be optional if the electrical drift was low during the scan.

5) Perform the procedure in section 9.10 above to determine the lowest noise level possible. This is the noise floor in $\mathrm{Z}$.

Figure 9.21 shows the images and the resulting determination of the noise floor for

Tip\#B-004 trace image. It's starting rms noise is $3.9 \mathrm{~nm}$. Once combined with 9.36

times the phase error image, the resulting rms noise is $1.28 \mathrm{~nm}$. 


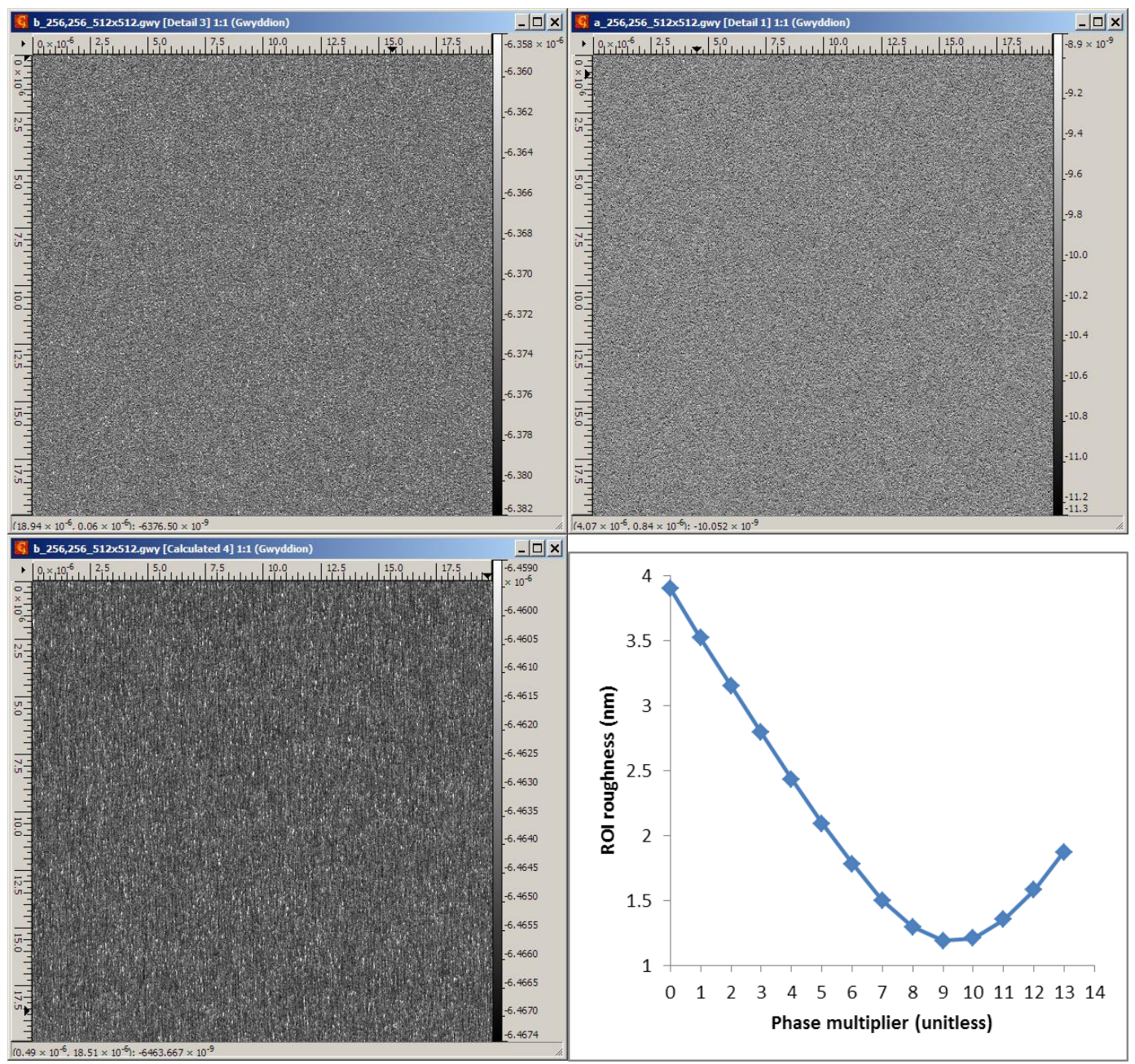

Figure 9.21. Determination of the system noise floor using a scan acquired while the tip is engaged but without XY scanning. The initial $R_{q}$ is measured as $3.9 \mathrm{~nm}$. It can be reduced to $1.18 \mathrm{~nm}$ using a multiplier of 9.36 to the phase data and adding to the height data. In this case, the proportional gain was 0.1 and the integral gain was 0.075 . The derivative gain was set to zero. (Tip\#B-004)

Table 9.3. Absolute $Z$ noise floor determination with $X Y$ scanning turned off. The minimum Rq values here are computed by adding a multiple of the phase error image.

\begin{tabular}{|c|c|c|c|c|c|c|}
\hline $\begin{array}{l}\text { Image } \\
\text { filenames }\end{array}$ & $\begin{array}{l}\text { Gain } \\
(P, I)\end{array}$ & $\begin{array}{c}\text { Trace } \\
\text { Measured } \\
\mathbf{R}_{\mathbf{q}}(\mathbf{n m})\end{array}$ & $\begin{array}{c}\text { Retrace } \\
\text { Measured } \\
\mathbf{R}_{\mathbf{q}}(\mathbf{n m})\end{array}$ & $\begin{array}{c}\text { Trace } \\
\min R_{q} \\
(n m)\end{array}$ & $\begin{array}{c}\text { Retrace } \\
\min R_{q} \\
(n m)\end{array}$ & Engaged? \\
\hline$a, b, c, d$ & $(0.100,0.075)$ & 3.9 & 3.91 & 1.180 & 1.185 & Yes \\
\hline a0, b0, c0, d0 & $(0.100,0.000)$ & 4.69 & 4.7 & 0.513 & 0.514 & No \\
\hline a1, b1, c1, d1 & $(0.050,0.005)$ & 1.94 & 1.95 & 0.384 & 0.384 & Yes \\
\hline$a 2, b 2, c 2, d 2$ & $(0.050,0.005)$ & 1.73 & 1.73 & 0.373 & 0.370 & Yes \\
\hline
\end{tabular}




\subsection{Noise assessment of AFM images on AITiC}

It was stated earlier that the AlTiC surface is polished to a surface roughness of about $1 \mathrm{~nm} \mathrm{R} \mathrm{q}_{\mathrm{q}}$. This would constitute a good validation of the ultimate noise floor being on the order of $0.384 \mathrm{~nm}$ using proportional and integral gains of 0.05 and 0.005 , respectively. Supposing our sample was likewise polished to a $1 \mathrm{~nm} \mathrm{R}_{\mathrm{q}}$ surface finish, our topography image would give similar results. This is only possible using the scheme of adding a multiple of the phase error presented above. The result is shown in Figure 9.22 .

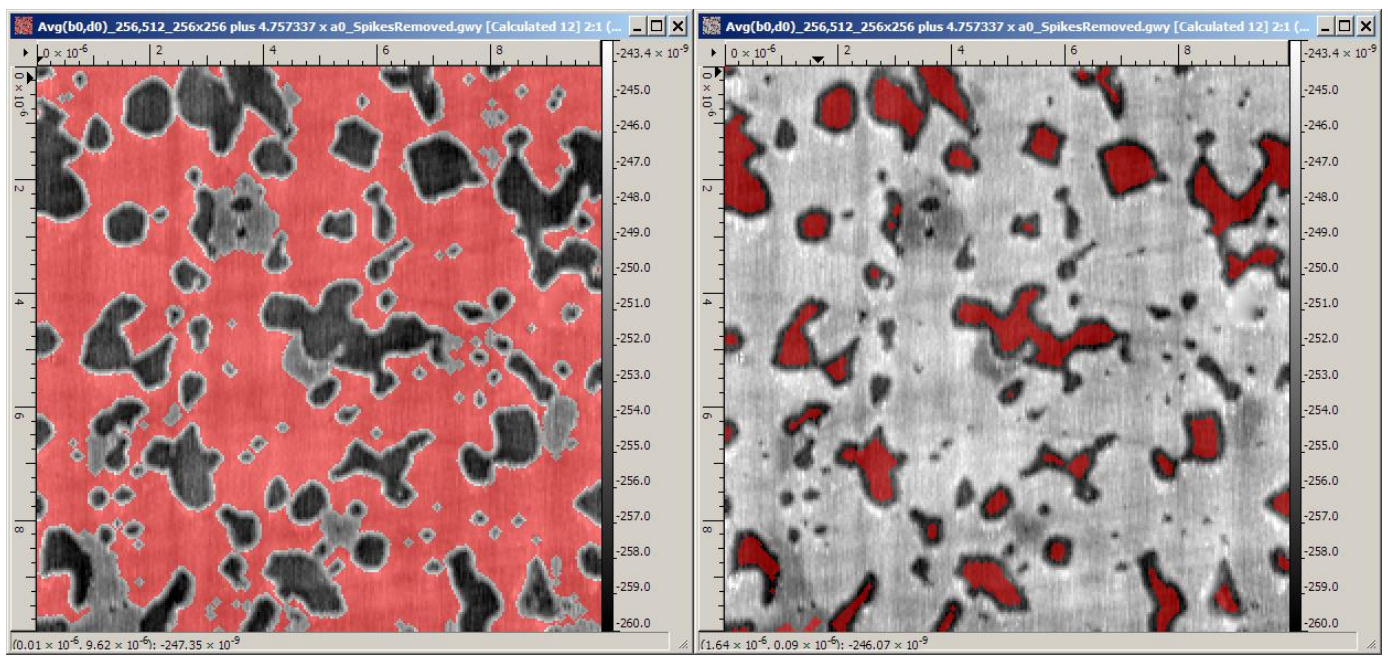

Figure 9.22. The result of masking the (left) $\mathrm{Al}_{2} \mathrm{O}_{3}$ surface and (right) $\mathrm{TiC}$ surface to compute their roughness. The $\mathrm{Al}_{2} \mathrm{O}_{3}$ and $\mathrm{TiC}$ roughness values are computed as $1.23 \mathrm{~nm}$ and $1.40 \mathrm{~nm} \mathrm{R}_{\mathrm{q}}$, respectively. On the $\mathrm{Al}_{2} \mathrm{O}_{3}$ image, the roughness of a $64 \times 64$ pixel rectangle having its origin at (105, 50) pixels has a roughness of $0.98 \mathrm{~nm} R_{\mathrm{q}}$. Individual TiC grains average to $0.98 \mathrm{~nm} R_{\mathrm{q}}$.

\subsection{Noise assessment summary}

Section 9.8 predicted a noise floor of $4.9 \mathrm{~nm} \mathrm{R}_{\mathrm{q}}$ for AFM scans, due only to controller Voltage noise and its resulting effect on Z. Many of the figures above illustrate 
that AFM scans having less than $4.9 \mathrm{~nm} \mathrm{R}_{\mathrm{q}}$ are possible on samples having topography whose islands are about $8.5 \mathrm{~nm}$ in depth. An extra $0.5 \mathrm{~nm}$ can be removed using the procedures stated above.

Table 9.3 illustrates the absolute noise floor scales with proportional gain and is actually larger when the tip is not engaged with the sample surface. Typical AFM scans are less than this and can be further reduced using the procedures of adding a multiple of the phase error to the topography.

\subsection{References}

National Instruments Manual, NI ELVIS II Series User. "NI Educational Laboratory Virtual Instrumentation Suite II Series (NI ELVISTM II Series) User Manual.” Accessed February 27, 2017.

http://www.ee.bgu.ac.il/ adcirlab/Experiments/book/08_Labview/ELVIS\%20User\%20M anual.pdf.

Nowak, Derek Brant. The Design of a Novel Tip Enhanced near-Field Scanning Probe Microscope for Ultra-High Resolution Optical Imaging, 2010.

http://adsabs.harvard.edu/abs/2010PhDT........38N.

Shrotriya, Archana. "Writing Win32 Dynamic Link Libraries (DLLs) and Calling Them from LabVIEW.” NI LabVIEW User's Manual, 1999.

http://citeseerx.ist.psu.edu/viewdoc/download?doi=10.1.1.452.8789\&rep=rep1\&type=pdf

West, Paul, and Natilia Starostina. "A Guide to AFM Image Artifacts." Pacific Nanotechnology, Inc. 3350 Scott Blvd \#29 Santa Clara, CA 95054-3105, n.d.

Whitehouse, D. J. "Nanometrology." Contemporary Physics 49, no. 5 (September 2008): 351-74. doi:10.1080/00107510802611251.

http://gwyddion.net/documentation/user-guide-en/multidata.html\#data-arithmetic 


\section{AFM-FIB operation}

AFM tips are mounted with a slight tilt so that their shanks can be imaged without the FIB coming too close to the quartz tuning fork. The tuning fork can become charged by the ion beam and will cause distortions which last for hours. Sometimes the tuning fork is imaged for one reason or another on purpose. When possible it is imaged near the mounting point of the tip. In general, the tip can be imaged and it still charges up, but it will inevitably lightly touch the sample surface at a few points during scans, losing its charge. The charged tip generally causes astigmation and a slight shift in the ion beam and tends to slowly go away.

Typically the FIB beam current is shut down during AFM operation, mainly because of the very long time to obtain scans. This is accomplished by dropping the FIB extractor to $\sim 6 \mathrm{kV}$. FIB electronics warm-up time is about 60 minutes, so all of the other FIB power supplies and controllers are left in their normal operating states during AFM operation.

\subsection{Coarse tip approach using FIB}

There is not a side-view camera to assist in coarse tip approach as was in the airbased configuration. Although it is very desirable to have one inside the vacuum chamber, it is really quite unnecessary. Once fabricated, each W tip is imaged using an SEM. Two important bits of information are obtained from these images:

1) Tip diameter. This establishes the absolute limit to the AFM's lateral resolution.

2) Shank to tip length. The tuning fork is usually installed at a slight angle so that it doesn't interfere with FIB imaging. If the taper to the tip is long enough, the tip 
itself can be imaged using a low current beam. Otherwise, this piece of information can be used to help determine a safe distance to manually move the coarse $\mathrm{Z}$ axis without crashing the tip.

To use the shank to tip length to assist in coarse approach, the nominal \# microns per Volt of FIB lens 2 focus should be known. Using a beam Voltage of $25 \mathrm{kV}$, a fairly safe estimate is $7 \mu \mathrm{m} /$ Volt. An illustrative example can be demonstrated using the coarse approach used for tip \#B-004. This tip is very blunt, but has a very sharp nub of about $18.9 \mathrm{~nm}$ on its very end. Its shank to tip distance can be conservatively estimated to be about $150 \mu \mathrm{m}$ (Figure 10.2). The tuning fork-tip assembly was mounted at a slight angle as shown in Figure 10.1. The shank-to-tip distance was short enough that the tip itself was not visible from the FIB.

Figure 10.1 shows that tip\#B-004 is a few mm from the sample surface. The FIB lens 2 values for the shank and sample surface are recorded. In this case they are, shank: $13586 \mathrm{~V}$, and sample surface: $13190 \mathrm{~V}$, the difference being $396 \mathrm{~V}$. Using $7 \mu \mathrm{m} / \mathrm{V}$ the estimated safe distance to move in $\mathrm{Z}$ is $-2772 \mu \mathrm{m}$. This brings the tip close enough to the sample to perform the automated approach sequence. Ultimately, the tip engaged at $\mathrm{Z}=-$ $3189 \mu \mathrm{m}$, giving about $8 \mu \mathrm{m} / \mathrm{V}$. In future coarse approaches, the newly determined value could be used instead. 


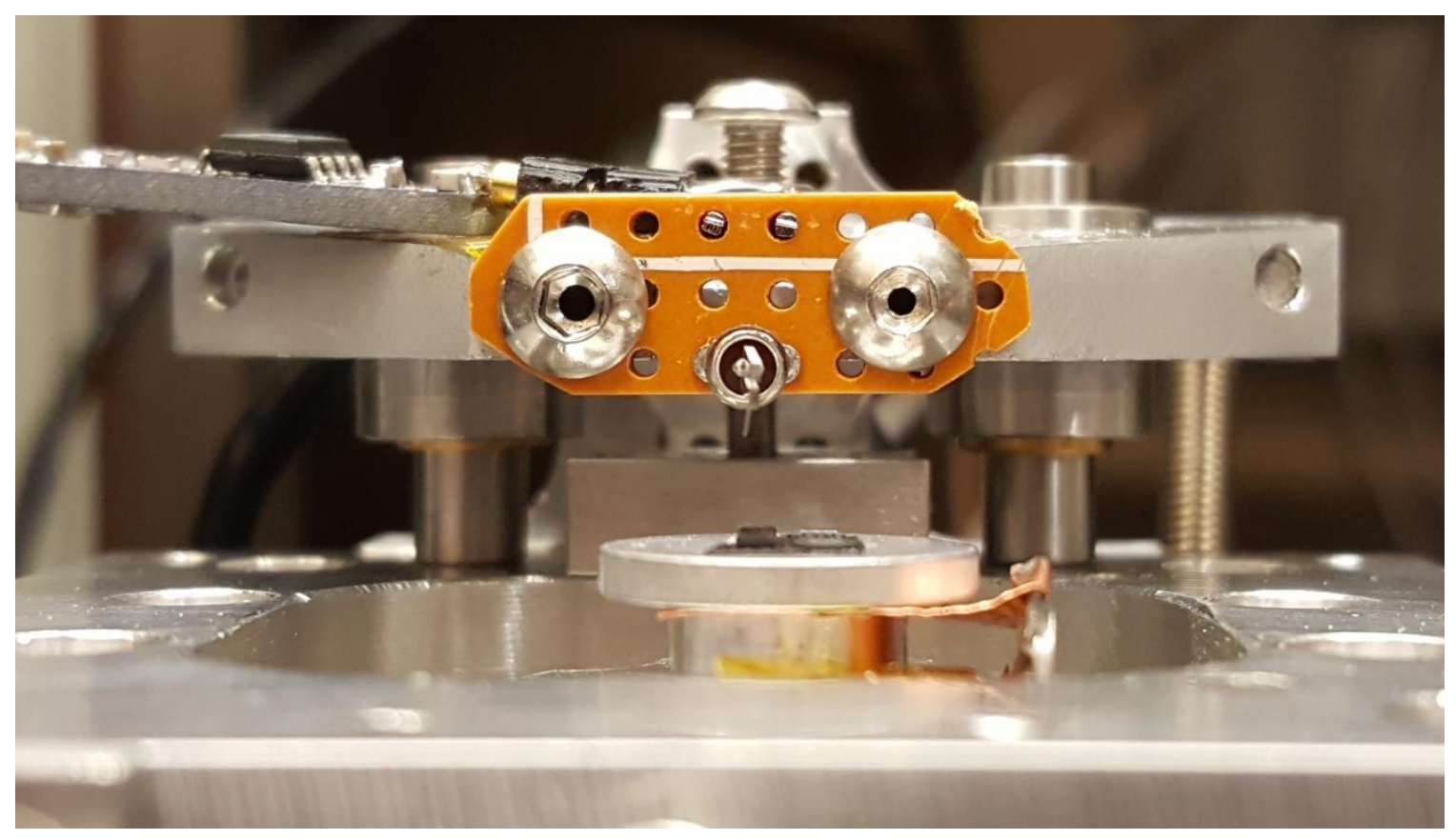

Figure 10.1. Close-up of the mounting of tip\#B-004, showing the slight angle from perpendicular. This tilt establishes a relatively safe distance between the quartz tuning fork and the ion beam path. With this particular tip, only the edge of the shank could be imaged using the FIB. The tip is shown approximately $4 \mathrm{~mm}$ from the sample surface. 


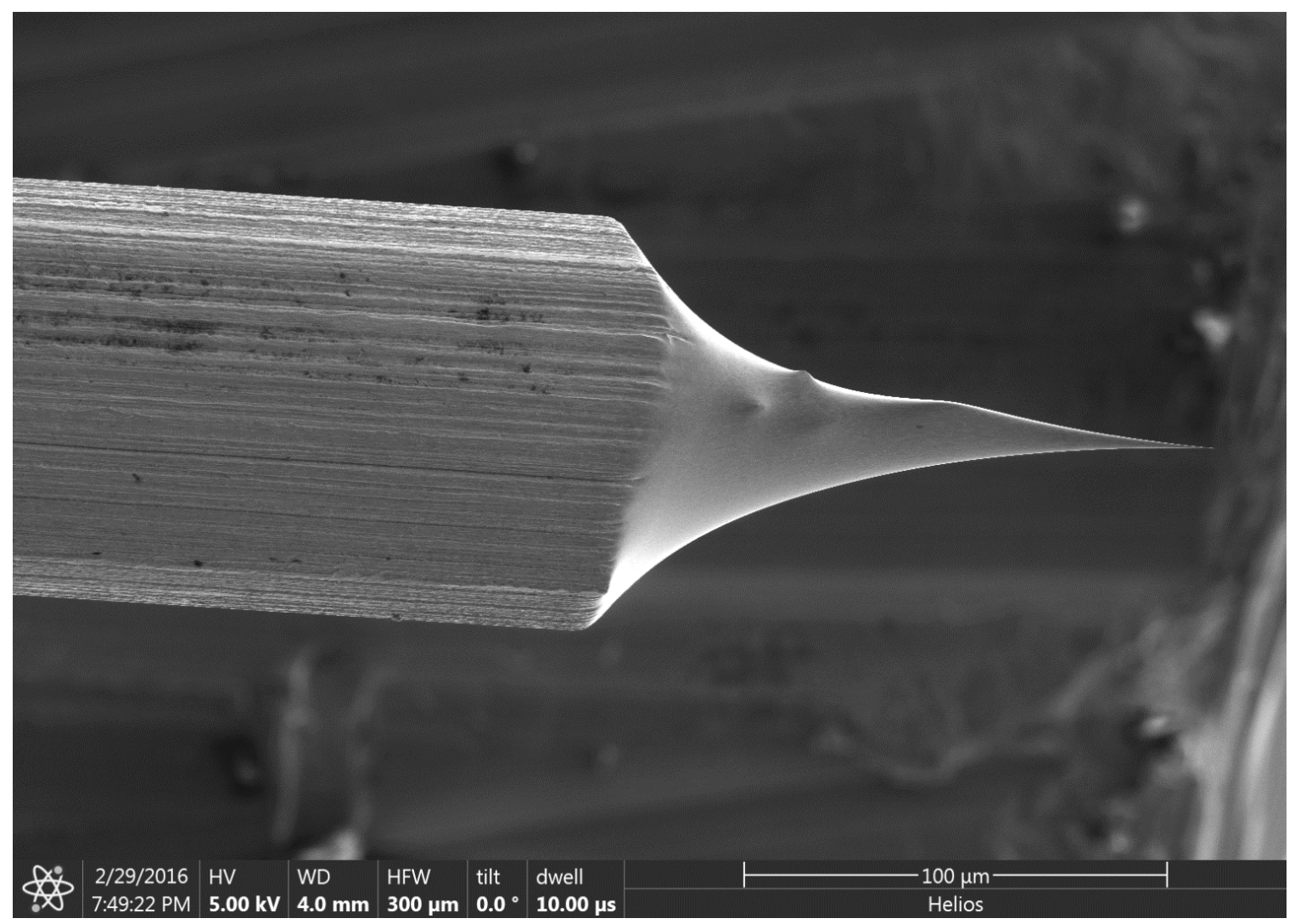

Figure 10.2. Tip\#B-004 showing that the shank to tip distance is about $150 \mu \mathrm{m}$. The FIB is imaged on the edge of the shank and an estimate to the surface is made by recording the FIB lens 2 Voltage on both, the shank and sample surface, applying $\sim 7 \mu \mathrm{m} / \mathrm{V}$. This is a very conservative estimate and the coarse $Z$-axis can be driven without the concern of crashing.
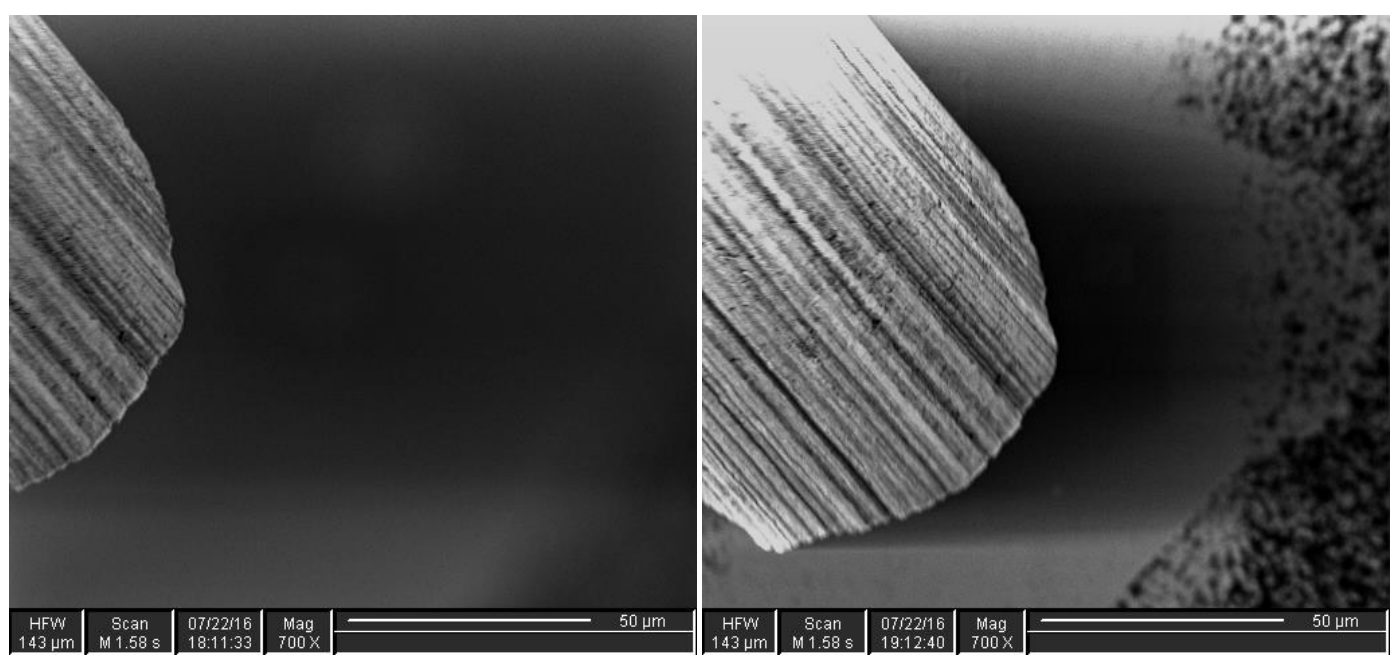

Figure 10.3. FIB images of the AFM tip shank (left) far from the sample surface making it look blurry and (right) engaged. The total distance in $\mathrm{Z}$ is $3190 \mu \mathrm{m}$ and the difference in lens 2 is about 396 V. 


\subsection{AFM tip end of life modes}

A particular AFM tip and tuning fork assembly can last for a long time. Fifty or

more AFM scans using a single tip are possible so long as the user is careful to avoid

some of the more common errors.

Common tip end of life modes are as follows:

1) Tip crashes. These happen usually from user error in setting the tuning parameters, approaching too fast, forgetting to turn on the Z-axis amplifier, or by making large lateral moves when the tip is close to the sample.

2) Tip wear. The tip may make contact with the sample surface many times during scans and can wear away the sharp point if the sample hardness is close to the tip's.

3) Tip contamination. The tip can pick up one of the many particles on a dirty surface and it can be difficult to affect its removal. Sometimes the FIB can be used to vaporize it, but contamination is usually as fatal as crashing.

4) Tip detachment from tuning fork. This is usually the mode of failure for longlasting tips. The Cyano-Acrolate adhesive outgases continually while in the vacuum and eventually the tip just falls off. This is usually preceded by excessive difficulty in tuning.

5) Tuning fork damage. Sometimes the tuning fork is damaged during the decanning process or during tip mounting/installation. Remarkably, this damaged tuning fork can often be tuned and used for AFM scans. A minor contact of the tip with the surface during scanning can reveal this damage. Once this defect has been "activated", the tip is then either retracted or smashed into the surface by the servo loop.

\subsection{Crashed tips}

A well-tuned closed-loop servo will generally protect the tip from abrupt steps in the sample, even during fast scans. And, Tungsten tips can withstand some rather hard impacts with the surface. However, AFM tips will eventually need to be replaced for one reason or another. During the debugging process of getting the AFM functional inside the vacuum, many AFM tips were crashed. Rather than making and mounting new tips, 
sometimes the old one was repaired using the FIB so that debugging could continue. Once crashed, the tip is no longer good for quality images due in part to the larger effective diameter and to its adverse effects on the servo feedback loop. Still, repairing damaged tips using the FIB can save some time when high quality images are not important.

Tips were crashed during the early stages of development for one more reason which may not be so obvious. Recall that the nominal operating Voltage for the Z-piezo is -150 Volts. The servo control then adjusts the full range of $300 \mathrm{~V}$ using a bipolar scheme. If the sample is tilted by more than a few hundred nm over the sample scan area, the final stopping point may end up below the starting point at 150 Volts, at say 140 Volts. If the servo is turned off at this point in an attempt to disengage, it will instead crash the sample into the tip about $500 \mathrm{~nm}$. Sometimes the tip can actually survive such a crash but this is usually not the case. A better scheme of operation would be to have the $\mathrm{Z}$ piezo always disengage towards zero Volts. This is actually a bigger change for the FPGA code than it sounds, so it has not yet been implemented. 


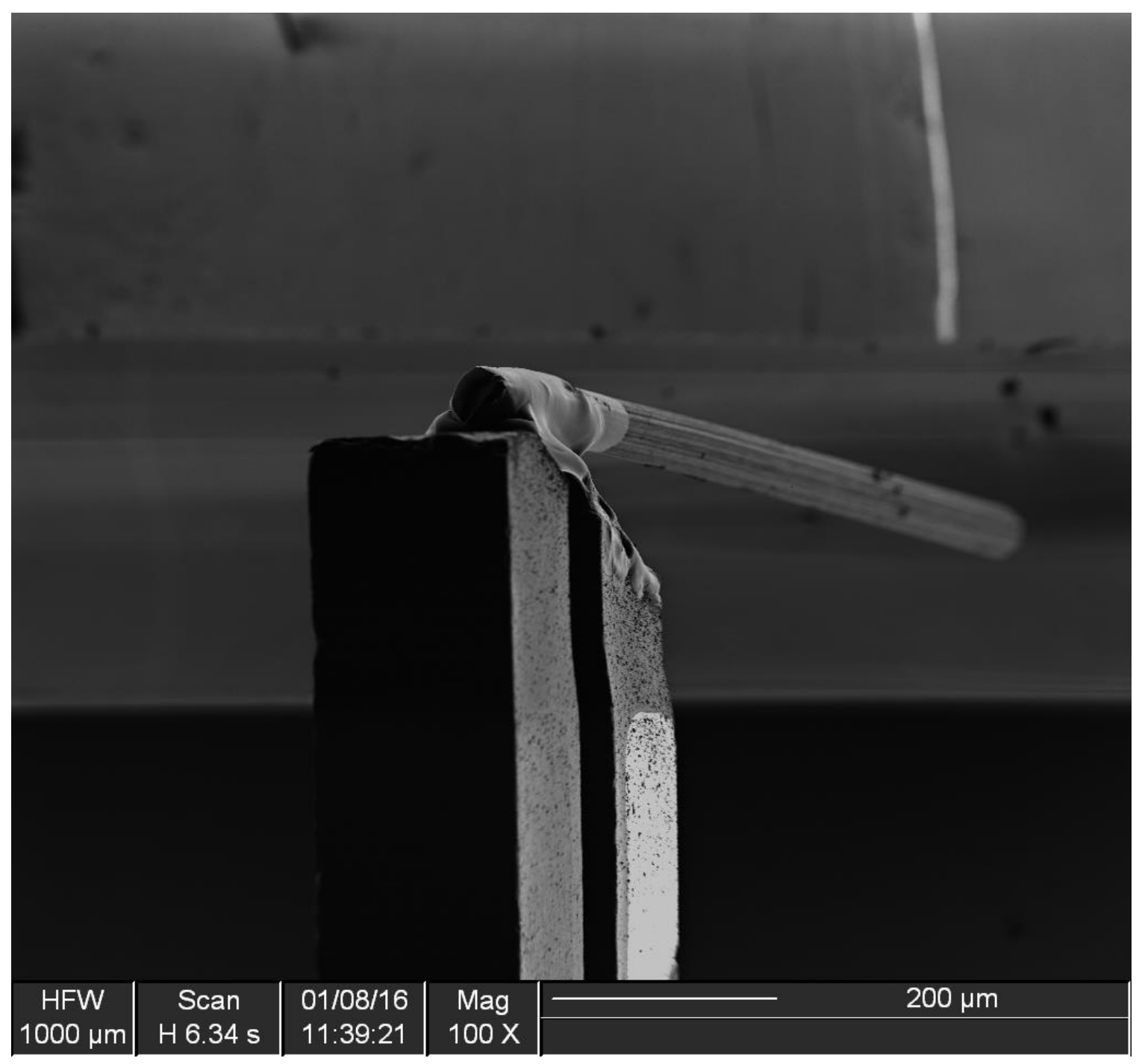

Figure 10.4. FIB image of a tip\#A-010 just after it was slammed into the sample while tuning the ZSquiggle motor. The motor had gotten stuck at $\mathbf{- 4 2 5 . 8} \boldsymbol{\mu m}$. During Squiggle motor tuning it moved another $-\mathbf{1 6 0 0} \mu \mathrm{m}$, driving the tip into the sample and breaking the adhesive. 


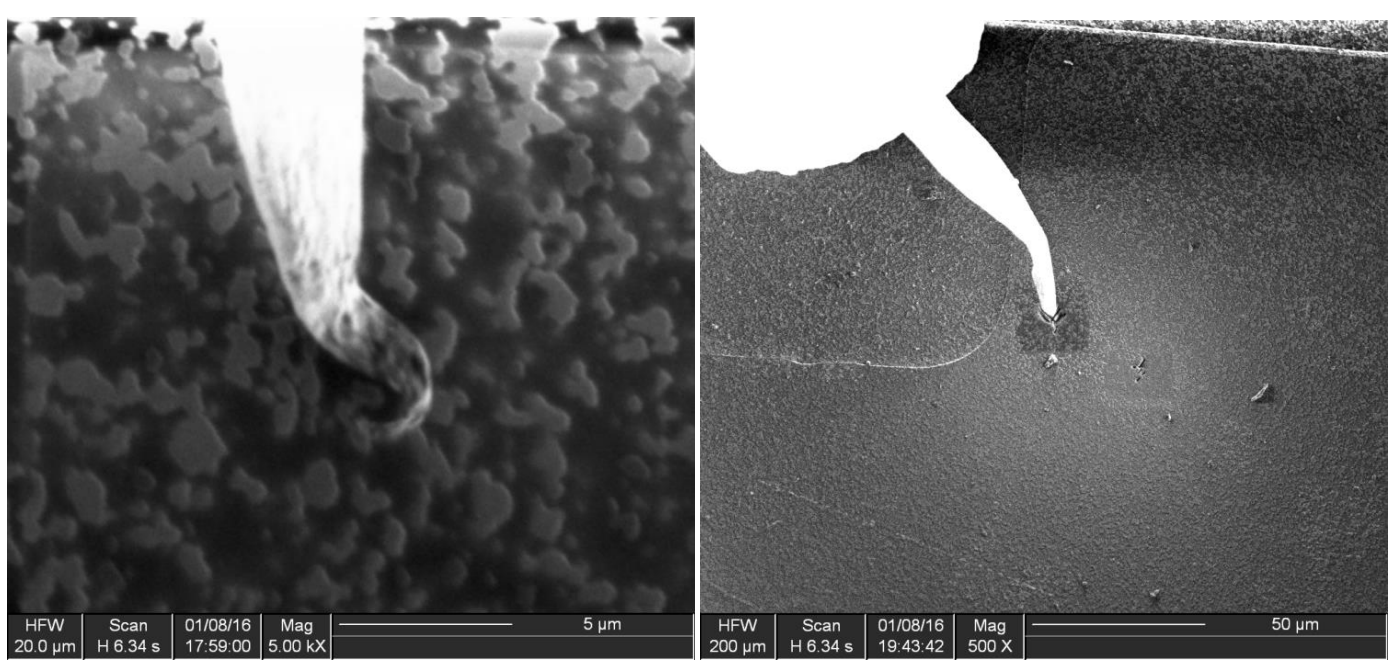

Figure 10.5. (left) This particular tip\#A-010 had touched the sample during installation and so was curled up at the very end. It was initially used for some testing of the XY Squiggle motors while scanning. (right) Later, it was repaired once by cutting off the curled portion in order to continue debugging the Z-Squiggle motor tuning. AFM scans after repair were better than the crashed tip but were still of low quality. This tip had a long enough taper that it was possible to view it using a FIB image. The original tip which was blunt, having a diameter of $179.7 \mathrm{~nm}$.

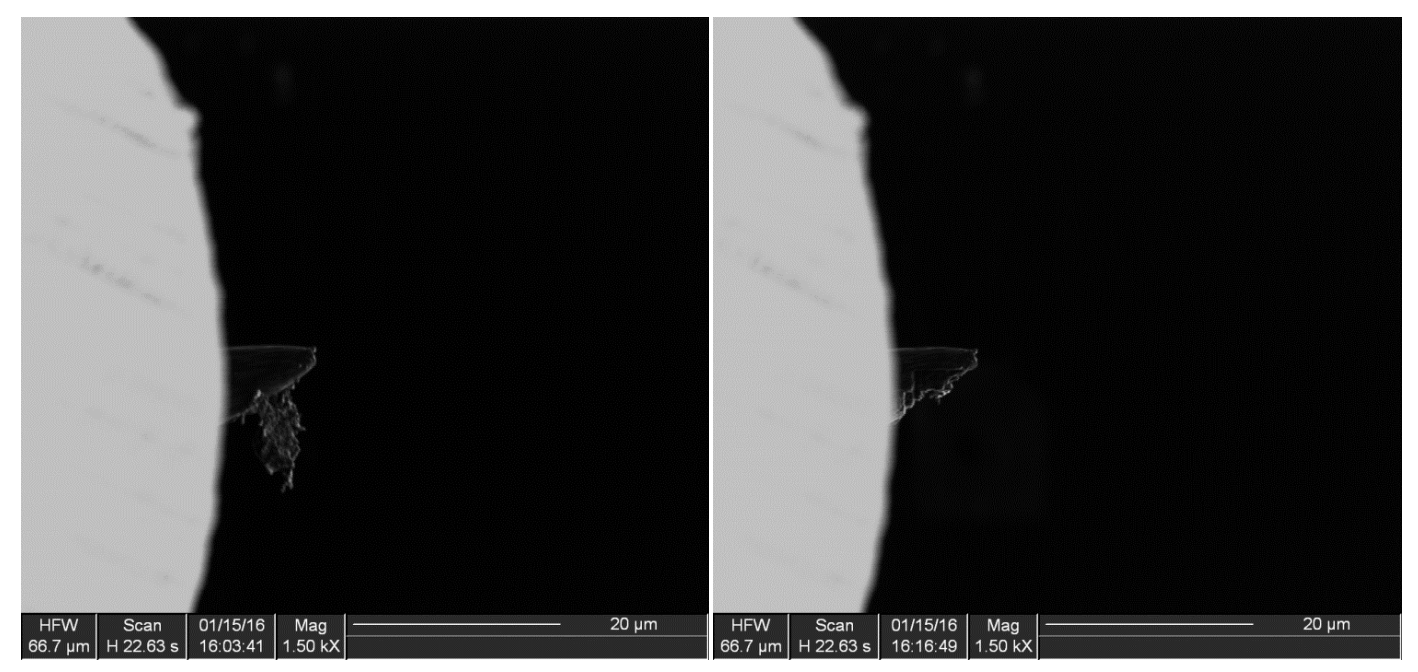

Figure 10.6. (left) AFM tip\#A-008 image of the tip's taper being pressed against a slider body to reveal the tip past its shank. It has picked up some contamination. (right) The same tip after milling with the FIB to remove the contamination. Later, it was revealed that the contamination simply moved to another location on this tip and still caused imaging artifacts. 


\subsection{Some AFM-FIB configuration details}

The AFM scan is transverse to the FIB. This allows the unambiguous observation of FIB scan artifacts using the AFM. For example, there will be times where the FIB deflection has a relatively large noise on it, causing the beam to jump around during imaging/milling. The result is that some of the scan lines receive more than one pass during imaging/milling, which leaves noticeable grooves in the sample. These grooves are unambiguously revealed in the AFM scan as the scan direction is roughly perpendicular to the FIB scan lines.

\subsection{Experiment \#1: Low beam current milling using 250 pA beam}

AFM tip\#A-005, having a diameter of $65.1 \mathrm{~nm}$ was installed and the $250 \mathrm{pA}$ FIB beam current was used to mill a crater in AlTiC. Ten FIB-AFM image pairs were taken. FIB images were acquired using Runscript in order to mill the crater without causing too much re-deposition. Auto focus was performed before each FIB acquisition.

FIB imaging conditions were as follows:

- $\quad$ Ibeam $=250 \mathrm{pA}$

- $\mathrm{HFW}=10 \mu \mathrm{m}$

- XY pixel count: $1024 \times 884$

- Pixel dwell: 1000 ns, 9000 ns

- \# frame acquisitions:

○ 16 at 1 us for the $1^{\text {st }}$ image (000)

○ 3 at 90 us for the next 9 images (001-009)

- \# auto focus frame acquisitions:

○ 14 at $800 \mathrm{~ns}$ for the $1^{\text {st }}$ auto focus (1024x884 pixels) (000)

○ 14 at $800 \mathrm{~ns}$ for the $2^{\text {nd }}$ auto focus (512x442 pixels) (001)

○ 14 at 90 us for the $3^{\text {rd }}$ auto focus (1024x884 pixels) (002) (human error)

○ 14 at $800 \mathrm{~ns}$ for the $4^{\text {th }}$ through $10^{\text {th }}$ auto focus (512x442 pixels) (003-009)

AFM imaging conditions were as follows: 
- XY field of view: $25.008 \mu \mathrm{m}^{2}$

- XY pixel count: $512 \times 512$

- Scan rate (Hz): 0.25

- $(\mathrm{P}, \mathrm{I}, \mathrm{D})=(0.050,0.005,0.000)$

- $\quad$ Scan time $(\mathrm{s})=2048 \mathrm{~s}=34.13$ minutes 

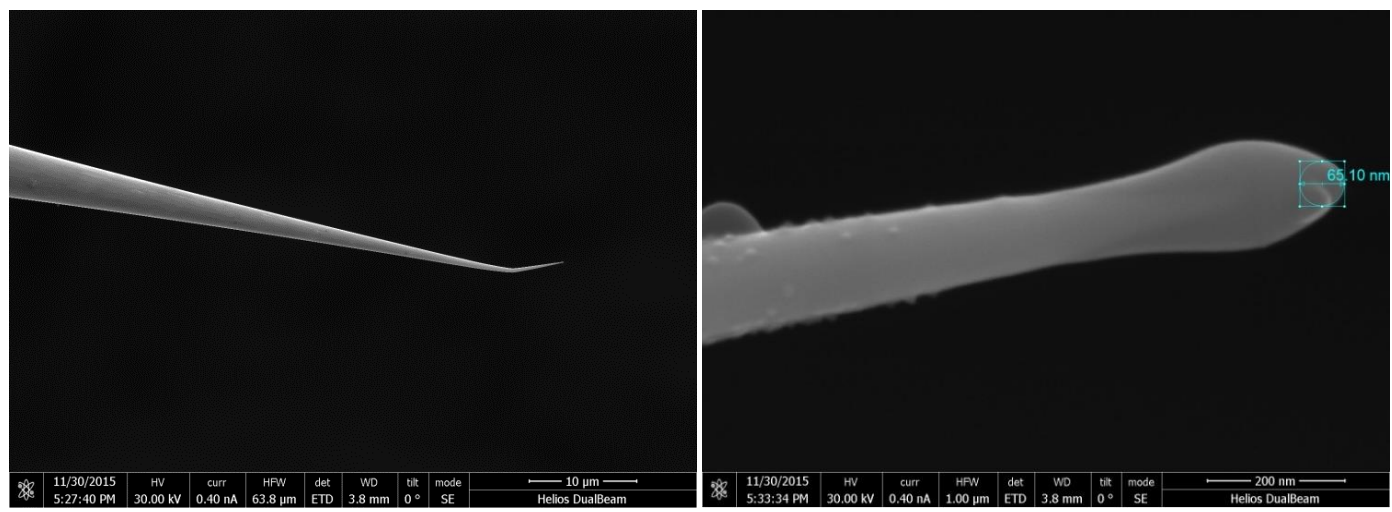

Figure 10.7. (left) Low-mag image of AFM tip\#A-005 showing a crook in the end. (right) The tip has a bulbous shape, but is still relatively sharp at about $65.1 \mathrm{~nm}$ diameter on the very end. This tip was fortunately mounted such that the crook caused it to be nearly perpendicular to the sample surface.

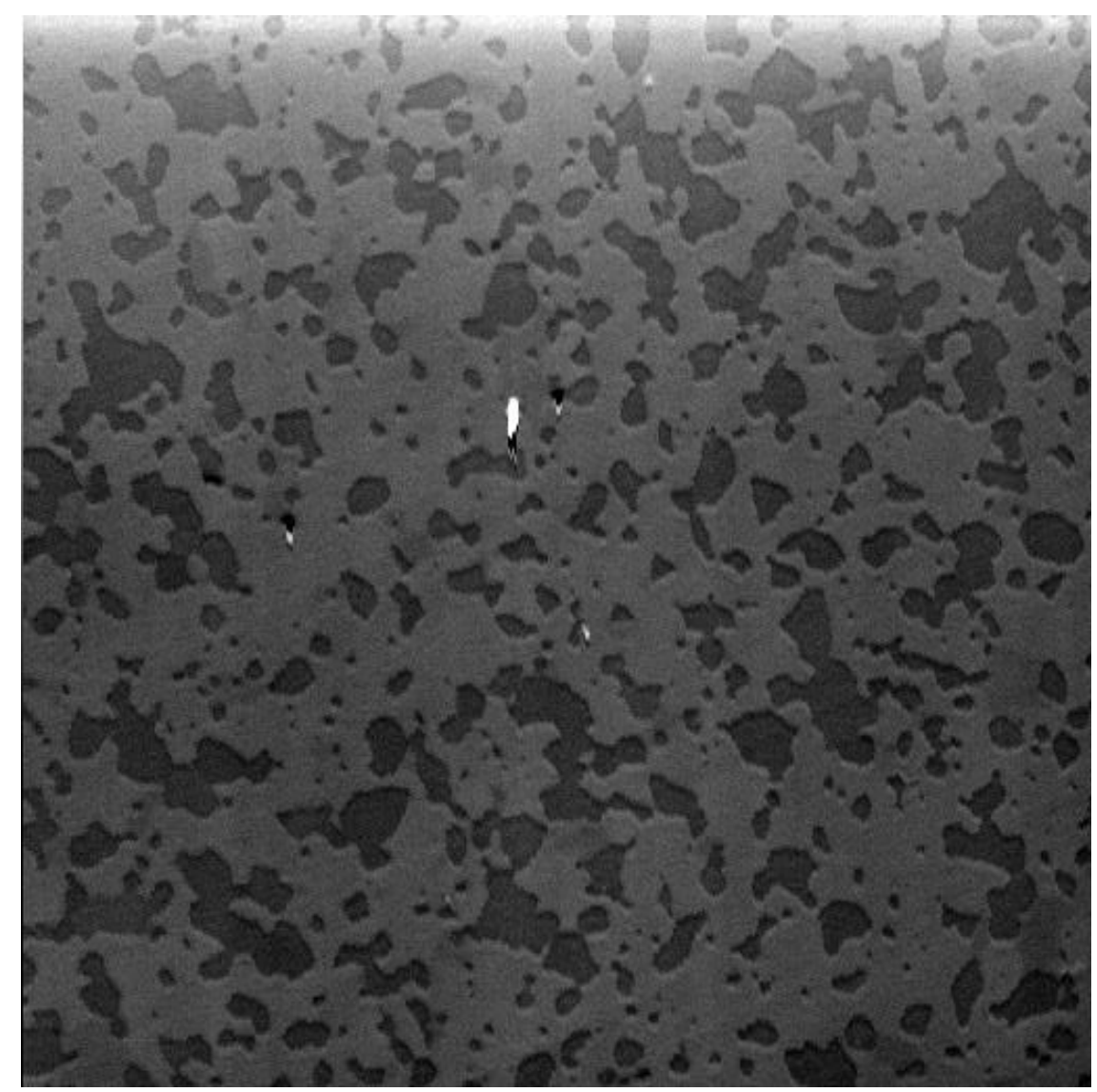

Figure 10.8. Starting AFM scan, using tip\#A-005, which is $25.01 \times 25.01 \mu^{2}$ before FIB imaging/milling. The vertical axis is the fast axis. At least two pinholes are apparent even before using the FIB. The darker islands are TiC and the surrounding material is $\mathrm{Al}_{2} \mathrm{O}_{3}$. These darker islands are recessed from the lighter regions by about $8.5 \mathrm{~nm}$. 

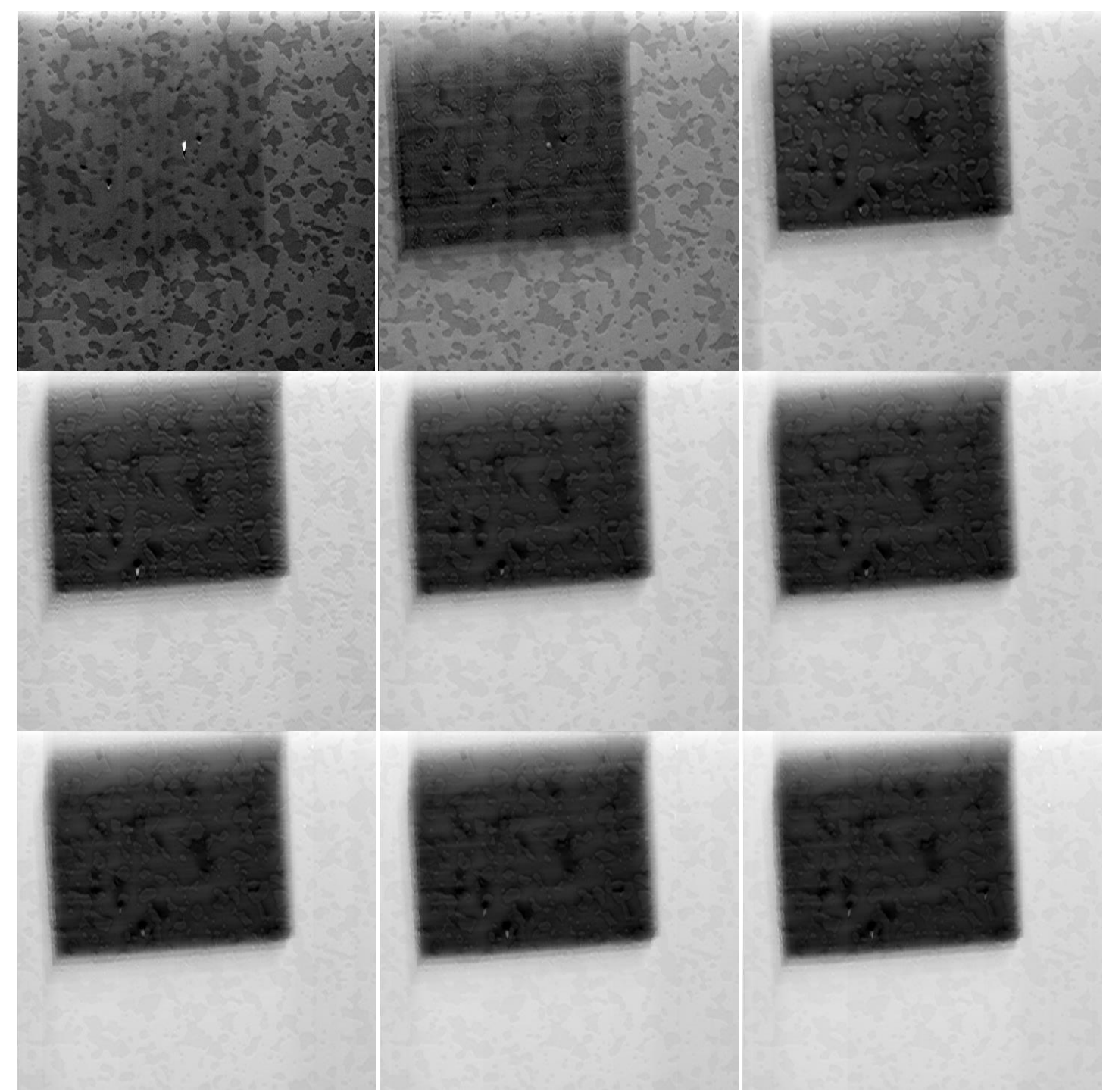

Figure 10.9. Sequence of nine $25 \times 25 \mu \mathrm{m}^{2}$ AFM scans, each following a FIB image. FIB images are scanned left-to-right, top-to-bottom and are not perfectly rotated to 90 degrees w.r.t. the AFM. FIB scanning artifacts are clearly visible as nearly horizontal streaks inside the crater. FIB HFW is 16 $\mu \mathrm{m}$. 


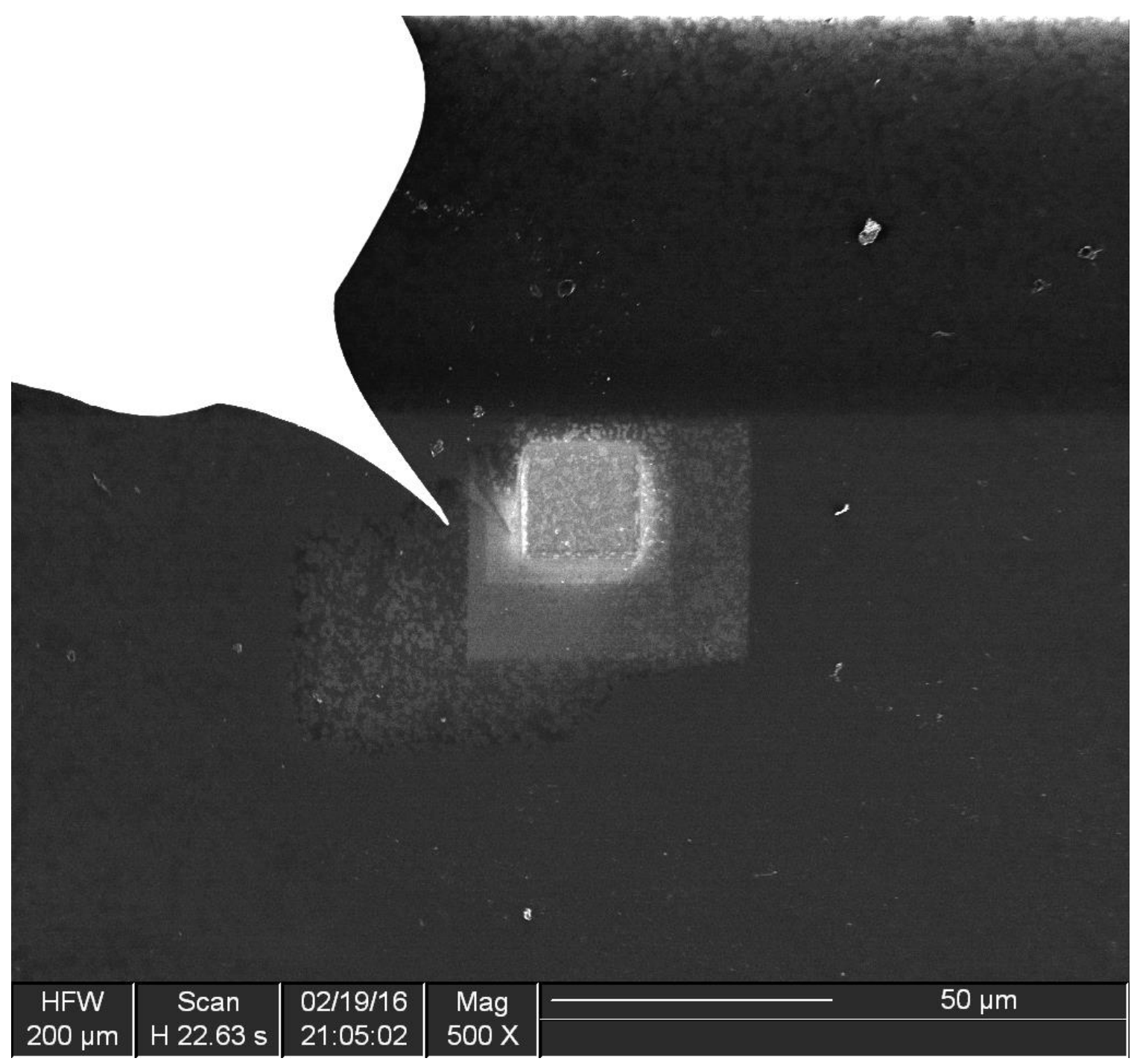

Figure 10.10. Image of the AFM tip lifted from the FIB crater edge. During the milling process, the AFM tip is parked on the left side of the crater, as can be seen by its shadow left by the FIB beam tails. The AFM scan slow axis is from left to right. This allows the AFM to scan to unambiguously detect FIB scan artifacts. Image contrast has been enhanced. 


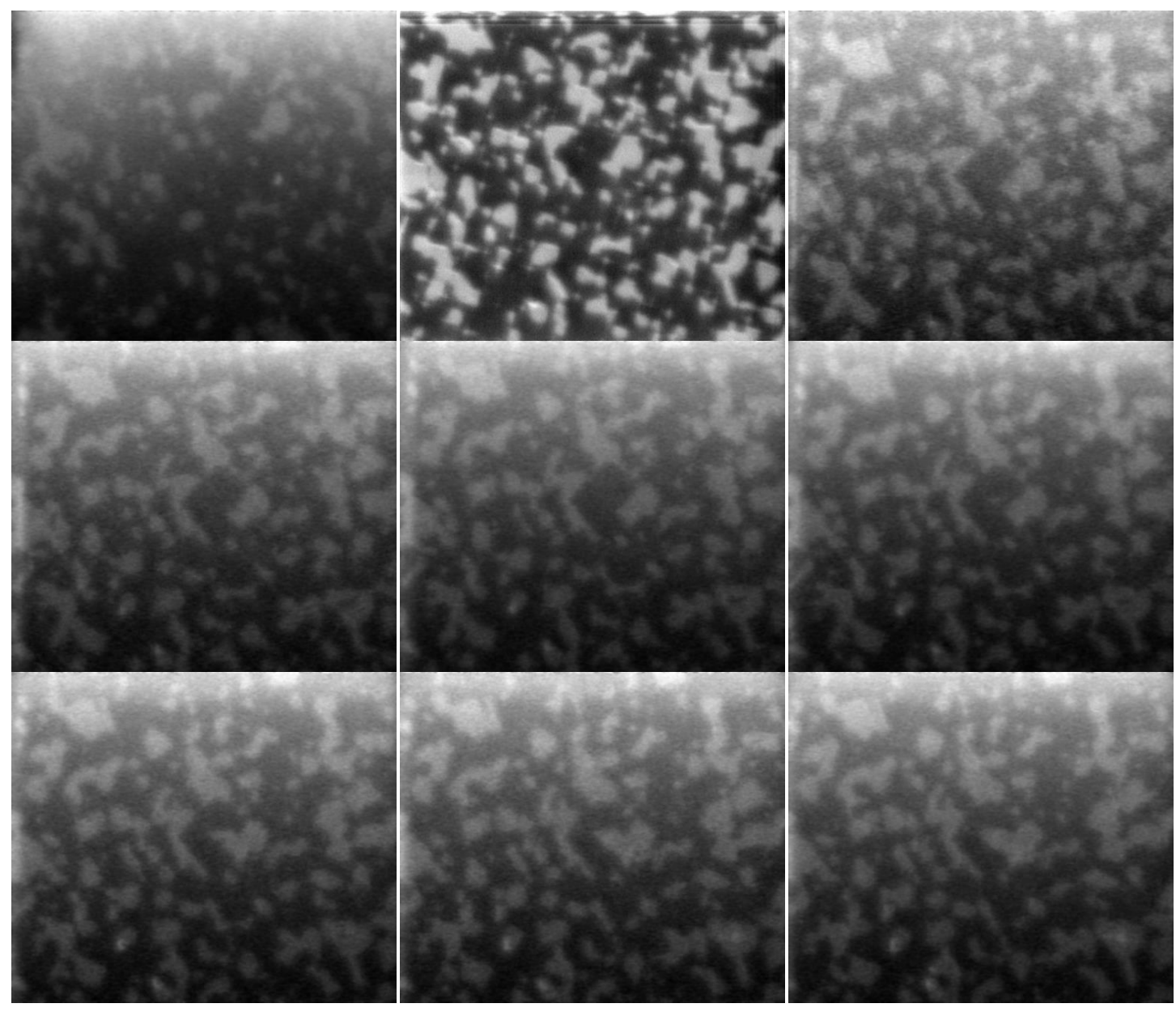

Figure 10.11. Auto focus image stacks averaged together. 


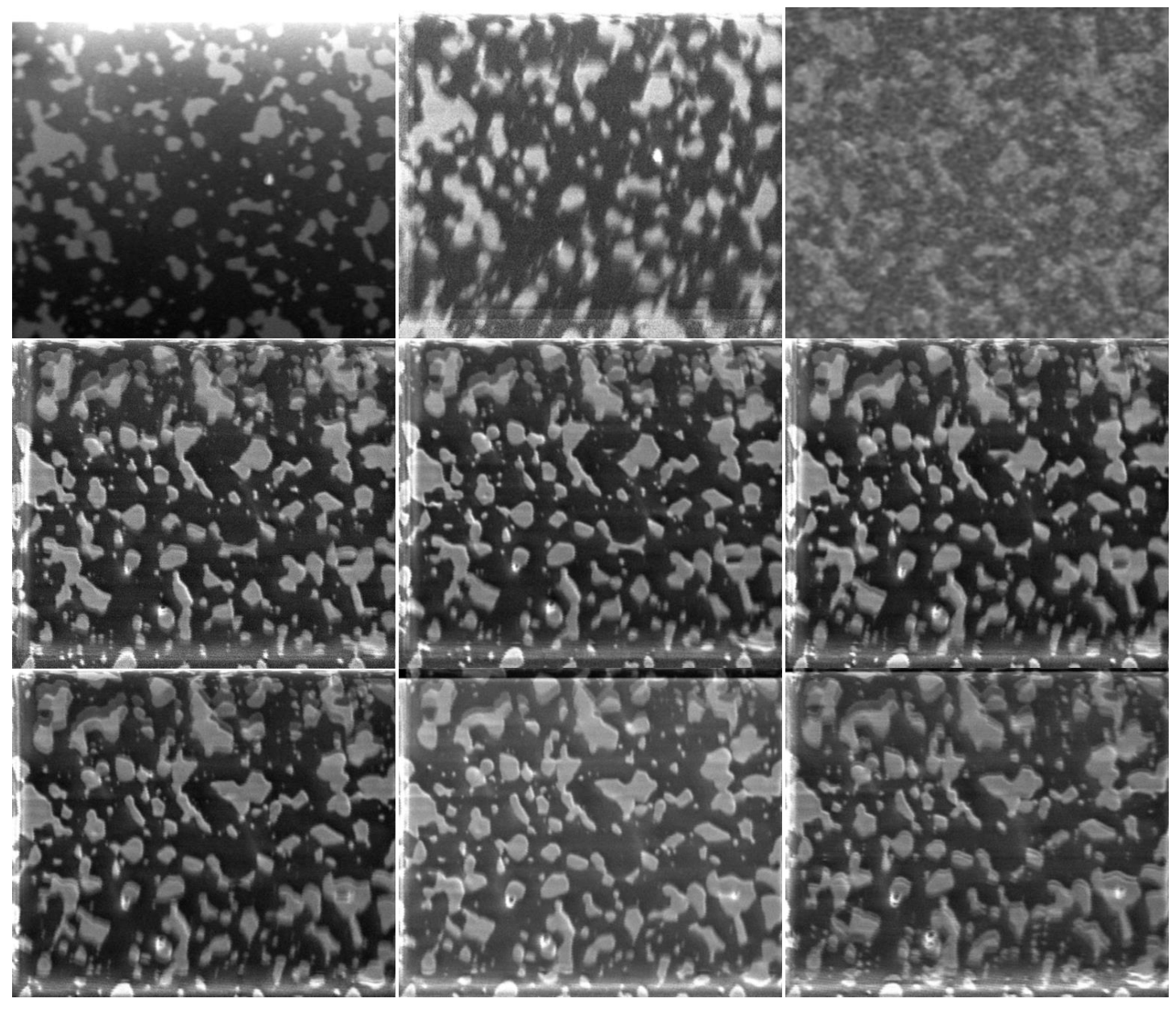

Figure 10.12. Runscript image stacks averaged together. 


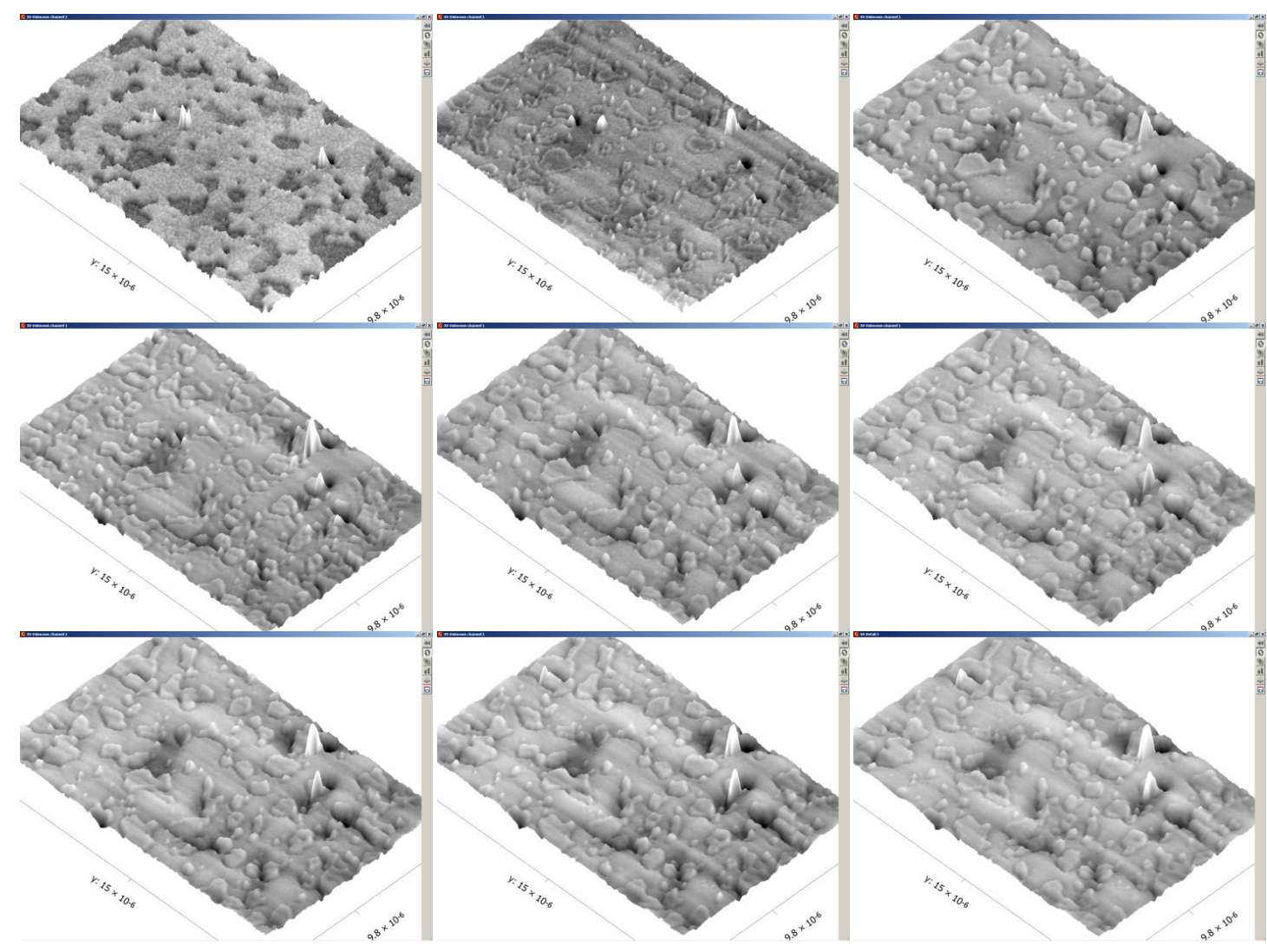

Figure 10.13. (left to right, top to bottom) AFM images inside the crater. 


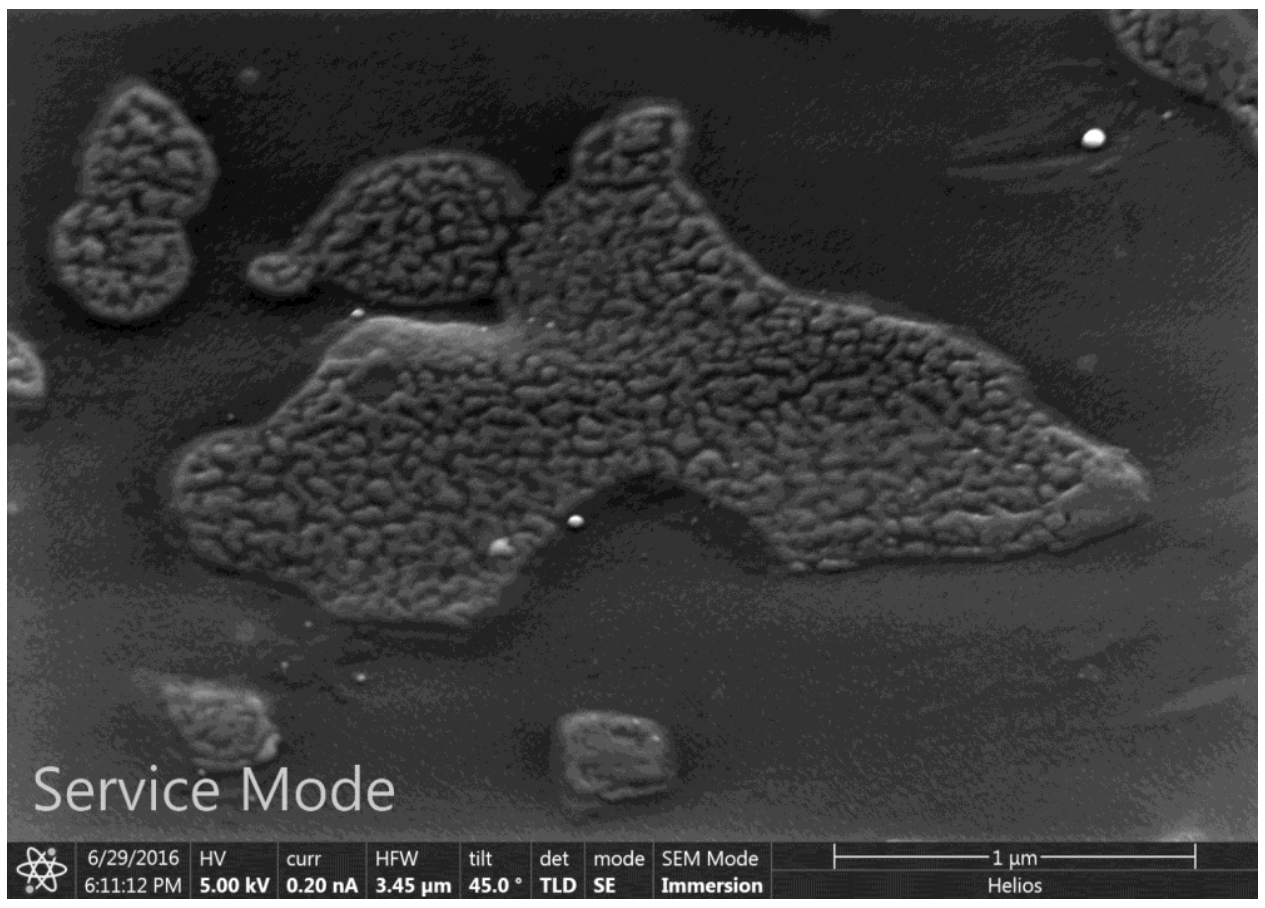

Figure 10.14. SEM image of a distinctive TiC island inside a FIB-milled crater. This feature is upside-down w.r.t. the corresponding FIB image. The bright circular features are Ga spheres remaining from the FIB imaging. These Ga spheres survived venting and exposure to air for months before this SEM image was taken.

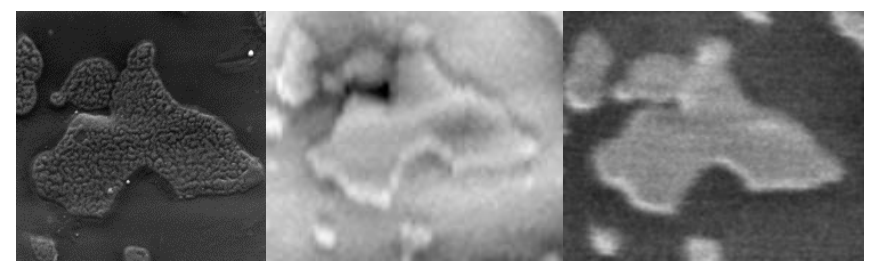

Figure 10.15. Comparative images of the distinctive feature in Figure 10.14. Each has been adjusted to have similar aspect ratios. (left) SEM image, (center) AFM image and (right) FIB image.

\subsection{Experiment \#1 results}

This experiment revealed some interesting facts about the FIB, the AFM, and the sample itself:

1) The TiC grain recession measured on the un-milled surface is about $8.5 \mathrm{~nm}$.

2) There are shallower recessions in the Al surface, possibly being regions where the TiC grains became too thin to stay interlocked and came out during polishing.

3) Scratches on the top surface of the sample are evident. These may have been 
created during the final cleaning/polishing after the DLC step of the manufacturing process.

4) The AFM scans reveal topography and artifacts not readily visible in the FIB images. Once the FIB images are re-viewed, some of these artifacts become evident.

5) Small pinholes in the sample appeared very early in the sequence. They are relatively deep and adversely affect the AFM servo loop.

6) AFM trace images were used for analysis. They exhibit a "curl" up in the data on the left side of the scans.

7) The FIB images are of low quality, displaying an excessive amount of deflection noise. This was originally attributed to excessive sample charging. Actually the noise was caused by dirty contacts in the Vernier selector switch on the deflection controller.

8) Some user errors in FIB image acquisition occurred.

a. The number of FIB images used for milling the crater was changed after the first one.

b. Auto focus for the $1^{\text {st }}$ image used more pixels than the others.

c. The $3^{\text {rd }}$ auto focus was accidentally run using 90 us pixel dwell and $1024 \times 884$ pixels, instead of the 800 ns which was used for the others. The FIB dose for this step was drastically larger than any other.

9) When imaged in an SEM having better resolution that either FIB or AFM, one of the distinctive $\mathrm{TiC}$ features inside the crater reveals texturing which may be a sign of the preferential milling which was predicted in chapter 6 .

Runscript is an effective tool for simultaneously making craters, avoiding redeposition while acquiring multiple images which can be averaged into one representing each milling step. It does however generate a lot of images which must be manually averaged offline. Integrated images can be acquired using $\mathrm{xP}$ directly and the resulting images have a high signal to noise ratio.

\subsection{Experiment \#2: High beam current milling using the $5.75 \mathrm{nA}$ beam plus image integration}

AFM tip \#B-002, having a diameter of $30.6 \mathrm{~nm}$ was installed and a FIB beam current of $5.75 \mathrm{nA}$, was used to mill a crater in AlTiC, alternately acquiring AFM topographic images. More than 50 of these FIB \& AFM images were acquired. Auto 
focus was performed between each pair of FIB \& AFM images. Experiment \#1 revealed that the deflection noise can cause streaks in the crater surface. This experiment will utilize image integration using short pixel dwells to reduce this effect.
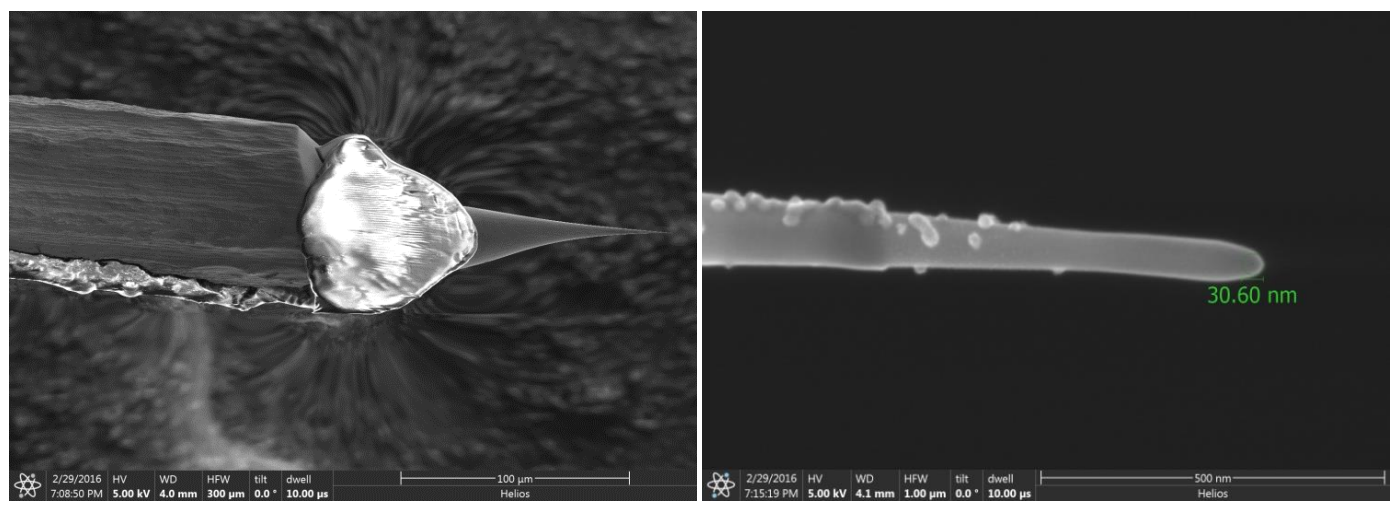

Figure 10.16. (left) Low magnification image of AFM tip\#B-002, showing a blob of insulating contamination on the tapered region. (right) High magnification image showing small bits of contamination on the taper, but a rather sharp tip, about $30.6 \mathrm{~nm}$ diameter. Installed inside the FIB, this blob blocked FIB imaging of the tip and charged excessively.

FIB imaging conditions were as follows:

- $\quad$ Ibeam $=5.75 \mathrm{nA}$

- $\mathrm{HFW}=20 \mu \mathrm{m}$

- XY pixel count: $1024 \times 884$

- Pixel dwell: 300 ns

- \# frame integrations:

○ 8 for the 1st 10 images (000-009)

○ 32 for the next 3 images (010-012)

○ 64 for the next 14 images (013-026)

- 128 for the next 16 images $(026-042)$

○ 256 for the next 8 images (043-050)

AFM imaging conditions were as follows:

- XY field of view: $29.607 \mu \mathrm{m}^{2}$

- XY pixel count: $512 \times 512$

- Scan rate $(\mathrm{Hz}): 0.25$

- $(\mathrm{P}, \mathrm{I}, \mathrm{D})=(0.075,0.035,0.000)$

- $\quad$ Scan time $(\mathrm{s})=2048 \mathrm{~s}=34.13$ minutes 


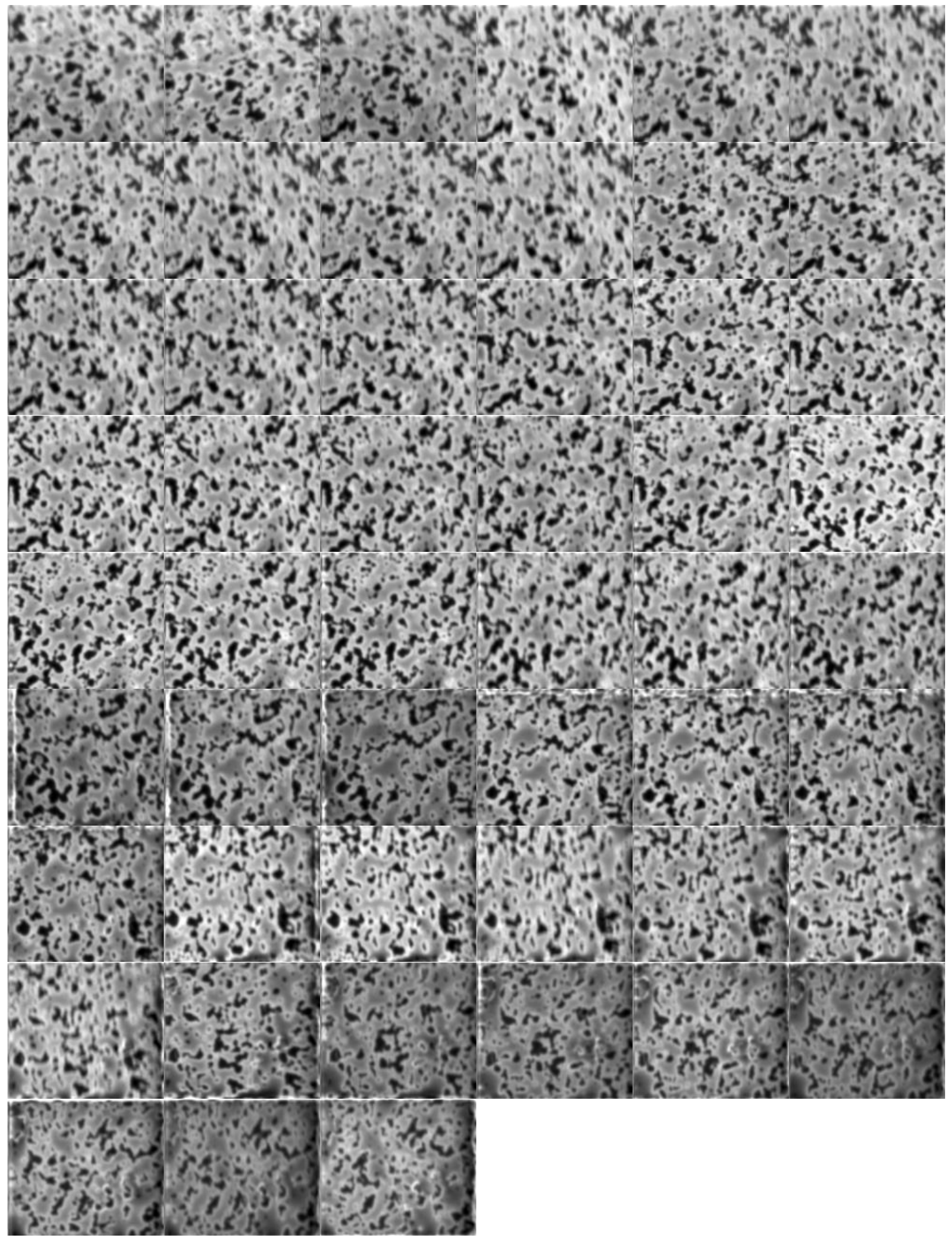

Figure 10.17. (left to right, top to bottom) FIB image acquisition of 51, $5.75 \mathrm{nA}$ images. Pixel dwell is $300 \mathrm{~ns}$ and HFW is $20 \mathrm{um}$. Images were acquired using increasing frame integration counts in order to determine the count necessary for $50 \mathrm{~nm}$ material removal. 


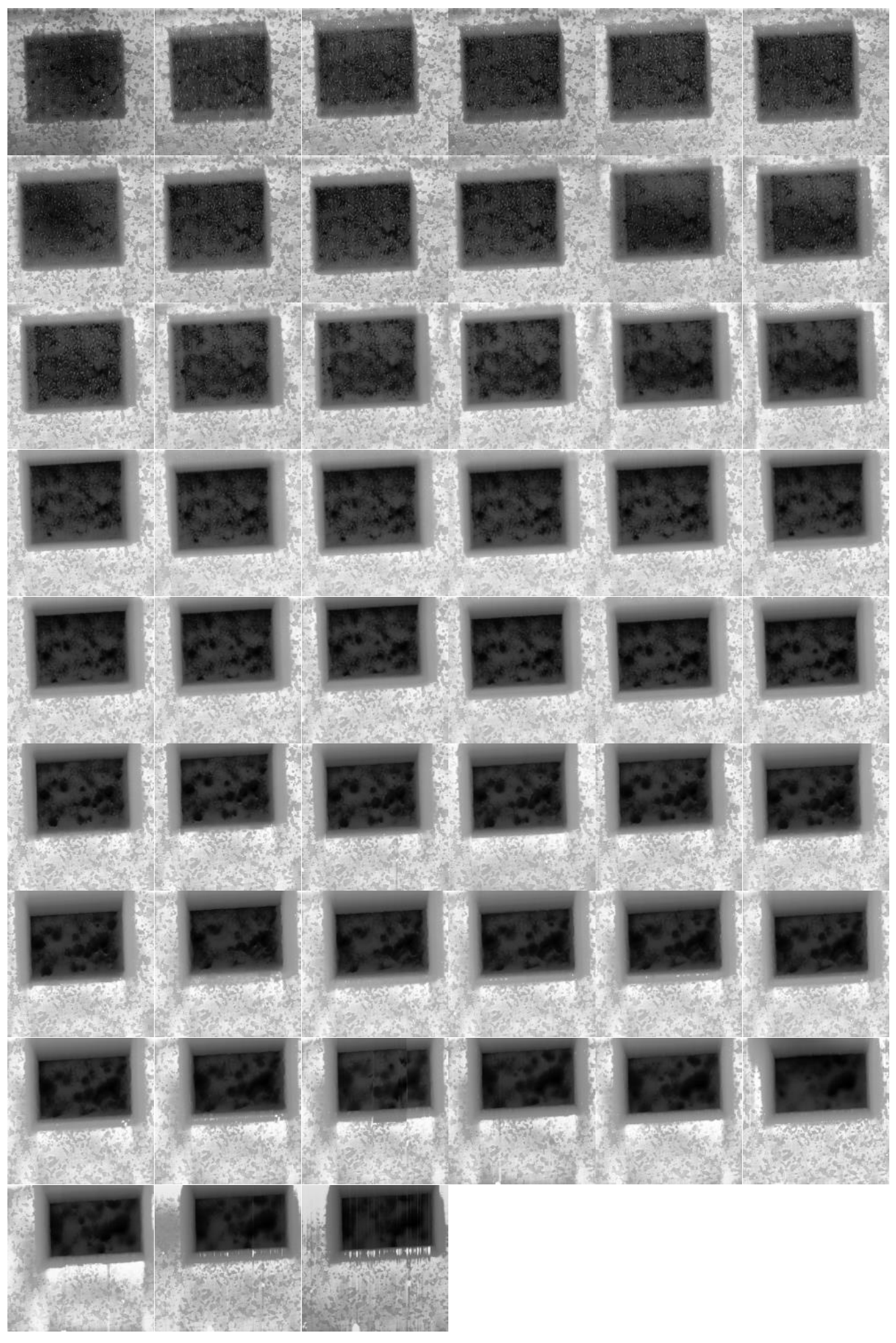

Figure 10.18. (left to right, top to bottom) AFM acquisition of 51 images, each occurring after its corresponding FIB image, shown in Figure 10.17. AFM images are $40 \times 40 \mu \mathrm{m}^{2}$ in size. Contrast has been normalized so that features can be seen on the sample surface and inside the FIB crater. 


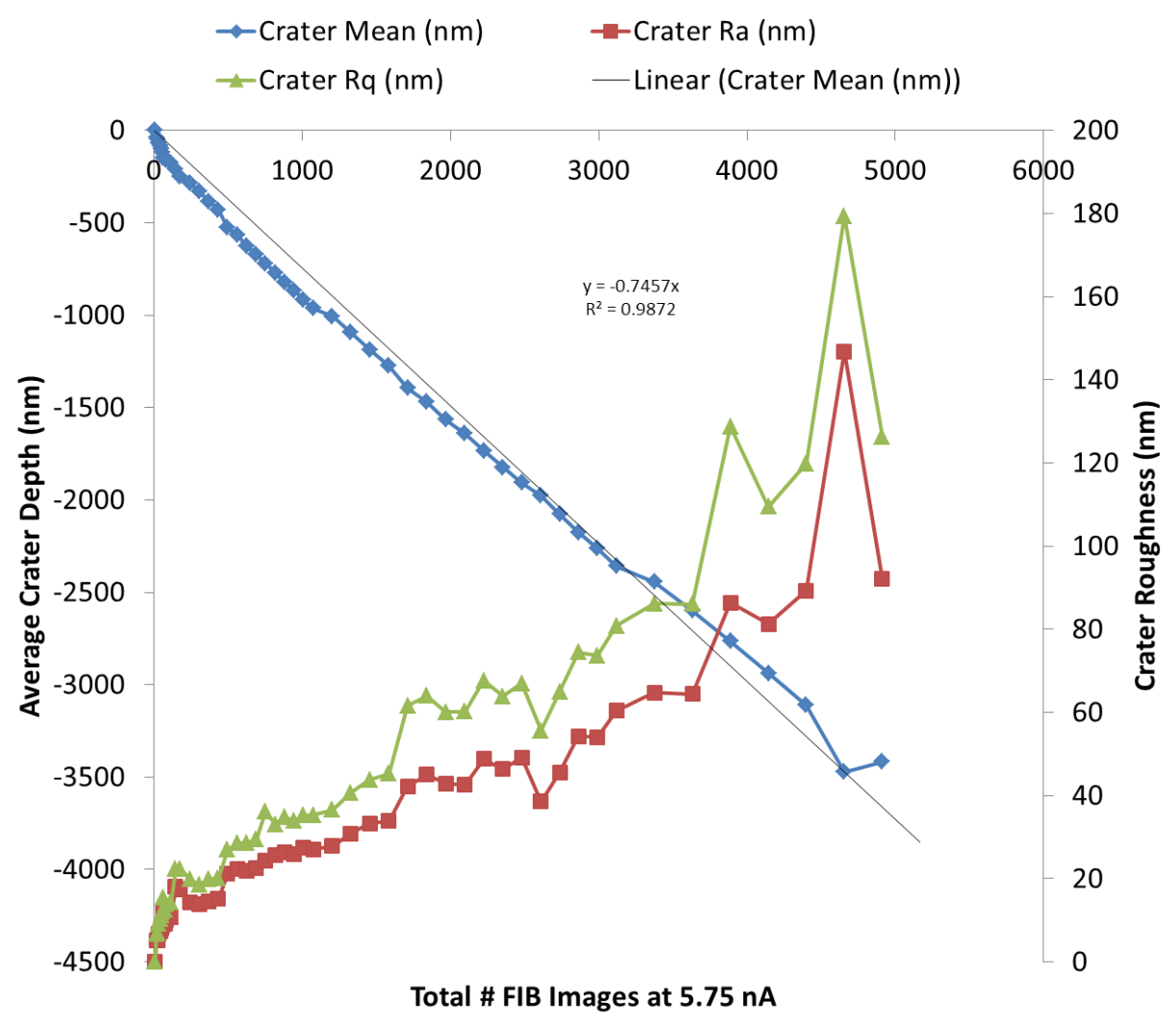

Figure 10.19. Analysis of the AFM images shown in Figure 10.18 for crater depth and roughness as a function of total integrated FIB image count. FIB images acquired for auto focus are not shown, because the goal for this experiment is to determine the number of integrations, being a power of 2 , which leads to an average removal of $50 \mathrm{~nm}$ to include the auto focus overhead. The result is 64 .

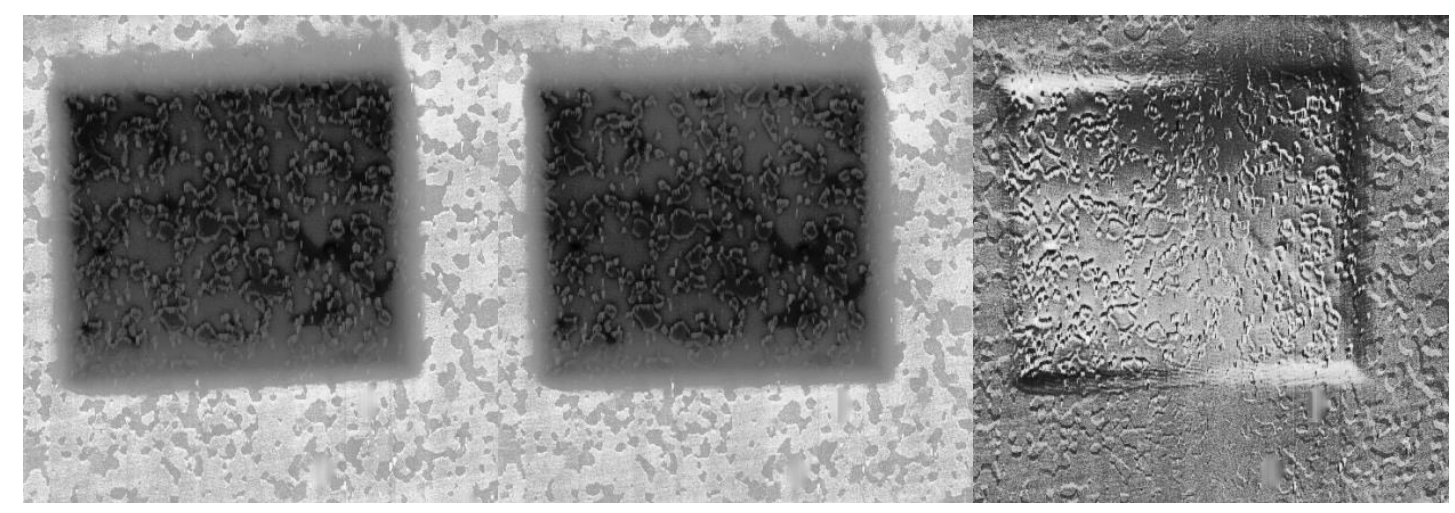

Figure 10.20. (left) AFM image \#10, taken as the last image before stopping for the day. (center) AFM image \#10a, taken as the first image of the next day before the FIB milling began. (right) Mutual crop difference image, highlighting the relative affine distortion between the two. These images have had their contrast levels normalized so that features on the surface and inside the crater can be viewed. 


\subsection{Experiment \#2 results}

Auto focus images are not shown for this experiment. They were acquired using the same pixel count and dwell as the integrated images, but were not integrated themselves. Careful inspection of the 51 FIB images reveals a lot of image stigmation variability. Although auto focus was used before each integrated image, stigmation was not adjusted so that the average dose per integrated image is constant. The stigmation variability is likely due to the large beam current being close enough to the AFM tip to charge it. Normally when a tip charges up, it tends to discharge slowly during an AFM scan as the tip makes somewhat frequent contact with the grounded sample at large steps. Recall that this particular tip had a large bulb of insulating contamination. Once charged from the beam, it would likely take a long time to fully discharge.

Chapter 6 predicts that the milling of dissimilar materials will lead to crater roughness growing without bounds. Figure 10.19 supports this conclusion for Ga FIB milling of this AlTiC sample. The roughness curves do not exhibit any flat bands for any of FIB settings used. Monotonic roughness growth with crater depth is not preferred but it appears to grow in a controlled fashion for the smaller image integration counts and so

may be dealt with in future experiments. The quality of the AFM data appears to degrade with deeper crater depths.

Inspection of Figure 10.19 shows the distinct groups of FIB image integration counts in the crater mean data. Determination of the optimal FIB image integration count was successfully determined to be 64 . The mean crater $\Delta \mathrm{Z}$ is $-50.9 \mathrm{~nm}$, with a standard deviation of $14.5 \mathrm{~nm}$ for the 14, 64 count, integrated FIB images. Including the auto focus images in the slope determination reduces it from $-0.7457 \mathrm{~nm} /$ image to -0.6079 
$\mathrm{nm} /$ image. It is not known for sure how the mostly blurry images acquired during auto focus contribute to the overall milling rate but we can conclude that the rate is bounded by these two slopes.

Data collection for this experiment spanned 15 days. At the end of each day, the FIB and AFM were shut down. The following day, at least one hour was allowed for system warm-up. Before a new FIB mill was begun, an AFM image was acquired so that it could be later compared with the one acquired earlier. Any system drift would be captured and the AFM's measurement stability can be assessed by differencing this new image with the last one from the day prior. Figure 10.20 shows the result for the $10^{\text {th }}$ image in the series. The difference in measured crater depth was only $-0.4 \mathrm{~nm}$. There was some relative affine distortion between the two images. This suggests that reconstructions for image stacks will require attention to lateral distortions.

\subsection{References}

Clark, B.K. "The effect of slider roughness on asperity testing of thin-film media." IEEE Trans. Magn. IEEE Transactions on Magnetics 29, no. 1 (1993): 235-40.

Cutler, Raymond A., Andrew C. Hurford, and Anil V. Virkar. "Pressureless-Sintered Al 2 O 3-TiC Composites." Materials Science and Engineering: A 105 (1988): 183-192.

FEI Company, "SD Gallium LMIS High Speed Blanking MVA Focusing Column with Electronics User's Guide”, PN 16887 rev A, 1993

Galileo Electro-Optics Corporation, "Channeltron Electron Multiplier Handbook for Mass Spectrometry Applications”, Galileo Park, P.O. Box 550 Sturbridge, MA 01566

Jannis Lübbe, Matthias Temmen, Holger Schnieder, and Michael Reichling. "Measurement and Modelling of Non-Contact Atomic Force Microscope Cantilever Properties from Ultra-High Vacuum to Normal Pressure Conditions." Measurement Science and Technology 22, no. 5 (2011). 
Li, Jing, Yan-sheng Yin, Rui-xia Shi, Lai-peng Ma, and Jian Li. "Microstructure and Mechanical Properties of Al2O3-TiC-4vol.\%Co Composites Prepared from Cobalt Coated Powders." Surface and Coatings Technology 200, no. 12-13 (March 2006): 3705-12. doi:10.1016/j.surfcoat.2005.02.165.

Liu, N., M. Shi, Y.D. Xu, X.Q. You, P.P. Ren, and J.P. Feng. "Effect of Starting Powders Size on the Al2O3-TiC Composites." International Journal of Refractory Metals and Hard Materials 22, no. 6 (November 2004): 265-69. doi:10.1016/j.ijrmhm.2004.09.001.

Sourty, E., J. L. Sullivan, and M. D. Bijker. "The Tribology of Advanced Digital Recording (ADR) Systems.” Tribology International 33, no. 9 (2000): 629-637.

West, Paul, and Natilia Starostina. "A Guide to AFM Image Artifacts." Pacific Nanotechnology, Inc. 3350 Scott Blvd \#29 Santa Clara, CA 95054-3105, n.d.

Whitehouse, D. J. "Nanometrology." Contemporary Physics 49, no. 5 (September 2008): 351-74. doi:10.1080/00107510802611251.

Yamamoto, Takayuki, Yoshiharu Kasamatsu, and Hiroyuki Hyodo. "Advanced StictionFree Slider and DLC Overcoat." Fujitsu Scientific and Technical Journal 37, no. 2 (2001): 201-11.

Zhang, Yanfeng, Lianjun Wang, Wan Jiang, Guangzhao Bai, and Lidong Chen. "Effect of Fabrication Method on Microstructure and Properties of Al2O3-TiC Composites." Materials Transactions 46, no. 9 (2005): 2015-2019. 


\section{FIB-SIMS Operation}

\subsection{Basic design elements for the Fei SIMS-I}

The actual SIMS-I configuration is actually slightly different than Figure 3.2. The entire assembly has a 3 degree tilt, reducing the chances of sample impact. The Ion Beam is aimed through a hole which goes all the way through the spherical energy analyzer. Ions are collected through a spherical electrostatic energy analyzer where the ones which pass the energy band are sent through an arc of 75 degrees. They then pass through three electrostatic lenses into the entrance of the Quadrupole mass analyzer. There are both entrance and exit electrodes and the ions finally reach the Channel Electron Multiplier (CEM).

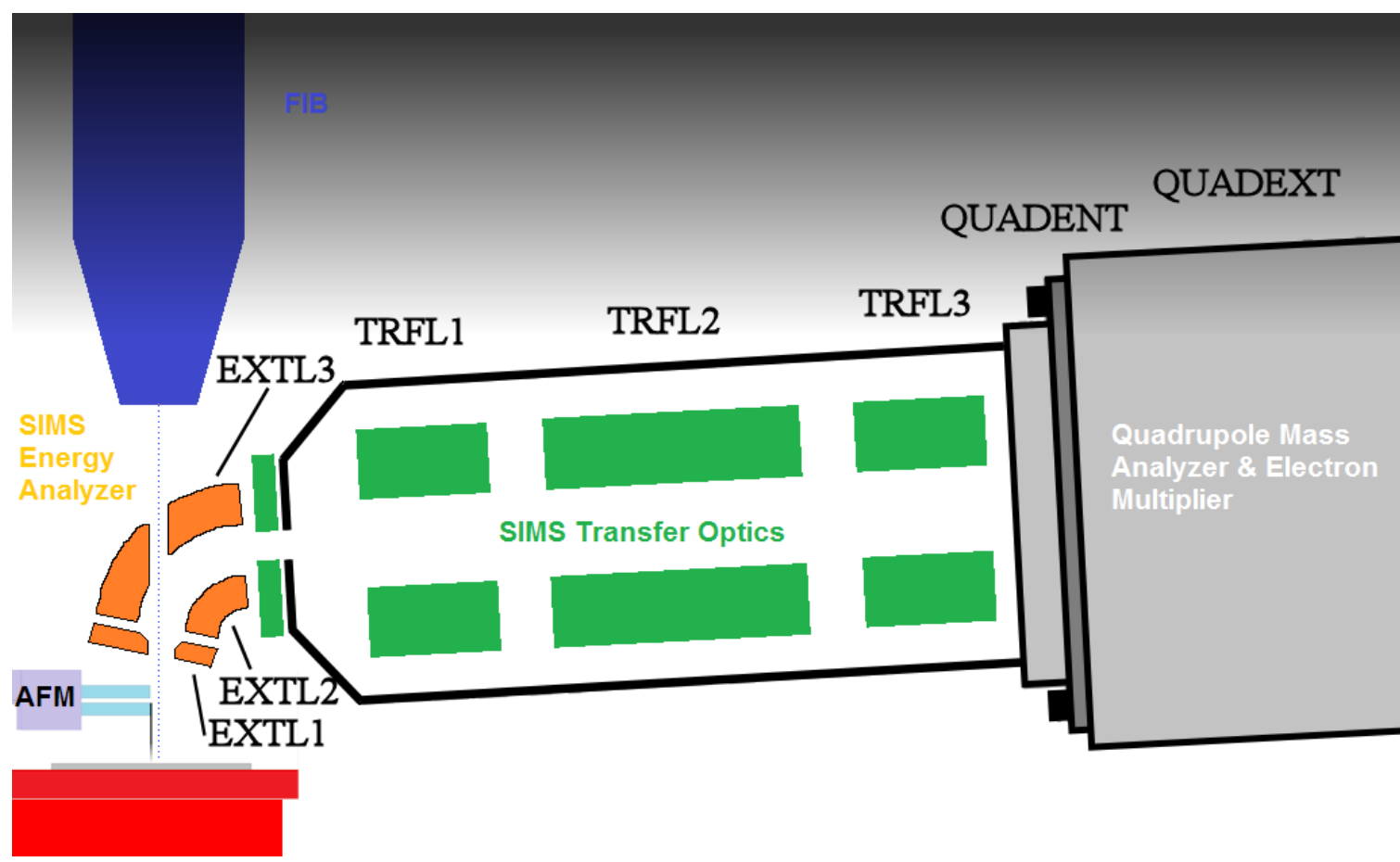

Figure 11.1. Rough illustration of the FEI FIB SIMS-I energy analyzer and ion transfer optics and AFM tuning fork/tip assembly. There are eight individual SIMS controls which need to be optimized for the highest possible ion count rate. Once the three elements comprising the energy analyzer, EXTL1, EXTL2 and EXTL3, are determined, the others are easily found. 
This configuration introduces unwanted beam shift and astigmation to the primary ion beam. Typical operating Voltages of the SIMS optics lead to an image shift of 20-25 $\mu \mathrm{m}$ in $\mathrm{Y}$. Thus the stage is used to center the sample when the SIMS is operating. Also, because the SIMS electrodes are generally energized with negative Voltages, the secondary electrons emitted from the sample are pushed back towards the sample and FIB image quality is greatly reduced.

Although the XP 2.25 software was implemented for the SIMS-III optics, the original code authors were careful to keep the same control elements as the SIMS-I. The only difficulty was in determining the control voltages for the SIMS-. These were not available in any on-hand documentation. 


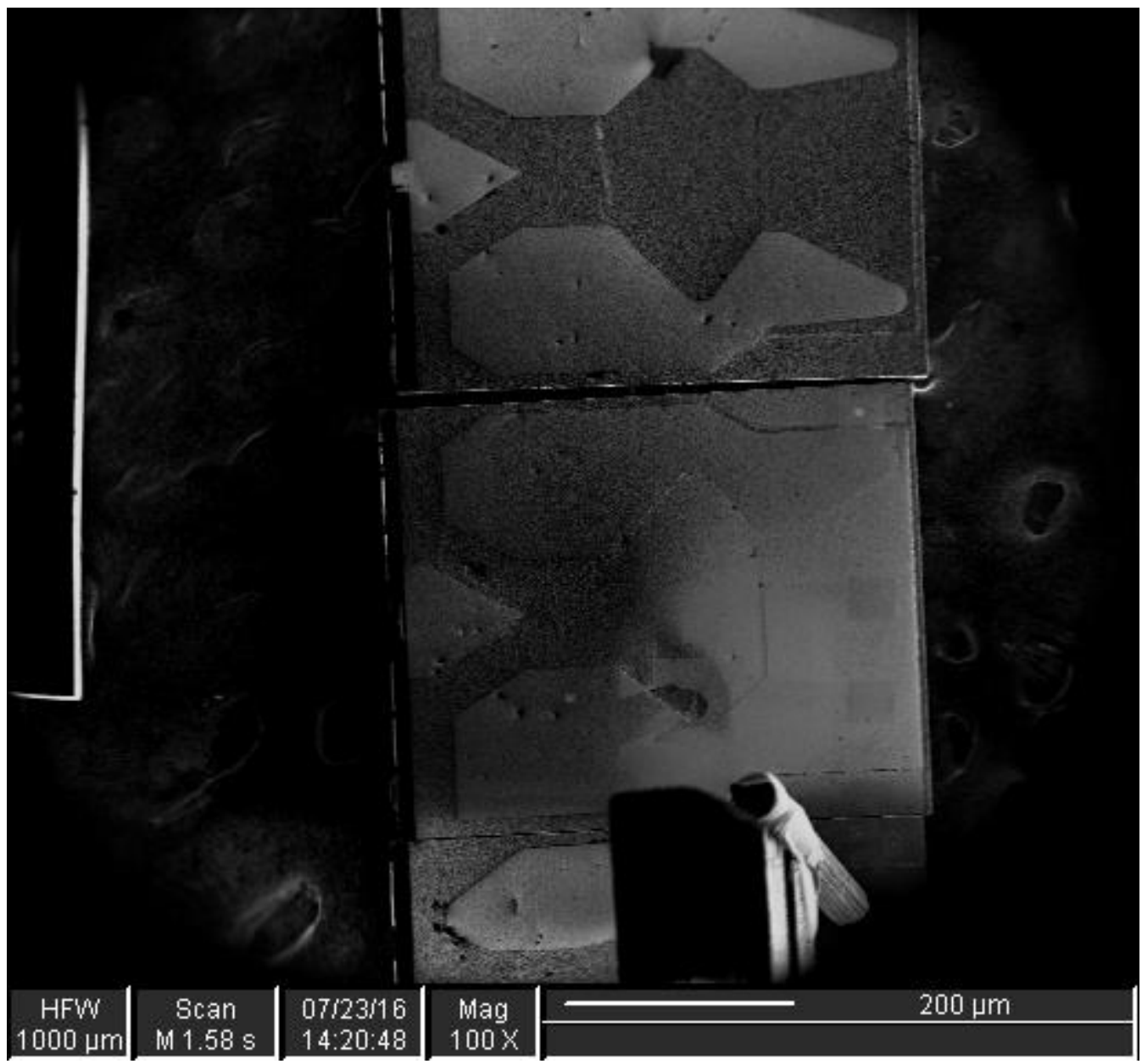

Figure 11.2. Low magnification image of two hard drive sliders which are made from AITiC. The air bearing surfaces are the triangular regions to the left and the upper \& lower smooth regions. The AFM tuning fork can be seen in the bottom right. The FIB's deflection controller Vernier setting has been turned all the way $\mathrm{CCW}$ for the largest possible field of view, so the scale shown is incorrect. The faint outline of the SIMS circular aperture is also visible.

\subsection{Benninghoven voltage ratios for SIMS energy filter}

Being FEI's first implementation, many details for the operation of the FEI SIMSI have been lost to obscurity. There are no user manuals available which indicate starting voltages for the SIMS-I. We have had occasion to use the electronics from the SIMS-I, including RF-Controller and Optics Controller, on our SIMS-III for debugging and 
achieved 2-4 times as many counts/s given similar beam currents and sample. Thus it was clear that the count rate was much lower for our SIMS-I.

The energy analyzer consists of a spherical electrostatic sector which redirects the ions leaving the surface into the quadrupole mass analyzer to a channel electron multiplier.

The maximum ion transmission through the analyzer occurs when the inner and outer electrodes are at potentials related as (Benninghoven eqn 4.97)

$$
\varphi_{s}^{ \pm}=2 V_{0}\left\{\frac{R}{R \mp d / 2}-\frac{1}{2}\right\}
$$

Where $\phi^{ \pm}$represents the inner and outer electrodes potentials, respectively. $\mathrm{R}$ is the average radius of the electrodes and $d$ is the difference between inner and outer radii. $V_{0}$ is the nominal energy of the incoming ions. For our configuration, this reduces to

$$
\varphi_{s}^{+}=\frac{5}{3} V_{0}
$$

and

$$
\varphi_{s}^{-}=\frac{3}{5} V_{0}
$$

This makes tuning the eight voltages of the SIMS optics much easier, as three of them are 
easily determined from this relationship. The other five voltages are also very easy to determine by trial and error.

The range in energies passed by the energy analyzer is (Benninghoven eqn 4.100)

$$
\left|\Delta \varphi_{s}\right|=2 V_{0}\left[\frac{d}{R}+\frac{1}{4}\left(\frac{d}{R}\right)^{3}\right]
$$

Secondary ion energy range is typically less than about $50 \mathrm{eV}$. For the SIMS-I configuration, $\mathrm{R} \sim 11.176 \mathrm{~mm}$ and $\mathrm{d} \sim 5.588 \mathrm{~mm}$. Thus a $\mathrm{V}_{0}$ of $125 \mathrm{~V}$ gives a dispersion of $132 \mathrm{eV}$, making it possible to collect the entire range of secondary ion energies.

\subsection{Optimization of the SIMS optics voltages}

An additional feature was implemented so that the tuning of the SIMS optics could be performed without the concern of the transmission efficiency of the quandrupole mass filter. The descriminator is powered by the SIMS RF/DC supply so that counts are only possible with it turned on. Power to the descriminator has been moved to an external bench supply so that the quadrupole acts only as a drift region which follows the SIMS optics, causing minimal reduction in pulse counts. For our SIMS configuration the signal names and descriptions are as follows:

- Bias Resistor: To ensure that the collector is the most positive element in the detector circuit so that electrons generated in the horn of the CEM are coerced towards the collector.

- Gain: Positive Voltage applied to CEM base to accelerate secondary electron cascade towards collector.

- Grid Bias: Negative voltage applied to accelerate positive ions to the horn of the CEM. A positive voltage is applied for negative ions. 


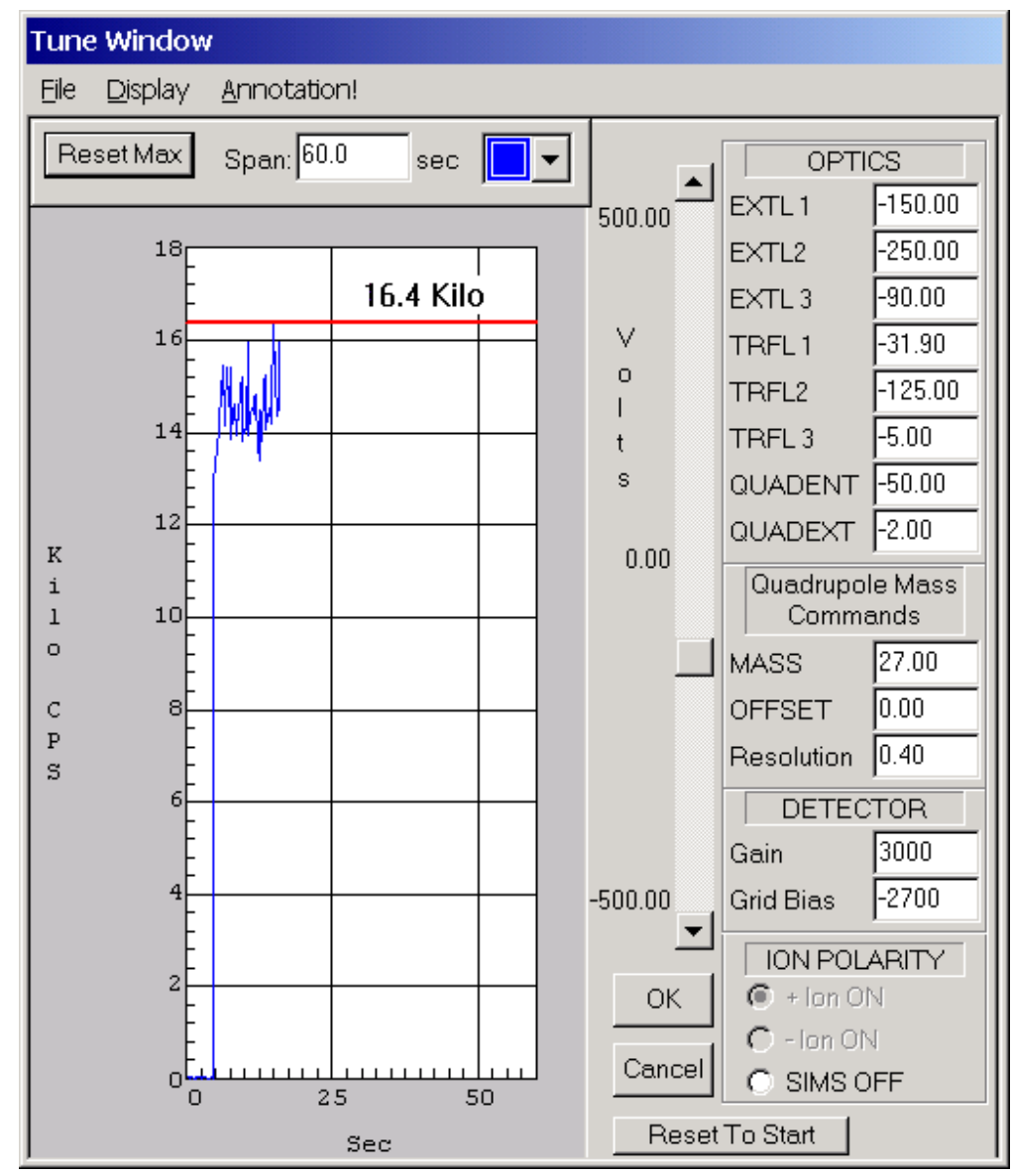

Figure 11.3. SIMS optics tuning window showing typical operating Voltages on the AITiC sample using $25 \mathrm{keV}$ and $250 \mathrm{pA}$.

\subsection{Practical considerations for optimal operation.}

FIB image shift occurs when the SIMS optics are turned on. The magnitude of this shift is illustrated in Figure 11.4, showing three separate pairs of image rectangles, each pair outlined in the same color. The lower image in each pair was acquired with the SIMS optics turned off and the upper rectangles resulted from using the SIMS optics settings shown in Figure 11.3. The magnitude of this image shift is also related to the alignment of the SIMS energy analyzer's aperture and ion beam. For this reason, the SIMS is usually inserted for the duration of an acquisition image stack to reduce 
variability in FIB-SIMS image relative shifts. This requires that the sample stage $\mathrm{Z}$ be low enough that the AFM can be engaged while the SIMS is inserted. Finally, since the SIMS analyzer is not rigidly attached to the chamber, the images are generally more sensitive to vibrations when the SIMS is inserted.

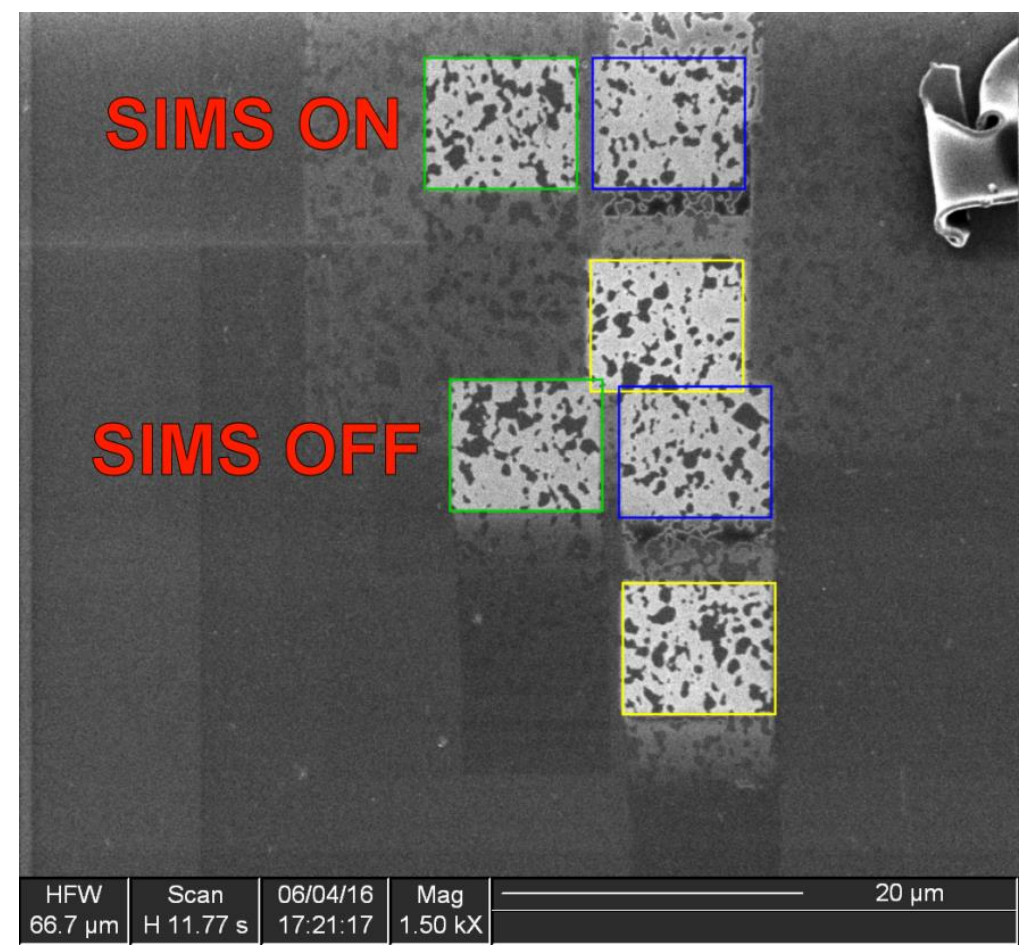

Figure 11.4. Low magnification images showing pairs of imaging rectangles associated with the deflection caused when the SIMS optics are turned on. Deflections shown resulted from using the SIMS optics settings shown in Figure 11.3. Deflection $(X, Y) \sim(-1.8 \mu \mathrm{m},+21.4 \mu \mathrm{m})$.

Focus of the ion beam is not strongly affected when the SIMS optics are energized. Auto focus is ultimately necessary during AFM-FIB-SIMS runs, but the images are affected more by the astigmatism created by energizing the SIMS optics. Once the FIB has been re-stigmated with the SIMS optics energized beam stigmation does not appear to be appreciably variable. 


\subsection{Possible artifacts related to SIMS-I geometry or by mixing of species}

The negative Voltage (EXTL1) applied to the SIMS energy analyzer, used for collecting positive ions, may be causing many of the negative ions to be pushed back to the surface as re-deposited material. An effort was made to determine if this is the case. Three SIMS Maps were made on a $20 \mu \mathrm{m}$ HFW using the $250 \mathrm{pA}$ beam, using a total of 9 ms per pixel. This translates to approximately 2037 seconds of FIB exposure with the SIMS on. The SIMS optics were using the same Voltages shown in Figure 11.3. Then the SIMS optics were turned off and a smaller, $10 \mu \mathrm{m}$ HFW rectangle inside the crater center was imaged until the $\mathrm{TiC}$ islands stood out, as judged by eye.

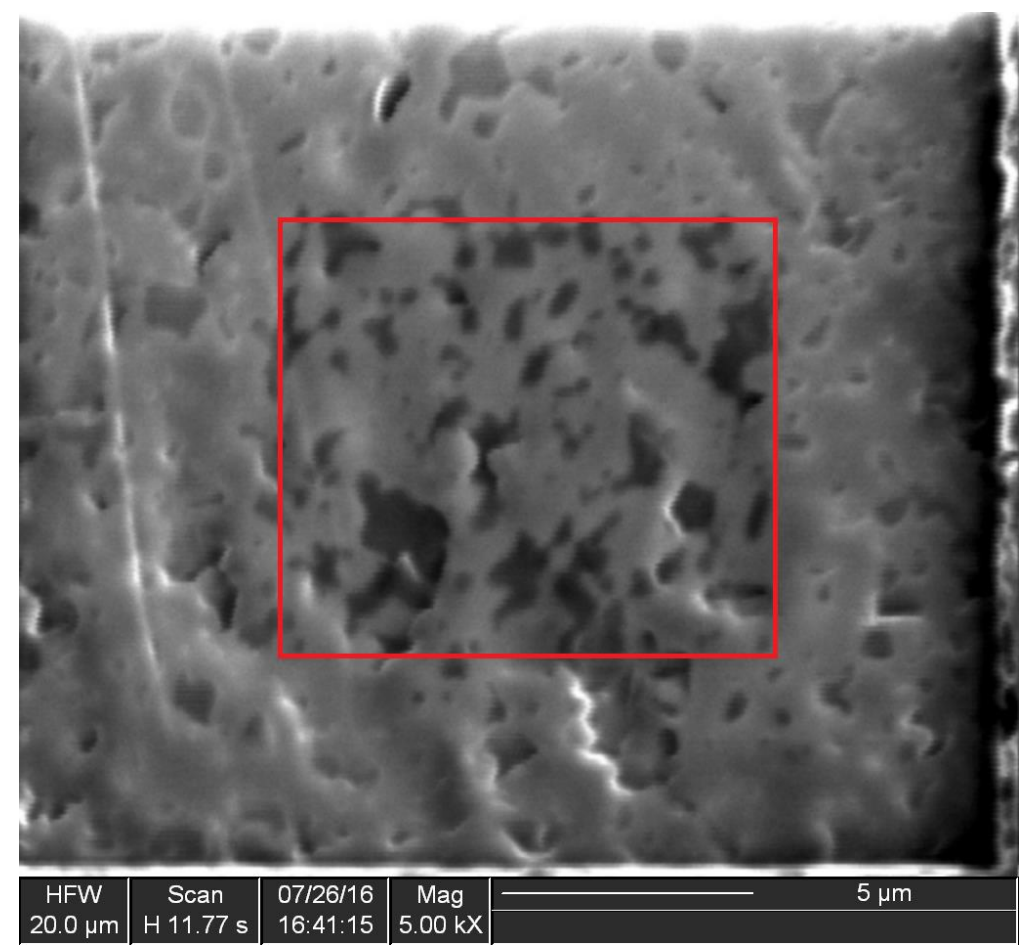

Figure 11.5. Example of possible re-deposition inside the crater during SIMS. The central area of the crater was imaged after 2037 seconds of FIB exposure with the SIMS optics turned off to reveal the higher contrast features under the re-depositied material. 
Figure 11.5 shows the result of this investigation. It appears to validate that some sort of amorphous layer has been somehow left inside the crater. This layer has the effect of reducing the image contrast of the distinct features. This action of amorphous deposition has been seen to be stronger for other FIB-SIMS settings which were not recorded. It is noted here as something to keep in mind when optimizing parameters. Because the SIMS acquisition utilizes long pixel dwells and a single raster in creating 2D maps, some care in the choice of FIB-SIMS operating parameters is needed to ensure that the milled crater has no artifacts which would ruin the analysis. Poor choice of FIB milling parameters, combined with long SIMS pixel dwell times can lead to massive re-deposition and scalloping on the crater floor. Figure 11.6 shows a crater in which this has occurred. Auto focus was not performed between the three SIMS maps, acquired using $4 \mathrm{~ms}$ pixel dwells. It is instructive to take the time to perform some sample SIMS maps using a range of FIB parameters on unimportant regions of the sample to establish robust operating conditions. 


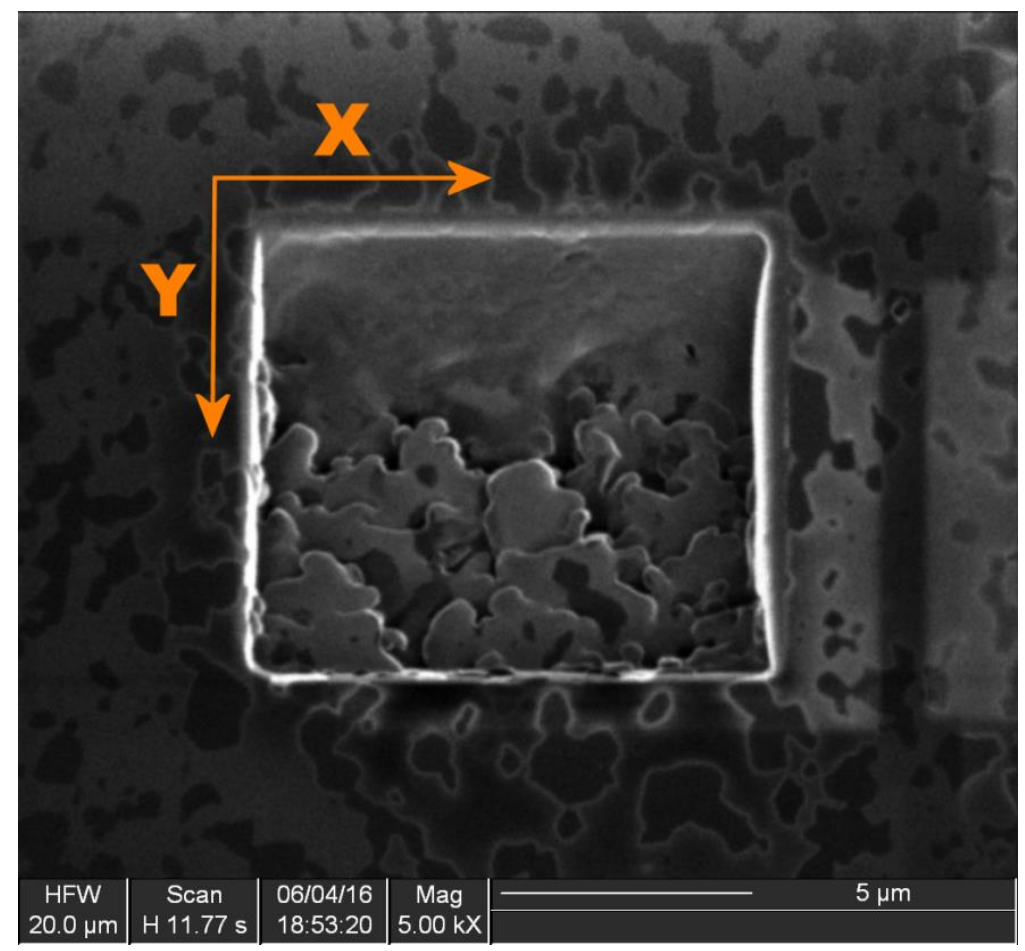

Figure 11.6. Massive sample re-deposition and scalloping inside the crater floor which occurred during three consecutive SIMS maps using $250 \mathrm{pA}$ at $4 \mathrm{~ms}$ pixel dwell and $10 \mu \mathrm{m}$ HFW.

\subsection{Experiment \#1: Low resolution, high milling rate SIMS}

The goal is to establish whether large beam currents can be used for making the crater and still give good lateral and vertical resolution. Thirty SIMS maps were acquired, having three species per level. Data were collected on 2016-07-09 using 25 $\mathrm{kV}, 5.75 \mathrm{nA}$ at $50 \mu \mathrm{m}$ HFW after some SIMS debugging. 
FIB-SIMS imaging conditions were as follows:

- Vbeam $=25 \mathrm{kV}$

- $\quad$ Ibeam $=5.75 \mathrm{nA}$

- $\mathrm{HFW}=50 \mu \mathrm{m}$

- XY pixel count: $512 \times 442$

- $\quad$ SIMS acquisitions: see Table 11.1

- Auto focus pixel dwell: $400 \mathrm{~ns}$

- Auto focus started to the right of the crater. The stage was moved to the left and auto focus was performed after the first set of 3 SIMS maps and was performed after each set of three thereafter.

Table 11.1. SIMS pixel dwells used for experiment \#1.

\begin{tabular}{|c|c|c|c|}
\hline run\# & Al dwell (ms) & Ti dwell (ms) & Mg dwell (ms) \\
\hline $\mathbf{0 0 0}$ & 0.9 & 3.6 & 0.47 \\
\hline $\mathbf{0 0 1}$ & 0.1 & 4 & 0.74 \\
\hline $\mathbf{0 0 2}$ & 0.1 & 2.49 & 0.73 \\
\hline $\mathbf{0 0 3}$ & 0.1 & 4 & 1.11 \\
\hline $\mathbf{0 0 4}$ & 0.11 & 4 & 1.12 \\
\hline $\mathbf{0 0 5}$ & 0.14 & 4 & 1.73 \\
\hline $\mathbf{0 0 6}$ & 0.15 & 4 & 1.76 \\
\hline $\mathbf{0 0 7}$ & 0.15 & 4 & 2.48 \\
\hline $\mathbf{0 0 8}$ & 0.1 & 4 & 3.23 \\
\hline $\mathbf{0 0 9}$ & 0.2 & 4 & 3.54 \\
\hline
\end{tabular}

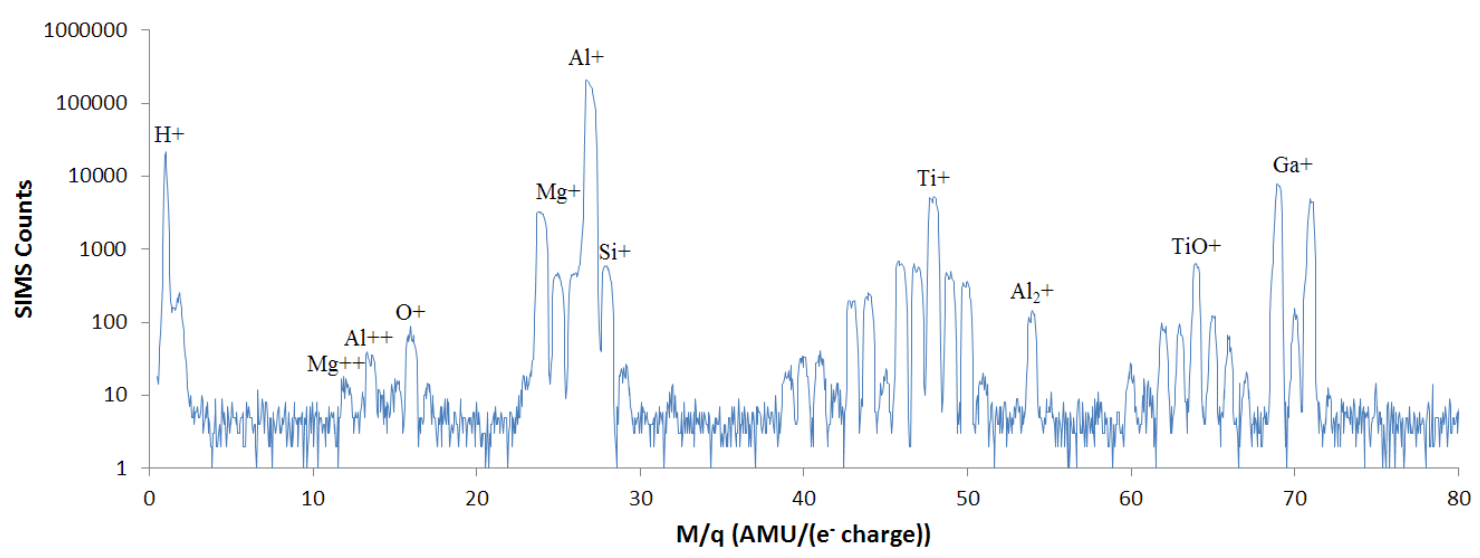

Figure 11.7. SIMS spectrum of AITiC using the $5.75 \mathrm{nA}$ beam. Peaks used for SIMS maps are the $\mathrm{Mg}+(24 \mathrm{amu}), \mathrm{Al}+(27 \mathrm{amu})$ and $\mathrm{Ti}+(48 \mathrm{amu})$. Traces of Carbon, Oxygen and doubly ionized Aluminum can be seen. 


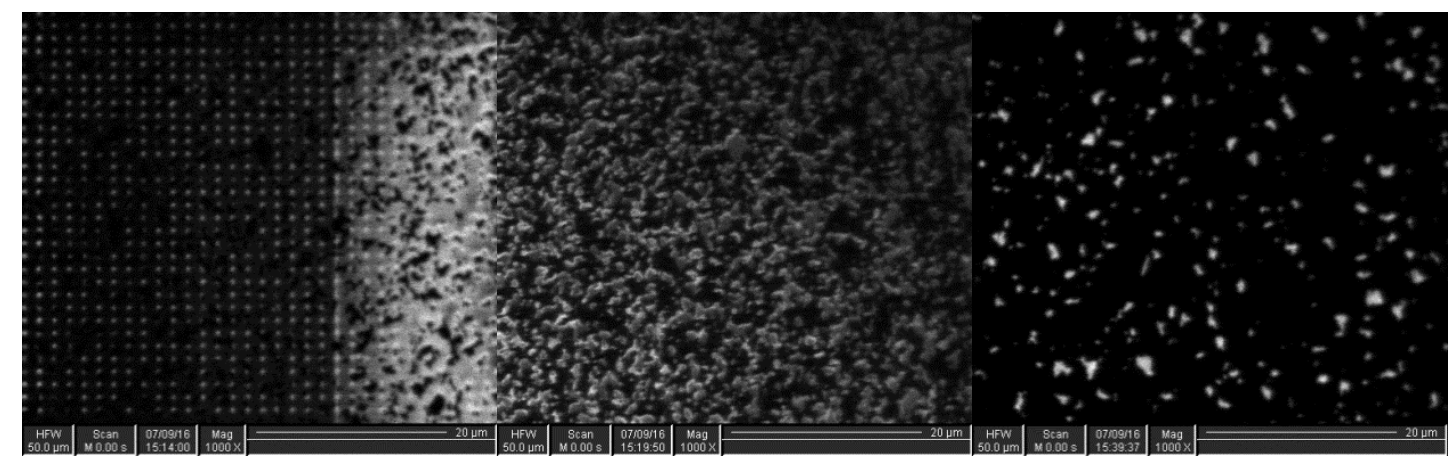

Figure 11.8. (left, center, right) Aluminum, Titanium and Magnesium SIMS maps shown in the order taken. These are the first set of SIMS maps to be merged into the first image of the 10-image sequence shown in Figure 11.12.

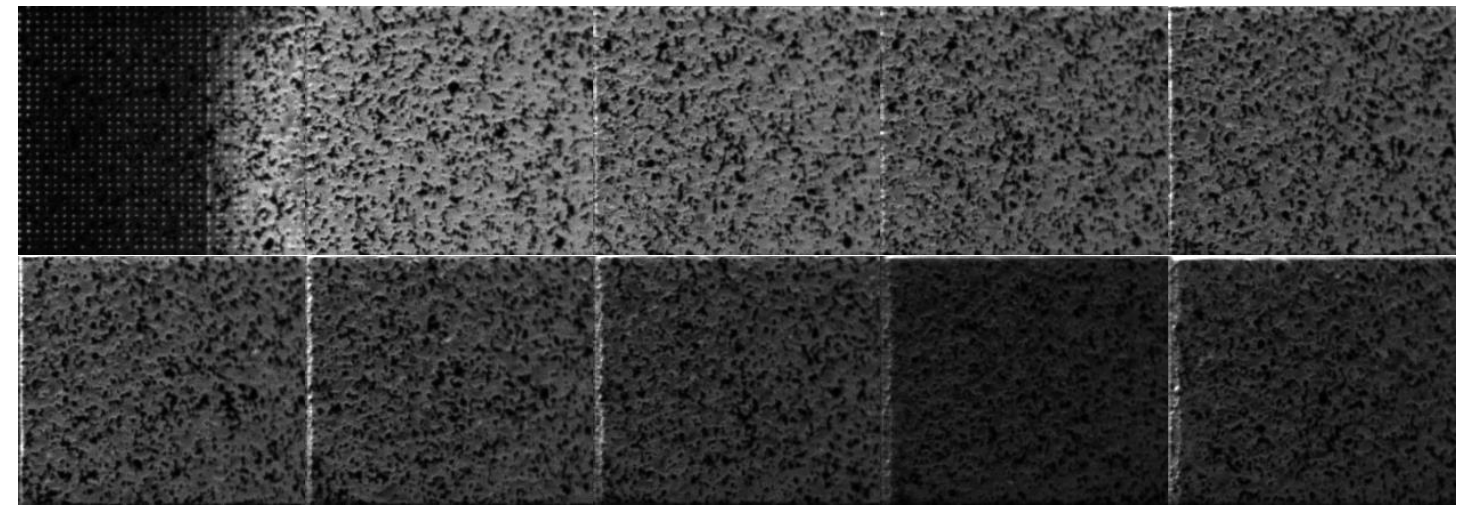

Figure 11.9. (left to right, top to bottom) Aluminum SIMS maps at $50 \mu \mathrm{m} \mathrm{HFW.} \mathrm{The} \mathrm{left} 2 / 3$ of the $1^{\text {st }}$ image is darkened by a thin layer of DLC. Raw images are shown.

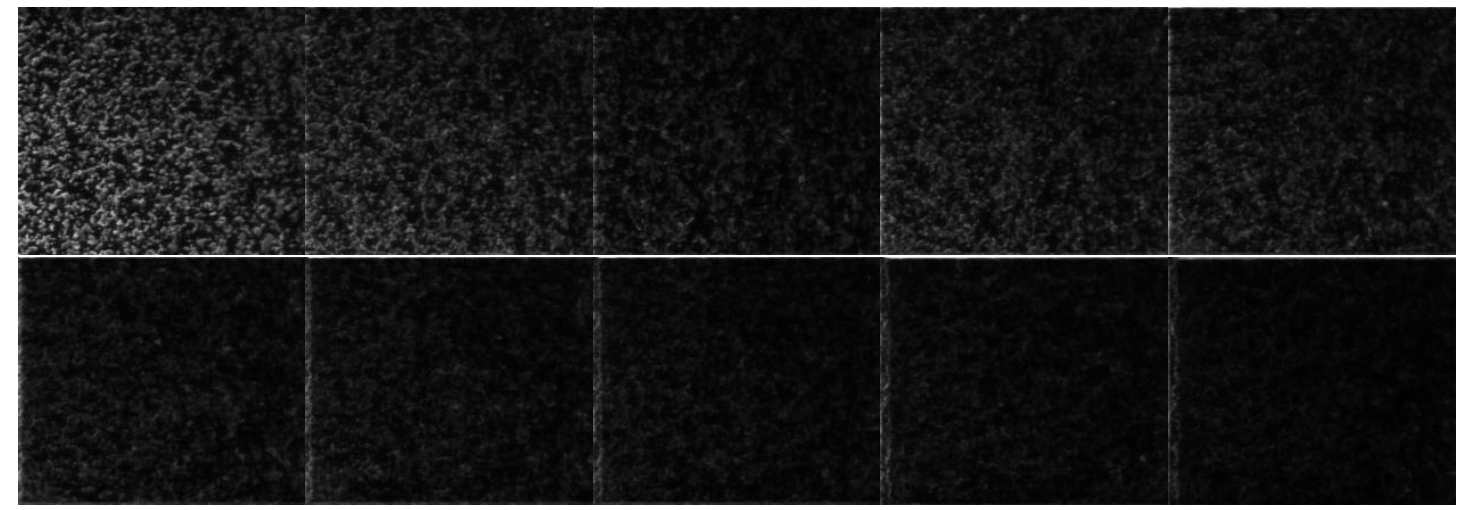

Figure 11.10. (left to right, top to bottom) Titanium SIMS maps at $50 \mu \mathrm{m}$ HFW. Raw images are shown. 


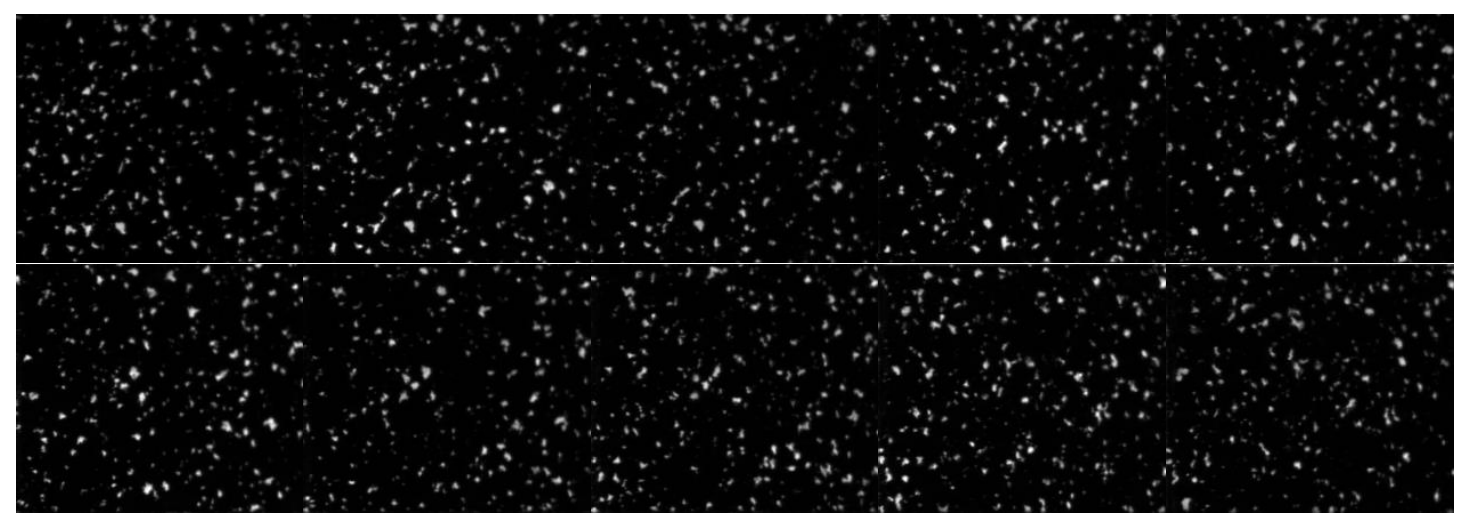

Figure 11.11. (left to right, top to bottom) Magnesium SIMS maps at $50 \mu \mathrm{m}$ HFW. Raw images are shown.

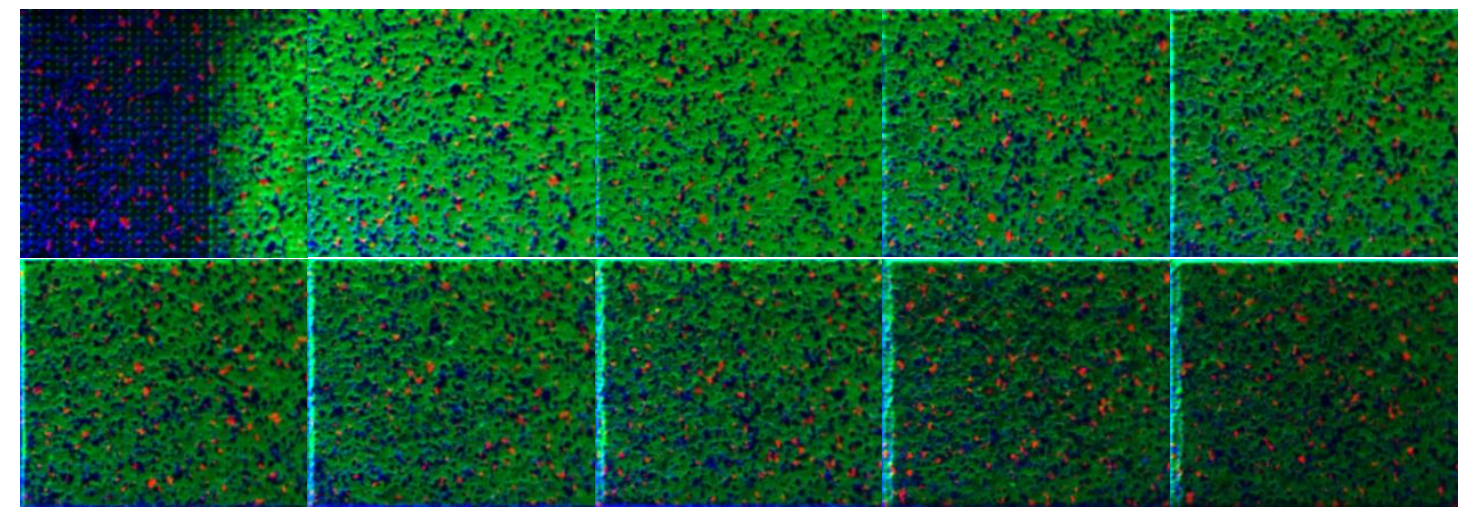

Figure 11.12. (left to right, top to bottom) SIMS maps using a large beam current of $5.75 \mathrm{nA}$ at 50 $\mu \mathrm{m}$ HFW. The first image shows the effect of the auto dwell determination as little dots on the DLC layer of the AITiC surface.

\subsection{Experiment \#1 results}

The first SIMS map revealed the effect of enabling the auto dwell determination option in the xP 2.25 SIMS software. When enabled, the system first acquires SIMS counts at every $16^{\text {th }}$ pixel. Once finished, it makes a determination for either a shorter or longer pixel dwell and presents the option to the user. The user can continue with the original dwell or take the suggested value. An interesting artifact in the form of these 
dots is evident on the first SIMS map in Figure 11.8.

The $5.75 \mathrm{nA}$ beam is large enough to use $50 \mu \mathrm{m} \mathrm{HFW}$, resulting in $100 \mathrm{~nm}$ pixels. The stronger SIMS signal allows for the $\mathrm{Al}$ and $\mathrm{Mg}$ pixel dwells to be less than the max, $4 \mathrm{~ms}$, for the duration of the milling, but $\mathrm{Mg}$ needs to be increased as the crater gets deeper. All of the species dropped in intensity as the crater got deeper. At this point it is difficult to determine if this is cause by enrichment due to nonstoichiometric milling or if this is an electrical problem. It is desirable to use the shortest pixel dwells possible.

Constant dwells for each species is preferred.

\subsection{Experiment \#2: High resolution, low milling rate SIMS}

Six sets of SIMS maps were acquired at masses 27, 24, 48 and 69 amu using the $250 \mathrm{pA}$ beam. The SIMS spectrum for $250 \mathrm{pA}$ is shown in Figure 11.13. The maximum count rate is about 20x smaller than for the $5.75 \mathrm{nA}$ spectrum shown in Figure 11.7 which results in longer pixel dwells for all species.

FIB-SIMS imaging conditions were as follows:

- $\quad$ Vbeam $=25 \mathrm{kV}$

- Ibeam $=250 \mathrm{pA}$

- $\mathrm{HFW}=20 \mu \mathrm{m}$

- XY pixel count: $512 \times 442$

- $\quad$ SIMS acquisitions: see Table 11.2

- Auto focus pixel dwell: $2400 \mathrm{~ns}$

- Auto focus started to the right of the crater. The stage was moved to the left and auto focus was performed after the first set of 3 SIMS maps and was performed after each set of three thereafter. 


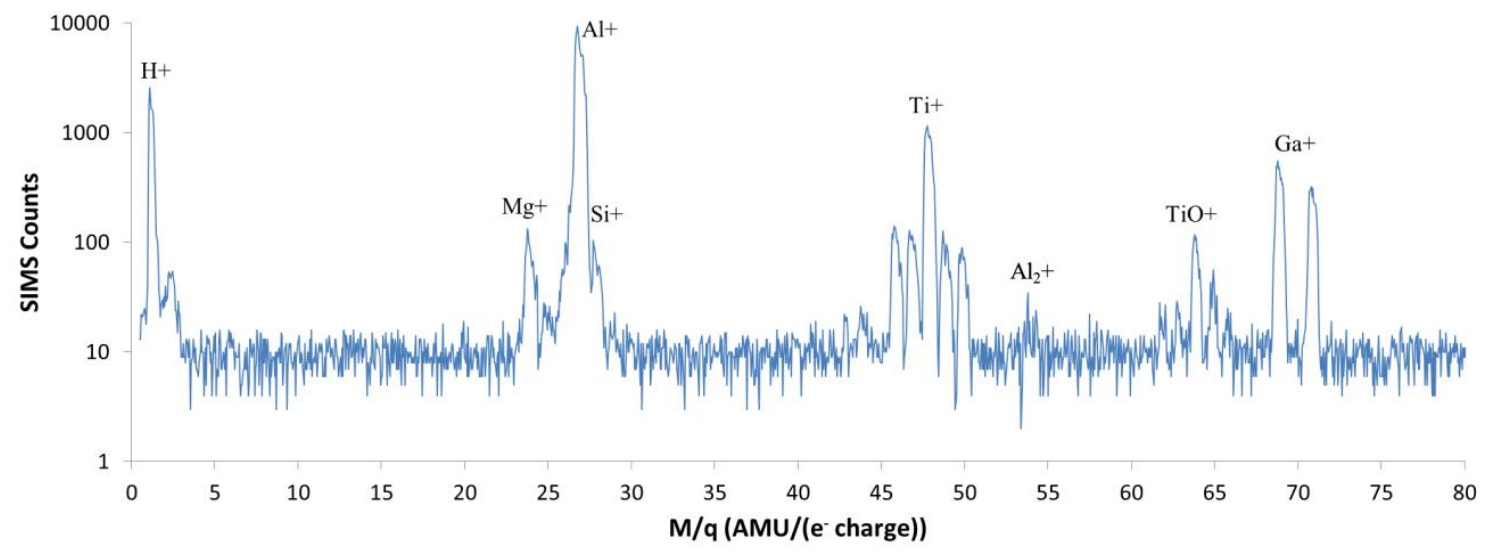

Figure 11.13. SIMS spectrum of AITiC using the $250 \mathrm{pA}$ beam. Peaks used for SIMS maps are the $\mathrm{Mg}+(24 \mathrm{amu}), \mathrm{Al}+$ (27 amu), Ti+ (48 amu) and Ga (69 amu).

Table 11.2. SIMS pixel dwells used for experiment \#2.

\begin{tabular}{|c|c|c|c|c|}
\hline run\# & Al dwell (ms) & Mg dwell (ms) & Ti dwell (ms) & Ga dwell (ms) \\
\hline $\mathbf{0 0 0}$ & 2 & 4 & 4 & 4 \\
\hline $\mathbf{0 0 1}$ & 1.5 & 4 & 4 & 4 \\
\hline $\mathbf{0 0 2}$ & 0.77 & 4 & 4 & 4 \\
\hline $\mathbf{0 0 3}$ & 1 & 4 & 4 & 4 \\
\hline $\mathbf{0 0 4}$ & 1 & 4 & 4 & 4 \\
\hline $\mathbf{0 0 5}$ & 1 & 4 & 4 & 4 \\
\hline
\end{tabular}

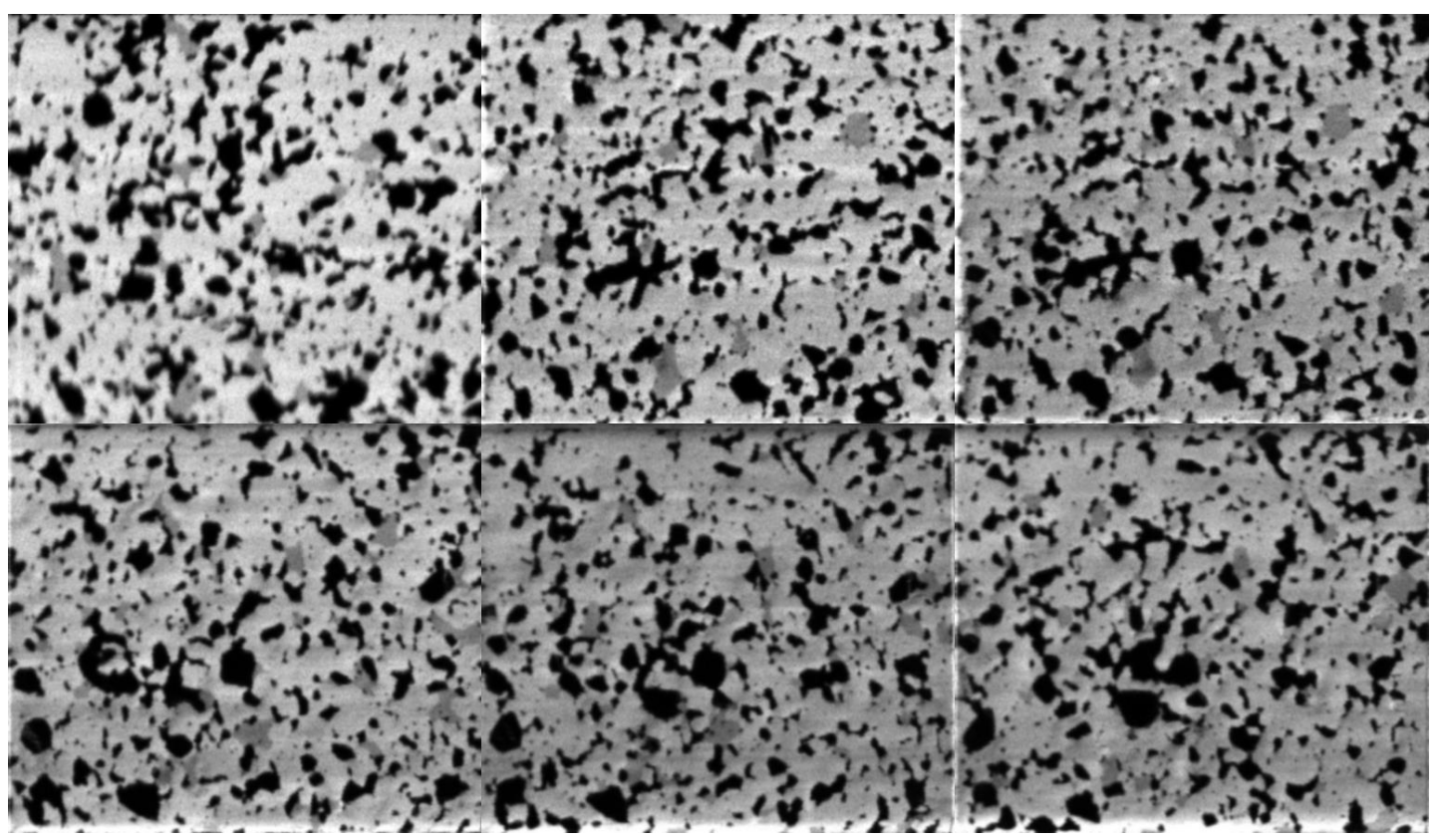

Figure 11.14. (left to right, top to bottom) Al SIMS maps. Images have been processed to boost contrast. 


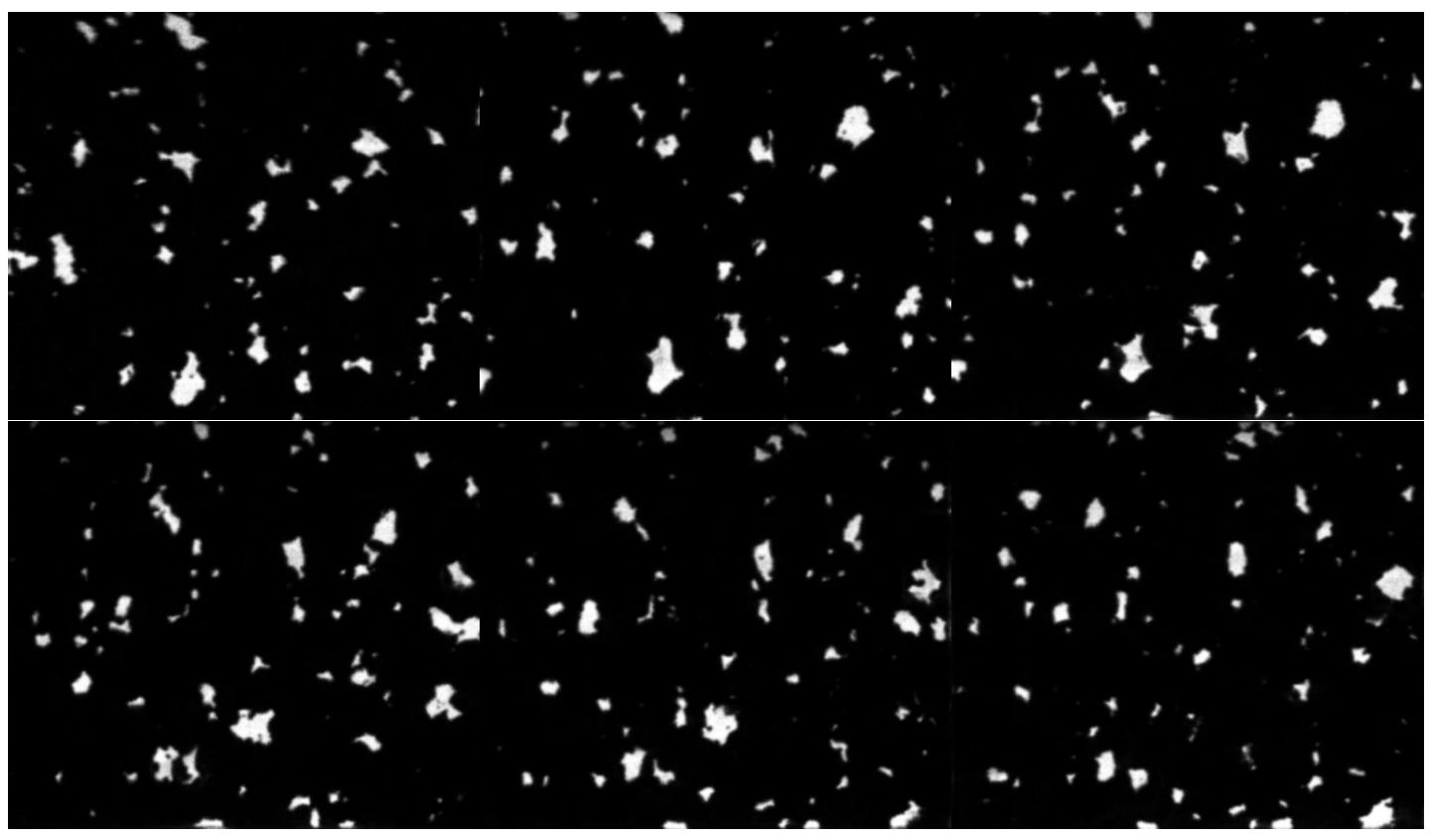

Figure 11.15. (left to right, top to bottom) Magnesium SIMS maps. Images have been processed to boost contrast.

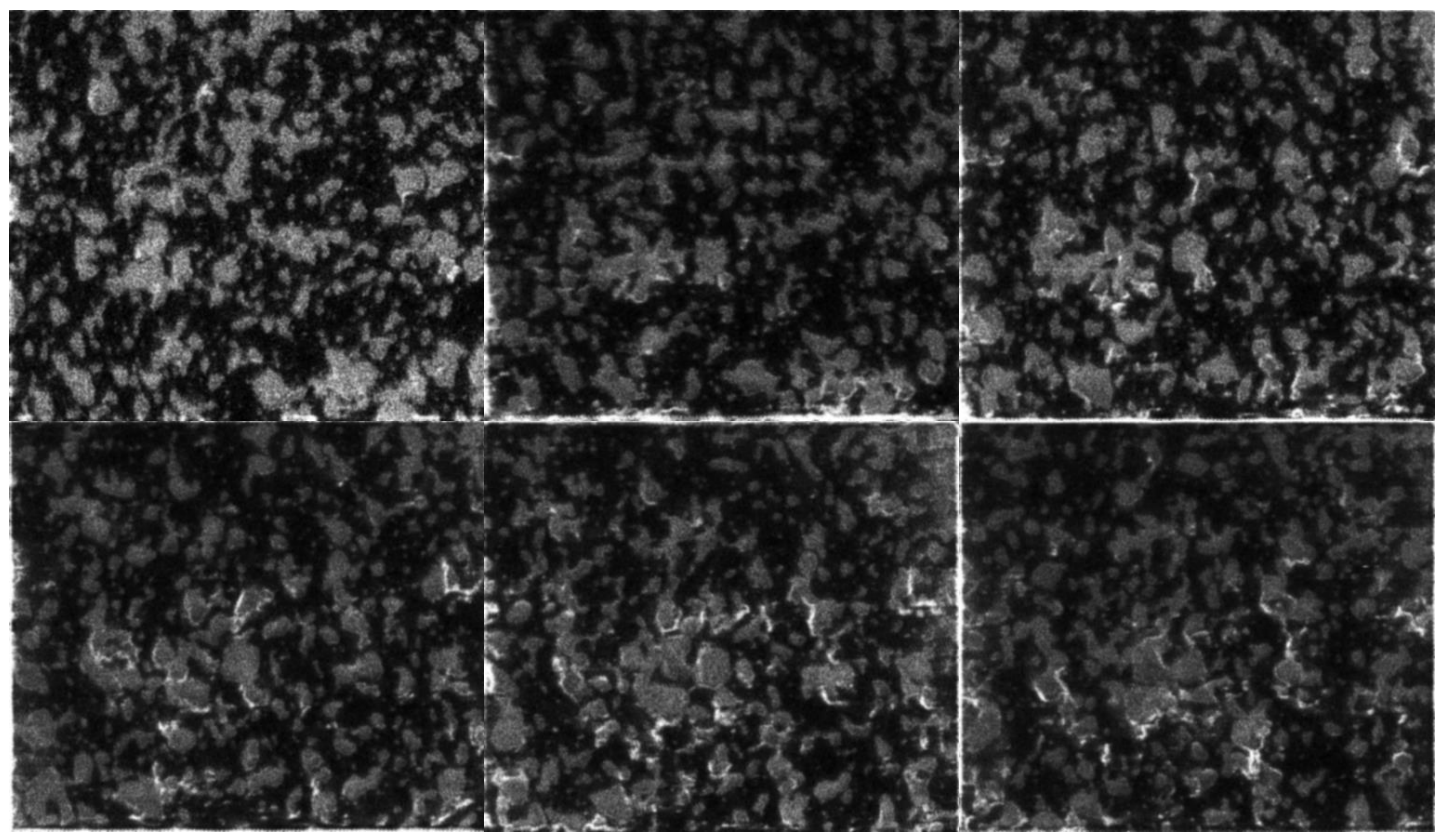

Figure 11.16. Ti SIMS maps. Images have been processed to boost contrast. 


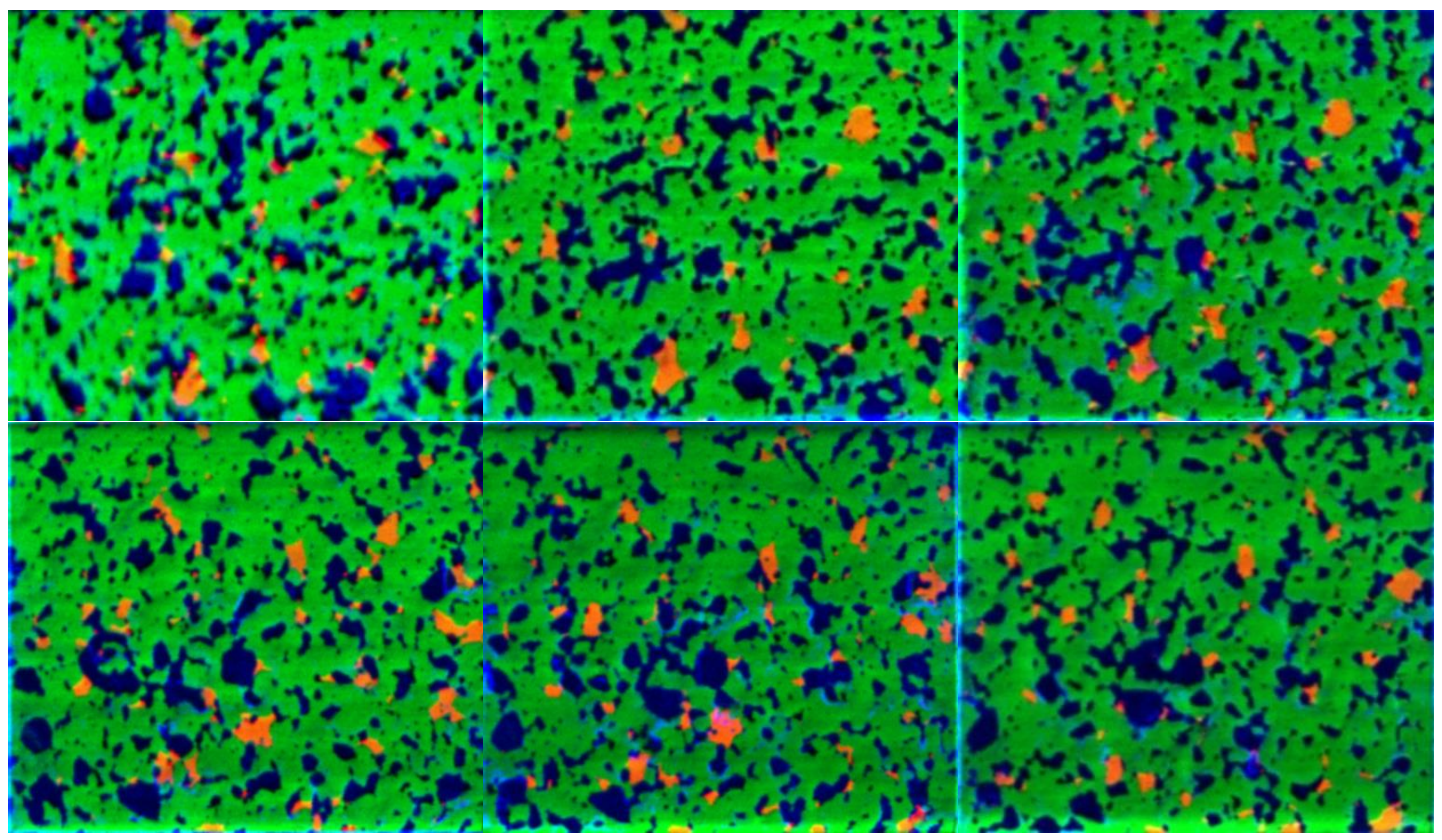

Figure 11.17. (left to right, top to bottom) SIMS maps showing Mg (red), $\mathrm{Al}$ (green) and Ti (blue).

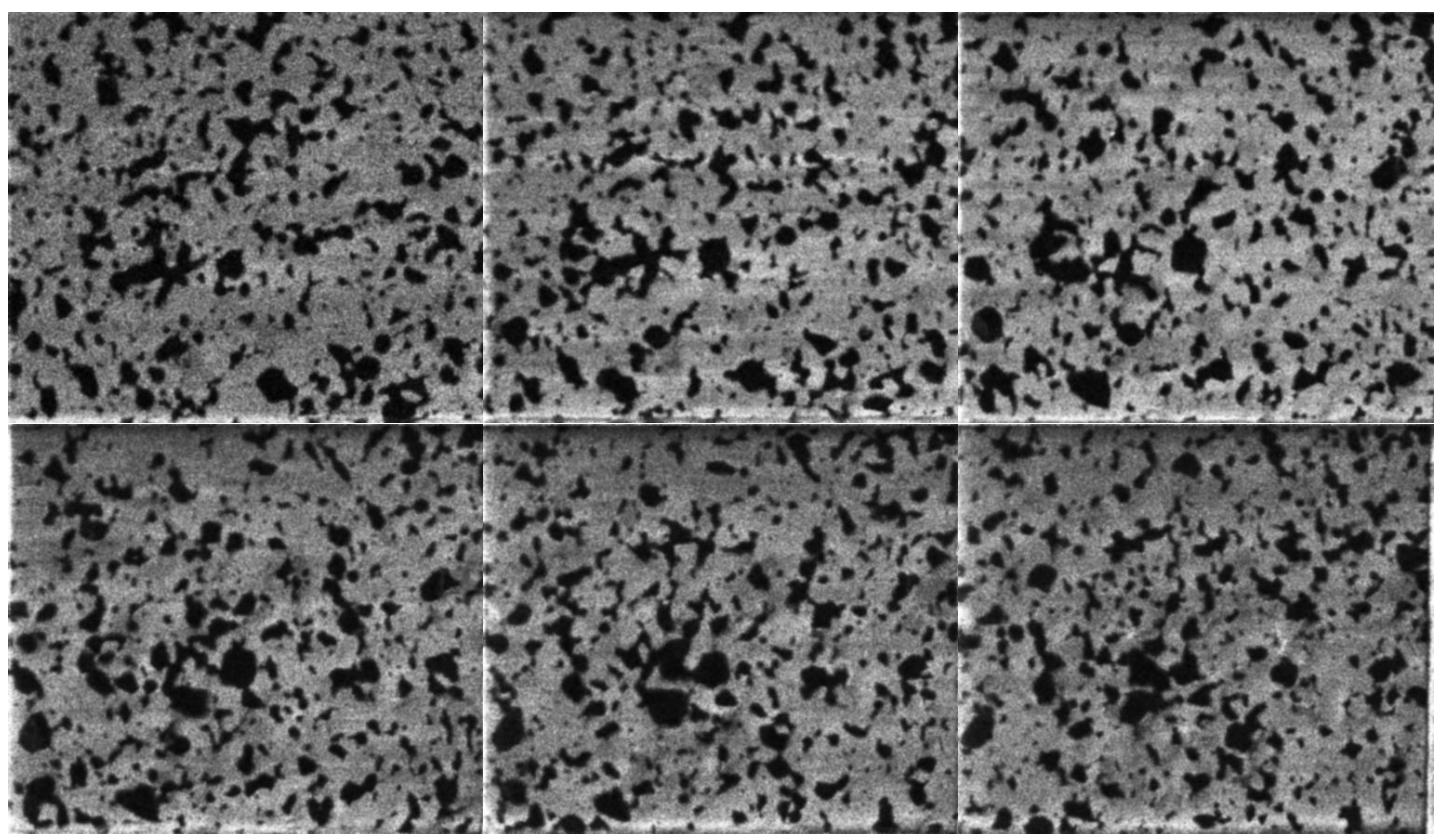

Figure 11.18. (left to right, top to bottom) Ga SIMS maps. Images have been processed to boost contrast. 


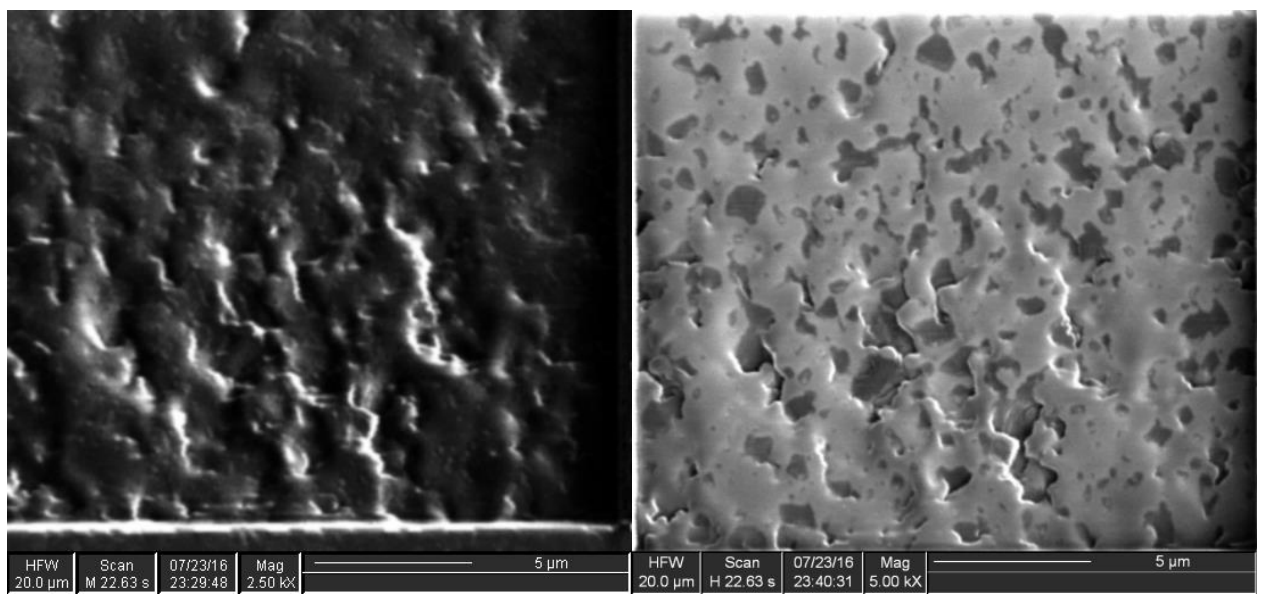

Figure 11.19. (left) Last FIB SE image acquired inside the crater with the SIMS optics turned on. Its contrast is adversely affected by energizing the SIMS optics. (right) FIB SE image after turning off the SIMS optics and re-centering using the stage. Both images have been processed to boost contrast.

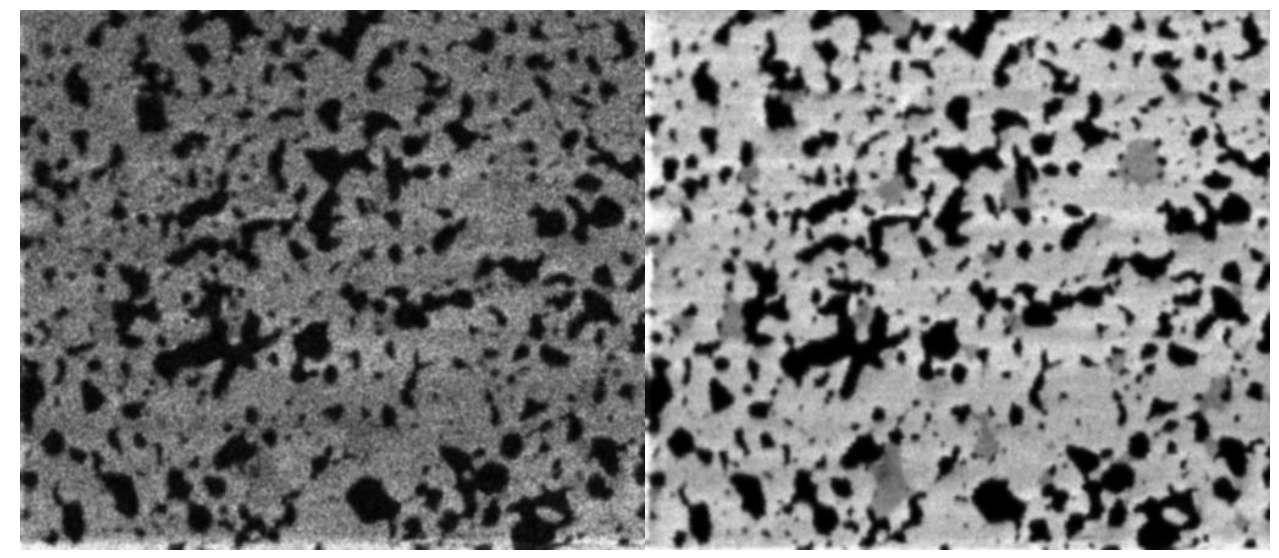

Figure 11.20. (left) Ga SIMS map for run \#000, which is the last one in the \#000 set. (right) Al SIMS map, which is the $1^{\text {st }}$ one in run \#001, thus being taken directly after the Ga SIMS map on the left. These images are very similar, showing nearly identical TiC regions as dark islands. The main differences are the light regions in the Al SIMS map, which are locations for Mg.

\subsection{Experiment \#2 results}

The 250 pA SIMS maps showed a lot of detail. However, the total milling depth per set of species is relatively high. Using low beam currents to mill the crater means minimizing the number of masses scanned to reduce the total dose per SIMS map. The number of masses scanned could be (\# species - 1), interpreting one of the un-scanned 
masses as the black regions left over from the others.

Taking the time to acquire Ga SIMS maps was worthwhile in that they look similar to the Al SIMS maps. They reveal that Ga is leaving the sample everywhere there is $\mathrm{Al}$ and $\mathrm{Mg}$. The spots where $\mathrm{Mg}$ causes reduced SIMS counts in the Al SIMS map are not evident in the Ga SIM map.

The crater showed signs of scalloping in the FIB SE images. Interestingly, the Al, Mg and Ga SIMS maps did not reveal it but the Ti SIMS maps did.

\subsection{Summary}

Although the intensity dropped as the crater got deeper using both $250 \mathrm{pA}$ and $5.75 \mathrm{nA}$, the quality of the SIMS maps allowed for histogram stretching to achieve good composite 2D maps for both currents. This SIMS system generally exhibits this behavior and may be in need of repair. There is some difficulty with interpretation of the SIMS data, making it difficult to determine how much the slow degradation of the acquisitions is due to physics and how much is electrical.

Combining these results with those of the AFM-FIB allows us to move forward. Since the AFM is the slowest part of this process, careful planning for each stack of SIMS maps and AFM images is needed to reduce the acquisition time.

\subsection{References}

Benninghoven, A, F. G Rüdenauer, and H. W Werner. Secondary Ion Mass Spectrometry: Basic Concepts, Instrumental Aspects, Applications, and Trends. New York: J. Wiley, 1987. 
Bhushan, Bharat. "Chemical, Mechanical and Tribological Characterization of Ultra-Thin and Hard Amorphous Carbon Coatings as Thin as 3.5 Nm: Recent Developments."

Diamond and Related Materials 8, no. 11 (1999): 1985-2015.

Bhushan, Bharat. "Micro/Nanomechanics of Magnetic Storage Devices," 1999. http://dlia.ir/nano.tech/english/Books/09/131.pdf.

Sourty, E., J. L. Sullivan, and M. D. Bijker. "The Tribology of Advanced Digital Recording (ADR) Systems.” Tribology International 33, no. 9 (2000): 629-637.

Whitby, James A., Fredrik Östlund, Peter Horvath, Mihai Gabureac, Jessica L. Riesterer, Ivo Utke, Markus Hohl, et al. "High Spatial Resolution Time-of-Flight Secondary Ion Mass Spectrometry for the Masses: A Novel Orthogonal ToF FIB-SIMS Instrument with In Situ AFM." Advances in Materials Science and Engineering 2012 (2012): 1-13. doi:10.1155/2012/180437.

Wirtz, Tom, Yves Fleming, Mathieu Gerard, Urs Gysin, Thilo Glatzel, Ernst Meyer, Urs Wegmann, Urs Maier, Aitziber Herrero Odriozola, and Daniel Uehli. 'Design and Performance of a Combined Secondary Ion Mass Spectrometry-Scanning Probe Microscopy Instrument for High Sensitivity and High-Resolution Elemental ThreeDimensional Analysis." Review of Scientific Instruments Review of Scientific Instruments 83, no. 6 (2012): 063702.

Yamamoto, Takayuki, Yoshiharu Kasamatsu, and Hiroyuki Hyodo. "Advanced StictionFree Slider and DLC Overcoat." Fujitsu Scientific and Technical Journal 37, no. 2 (2001): 201-11. 


\section{Approximate cost, schedule and future plans}

The original projected cost in 2006 for this project was for $\$ 76,015.50$, which included a $\$ 30,000.00$ coarse tip positioning stage made by AttoCube for the AFM's coarse positioning stage. At the time of my prospectus defense, November 2012, I had instead agreed to construct the coarse positioning stage myself. This reduced the estimate to

$\$ 47,785.00$.

\subsection{Projected vs actual costs}

Table 12.1. AFM-SIMS project projected costs.

\begin{tabular}{|c|c|c|}
\hline \multicolumn{3}{|l|}{ ITEM } \\
\hline Main System Controller (FIB and SIMS) & $\begin{array}{c}\text { Projected Cost at } \\
\text { time of Project } \\
\text { Proposal }\end{array}$ & Actual Cost \\
\hline 1- NI FPGA Card -PCI-7833R & $\$ 4,950.00$ & $\$ 4,950.00$ \\
\hline Fast Computer w/ 3 monitors + quad video card. & $\$ 4,000.00 *$ & $\$ 0$ \\
\hline NI Cables and connectors & $\$ 1000.00$ & $\$ 1000.00$ \\
\hline \multicolumn{3}{|l|}{ Scan Head \& Controllers (AFM ) } \\
\hline $\begin{array}{l}1 \text { - P-010.40H PICA Piezo stack (see Physik Instrumente } \\
\text { quote) }\end{array}$ & $\$ 1160.00$ & $\$ 1160.00$ \\
\hline $\begin{array}{l}2 \text { - E-610.S0 Controller for P-612.2SL (see Physik } \\
\text { Instrumente quote) }\end{array}$ & $\$ 4,850.00$ & $\$ 4,850.00$ \\
\hline $\begin{array}{l}1 \text { - P-612.2SL Mini XY } 100 \mu \mathrm{m} \text { x } 100 \mu \mathrm{m} \text { scan stage (see } \\
\text { Physik Instrumente quote) }\end{array}$ & $\$ 7,500.00$ & $\$ 7,500.00$ \\
\hline $\begin{array}{l}2 \text { - SQL-100 Squiggle motor and controller for coarse XY tip } \\
\text { placement (Newscale technologies) }\end{array}$ & $\$ 2,000.00$ & $\$ 2,000.00$ \\
\hline $\begin{array}{l}1-\text { SQL-3.4-E squiggle motor and controller for coarse tip } \\
\text { approach (Newscale technologies) }\end{array}$ & $\$ 825.00$ & $\$ 825.00$ \\
\hline HV Power supplies for main controller & $\$ 1,500.00 *$ & $\$ 0$ \\
\hline \multicolumn{3}{|l|}{ MISC } \\
\hline $\begin{array}{l}\text { Circuit Boards \& components (HV op-amps, connectors, } \\
\text { chips, etc..) }\end{array}$ & $\$ 5,000.00 * *$ & \multirow[t]{4}{*}{$\$ 1000.00$} \\
\hline Machining Shop time & $\$ 5,000.00^{*}$ & \\
\hline Microscopy center time for SEM, TEM usage & $\$ 8,000.00^{*}$ & \\
\hline Misc. chemicals and perishables for sample fabrication & $\$ 2,000.00 * *$ & \\
\hline Totals: & $\$ 47,785.00$ & $\$ 23,285.00$ \\
\hline
\end{tabular}

Many of the items on this list $(*)$ were not purchased, but were instead modified 
from non-working equipment found around the laboratory, from my personal collection, or from castoffs from the company I work for. Most of the machining was done in my home shop. I have a small CNC milling machine and a 12"x36" metal-working lathe fitted with digital readouts (DRO's). My time was not counted. The summed amount spent on items $\left({ }^{*}\right)$ was less than one thousand dollars. As a result, the final cost to the University for the development of this instrument was actually less than $\$ 24,000.00$.

Below is the schedule, as defined in the original grant proposal for this project.

For all practical purposes, it began at the start of year 2 .

Table 12.2. AFM-SIMS proposed schedule

\begin{tabular}{|l|r|r|r|}
\hline Process & Year 1 & Year 2 & Year 3 \\
\hline Purchasing & & & \\
\hline Design and \& Machining Parts for AFM & NFM control \& Misc. Circuit board Design and & & \\
\hline Fabrication & & & \\
\hline Software Development & & & \\
\hline Testing, Calibration \& Debugging & & & \\
\hline Analyzing Samples & & & \\
\hline $\begin{array}{l}\text { Development and deployment of the webpage for } \\
\text { dissemination of design }\end{array}$ & & & \\
\hline
\end{tabular}

\subsection{Decision not to open source the AFM-SIMS design}

The ANSOM design was open sourced so that others may construct their own apparatus. This AFM/SIMS system was not. The decision not to open-source this design comes from multiple considerations. First is that some of the hardware/software used is no longer available. This includes the unavailability of Squiggle motors which were central to the coarse stage design. Second is that the availability of lower cost electronics 
and control/development software makes it worthwhile to redesign some of these components. Third is that a better electronics design is needed for reduced noise during operation. Finally, an open-source design can be a lot of effort to maintain without dedicated resources and budget.

\subsection{Possible future work}

\subsubsection{AFM}

The AFM was based on an open source design, and while it works quite well, it uses technology which is now ten or more years old. Certain elements of this technology have either become drastically cheaper or have increased performance for the same cost. Such items include:

1) FPGA has now reached the mainstream, including low cost development boards and software tools.

2) High performance op-amps, ADC's and DAC's are now available for a few dollars each. System cost for new designs can be drastically reduced.

3) Computing hardware and software are literally orders of magnitude faster than they were in 2006. It is now more expensive to maintain old hardware/software than it is to create new.

Much effort was put into making this AFM design so that it can be easily

replicated. Unfortunately, the Squiggle motors are no longer available in the formats used in this design, a new design is needed for assembly of new AFM coarse XY stages.

The controller electronics are constructed using single-ended analog op-amps. This has led to relatively large ground plane noise creeping into the control signals. These circuits will eventually be redesigned using the latest in op-amp technology and 
differential signaling.

The controller FPGA cost can be reduced greatly by switching to a newer, cheaper design. The FPGA software cost can similarly be reduced by using something like webPack from Xilinx.

\subsubsection{FIB -SIMS}

The Fei FIB 611 is a robust platform to build on. For the most part, it is constructed of hardware and electronic components which are still available today. However, the machine is almost 25 years old now and some things may become problematic in the future.

The vacuum console is driven from a PAL-based state machine and its design may be difficult to replace directly. Instead, a microcontroller-based state machine, combined with discrete logic components would be simpler to implement when the need arises.

The control PC \& Software can be found on the surplus marked from time to time when replacements are needed. However, this is not sustainable or even desirable for evolution of this AFM-SIMS instrument. Computing technology has advanced to the point where the entire microscope control can be handled by a modern embedded controller. Similarly, the DAQ cards used to control the SIMS can be replaced with cheaper, higher performance designs. Eventual software enhancements to include: 
1) Integrated FIB control to include imaging patterning and special functions.

2) Remote control/handshaking operation for complete integration with other instruments.

3) Integrated optical imaging.

4) Integrated stage control.

5) SIMS optics control to accommodate formulaic relationships between optical elements for faster optimization.

6) SIMS maps to accommodate multiple masses on a line-by-line basis, each with its own pixel dwell, to reduce the effect of drift.

7) Image shift and stigmation automatically corrected for during SIMS operation.

As stated earlier, the FIB 611 platform is a robust design and so can in principle be used indefinitely. The stepper motor stage can be repaired as needed and its motor drivers can easily be replaced using the latest offerings.

Suggested enhancements to the existing system include:

1) Computer control of stigmators

2) Computer control of beam shift

The SIMS-I generally has a lower transmission capability than the SIMS-III. Its energy analyzer, while useful for certain surface studies is not as useful for SIMS nanotomography since it possibly blocks many of the ions created at the sample. More ions can be generated by introducing $\mathrm{O}_{2}$ at the site of FIB milling. The SIMS-I contains a nozzle for this and it should be evaluated before deciding to switch to another SIMS design.

Being a single beam system, it takes practice to get good at coarse tip placement using the FIB. A side-mounted optical telescopic microscope would be a welcome addition to this instrument. Among the GIS ports on this system, there is one of them available for mounting an optical system. It is in a relatively safe spot for mounting such a device, virtually free of interference with any of the stage elements. 
Lastly, it is worth mentioning that the machine can be used as-is for some time without any upgrades at all.

\subsection{References}

Castaldo, V., C.W. Hagen, and P. Kruit. "Simulation of Ion Imaging: Sputtering, Contrast, Noise." Ultramicroscopy 111, no. 8 (July 2011): 982-94.

doi:10.1016/j.ultramic.2011.03.019. 


\section{BIBLIOGRAPHY}

Altermann, Wladyslaw, and Józef Kazmierczak. "Archean Microfossils: A Reappraisal of Early Life on Earth.” Research in Microbiology 154, no. 9 (November 2003): 611-17. doi:10.1016/j.resmic.2003.08.006.

Ando, Masaaki, and Julius J. Muray. "Spatial Resolution Limit for Focused Ion-Beam Lithography from Secondary-Electron Energy Measurements." Journal of Vacuum Science \& Technology B: Microelectronics Processing and Phenomena 6, no. 3 (1988): 986-988.

Andrews, Harry C, and Bobby Ray Hunt. Digital Image Restoration. Englewood Cliffs, N.J.: Prentice-Hall, 1977.

Azarian, M. H., C. L. Bauer, and M. S. Jhon. "Tribology of Thin-Film Media in Both Flying and Sliding Modes." Wear 168, no. 1 (September 1, 1993): 59-76.

doi:10.1016/0043-1648(93)90199-V.

Bardi, U, S.P Chenakin, A Lavacchi, C Pagura, and A Tolstogouzov. "Sputter Depth Profiling by Secondary Ion Mass Spectrometry Coupled with Sample Current Measurements.” APSUSC Applied Surface Science 252, no. 20 (2006): 7373-82.

Baroody, E. M. “A Theory of Secondary Electron Emission from Metals.” Physical Review 78, no. 6 (1950): 780.

Barth, J. E, and P Kruit. "Addition of Different Contributions to the Charged Particle Probe Size." OPTIK -STUTTGART-101, no. 3 (1996): 101-9.

Benninghoven, A, F. G Rüdenauer, and H. W Werner. Secondary Ion Mass Spectrometry: Basic Concepts, Instrumental Aspects, Applications, and Trends. New York: J. Wiley, 1987.

Bielefeldt, H, and F J Giessibl. “A Simplified but Intuitive Analytical Model for Intermittent-Contact-Mode Force Microscopy Based on Hertzian Mechanics." Surface Science. 440, no. 3 (1999): L863.

Bhushan, Bharat. "Chemical, Mechanical and Tribological Characterization of Ultra-Thin and Hard Amorphous Carbon Coatings as Thin as 3.5 Nm: Recent Developments." Diamond and Related Materials 8, no. 11 (1999): 1985-2015.

Bhushan, Bharat. "Micro/Nanomechanics of Magnetic Storage Devices," 1999. http://dlia.ir/nano.tech/english/Books/09/131.pdf. 
Bigler, Jeff. "Periodic-Table.xls," n.d. http://www.mrbigler.com/documents/PeriodicTable.xls.

Binnig, Gerd, Calvin F. Quate, and Ch Gerber. "Atomic Force Microscope.” Physical Review Letters 56, no. 9 (1986): 930.

Bracewell, Ronald N. Fourier Analysis and Imaging. New York: Springer, 2006.

Brasier, Martin D, Owen R Green, John F Lindsay, Nicola McLoughlin, Andrew Steele, and Cris Stoakes. "Critical Testing of Earth's Oldest Putative Fossil Assemblage from the 3.5 Ga Apex Chert, Chinaman Creek, Western Australia." PRECAM Precambrian Research 140, no. 1 (2005): 55-102.

Cady, Sherry L., Jack D. Farmer, John P. Grotzinger, J. William Schopf, and Andrew Steele. "Morphological Biosignatures and the Search for Life on Mars." Astrobiology 3, no. 2 (2003): 351-368.

Cai, K. F., D. S. McLachlan, N. Axen, and R. Manyatsa. "Preparation, Microstructures and Properties of Al 2 O 3-TiC Composites." Ceramics International 28, no. 2 (2002): $217-222$.

Castaldo, V., C.W. Hagen, and P. Kruit. "Simulation of Ion Imaging: Sputtering, Contrast, Noise." Ultramicroscopy 111, no. 8 (July 2011): 982-94.

doi:10.1016/j.ultramic.2011.03.019.

Castleman, Kenneth R. Digital Image Processing. Englewood Cliffs, N.J.: Prentice-Hall, 1979.

Clark, B.K. "The effect of slider roughness on asperity testing of thin-film media." IEEE Trans. Magn. IEEE Transactions on Magnetics 29, no. 1 (1993): 235-40.

Curnutt, J, E Gomez, and K.E Schubert. "Patterned Growth in Extreme Environments." ASTRONOMICAL SOCIETY OF THE PACIFIC CONFERENCE SERIES 420 (2009): $233-38$.

Cutler, Raymond A., Andrew C. Hurford, and Anil V. Virkar. "Pressureless-Sintered Al 20 3-TiC Composites." Materials Science and Engineering: A 105 (1988): 183-192.

Doughty, Jeffrey Jon. "Symmetric Near-Field Probe Design and Comparison to Asymmetric Probes," 2010. http://pdxscholar.library.pdx.edu/open_access_etds/390/.

Edwards, Hal, Larry Taylor, Walter Duncan, and Allan J Melmed. "Fast, HighResolution Atomic Force Microscopy Using a Quartz Tuning Fork as Actuator and Sensor." Journal of Applied Physics. 82, no. 3 (1997): 980. 
Elektros. “ETEM User Manual.pdf.” Elektros, circa 1975.

Extrel Corporation, "Model C50 Mass Spectrometer User Reference Manual”, P.O. Box 11512 Pittsburgh, Pennsylvania 15238

FEI Company, "SD Gallium LMIS High Speed Blanking MVA Focusing Column with Electronics User's Guide", PN 16887 rev A, 1993

Fowles, Grant R. Analytical Mechanics. Philadelphia: Saunders, 1986.

Fox Electronics, “Thru-Hole Tuning Fork Data Sheet”, 2012

Fraser, Stephen. Pro Visual C++/CLI and the .NET 2.0 Platform. Berkeley, CA; New York: Apress ; Springer-Verlag [distributor], 2006.

Galileo Electro-Optics Corporation, "Channeltron Electron Multiplier Handbook for Mass Spectrometry Applications”, Galileo Park, P.O. Box 550 Sturbridge, MA 01566

Giessibl, F. J, and M Tortonese. "Self-Oscillating Mode for Frequency Modulation Noncontact Atomic Force Microscopy.” APPLIED PHYSICS LETTERS 70, no. 19 (1997): 2529-31.

Giessibl, F. J. "A Direct Method to Calculate Tip-Sample Forces from Frequency Shifts in Frequency-Modulation Atomic Force Microscopy." APPLIED PHYSICS LETTERS 78 (2001): 123-25.

Giessibl, F. J. “AFMs Path to Atomic Resolution.” MATERIALS TODAY -OXFORD THEN KIDLINGTON- 8, no. 5 (2005): 32-41.

Giessibl, Franz J. "Forces and Frequency Shifts in Atomic-Resolution Dynamic-Force Microscopy." Physical Review. B, Condensed Matter. 56, no. 24 (1997): 16010.

Giessibl, Franz J. “Atomic Resolution on Si (111)-(7x7) by Noncontact Atomic Force Microscopy with a Force Sensor Based on a Quartz Tuning Fork." Applied Physics Letters 76, no. 11 (2000): 1470-1472.

Giessibl FJ, Herz M, and Mannhart J. "Friction Traced to the Single Atom." Proceedings of the National Academy of Sciences of the United States of America 99, no. 19 (2002): 12006-10.

Giessibl F.J, Pielmeier F, Eguchi T, An T, and Hasegawa Y. "Comparison of Force Sensors for Atomic Force Microscopy Based on Quartz Tuning Forks and LengthExtensional Resonators." Phys. Rev. B Condens. Matter Mater. Phys. Physical Review B - Condensed Matter and Materials Physics 84, no. 12 (2011). 
Giannuzzi, Lucille A, and F. A Stevie. Introduction to Focused Ion Beams:

Instrumentation, Theory, Techniques, and Practice. New York: Springer, 2005.

Giannuzzi, L.A, and M Utlaut. "Non-Monotonic Material Contrast in Scanning Ion and Scanning Electron Images." Ultramicroscopy Ultramicroscopy 111, no. 11 (2011): 156473.

E. K. Gibson Jr., F. Westall, D. S. McKay, K. Thomas-Keprta, S. Wentworth, and C. S. Romanek, "EVIDENCE FOR ANCIENT MARTIAN LIFE", Mail Code SN2, NASA Johnson Space Center, Houston TX 77058, USA.

Gonzalez, Rafael C, and Richard E Woods. Digital image processing. Reading, Mass.: Addison-Wesley, 1993.

Grober, Robert D., Jason Acimovic, Jim Schuck, Dan Hessman, Peter J. Kindlemann, Joao Hespanha, A. Stephen Morse, Khaled Karrai, Ingo Tiemann, and Stephan Manus. "Fundamental Limits to Force Detection Using Quartz Tuning Forks." Review of Scientific Instruments 71, no. 7 (July 2000): 2776-80. doi:10.1063/1.1150691.

http://gwyddion.net/documentation/user-guide-en/multidata.html\#data-arithmetic

Hagen, C. W., E. Fokkema, and P. Kruit. "Brightness Measurements of a Gallium Liquid Metal Ion Source." Journal of Vacuum Science \& Technology B: Microelectronics and Nanometer Structures 26, no. 6 (November 2008): 2091-96. doi:10.1116/1.2987958.

Hansen, Per Christian. Discrete Inverse Problems: Insight and Algorithms. Philadelphia: Society for Industrial and Applied Mathematics, 2010.

Harriott, L. R. "Focused ion beam secondary ion mass spectrometry: Ion images and endpoint detection." J. Vac. Sci. Technol. B Journal of Vacuum Science \& Technology B: Microelectronics and Nanometer Structures 7, no. 2 (1989): 181.

Herz, M, F. J Giessibl, and J Mannhart. "Probing the Shape of Atoms in Real Space." Phys. Rev. B Physical Review B 68, no. 4 (2003).

T. Ihn, T. Vancura, A. Baumgartner, P. Studerus, K. Ensslin, “ Operating a phase-locked loop for controlling a high-Q tuning fork sensor for scanning force microscopy", arXiv: cond-mat/0112415v1 [cond-mat.mes-hall] 21 Dec 2001

IMSI. TurboCAD Version 19 User's Guide. 25 Leveroni Court Novato, CA 94949: IMSI/Design, 2012. www.TurboCAD.com.

Ishitani, T, T Yamanaka, K Inai, and K Ohya. "Secondary Electron Emission in Scanning Ga Ion, He Ion and Electron Microscopes." Vacuum Vacuum 84, no. 8 (2010): 1018-24. 
Itay Halevy1,2, Woodward W. Fischer, and John M. Eiler, "Carbonates in the Martian meteorite Allan Hills 84001 formed at $18+/-4{ }^{\circ} \mathrm{C}$ in a near-surface aqueous

environment”, PNAS | October 11, 2011 | vol. 108 | no. 41 | 16899

Jannis Lübbe, Matthias Temmen, Holger Schnieder, and Michael Reichling. "Measurement and Modelling of Non-Contact Atomic Force Microscope Cantilever Properties from Ultra-High Vacuum to Normal Pressure Conditions." Measurement Science and Technology 22, no. 5 (2011).

Jansson, Peter A. Deconvolution of Images and Spectra. San Diego: Academic Press, 1997.

Kanaya, Koichi, and Susumu Ono. "Secondary Electron Emission from Solid Surfaces by Bombardment with Charged Particles." Jpn. J. Appl. Phys. Japanese Journal of Applied Physics 13, no. 6 (1974): 944-49.

Khaled Karrai, Robert D. Grober, "Piezoelectric tip-sample distance control for near field optical microscopes”, 1942 Appl. Phys. Lett. 66 (14), 3 April 1995

Khaled Karrai, Robert D. Grober, "Piezo-electric tuning fork tip-sample distance control for near field optical microscopes”, Ultramicroscopy 61 (1995) 197-205

Khursheed, Anjam. Scanning Electron Microscope Optics And Spectrometers. World Scientific, 2011.

Lawrence Livermore National Laboratory, United States, Department of Energy, United States, Department of Energy, and Office of Scientific and Technical Information. Phase Separation of Lipid Membranes Analyzed with High-Resolution Secondary-Ion Mass Spectrometry. Washington, D.C: United States. Dept. of Energy, 2009. http://www.osti.gov/servlets/purl/973328-jjrQQb/.

Li, Jing, Yan-sheng Yin, Rui-xia Shi, Lai-peng Ma, and Jian Li. "Microstructure and Mechanical Properties of Al2O3-TiC-4vol.\%Co Composites Prepared from Cobalt Coated Powders." Surface and Coatings Technology 200, no. 12-13 (March 2006): 3705-12. doi:10.1016/j.surfcoat.2005.02.165.

Jinquan Liu, Andrea Callegari, Marting Stark, Majed Chergui, “A simple and accurate method for calibrating the oscillation amplitude of tuning-fork based AFM sensors", UltraMicroscopy 109 (2008) 81-84

Liu, Jinquan, Andrea Callegari, Martin Stark, and Majed Chergui. “A Simple and Accurate Method for Calibrating the Oscillation Amplitude of Tuning-Fork Based AFM Sensors." Ultramicroscopy. 109, no. 1 (2009): 81. 
Liu, N., M. Shi, Y.D. Xu, X.Q. You, P.P. Ren, and J.P. Feng. "Effect of Starting Powders Size on the Al2O3-TiC Composites." International Journal of Refractory Metals and Hard Materials 22, no. 6 (November 2004): 265-69. doi:10.1016/j.ijrmhm.2004.09.001.

Jannis Lübbe, Matthias Temmen, Holger Schnieder, and Michael Reichling. "Measurement and Modelling of Non-Contact Atomic Force Microscope Cantilever Properties from Ultra-High Vacuum to Normal Pressure Conditions." Measurement Science and Technology 22, no. 5 (2011).

Morawski, Ireneusz, Józef Blicharski, and Bert Voigtländer. "Voltage Preamplifier for Extensional Quartz Sensors Used in Scanning Force Microscopy." Review of Scientific Instruments 82, no. 6 (June 2011): 063701. doi:10.1063/1.3594103.

National Instruments Manual, NI ELVIS II Series User. "NI Educational Laboratory Virtual Instrumentation Suite II Series (NI ELVISTM II Series) User Manual." Accessed February 27, 2017. http://www.ee.bgu.ac.il/ adcirlab/Experiments/book/08_Labview/ELVIS\%20User\%20M anual.pdf.

Novotny, Lukas, and Bert Hecht. Principles of Nano-Optics. Cambridge: Cambridge University Press, 2006.

Nowak, Derek Brant. The Design of a Novel Tip Enhanced near-Field Scanning Probe Microscope for Ultra-High Resolution Optical Imaging, 2010. http://adsabs.harvard.edu/abs/2010PhDT.........38N.

Ohya, K, K Inai, H Kuwada, T Hayashi, and M Saito. "Dynamic Simulation of Secondary Electron Emission and Charging up of an Insulating Material." Surface \& Coatings Technology. 202, no. 22 (2008): 5310.

Ohya, K, and T Ishitani. "Simulation Study of Secondary Electron Images in Scanning Ion Microscopy." Nuclear Instruments and Methods in Physics Research Section B: Beam Interactions with Materials and Atoms Nuclear Instruments and Methods in Physics Research Section B: Beam Interactions with Materials and Atoms 202 (2003): $305-11$.

Ohya, K., T. Yamanaka, K. Inai, and T. Ishitani. "Comparison of Secondary Electron Emission in Helium Ion Microscope with Gallium Ion and Electron Microscopes." Nuclear Instruments and Methods in Physics Research Section B: Beam Interactions with Materials and Atoms 267, no. 4 (February 2009): 584-89.

doi:10.1016/j.nimb.2008.11.003.

Ohya, K. "Monte Carlo Simulation of Heavy Ion Induced Kinetic Electron Emission from an Al Surface." Nuclear Instruments \& Methods in Physics Research. Section B, Beam Interactions with Materials and Atoms. 195, no. 3 (2002): 281. 
Ohya, K. "Comparative Study of Target Atomic Number Dependence of Ion Induced and Electron Induced Secondary Electron Emission." Nuclear Instruments \& Methods in Physics Research. Section B, Beam Interactions with Materials and Atoms. 206, no. 1 (2003): 52.

Ohya, Kaoru, and Tohru Ishitani. "Monte Carlo Study of Secondary Electron Emission from SiO2 Induced by Focused Gallium Ion Beams." Applied Surface Science 237, no. 1-4 (October 2004): 602-6. doi:10.1016/j.apsusc.2004.06.133.

Oregon Micro Systems, Inc., 'User's Manual Intelligent Motor Controllers PC38 Family", 1989, 1990, 1996, 1997, 1999 Oregon Micro Systems, Inc., A Pro-Dex Company.

Orloff, Jon, L W Swanson, and M Utlaut. "Fundamental Limits to Imaging Resolution for Focused Ion Beams." Journal of Vacuum Science \& Technology. B, Microelectronics Processing and Phenomena 14, no. 6 (1996): 3759.

Orloff, Jon. Handbook of Charged Particle Optics. Boca Raton, Fla.: CRC Press, 1997.

Osterhout, Jeffrey T. "Diversity of Microfossils and Preservation of Thermally Altered Stromatolites from Anomalous Precambrian Paleoenvironments.” University of Cincinnati, 2016. http://rave.ohiolink.edu/etdc/view?acc_num=ucin1470753351.

Russo, Michael F, Mostafa Maazouz, Lucille A Giannuzzi, Clive Chandler, M Utlaut, and Barbara J Garrison. "Trench Formation and Lateral Damage Induced by Gallium Milling of Silicon.” Applied Surface Science. 255, no. 4 (2009): 828.

K Saitoh, K Hayashi, Y Shibayama, and K Shirahama. "A Low Temperature Scanning Probe Microscope Using a Quartz Tuning Fork." Journal of Physics: Conference Series 150 , no. 1 (2009).

Sakai, Y., T. Yamada, T. Suzuki, and T. Ichinokawa. "Contrast Mechanisms of Secondary Electron Images in Scanning Electron and Ion Microscopy." Applied Surface Science 144 (1999): 96-100.

Sakai, Y, T Yamada, T Suzuki, T Sato, H Itoh, and T Ichinokawa. "Contrast Mechanisms in Scanning Ion Microscope Imaging for Metals.” APPLIED PHYSICS LETTERS 73, no. 5 (1998): 611-13.

Sanguinetti, B, and B.T.H Varcoe. "Use of a Piezoelectric SQUIGGLE ${ }^{\circledR}$ Motor for Positioning at $6 \mathrm{~K}$ in a Cryostat.” JCRY Cryogenics 46, no. 9 (2006): 694-96.

J. William Schopf, Anatoliy B. Kudryavtsevb, Andrew D. Czaja, Abhishek B. Tripathi, "Evidence of Archean life: Stromatolites and microfossils", Precambrian Research 158 
Schopf, J. William, and Anatoliy B. Kudryavtsev. "Biogenicity of Earth's Earliest Fossils: A Resolution of the Controversy." Gondwana Research 22, no. 3-4 (November 2012): 761-71. doi:10.1016/j.gr.2012.07.003.

Serway, Raymond A. Physics for Scientists and Engineers. Philadelphia [etc.: Saunders College Publishing, 1992.

Shrotriya, Archana. "Writing Win32 Dynamic Link Libraries (DLLs) and Calling Them from LabVIEW." NI LabVIEW User's Manual, 1999.

http://citeseerx.ist.psu.edu/viewdoc/download?doi=10.1.1.452.8789\&rep=rep1\&type=pdf

Sigmund, Peter, and Andreas Schinner. "Binary Theory of Electronic Stopping." Nuclear Instruments and Methods in Physics Research Section B: Beam Interactions with Materials and Atoms 195, no. 1 (2002): 64-90.

Sigmund, Peter. "Stopping Power in Perspective." Nuclear Instruments and Methods in Physics Research Section B: Beam Interactions with Materials and Atoms 135, no. 1 (1998): 1-15.

Silberman, Donn M. "Dynamic Alignment, Tolerances, and Metrology Fundamentals at the Nano and Micro Scales." In SPIE Optical Engineering+ Applications, 95820B95820B. International Society for Optics and Photonics, 2015. http://proceedings.spiedigitallibrary.org/proceeding.aspx?articleid=2436526.

Smith, Roger. Atomic and Ion Collisions in Solids and at Surfaces: Theory, Simulation and Applications. Cambridge, U.K.; New York, NY, USA: Cambridge University Press, 1997.

Sourty, E., J. L. Sullivan, and M. D. Bijker. "The Tribology of Advanced Digital Recording (ADR) Systems.” Tribology International 33, no. 9 (2000): 629-637.

Steele, Andrew, David T. Goddard, Dave Stapleton, Vanessa Peters, Virginia Bassinger, George Sharples, David d Wynn-Williams, and others. "Investigations into an Unknown Organism on the Martian Meteorite Allan Hills 84001." Meteoritics \& Planetary Science 35, no. 2 (2000): 237-241.

Thomas STEPHAN, Elmar K. JESSBERGER, Christian H. HEISS, and Detlef ROST, "TOF-SIMS analysis of polycyclic aromatic hydrocarbons in Allan Hills 84001", Meteoritics \& Planetary Science 38, Nr 1, 109-116 (2003)

Sternglass, E. J. "Theory of Secondary Electron Emission by High-Speed Ions." Phys. Rev. Physical Review 108, no. 1 (1957): 1-12. 
Tambade, Popat S. "Harmonic Oscillator Wave Functions and Probability Density Plots Using Spreadsheets." Latin-American Journal of Physics Education 5, no. 1 (2011): 7.

Ternes, Markus, Christopher P. Lutz, Cyrus F. Hirjibehedin, Franz J. Giessibl, and Andreas J. Heinrich. "The Force Needed to Move an Atom on a Surface." Science 319, no. 5866 (2008): 1066-1069.

K.L. Thomas-Keprta, S.J. Clemett, D.S. McKay b, E.K. Gibson, S.J. Wentworth, "Origins of magnetite nanocrystals in Martian meteorite ALH84001", Geochimica et Cosmochimica Acta 73 (2009) 6631-6677

Toporski, J. K. W., A. Steele, F. Westall, R. Avci, D. M. Martill, and D. S. McKay. "Morphologic and Spectral Investigation of Exceptionally Well-Preserved Bacterial Biofilms from the Oligocene Enspel Formation, Germany." Geochimica et Cosmochimica Acta 66, no. 10 (2002): 1773-1791.

Torbrugge S, Lubbe J, Troger L, Cranney M, Reichling M, Eguchi T, and Hasegawa Y. "Improvement of a Dynamic Scanning Force Microscope for Highest Resolution Imaging in Ultrahigh Vacuum." Rev. Sci. Instrum. Review of Scientific Instruments 79, no. 8 (2008).

Tsipinyuk, Ba, and Vi Veksler. "Energy Spectra of Secondary Ions and Secondary Ion Emission (SIE) Mechanisms.” Vacuum 29, no. 4-5 (January 1979): 155-67. doi:10.1016/S0042-207X(79)80747-2.

Volkert, Cynthia A., and Andrew M. Minor. "Focused Ion Beam Microscopy and Micromachining." MRS Bulletin 32, no. 05 (2007): 389-399.

Werner, H. W., and P. R. Boudewijn. "A Comparison of SIMS with Other Techniques Based on Ion-Beam Solid Interactions." Vacuum 34, no. 1-2 (1984): 83-101.

West, Paul, and Natilia Starostina. "A Guide to AFM Image Artifacts." Pacific Nanotechnology, Inc. 3350 Scott Blvd \#29 Santa Clara, CA 95054-3105, n.d.

Whitby, James A., Fredrik Östlund, Peter Horvath, Mihai Gabureac, Jessica L. Riesterer, Ivo Utke, Markus Hohl, et al. "High Spatial Resolution Time-of-Flight Secondary Ion Mass Spectrometry for the Masses: A Novel Orthogonal ToF FIB-SIMS Instrument with In Situ AFM." Advances in Materials Science and Engineering 2012 (2012): 1-13. doi:10.1155/2012/180437.

White, Lauren M., Everett K. Gibson, Kathie L. Thomas-Keprta, Simon J. Clemett, and David S. McKay. "Putative Indigenous Carbon-Bearing Alteration Features in Martian Meteorite Yamato 000593.” Astrobiology 14, no. 2 (February 2014): 170-81.

doi:10.1089/ast.2011.0733. 
Whitehouse, D. J. "Nanometrology." Contemporary Physics 49, no. 5 (September 2008): 351-74. doi:10.1080/00107510802611251.

Wirtz, Tom, Yves Fleming, Mathieu Gerard, Urs Gysin, Thilo Glatzel, Ernst Meyer, Urs Wegmann, Urs Maier, Aitziber Herrero Odriozola, and Daniel Uehli. "Design and Performance of a Combined Secondary Ion Mass Spectrometry-Scanning Probe Microscopy Instrument for High Sensitivity and High-Resolution Elemental ThreeDimensional Analysis." Review of Scientific Instruments Review of Scientific Instruments 83, no. 6 (2012): 063702.

Yamamoto, Takayuki, Yoshiharu Kasamatsu, and Hiroyuki Hyodo. "Advanced StictionFree Slider and DLC Overcoat." Fujitsu Scientific and Technical Journal 37, no. 2 (2001): 201-11.

Yi, Whikun, Taewon Jeong, Segi Yu, Jeonghee Lee, Sunghwan Jin, Jungna Heo, and J M Kim. "Study of the Secondary-Electron Emission from Thermally Grown SiO2 Films on Si.” Thin Solid Films. 397, no. 1 (2001): 170.

Young, Warren C, Richard G Budynas, and Ali M Sadegh. Roark's Formulas for Stress and Strain. New York: McGraw-Hill, 2012.

Zhang, Yanfeng, Lianjun Wang, Wan Jiang, Guangzhao Bai, and Lidong Chen. "Effect of Fabrication Method on Microstructure and Properties of Al2O3-TiC Composites." Materials Transactions 46, no. 9 (2005): 2015-2019.

Ziegler, J. F, J. P Biersack, and Matthias D Ziegler. SRIM, the Stopping and Range of Ions in Matter. Chester, Maryland: SRIM Co., 2008. 
Appendix A: Miscellaneous software

Machine control:

- $\mathrm{xP} 2.25$

- Stage controller app, written in C++ using MSVS 2008

- Picoammeter app, written in C++ using MSVS 2008

- $\quad$ autolib control application, written in C++ using MSVS 2008

- National Instruments Labview 2010 SP1, including RIO for FPGA development

- NewScale Pathway V2.8.4 for Squiggle motor control

Analysis:

- Gwyddion 2.43

- ImageMotionRemover.exe, written in C++ using MSVS 2008

Productivity:

- $\quad$ MS Windows 3.1, nT 4.0, XP SP3

- MS Office 2010

- MS Paint

- Paint.Net

- Paint Shop Pro versions 8.0, X3 and X5

- MS Visual Studio versions 1998, 2005, 2008, 2010, 2015

- Depends.exe

- Bulk Rename Utility Version 2.7.1.2

- Notepad++ Version 7.2.2

- VirtualCloneDrive Version 5.4.5.0

- Drive Image XML for system backups

- Doodle Poll for meeting coordination

- Data Thief for acquiring data points from scanned graphs

- TurboCAD versions 17,19 


\section{A.1 Auto focus code listing}

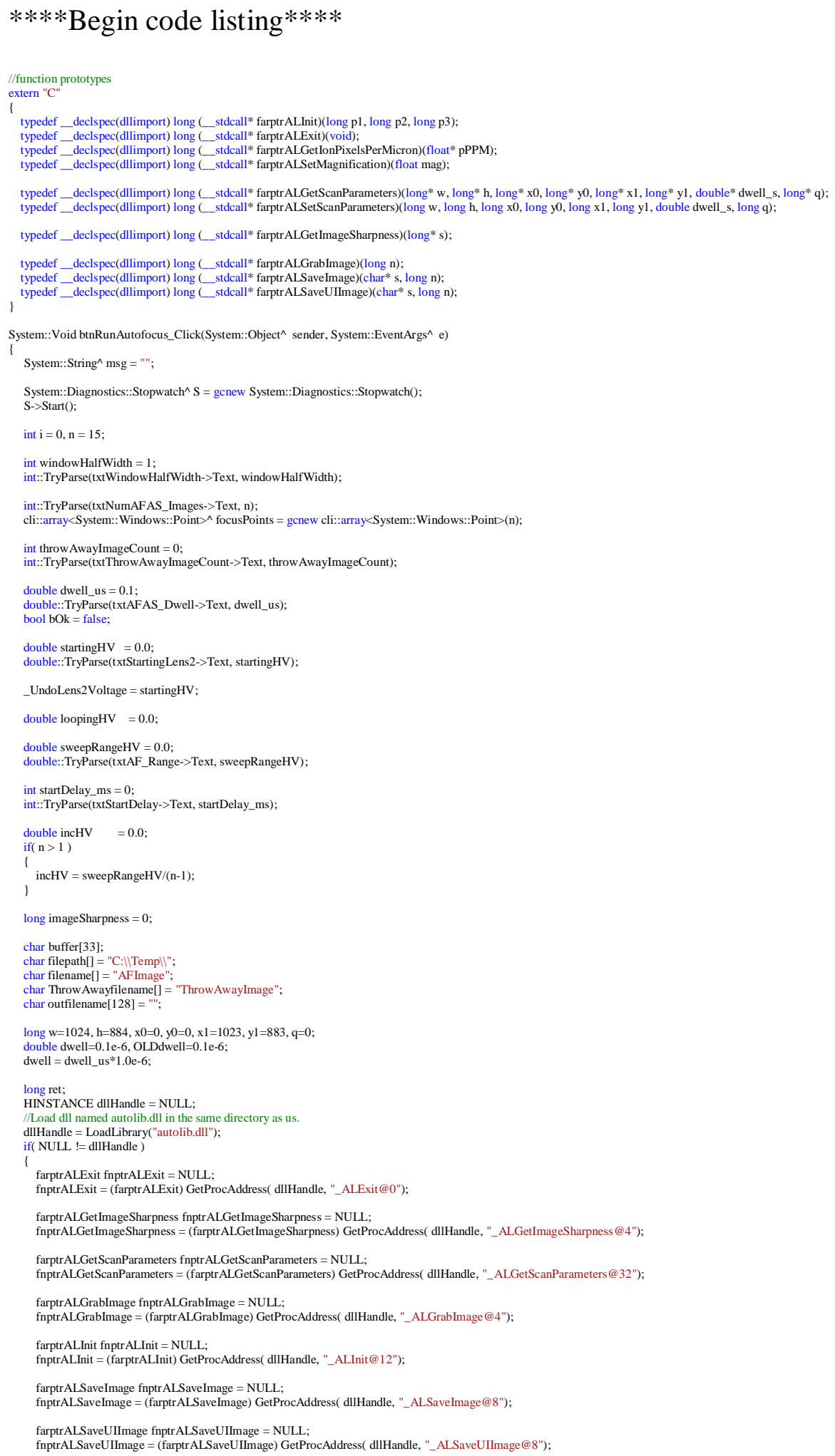




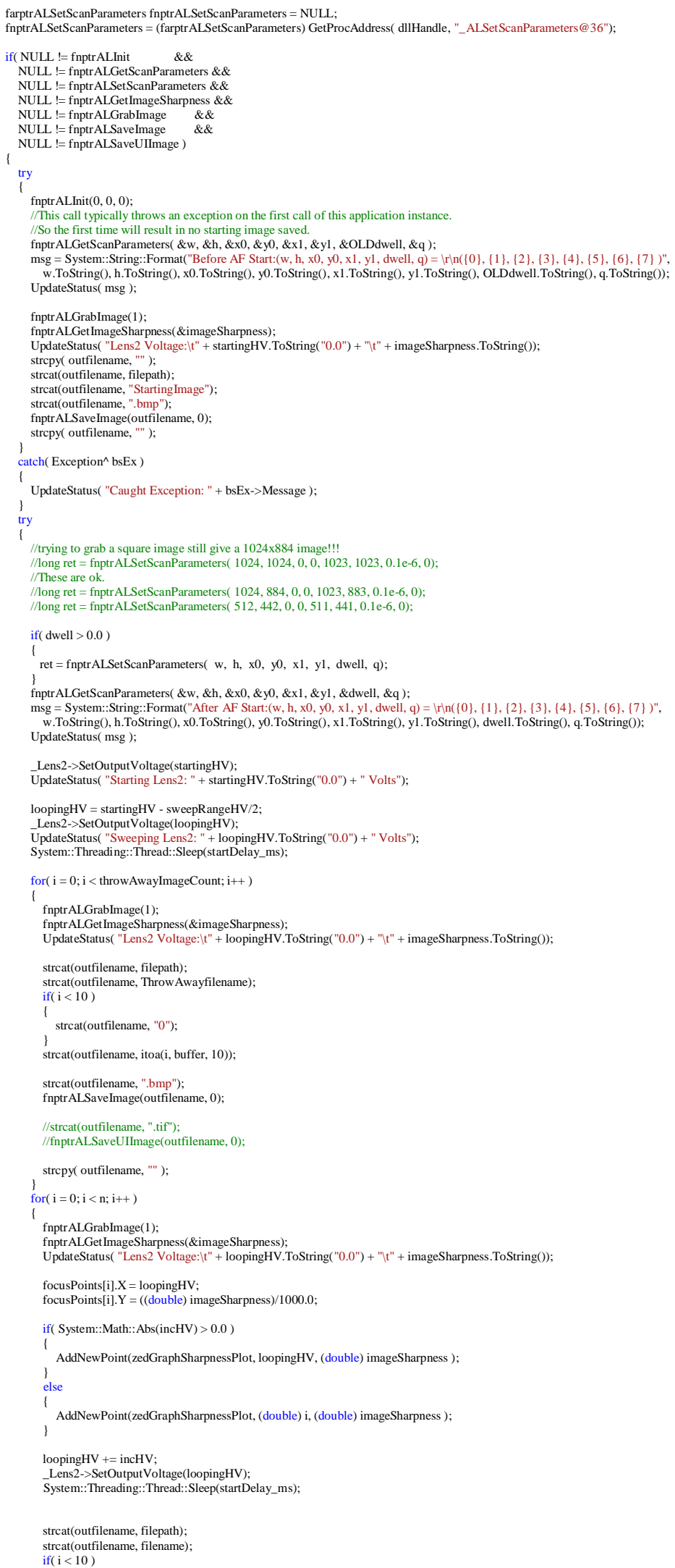




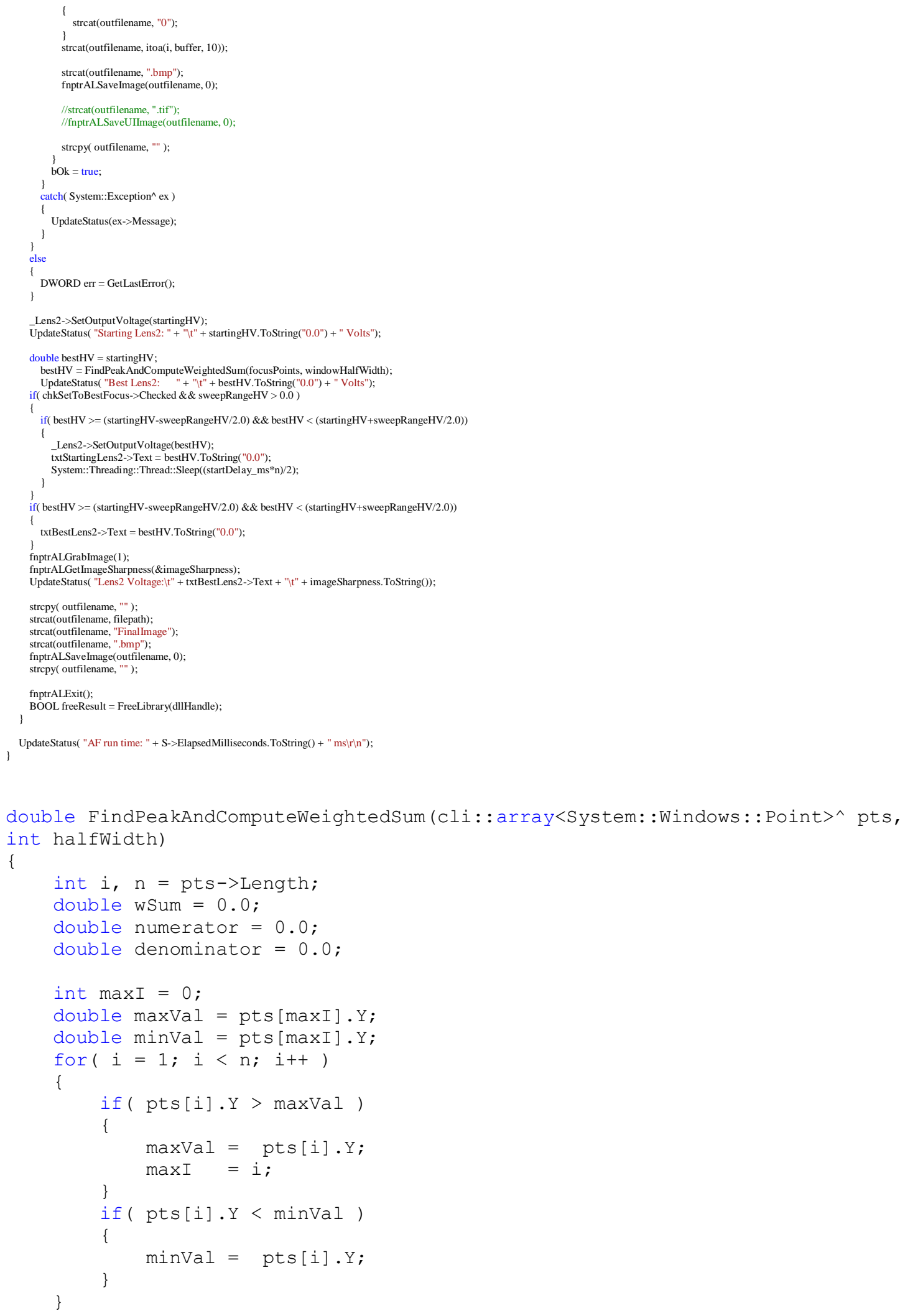




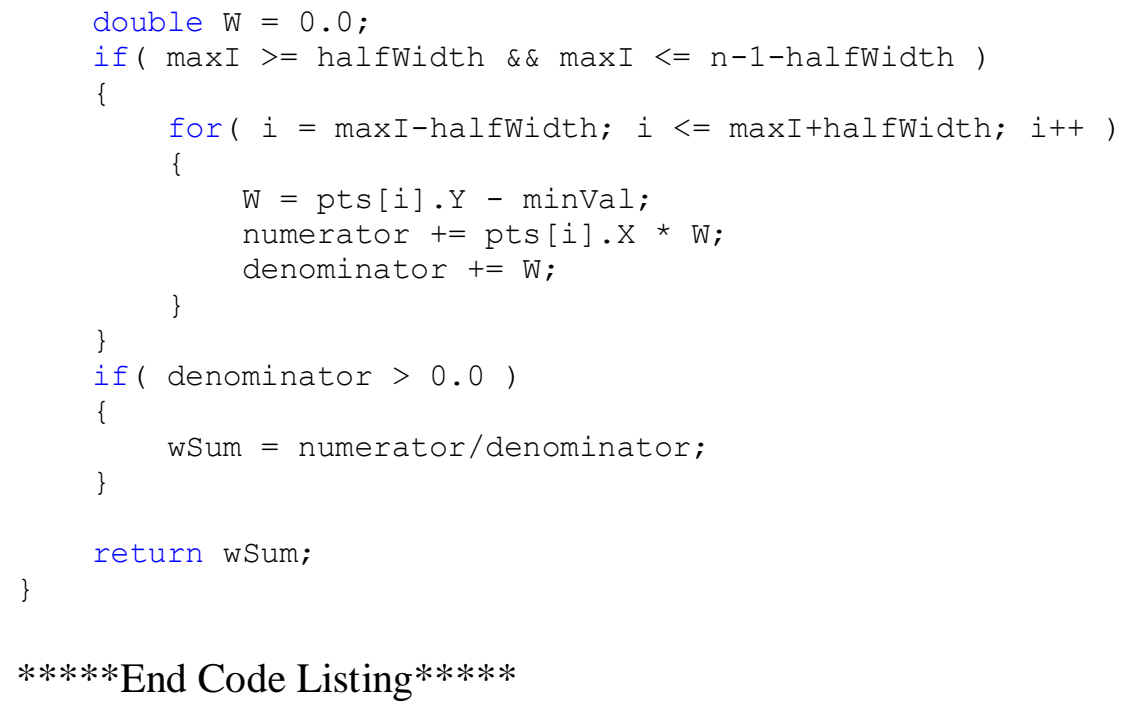

The output from the function above is displayed on the "Results" tab. It looks like this:

(Text in green is not a part of the actual output)

******B Begin Text Output to UI******

Before AF Start:(w, h, x0, y0, x1, y1, dwell, q) = $(512,442,0,0,512,442,4 \mathrm{E}-07,0)$

Lens2 Voltage: $\quad 13001.1 \quad 180947$

After AF Start:(w, h, x0, y0, x1, y1, dwell, q) = $(512,442,0,0,512,442,4 \mathrm{E}-07,0)$

Starting Lens2: 13001.1 Volts

Sweeping Lens2: 12951.1 Volts Sharpness

Lens2 Voltage: $\quad 12951.1 \quad 47309$

Lens2 Voltage: $\quad 12951.1 \quad 39563$

Lens2 Voltage: $\quad 12951.1 \quad 36673$

Lens2 Voltage: $\quad 12951.1 \quad 34185$

Lens2 Voltage: $\quad 12961.1 \quad 36607$

Lens2 Voltage: $\quad 12971.1 \quad 48956$

Lens2 Voltage: $\quad 12981.1 \quad 70803$

Lens2 Voltage: $\quad 12991.1 \quad 101821$

Lens2 Voltage: $\quad 13001.1 \quad 121678$

Lens2 Voltage: $\quad 13011.1 \quad 110030$

Lens2 Voltage: $\quad 13021.1 \quad 80464$

Lens2 Voltage: $\quad 13031.1 \quad 55705$

Lens2 Voltage: $\quad 13041.1 \quad 38295$

Lens2 Voltage: $\quad 13051.1 \quad 28517$

Starting Lens2: $\quad$ 13001.1 Volts

Best Lens2: $\quad 13001.9$ Volts

Lens2 Voltage: $\quad 13001.9 \quad 122285$

AF run time: $32904 \mathrm{~ms}$

$\leftarrow$ Throwaway image \#0

$\leftarrow$ Throwaway image \#1

$\leftarrow$ Throwaway image \#2 
In all, 16 images are acquired for an 11-image auto focus. The start image is taken at the starting focus Voltage. Then three throwaway images are acquired at the focus Voltage corresponding to the beginning sweep defocus. The auto focus images are acquired at incrementally increasing focus values until the sweep finishes. The best focus is determined and the focus is set to this optimal value and the final image is acquired.

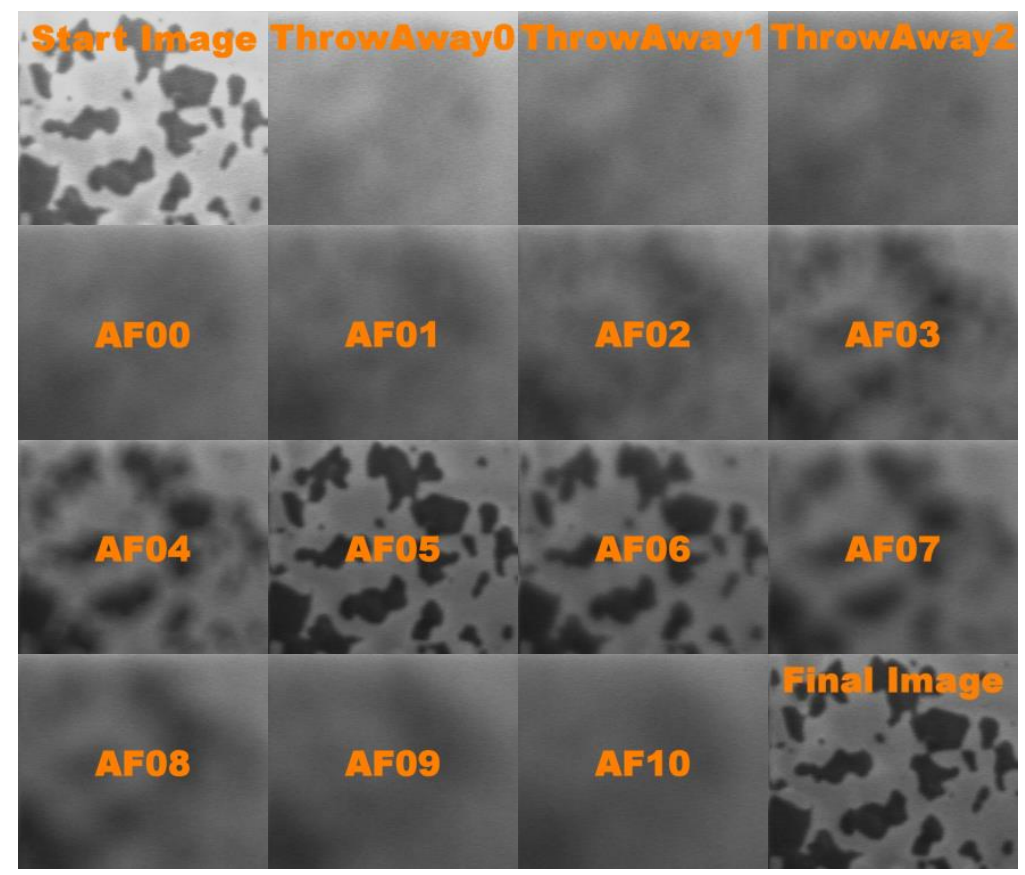

Figure A.1. Illustration of an 11-image auto focus from top to bottom and left to right. The three throwaway images clearly show a trend of decreasing mean value even though they are acquired at the same focus value. 
The result is displayed in this UI screen shot

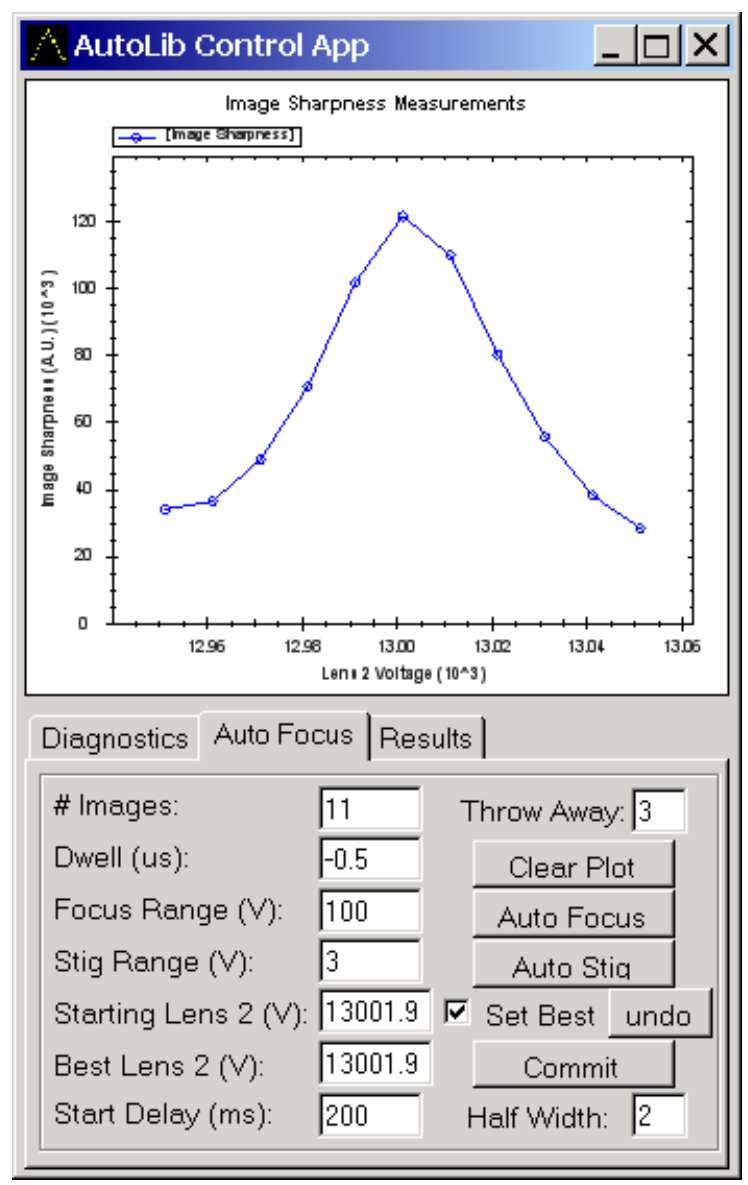

Figure A.2. Auto focus screenshot showing 3 Throw Away images, 11 sweep images. Focus range is 100 Volts. The negative value for Dwell (us) tells it to use the same dwell as xP. 


\section{A.2 Autolib depends.exe listing}

??0CRegEdit@@QAE@ABV0@ @Z

??0CUI1280AppRegistry@@QAE@ABV0@@Z

??0CUI1280UsrRegistry@@QAE@ABV0@@Z

??0CUI1280UsrRegistry@@QAE@XZ

??0CUsrRegistry@@QAE@ABV0@@Z

??0CUsrRegistry@@QAE@VCString@@@Z

??0CUsrRegistry@@QAE@XZ

??1CRegEdit@@UAE@XZ

??1CUI1280AppRegistry@@UAE@XZ

??1CUI1280UsrRegistry@@UAE@XZ

??1CUsrRegistry@@UAE@XZ

??4CRegEdit@@QAEAAV0@ABV0@@Z

??4CUI1280AppRegistry@@QAEAAV0@ABV0@@Z

??4CUI1280UsrRegistry@@QAEAAV0@ABV0@@Z

??4CUsrRegistry@@QAEAAV0@ABV0@@Z

??_7CRegEdit@@6B@

??_7CUI1280AppRegistry@@6B@

??_7CUI1280UsrRegistry@@6B@

??_7CUsrRegistry@@6B@

??_C@_03MIAH@Exe?\$AA@

??_C@_04GNHP@Help?\$AA@

??_C@_06EMFJ@Images?\$AA@

??C@07CMIM@Acctlog?\$AA@

??_C@_07NNN@Pattern?\$AA@

??_C@_0L@DIBM@StageFiles?\$AA@

??_C@_0L@GEAH@CNeutFiles?\$AA@

??_C@_1EA@KKKL@?\$AAS?\$AAO?AAF?\$AAT?\$AAW?\$AAA?\$AAR?\$AAE?\$AA?2?\$AAM?\$A

AI?\$AAC? \$AAR?\$AAO?AAS?AAC? \$AAO?AAP? \$AAE? \$AA?2@

?GetAcctlogDir@CUI1280AppRegistry@@QAEKAAVCString@@@Z

?GetCNeutFilesDir@CUI1280AppRegistry@@QAEKAAVCString@@@Z

?GetExeDir@CUI1280AppRegistry@@QAEKAAVCString@@@Z

?GetHelpDir@CUI1280AppRegistry@ @ QAEKAAVCString@@ @Z

?GetImageDir@CUI1280AppRegistry@@QAEKAAVCString@@@Z

?GetPatternDir@CUI1280AppRegistry@@QAEKAAVCString@@@Z

?GetStageFilesDir@CUI1280AppRegistry@@QAEKAAVCString@@@Z

?SetAcctlogDir@CUI1280AppRegistry@@QAEKVCString@@@Z

?SetCNeutFilesDir@CUI1280AppRegistry@@QAEKVCString@@@Z

?SetExeDir@CUI1280AppRegistry@@QAEKVCString@@@Z

?SetHelpDir@CUI1280AppRegistry@@QAEKVCString@@@Z

?SetImageDir@CUI1280AppRegistry@@QAEKVCString@@@Z

?SetPatternDir@CUI1280AppRegistry@@QAEKVCString@@ @Z

?SetStageFilesDir@CUI1280AppRegistry@@QAEKVCString@@@Z

_ALAddLogEntry@4

_ALAddLogEntryNoLF@4

_ALAutoCB@0

_ALAutoFocus@0

_ALAutoStig@0

_ALCNGetAutoMonitor@12

_ALCNGetBeamCurrent@4

_ALCNGetBeamEnergyRange@8
_ALCNGetBeamEnergyVoltageSetting@4

_ALCNGetBlanking@4

_ALCNGetDetectorMode@4

_ALCNGetDetectorType@4

_ALCNGetEnergy@4

_ALCNGetFilament@4

_ALCNGetFilamentRange@8

_ALCNGetFilamentRangeService@8 
_ALCNGetFilamentVoltageSetting@4

_ALCNGetGridOffSet@ 4

_ALCNGetGridRange@8

_ALCNGetGridVoltage@4

_ALCNGetGridVoltageSetting@4

_ALCNGetLastError@8

_ALCNGetState@4

_ALCNGetVacMode@4

_ALCNHWVersionString@4

_ALCNSetAutoMonitor@20

_ALCNSetBeamEnergyVoltageSetting@8

_ALCNSetBlanking@4

_ALCNSetEnergy@8

_ALCNSetFilament@8

_ALCNSetFilamentService@8

_ALCNSetFilamentVoltageSetting@8

_ALCNSetGridOffSet@4

_ALCNSetGridVoltage@8

_ALCNSetGridVoltageSetting@8

_ALCNSetState@4

_ALCheckStageReadyByTimeout@0

_ALClearLaserAlign@0

_ALCreateDummy@4

_ALCreatePattern@72

_ALDbgStartTimer@0

_ALDbgStopTimer@0

_ALDeGauss@0

_ALDeleteAllPatterns@0

_ALDeletePattern@4

_ALDimButtons@0

_ALDoMatch@68

_ALExit@0

_ALFinishLaserAlign@0

_ALGetAVA@8

_ALGetActiveImage@4

_ALGetAlignAngleSnor@8

_ALGetAlignBeamShift@8

_ALGetAlignFocus@0

_ALGetAlignLens1@0

_ALGetAlignStig@0

_ALGetAperture@4

_ALGetApertureInfo@16

_ALGetBeamCurrent@4

_ALGetBeamRotation@4

_ALGetBeamShift@8

_ALGetBrightness@4

_ALGetCapProbeEnabled@4

_ALGetChamberPressure@4

_ALGetCircleCenter@12

_ALGetColumnPressure@4

_ALGetContrast@4

_ALGetControlBeam@4

_ALGetCosSinScanRot@8

_ALGetDacSnorA@4
_ALGetDeflDistY@4

_ALGetDetBrightness@4

_ALGetDetContrast@4

_ALGetDetFrontEndVoltage@4

_ALGetDetGridVoltage@4

_ALGetDetMode@4

_ALGetEmission@4

_ALGetEnableJoystick@4

_ALGetEnableMUI@4

_ALGetErrorText@8

_ALGetEucentricOnOff@4

_ALGetEventLogText@16

_ALGetExtractor@4

_ALGetFocus@4

_ALGetGIS@8

_ALGetGISLifeTime@8

_ALGetHTState@4

_ALGetHV@4

_ALGetHistogram@4

_ALGetImageSharpness@4

_ALGetImagingState@4

_ALGetInnerRadius@8

_ALGetIonBeamUnBlankStatus@4

_ALGetIonPixelsPerMicron@4

_ALGetIsMilling@4

_ALGetLLStatus@4

_ALGetLaserCounts@8

_ALGetLaserEnabled@4

_ALGetLaserPos@20

_ALGetLens1@4

_ALGetLens2@4

_ALGetLifeTime@4

_ALGetMagnification@4

_ALGetMillCheck@8

_ALGetMillingState@4

_ALGetOuterRadius@8

_ALGetParallelMode@4

_ALGetPatternParams@96

_ALGetPatternRect@20

_ALGetPixelsPerMicron@4

_ALGetPrimaryBeam@4

_ALGetQuad@8

_ALGetRotation@8

_ALGetScanParameters@32

_ALGetScreenPixelsPerMicron@8

_ALGetScriptDialogText@8

_ALGetSelectDet@4

_ALGetStageCurrent@4

_ALGetStageTimeoutRetries@0

_ALGetStigmator@8

_ALGetSuppressor@4

_ALGetUUEnabled@4

_ALGetUUPoint@24

_ALGetUnBlankBeamCurrent@4 
_ALGetV21V22Status@8

_ALGetVISlope@4

_ALGetVacuumStatus@4

_ALGetVariable@8

_ALGetWorkDistEucentric@4

_ALGetWorkDistInt@4

_ALGetX@8

_ALGetY@8

_ALGetZ@8

_ALGrabImage@4

_ALHeightMapAddPoint@4

_ALHeightMapAddPointExplicit@44

_ALHeightMapClearMap@0

_ALHeightMapEnable@4

_ALHeightMapRemovePoint@8

_ALHeightMapUpdatePoint@8

_ALInit@12

_ALInputDialog@20

_ALInsertGIS@4

_ALIsOMIOptionConfigured@0

_ALIsStageHomed@4

_ALLaserBeamShiftAdjust@0

_ALLoadOmiImage@0

_ALLoadOmiImageFile@4

_ALLoadOmiProject@4

_ALLoadPatternFile@4

_ALLoadPatternMatlFile@8

_ALNextPattern@4

_ALOMICNLSearch@20

_ALOMIPMTool@20

_ALOMISetCoorCNLsModel@20

_ALOMISetCoorPMToolPattern@20

_ALOMITrainCNLSearch@4

_ALOMITrainPMTool@4

_ALOmiObjManShowDialog@4

_ALOpenImage@8

_ALPump@0

_ALQueryCapProbe@8

_ALQueryCapProbeNumber@8

_ALReadCapProbe@4

_ALReadCapProbeNumber@4

_ALReadDBFloat@8

_ALReadDBInt@8

_ALReadDBItem@8

_ALReadOmiOCR@20

_ALReadPatAlignment@16

_ALRegresionOneOptOutlier@40

_ALRetractGIS@4

_ALSaveImage@8

_ALSaveOmiProject@4

_ALSavePatternFile@4

_ALSaveUIImage@8

_ALScriptClear@4

_ALScriptRun@8
_ALScriptStop@0

_ALSelectDet@4

_ALSetAVA@8

_ALSetAlignAngleSnor@8

_ALSetAlignBeamShift@0

_ALSetAlignFocus@0

_ALSetAlignLens1@0

_ALSetAlignStig@0

_ALSetAperture@4

_ALSetBeamRotation@4

_ALSetBeamShift@8

_ALSetBrightness@4

_ALSetCircleCenter@20

_ALSetContrast@4

_ALSetControlBeam@4

_ALSetDeflFilters@4

_ALSetDetBrightness@4

_ALSetDetContrast@4

_ALSetDetFrontEndVoltage@8

_ALSetDetGridVoltage@8

_ALSetDetMode@4

_ALSetEnableJoystick@4

_ALSetEnableMUI@4

_ALSetEucentricOnOff@4

_ALSetExtractor@4

_ALSetFocus@4

_ALSetGraphicWnd@4

_ALSetHTState@4

_ALSetInnerRadius@12

_ALSetInstance@4

_ALSetLaserCounts@16

_ALSetLaserEnabled@4

_ALSetLaserPoint@36

_ALSetLens1@4

_ALSetLens2@4

_ALSetLightTowerState@4

_ALSetLog@4

_ALSetLogfile@8

_ALSetMagnification@4

_ALSetMatchWinMinimize@4

_ALSetMaterialFile@8

_ALSetMillCheck@8

_ALSetOuterRadius@12

_ALSetParallelMode@4

_ALSetParentWnd@4

_ALSetPatternParams@156

_ALSetPatternRect@36

_ALSetPrimaryBeam@4

_ALSetQuad@8

_ALSetRotation@12

_ALSetSaveImageId@4

_ALSetScanParameters@36

_ALSetStageTimeoutRetries@4

_ALSetStigmator@8 
_ALSetStreamDisplayPixels@4

_ALSetStringVariable@8

_ALSetSuppressor@4

_ALSetUUEnabled@4

_ALSetUUPoint@40

_ALSetVariable@12

_ALSetX@12

_ALSetY@12

_ALSetZ@12

_ALSigFigs@8

_ALStageGetPos@20

_ALStageGetPosEx@24

_ALStageMove@44

_ALStageMoveDelta@44

_ALStageMoveDeltaNoWait@44

_ALStageMoveEx@48

_ALStageMoveExNoWait@48

_ALStageMoveNoWait@44

_ALStagePosRefresh@4

_ALStageWaitEndMove@0

_ALStartImaging@4

_ALStartMilling@0

_ALStopImaging@4

_ALStopMilling@0

_ALTestMathErrs@0

_ALTimeOfDay@0

_ALUnSetLaserCounts@0

_ALUpdateLink@28

_ALVent@0

_ALWaferCancelOp@0

_ALWaferGetCassetteSlotStatus@8

_ALWaferGetCassetteStatus@12

_ALWaferHomeRobot@0

_ALWaferInventory@0

_ALWaferLoad@8

_ALWaferLoadCassetteToOCR@8

_ALWaferLoadOCRToTool@0

_ALWaferLoadPort@4

_ALWaferLoadlockLoad@4

_ALWaferLoadlockUnload@4

_ALWaferLockPort@4

_ALWaferRecover@12

_ALWaferStatus@48

_ALWaferTransfer@16

_ALWaferUnload@8

_ALWaferUnloadOCRToCassette@8

_ALWaferUnloadPort@4

_ALWaferUnlockPort@4

_ALWriteCapProbe@4

_ALWritePatAlignment@16

_ALeAutoEucentric@0

_ALeAutoStig@0

_ALeGetAperture@4

_ALeGetColMode@4
_ALeGetColumnPressure@8

_ALeGetEmission@4

_ALeGetHV@4

_ALeGetHVOnOff@4

_ALeGetModeMinMaxWD@12

_ALeGetSpot@4

_ALeGetWD@4

_ALeIsColModeAllowed@8

_ALeReadDBFloat@8

_ALeReadDBInt@8

_ALeReadDBItem@8

_ALeSetAperture@4

_ALeSetColMode@4

_ALeSetHV@4

_ALeSetHVOnOff@4

_ALeSetSpot@4

_ALeSetWD@4

_InternalScriptRun@4

_OpenDialog@8

_ScriptSleep@4 


\section{Appendix B: Samples of selected G-Code for CNC operation}

Most of the AFM fabrication and ancillary hardware were created using a CNC milling machine. This machine uses Linux $\mathrm{CNC}$, sometimes known to hardcore fanatics as emc2 or just "axis". Each piece of the AFM hardware was cut utilizing one or more g-code files. In general, tool changes and part orientation changes resulted in unique g-code files. For example, when making something simple like a plate with holes drilled in some defined pattern, one g-code file was used for pre-drilling the hole centers using a center drill and another was used to drill the holes using a drill bit. Another example is the fabrication of a block having same-sized holes drilled on its faces. Each unique face gets its own g-code file. As a final example, the coarse stage's center block serves as the central structural member and required 18 individual g-code files to construct it. The number of g-code files used for the complete AFM construction are too numerous to present here but there are a few which deserve mention.

The three categories of files discussed here are:

1) Making circles without looping

2) Looping for making d-sub connectors

3) Creating and calling functions using G-Code

Sometimes non-linear shapes are desirable for certain assemblies made using CNC. Computer Aided Machining (CAM) plug-ins are available for CAD packages. The TurboCAD package was used for this AFM construction and sometimes irregular shapes were needed such as the cutouts for the XY encoders in the middle block of the coarse $\mathrm{XY}$ tip positioning stage. The CAM plug-in generated many lines of code $(46 \mathrm{kB})$ which could only be practically modified by regenerating with the CAM plug-in. TurboCAD runs in Windows and so there is some disconnection in g-code generation and the machining operations. Even minor changes to the design can result in time-consuming gcode generation via CAM. In many cases, the geometric shapes are simple enough to program by hand and can instead be made directly on the CNC milling machine as needed.

\section{B.1 Making circles without looping}

Using a CAM plug-in to create g-code for circles will often lead to the generation of a long raw list of coordinates. While this if perfectly fine in a production scenario, amateurs may want more control of the evolution of their g-code libraries and so will want the simplest representations possible. The G2 and G3 commands are specifically designed for making circles and can be made to take multiple passes in doing so. 
***********Begin Code Listing $* * * * * * * * * *$

( Circles.txt )

( LinuxCNC 2014-03-07 )

( This program mills a circle $0.1 \mathrm{~mm}$ deep, taking two turns to do so. )

( The circle begins on the $\mathrm{X}$-axis )

( Millimeters, Absolute, Continuous, Cancel Cutter Radius Compensation )

G21 G90 G64 G40

G0 Z0.5

( engraving )

G17

( Spindle CW )

M3 S10000

( Make a $9 \mathrm{~mm}$ hole using as $6 \mathrm{~mm}$ end mill )

G0 $\mathrm{X}+3 \mathrm{Y}+0 \mathrm{Z}+0.5$ (begin arc on the $\mathrm{X}$-axis)

G0 Z+0.0

( Draw a circle )

$(\mathrm{X}+3 \mathrm{Y}+0 \mathrm{Z}-0.1$ is the end point)

( $\mathrm{I}-3 \mathrm{~J}+0$ is the relative center from the starting point)

(P2 means take two turns to make this cut)

(F150 is the feed rate in $\mathrm{mm} / \mathrm{min}$ )

G2 X+3 Y+0 Z-0.1 I-3 J+0 P2 F150 (finish at the same location we started)

G0 Z+0.5

M5 ( Stop Spindle )

M2 ( End Program )

***********End Code Listing $* * * * * * * * * *$

B.2 Looping for making d-sub connectors

***********Begin Code Listing $* * * * * * * * * *$

( DB9-Horizontal_Loop.txt )

( 2014-05-19)

( Use a 1/4" end mill )

( Origin is part center. ) 
( Mounting holes are 1/8" in diameter and are 1" apart )

( Mounting holes may be instead be tapped with \#4-40 threads )

( Inches, Absolute, Continuous, Cancel Cutter Radius Compensation )

G20 G90 G64 G40

( engraving )

G17

( Spindle CW )

M3 S10000

G1 Z0.020 F8.0

$\# 1=-0.01$ ( Loop counter initialized $)$

o100 do

G1 X+0.25 Y+0.07 Z\#1

G1 X-0.25 Y+0.07

G1 X-0.225 Y-0.07

$\mathrm{G} 1 \mathrm{X}+0.225 \mathrm{Y}-0.07$

$\# 1=[\# 1-0.01]$ (increment counter $)$

o100 while[\#1 GT -0.15]

G1 Z+0.020

M02

***********End Code Listing $* * * * * * * * * *$

\section{B.3 Creating and calling functions using G-Code}

The g-code specification allows for the creation of functions which can be called with parameters. The coding style sort of resembles BASIC and the parameters are passed in what resembles a stack. Parameters are referenced by their respective position on the stack, i.e. variable \#1 on the stack is simply called ""\#1" and so on. With this scheme it is important to comment the code in each subprogram so that it is clear how to use it at later times.

D-sub connectors are very common in electrical equipment so it is useful to have a library of functions for creating them.

************Begin Code Listing $* * * * * * * * * * *$ 
( DB9-Horizontal_SubProgram-Loop-PostRecess.txt )

( 2014-05-19)

( Use a $1 / 4 "$ end mill )

(Origin is part center.)

( Mounting holes are 1/8" in diameter and are 1" apart )

( Mounting holes may be drilled using a 0.110" drill for a tight fit )

( Mounting holes may be instead be tapped with \#4-40 threads )

( Inches, Absolute, Continuous, Cancel Cutter Radius Compensation )

G20 G90 G64 G40

( engraving )

G17

( Spindle CW )

M3 S10000

G1 Z0.020 F8.0

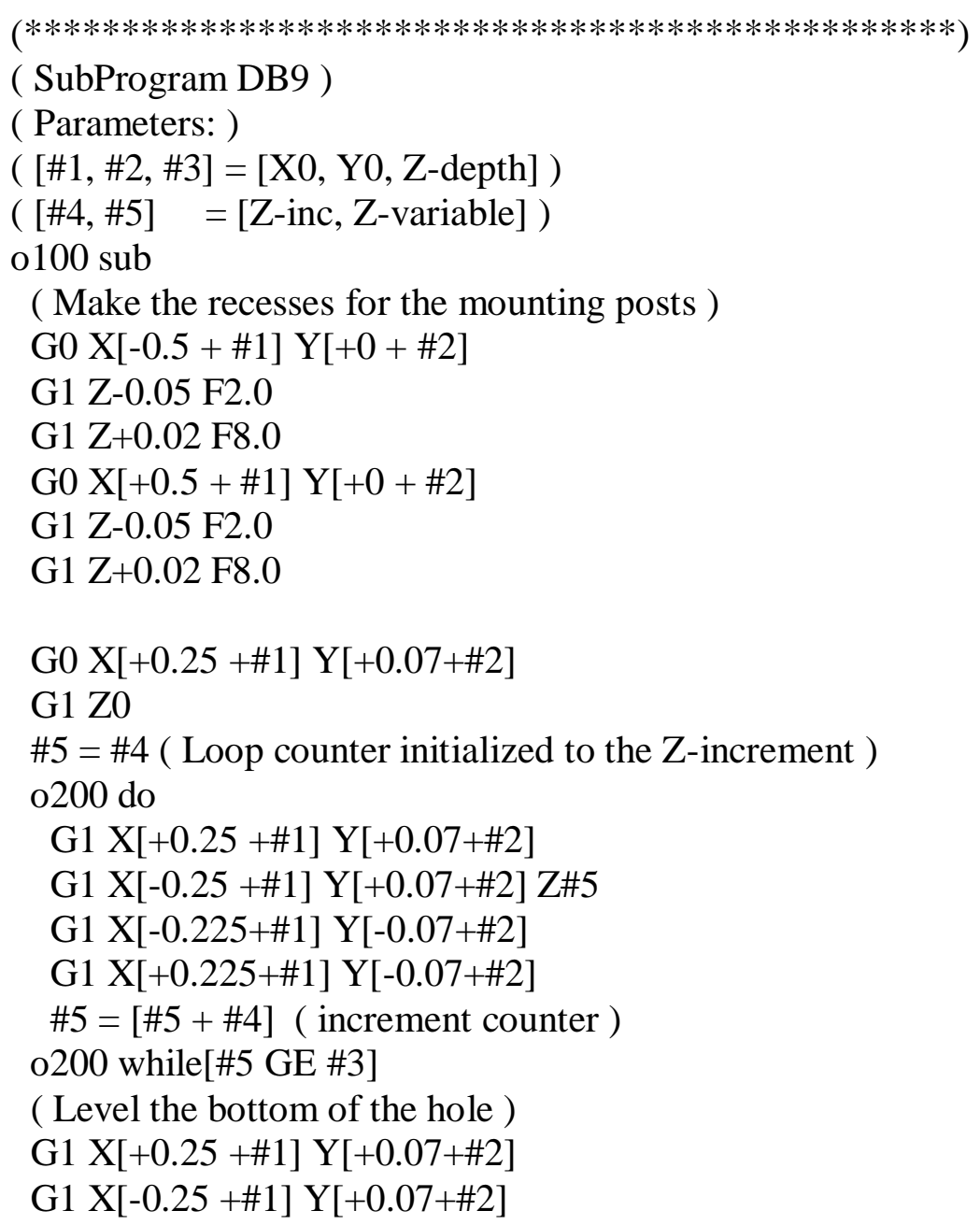


G1 Z+0.020

o100 endsub

( End of SubProgram DB9)

$(* * * * * * * * * * * * * * * * * * * * * * * * * * * * * * * * * * * * * * * * * * * * * * * *)$

$(* * * * * * * * * * * * * * * * * * * * * * * * * * * * * * * * * * * * * * * * * * * * * * * *)$

( SubProgram DB37 )

( Parameters: )

$([\# 1, \# 2, \# 3]=[\mathrm{X} 0, \mathrm{Y} 0, \mathrm{Z}-\mathrm{depth}])$

$([\# 4, \# 5]=[$ Z-inc, Z-variable $])$

o300 sub

(Make the recesses for the mounting posts )

G0 X[+0.0 + \#1] Y $[-1.25+\# 2]$

G1 Z-0.05 F2.0

G1 Z+0.02 F8.0

G0 X[+0.0 + \#1] Y $[+1.25+\# 2]$

G1 Z-0.05 F2.0

G1 Z+0.02 F8.0

$\# 5=\# 4$ ( Loop counter initialized to the Z-increment )

G0 X[-0.08 + \#1] Y $[+1.01+\# 2]$

G1 Z0

0400 do

$\mathrm{G} 1 \mathrm{X}[-0.08+\# 1] \mathrm{Y}[+1.01+\# 2]$

G1 X[-0.08+\#1] Y[-1.01 + \#2] Z\#5

G1 X[+0.08+\#1] Y[-0.987+\#2]

G1 X $[+0.08+\# 1] \mathrm{Y}[+0.987+\# 2]$

$\# 5=[\# 5+\# 4]$ (increment counter)

o400 while[\#5 GE \#3]

( Level the bottom of the hole )

G1 X[-0.08 + \#1] Y $[+1.010+\# 2]$

G1 X[-0.08 + \#1] Y[-1.010 + \#2]

G1 Z+0.020

o300 endsub

( End of SubProgram DB37)

$(* * * * * * * * * * * * * * * * * * * * * * * * * * * * * * * * * * * * * * * * * * * * * * * *)$

( RS232 Connectors )

( call [X0] [Y0] [depth] [depth increment] [NA] )

o100 call [-1.3125] [-1.625] [-0.11] [-0.01] [0]

o100 call [-1.3125] [-0.6875] [-0.11] [-0.01] [0]

o100 call [-1.3125] [+0.25] [-0.11] [-0.01] [0] 


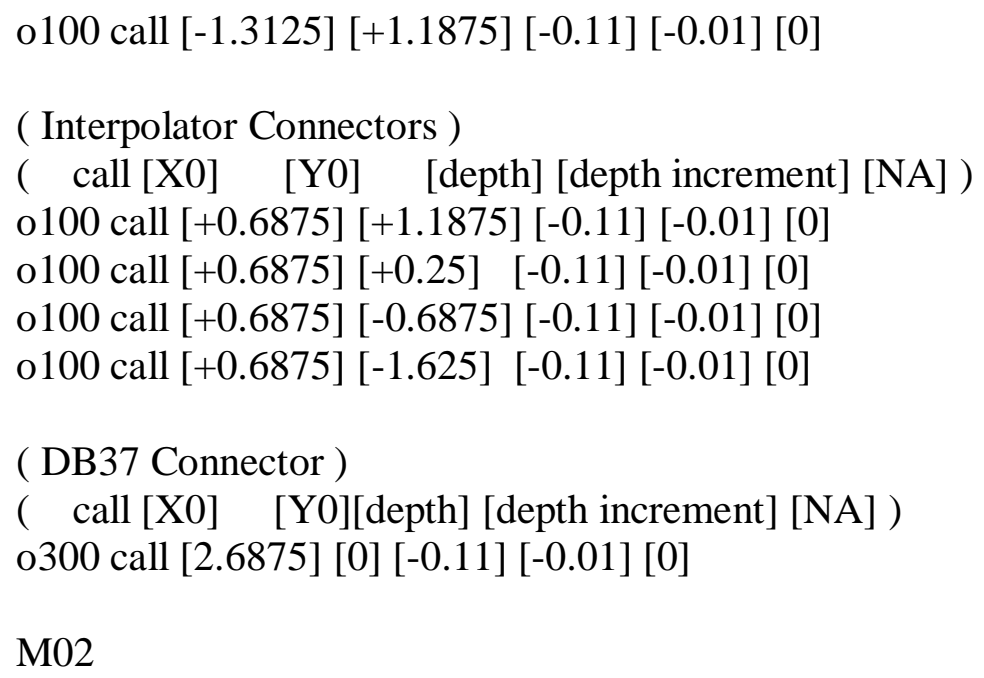

